



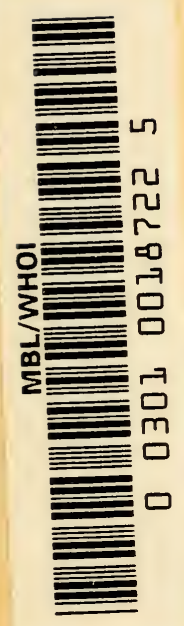





\section{STRUCTURE OF THE VERTEBRATES}





\section{Structure}

\section{of the Vertebrates}

MALCOLM E. LITTLE

Assistant Professor of Biology

New York University

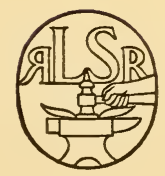

Ray Long \& Richard R. Smith, Inc.

New York

1932 
Copyright, May, 1932, by

Ray Long \& Richard R. Smith, Inc.

All rights reserved.

PRINTED IN THE UNITED STATES OF AMERICA

BY STRATFORD PRESS, INC., NEW YORK 
To the Memory of

George Otis Schoonhoven 



\section{ACKNOWLEDGMENTS}

FREE and unacknowledged use has been made of the many excellent texts and original papers on the subject. Few conclusions, even minor ones, have been contributed by a single worker; and all of the older results have now become common property, a part of our scientific heritage. Further, complete acknowledgment in the text would be confusing to more advanced students than those for whom this text is designed.

The author owes thanks to his colleagues who helped plan the course, and who assisted in re-shaping the outline and content of this book. I am particularly grateful to Dr. H. A. Charipper who read Parts I and II, and to Mr. R. T. Kempton who read the entire manuscript. Dr. H. E. Wood, 2nd., read Part III, and Mr. Everett Lyne the entire book.

The drawings for Chapter XV were made by Dr. Florence D. Wood. Mr. Anthony Barbanera illustrated Chapters VIII, XI, XII, XVII, and XVIII, and made some drawings for other chapters.

In the preparation of the manuscript for publication, and in reading the proofs, the author has had the assistance of Miss Lillian Fuellhart, Miss Charlotte Rowe, and Miss Katharine Twichell; and of his assistants, Robert H. Ringewald, Thomas G. Ierardi, James G. Meyers, and Herman Littman. Dr. John J. Schoonhoven edited the glossary.

Many of the drawings are from the publications of, or material in, the American Museum of Natural History. Several of the birds were copied from paintings; many of the natural history and skeletal drawings were made from specimens. The author is grateful for the permission to work at the Museum.

Although the manuscript was carefully edited by men who have had long experience in teaching anatomy, numerous minor errors have undoubtedly escaped their attention. Wherever there was a disagreement as to method of treatment or the inclusion of material it was necessary for the author to depend upon his own judgment. For any errors of fact, and for the general policies, the author takes entire responsibility. 



\section{PREFACE}

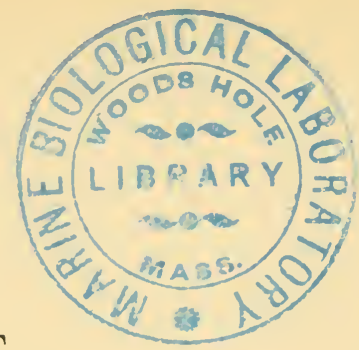

THIS text is designed primarily for use in a half-year course in Comparative Anatomy. To attain this end it was necessary to reduce the amount of detail that is included in the usual text and to arrange the material for flexibility. Additional material for those who desire it will be found in separate sections. The author makes no apology for the omission of anatomical minutiae. Such technicalities usually do not have wide application, and often so confuse the student that the central theme is lost.

An attempt has been made to simplify the descriptions of development from the generalized to the specialized, yet, at the same time, to emphasize the essentials of anatomy. For this reason enough natural history is included to make clear the comparison of structures and to show the evolutionary implications of anatomy.

The result of a desire for simplification and flexibility is a book in three parts, each to a certain extent independent of the others. Chapters are numbered consecutively, and each is divided into sections, so that the instructor may omit certain chapters or sections without destroying the continuity of the book.

Vertebrate Zoology, part i, considers the systematic relationships of the groups of vertebrates. A study of the comparative anatomy of organs (systemic anatomy) presupposes this knowledge of the vertebrates, for it is impossible to compare unknown entities. In addition, the author is sufficiently attached to the "Old School" to believe that a student, whether pre-professional or general, should know the major groups of animals as he knows the major periods of history.

Each vertebrate class is included in a separate chapter. At the beginning of each chapter is a general discussion, and this is followed by discussions of the more important orders. Those orders which have a direct bearing upon the evolution of man are in- 
cluded first; the others are added for those instructors who can give more time to natural history. At the end of each chapter is included a brief discussion of the embryology of the class, placed in a distinct section, so that it may be conveniently omitted. This omission will probably be desirable in curricula where a course in embryology follows comparative anatomy.

Comparative Morphology, part il, is a study of the comparative development and form of the organic systems. Reference is made to fossil evidence in places where it does not entail a knowledge of stratigraphy. Emphasis is placed upon the dogfish and the mammal, as most half-year courses are limited to a laboratory study of these two forms. It is assumed that most of Part II will be covered in the course. The chapter on "Mechanics of Development" is included, as this phase of anatomy has gained unusual prominence in anatomical research during the past few years.

Evolution of the Vertebrates, Part iII, takes up the palaeontological evidence and some other points of interest which are necessarily omitted in the first two parts, and includes a chapter on Geographical Distribution. This material may be assigned as collateral reading, or omitted. The chapter entitled "Adaptive Radiations of the Vertebrates" is designed as a summary of the courses which vertebrate evolution has followed. It is felt that a review of the lines of evolution tends to coordinate the evidence which has been gained in a study of individual systems.

It is $m y$ hope that the book in part meets the objections of $m y$ students, both pre-medical and general, to the books previously assigned to them; and that it complies with the following requests: (1) that unnecessary detail be omitted, particularly those details which apply to small groups and have little comparative significance; (2) that ordinal relationships rather than specific comparisons be given, so that laboratory work can be correlated more easily; (3) that generic names be omitted wherever possible and explained when used, as few students are taxonomists; and (4) that less be left to the imagination when comparing generalized mammalian and human structures. 
Compliance with the above requests might seem to be limiting the individual initiative of the student, and putting class emphasis upon the middle group of students rather than the selected few. The defense for this is: (1) the belief that the interested and brilliant student will discover enough for himself to stimulate his imagination; (2) the pragmatic statement that the average student goes to professional school with little idea as to the bearing of comparative anatomy upon human structures; and (3) the fact that this method has proved its efficiency with our students at New York University.

Although most scientific terms are defined when first used, a glossary is appended for convenience. In it both the derivations of the words and their definitions are given. A second appendix gives a more complete classification of the vertebrates than is included in the body of the text. A generic name which is used in the text will be found defined in the glossary. Then by referring to the classification, its relationships and also all the other members of the group can be located.

Instead of footnotes, cross references are made in the text. This arrangement appears to be less distracting to the reader and will also, it is hoped, bring the chapters into closer unity. Malcolis E. Little.

New York University,

May, 1932. 



\section{CONTENTS}

PART I. VERTEBRATE ZOOLOGY

PAGE

Preface . . . . . . . . . . . $\mathrm{ix}$

\section{CHAPTER}

I. The Chordate Phylum . . . . . . . 3

II. The Early Chordates . . . . . . . 11

III. Crclostones . . . . . . . . . . . . 29

IV. FISHeS . . . . . . . . . . . . 35

V. Aliphibia . . . . . . . . . . 48

VI. Reptilia . . . . . . . . . 56

VII. Aves . . . . . . . . . . . . 71

Vili. Mimnalia . . . . . . . . . . . 76

PART II. COMPARATIVE MIORPHOLOGY

IX. Tisstes of the Body . . . . . . . . . . 99

X. Istegumentari Structures . . . . . . 107

II. Stpporting Structures . . . . . . . 125

XII. Mescular System . . . . . . . . . . . 175

XiII. Digestire Systen . . . . . . . . . . 192

IIV. Respiratory System . . . . . . . . 205

IV. Vascular Sisteni . . . . . . . . . 221

Xvi. The Urinogenital Sistedi . . . . . . 250

XViI. Nervous Srsteni . . . . . . . . . 271

Xvili. Organs of Special Sexte . . . . . . 300

Xix. Mechanics of Developient . . . . . 313 xiii 



\section{CHAPTER I}

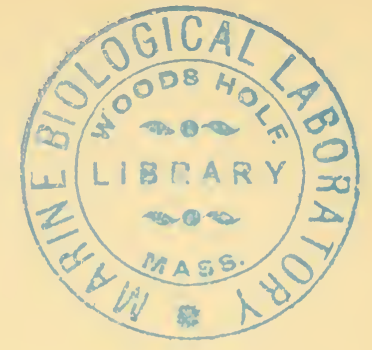

\section{THE CHORDATE PHYLUM}

IN CLASSIFYING living organisms it was found necessary to divide them into Plant and Animal KingDosis. Each of these, in turn, was divided into large groups, or PHYLA, each phylum separated from some other by only a few fundamental structural characteristics. On this basis all animals with a vertebral column were placed in the phylum Vertebrata. However, as research in natural history and embryology continued, several primitive groups of animals were discovered which lacked vertebrae but which were evidently closely allied to the vertebrates and not to any other known phylum. As a result the phylum now includes the vertebrates and these primitive forms, and is called the Phylum Chordata.

Considered either as to range in size or complexity of structure, the Chordates form the most diverse phylum of animals which we know. Its members range in size from animals barely visible to the naked eye to the whale and the extinct dinosaur; in structure from worm-like, swimming creatures to the elephant and the bird; and in physiological organization and function from stationary animals with a degenerate nervous system (their only apparent activity being the digestion of passively acquired food, and the reproduction of their kind) up to the anthropoid apes and man.

The chordates have only three characteristics which are not found in any other group, and as two of these disappear in the adults of some vertebrates, we must state that embryologically all chordates have (1) a notochord which gives the group its name; (2) a dorsal hollow nerve cord; and (3) gill slits in the pharynx. Thus the phylum is linked by fundamental developmental characters, not by structures which are acquired during growth and later development. Man, for example, is as surely a 
chordate as a fish with persistent notochord and gills, although the notochord is crowded out of existence during late foetal life or infancy, and the gill slits are present for only a short period.

Notochord. The notochord is an elastic rod of tissue acting as a supporting structure of the body. It is the only dorsal support of the primitive chordates, but in the vertebrates it becomes surrounded by the cartilage or bone which forms the vertebrae, and it is completely lost in the adults of the higher groups.

The notochord arises from endodermal cells which lose their nuclei, and it then becomes an apparently homogeneous, noncellular mass of tissue surrounded by sheaths of connective tissue. Its position is dorsal to the primitive gut and ventral to the nerve cord.

Nerve Cord. The vertebrate brain and nerve cord, which together form the central nervous system, are hollow and dorsal in position. In both characteristics they differ from all other animals, the invertebrates having a solid nerve cord, ventral in position.

The chordate nervous system arises from ectodermal tissue as a groove along the dorsal surface of the developing embryo. The significance of this dorsal position will be apparent to the student if he will consider the great development of the brain in the higher vertebrates, and its position in relation to the digestive tract.

Pharyngcal Gill Slits. All chordate embryos develop gill slits in the pharynx, that region of the digestive tract between the mouth cavity and the esophagus. These gill slits are retained by the adults in all the primitive chordates; and, among the vertebrates, in the fish and some of the salamanders. In frogs they are found in the tadpoles but are lost in the adults. In higher vertebrates the gill slits disappear in the embryo, except for the first slit which remains as the cavity of the middle ear.

The slits arise in the embryo as paired, endodermal outpocketings from the pharynx, extending laterally toward the sides of the animal. As each pouch approaches the ectoderm the latter forms a depression at the point of contact. The membrane formed at this point by the two layers breaks, forming a tube connecting the pharynx with the outside. 
The student should distinguish between gills and gill slits. Gills are respiratory organs which in primitive animals are contained within the gill slits. However, other types of gills exist; and in three groups of vertebrates (reptiles, birds and mammals) gills are never present, eren in the embryo, although slits are always formed.

Other Characteristics. In addition to the above three characteristics, which may be called the diagnostic structures of the chordates, there are others which are common to the vertebrates and other phyla. Among the more important of these are the following.

1. Metamerism, or segmentation, is the division of the animal into definite somites or segments, the condition arising in the mesoderm as a serial repetition of parts. Among invertebrates segmentation can be studied in the earthworm and the insect; and can easily be seen in the lower groups of vertebrates. It is, however, obscured in the more specialized forms. This apparent loss is due to the derelopment of fins or legs with their large muscles which cover the fundamental structure.

2. Coelomic Cavity, or body cavity, is found in several invertebrate groups and in the chordates. It arises as a splitting of the early mesodermal segment, the inner layer of mesoderm lying next the digestive tract, the outer layer in contact with the body wall. The coelom is between.

3. Bilateral Symmetry is found in the chordates and in most groups of the invertebrates. If a median plane is drawn from anterior to posterior (from head to tail) either half of the animal will be a mirror image of the other. Due to growth of organs complete symmetry is soon lost in the developing embryo; but in most vertebrates the skcleton, muscles and nervous system keep their symmetry throughout life.

4. Cephalization is the tendency of the nervous system, the sensory and coordinating mechanism, to concentrate in the anterior or head end. Within the chordate phylum there is a progressive tendency toward a concentration and shortening of the central nervous system.

5. Blood Flow, or the direction of circulation, in the rertebrate is the opposite of that of the invertebrate. The chordate heart is 
ventral in position, and blood from the heart is forced dorsally and then posteriorly, the large veins returning the blood being ventrally located.

Nomenclature. Scientific terms can best be explained when they first appear in the text. In addition, a glossary is appended to assist the student when these definitions have been forgotten. A few terms, however, which involve evolutionary principles, are defined here before the classification of the phylum is given.

1. Primitive, when used to apply to organisms, refers to that which is racially ancient. Technically, any animal which is living today is "recent"; but if it retains many of the characteristics which were typical of its early ancestors, it is referred to as a primitive animal.

2. Specialized animals are those which, during the course of evolution, have become adapted to life in a limited environment; or those which are specialized for limited activity. The entire animal may be spokien of as specialized, or one may refer to specialized individual structures. Therefore an animal may be primitive, and yet specialized in many of its characteristics.

3. Generalized animals or characters are those which are capable of modification, those which are not specialized toward one particular function. Man's hand is more generalized than his foot.

It is clear that any one of these terms may be applied to an individual structure, or to an animal as a representative of a group. If the latter, the entire sum of characters must be considered. Consequently, a generalized animal may have many specialized characteristics; and a primitive animal may be either generalized or specialized. Further, each of the three terms is used as a limiting adjective, and any statement must be applied to a specific group and considered in relation to other similar races. We may speak of a primitive man, though he would not be a primitive rertebrate; or, a primitive vertebrate would not be primitive in relation to the lower chordates. Limiting the term thus is necessary due to the very nature of the evolutionary process. For, during millions of rears of life, it is inconceivable that any large group of animals could have existed without changes and specializations; and these changes would 
proceed in different ways and at different rates in isolated races of the group.

4. Adaptive Radiations refers to the specializations which adapt different divisions of a group to various environments. The whale and monkey are both mammals though adapted to entirely different conditions. This tendency of an ancestral stock to radiate into many evolutionary lines is frequently called divergence. Any group which gives rise to another is ancestral to those which follow. However, the process of divergence cannot be carried on indefinitely for races, like individuals, die. They may become so specialized that they cannot survive the slightest change of environment, and it is therefore apparent that the possibilities of divergence are greatest in a generalized race.

5. Degenerate conditions are merely a phase of specialization. It implies a loss of functions which once were present. If an animal during its embryonic life has a well developed nervous system, and then during the course of its development loses much of the function of its coordinating structures, we think of it as a degenerate adult. In racial history the same applies. A Boa constrictor, with only diminutive structures in place of hind legs, is a specialized reptile with degenerate legs.

6. Homology. The above necessitates a consideration of homology, for before one can say that a snake has degenerate legs it must be admitted that his racial ancestors had functional legs. The proof of this lies in the fact that the tiny appendages of a Boa are developmentally similar to those of a lizard, and the fossil history confirms the embryological evidence. As a result the evolutionist thinks of them as homologous structures. Homology does not necessarily imply any functional relationship, but a developmental similarity; that is, two structures are homologous if they evolved from the same organ in a racial ancestor. In this way the wing of a bird and the hand of man are homologous, though functionally different. Their racial and embryological history show that they arose from a similar primitive structure.

7. Analogy. Analogous structures are those which serve a similar function, for example the wing of a bird and the wing of a butterfly. They are structurally, racially and embryologically 
different, though both are used for flying. But the wings of the bat and the bird are not only analogous but skeletally homologous. They are functionally similar and evolved from the same ancestral vertebrate limb.

Classification of Chordates. Like all other phyla, chordates are classified according to the Linnean system. The heterogeneous assemblage included in a phylum must be divided into smaller and more coherent groups according to their fundamental structures. The simplest method of classification is to split the phylum into the necessary number of Classes; each class into Orders, an order being a fairly large group of animals with numerous similarities of development and structure; the order is in turn divided into Fanilies; a family into Genera, a genus being a coherent small group, and the genus includes a varying number of Species. The species is the smallest group that can be accurately set apart from others.

It is often impossible to carry out a scheme as simple as the above and maintain accuracy. Particularly is this true in a diverse group like the chordates. It is necessary to set up Subphyla or Sub-classes which may include only a few forms. An illustration is a Sub-phylum of chordates which contains less than a dozen species. These few species are so closely related that they form only one family; but as a group they differ so widely in structure from all other chordates that they must be given an independent position equivalent to that of the vertebrates, which contains six classes and several hundred orders. In technical works it is often necessary to include numerous other divisions in an attempt to keep equivalent groups in a proper balance. As these divisions often become confusing even to the technically trained they have been omitted as far as possible, for they are of more academic than practical interest.

The following are the major divisions of the phylum with the classes of each. Under the separate chapter headings a more detailed classification will be given, but in each case the author has taken the liberty of excluding any order not considered necessary for the continuity of the discussion. 
Phylum. Chordata

Sub-phylum I. Hemichordata. Primitive animals with a poorly developed notochord located in the anterior end. The type order is composed of worm-like animals, the genus Balanoglossus being the usual form for study. The other two orders are aberrant and difficult for the beginner.

Sub-phylum II. Urochordata. A degenerate group in which the embryos have a well developed notochord in the tail region (uro, tail). One order remains free-swimming and retains the tail in the adult. The other two orders lose the tail after the larval period. Embryology is necessary to show the affinities of most of the group.

Sub-phylum III. Cephalochordata. A small group, closely allied to the vertebrates, and often included with them as the Euchordata ("complete" notochord). Amphioxus is the type form, and is the simplest expression of vertebrate characteristics known to naturalists. The notochord extends from tip of head to end of tail.

Sub-phylum IV. Vertebrata or Craniata. Animals with vertebrae surrounding, incompletely or completely, the notochord. A cranium, or brain case, is also typical. The sub-phylum is very large, and is divided into the following classes.

Class 1. Cyclostomata are round-mouthed animals, as the name indicates. The cyclostomes have no jaws, no appendages, and very incomplete vertebrae. The larva closely resembles Amphioxus.

Class 2. Pisces, or fish, have jaws, vertebrae completely surrounding the notochord, and fins as appendages. All known fish differ widely from the cyclostomes.

Class 3. Amphibia include the frogs and salamanders, animals with a "double" life. The larva is a water-living form with gills and many other fishlike characteristics, and usually metamorphoses into a lung-breathing, land-living animal.

Class 4. Reptilia. The reptiles are the turtles, lizards, snakes and alligators. They are covered with 
scales of ectodermal tissue, and agree with the following two classes in having fluid-filled embryonic membranes surrounding the embryo; and in being lung-breathing animals which never develop gills.

Class 5. Aves or birds are vertebrates with feathers. Structurally they can be described as warmblooded reptiles with feathers.

Class 6. Mammalia, the mammals, are warm-blooded animals with milk glands, and are usually covered externally with hair. All mammals have some hair, though it may be reduced to a few whiskers. Their name is from "mamma", a breast.

The classification of man illustrates the method used, and how any species can be identified with the use of a key to the particular phylum.

Classification of Man:

Phylum-Chordata

Sub-phylum-Vertebrata

Class-Mammalia

Order-Primates (Monkeys, Apes and Man)

Family-Hominidae (Extinct and living genera of men)

Genus-Homo (A few fossil types and all living men)

Species-sapiens (all living men.)

For proper identification both the genus and species are always given, the genus capitalized, and written Homo sapiens. The specific name may be given to other, widely different, species; but by agreement there can be only one genus with the name.

A species is often divided into varieties or races. Living men are all included in one species, though divided into four races: Caucasian or white; Mongolian, the yellow, brown and red; Ethiopian or African dark-skinned people; and the Negrito, or blacks of the Pacific islands. Similar racial divisions may be used whenever a species shows variations in different parts of its range of habitat. 


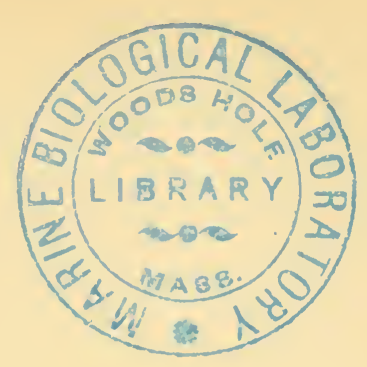

\section{CHAPTER II}

\section{THE EARLY CHORDATES}

THE cephalochordates and the vertebrates form a closely linked, progressive series; but the hemichordates and urochordates differ so widely from the others that they are included here merely to show the trial and error method on the part of nature in developing the fundamental characteristics of the vertebrate form.

Hemichordata. Considered as a whole the Hemichordates form a rather artificial group, the lower forms being included with the chordates because of certain affinities with Balanoglossus. The latter has unmistakable chordate form. The student should not consider this inclusion as a feeling of insecurity on the part of naturalists, but as the natural result of adaptive radiations. The very existence of these intermediate forms with doubtful affinities indicates the development of the higher chordates from worm-like animals whose descendants evolved in many directions.

Balanoglossus is a burrowing animal, varying in size from a few centimeters to a meter or more in length. These chordates are often called the Acorn Worms, though their worm-like characteristics are all external. The burrowing proboscis (or "acorn") is a tough, distensible organ with muscular and connective tissue bands running in both circular and longitudinal directions. At the posterior end of the proboscis is the collar, with the mouth within its ventral lip. The mouth empties almost immediately into the pharynx, in which are located the gill slits and gills. The intestine is a straight tube leading to the posterior anus. The nerve cord with its small anterior ganglion, or brain, is located on the dorsal side. The notochord is poorly developed. 

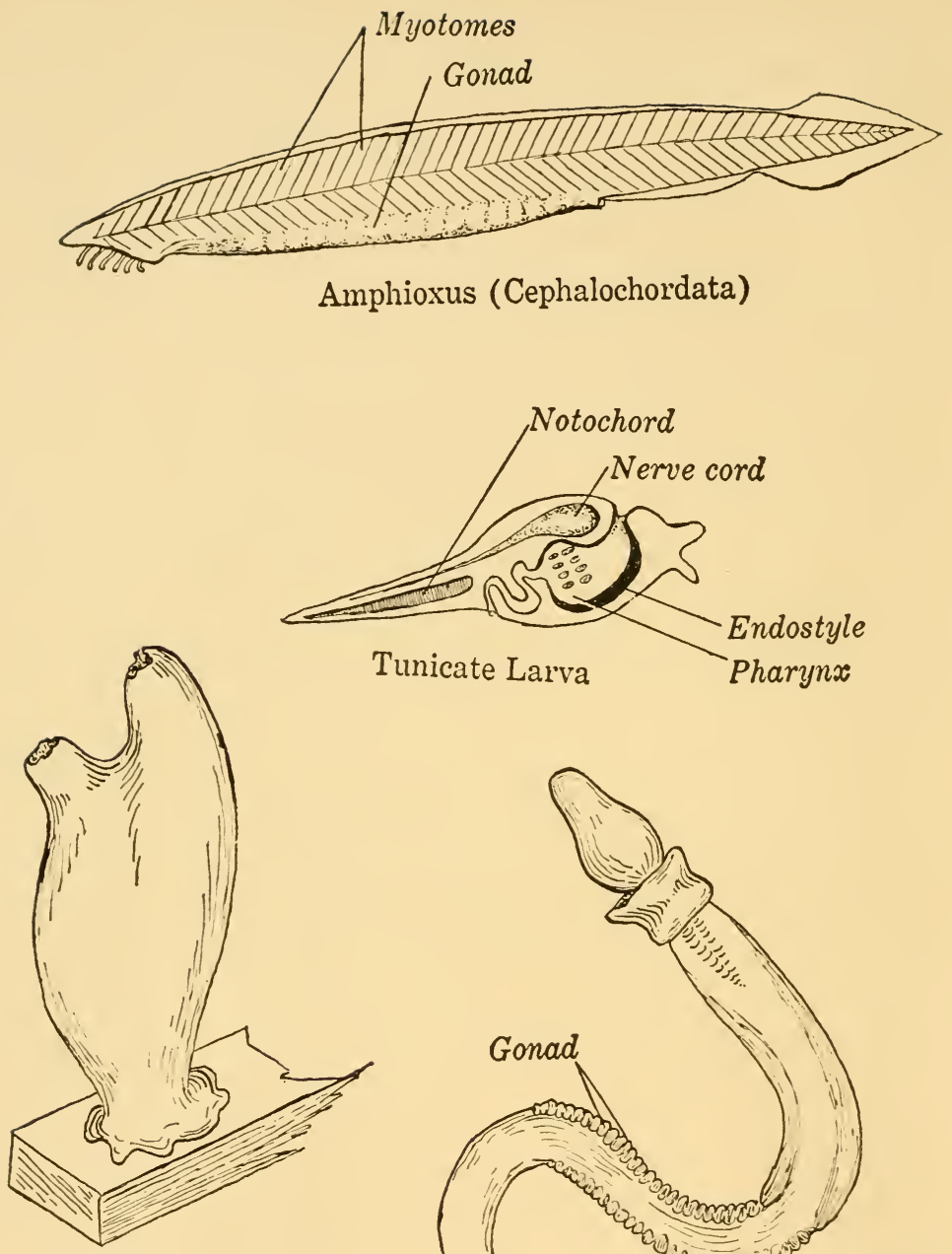

Adult Tunicate

(Urochordata)
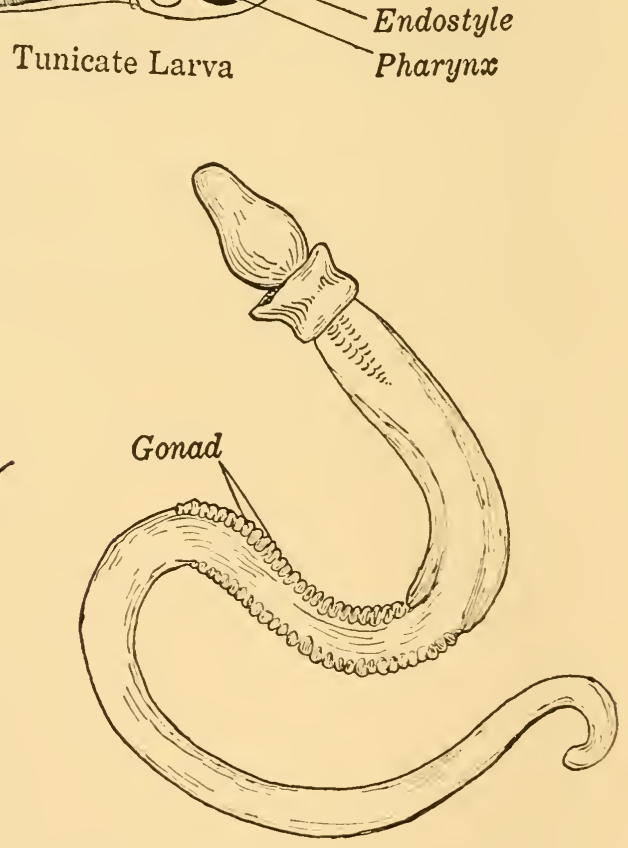

Balanoglossus (Hemichordata)

FIG. 1. Types of primitive Chordata. The Hemichordata are most primitive; the two diagrams of the Tunicate show the degenerative specialization of the adult; and at the top is Amphioxus, which is almost vertebrate in structure. 
Urochordata. The Urochordates, or Tunicates, are a large group showing intimate connections with each other, and distinct relationship to the Cephalochordates. The larger forms are familiar objects in shallow water along the sea coast, and are there known as "Sea Squirts". Until their embryology was studied the affinities of these animals were unknown, due to the degeneration which they undergo in metamorphosis from larva to adult.

The larva of a typical tunicate has clearly defined chordate characteristics. The mouth opens into a relatively large pharynx with a few gill slits. Within the pharynx there is a median, ventral groove, the endostyle, which collects food particles and passes them into the short intestine. The intestine becomes spiralled early in development. The nerve cord shows marked advances over the hemichordate condition. The anterior ganglion is well developed and often called a brain. Connected with it is a light-sensitive organ, the so-called eye, and an otocyst which functions as a balancing organ. The notochord is also well developed, though it extends no farther anteriorly than the posterior end of the pharynx.

The larva leads a free-living existence for a short time and then undergoes rapid metamorphosis into the adult form. At this time it settles to the bottom and becomes attached to a stone, wharf pile, or other object. The nerve cord rapidly degenerates; the pharynx increases in size with a multiplication of gill slits; and the tail, with the entire notochord, is absorbed, the animal feeding on its own tissues during the metamorphosing process. Synchronously with the degenerative changes a tunic, or cover, is secreted, surrounding the animal as a lifeless mantle of animal cellulose. This bears the same relationship to the animal as does the shell to a snail. The tunic has two openings, one an incurrent pore, the other excurrent. The animal is then a completely stationary organism carrying on nutritive and reproductive processes.

The tunicates, with the exception of the hemichordates, are perhaps the most ancient line of the phylum. The larvae show their group relationships, but during their evolution the adults have become specialized in a degenerative manner. 


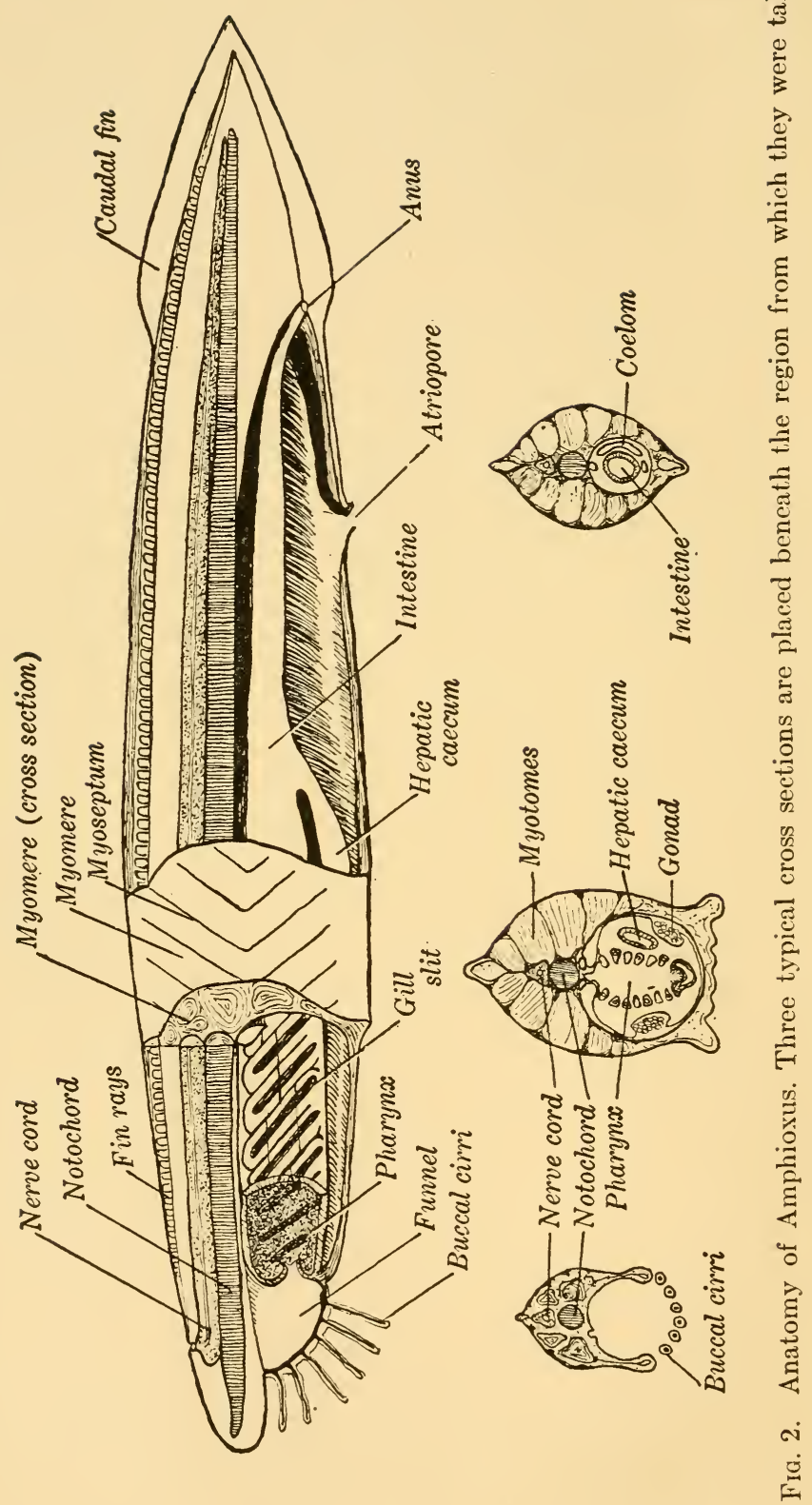


Cephalochordata. The cephalochordates are small, lanceshaped animals living on sandy shoals of the ocean, near the coasts of Europe, Asia and North America. The type form is Amphioxus, its "double pointed" structure being an adaptation to burrowing in the sand. The anterior end is braced by the extension of the notochord into the rostrum which projects in front of the mouth. The animals are active during the night, but in the day burrow into the sand with only the anterior end projecting above the surface. In this position they wait for currents of water to wash food into their mouths.

External Anatomy. The adult Amphioxus varies in length from four to six centimeters, about two inches. The anterior end can be determined by the pointed rostrum, and the ventral mouth a few millimeters from the front. The latter is surrounded by a circle of buccal cirri. In cross section the animal is roughly triangular in shape, the base being the ventral side.

In a preserved animal the oral hood and funnel can be seen within the circle of cirri. The structures are formed by a fold of tissue extending downward and backward from the rostrum, so that a cross section through the hood would be circular. At the bottom of the funnel is the mouth, which can be studied only microscopically. The "fins" of the animal are simple in structure; a dorsal fin extends from the base of the rostrum to the posterior end of the animal, where it becomes wider and swings around the posterior tip to form the caudal fin, and the narrower ventral fin. With the fins may be included the metapleural folds, which will be discussed later. These folds are paired, extending along the lateral ventral sides of the animal to a point just anterior to the rentral fin, where the two folds meet. Examination will show that at the confluence of the folds there is an opening, the atriopore. The anus can be located posterior to the atriopore, on the left side of the ventral fin.

The animal is covered with a thin skin, composed of an ectodermal layer one cell thick, and a dermis, which is a loose, gelatinous, mesodermal connective tissue. A non-cellular cuticle is secreted by the ectodermal cells, acting as a transparent protective layer on the outside. Through the thin skin can be seen the distinctly metameric, V-shaped muscle segments, separated from each other by thin sheets of connective tissue, the 
myosepta. These structures are important, for the muscle fibers are attached to them, and they bind the myotomes to each other and to the notochord. If the animal is thoroughly adult the metameric gonads, or reproductive organs, will be visible. The sexes cannot be distinguished except microscopically. In sections the female ovary shows the spherical eggs or ova, and the male testis has very minute sperms.

Digestive Tract and Accessory Structures. Beginning at the anterior end, the first nutritive structures are the buccal cirri. In the living animal these are constantly in motion, and in addition to a sensory function, probably assist in carrying a stream of water with suspended food particles into the funnel, and thus into the mouth. Within the funnel are two structures which are supposed to have a sensory function: (1) a ciliated groove (Groove of Hatschek) extending the length of the funnel, slightly to the right of the dorsal midline; and (2) the "wheel organ", patches of thickened, ciliated epithelium surrounding the mouth and extending anteriorly like spatulate fingers. The latter probably assists in passing the water toward the mouth and pharynx.

The mouth is guarded by a velum, or curtain, hanging from the dorsal wall of the rounded opening. The mouth opens directly into the pharynx, the largest single organ of the entire animal. As the water which is forced through the mouth becomes more or less still in the enlarged cavity, the food particles tend to drop to the ventral floor where they are caught in a groove along the mid-ventral line, the endostyle. This groove is lined with cilia and mucous secreting cells, so that the food is caught in the mucus and passed anteriorly toward the mouth. At the level of the mouth the endostyle splits into two grooves, the peripharyngeal grooves, which pass dorsally around the opening and unite in the dorsal-median line as the epipharyngeal groove. In this groove the cilia beat backward, carrying the mucus with its attached food particles to the opening of the stomach-intestine, a straight tube in which digestion and absorption take place. The intestine opens to the outside at the anus.

Immediately posterior to the pharynx there is a single rentral 
outpocketing of the stomach-intestine, the hepatic caecum. This blind pouch grows forward on the left side of the pharynx, and assists digestion. From its method of origin it has been identified with the liver of the vertebrates.

Within the pharynx are the gill slits and their gills. In the larva these are metameric, paired openings; but in the adult metamerism is soon lost, and new gills are added until there may be more than a hundred pairs. The gills serve as respiratory organs, the water which carries food particles also carrying dissolved oxygen. The course of the water is, therefore, important. It is forced through the mouth into the pharynx. There a portion of its food is dropped, and the oxygenated water is carried out through the gill slits, supplying oxygen to the vascular gills and taking up the carbon dioxide. In the adult an atrium is present, a specialized external covering which protects the gills (page 27).

Supporting Structures. The main supporting element of Amphioxus is the notochord. This rod of spongy tissue is surrounded by two concentric sheaths of connective tissue, the notochordal sheaths, which give strength to the notochord. Fibers pass out from these sheaths between the muscles, supporting them and giving points of attachment to the muscle fibers. Any contraction of the latter exerts a pull on the notochord and affects the entire animal.

The cartilage of Amphioxus is a very soft tissue having few of the characteristics of true cartilage. These cartilages can be seen in the dorsal fin as the segmental fin rays. A horseshoeshaped buccal cartilage surrounds the funnel, the open side at the bottom. From this cartilage a small ray extends out into each cirrus. The gill slits are also supported by cartilaginous gill bars which lie between the slits. The illustrations give the details of these structures.

Muscular System. The muscles are of the simplest nature. The myotomes, or muscle bands, are V-shaped with the apex pointed forward. The muscle fibers run longitudinally, groups of fibers being caught into bundles by connective tissue sheaths, and a large number of bundles forming a myotome. Between each myotome is the myoseptum which is intimately connected 


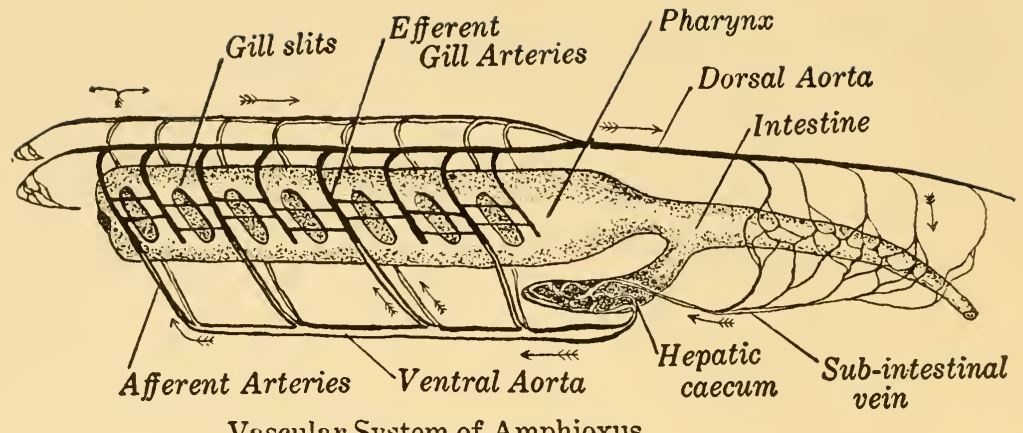

Vascular System of Amphioxus

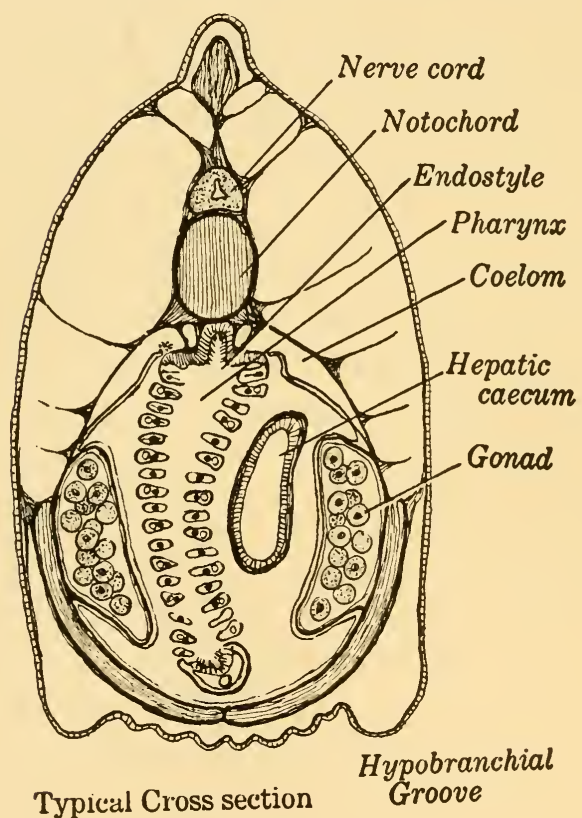

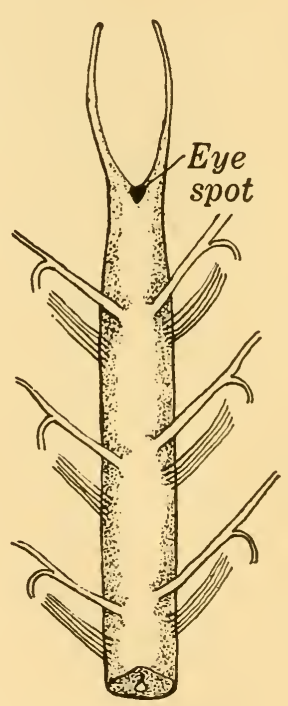

Nerve cord of Amphioxus

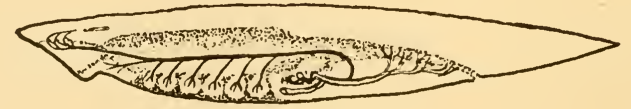

The Cardinal Veins

FIg. 3. Internal Anatomy of Amphioxus. The cross section shows the relative position of the structures. The top diagram gives the relationship between the digestive and vascular systems. 
with the notochordal sheaths. Each fiber is attached at either end to the myoseptum, so that the myotomes are functionally continuous although broken into definite metameres.

Any muscular contraction exerts a pull on the anterior-posterior axis, so that the range of movement is limited to a side to side motion. Forward movement of the animal is caused by the larger size of the anterior end. The tail region is more flexible, and an alternate contraction of the muscles of either side causes the tail to act as a propeller both in swimming and in burrowing.

Vascular System. The blood system of Amphioxus is the perfect starting point for a study of the vertebrate system. A glance at the diagrammatic drawing will assist in understanding the description. Recall that the vascular system has the functions of (1) taking up absorbed food materials from the digestive tract; (2) carrying waste products from the cells to the organs of excretion (and respiration); (3) taking up oxygen from the gills; and (4) distributing food and oxygen to the tissues of the entire body. Further, Amphioxus, like the vertebrates, has a closed system. The blood vessels are continuous, and the blood remains within the lining of the vessels; therefore, when an artery divides into capillaries, these same capillaries re-collect into veins without any definite break. Consequently it is impossible to make any definite distinction between arterial and venous capillaries.

Amphioxus has a slightly enlarged, pulsating ventral aorta, homologous with the vertebrate heart. From this point the aorta continues anteriorly, giving off a pair of afferent branchial arteries at alternate gill supports. These arteries break into smaller vessels in the gills, and then collect into efferent branchial arteries. Lateral branches pass into the secondary gill supports, with the result that there is an efferent artery for each gill. Passing through the gills the blood has been aerated. The efferent branchials enter the paired dorsal aortae, one on either side of the notochord. Branches go forward into the head region, the two dorsal aortae extending backward and fusing to form a single vessel at the posterior end of the pharynx. The aorta then continues al wo the body and into the tail, giving off metameric vessels to the muscles and the digestive tract. 
The capillaries draining the intestine and muscles collect into veins, and these fuse to form a ventral subintestinal vein. At the hepatic caecum this vein breaks into smaller vessels which spread around the caecum and re-form into a continuation of the ventral vein. This is the equivalent of a hepatic portal system and a hepatic vein. The latter is continuous with the ventral aorta.

A minute group of vessels, draining the gonads and part of the head region, has been described and is considered the homologue of the cardinal system of vessels found in the higher groups.

Observe that the blood flows forward in the ventral vessels and backward in the dorsal. This is the typical vertebrate system. The student should work out for himself the relative amounts of food, oxygen and waste matter contained in the blood of each region of the body.

Excretory System. The organs of excretion are paired nephridia, each of which has a head in the coelomic cavity and a short nephric tubule which embryologically empties to the outside of the body. This is slightly modified in the adult by the development of a protective atrium (see page 27). In their metameric arrangement the nephridia may be considered as primitive structures; but the individual nephridium is a highly specialized organ.

Reproductive System. The gonads (ovaries and testes) are metameric, very numerous, and lie along the ventral half of each side of the animal. As in the vertebrate the gonads arise in the dorsal mesodermal tissues, outside the coelomic cavity and covered by the peritoneum, the lining of the body cavity. As the atrial folds develop, the gonads, each surrounded by a part of the coelomic cavity and peritoneum, are pushed ventrally. Therefore, as the sperm and ova ripen they break through the covering membranes and fall into the atrial cavity, from which they pass to the outside through the atriopore and fertilization takes place externally. This is a specialization in Amphioxus, in correlation with the development of the atrium (page 27), and differs from the condition found in the vertebrates. In the most primitive vertebrates the ova and sperm fall directly into the coelomic cavity; in all others the sperms 
have tubes which conduct them to the outside, while the sperms retain the primitive condition.

Nervous System. Amphioxus has no brain, the anterior part of the nerve cord being even smaller than that lying farther posteriorly. This is considered a specialized condition, for the tunicate has a better developed brain than the cephalochordates. At the extreme anterior end of the nerve cord are two so-called cranial nerves, and between them a light-sensitive, pigmented eye spot. Posterior to this region are the metameric spinal nerves. Each has two roots arising from the spinal cord, a dorsal root and a ventral root. The dorsal is a mixed nerve, being partly sensory and partly motor, while the ventral is entirely motor. Differing from the vertebrate, all the nerve cells are contained within the cord; and the roots do not unite into a single spinal nerve, but each retains its independence and ramifies into the tissues of the body.

Embryology of Amphioxus. Amphioxus in its development is properly considered the basis for all vertebrate embryology. Its study has cleared many obscure points due to its perfectly generalized developmental stages, and the leisurely manner of organ formation in the early embryo.

The ova are transparent objects on the verge of human vision. Both eggs and sperms are thrown into the water about dusk during the breeding season, where fertilization takes place. The ovum has a small amount of yolk material slightly concentrated at the ventral pole. This is of more than passing significance in cleavage, as it is a general rule that cells loaded with inert material cleave more slowly than others.

About an hour after fertilization the egg divides, the first cleavage being through the dorso-ventral axis. The second is in the same direction, at right angles to the first. The third cleavage is horizontal, perpendicular to the first two, and slightly asymmetrical, the four dorsal cells being slightly smaller than the four ventral. Due to the use of cellular materials to supply energy for cleavage, and the natural tendency of a liquid to assume a spherical shape, a cavity has appeared in the center of the eight-celled embryo, called the segmentation cavity. 
After the fourth or fifth cleavage there is a slightly more rapid rate of cleavage in the dorsal hemisphere, and these cells are distinctly smaller. The segmentation cavity has also enlarged, so that at about the 256-cell stage the embryo is a hollow sphere, the blastula. The process of gastrulation begins immediately after, and may be illustrated by pushing a finger into a rubber ball until a cup-shaped, two-layered structure results. In Amphioxus gastrulation is caused by two synchronous processes: (1) the more rapid growth of the dorsal cells causes this hemisphere to become larger than the ventral, and the blastula assumes a hemispherical shape; and (2) the ventral cells invaginate, or push inward, until they come in contact with the dorsal layer. The earlier segmentation cavity is practically obliterated, and a new cavity is formed inside the cup. Thus the early gastrula is two-layered, the outer layer of cells being the ectoderm, the inner layer (derived from the ventral hemisphere) being the endoderm. The inner cavity, surrounded by endoderm, is the archenteron or primitive gut. Continued growth of the gastrula causes it to elongate, and the lips of the cup to narrow toward a central point. The opening left after

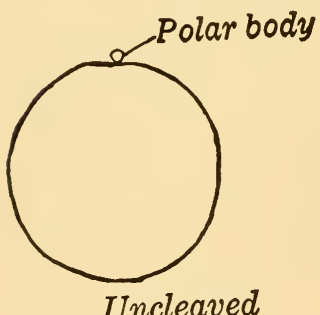

egg

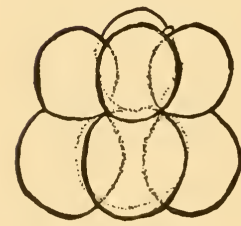

8 Cells

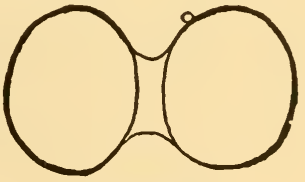

2 Cells

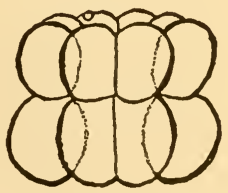

16 Cells

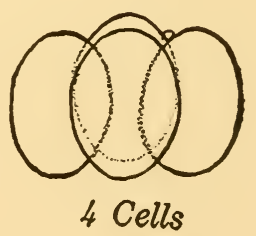

4 Cells

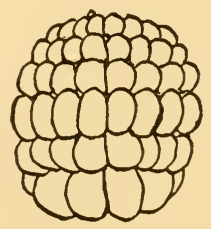

Blastula

Fig. 4. Cleavage of Amphioxus from egg cell to blastula. 

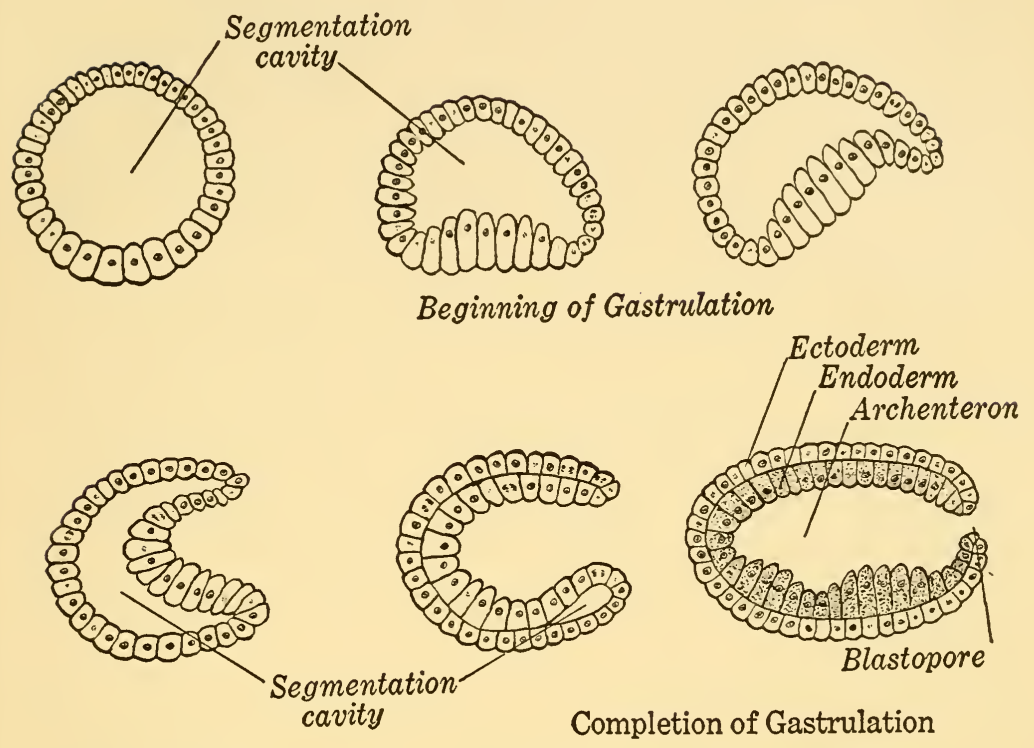

Frg. 5. Sagittal Sections of Amphioxus showing gastrulation. The ventral (endodermal) pole invaginates synchronously with the overgrowth of the dorsal (ectodermal) pole.

gastrulation is complete is the blastopore which eventually gives rise to the anus.

Organogenesis, or organ formation, begins almost at once. The development of the ectodermal dorsal nerve cord, the endodermal notochord, and the mesodermal tissues are practically synchronous. They are considered separately for convenience, and constant reference should be made to the drawings.

The nerve cord begins as a plate of cells (the neural or medullary plate) along the dorsal, median line of the embryo. The lateral margins of the plate soon break away from the ectodermal cells covering the remainder of the embryo. The ectoderm along either side of the plate becomes elevated, forming neural folds, and these folds grow toward each other along the dorsal midline. When the epidermal covering of the embryo is about complete the neural plate sinks in the middle to form a neural groove, and the continued infolding of the groove and the upgrowth of the edges of the plate soon develop the dorsal 
neural tube which becomes the nerve cord of the animal.

The notochord begins its development slightly later than the nerve cord. It is formed from the dorsal cells of the archenteron. These endodermal cells develop a groove, the curve being exactly opposite to that of the nerve cord, and the lateral margins grow downward until they meet. The notochord becomes a continuous loose rod of cells beneath the neural groove.

The mesoderm arises from the primitive endoderm as paired, lateral pouches from the gut. It should be kept in mind that though the nerve cord and notochord are continuous structures, the mesodermal pouches are a series of discontinuous outgrowths, or enterocoels (literally "gut pouches"). Thus metamerism, or segmentation, has its origin in the mesoderm. Only three pairs of pouches arise directly from the archenteron, the others developing by the growth and division of the third (most posterior) primitive pouch.

Soon the lining of the gut fuses along the dorsal border cutting the notochord off as a separate structure; and following this the mesodermal somites lose their connection with the enteron. The embryo is now an ellipsoid organism completely enclosed by ectoderm, for as the neural folds closed along the dorsal midline they also overgrew the blastopore. Therefore, for a short time, the neural canal is continuous with the enteric canal. Later the posterior end of the nerve cord closes, and the blastopore breaks through the ectoderm to form the anus, or posterior opening of the enteron.

A coelomic cavity develops through the enlargement of the original enterocoel cavities. As the metameres grow downward the ventral portion becomes thin-walled, and the dorsal part of each thickens into a mass of tissue. The dorsal segment is the epimere which gives rise to the muscle tissues and the connective tissues; the ventral hypomere forms the smooth muscle of the digestive tract, the heart, the mesenteries, and the lining of the coelomic cavity. These relationships can be studied in the illustrations. Observe that as the hypomere pushes ventrally the median layer of cells is pushed against the gut, which has separated from the notochord. During this process the dorsal epimere has grown around the notochord and also ventrally, forcing its way between the ectoderm and the lateral layer of 

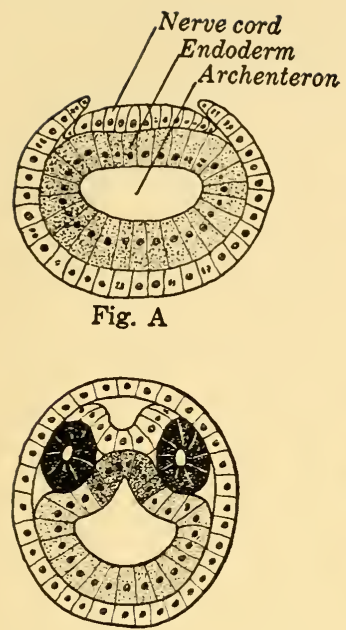

Fig. D

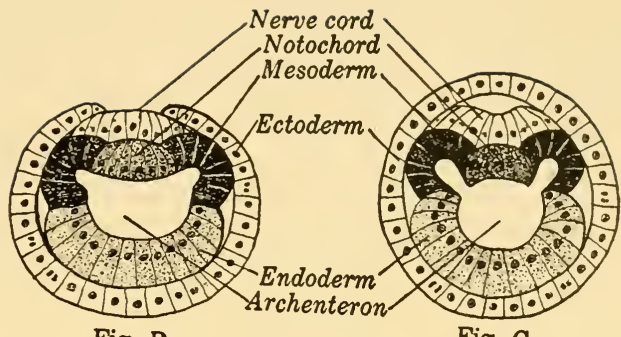

Fig. B

Fig. C

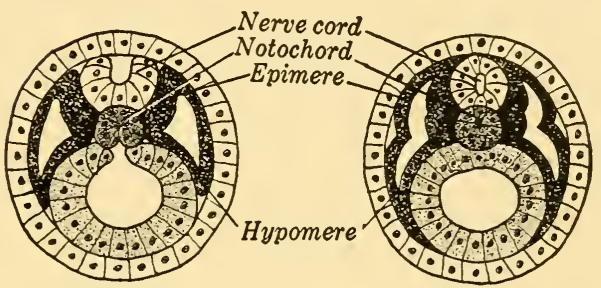

Fig. F

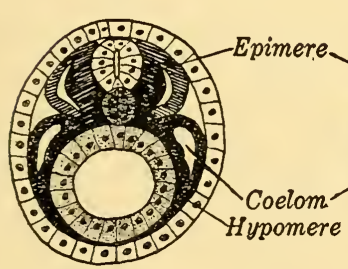

Fig. G

Fig. E

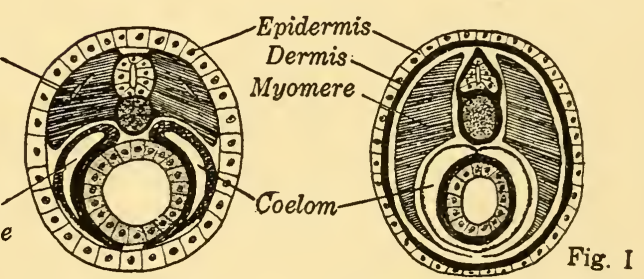

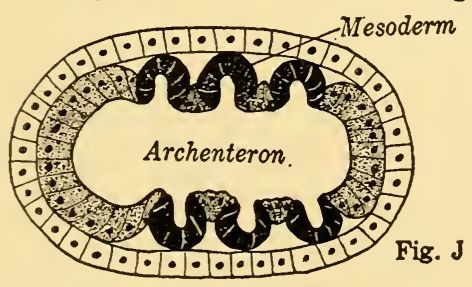

Longitudinal Section Through Stage "C"

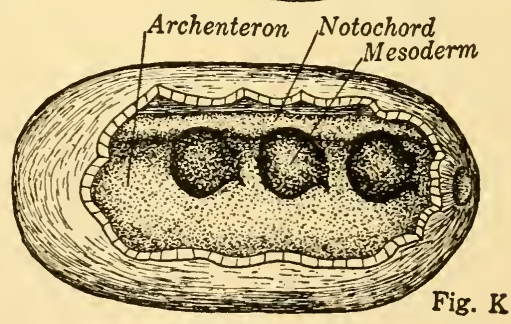

Dissected Embryo at Stage "C"

Fig. 6. Organogenesis in Amphioxus. Diagrams A to I are cross sections to show the development of the continuous nerve cord and notochord, and the discontinuous enterocoels. Drawing $\mathrm{J}$ is a horizontal section. Drawing $\mathrm{K}$ has the outer ectoderm dissected away to show the relationship between the archenteron and the enterocoels. 
the hypomere. Eventually, both the epimere and the hypomere reach the mid-ventral line, each meeting its mate which developed on the other side. The coelomic cavity is now almost complete. Further development consists in strengthening the dorsal mesentery which supports the gut; the absorption of most of the ventral mesentery; and the disappearance of the septa which up to this time have divided the coelomic cavity into separate pockets. When complete the coelom is a continuous cavity from anterior to posterior, with no separation of the two sides at the bottom, but divided dorsally by the mesentery.

Gill Slits develop in the anterior, pharyngeal part of the enteron. These begin as paired outpocketings of the endoderm, the bubble-like structures growing laterally until they reach the ectoderm. The ectoderm pits in at these points, fuses with the endoderm, and then breaks through to form the gill slit which connects the pharynx with the outside. At first the slits are metameric, but during development metamerism is lost in these structures.

The mouth is formed by the anterior wall of the gut coming in contact with the ectoderm and breaking through. In Amphioxus the mouth opening is at first asymmetrical, but it later moves into its normal terminal position. The heart, or ventral aorta, is at first a thin tube of mesodermal cells lying ventral to the gut, but later it is surrounded on either side by the hypomere. The latter develops into the cardiac muscle which gives the heart its strength and rhythmic beat.

Importance of Amphioxus. More time has been given to the structure and development of Amphioxus than the student may feel is necessary, but the group illustrates perfectly the fundamental plan of the entire chordate group. Its embryology is so generalized that it clears many doubtful points in the development of the true vertebrates, and the developmental approach to comparative anatomy needs no defense.

The importance of the group lies in the fact that in no other animals are the essential chordate characteristics shown as simply and with so few complications. Particularly is this true of the young animal. The adult has numerous specializations, and the student should review the meaning of "generalized" and 
"primitive" in relation to Amphioxus. The group is primitive because it is fundamentally similar to the earliest ancestors of the phylum. The young Amphioxus is generalized because its characters apply to the chordates as a whole. However, the group has many specializations, or characters which have been added to serve a particular function. The student has but to consider the enormous length of time since the origin of the first Amphioxi to understand this. The constant mutations which occur in all animals; constantly changing environmental conditions which place new hazards upon existence; and the destruction of the least adapted types due to natural selection, explain why a group rarely exists for long periods without adding specializations which adapt it for particular conditions.

Specializations of Amphioxus. An incision in the ventral body wall of a frog or other vertebrate would open the coelomic cavity; but a similar incision in the body of Amphioxus would open an ectoderm lined cavity, the atrium, which serves to protect the gills of the burrowing animal. This cavity is closed at the anterior end and opens posteriorly through the atriopore. Therefore, water entering the mouth of the adult passes through the gills into the atrium, and out through the atriopore. In this way the circulation of water is not hindered by the surrounding sand. Its development is illustrated by the drawings. The atrial folds of the larva begin in the anterior dorsal region and pass posteriorly and ventrally until they nearly meet anterior to the anus. These continuous folds then grow ventrally, being covered on both sides by ectoderm. Eventually they meet along the midventral line, fuse for their length, and develop the new cavity. The metapleural folds are simply longitudinal folds of skin along the lateral walls of the atrial walls.

The coelomic cavity of the pharyngeal region is also highly specialized. This is due to (1) the increased size of the atrium, and (2) more especially to the great number of gills. The body of a vertebrate can be compared to a room with its inner layer of plaster (the endoderm), the outer layer of brick (the ectoderm), and the cavity between (the coelomic cavity). If the windows are compared with the gill slits, it will readily be seen that every window destroys a certain portion of the wall 
cavity. Continue the multiplication of windows, and eventually the internal cavity remains only in the region above the windows, as narrow strips between them, and ventral to the inner cavity. As the gill slits of Amphioxus are very long and close together the coelomic cavity of the adult is left (1) as a ventral tube; (2) two dorsal tubes separated by the mesentery; and (3) long, very narrow tubes, between the primary gills, which connect the dorsal and ventral portions. During development each of these primary gills is divided into two by the downgrowth of a septum which naturally does not carry any of the coelomic cavity with it. Therefore, only every alternate gill support has a tube of coelom.

The rostrum and the anterior extension of the notochord are also considered as specializations. This is based on the fact that in all vertebrates the notochord extends no farther than the middle of the brain, and in Amphioxus the rostrum is a later growth. Unfortunately the notochord of the more primitive chordates offers no solution, for in the tunicates the notochord is limited to the tail; and in the more primitive hemichordates the notochord is rudimentary and found in the anterior region.

The asymmetry of the mouth opening is correlated with a bilateral slippage during development. One half of the embryo pushes forward about half the distance of one body segment, and though the mouth finally corrects its position, the myotomes and the gonads maintain this asymmetry throughout life. This accounts for the fact that when cross sections of Amphioxus are studied a pair of gonads is never cut through the same region, one section always being larger than the other. 


\section{CHAPTER III}

\section{CYCLOSTOMES}

Cyclostomes are the most primitive vertebrates, in many respects intermediate between the Amphioxi and the fish. They are popularly called "round mouthed eels", the name being derived from a superficial eel-like structure, not from any anatomical similarity. The animals inhabit both fresh and salt water and are very widely distributed.

The characteristics of the group are:

1. No paired appendages. The cyclostomes entirely lack the two pairs of appendages typical of the vertebrates. The student should not confuse the leglessness of snakes, some fish, and other animals with the inherent absence of paired fins in this class of vertebrates. In the higher legless forms there are always embryonic limb buds, which may remain as rudiments in the adult. The cyclostomes have no embryonic evidence of ever having had fins.

2. Round mouth. The word cyclostome means "round mouth", in reference to the round oral hood, somewhat similar to that of Amphioxus. A rasping "tongue" scrapes the food into fine fragments, while the sucking oral hood attaches the animal to its prey.

3. No jaws. Lacking jaws, the oral hood remains permanently open, the two characters being interdependent. The absence of jaws is a very primitive characteristic, as is shown by both embryological and fossil evidence.

4. Gill structure. One of the orders of living cyclostomes has seven gill openings, the other has as many as fourteen. As five gill slits is the typical number of the higher vertebrates, and the most primitive fish known has seven, the cyclostome condition is considered primitive. The internal and external openings 
of the gill pouches are round, agreeing with the structure of the primitive fossils, but differing from the fish.

5. Nervous system. The brain is primitive compared with that of the fish, although highly developed compared with Amphioxus. The olfactory lobes are highly developed, but there is no distinct cerebrum or cerebellum. The other organs of special sense are poorly developed. The lampreys have two semicircular canals in the inner ear, agreeing with the fossil forms; the other order (myxinoids) have a single inner ear canal, which is considered by some anatomists to be a degenerative specialization. Most of the cyclostomes are blind, or nearly so, the eyes appearing to be degenerate and not primitive.

6. Supporting structures. The notochord is covered with sheaths, as in Amphioxus, and is the main support of the body. The vertebrae, which appear first in this class, are minute cartilages which could serve no possible function. A chondrocranium, or primitive cartilaginous "skull" has developed. It is an open trough without a roof, in which the brain rests.

7. No bone. The living orders of cyclostomes lack bone entirely. The "teeth" of the oral hood are ectodermal horny structures, and have no resemblance nor relationship with the teeth of higher vertebrates.

8. Nasal opening. There is a single, dorsal, nasal opening connecting the medium with the olfactory sacs. As the tube extends past the olfactory region it is usually called the nasopituitary opening.

Classification of the Cyclostomes. The living cyclostomes are divided into two orders which vary widely in development and structure. The fossil forms include three orders which are grouped together as the Sub-class Ostracodermi. From the embryological evidence (Dean) and the fossil evidence (Stensio) each of the living orders may be considered as a sub-class, equivalent to the Ostracodermi.

With the cyclostomes is often included a group of very small, extinct, animals known from a single genus (Palaeospondylus). If it is an adult rather than a larval stage, it gives evidence of the early segmentation of the notochordal supporting structures. 
Petromyzontea, or lampreys, include Petromyzon marinus, the form ordinarily used in laboratories for dissection. The lampreys have a complete cartilaginous branchial basket and seven gill pouches. During development a longitudinal constriction separates the dorsal gullet from the ventral respiratory

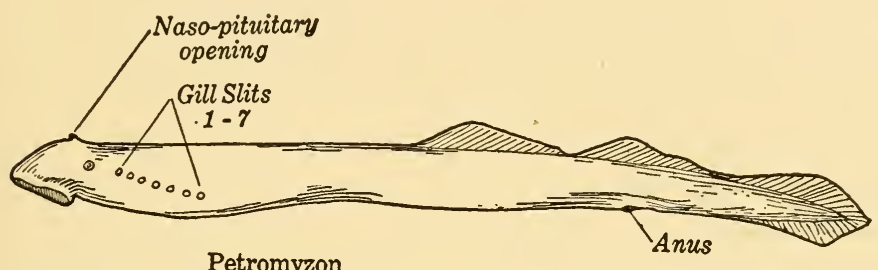

Petromyzon
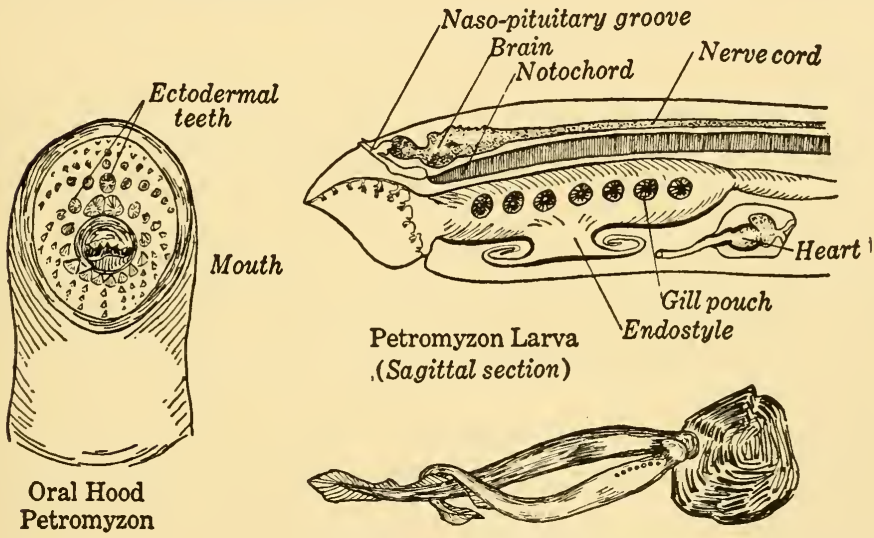

(Sagittal section)

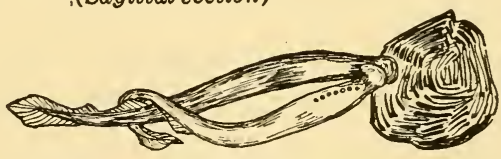

Mating Brook Lampreys

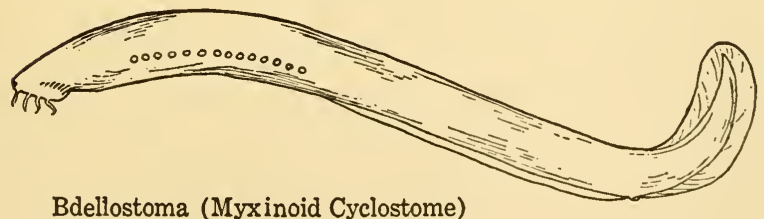

Fig. 7. Cyclostomes. Bdellostoma belongs to the more primitive order, the Myxinoidea. The section of the lamprey larva (Petromyzontia) shows the pinching off of the endostyle to form the thyroid. 
tube. The naso-pituitary pouch ends blindly under the brain. There are two semicircular canals in the inner ear. The eyes are better developed than those of the following order.

Myxinoidei, the hag-fishes, are ecto-parasitic and a serious menace to fish. They are limited to the Pacific Ocean. This order has a reduced branchial basket and fourteen gill openings. These may open directly to the outside, or unite as a single ventral outlet. The oral hood of some forms is surrounded by fleshy barbels. The eyes are degenerate, and there is a single semicircular canal in the ear.

Ostracodermi. These are fossil forms which have clear-cut cyclostome affinities (Stensio, 1927 and 1931). They lack jaws and teeth, and have no paired appendages. Sections of the ani-

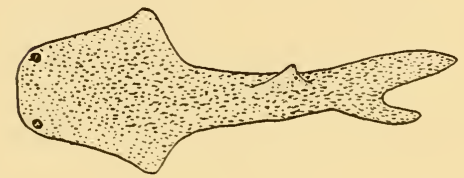

A. Thelodus

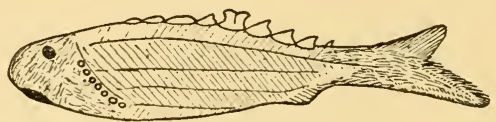

B. Birkenıa

FIG. 8. Ostracoderms; extinct cyclostomes with dermal plates. Birkenia is a free-swimming type with a few dorsal spines. Thelodus has numerous dermal denticles and is specialized for bottom living.

mals show the following agreement with the living cyclostomes: (1) round gill openings; (2) the structure of the brain and spinal nerves; (3) the arrangement of the cranial circulatory system; (4) two semicircular canals in the inner ear; (5) a single dorsal nostril; and (6) an unconstricted notochord. The apparently well developed eyes, the round mouth, and the lack of jaws can be determined from a study of the external anatomy.

The ostracoderms differ from the living cyclostomes in having dermal denticles, scales, or plates covering the body. The more primitive genera had small bony denticles without a broad base; the more specialized groups developed a broad base to the denticle, and the most specialized forms had plates covering the body.

Development of Petromyzor. The egg is approximately a millimeter in diameter, and the early stages of development 
are sufficiently like those of Amphioxus to be understood without repetition. The embryo hatches as a larva which shows relationship with the primitive chordates.

The larva is strikingly like Amphioxus in body shape and in structure, though (1) the reduced number of gill slits, (2) the more advanced brain, and (3) the chondrocranium are typically vertebrate. Even in the early larva the ventral hepatic outpocketing, which remains in Amphioxus as a caecum, has become a glandular liver. The similarities are in the oral hood, the myotomes, and the pharyngeal structures. The food getting structures of the pharynx are a ventral endostyle and a dorsal epipharyngeal groove. Both are ciliated and secrete mucus. After three or four years the larva metamorphoses into the adult type. The dorsal part of the pharynx, including the upper groove, pinches off as an esophagus, leaving the pharynx as a blind sac. The endostyle also closes, becomes at first a blind pouch with a slight pharyngeal connection, and then the connection is lost as the pocket becomes glandular. The endostyle is then the thyroid gland. This is one of the clearest homologies in vertebrate anatomy, the thyroid of higher types always arising as a median, ventral outpocketing of the pharynx.

Specializations of Cyclostomes. The larva of the lamprey shows such definite relationships with Amphioxus, and the fossil forms are fundamentally so similar to the living representatives, that most anatomists consider the class as very primitive, although highly specialized, vertebrates. Other anatomists consider them as completely degenerate vertebrates. The evident degeneracy of the myxinoids as regards the branchial basket, the eyes, and the fins is used as evidence for the latter theory. The research on the ostracoderms, on the other hand, gives weight to the older theory.

The fact that all known ostracoderms had some form of skin, or dermal, bone indicates that these structures may have once existed in all cyclostomes and have been lost in the living orders. According to this theory the ostracoderms lost their free-swimming ability with the development of armor and became extinct. 
Although the living cyclostomes are far removed from the fish, the lines of evolution evidently having split far back in the history of the vertebrates, certain of the ostracoderms are sufficiently generalized to have been the ancestors of the more highly developed fish. 


\section{CHAPTER IV}

FISHES

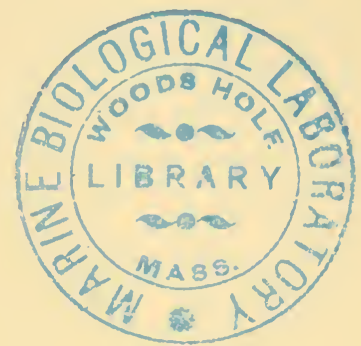

The Class Pisces, or fishes, is the largest class in number of genera. With a few exceptions they are completely aquatic in habit, and are distributed throughout the world in both fresh and salt water. In their anatomical characteristics they show a wide variation, as would be expected from their wide geographical distribution, and the enormous length of their racial history. The group may be defined as aquatic vertebrates with fins, gills, and dermal scales. The fish evolved from a cyclostome type of ancestor at such an early period in vertebrate history that they may almost be considered as parallel lines of development. The following list of characters will help to show their advance over the cyclostomes, and give an idea of their fundamental structure.

1. Fins. The fish have two pairs of appendages, pectoral and pelvic, in addition to the dorsal, ventral and caudal fins. The fins are characteristic in having the distal (outer) border of dermal rays, entirely unlike the feet and toes of more advanced vertebrates. In several highly specialized recent fishes the fins have been lost. Such cases are degenerative specializations.

2. Jaws. Like all higher vertebrates, the fish have developed jaws for holding, biting, or crushing food. The jaws are modified gill arches.

3. Scales. Dermal scales are found in the fish and set them apart from the cyclostomes. Such scales arise in the mesodermal tissues of the skin (the dermis) and are developmentally different from epidermal structures. Fish scales vary from minute bony dermal denticles in the most primitive group of fish to heavy bony plates, or flexible dermal scales in the typical food fish. In a number of specialized forms the scales are almost, or 
quite, absent. This degeneration of the scales often accompanies a loss of fins, and is correlated with mud-living habits.

4. Gills. The typical fish has internal gills located in the gill slits, serving a respiratory function. In two groups of fish, including the order which is considered ancestral to the higher vertebrates, the larvae have external gills similar to those of the amphibia.

5. Vascular System. The heart, a twisted, S-shaped structure, has one auricle and one ventricle. The circulatory system is built around the gills, essentially like that of Amphioxus, although with many modifications.

6. Nervous System. The brain is very simple in character. The cerebral hemispheres are functionless, but the cerebellum is well developed. The eyes and organs of balance are typical of vertebrates from fish up. The latter structure is the only "ear" of the fish, with the result that none of these animals hear as it is understood by man.

7. Skeleton. The primitive fish have only a cartilage skeleton, but in the higher fish this is replaced by bone. The vertebrae are advanced over the cyclostome condition in having the separate cartilages fused to form a solid vertebra surrounding the endodermal notochord. In all fish the notochord is constricted by the vertebrae, and in the higher forms is entirely obliterated in the adult.

The class is divided into three Sub-classes.

I. The Elasmobranchii, or "plate gilled" fish, which includes the sharks, skates and rays, and are the most primitive fish still existing.

II. The Teleostomi, or fish with a complete, terminal mouth. It includes practically all the food and game fish-all the fish known to most students, with the exception of the elasmobranchs.

III. The Dipnoi or lung fish. The name, "double breathing", refers to the fact that they have gills and a lung-like swim bladder. At present there are only three genera in the tropical regions of three continents.

Elasmobranchil. The elasmobranchs are characterized by a skeleton made entirely of cartilage; and although the group 
includes the largest known fish, some reaching a length of sixty feet, even these animals lack bone in the skeleton. There are, horrever, bony scales as outgrowths of the skin. These are the forerunners of the dermal or skin plates of the higher groups. The teeth, which are found first in the elasmobranchs, are continuous with the scales, and are modified dermal denticles. The mouth is ventral in position.

The sub-class is divided into several extinct orders, and two recent orders. Of the former, only the type genus of one order will be discussed in this text. This is Cladoselache, an animal that gives an indication as to the evolution of the fins. One of the living orders (Holocephali) may be considered an end group and far from the line of evolution of the higher types. The remaining order, the Selachii, are divided into two suborders, one including the sharks, the other the skates and rays.

The elasmobranchs, like the higher fish and the reptiles, have two methods of reproduction. The eggs are large yolked and fertilized internally. However, some of the selachians secrete a horny shell around the egg and discharge them into the water. These elasmobranchs are oviparous, or egg laying. Other members of the order retain the eggs in the oviducts until the embryo is capable of independent existence, at which time the young fish is discharged into the water. This condition is called ovoviviparous to distinguish it from the viviparous development of the mammals. In ovo-viviparous animals the young depends upon the yolk for its nourishment, and grows by drawing on the food stored in the egg. The mammalian development will be discussed under the heading Mammalia.

The skates and rays are a specialized group, and are of interest to the naturalist as showing the adaptive radiations of the order. An almost complete series can be made showing the course of evolution from the sharks. Beginning with a sharklike skate, these animals have become flattened dorso-ventrally and proportionately widened. In this process the pectoral fins have become fused to the body wall, and the tail has degenerated until it is only a whip-like structure. Specimens of the ray have been captured measuring more than twenty-five feet across. These fish are an interesting offshoot of a primitive stock. 


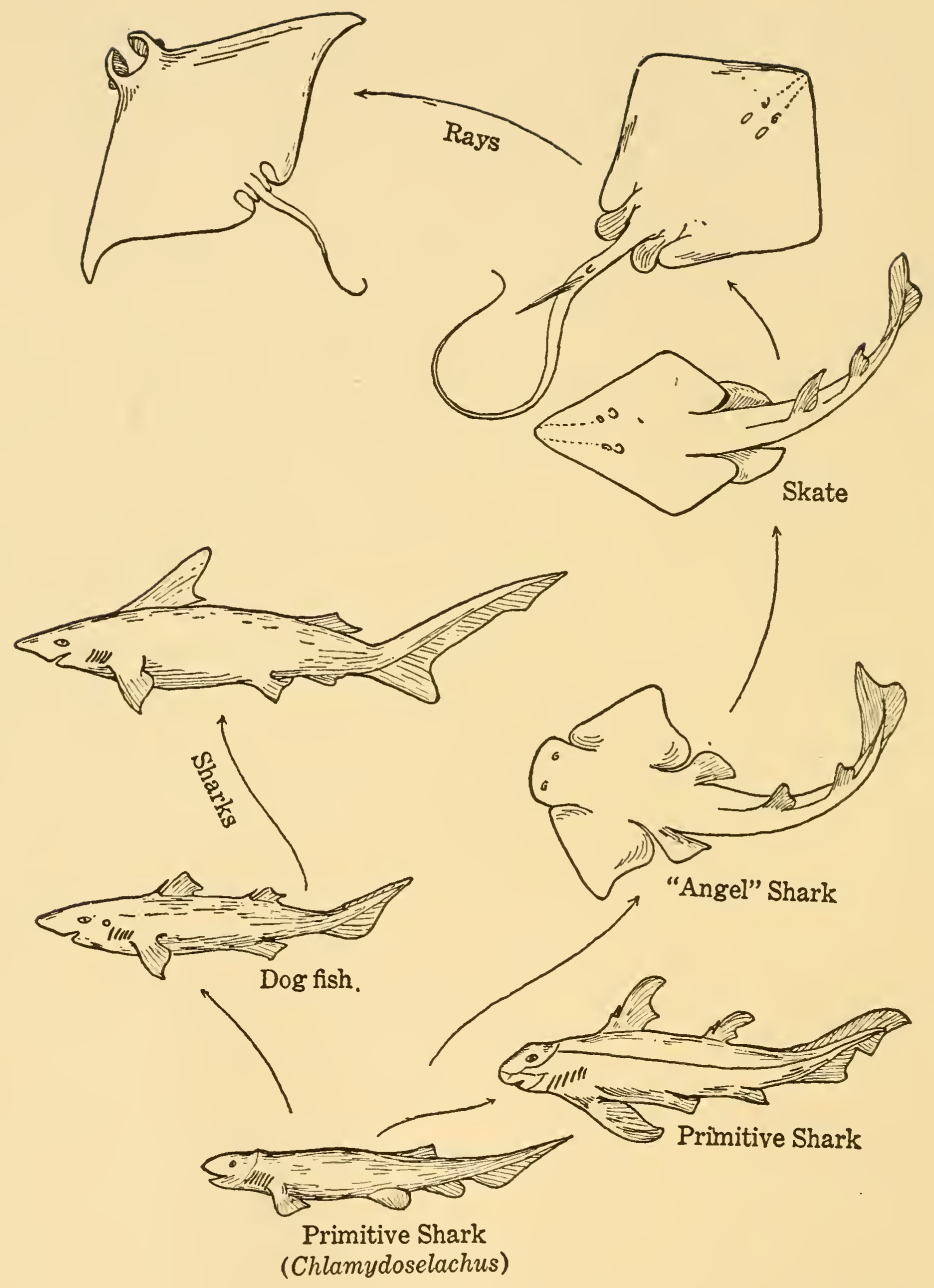

FIG. 9. Adaptive Radiations of the Elasmobranchs. The types illustrated are all recent forms. The arrows indicate the two main lines of evolution and the probable course of specialization. 
The sharks are the most primitive fish known, some of them having six or seven gills. The most anterior slit has become modified in the elasmobranchs and a few higher fish into a small opening, the spiracle. This structure is of importance in the evolution of the land vertebrates. With the exception of these two families, the fish have five gills.

The dogfish, a small shark, is an "epitome of vertebrate anatomy", and is usually used in courses of comparative anatomy for dissection.

1. External Anatomy. The dogfish has a fusiform body adapting it for rapid locomotion in the water. A pointed rostrum projects in front of the mouth; and at the end of the tapering body the vertebral column turns up to form an asymmetrical, heterocercal, tail. The pelvic fins are in the primitive position immediately anterior to the anus; and, with the pectoral fins, are used as balancing organs in swimming. The body is covered with dermal denticles which have a wide basal plate with a spine which projects through the skin to the outside. The cloaca is the outlet of the digestive and urino-genital ducts.

2. Internal Anatomy. The chondrocranium is roofed and encloses the brain. The jaws are not fused to the chondrocranium but are suspended by ligaments and cartilages. The pharynx is large. As the food is swallowed whole the stomach carries on a very large part of the protein digestion, and the absorptive small intestine is highly coiled and fused to form a spiral valve. The oviducts, into which the eggs pass from the ovary, are large and vascular. As the eggs remain in the oriducts until the young have absorbed the yolk mass, the vascular supply undoubtedly assists in respiration and the removal of waste products.

Teleostomi. The name refers to the fact that the mouth of these fish, like that of the higher vertebrates, has the jaws encased in bone, with the upper jaw fused to the skull. This makes the upper jaw immovable and is a definite advance over the elasmobranch condition. In this group the dermal denticles have developed into dermal plates or scales, and in the head region the plates cover the chondrocranium as the dermal bones of the skull. The sub-class is very large and 
ordinal differences will be considered in the following discussion of group characteristics.

Order Chondrostei, the most primitive of the teleostome fish, include the sturgeons and the spoonbill fish. The latter are found in the Mississippi valley and in China. The former are found in the fresh and salt waters of the northern hemisphere. Caviar is the egg roe of the sturgeon, and comes from Russia, the Great Lakes region of America, and Alaska.

The sturgeon resembles the shark in many respects. The rostrum projects in front of a ventral mouth; the tail is heterocercal; the digestive tract has a spiral valve; the skeleton is cartilage; and, in the type genus, the spiracle is retained. The bony plates cover the entire head, and the ordinal name refers to the fact that there is a perfect cartilaginous chondrocranium encased in bone. Posterior to the head the plates are arranged in five rows, one dorsal, and two on either side. Between them, and on the lateral-ventral regions, are many dermal denticles. There are no teeth in the recent forms, though the fossil ancestors had a proper supply.

This group of fish developed a swim bladder, a structure of great phylogenetic interest. The bladder arises in the animal as a dorsal outpocketing of the end of the pharynx, and the connection with the digestive tract is maintained throughout life. It can be inflated or deflated, serving as a hydrostatic organ by lessening the specific gravity of the fish; and in poorly aerated water it has a respiratory function.

Order HoLostei. The holosteans are fish with an entire bony skeleton, but with sufficiently typical characters to set them apart from the following order, although the two merge almost imperceptibly. The most primitive recent form is the Gar-pike (Lepidosteus, not related to the pike or pickerel) which has bony scales covering the body in a tile-like manner. The rhomboidal scales fit edge to edge, and do not overlap as in most fish. The teeth are well developed; the tail is partly heterocercal; the spiracle is only a pit; and the digestive tract retains many primitive characteristics. The swim bladder retains its connection with the gut and has a vascular supply. The Bowfin has over-lapping scales, and in all other structures shows the line of evolution toward the following order. 


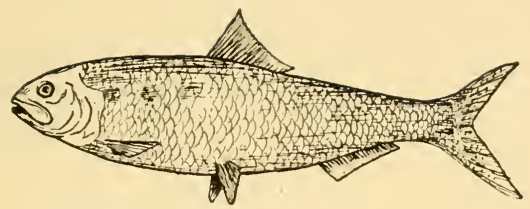

Shad (Teleostei)

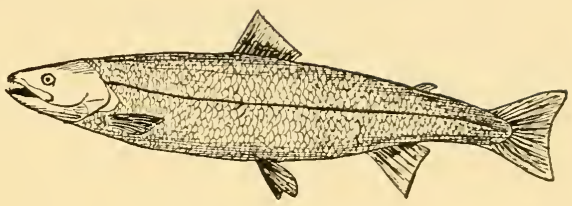

Salmon (Teleostei)
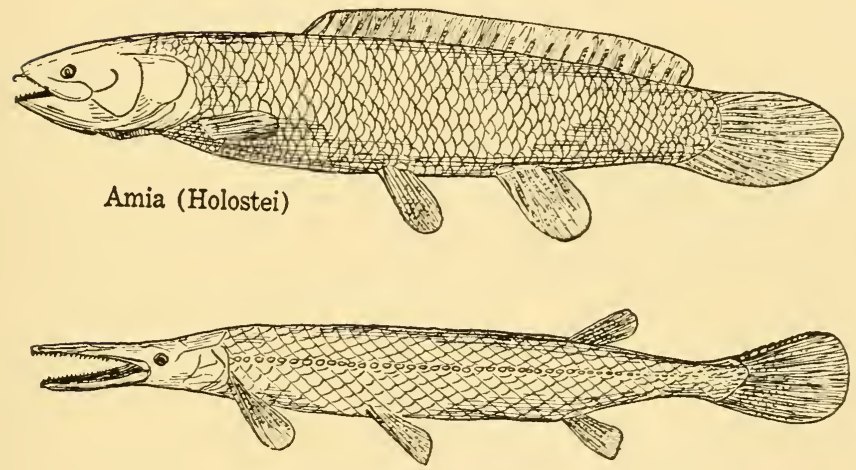

Gar-pike (Holostei)

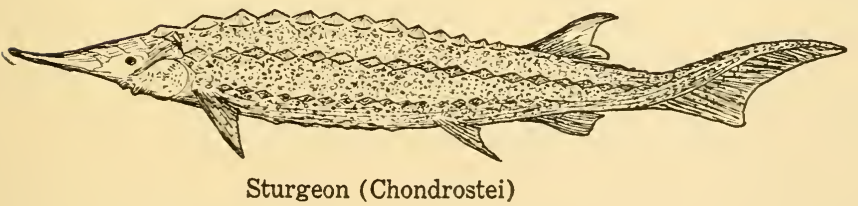

FIG. 10. Types of Bony Fish. The Sturgeon is the most primitive living bony fish. The drawings show the change of the tail from heterocercal to homocercal, and the shift of the pelvic fins to an anterior position. 
Order Teleostei form the largest group of the fish, and express the characteristics which one thinks of as "fishy". They include all the common food and game fish, from the more primitive Tuna to the highly specialized globe-fish and the sea horse. Though they lie entirely out of the line of evolution of the higher

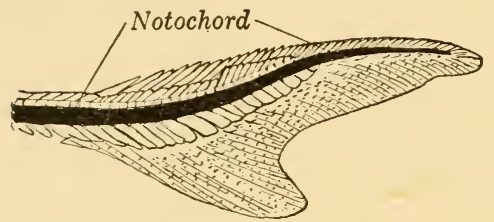

FIG. 11. Tail Skeleton of the Sturgeon. The notochord extends almost to the tip of the tail and the radial cartilages are practically equal on dorsal and ventral sides. A typical heterocercal tail.

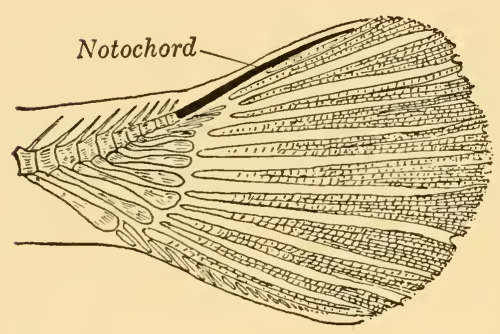

Fig. 12. Tail Skeleton of the Garpike. The notochord has shortened, and the ventral radials have elongated, giving a homocercal appearance.

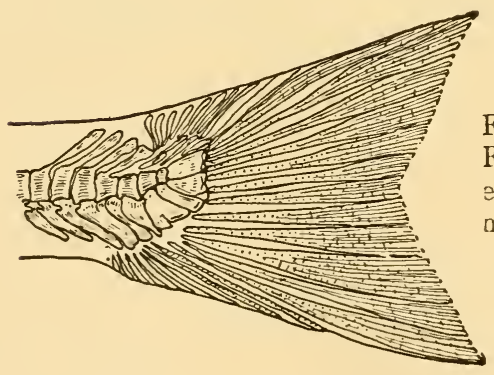

Fig. 13. Tail Skeleton of a Teleost Fish. The notochord has been obliterated and the caudal bones are almost homocercal. Other teleost fishes carry the modification further.

groups, there is no more perfect order for a study of adaptive radiations and specializations. Technically they are divided into thirty or more orders, and numerous sub-orders; and there are more than two hundred families.

The scales typically overlap and have lost the heavy bony structure which is found in the more primitive forms. The tail 


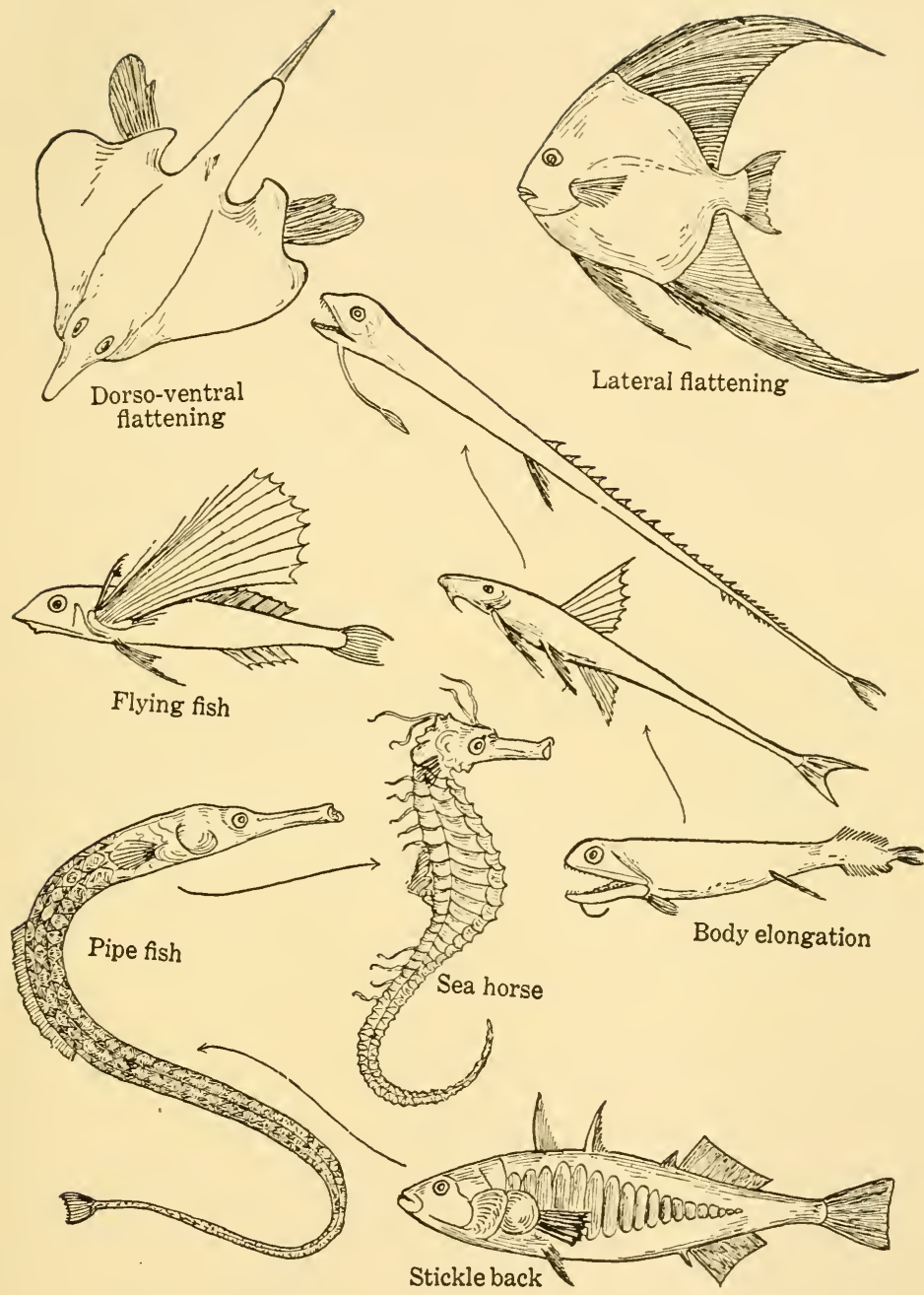

FIG. 14. Adaptive Radiations of the Teleostei. The primitive teleosts resembled the Salmon, Fig. 10, and evolution has proceeded in many directions. Several of the modifications are illustrated. 
is normally homocercal, although it may assume weird proportions in some families. The mouth is terminal and teeth are present except in a few families. In the most primitive family the swim bladder retains a small connection with the gut. In the others the connection is lost and the organ serves only a hydrostatic function.

Order Crossopterygir, which today is limited to a very few genera in the Nile, Niger and Congo rivers, was once a widely distributed and flourishing group. It is of particular interest to the anatomist and evolutionist as it is now agreed that the
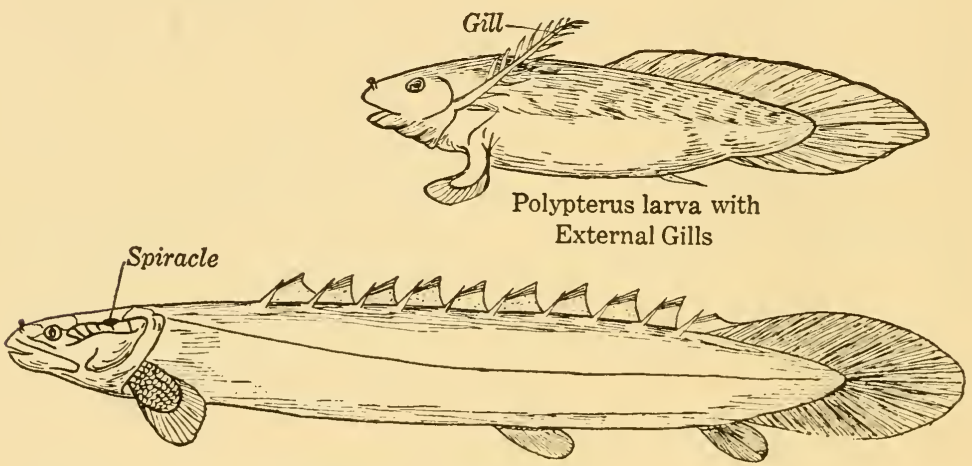

Polypterus (Crossopterygii)

Fig. 15. A Recent Crossopterygian Fish. The larra has the external gill and assumes the foot position typical of the amphibia. The order is characterized by the fleshy lobe of the pectoral fin.

amphibia arose from this order of fishes. In fact, by some anatomists, it is considered the stem form from which all the Teleostomi evolved. The name means "fringe fin", although "lobe fin" is more descriptive of the fin condition. Instead of a fin supported by three parallel cartilages or bones, these animals have appendages with a fleshy lobe surrounded by a fringe of dermal rays. The recent forms have a rather degenerate skeleton in the lobe, but their fossil ancestors had three large bones and several small ones, which are clearly homologized with the humerus, radius and ulna, and carpels of the land animals.

The head plates strongly resemble those of the earliest amphibia. The primitive crossopterygians had bony plates on their 
bodies, although the recent forms have overlapping scales. The spiracle is retained. The swim bladder is a ventral outpocketing of the gut, and may be either one or two lobed. The bladder is highly vascular, the blood supply coming from the last (sixth) gill artery in much the same way that the lungs of the higher forms is supplied.

Aside from the fossil and anatomical evidence, the larva of the recent forms shows relationship with the amphibia. Unlike most fish, the crossopterygian hatches as a larval form with external gills in addition to the normal internal. In this they resemble the amphibian tadpole. Equally as interesting is the fact that the larva settles to the muddy bottom with its front fins drawn under the body, using them as a support, not merely as a balancing organ as does the typical fish. After metamorphosing from the larva to the adult type, the fish makes constant use of its respiratory swim bladder, for its normal habitat is in the muddy rivers and lakes which tend to become de-oxygenated and unhealthy for gill breathing animals. Due to its importance in the scheme of human evolution, frequent reference will be made to this order in the later chapters.

Dipvor. This small group has been given equal rank with the two great sub-classes, although the living representatives are limited to three genera. This is on account of their wide divergence anatomically, and their ancient lineage as a widely distributed, definite, easily defined division of vertebrates. All recent forms live in tropical regions which have seasonal periods of rain and drought, one in Africa, one in Australia, and one in South America. Most anatomists agree that they are a group which has paralleled the amphibia in evolution; a trial, and it appears now largely an error, on the part of nature in evolving land animals.

From earliest times these animals were highly specialized as to teeth and fins, two characters which effectively prevented further evolution toward land life. The eggs hatch as larvae with external gills. During larval life the lungs develop from a ventral, paired, highly vascular swim bladder. These lungs are more lobulated, larger, and more efficient than those of most amphibia. This is an adaptation to the dry seasons during which the 
animals burrow into the mud and aestivate, a condition physiologically similar and seasonally opposite to hibernation. As the water in the lakes recedes the fish burrows into the ground, secretes a mucous capsule around itself, and remains there until the return of wet weather. Normally this is not more than six or seven months, although specimens have been kept in the laboratory encased in dry mud for more than eighteen months without any marked loss in weight. On being broken out of the capsule and placed in water, respiration begins in a few seconds,
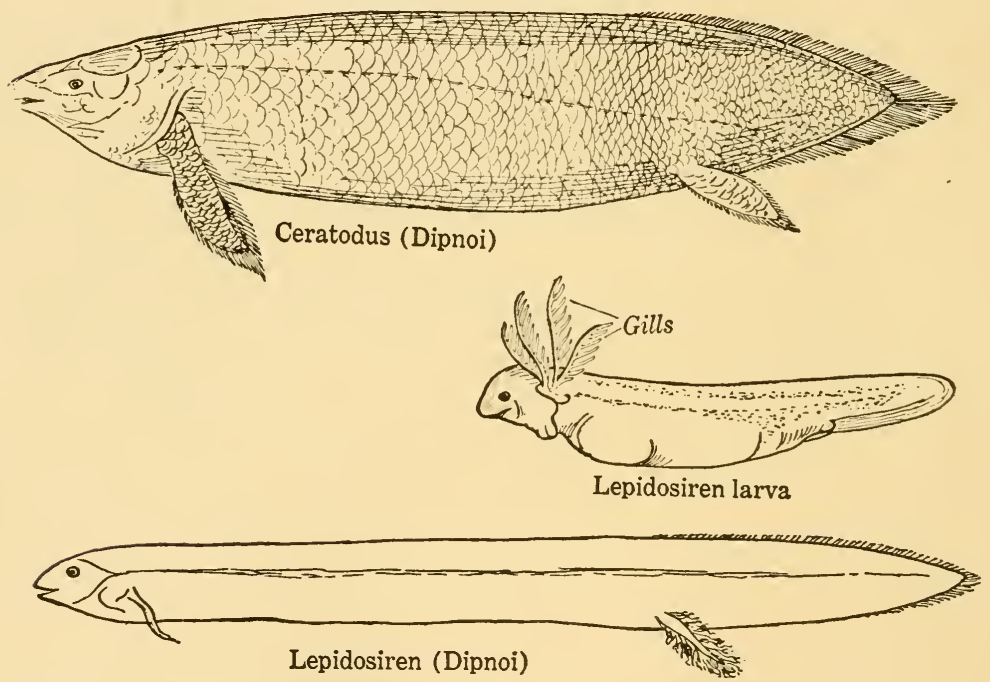

FIG. 16. Recent Lung-fish. Ceratodus is more primitive. Lepidosiren has degenerate fins and an elongated body. The specimen illustrated has the vascular outgrowths which appear on the pelvic fins of the male during breeding time.

and the fish is apparently none the rorse for the experience. These modifications have gone so far in the South American form that the animal is incapable of supplying itself with sufficient oxygen through its gills, and drowns when kept immersed in water. During the breeding season the male of this genus (Lepidosiren) develops accessory gills on its pelvic fins, and is thus able to remain under water and guard the eggs. 
Evolution of the Fish. The student should keep in mind that no living group of animals can be the direct ancestors of another. From this standpoint "higher" and "lower" are rather meaningless. The terms are based on the human concept of superiority, and to a large extent refer to the evolution of the nervous system; although we may say that "higher" animals are those which have evolved farthest from the fundamentai, primitive characteristics.

We may also logically speak of the fish ancestors of men, not meaning any living group, but some common ancestral type which evolved in several directions. One group of fish became better adapted for certain aquatic conditions, another for land conditions; or, stated differently, a group which was generalized had a far better chance of developing in several directions than did a more specialized type.

All the above conditions are abundantly illustrated by the fish. The earliest ancestor was an amphioxus-like chordate. From this animal numerous varieties developed, the recent Amphioxi having remained most nearly like the ancestral form. Another variation from the original ancestor became more cyclostome in structure, and from this group new variations appeared. Those which most resemble the ancient stem form are the Cyclostomes, while other groups which added new characters enabling them to cope better with environmental conditions evolved into primitive fish.

All the earliest known fossil fish belong to the elasmobranchs, and there again we see evolution proceeding in many directions. The generalized sharks were not only capable of surviving in more varied environments, but were more liable to develop new mutations having a survival value.

Some of the adaptations have been mentioned. Those which tended toward respiration and locomotion on land, as did the specializations of the Crossopterygii, naturally opened up an enormous area as a habitation for the vertebrates. They were poor fish but excellent ancestors; and therefore Darwin's "survival of the fittest" has been modified to "survival of the best adapted for a particular environment". 


\section{CHAPTER V}

\section{AMPHIBIA}

The Class Amphibia consists of those four-footed animals which have a double life, one adapted for water living in the larval stage, and an adult phase when they take on the characteristics of land animals. The newts, salamanders, frogs and toads are representative of the class. The living examples make a very homogeneous group, although the earliest forms resembled the Crossopterygii on the one hand, and the reptiles on the other. They are in every respect more closely allied to the fish than to the reptiles. The most important difference from other land animals lies in their embryology, the eggs always being laid in water or moist places, where they hatch as gilled larvae.

The first amphibia varied from their fish ancestors in several respects, most largely in the development of four walking appendages with toes. The larva retained the external gills and an internal anatomy so like their ancestors that the tadpole is essentially a fish in structure and mode of life. At metamorphosis the early amphibia evidently lost their gills and breathed with lungs, but the fossil evidence indicates that they remained mudliving animals. The bones of the skull and the dermal covering of the body were essentially similar to those of the fossil Crossopterygii. The spiracle, which is found in the ancestral fish, is modified in the amphibia into the middle ear. As can be seen in a frog, the middle ear is a slightly enlarged cavity lying next the internal ear, and covered on the outside by a skin membrane (the tympanum or ear drum). The wide inner part opens directly into the pharynx.

The recent amphibia can be identified by their smooth skin, usually well supplied with mucous glands; and, except in forms with degenerate legs, by four front toes and five on the hind legs. 
Their nervous system shows definite advances over the fish type, except for the cerebellum which is poorly developed in recent amphibia. The vascular system has several important structures added which in later evolution became of supreme importance. However, the system is poorly adapted for land life, and the steady disappearance of species as soon as more efficient land
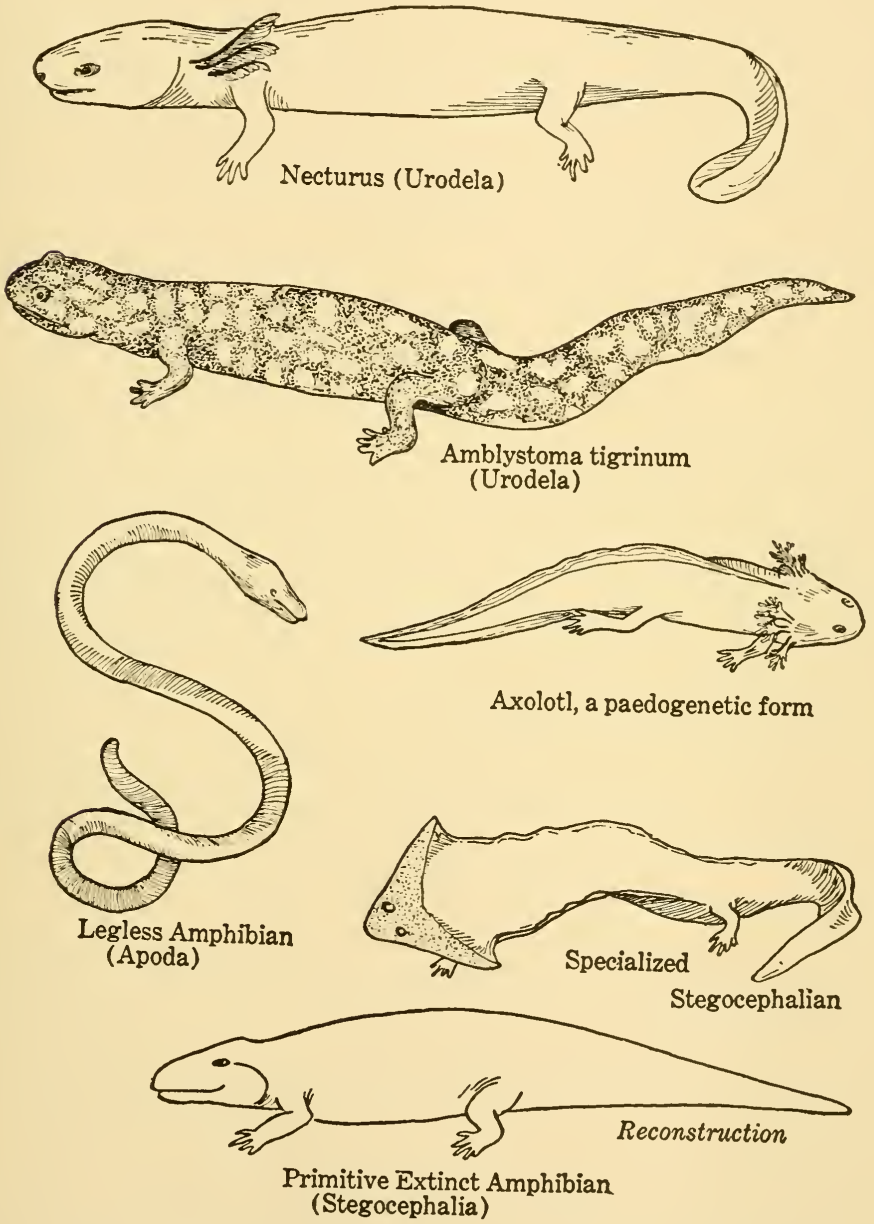

FIg. 17. Types of Amphibia. The extinct Stegocephalia gave rise to the recent orders. 
structures developed shows that the class was poorly adapted for land existence, and could not stand the competition with the reptiles which evolved from them.

Order Stegocephalia. For simplicity these fossil amphibia have been classified as one order. The Stegocephalia vary widely in structure, the earliest known form (Archegosaurus) having a skull with several bones found nowhere else but in the fish. During the years when there was no competition on land the amphibia multiplied and evolved in many directions. They were well encased in an armor of bone, strong enough to protect them from each other and from any predaceous fish; but too heavy to permit of great activity. The evidence indicates that the primitive amphibian foot had six toes, which soon became reduced to the typical five of the land vertebrates. Some of the Stegocephalia have a further reduction on the pectoral foot, like that of recent forms.

Order ApodA. These are legless amphibia. Their burrowing habits are their only defense against more active animals and came with the loss of appendages. In fundamental structure they are the most primitive of living amphibia. Their skull bones are less modified than those of other orders; they have minute dermal scales beneath the skin; and their development is an important link in comparative embryology. The order is rather widely distributed in the sub-tropical regions of the earth.

Order Urodela. The urodeles, as the name implies, are the tailed amphibia, and in body shape are more nearly like the Stegocephalia than any other recent order. They may be completely aquatic, mud-living, or intermediate in their habits. None survive in very dry regions, nor in the tropics.

The largest living amphibian is Cryptobranchus of China and Japan which attains a length of more than six feet. A closely related species of the same genus inhabits the Mississippi valley. Other large forms live in the southeastern part of the United States. These are very specialized, particularly as to gills and appendages.

Most urodeles are small and lack gills in the adult. These are the newts and salamanders which can be found in streams and muddy places in most parts of the Northern Hemisphere. Some of the genera are highly modified, one or two lacking both gills 
and lungs and breathing entirely through the skin. Others retain the external gills throughout life.

Certain of the urodeles remain in the larval condition, reaching sexual maturity without metamorphosis. This permanent

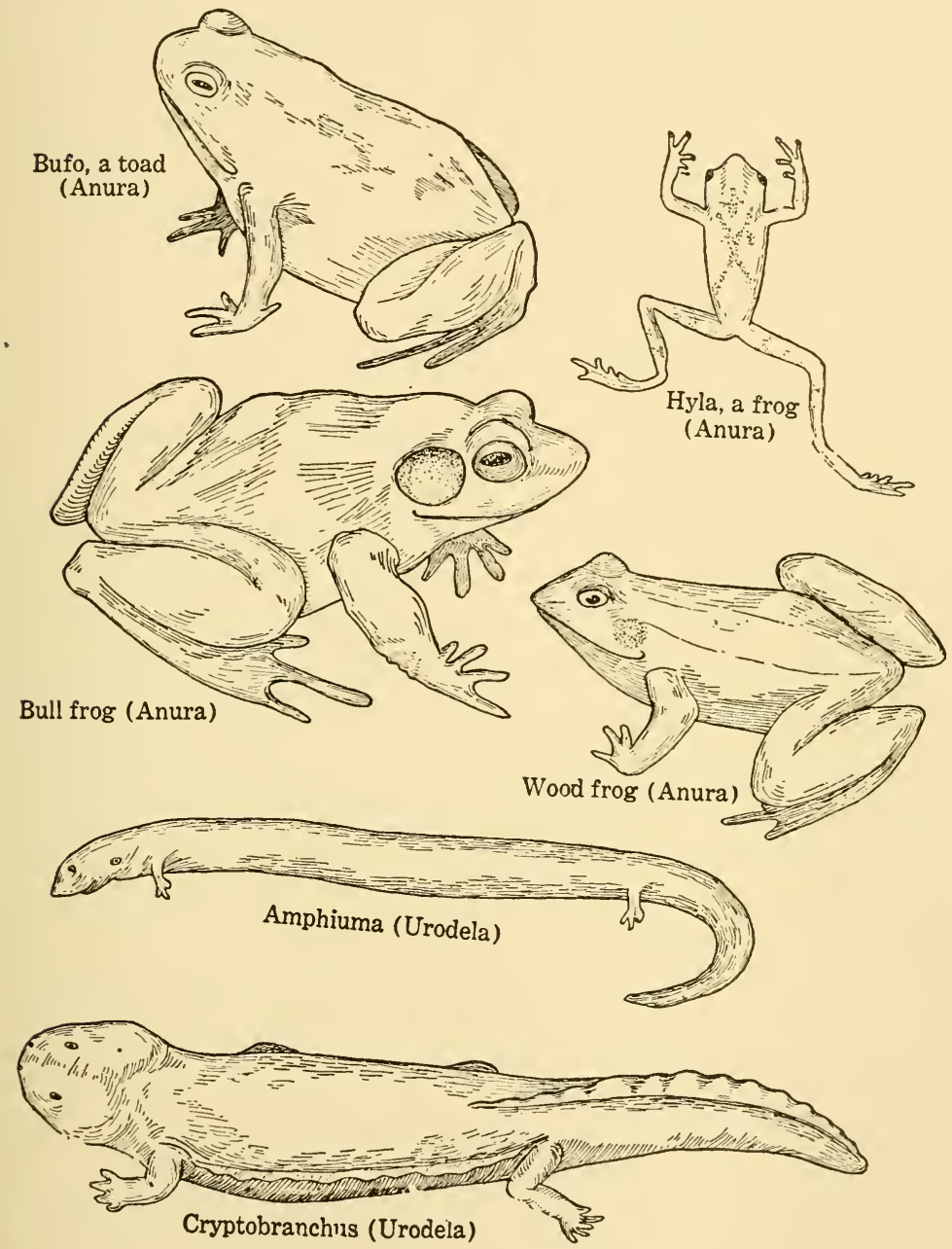

FIa. 18. Types of Amphibia. Cryptobranchus and Amphiuma are the largest living American amphibians. The frogs and toads are highly specialized forms. 
larval condition is called paedogenesis and was discovered in the Axolotl of Mexico. The animals had been classified as a separate genus and family, but when the environment was changed the young metamorphosed into a well known salamander (Amblystoma). Many students of the subject today interpret the retention of external gills in other forms as paedogenesis, although experiment has so far failed to confirm the theory.

Order Axura. The tailless amphibia are the frogs and toads, and are distributed throughout the world. They are in many respects the most specialized living amphibia. Most of the genera are capable of living their adult life away from water. This is particularly true of the toads, whose warty skin, containing relatively few mucous glands, adapts them for life in semi-arid regions. But although the adult may live in her burrow on a dry hillside, the eggs are invariably laid in water where they hatch as tadpoles. In the smaller toads the Iarval life is very short and they will hatch into tiny toads in a few weeks, giving rise to the myth that it rains toads. Other myths relating to their longevity are also without foundation. They may live for long periods without food or water, but to believe that they are found embedded in solid rock demands credulity rarely found in a scientist.

Erolution of the Amphibia. Almost from the beginning of amphibian history two lines of evolution are apparent. Some families retained rather generalized amphibian characteristics, and probably gave rise to the amphibia of today; other families became highly specialized with enormous heads, or other structures which eventually rendered them unfit for survival in a changing environment; and others assumed characteristics which appear reptilian. Some of these pro-reptilian forms were undoubted amphibia (Eryops, for example) for the skeletons of gilled larvae have been discovered.

Embryology of the Frog. The frog has a very generalized development, more primitive than the recent fish. For this reason it is selected for discussion in most texts, as it logically follows the developmental processes of Amphioxus. The Apoda have a development which verges toward the reptilian and, as was men- 
tioned, is used to illustrate an intermediate stage between the amphibia and the higher vertebrates.

The student is undoubtedly familiar with the processes of maturation of the egg and sperm. The mature eggs of frogs and toads are fertilized by the male at the time of deposition, before the thin coating of gelatinous material swells by the addition of water. The toad lays its eggs in a string, the frog and salamander eggs are laid in masses. The frog egg is spherical, about one millimeter in diameter, and has more inert yolk material than is found in Amphioxus. These yolk granules are also more concentrated at the ventral pole. The plans of the first two cleavages are dorso-ventral, and that of the third is transverse. This is similar to the first three clearages of Amphioxus, with the slight exception that the third is more asymmetrical, the dorsal four cells of the frog being decidedly smaller than the ventral. The general principle that a cell loaded with inert matter cleaves more slowly than one with relatively more protoplasm has been stated; therefore in the frog clearages after the fourth proceed more rapidly in the dorsal region of the embryo. The result is a blastula with the segmentation carity entirely in the upper half of the egg.

Gastrulation in Amphioxus was described as due to both invagination and the overgrowth of the rapidly dividing dorsal cells. In the frog invagination is largely suppressed, and the latter process greatly increased. This overgrowth is more rapid at the anterior end. The student can visualize the process if he will think of the equator of the egg remaining static while the rapidly dividing upper cells flow ventrally as a double layer, more rapidly on one end than the other. As this two-layered structure approaches the posterior pole the opening tends to constrict; and when gastrulation is complete, only a narrow aperture is left as the blastopore. From this it is clear that most of the endodermal lining of the archenteron has been derived from the dorsal pole cells. The remainder of the endoderm developed from the invaginating ventral pole cells. During gastrulation the segmentation cavity was practically crowded out of existence.

At the completion of gastrulation the process of elongation begins, and then the dorsal neural groove is formed. The hollow 
nerve cord develops by the dorsal and median growth of the neural folds; but the frog differs from Amphioxus, and agrees with all other vertebrates, in having the folds meet at the middorsal line before the neural plate breaks away from the epidermal cells.
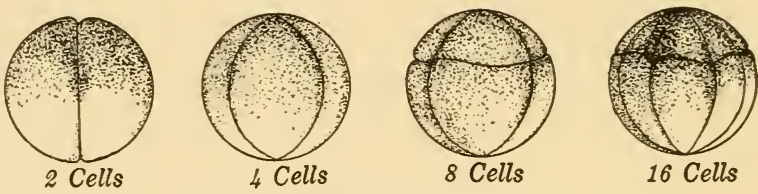

\section{Cleavage Stages of Frog's Egg}

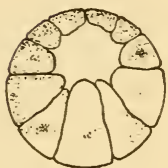

32 Cells

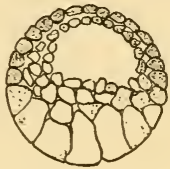

Blastula

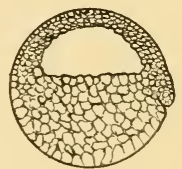

Beginning of Gastrulation

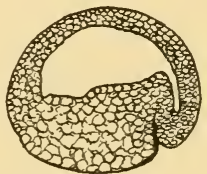

\section{Longitudinal Sections of Early Embryo}
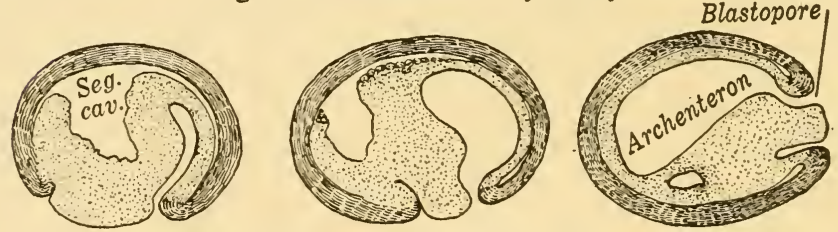

Longitudinal Sections: Completion of Castrulation

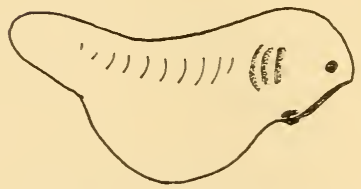

Tadpole at time of hatching
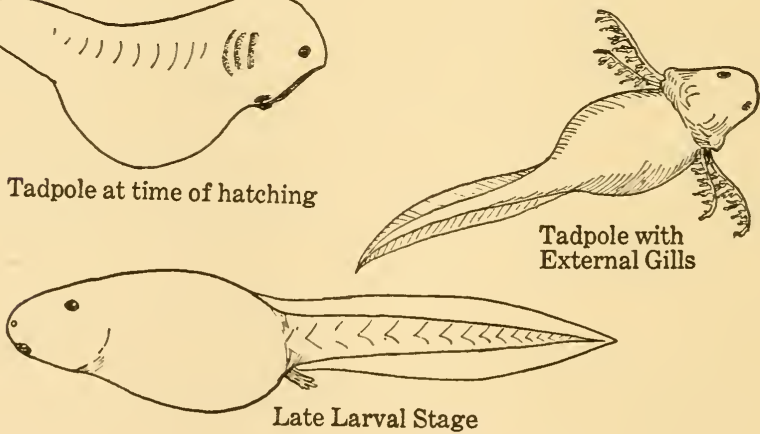

FIG. 19. Embryology of the Frog. The diagrams show the holoblastic cleavage and gastrulation. Gastrulation is almost entirely an overgrowth of the dorsal pole cells. 
The stages in the development of the notochord are telescoped. Instead of infolding as in Amphioxus, the endodermal cells multiply rapidly along the dorsal line of the archenteron and split off to form the continuous rod of notochordal tissue. The mesoderm also goes through a compressed development. Unlike the metameric enterocoels of Amphioxus, the frog mesoderm arises as a proliferation of cells on either side of the notochord, the continuous band of tissue soon dividing into metameric blocks. A cavity develops within each mesodermal mass, homologous with the enterocoelic cavities of Amphioxus. Later development of the organs is sufficiently similar to that of Amphioxus not to need further discussion in a text on anatomy.

As the embryo grows the nervous system and the organs of special sense develop. The mouth breaks through, and the embryo, now a young larva, ceases to depend upon its yolk mass for food and begins eating. Up to this time respiration has been through the epidermis to the blood of the already functioning vascular system, but as the larva develops in mass gills develop in correlation with changes in the branchial arches. These gills are external and three in number. In time the external gills are lost, internal ones having developed within the gill slits, and the frog tadpole pursues a fish-like existence in the water. Hind legs make their appearance, getting larger as the tadpole grows older.

The larval stage varies in length in different species, lasting from six weeks in the wood frog to as much as three years in the bull frog. Temperature causes a variation in time, heat speeding metamorphic processes. When the tadpole approaches metamorphosis it stops eating; the front legs, which have been enclosed in an atrium analogous with that of Amphioxus, break through; the gills are lost and the animal makes more use of its lungs; and the tail is absorbed and used for food. When metamorphosis is complete the young frog hops out on land, weighing approximately half as much as did the tadpole. From this time until maturity development is only a matter of growth and differentiation of certain organs, as in the land vertebrates. 


\section{CHAPTER VI}

\section{REPTILIA}

THE reptiles are represented today by the turtles, alligators, lizards, snakes, and a small group of New Zealand reptiles. These are divided into five orders. The numerous fossil orders and the recent reptiles form four distinct sub-classes. Three of these are represented today. Most of the reptiles disappeared after the mammals arose and the reptiles ceased to rule the land areas of the earth. Reptiles can be identified in the field by their ectodermal scales which give the animal a rough feeling when the hand is drawn from the tail toward the head. There is no truth in the belief that snakes and lizards are slimy, their skins being remarkable for the lack of integumentary glands.

Anatomically the living reptiles vary widely from each other, and from other living groups. The great gulf which separates amphibia from reptiles is based upon their embryology. All reptiles lay Iarge yolked eggs (which may be retained in the oviducts until time of hatching from the egg membranes) and the young begins life as a small adult. There is never a larval period; and though the reptiles, like all other vertebrates, have gill slits during the embryonic period, there is never a sign of gills. Embryonic respiration takes place through the shell, and the blood is oxygenated through a specialized breathing structure, the allantois, which is an outpocketing of the posterior end of the primitive gut and disappears at the time of hatching. Reptiles also develop an amnion, an enveloping, fluid-filled, protective embryonic membrane. This is in correlation with their land life, the surrounding fluid acting as a buffer against any chance blow and preventing drying of the embryo. These membranes are also found in the birds and mammals, with the result that all three of the higher classes of vertebrates are included together as the Amniotes. 
Sufficient identification of the class Reptilia is: (1) the covering of ectodermal scales; (2) large, yolk laden eggs; (3) a variable temperature, depending to a large extent on the environment; and (4) the allantois and amnion. Other differences will become clear in later discussions.

For a study of mammal evolution, only the first three of the following orders are necessary. The others will assist in understanding the general scheme of vertebrate evolution. The first order is usually studied in anatomy courses as representative of the earliest reptiles. The other two are extinct ancestral stocks from which the mammals evolved.

Order Chelonia. The turtles, tortoises and terrapins are the most primitive, and at the same time the most superficially specialized of living reptiles. They are easily identified by their bony covering, forming a dorsal and a ventral "shell". This is composed of dermal plates which become fused with the ribs, and serves as a protection to otherwise inefficient, lethargic animals.

The skull shows relationship with the earliest reptiles, and this is corroborated by the simple development of their vascular and nervous systems. The group, in their present form, is very ancient; and during their history they have become adapted to almost every environment. Some inhabit dry, sandy regions, their burrows giving them the popular name of "gophers"; others, like the Box Tortoise, are found in more or less moist regions; the small pond turtles and the terrapins are largely aquatic; and the Sea Turtle is so highly modified for life in the ocean that it rarely, or never, leaves the water except at breeding time. In this the reptiles reverse the breeding habits of the amphibia. No matter how specialized a turtle is for aquatic life, it always goes to the land to lay its eggs. The degree of specialization for water life can, within limits, be determined by the cross section of its shell: the deeper the shell in proportion to its width, the greater its adaptation for land; and conversely, the flatter the shell, the more modified for water.

In size the turtles vary through equal limits. The largest living specimen is the Galapagos tortoise which is found only on the barren islands off the coast of South America. But even this huge chelonian is dwarfed by some of the fossil specimens which have been found in India and other regions. 
The breeding habits are rather consistent, all turtles laying their eggs in the sand and depending on the sun's heat to hatch them. There is no evidence of care of the young, the newly hatched animal having to depend upon its own resources for food and protection.

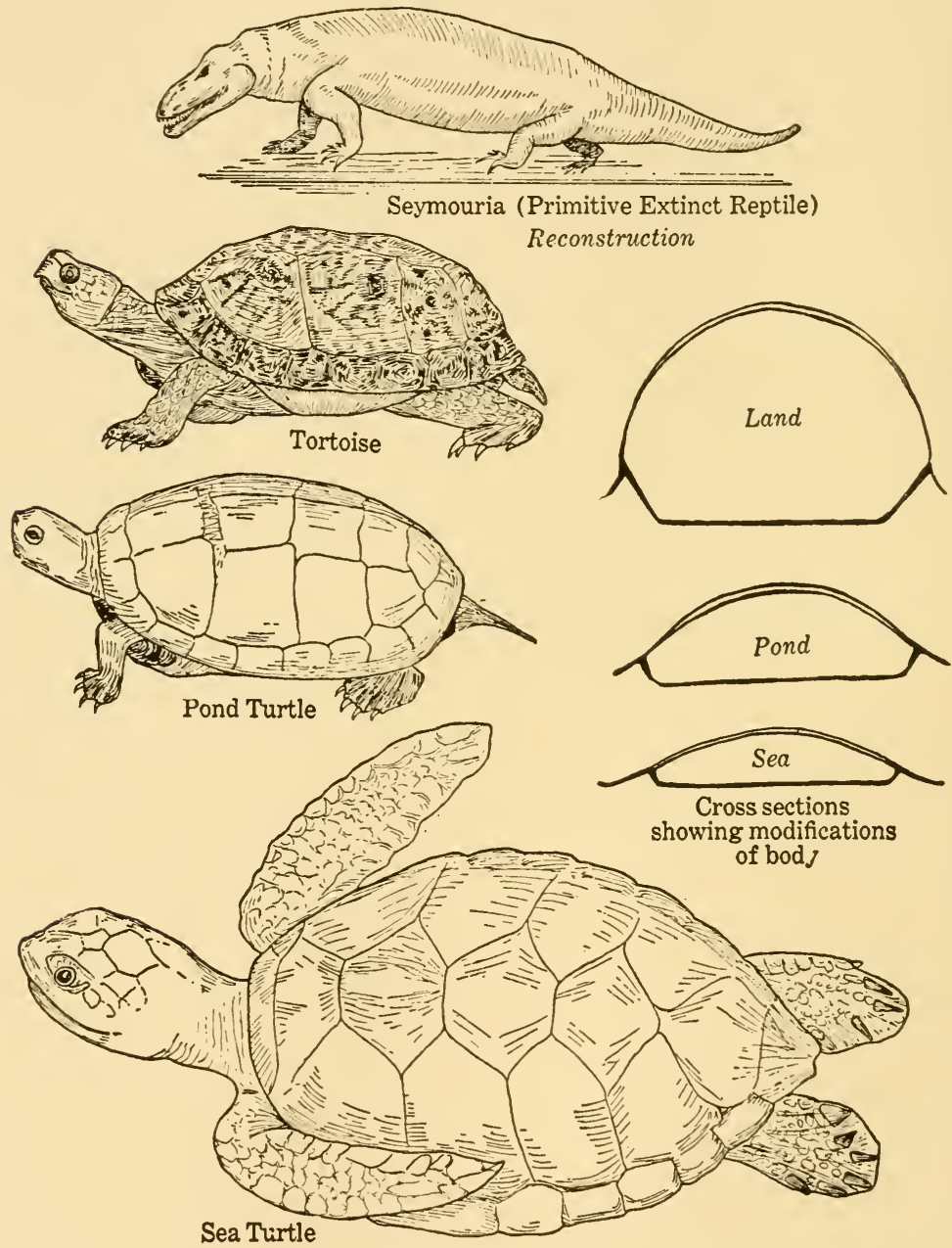

FIg. 20. Anapsid Reptiles. Seymouria (at top) represents the most primitive reptiles. The turtles and tortoises are specialized representatives of the Sub-class Anapsida. 
Mamalatlike Reptiles. These animals are all extinct and known only as fossils. Two orders are recognized, which are classified together as the sub-class Synapsida; and from the standpoint of human evolution are the most interesting of the
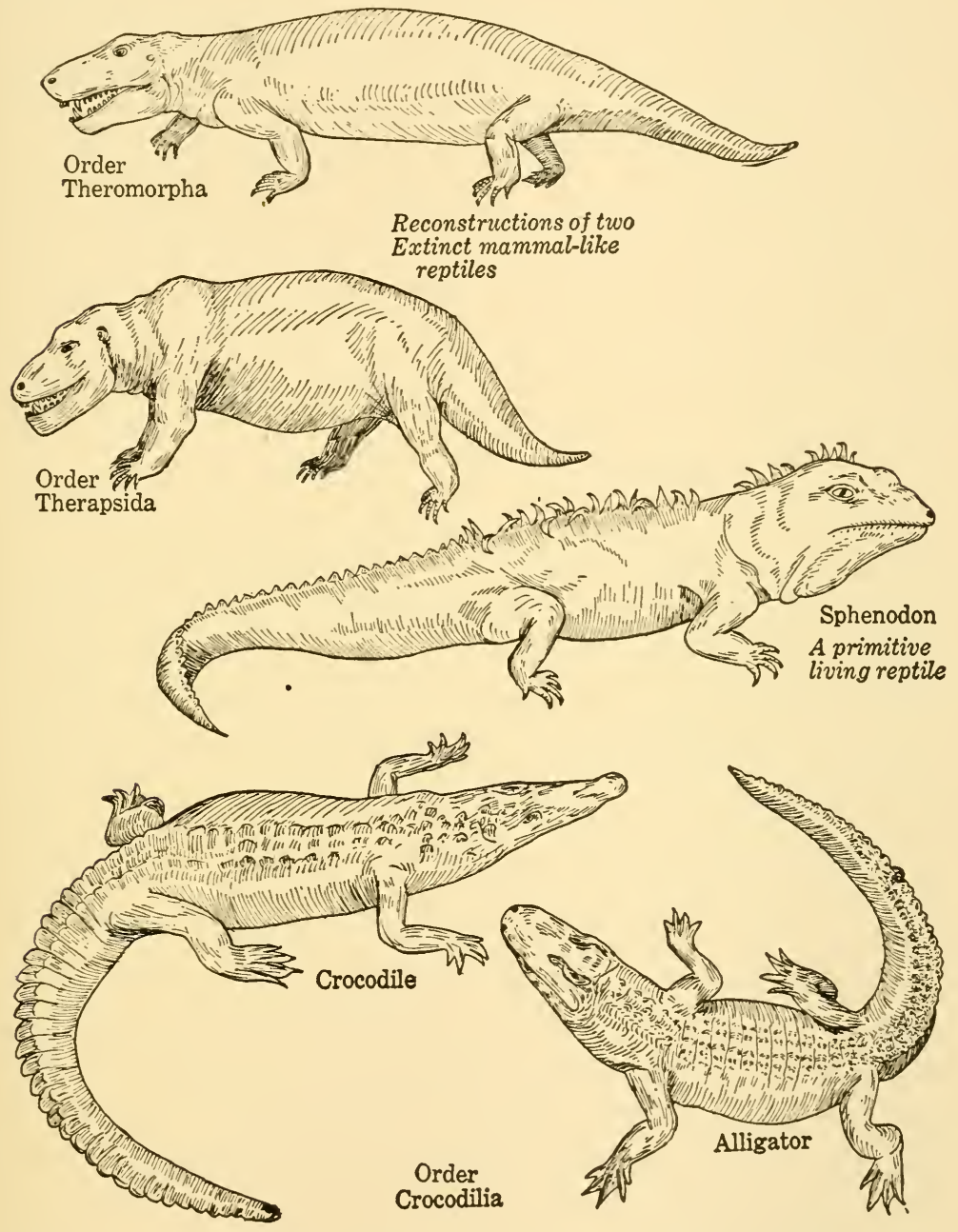

FIG. 21. At the top are extinct mammal-like reptiles (Sub-class Synapsida) which gave rise to the Class Mammalia. The other three are recent Diapsid reptiles. 
reptiles. They branched directly from the early type, of which the turtles are the only living representatives. Some of these synapsid reptiles carried their legs straighter under the body, not in the typical reptilian position, and the skull assumed mammal-like proportions. Skulls of these orders are illustrated on page 136 .

Order Theronorpha. The Theromorpha were reptiles of more or less "mammal form". It is the more primitive of the two orders of synapsid reptiles.

Order Therapsida. The mammal-like characters which showed their beginning in the former order were carried to their logical conclusion in the Therapsida. In size they varied from that of a rat to animals as large as a bear. The legs were held in the mammal position, with the elbows against the body instead of at right angles; and the skull was almost completely mammalian. By comparison with existing orders of reptiles and mammals, they are much nearer the latter than the former.

Evolution of the Reptiles. The first reptiles had a body form resembling a shortened lizard and came directly from the amphibian stock. With their adaptations for land life they soon migrated widely and began their own adaptive radiations as the masters of the earth. These early ancestral reptiles and the turtles together form a sub-class, the Anapsida. From this primitive stock arose three lines of evolution, giving rise to the three other sub-classes of the Reptilia.

1. The Synapsida, or mammal-like reptiles, are known from slightly earlier times than the following groups, although it is probable that the three lines arose almost synchronously.

2. The Parapsida include the snakes and lizards of the present. Several fossil orders are known. The snakes are the most specialized living reptiles.

3. The Diapsida include the Crocodilia (crocodiles and alligators), Rhynchocephalia, and the extinct pterodactyls and dinosaurs. In the great number of forms which have evolved, this is the largest of the reptilian groups, and the dominant animals during the Age of Reptiles. The birds (Class Aves) evolved from a primitive diapsid stock.

Order Rhynchocephalia. The order is represented today by 
one genus (Sphenodon) found in New Zealand. It is an ideally generalized reptile, having been described as a "living fossil". In its skeletal structure and body shape it is the last remaining specimen of a lost race.

Order Crocodilia. This order includes the alligators of the United States and China; the crocodiles, which are distributed throughout the warmer regions of the earth; the caimans of Central and South America; and the gavials of southern Asia. These animals are carnivorous in their habits, and in certain regions are dangerous to man. This is particularly true in the Ganges River.

This is also an ancient order, related to the dinosaurs. The skull and bony skeleton are generalized; the back is well supplied with dermal plates which are hidden under the ectodermal scales; and the soft anatomy is typically reptilian, though highly modified. The brain is the best developed among the reptiles, and the heart is four-chambered.

Their breeding habits are much like those of the turtles, the eggs being laid in nests near the water. The American alligators build large nests of sticks or mud, and after the eggs are laid they are covered by the mother and left for the atmospheric heat to hatch them. They grow slowly after hatching, and have a long span of life. This characteristic of growth, coupled with the value of their skin for leather, has rapidly depleted their ranks.

Order Lacertilia. The lizards, which are included in this order, are so nearly related to the snakes that they are often classified together. Most of the lizards have four legs with five toes on each foot, although some genera have degenerate legs or lack them altogether. A legless lizard can be distinguished from a snake by its eyelids, which are lacking in the snakes, and the small ventral scales. The smaller lizards are popularly confused with the salamanders, but their scales and their rapidity of motion make identification easy.

Lizards are either oviparous or ovo-viviparous (page 37 ). With one exception they are non-poisonous, the genus Heloderma (Gila Monster) of Mexico and the southwestern part of the United States having poison glands. There is wide disagreement as to the violence of their poison, but it is doubtful if their 
bite would be fatal to man. The ability to change color is another characteristic of several genera. The best example of this is the chameleon of Europe, Asia and Africa. The small green lizard (Anolis) of the southern United States has similar, although less marked, ability to change under differing environ-
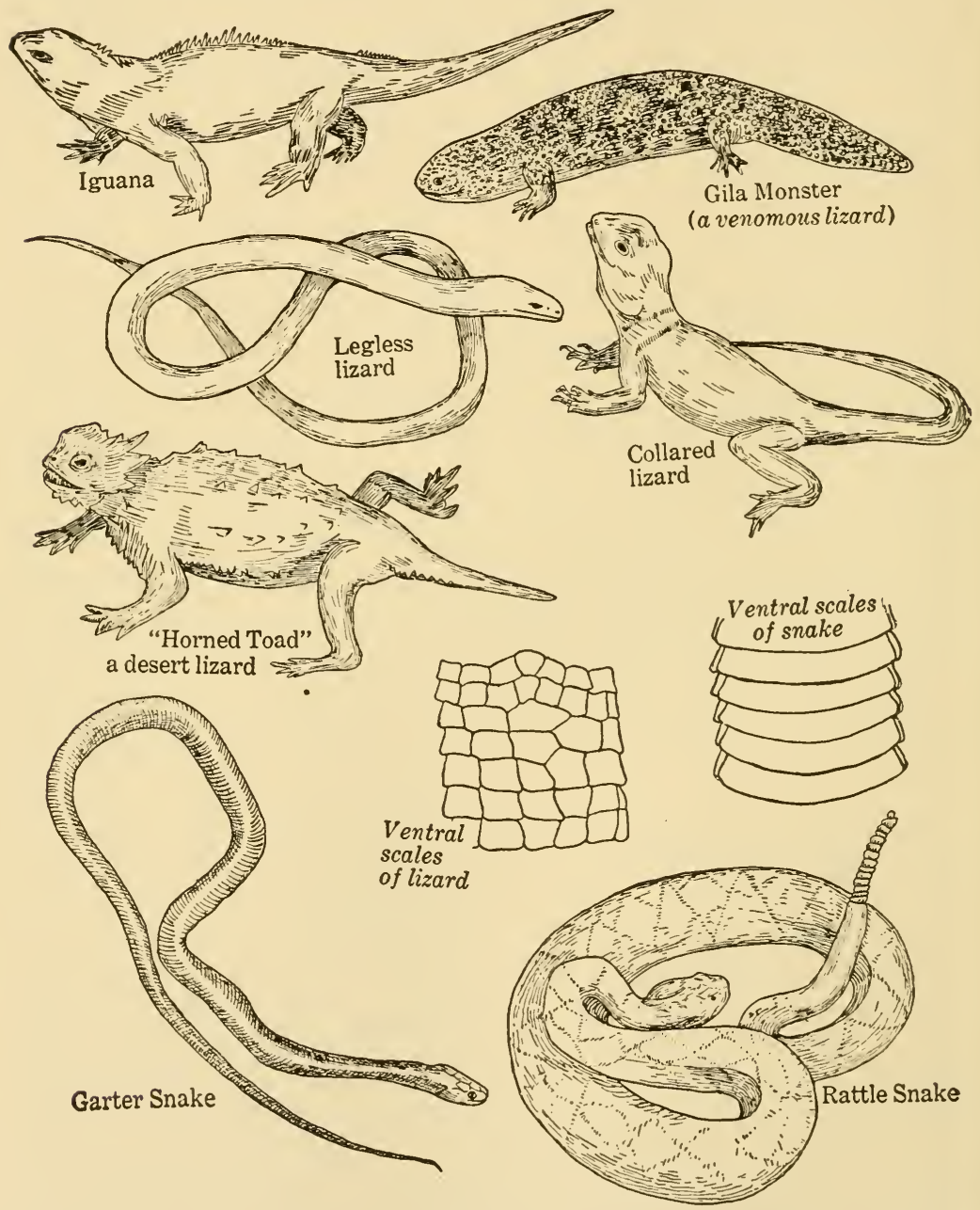

Fig. 22. Types of Lizards and Snakes (Sub-class Parapsida). The lizards show the specialization of the order toward leglessness, bony armor, protective poison, and the running modification. 
mental conditions. The mechanism is similar to that of the tree frogs, depending upon the shrinkage or expansion of the pigment cells.

In size the lizards range from a few inches to several feet; a species recently captured in the Malayan Islands measuring more than twelve feet. They are carnivorous in diet, the smaller ones depending upon insects, the larger eating small mammals or birds, particularly the eggs of the latter.

Order Ophidia. The snakes are legless reptiles. Most genera have even lost the girdles, although the boas and pythons have minute vestiges of hind legs. They are all elongated in body shape, and their skeleton and internal organs are highly specialized for the condition. The long ribs are attached to the ventral plates. Locomotion is attained by the motion of the ribs and by lateral coiling, bracing, and pushing forward. There are no records of snakes moving by dorso-ventral coiling.

The skull is the most specialized among the reptiles. The bones of the mouth and skull are so arranged that the jaws can be stretched downward and laterally to enormous limits. A small boa, with a transverse head measurement of a centimeter, is able to swallow a grown mouse. The soft anatomy is almost as modified. Usually there is a single, highly elongated lung, and the digestive glands and tract are built on the same long lines. The skin has few glands, but most snakes have anal glands which secrete fluids of such distinctive odors that a number of genera or families can be identified by the scent. Like the lizards, the snakes are both oviparous and ovo-viviparous, the latter having given rise to the myth that snakes swallow their young in times of danger.

The poison glands are highly developed in a number of snakes. In correlation with this there are modifications of certain teeth, the fangs. These may have simply a groove down one face, or the edges of the groove may grow over to form a hollow tube. The glands lie in close contact, and usually open into the cavity of the tooth. The result is a natural hypodermic syringe, the poison being forced under the skin. There is no sure rule for telling poisonous from non-poisonous snakes except by close inspection, and this is often inconvenient in the field. In the United States the only poisonous snakes of any importance are the rattle- 
snakes (Crotalus); the "copper head" and the "cotton mouth moccasin", the last two belonging to the same genus (Agkistrodon).

The smallest snakes are only a few inches in length, the largest more than thirty feet. The latter are the python of the Old World, and the anaconda of America. Both are tropical in range, and not poisonous. They throw coils around their prey and constrict until the animal is dead.

Embryology of the Reptiles. Fertilization in the reptiles is always internal, and takes place before the membranes and shell are laid around the yolk. As the egg approaches maturity in the ovary yolk granules develop, and when the egg is mature it is large and heavily loaded with yolk. This inert material is centered at the ventral pole, with the active protoplasm only a small drop located at the dorsal side. It is apparent that the protoplasm cannot exert sufficient force to cleave the entire mass, and the result is that the cleavages are limited to the drop of protoplasm. This type of cleavage is called meroblastic, as opposed to the holoblastic cleavage of eggs which divide entirely, as illustrated by Amphioxus and the frog.

The first and second cleavages cut the protoplasm, or germinal disc, at right angles to each other. But the protoplasm is so thin that transverse cleavage is impossible, and the third cleavage is in the same direction as the first two. The result is that the eight cells are in one horizontal plane. Future divisions continue to cut the disc, until a blastula is formed as a flat plate of cells spread out over the yolk. The student can visualize this and later processes by breaking a fertilized hen's egg and looking for a white spot about three millimeters in diameter on top of the yolk. If the slightest bit of red appears it shows that the blood vessels have developed and the embryo is fairly well advanced. The average kitchen is an excellent embryological laboratory if a hand lens is available.

Gastrulation is due to the ventral growth of cells at one point on the blastula. Comparing Amphioxus and the frog, it will be seen that gastrulation in the latter proceeds almost entirely by the overgrowth of dorsal pole cells, invagination of the ventral cells playing a minor part. The reptile and bird have carried the 
process to the extreme limit, the ventral yolk being non-cellular and serving only as a supply of food.

At the beginning of gastrulation the blastula is a plate of cells, with an inner thicker portion which gives rise to the em-

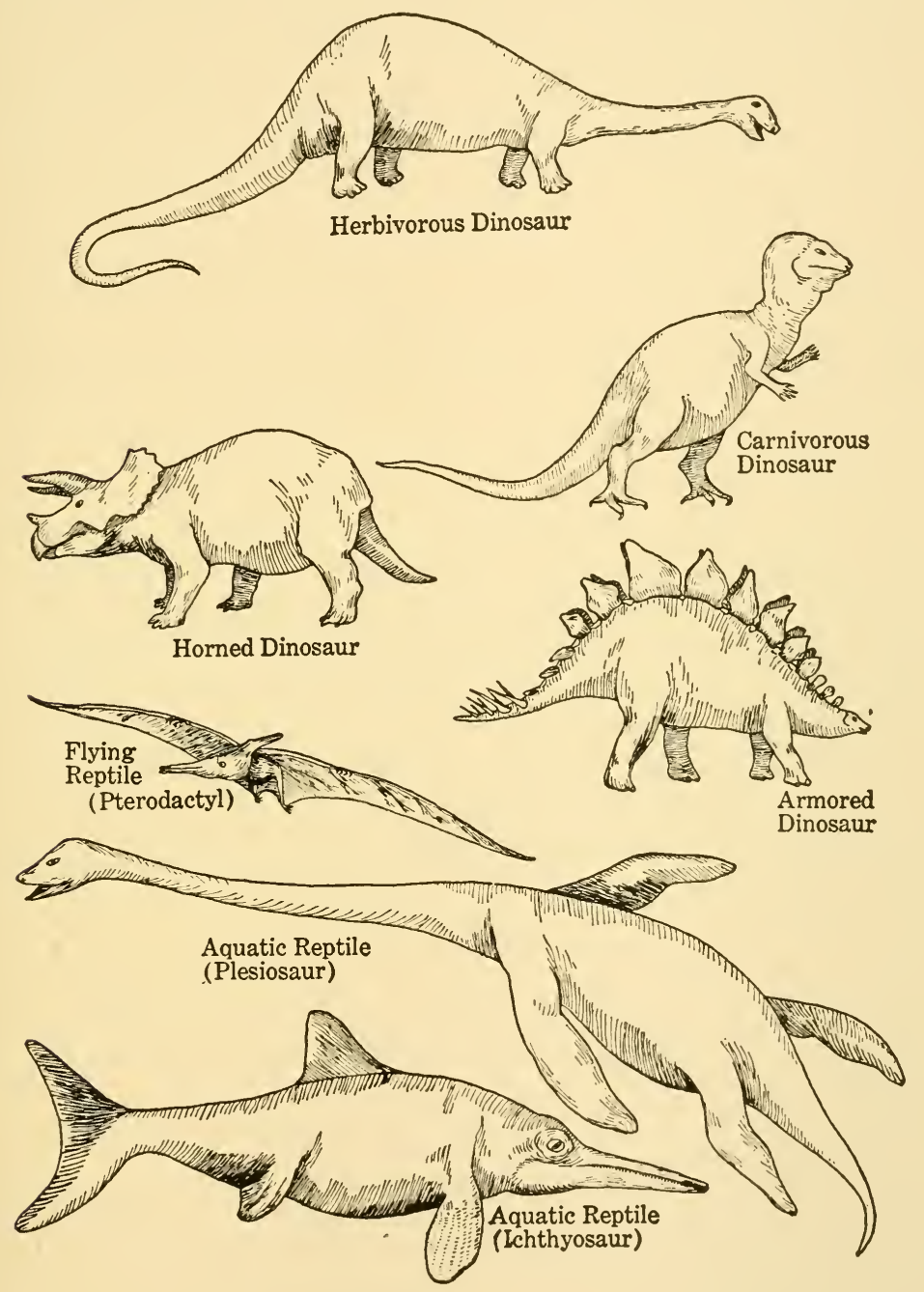

FIG. 23. Adaptive Radiations of the Reptilia. A group of extinct reptiles showing a few of the major specializations. 
bryo, and an outer ring of cells which are beginning to spread around the yolk as extra-embryonic tissue. At the edge of the inner disc a ventral inpocketing of the marginal cells begins, and by continued growth folds under and spreads anteriorly and laterally, paralleling the original blastula cells. The point of inpocketing is homologous with the blastopore.

Thus the gastrula is composed of two parallel, flat plates of cells, the original plate being the ectoderm, the ventral layer next the yolk being the endoderm. The slight cavity between the
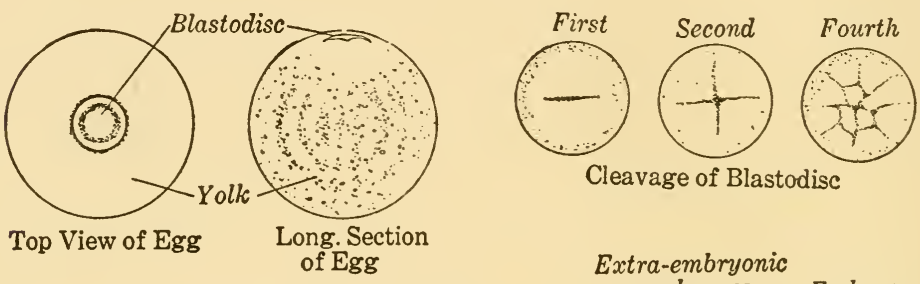

Cleavage of Blastodisc

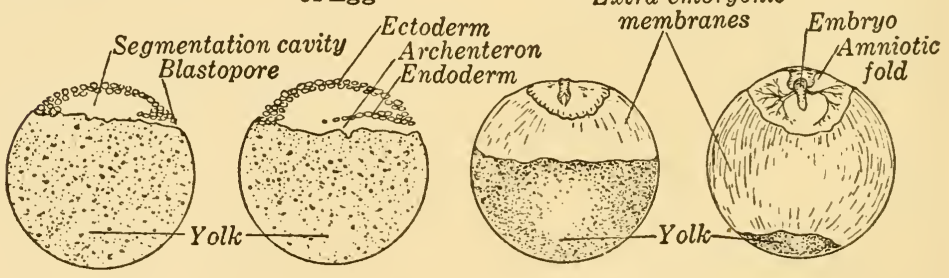

FIG. 24. Early Embryology of the Chick. The egg has a large yolk with a dorsal drop of protoplasm. Clearage is meroblastic. The longitudinal sections (bottom, left) show gastrulation. The diagrams at the right show the overgrowth of the extra-embryonic membranes, the embryo limited to a small area at the top.

endoderm and the yolk is the archenteron. It is spread out over the yolk, and only takes shape with the development of the embryo.

The neural groove appears as a faint line extending anteriorly from the blastopore. The median line of cells sinks while the lateral neural ridges rise toward the dorsal median line, much as in the frog. Synchronously with the above, the notochord makes its appearance as a proliferation of endodermal cells, forming a continuous structure ventral to, and parallel with, the neural groove. 
The mesoderm follows immediately after the beginning of the notochord. It arises as two groups of somites on either side of the notochord, and grows laterally. As the two sheets of mesoderm spread between the ectoderm and endoderm the outer portion splits (recall the enterocoel of Amphioxus, and the cavity in the frog mesoderm). As growth proceeds the upper layer of mesoderm follows the ectoderm, and the lower adheres to the endoderm.

The embryo is now taking shape, and as differentiation of organs proceeds the peripheral cells divide, carrying embryonic tissues around the yolk. Eventually these membranes of embryonic origin completely surround the yolk mass, the inner membrane composed of endoderm and one layer of mesoderm being the yolk sac. The outer membrane is mesoderm and ectoderm. However, although these membranes are of embryonic origin, only the original region forms the embryo, the outlying tissues being extra-embryonic and are lost at hatching time.

The embryo grows more rapidly at the anterior end, and as the neural groove closes and enlarges to form the brain it lifts up from the surrounding tissues. The posterior end then lifts off and the sides of the embryo bend downward and constrict ventrally. These processes lift the embryo from the extra-embryonic membranes, so that the embryo is raised above the still spherical yolk. Recall that the ectoderm, mesoderm and endoderm of the embryo are continuous with the membranes which spread around the yolk mass, and the archenteron is still open ventrally above the yolk sac. Anterior and posterior growth accomplishes two results: (1) the two ends of the archenteron are pulled out so that a distinct gut is formed; and (2) the original point of connection between the embryo and yolk is left as a circular body stalk. The connection between the gut and the yolk sac continues until the time of hatching, but as the body stalk increases only slightly in diameter and the embryo grows rapidly, the connection is relatively very slight after the first few days.

Annion and Allantois. These two distinctive structures of the amniotes (reptiles, birds and mammals) have begun their development at this time. Recall that the extra-embryonic tissues, continuous with those of the embryo's body, form two sheaths 
around the yolk with a cavity between them. The cavity was formed by the splitting of the mesoderm, the portion lying within the embryonic body being the coelomic cavity and the larger outer cavity the extra-embryonic coelom. The two are connected through the body stalk. The inner sheath or membrane surrounds the yolk as the yolk sac, the endodermal layer lying next the yolk with the visceral layer of mesoderm next the cavity. The outer membrane is composed of mesoderm and ectoderm, the latter being external.

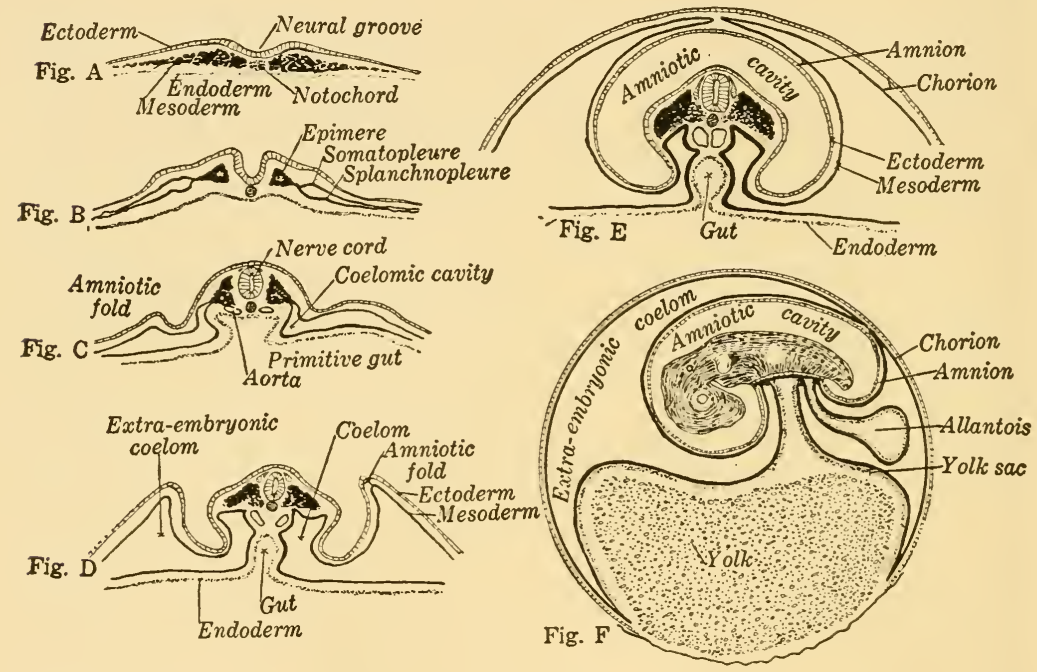

FIg. 25. Organogenesis in the Chick. Figures $\mathrm{A}-\mathrm{E}$ are cross sections through the embryonic region, the yolk omitted. Fig. F shows a total embryo, with a hemi-section of the extra-embryonic membranes.

The amnion slightly precedes the allantois in development. It is formed by two synchronous processes: (1) the embryo sinks as the yolk materials below it are absorbed and the weight of the embryo increases; and (2) the extra-embryonic tissue grows dorsally and medially over the embryo. The anterior region of the amnion develops more rapidly and the embryo sinks head foremost. The result is that the amniotic fold progresses posteriorly as a crescentic, double membrane. The posterior fold grows more slowly than the anterior and lateral folds. Fusion eventu- 
ally takes place near the posterior end, and is due to a steady narrowing of the circular opening caused by the growth of the folds toward a common point. When fusion occurs there are two encircling membranes which have developed from the original outer membrane of ectoderm and mesoderm. (1) The inner is the amnion, lined with ectoderm which is continuous with that of the embryonic body, and completely surrounds the embryo except at the body stalk. Fluids diffuse into the amniotic cavity, and the embryo continues its development in a fluid medium. (2) The outer membrane is the chorion and lies next the porous shell. At this stage the embryo is surrounded by the amnion; the yolk sac is ventral; and the chorion surrounds the entire structure.

The allantois begins its development before the completion of amnion formation. It develops as an outpocketing of the posterior end of the gut and grows outward toward the chorion between the amnion and the yolk sac, lying in the extra-embryonic coelom. At this stage the gut is covered by a layer of mesoderm, and the allantoic outpocketing naturally is covered by a mesodermal layer. The allantois becomes enlarged at the outer end, and as it reaches the chorion (lying against the shell) it spreads until it almost fills the extra-embryonic coelom.

Blood vessels pass outward from the embryo with the allantois and come into contact with the porous shell. Respiration takes place by osmosis through the thin membranes of the shell into the blood vessels of the chorio-allantoic membrane. The circulating blood transports the carbon dioxide from the embryo to the distal capillaries, where waste products are given up and oxygen is received. During embryonic life the aerated blood goes to the embryo through the allantoic veins and is mixed with the unaerated blood of the embryo before reaching the heart. The complete separation of aerated and unaerated bloods does not take place until after the animal hatches and the lungs become functional.

Later Growth. After the nervous system and the vascular systems are laid down, the organs of excretion begin their development. The mesoderm gives rise to the muscles, connective tissues and bone, and the glands begin their development. It is a 
differentiation of the primitive germinal layers into tissues and organs, and their individual histories are traced in Part II.

Continued growth increases the size of the embryo and decreases the amount of yolk. At the time of the completion of the amnion the embryo is a small structure on top of a large yolk mass; but by the time of hatching their relative sizes have been reversed and the yolk sac is a small mass on the ventral side of the young animal. In hatching the shell and surrounding membranes are broken and the latter are sloughed off in a short time.

LABoratory Study. The great majority of laboratories find it impractical to get reptiles for a study of embryology and use the chick instead. The bird is essentially similar to the reptile in its developmental processes, and due to the ease with which fertilized hen's eggs are obtained and hatched in the laboratory, they make ideal material for a study of meroblastic cleavage and the principles of amniote development. For this reason the illustrations used show chick development. 


\section{CHAPTER VII}

\section{AVES}

The birds (Aves) are feathered vertebrates; or, as Huxley expressed it, "glorified reptiles". The class evolved from the diapsid reptiles, but are far more specialized than any living reptilian group. In fact, it is the most coherent class of vertebrates; and when considered as an entire class, is the highest major group of the vertebrates. Nan and certain other mammals surpass them in the specialization of the nervous system, although the average of the birds is almost as high as that of the lower mammals.

Birds are warm blooded and have a constant temperature; the skeleton is light and strong, a modification in correlation with their flying habits; the heart is completely four-chambered like the mammals; and the fore limbs are highly modified from the primitive arm of the land vertebrates.

Little is known about the evolution of the flying mechanism in birds. Two fossils from the lithographic limestone of Germany tell something as to the evolution of the birds from the reptiles, but it is still in doubt as to how flying originated. Various theories have been advanced. The fossils from Germany are called Archaeopteryx, or "ancient wing", and these fossils indicate that the early birds were not powerful fliers. Study of the illustration will show a number of interesting features. The skull is heavy and reptilian, not light as in living birds; there are conical teeth, much like those of a reptile; the digits of the wing are well developed with claws at the end; and the tail is long like that of a lizard, but with a row of feathers on either side. Evidently this animal was hardly more than a feathered reptile.

Two later fossil birds are known, which are more distinctly bird-like in their anatomy. The tail, wing, and hind leg had become modified to the avian condition before the teeth were lost 
in the course of evolution. This fact is of interest as no known living bird has any sign of teeth, the bill being made of thickened epidermis like that of the claws. The so-called "egg tooth" of the newly hatched bird is an epidermal growth of the bill and is lost soon after the embryo pecks its way out of the shell.

The birds of today are divided into two great groups showing two distinct lines of evolution: (1) the flying birds which have a keel (carina) on the breast bone, and (2) the running birds with degenerate wings and a smooth breast bone. The latter undoubtedly evolved from the former, but for ease of discussion will be considered first.

RATite birds are those with smooth breast bones, including several extinct species and the living ostrich, rhea, cassowary, emu, and the kiwi. The roc of Sinbad the Sailor belonged to this group, and was an enormous bird which laid an equally enormous egg. As the skeleton and egg only were known, it was easy for the layman to draw the conclusion that it would have great powers of flight, despite the fact that it was a non-flying species.

The ostrich is a native of Africa, but the animal has been naturalized in many of the warmer regions of the earth, the plumes from the farm animals being of better quality than those taken from wild birds. It stands about eight feet high, with small wings and powerful legs. 'The egg has the content of about two dozen hens' eggs, and is the largest single cell known.

The rhea is South American in distribution. The emu and cassowary inhabit Australia, and like the former, occupy open grassy plains. The Kiwi or Apteryx (without wings) is the most specialized of the ratites, and is confined to New Zealand. The wings are small boned and are not visible externally. For their size, these birds lay huge eggs, each being approximately onefourth of the body weight.

Carinate birds are world-wide in distribution. In size they range from the smallest humming bird to the albatross and condor. In flying ability they vary from the non-flying penguins to the great speeds attained by migratory birds. Modern airplanes have demonstrated speeds of more than a hundred miles an hour in some birds, but whether this could be maintained for long periods is not proved. 
During their evolution the orders of birds have developed a wide number of adaptive radiations. Some are typically wading animals with long legs and beaks, as in the cranes, storks and flamingoes; others are adapted for swimming, as the ducks, swans and penguins; the carnivorous birds are adapted for tear-
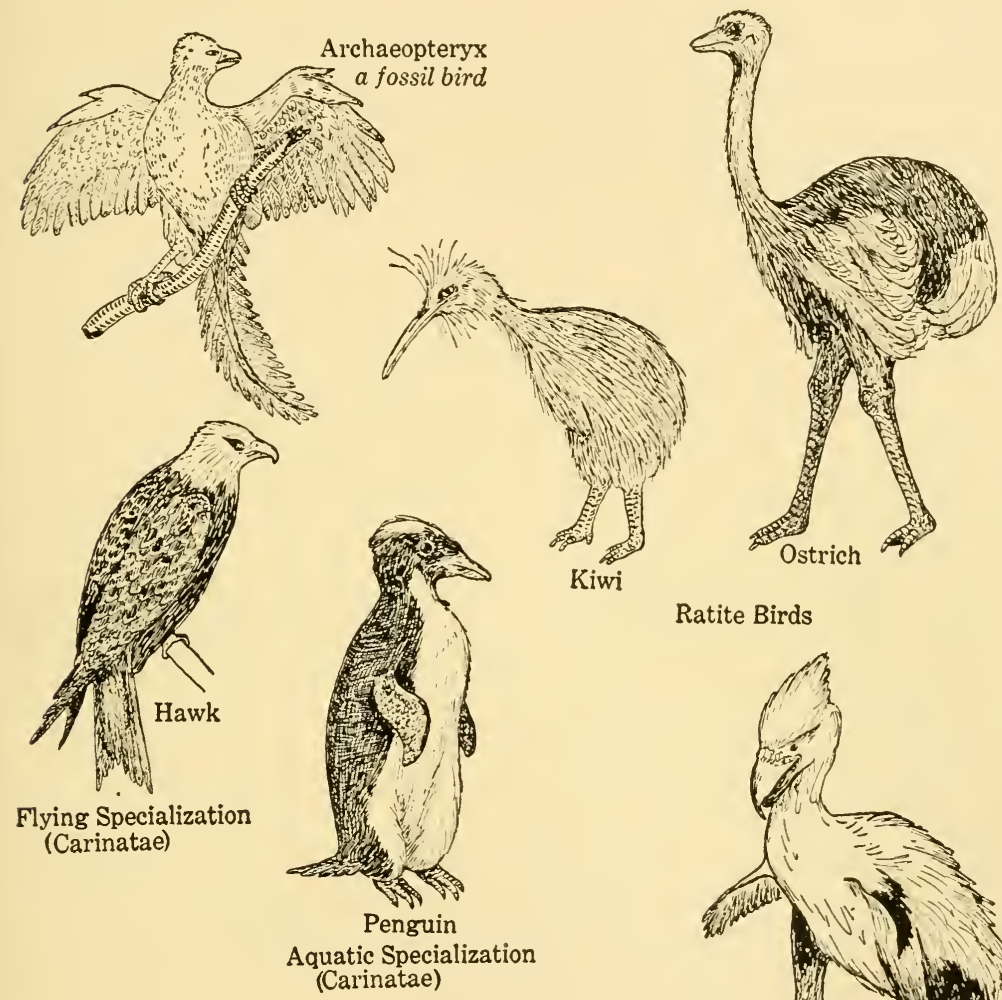

Ratite Birds

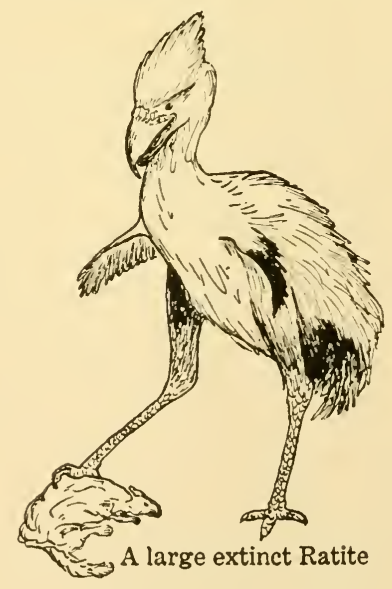

FIg. 26. Adaptive Radiations of the Birds. Archaeopteryx was a reptilelike bird. Recent evolution has proceeded along two major lines, represented by the Hawk and Kiwi. 

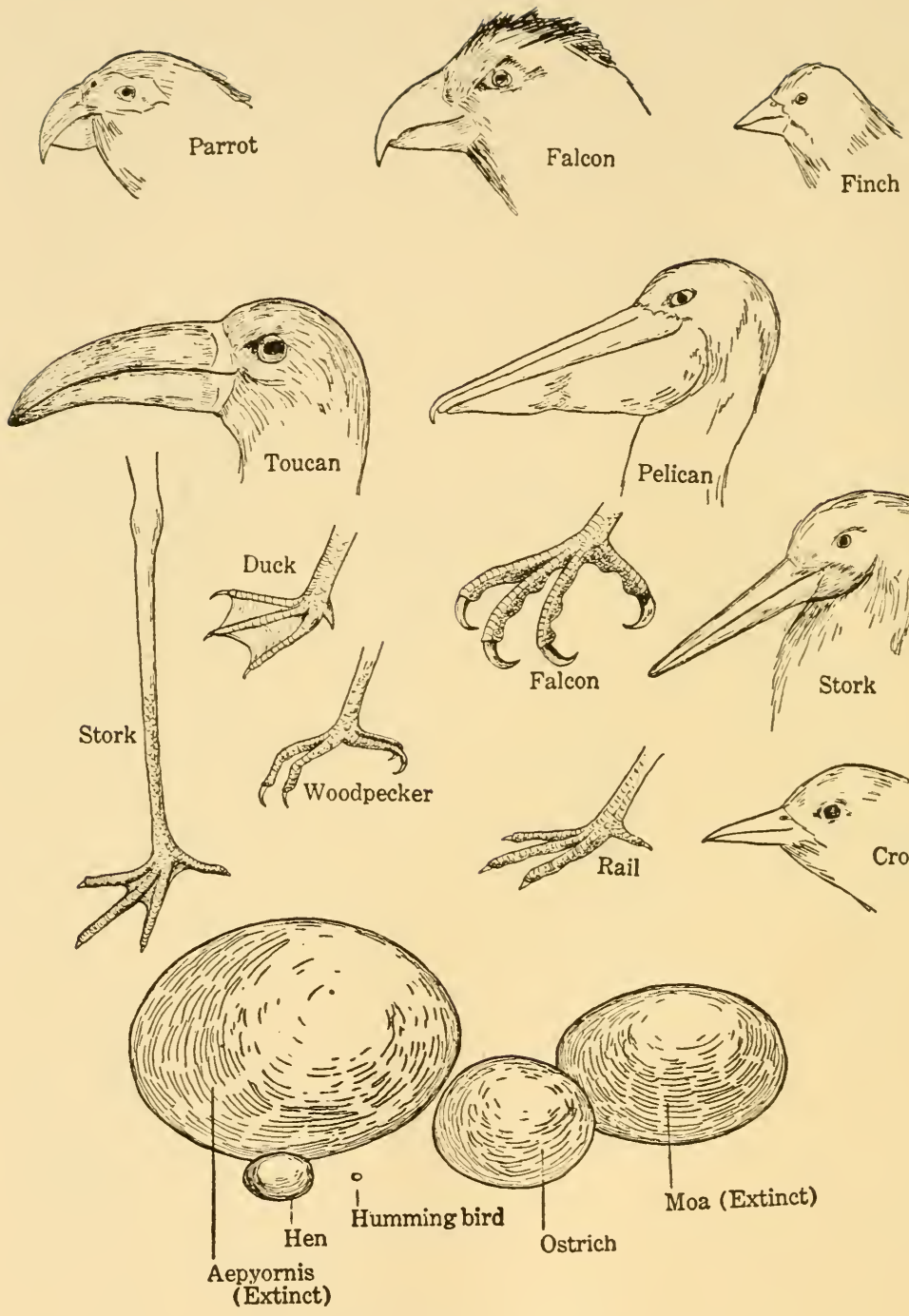

Fig. 27. Specializations of the Birds. The beak is modified for pecking. tearing, or catching fish. The legs and claws are adapted for correlated functions: walking, grasping, or wading. The eggs of different birds (at bottom) show the comparative sizes. 
ing flesh with both claws and bill, for example the owls, hawks, eagles and vultures; the sand-piper, secretary bird, and the common fowl, while well equipped for flying, can run swiftly along the ground; and the opposite of the last adaptation is found in the humming birds, which have almost no ability for ground locomotion, but are able to remain on the wing, poised in one place before a flower, for a sustained period.

It has been often stated that anatomically a "bird is a bird", but this applies only to the soft anatomy. For the many modifications, and the numerous orders, the student is referred to books of natural history. As the birds are far removed from the line of human evolution a full discussion cannot be attempted.

Embryology of the Bird. Avian development is more specialized than that of the reptile, but is the same in all essential details. This similarity was discussed under reptilian embryology. Most birds sit upon the eggs during the entire time of incubation, only a few genera which live in dry sandy regions depending at all upon the heat of the sun. The body temperature is higher than that of the typical mammal, being about 103 degrees Fahrenheit, and this temperature must be maintained for the proper development of the embryos.

When the egg is laid development has already begun, but cleavage is suppressed when the egg cools. Embryos may remain in this arrested condition for several weeks, and when heat is again applied begin growth. In this way all the eggs, which were laid at different times, begin development together and hatch at about the same time. 


\section{CHAPTER VIII}

\section{MAMMALIA}

Mammals can be defined as animals with hair and milk glands. Either part of the definition will serve, for only mammals secrete milk for their young; and although some mammals are almost lacking in hair, even the whale has a few whiskers. One extinct reptile has been described as having hair, but this is probably a hair-like structure, rather than true hair. However, it is not beyond the realm of possibility that some therapsid reptiles had hair between the scales. In any event, the above definition will serve to identify the living mammals.

Mammals include a heterogeneous assemblage of animals. Some lay eggs like a reptile, others give birth to very immature young, and others, which make the majority group, gire birth to young in a well developed condition. The last group includes the mice, porcupines, cats, dogs, seals, whales, elephants, pigs, monkeys and men-in fact, practically all the mammals which any student outside of Australia would know. The mammals are divided into three sub-classes and a number of orders.

Sub-class Prototheria. This group includes one order and a few genera. They are the most primitive of living mammals and are restricted in their distribution to Australia, New Zealand, and the adjacent islands. The animals have the body covered with hair, and there are milk glands. The latter have no teats, the secretory ducts opening separately into depressions on the mother's abdomen, and the young lick the milk off.

The order is called the Monotremata (one opening) in reference to the cloaca into which the digestive and urinogenital openings empty. This structure is typical of the amphibia, reptiles and birds, and is not found elsewhere among the mammals. 
The monotremes lay small, reptile-like eggs which are hatched in a nest built by the parents. The young are very immature at the time of hatching, and are kept in the nest for some time. The brain is better developed than that of any reptile, but very primitive compared with other mammals. The body temperature is not constant, varying widely with the environment. The skeleton is very reptilian in character, the legs being held laterally at right angles to the body. These characters have given rise to the theory that they arose from the reptilian stem as a separate line of evolution, and that they are not as closely related to the other mammals as are the Therapsida. The monotremes have been described as a "museum of reptilian characteristics".

Ornithorhynchus, or the Duck-billed Platypus, is the best known, although rarely seen in captivity, and it has never been successfully kept in zoos in America or Europe. It is a waterliving mammal, inhabiting the ponds and quiet streams of New Zealand. The nests are dug into the bank above the water level, the opening being protected by the water which fills the tunnel.

The more widely spread Echidnas live in drier regions and, due to their spiny covering, are erroneously called hedge-hogs. They are also called spiny ant-eaters, and though many do live on ants, the term is misleading for the name was long before given to an entirely different group. Specimens have been kept for long periods in zoological gardens.

Sub-class Metatheria. One order, the Marsupialia, comprises the entire sub-class. Marsupial refers to the marsupium or breeding pouch, which is simply a deep fold of skin on the lower abdomen. The teats are within the pouch, and immediately after birth the immature young climb into the pouch and remain there until able to maintain independent existence.

With the exception of the opossums of the American continent, and obscure South American forms, the order is limited to Australia and the nearby territory. The American opossum is perhaps the most generalized mammal in existence, its skeleton having remained practically unchanged since the early history of the race. It has survived in competition with the higher mammals by remaining in hiding except at night. For the causes 

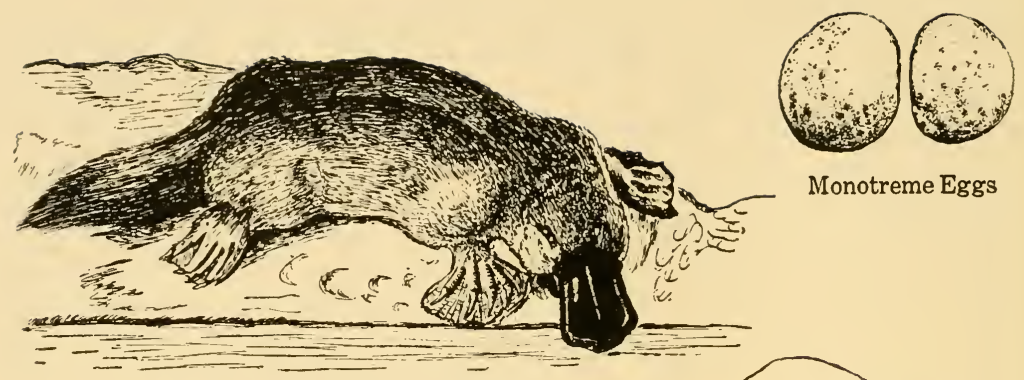

Monotreme Eggs

Ornithorhynchus (Monotremata)
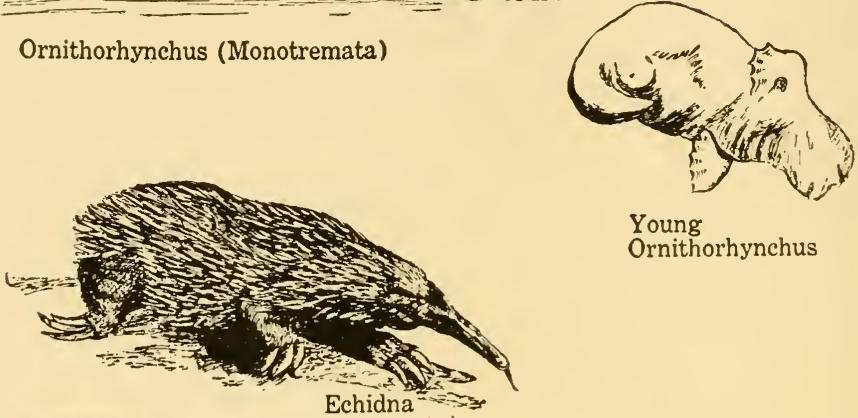

Young

Ornithorhynchus

(Monotremata)

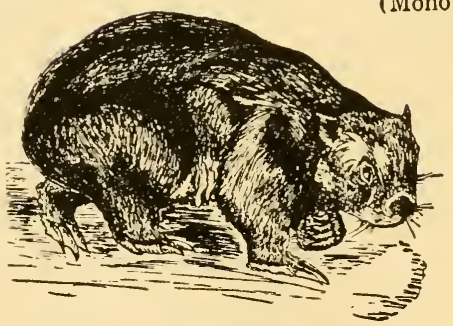

Wombat (Marsupialia)
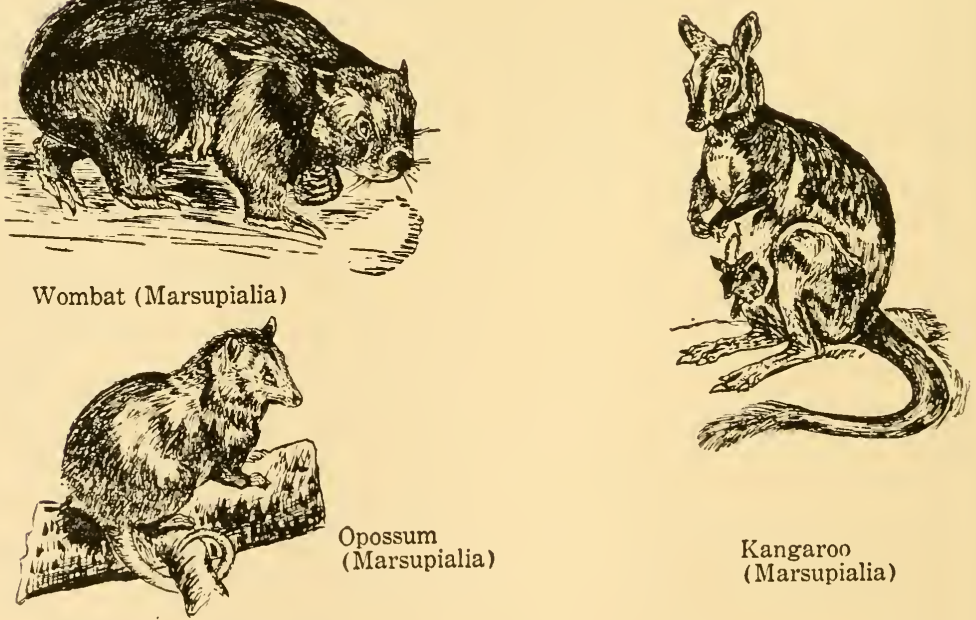

Kangaron

(Marsupialia)

Fig. 28. Primitive Living Mammals. At top are shown the two families of egg-laying mammals. Below are types of Marsupialia. 
of preservation of the marsupials in Australia, the student may refer to Chapter XX.

Among the better known Australian marsupials are the kangaroos. The student need not be reminded of their small fore feet, their large hind legs and their powerful tails which assist the animals in jumping. The largest stand more than six feet high. The wombat is a herbivorous marsupial resembling a ground-hog. Other herbivorous species are no larger than rats, and much resemble these rodents in external appearance. Other marsupials are carnivorous, the better known being the small Tasmanian Devil and the Tasmanian Wolf. The latter is about the size of the gray wolf, and but for its fundamental marsupial structure could be mistaken for it. However, no matter how closely one of these animals may resemble a higher (placental) animal, it is structurally and developmentally a marsupial.

Developuent. The marsupials have small eggs which are almost without yolk, and of microscopic size. Fertilization is internal, as in all amniotes. The fertilized eggs are retained in the modified posterior part of the oviducts. This portion of the ducts becomes the uteri (and later a single uterus) in the two higher groups of mammals. The uterine wall secretes a nutrient fluid, the so-called "uterine milk", upon which the embryos live by absorption. As there is little or no yolk, and no connection with the mother, the young are born in a very immature condition.

The new born marsupial is merely an embryo with a few well developed, specialized characters. At birth an opossum, which when adult is about the size of a house cat, is not larger than a bean. It weighs approximately four grains; or, to state it more graphically, 1750 of them weigh a pound. A few reflexes are present, including a negative geotropism, and the young climb up the side of the mother and into the pouch with the aid of front claws which are later shed. There they attach themselves to the nipples, which are long and about the diameter of broom straws. The epithelium of the lips fuses to the nipple and they remain firmly attached for a week or more. By that time they are able to see, and soon begin climbing out of the pouch on to the mother's back. 
As with most transitional groups, there are many characters which, by comparison with the higher types, seem very inefficient; but when compared with lower forms their selective value is apparent. This is true of the development of the marsupials. They managed to survive the Age of Reptiles due to higher intelligence and some care of the young; but when their more highly developed descendants arose they all but disappeared. And the majority of those which survived were in a region protected from the higher mammals. There is a single exception to the above description of development, but this will be mentioned in the following chapter.

Sub-class Eutheria. This group is also known as the Placentalia on account of the placenta, an embryological development
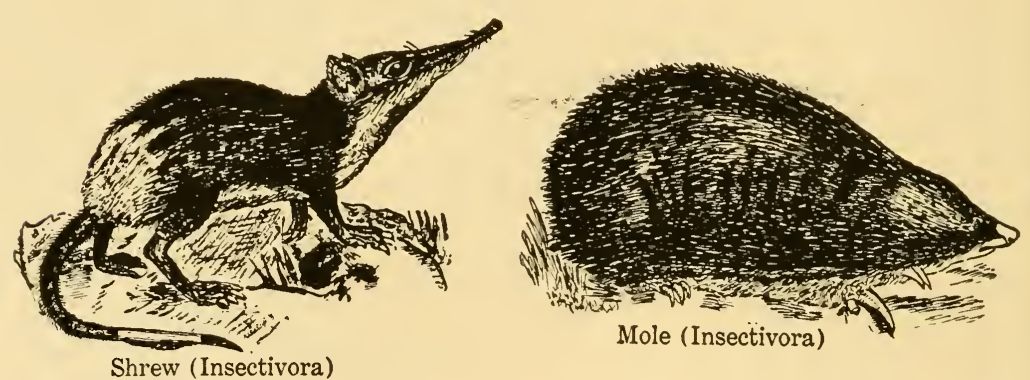

FIG. 29. Order Insectivora. The living representatives of the most primitive placental order are externally specialized. The Order Primates rose directly from the Insectivora.

of the allantois which attaches the young to the wall of the mother's uterus. Their distribution is world-wide except for Australasia, where only the flying mammals were found until others were naturalized by white men. Various types of placentals were mentioned at the beginning of the chapter.

The eggs are microscopic in size and almost lacking in yolk. Growth depends upon food and oxygen being absorbed from the blood stream of the mother, and the young are born in a fairly . well developed condition. The following grouping of orders is simplified. Those which bear upon human evolution, or which 
are used for dissection in most courses in anatomy, are included first.

Order Insectivora. This order was the first to appear in the evolution of the placentals, and is still the most primitive orcler. Externally they have numerous specializations, for example: the spines of the European hedge-hog, the jumping habits of the African shrews, and the blindness and digging claws of the moles. Their teeth are very generalized and adapted for insect eating. One family (often set up as a separate order) appears
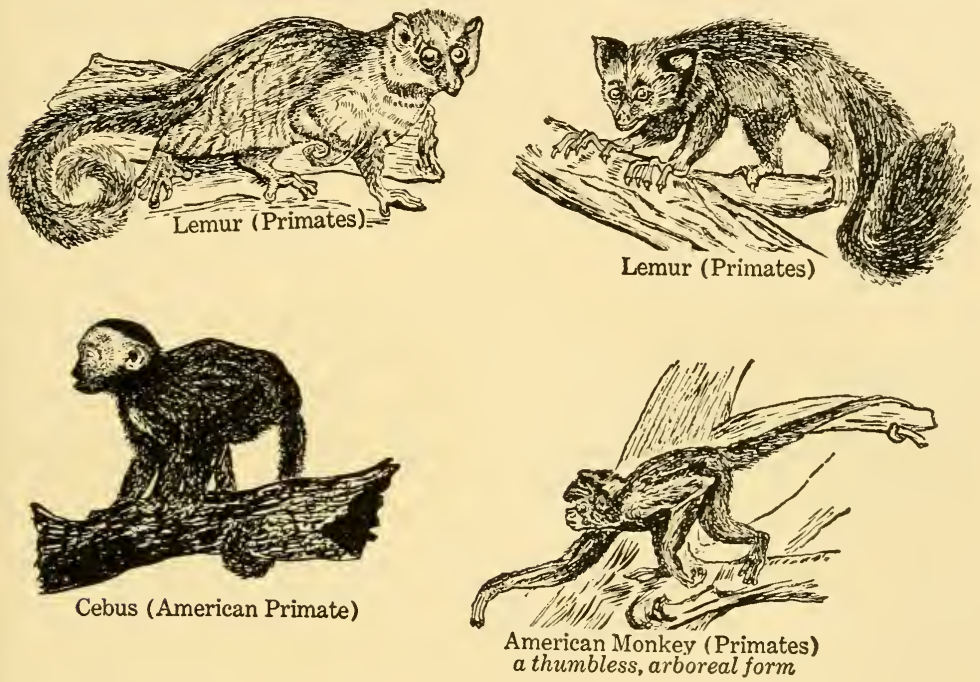

FIG. 30. Order Primates. The Lemurs (top) are the most primitive members of the order. The American monkeys are an early branch from the parent stem.

about mid-way between the insectivores and the most primitive primates in several characters.

Order Primates. The primates are the lemurs, monkeys, apes and man; and a few less well known families. They evolved from the insectivores and are therefore one of the oldest orders of mammals. Most of them are arboreal in habit, and all of them show unmistakable specializations for tree living conditions. Man is the least adapted for this ancestral type of life. The order is divided into the following sub-orders. 
Sub-order Lemuroidea. The lemurs were so named for their nocturnal and "ghostly" habits. The most primitive members almost merge into the insectivores. Fossil lemurs were widely distributed, but of the fifty existing species thirty-six are limited to Madagascar, the others being found in tropical Africa and the Orient. The different species indicate the course of evolution from the most primitive to the monkeys.

Sub-order Anthropoidea. This sub-order carries the specialization of the nervous system and other body characters from the primitive condition to man. In this group the teats are always in the pectoral region; the embryological structures of nutrition (placenta) are similar; the skeleton shows the course of evolution toward the upright position; and the nervous system shows the steady advance from the lower monkeys to the human.

The South American monkeys are an early off-shoot from the original stem, including the marmosets and the typical organ grinder's monkey. They can be identified by the distance between the nostrils, the septum being wide and the apertures directed outward. Also, many of them are able to hang by their prehensile tails.

The Old World monkeys are more man-like, the nostrils being close together and separated by a narrow septum. The tail is never prehensile. The teeth are thirty-two in number, the dental formula being the same as in the higher families. The family includes both the tailed monkeys and the apes, and members are found in Africa, Southern Europe and Asia. The apes are the most specialized of the primates, their dog-like faces and vivid coloration making them unpleasantly ugly. These apes and baboons have little relationship to the groups which gave rise to the human family.

The simian family includes the gibbons, which walk in an almost upright position; the orang outans of the Malayan regions; and the chimpanzees and gorillas of Africa. The last two genera are the nearest relatives of man among living animals. The muscular system and the nervous system are almost identical with those of man. In judging similarities between groups the young should be studied, as older animals tend to 

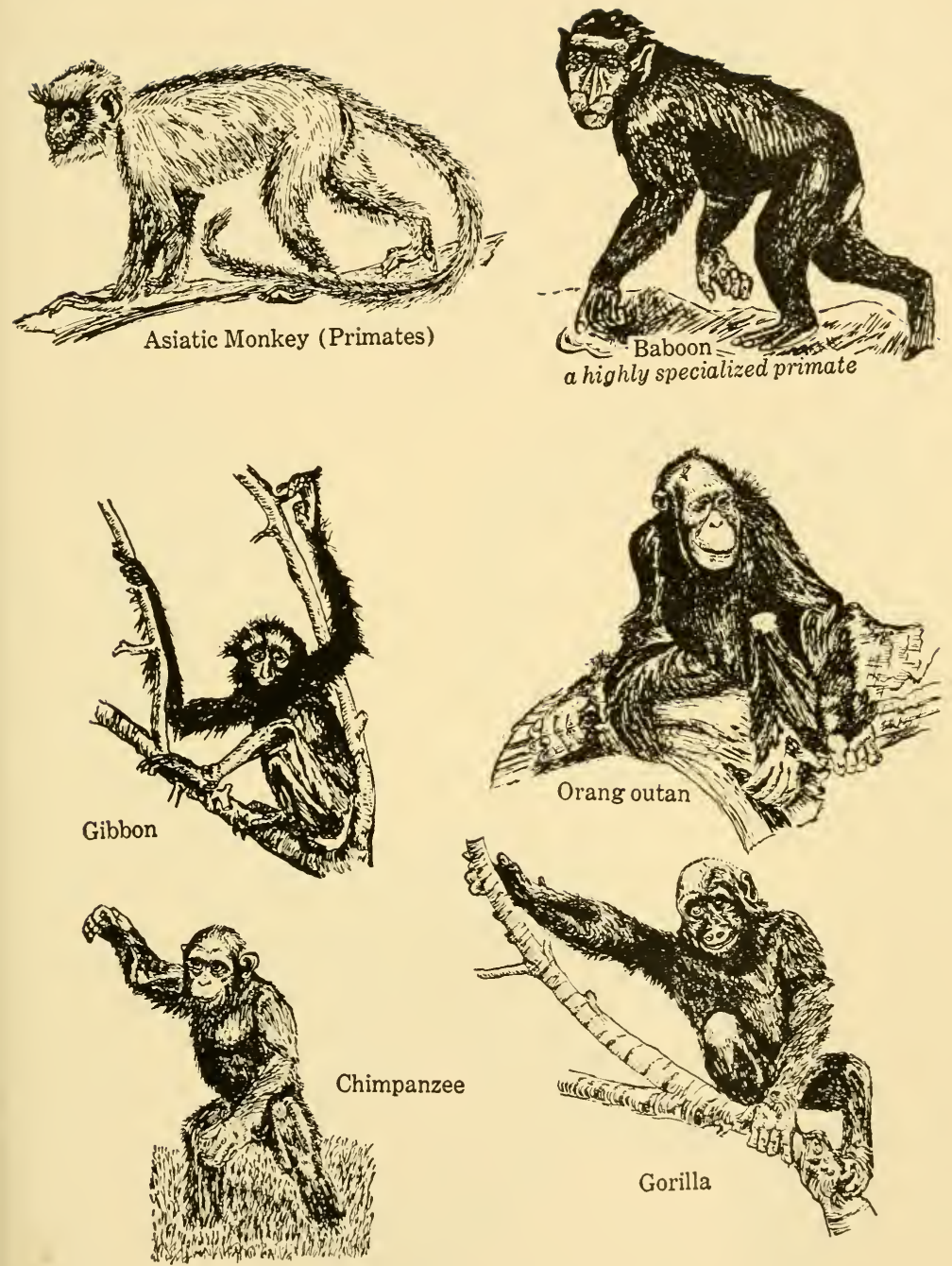

FIG. 31. Old World Primates. 
develop their own characteristics, making them appear more divergent than they are in reality.

The Hominidae include among existing animals only the races of man. All existing men are classified as one genus and one species, Homo sapiens. Several genera of fossil men are known, and these connect the present representatives of the genus Homo with the earlier anthropoids. And even among the living representatives of the higher anthropoids the relationships are so close that there is a tendency on the part of recent investigators to include man and the gorilla in one family.
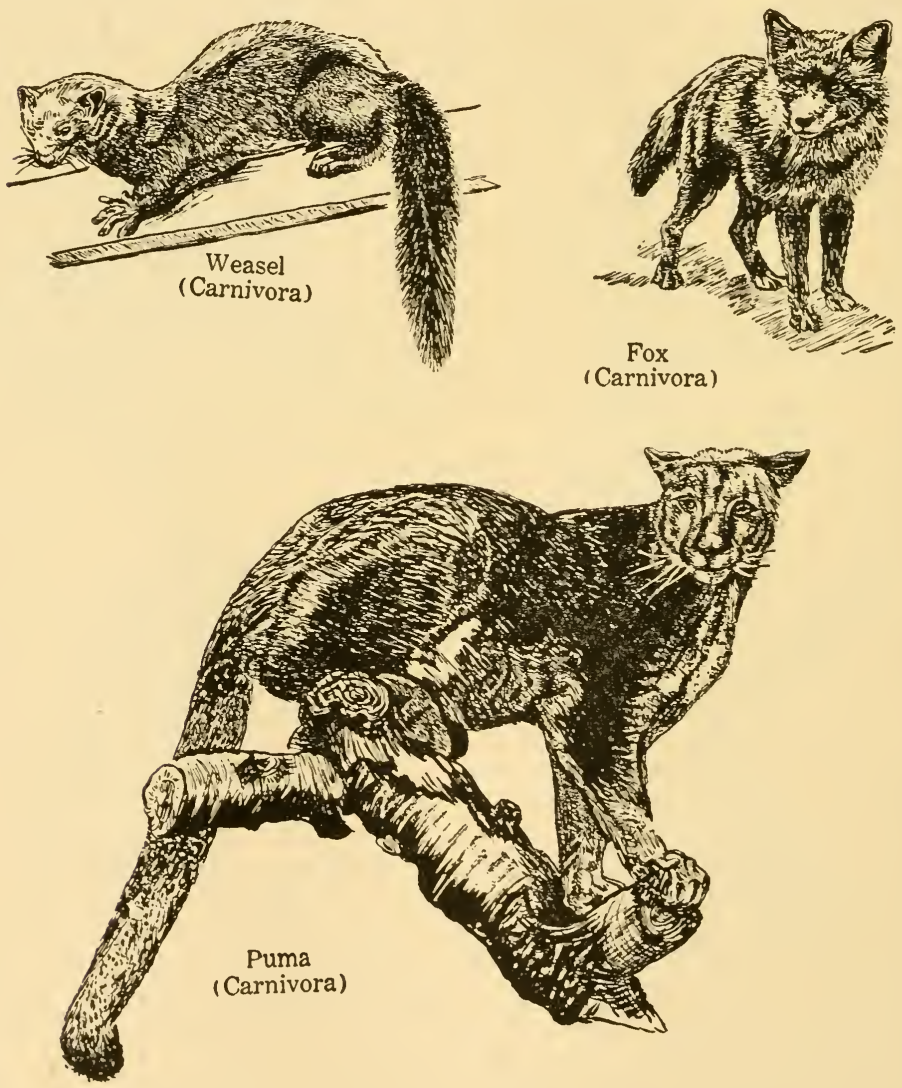

FIG. 32. Carnivora. Three types of land living carnivores (Fissipedia). 


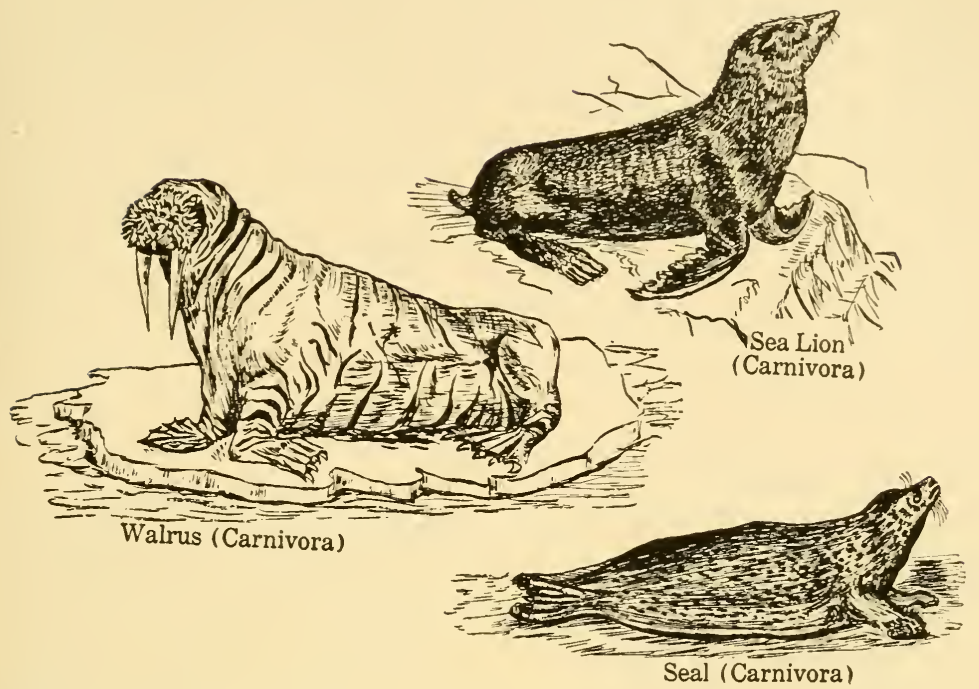

FIG. 33. Carnivora. Aquatic or partially aquatic carnivores (Pinnipedia). A comparison of the Sea Lion and Seal shows the latter's increased specialization for water life.

Order Carnivora. The carnivores, or flesh eaters, are distinguished by the enlargement of the canine or "eye" teeth. The posterior teeth (premolars and molars) are usually modified for cutting, and the animal is able to tear and cut his food. The order is divided into two large groups. (A) The land carnivores include the dog family, the cats (lions, tigers, pumas, and the smaller cats), the hyenas, the bears, and a few others which will be unknown to most students. (B) The carnivores modified for swimming form the second group: the sea-elephant, sealion, walrus, and the Atlantic seals. The last are the most highly modified for aquatic life.

Order Rodentia. The rodents are gnawing animals and have the two median incisor teeth of both jaws highly developed. The posterior teeth are modified for grinding. The animals were originally herbivorous, although the rats and others are omnivorous. In this order are the mice, rats, guinea-pigs, and the large South American capybara which is the size of a hog. By some anatomists the rabbits are included in another order, due 
to the presence of the lateral incisors in addition to the median, and a history which indicates early separation from the typical rodent stock. The rabbit is a favorite laboratory animal for dissection.

Order Cheiroptera. The bats, which form this order, are flying mammals. The name literally means "hand wing", the digits being enormously elongated with a leathery membrane stretched between them. Their dentition and internal anatomy show them to be closely related to the insectivores. The existing members-
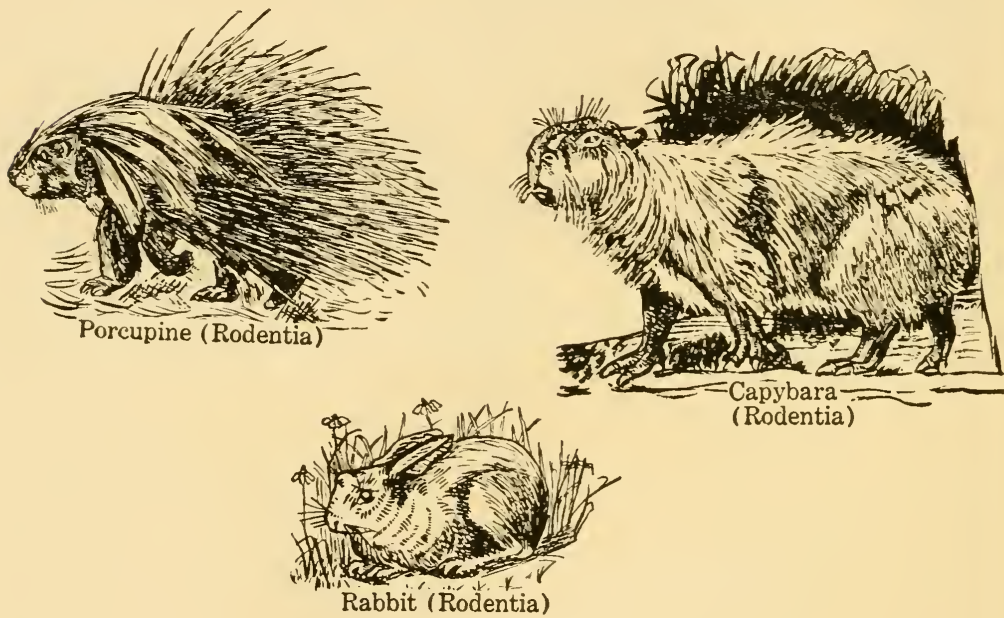

(Rodentia)

FIG. 34. Rodentia. Capybara is the largest living rodent. The rabbit is more technically placed in a different order, Lagomorpha. The porcupine is a true rodent with long ectodermal spines.

are mostly insect eaters, although some are fruit eating, and a few (the vampire bats) cut the skin of other animals and drink the blood. This genus of South American bats has sharp canines and incisors, a gullet which permits only liquid food, and an almost straight digestive tract forming a "stomach-intestine". The distribution of the order is world wide, their flying habits having permitted them to invade Australia and other insular regions.

Order Edentata. The edentates are not all entirely toothless, a few having small teeth, and some have teeth in the young 
which are later lost. This order is heterogeneous and should be divided into three orders. Simplicity of classification is the only excuse for grouping them. Included here are the South American ant eaters, sloths, armadillos; the genus Manis of Africa and the Orient; and the Aard varks, or Gape ant eaters, of Africa.

The armadillos are unique among living mammals in possessing a heavy bony covering. This is arranged as scutes, or plates, placed transversely to the axis of the body. The species are classified by the number of plates. When frightened the animal rolls up, presenting a heavy defensive armor to any predaceous animal.

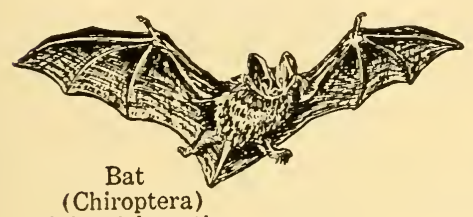

Flying Adaptation
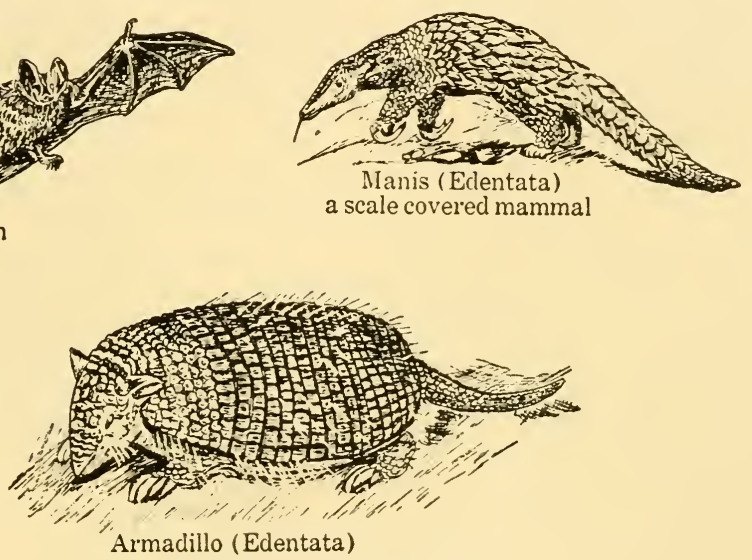

FIG. 35. Specializations of Mammalia.

Manis is of particular interest to the anatomist on account of the covering of ectodermal scales. These scales are reptile-like in character and cover the entire body from head to tail. Between the scales are the hairs, usually in groups of three, one group under each scale. Due to the evidence regarding the evolution of the hair, it is thought that this represents the condition found in the earlier mammals.

Order ARtiodactrla. The even-toed, herbivorous animals include the camels, sheep, cattle, deer, antelopes, pigs and hippopotami. These animals can be identified by the fact that the weight is carried on the third and fourth digits, with the axis 


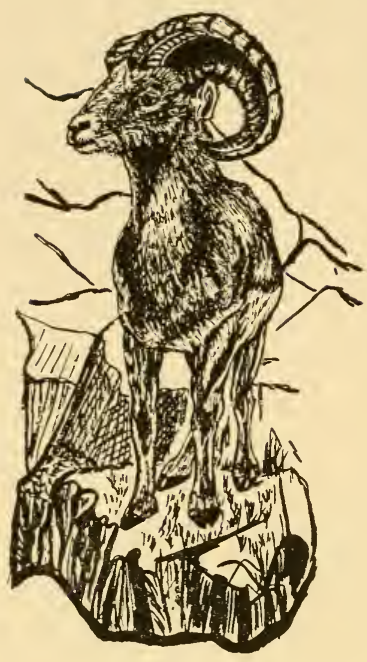

Mountain Sheep (Artiodactyla)
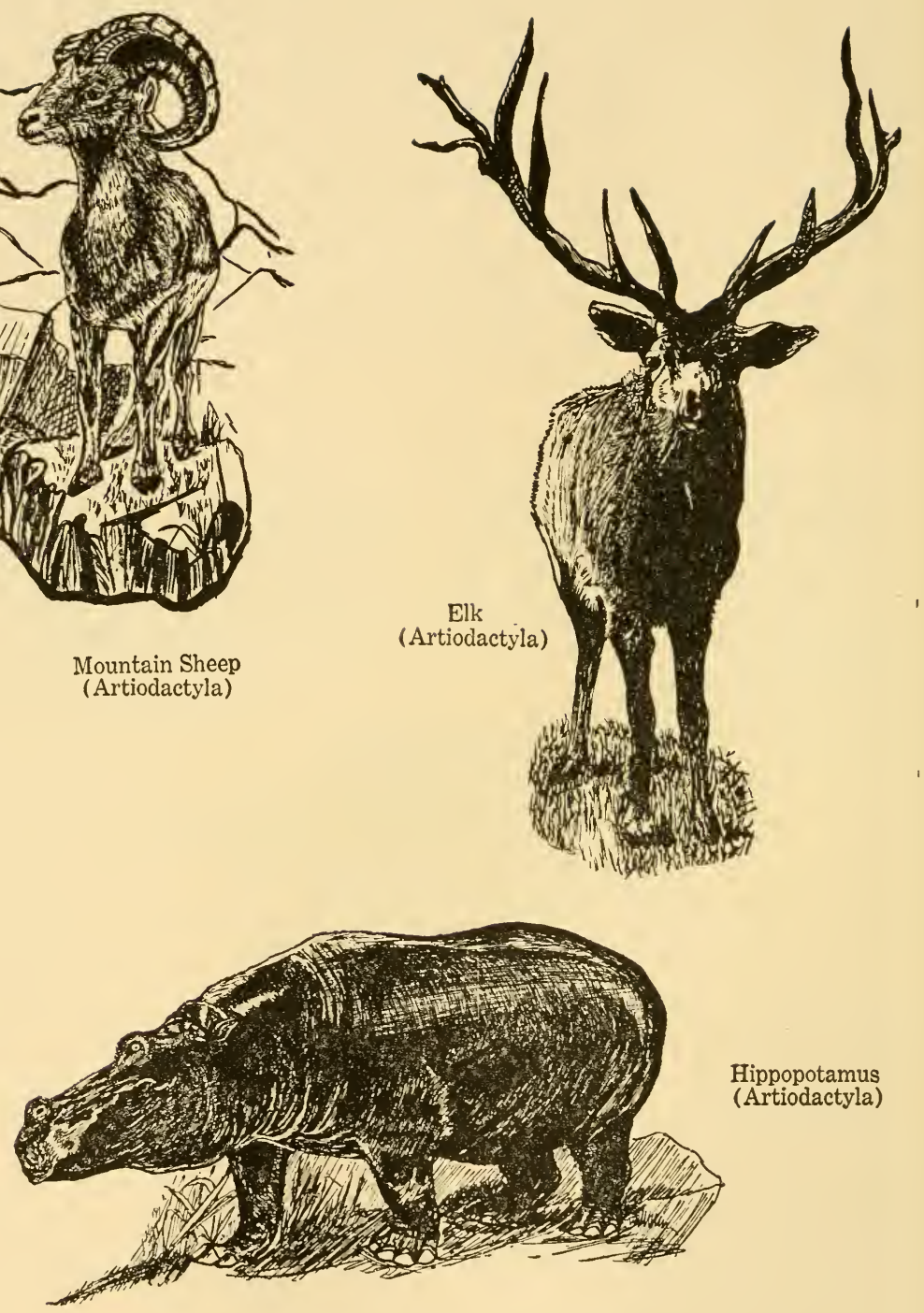

Fig. 36. Order Artiodactyla. The order of even-toed placentals has become specialized toward size, a heavy protective skin, or defensive horns or antlers. 
of the leg passing between them. This order and the following were formerly classified together as the ungulata, but their evolutionary history shows them to have been separate groups since very early in the history of mammals.

Order Perissodactila. These are the odd-toed herbivorous mammals. The axis of the leg passes through the third digit, with the weight resting upon it. The rhinoceros, horse, zebra,

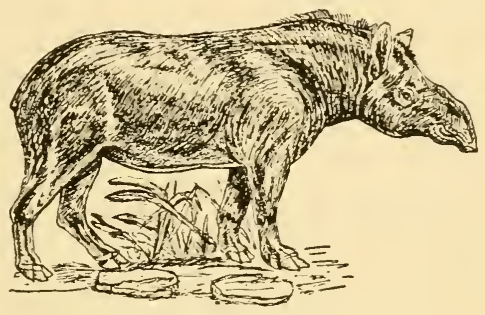

Tapir (Perıssodactyla)

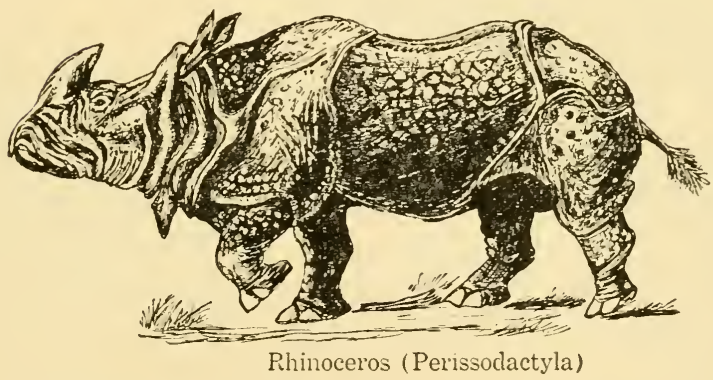

FIG. 37. Order Perissodactyla. The order includes the horse, ass, zebra and the above forms.

tapir and ass are members of the order. The horse is a very highly specialized member of the group, the number of digits having been reduced to one in the adult. There is, perhaps, no evolutionary history better worked out than that of the horse.

Order Proboscides. The proboscidea are animals with a great elongation of the nose forming a proboscis, and the living members of the order are usually included in one genus, Elephas. The evidence warrants the separation of the African elephants as a different genus from the Asiatic elephant. The elephants 
are the largest land animals, the legs being modified into straight columns with little flexion at the joints, a specialization in correlation with the great weight of the body. The Indian elephant is more easily tamed, although progress has been made in training the African species for work. Most circus elephants are of the Indian variety. Judging by their brain capacity the elephant is a rather low order of mammal, and experiments indicate that stories of their intelligence are highly colored. Several extinct families of proboscideans are known, including the Mastodon and Mammoth, the latter a true elephant more highly specialized than the recent forms.

Order Sirenia. The manatees and dugongs are herbivorous aquatic mammals. Most of them inhabit the sea, although they

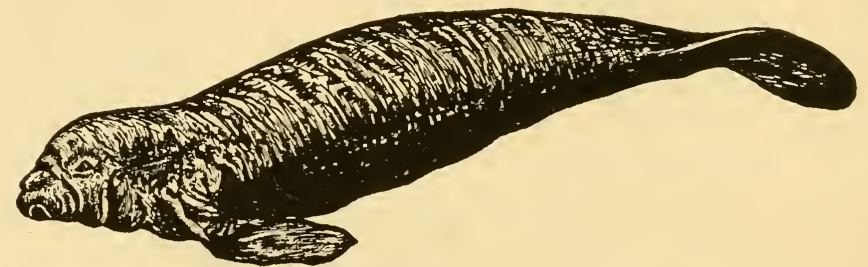

Manatee (Sirenia)

FIG. 38. Order Sirenia, aquatic, herbivorous placentals.

may go farther up rivers than the brackish waters; and some are apparently limited to fresh water. The manatees are found in the Atlantic Ocean and its tributaries on the shores of Africa and the warmer parts of America. The sirenia gave rise to the mermaid myth, for when a dark head rises above the water, and the mother is seen holding her young in her flippers, the illusion is sufficient to mislead the average seaman. And the illusion is complete when the animal dives and the flukes of the tail are seen.

Order Cetacea. The cetacea are the whales and their allies. They are the largest living animals, and from the standpoint of mass are probably the largest which have ever existed. The herbivorous dinosaurs were longer but not so large. Some whales reach a length of ninety feet or more, while the smaller porpoises are only a few feet long. The young are born with the 
swimming instinct well dereloped, but when speed is required the young are carried on the front flipper of the mother. The teats of some species are partly enclosed by a fold of skin so that the young can be suckled while the mother is swimming slowly.

The skeleton is highly modified, the digits being enclosed in skin, forming a flipper. The number of phalanges is always increased. The pelvic limbs are lost, but a vestigial pelvis is
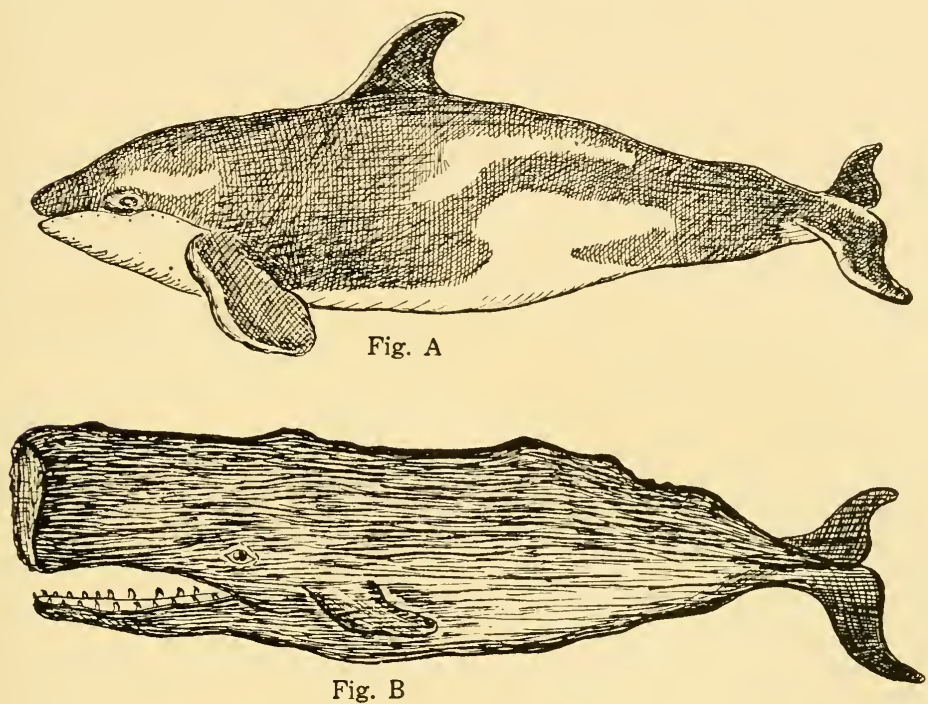

Fig. 39. Order Cetacea. Aquatic, carnivorous placentals including the porpoises and whales. They are the largest known mammals.

present, and femori have been found in several specimens. The skull is modified, with the nostrils on top of the head, and the frontals reduced. Some of the whales have teeth which are small, very numerous, and similar from front to back; others have only a few, or entirely lack teeth. The last have an epidermal strainer in the mouth, the so-called "whale bone", which assists the animal in catching its food. This consists of enormous numbers of small fish and squid which are taken into the mouth with the water and held by the strainer as the water is ejected. The whales are rapidly disappearing because of modern whal- 
ing apparatus, and the increasing demand for oil from the thick layer of subcutaneous fat. Ambergris, a cholesterol substance used in the manufacture of perfume, is a secretion of the intestinal tract. A great deal of mythology has grown up around the whale. The "blowing" of a whale is the condensation of the breath in cold air, not a jet of water as usually depicted in story books; and the belief that it is a fish goes back to biblical times. Even as late as the nineteenth century natural histories called it a fish, a great convenience, as the flesh of the porpoise was a royal dish and with this classification could be eaten during Lent.

Embryology of the Mammals. The Monotremes, with their large yolked eggs, have developmental processes essentially like those of reptiles. The two higher groups, however, have lost the large amount of yolk in the egg, and specializations of fundamental importance have developed. The following description of early embryology applies generally in its essentials, but each order has its own modifications which come properly in a study of comparative embryology. The later development of marsupials has been mentioned, and the present discussion is limited to the placental, using the rabbit as a type.

The minute egg is fertilized in the "oviduct", the upper end of the uterine tube, and passes into the uterus. It soon becomes attached to the uterine wall. The early cleavages are holoblastic, and the cleaving ovum forms a hollow, elliptical organism with a small knot of cells at the dorsal pole. This hollow sphere is not a blastula, but the placental homologue of the yolk sac. the knot of cells at the top being the equivalent of the embryonic disc. The embryo, having formed its own yolk sac, proceeds to develop in a typically amniote manner.

The dorsal embryonic cells, or embryonic knot, begins division and forms a flat plate of cells, the true blastula of the placental mammal. Gastrulation is by an invagination near one edge of the blastula, much like that of the reptile. The amniotic folds grow over the embryo, the inner layer forming the amnion enclosing the amniotic cavity; and the outer layer, encircling the amnion and yolk sac, is the chorion. The latter is in contact with the wall of the uterus. The allantois develops as an 
outpocketing of the hind gut and grows outward toward the chorion, lying between the amnion and yolk sac.

Up to this time the more fundamental processes of the placental have parallelled those of the reptile; but the later development of nutritive processes differs materially from those of other
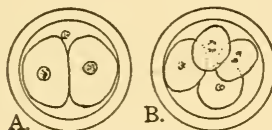

B.

Cleavage of Mammalian Ovum

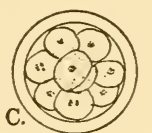

D. -20

\section{Embryonic knot}

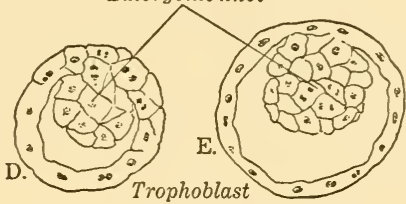

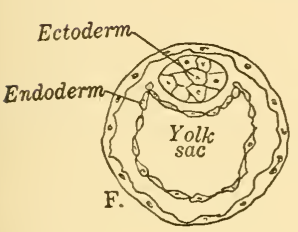
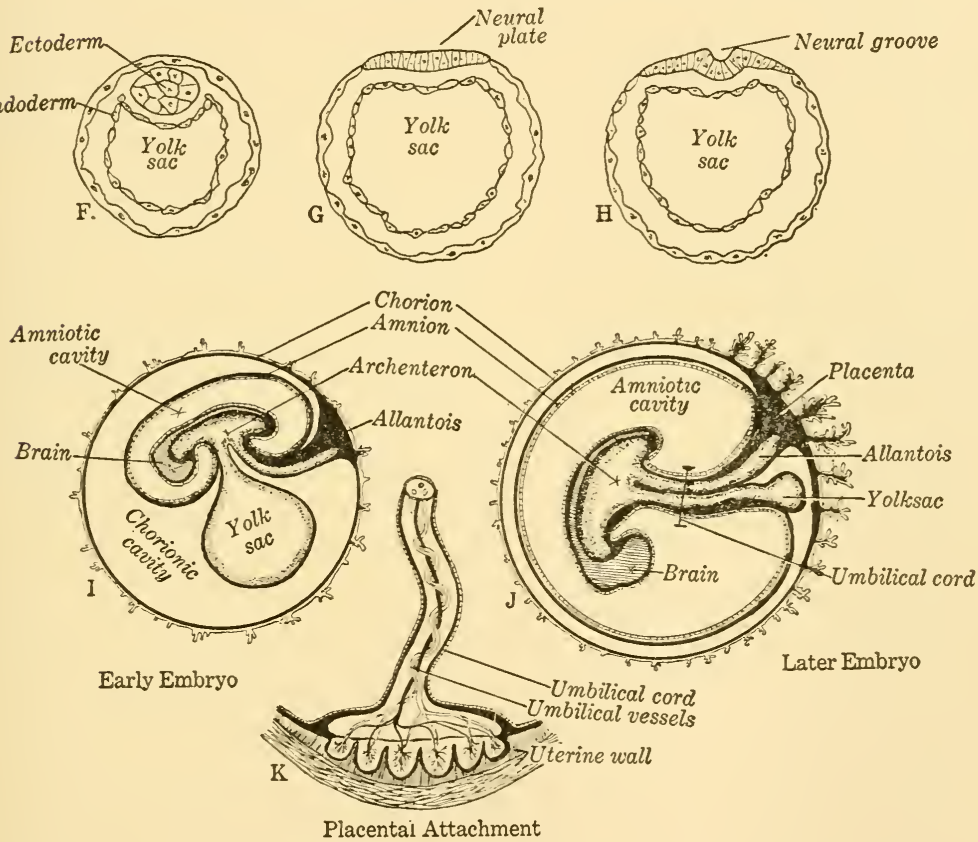

Fig. 40. Embryology of the Mammal. Diagrams D to $\mathrm{H}$ inclusive are cross sections to show the development of ectoderm, endoderm and yolk sac from the embryonic knot of cells. Drawing $I$ and $J$ are longitudinal sections to show the development of the embryo, the umbilical cord, and placenta. Drawing $\mathrm{K}$ shows the attachment of the embryonic placenta to the maternal uterine wall, and the placental villi extending into the blood sinus of the uterus. 
vertebrates. The chorion of meroblastic embryos lies in contact with a porous shell, oxygen being absorbed by the capillaries of the allantois from diffused air, and food from the yolk granules. The essentials of nutrition are supplied the placental embryo by the development of a placenta.

Hair-like villi develop on the chorion. These processes secrete an enzyme which eats into the maternal tissue to form numerous cavities which eventually fuse to form a relatively large sinus in the thickened wall of the uterus. When the allantois reaches the chorion the growth of the villi is accelerated in this region and the allantoic tissue grows into the villi. Synchronously the villi of the other parts of the chorion degenerate. As the allantoic villi burrow into the maternal sinus numerous veins and arteries are cut, so that the sinus fills with maternal blood. The placenta is the mass of tissue formed by the chorion and allantois, with its villi and blood ressels. In the rodents and most primates the placenta is discoidal in shape, and is connected with the embryo by the body stalk.

Blood vessels pass downward from the embryo to the placenta and there break into capillaries and enter the villi. By osmosis through the membranes of the placenta waste products are given out into the maternal blood sinus, and oxygen and food are taken up from the blood entering the sinus from the cut arteries. The blood is constantly being drained from the sinus by the veins with the result that the supply is always fresh.

The connection between the placenta and the body of the embryo is the elongated body stalk, known as the umbilical cord. After birth the cord and placenta slough off and are lost. The point of attachment of the embryo remains as the umbilicus or navel.

The sub-class Placentalia receired its name from the placenta, which is typical of the group. The structure is not found in any other mammal, with the exception of a primitive homologue dereloped by one marsupial (Perameles). There are several types of placentae, apparently equally efficient. (1) The discoid placenta is found in the rodents, the higier primates, and in other orders; (2) the diffuse placenta, spread evenly over the chorion, is found in the horse, pig, and lemurs; (3) a cotyle- 
donary placenta, consisting of rounded masses of villi spaced at intervals over the chorion, develops in the cow, sheep and other herbivorous animals; (4) an intermediate type, combining types (2) and (3) is present in a few herbivores; and (5) a zonary placenta develops in the carnivores, consisting of a median band around the chorion.

With its efficient feeding mechanism the placental mammal is born in a fairly well developed condition. The degree of development varies with different orders and even the genera within an order. In general terms, the more primitive mammals are born in a less mature condition, but even there one must distinguish between total derelopment and the specialization of certain characters. The newborn rat, for example, is able to crawl although its eyes are not open; a newborn monkey has its eyes open but has very limited body coordinations. The length of the embryonic period is fairly correlated with size at birth, although the body size of the parents is a contributing factor. Further, the length of this period of gestation and the rapidity of post-natal development are correlated with the total life span of the race. The poor development of the human at birth, and the prolonged period of development have been variously interpreted. 



\section{PART II}

\section{COMPARATIVE MORPHOLOGY}

Axatomy can be studied either by taking type forms of the different classes and completing one before beginning the next; or by studying all of the types comparatively, one system of organs at a time. The former is systematic anatomy and was the method used in the first part of this text. The latter is systemic anatomy, and presupposes some knowledge of the animals to be studied. The following chapters on morphology, or form, consider the organs and systems of organs in relation to their development and completed structure.

Each system is traced from the simpler to the more complex, both from the standpoint of individual development (ontogeny) and racial development (phylogeny). Sufficient information has been given about natural history and embryology to show that each organism is an individual, and that each group is more or less definitely separated from others; but the student has undoubtedly realized that there are underlying relationships between groups, just as there is a relationship between the organs of the body. Thus, from the standpoint of evolution, the classes and orders of the vertebrates are but units in a coordinated whole.

The fundamental unity of development that is found in the races of vertebrates is also true of the organs of the body. In the preceding embryological discussions, and in the chapters which follow, each system has been considered as a unit. But, although this is convenient for study, independence of organs is never true functionally for each system is dependent upon every other; and the individual is not a group of discrete organic units, but a coordinated whole.

The stress which is generally placed upon laboratory dissections applies particularly to the study of comparative morphology. The anatomical facts should be gained at first hand from a study of the animals themselves, and the best that a book can do is to correlate these more or less isolated details into a coherent scheme. A text, however, cannot replace the knowledge gained in the laboratory. 



\section{CHAPTER IX}

\section{TISSUES OF THE BODY}

THE tissues and organs of the body illustrate perfectly the principle of unity of structure in the individual organism. We speak of the body as being made of tissues, each specialized for certain particular functions. Various tissues make up the organs and systems of organs, and the systems make the individual. It is therefore well to understand the fundamental tissues before considering organs.

Ordinarily five types of tissues are recognized: (A) epithelial or covering tissues which cover the body and line all cavities; (B) connective tissues which include all the supporting elements of the body; (C) fluid tissues, the blood and lymph, which are often included with the connective tissues; (D) muscular tissues, those which are specialized for contractility; and (E) the nerve tissues, the most highly specialized cells of the body, functioning as carriers of stimuli. These five types are treated separately below.

A. Epithelial tissues are perhaps the most diverse in structure and function of any found in the body. Most epithelial cells retain the ability to reproduce themselves and replace those cells which are lost. Epithelia include the outer layers of the skin; the lining cells of the blood vessels and digestive tract; and the secretory cells in the glands of the body. In fact, all cells are included which act as a cover to other tissues. These cells differ widely in origin and structure. The epithelium covering the body is ectodermal; that which lines the digestive tract and the glands derived from it are endodermal; and those lining the blood vessels, the coelomic cavity, and other spaces, are mesodermal in origin. Structurally several types are recognized.

1. Squamous tissues have flat polygonal cells which are very 
thin, and lie against each other in a tile-like manner. Those in the skin lose the power of division.

2. Cuboidal epithelium, as the name implies, is composed of cells which are about as deep as they are in width.

3. Columnar cells are long, one of the smaller faces lying in contact with the opening. This type is naturally the thickest of those epithelia which are only one cell deep.

Any surface may be covered by epithelium which is a single layer in thickness, or by stratified epithelium composed of several layers of cells. The former is simple and is easily classified by its type of cells. In stratified epithelia it is not uncommon to have a base of columnar cells with several layers of cuboidal cells above them. These tissues vary in thickness and in type

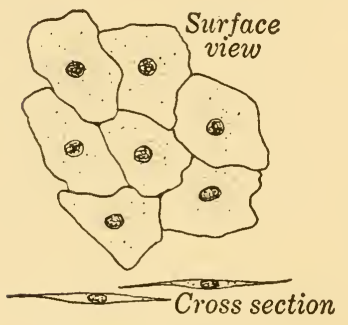

Squamous cells
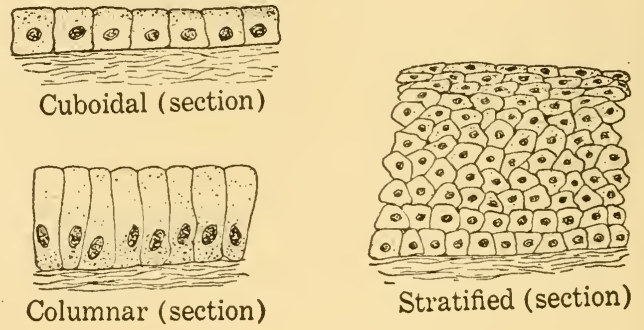

Fig. 41. Epithelial Tissues.

of cells composing the strata. Also, epithelial cells may change their shape when covering a distensible membrane. The squamous cells of the arteries become thinner under force of blood pressure, and the cuboidal cells of the bladder become squamous when the organ is enlarged.

B. Connective Tissues. These are mesodermal tissues which support the other tissues. They are the most abundant tissues of the body, ramifying through every gland and muscle. It is customary to divide them into (1) connective tissues proper and (2) cartilage and bone.

1. Embryonic tissues are of little importance in a study of the adults of any group, except in pathology. The healing proc- 
ess in a cut or wound depends upon other connective tissues reverting to the embryonic condition, filling the opening, and then re-developing into the original type.

2. Reticular connective tissue fills every available space in the body. A section of a gland or muscle stained for connective tissue will demonstrate this. In either a gland or muscle the reticular fibers surround every cell, interlacing to form a firm network throughout the structure. These fibers are usually continuous with the following, thus binding the tissues and organs strongly together.

3. White fibrous tissues form the loose areolar tissues found under the skin and most of the tendons and ligaments of the body. The fibers of the latter lie parallel, are strong and tough, and allow of little stretching.

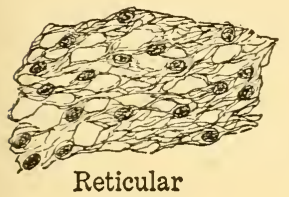

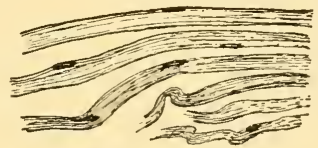

White fibrous

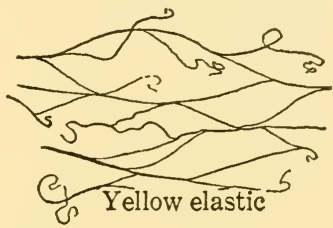

FIG. 42. True Connective Tissues.

4. Yellow Elastic fibers are less abundant than the preceding, and are usually mixed with other types. The fibers split and fuse again, and are elastic in nature. They are found in the walls of arteries, in the connective tissues under the skin, and in other places where they automatically pull a structure back into shape after displacement. Most mammals have a strong bundle of these fibers in the dorsal part of the neck.

5. Cartilage is a strong, firm connective tissue. The cells of cartilage are essentially like those of the true connective tissues, but instead of developing fibers as do the others, they secrete a non-cellular, translucent substance around themselves. As more of this substance is formed the cells are pushed farther apart until the cells lie isolated from each other in the surrounding matrix.

The above description applies to the basic structure of all cartilage, and to the adult condition found in hyaline (clear) 
cartilage, which is simplest and most abundant. In the discs between the vertebrae and other parts of the body the simple cartilage is strengthened by white connective tissue fibers, and is known as fibrous cartilage. Elastic cartilage, containing elastic fibers, is found in the larynx and other isolated parts of the vertebrate.

6. Bone is the hardest of the connective tissues. The bone cells develop fibers which finely interlace, and then salts of calcium and potassium are laid down in the spaces between the fibers. So intimately are the organic and inorganic elements mixed that either may be destroyed without affecting in the slightest

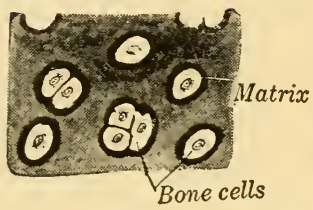

Hyaline cartilage

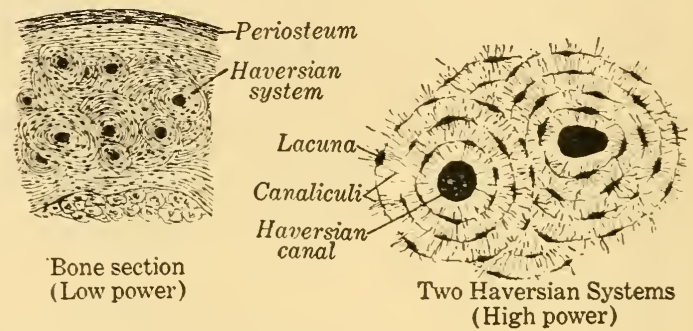

(High power)

Fig. 43. Cartilage and Bone.

the appearance of the minutest structure of the tissue. The cells lie in spaces (lacunae) in the matrix, with microscopic canals (canaliculi) radiating in all directions and joining the canals from other cells. This ? akes a protoplasmic connection throughout the entire bone. The blood supply is secured from small arteries and veins which pass longitudinally through the matrix, a pair (artery and vein) lying in a Haversian canal.

A cross section of a long bone shows that the matrix is laid down as concentric layers or lamellae. The lamellae in contact with the membrane surrounding the bone extend completely around the shaft; but within these few concentric layers, the lamellae surround the Haversian canals, forming Haversian systems. Each canal is surrounded by concentric lamellae, each system being microscopic in size. The cell spaces lie between the lamellae. A more complete discussion is given in Chapter XI, page 172 .

Although the bones are structurally similar, there are two de- 
velopmental types in the higher vertebrates. The oldest, from the standpoint of evolution, are the dermal bones which are homologous with the dermal denticles of the dogfish, and in all cases develop in the mesodermal layers of the skin. The others are replacement bones which originate in cartilage. The cartilage is not transformed but is first destroyed, and bone is laid down by bone building cells. This process is very gradual, much skeletal cartilage being left in the new-born human, and the replacement in the long bones is not complete until growth has ended. Especially in the lower rertebrates, large regions are never completely ossified.

C. Fluid Tissues. The blood of the vertebrate is composed of a fluid medium with cells, or corpuscles, floating in it as discrete units. The fluid is the plasma. As most of the corpuscles arise in the long bones, these tissues are often included as fluid connective tissues.

Blood corpuscles are of two main types: (1) red cells or erythrocytes, containing the haemoglobin which has an affinity for oxygen; and (2) the white cells, or leucocytes, which are ameboid in nature and assist in freeing the body of foreign particles. A more detailed description is given in the chapter on the vascular system.

D. Muscle Tissues are highly specialized for a contractile function. In the higher vertebrates, at least, they lose the ability

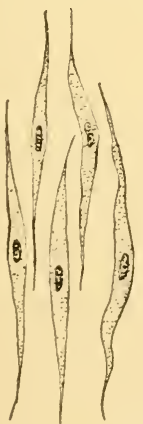

Smooth

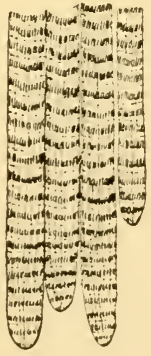

Striated

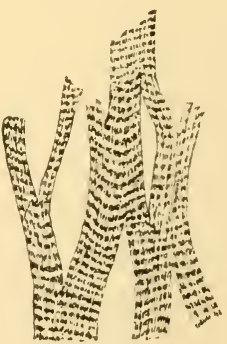

Cardiac

Fig. 44. Muscle Tissues. 
in the adult to divide and reproduce themselves, although cut ends of fibers may be regenerated. The "growth" of a muscle as a result of exercise is not true growth in the sense that the number of fibers is increased, but depends upon the individual cells adding fluids. The muscle increases in size without an increase of the elements of which it is made. The three types of muscle tissue differ functionally and structurally.

1. Striated or skeletal muscle forms the greater part of the bulk of the body. Each individual fiber is very long, some being twelve or fifteen centimeters in length, and is syncytial in structure. Under the microscope these fibers appear transversely striped, light and dark bands of protoplasm alternating. The fiber is surrounded by a very thin sheath of connective tissue; and in the protoplasm are the numerous nuclei. These muscles are voluntary in function, that is, they are under the control of the central nervous system, and can be contracted at will.

2. Smooth or visceral muscles are those which are involuntary in function. They contract slowly when stimulated and are not under the direct control of the brain. The cells are small, spindle-shaped, and each has a single nucleus.

3. Cardiac muscle is found in the heart and combines the functions of striated and smooth. It is involuntary in action, but responds rapidly to stimuli. Cardiac muscle is usually described as a syncytium, the protoplasm extending continuously throughout the mass. Microscopically the tissue appears divided into irregular cells which are striated, each with a single nucleus. Physiologically the heart is a compact unit, nerve impulses traveling rapidly over its entire area.

E. Nerve Tissues are ectodermal in origin and are discussed fully in the proper chapter. The individual nerve cell has a cell

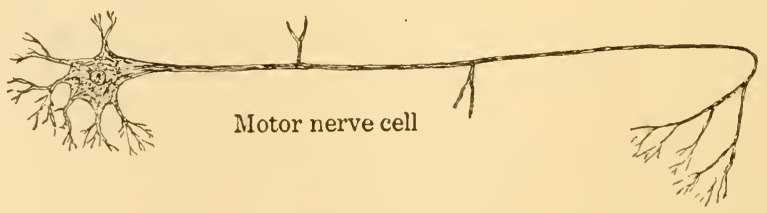

FIg. 45. A typical Nerve cell (Neurone). 
body with processes at either end. One set of fibers (the dendrites) receive impulses which are passed through the cell body and out to nerve cells or other tissues through the long axone. These tissues are the coordinating mechanism of the body. The processes may be re-developed, but the destroyed cells cannot be replaced.

The student cannot be told too often that the organism is a unified mechanism and depends upon the proper coordination and function of all its parts. Proper function depends upon proper balance between tissues and organs. This interdependence

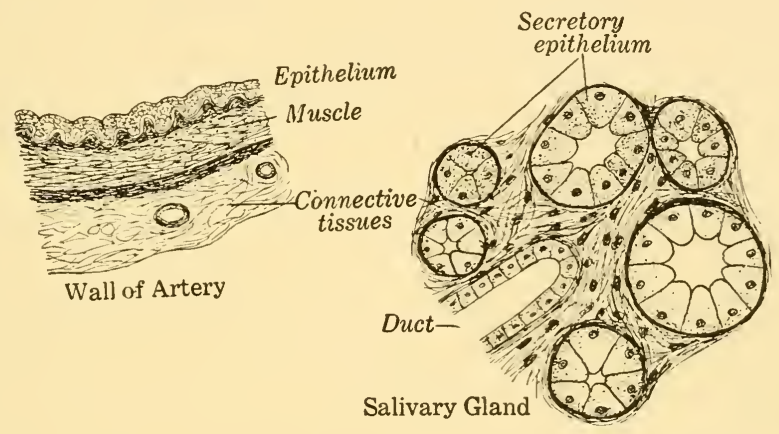

Fig. 46. Organ Formation, showing different tissues combined in a single organ.

of the tissues can be illustrated with any organ of the body. We speak of muscle tissues, but a muscle is an organ, not a tissue. The muscle tissues are the contractile elements, but for their proper growth and function they must be bound securely into a functional unit by connective tissues; the impulses which cause them to contract must pass over the nerve fibers which innervate them; and for food and oxygen they depend upon the fluid tissues to transport these substances to them. And the thin epithelial walls of the capillaries permit the plasma to pass out to the muscle fibers.

Similarly, every organ of the body is made of several types of tissue. The blood vessels are composed of muscular and connective tissues, lined by epithelia, and controlled by nerve stim- 
uli. In the same manner the individual is a group of organs and systems which must function as a whole.

In studying the following chapters the student will do well to keep function in mind, and remember that the individual is the sum of all its parts. Without this, anatomy becomes a mass of labelled structures, each unrelated to any other. 


\section{CHAPTER X}

\section{INTEGUMENTARY STRUCTURES}

THE integument, or covering, of the vertebrate consists of the skin and its derivatives. Amphioxus has the simplest possible skin structure, consisting of a thin layer of mesodermal connective tissue, with an ectodermal layer of epidermis only one cell thick. The latter cells secrete a thin cuticle of non-cellular material which covers the animal externally. The vertebrate skin is based on the same principle although it is thicker, lacks the cuticle, and is complicated by other structures which grow from either layer.

The skin is essentially protective in function. The cyclostomes secrete a protective layer of mucus, the fish have protective scales which arise from the skin, and the reptiles, birds and mammals have a dry integument which is impervious to pathogenic bacteria.

The ectodermal part of the skin is the epidermis and lies upon the dermis (corium) which develops from the epimere of the mesodermal somite. The dermis is a fibrous structure resembling the trpical connective tissues, and is usually a close-packed structure well supplied with nerves and blood vessels. It is connected with the underlying muscles by connective tissue fibers which freely interlace with the reticular fibers. There is a layer of subcutaneous connective tissue lying betreen the well defined skin and the muscles. This permits some movement of the skin without affecting the muscles, and explains the ease with which most vertebrates are skinned. In addition, the subcutaneous tissues are normally loaded with droplets of fat, which assists in heat regulation and supplies a store of food for the animal. This becomes of great importance in animals which hibernate or aestivate.

The epidermis is several layers thick. Lying next the dermis is a growth layer, the stratum germinativum (stratum Malpi- 
ghii) which is in constant cellular division. Above this is a pigmented area, not well defined in most vertebrates, and then a various number of layers considered together as the stratum corneum. The cuboidal cells of the stratum germinativum become flattened in the corneous layer, the outer ones dead. Due to continual mitoses the skin would become progressively thicker if the outer dead cells were not discarded. In the amphibia and reptiles this is accomplished by periodic ecdyses or moults, depending to a large extent upon the amount of food and the consequent more rapid growth. In the birds and mammals the outer cells are shed as small flakes. Dandruff and the minute rolls of epidermis removed with a rough towel are illustrations of the mechanism. Death in these cells is in large part due to lack of nutrition, nerves and blood ressels being lacking in the epidermis.

In the making of leather the epidermis is lost. The remaining thick mat of dermal fibers is softened, cured and used in manufacture. Practically all commercial leather is from the mammal, though alligator, snake and lizard skins are widely used for ornamental leathers; and ostrich skin is frequently used for bill-folds and other small articles. The dermis retains practically all of the original structure, the scales of the reptile or the feather pits of the bird.

\section{A. Dermal Derivatives of the Skin}

Structures derived from the skin mesoderm appeared first in the most primitive fish. The primitive condition has been retained by the sharks (Elasmobranch fish) where they are found as isolated dermal denticles. These minute bony plates cover the body, varying slightly in size, and continue over the lips where they have become modified into teeth. They are, therefore, homologous with the teeth of all vertebrates. Embryologically dermal denticles and teeth are composed of both mesoderm and ectoderm, the basal plate and body of the denticle being mesoderm, while the outer covering, or enamel, is of ectodermal origin. The student should study the diagrams of the denticles and compare them with the drawings of developing teeth on page 142 . 


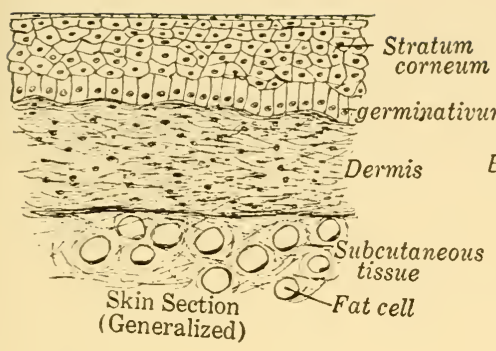

Stratum germinativum

Stratum corneum

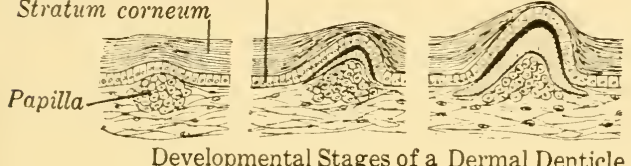

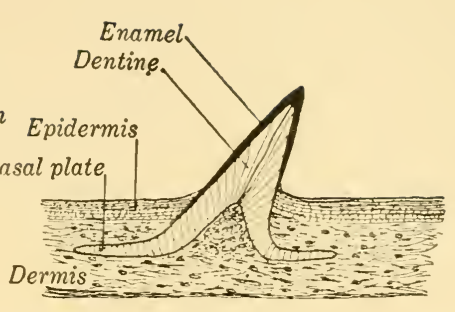

Dermal Denticle

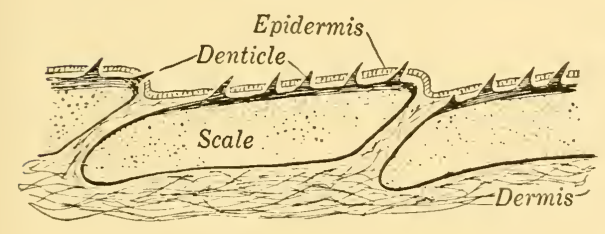

Section of a Bony Scale (Lepidosteus)

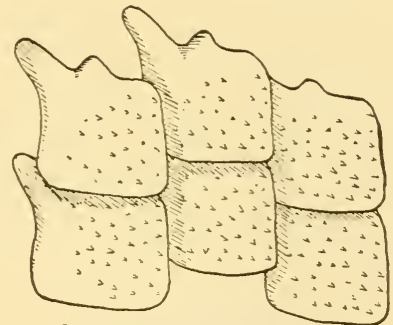

Surface View of Bony Scale (Lepidosteus)

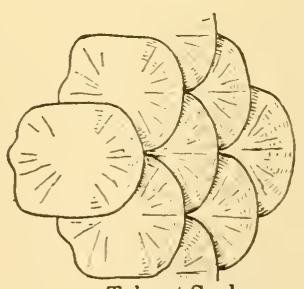

Teleost Scales (Surface View)

FIG. 47. Skin and Dermal Scales of the Fish. 
In the chondrostean fish (the sturgeon and its relatives) the denticles are fused into plates which are relatively large, covering the head with a close-set group of dermal bones, and the body with bony plates. In this and the higher groups the ectodermal part of the dermal plates is lost. The head plates and the body plates are continuous in these fish, and the former are not fused to the underlying chondrocranium. The teeth in the bony fish and the land vertebrates have become well differentiated from the dermal plates, and retain the enamel covering.

In the Holostean fish (page 40) there is a more marked difference between the head and body plates. The former fuse to the skull bones which developed from the chondrocranium, forming a definite skull derived from both dermal and replacement bone. This same relationship is carried through all the higher classes of vertebrates. The Teleostei (page 42) are essentially like the Holostei, though the dermal scales lose the heary bony characteristics of the earlier fish, and overlap as flexible scales.

The primitive Crossopterygii (page 44), which gave rise to the Amphibia, had large heary scales practically continuous with the head plates. Naturally this primitive relationship was carried over to the Stegocephalia. These extinct amphibia had a completely ossified skull, a ventral group of dermal plates, and a dorsal covering. The recent amphibia have lost all signs of body plates, except for very minute ones buried in the skin of the Apoda (page 50).

The amphibian line which branched toward the reptilian line of ancestry retained the body plates. These were a protective mechanism and were of selective value in the constant struggle for food. Practically all the early reptiles had these dermal plates, which, by analogy with the recent forms, we presume were embedded in the skin and covered by the ectodermal layer. Two lines of evolution, as far as the dermal structures are concerned, are apparent. The large, sluggish reptiles tended to keep the plates, for in these animals they are of protective value; the smaller, more active animals tended to lose them, for the race in life is to the swift or well protected. And the student should remember that racial survival depends upon individuals remaining alive. Among the recent reptiles the snakes and lizards lack the plates, the other three orders retain them. 
There is no evidence that the body plates were carried over to the mammals, although the bones of the top of the head and face are homologous structures. In certain small whales there are calcified plates on the dorsal surface; and the armadillos (page 87) are well armored with bony shields. Most anatomists, however, consider these cases as secondary developments, not as homologous structures. The evidence is too complicated for a discussion here.

\section{B. Ectoderial Derivatives of the Skin}

IVith the exception of the dermal denticles of the elasmobranchs, there is a clean-cut distinction between dermal and epidermal derivatives of the skin. The difference is based upon

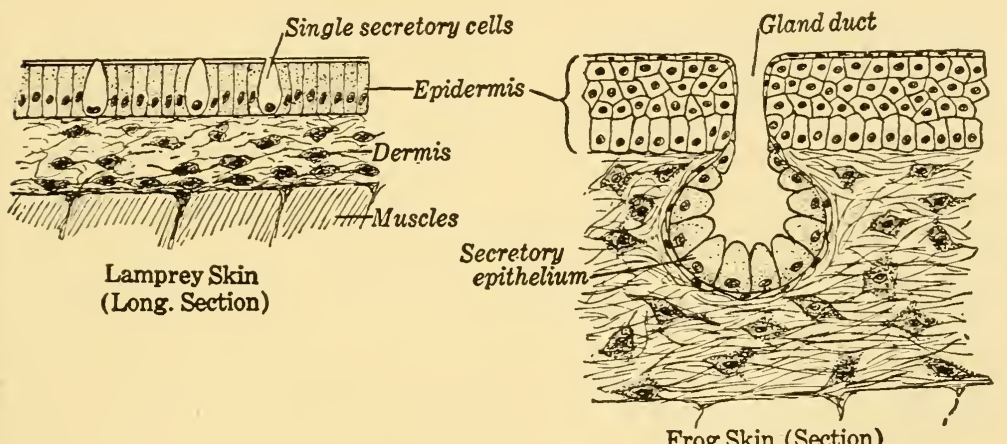

Frog Skin (Section)

Simple aciniform gland

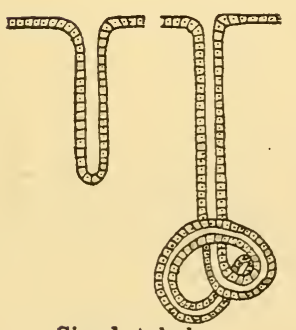

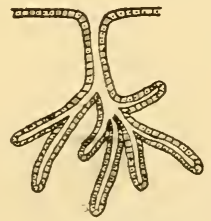

Branching tubular

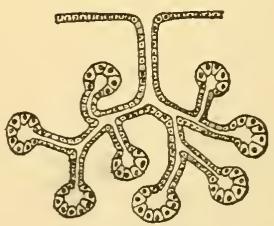

Branching Aciniform

Simple tubular

FIG. 48. Gland Types. The skin drawings (top) show the relationships with the dermis and muscles. The diagrams (bottom) show the branching of simple glands to form more complicated structures. 
origin. Functionally all the ectodermal structures depend upon the dermis for support, and as the blood vessels lie in the dermal layers the nutritive supply is also from these tissues.

Phylogenetically, the earliest ectodermal structures are glands. In the three most primitive classes (cyclostomes, fish and amphibia) there are no other structures of importance. In the cyclostomes and fish these are mucous glands which keep the skin clear of accumulated dirt, and are to a certain extent protective. In the amphibia the same functions are present, and in addition most amphibia depend upon the mucous to keep the skin moist, which is necessary for the skin's respiratory function.

All glands of the vertebrates are based upon three simple types: (1) single secreting cells; (2) tubular glands which are straight or coiled impocketings of the ectoderm; and (3) aciniform glands which are flask-shaped, the neck being the duct to the outside and deeper part the acinus. Either of the last two types may be branched, (1) as simple branches, or (2) into highly complex structures.

Functionally all glands go back to the individual, secreting, epithelial cell. These cells are modified for secretory activity; and the most complex gland is merely a collection of cells specialized for the same function. The duct serves as an outlet, the lining cells being flattened and not secretory.

Reptiles. The reptile skin is almost free of glands. A few buccal, or mouth, glands are present; and most reptiles have scent glands in the anal region. In a number of turtles and snakes the odor is so distinctive that the animal can be detected and identified without seeing it.

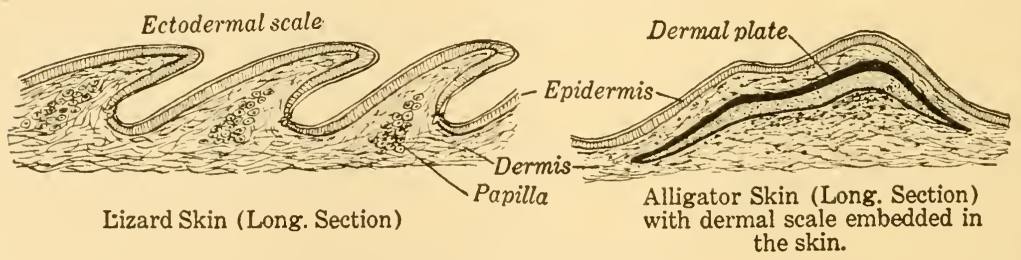

FIg. 49. Sections through Reptile Scales. Reptiles are characterized by ectodermal scales, although in some forms these are supported by dermal plates. 
Ectodermal scales are an identifying character of the reptiles. The lizard skin is typical of the primitive type. In this group the scales are usually sharply pointed at the posterior tip, overlapping the one behind. Under each scale is a mesodermal papilla, and so intimately connected are the two that the epidermis may be removed without destroying the appearance of the skin. In the turtles the scales are wide and flat, but are essentially like those of the lizard. The snakes have dorsal scales like the lizard, but the ventral scales, or scutes, are wide with a rib attached at either side.

Claws arose first in the reptiles as modified areas of epidermis on tle tips of the toes. They are present in all reptiles with feet. The development of claws is discussed under the mammal (page 115).

Structures of particular interest to the evolutionist are sensory organs in the skin of lizards. Under each scale there is a minute invagination with a nerve ending at its base, and from the slight pit a hair-like sensory thread projects. The evidence indicates that this is the reptilian homologue of the mammalian hair.

Binds. Like the reptiles, the birds are almost devoid of skin glands. Some have scent glands around the anus, and most of them have oil glands at the base of the tail feathers. Scales and claws were carried over to the bird, the legs of most birds being encased in typical reptilian scales, with their toes terminated by claws. The horny beak is also an epidermal derivative.

Feathers are modified scales, although highly different in the full grown condition. There are two major types of feather: (1) doun feathers, found in the young and scattered under the larger feathers of the adult; and (2) contour feathers. The latter are the typical feathers, the flat vanes spreading on either side of the central quill. These vary in size and structure from the hairlike filoplumes to the large quills of the wing and tail.

Mammals. The mammalian skin and its derivatives are so highly modified that only a few of the latter can be thought of as directly inherited from the reptiles. The claws, nails and hoofs; the scales of the primitive orders; and the pigment are 
inherited. The hair has a possible homologue, but the skin glands are unlike anything in the ancestral groups. In addition, most orders have some epidermal modification which arose independently.

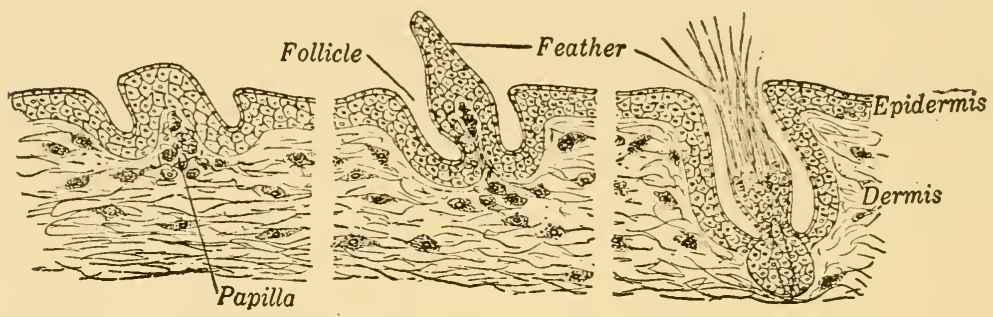

Developmental Stages of Feather

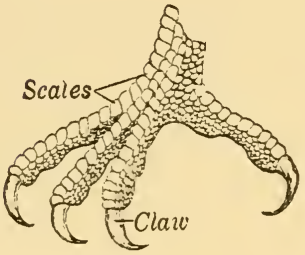

Hawk's Foot

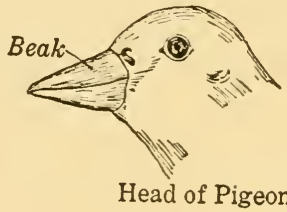

Head of Pigeon

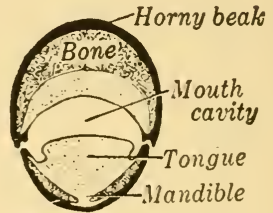

Cross Section of Bird's Beak

Fig. 50. Integumentary Structures of the Bird. The scales are homologous with the feathers. The horny beak is a modification that has appeared in other toothless forms.
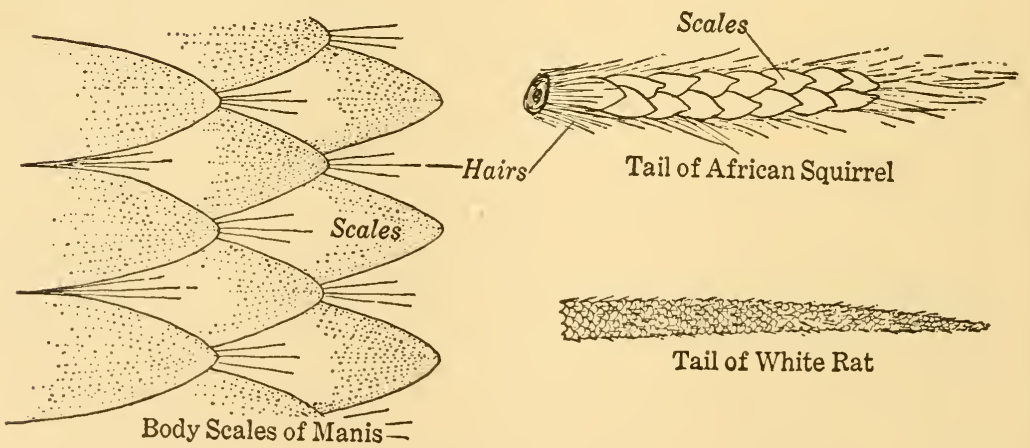

Fig. 51. Scales of the Mammal. Scales are usually limited to the tail region in the Mammalia. Manis (Fig. 35) is covered with orerlapping ectodermal scales. 
1. Scales. The earliest mammals were undoubtedly supplied with scales. Among the primitive orders today there are many illustrations of their survival, particularly in the tail region which is most liable to retain primitive characters. A casual examination of the tail of a rat or mouse will demonstrate the condition. Manis, an edentate (page 87), is completely covered with large scales. The hairs, in groups of three, grow out from the under side of the scale, in the same relative position as the sensory threads of the lizard. Scales are absent in the human, such freaks as Barnum's "Alligator Boy" being diseased conditions and not reversions to a primitive type.

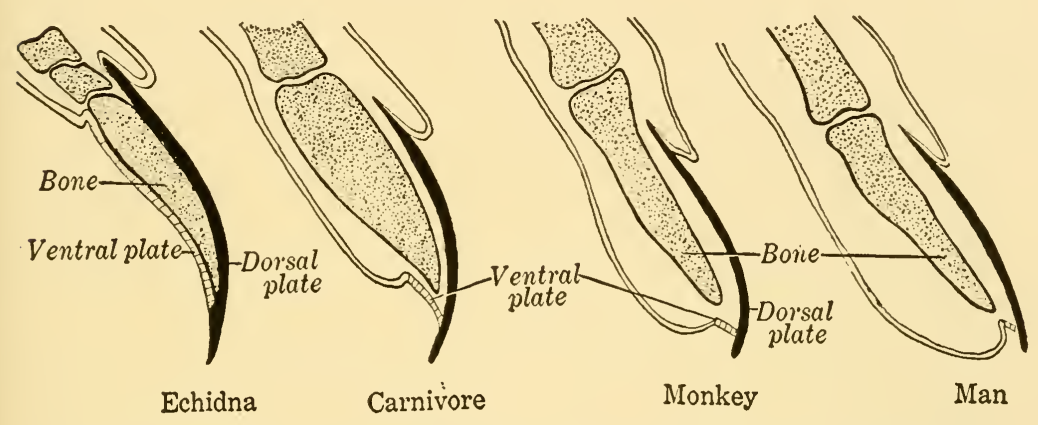

FIG. 52. Sections of Claws and Nails, showing the forward growth of the ball of the finger, and the shortening of the ventral plate of the claw.

2. Claws, Nails, and Hoofs. The primitive ending of the mammal's digits is the claw, similar in origin and structure to that of the reptile. Claws are retained by the insectivores, rodents, carnivores and others. During the course of evolution modification has proceeded in two directions: (1) in the herbivorous, running animals the claws have become rounded hoofs, and (2) in the primates the claws have gradually become flattened into nails, which reach their culmination in the human.

3. Pigment. Although the pigment of the lower vertebrates is more often located in the dermal layer as pigmented connective tissue cells, pigment is extensively scattered through the epidermis of reptiles and birds. In the mammals the pigment layer is apparently limited to the epidermis of the skin. The pigment granules are located within the cells and give the skin 
and its derivatives their color. The baboons have patches of brilliant scarlet or blue (the combination giving a purplish color). In most mammals the pigment is a dark brown, the amount and position giving the varying shades. In the human hair red is not a true pigment but a soluble dye. A person, therefore, might be genetically red headed, with the dye obscured by a layer of brownish pigment.

Apparently too wide conclusions have been drawn as to skin pigment and climate. In general terms the lighter races live in colder climates. But the Eskimos are darker than their white neighbors to the south; the Congo pigmies are lighter than the taller tribes in the mountains; and the white Sicilians are darker than some of the Negro tribes. It would appear, therefore, that it is inherited-character, and the lighter races have migrated away from the points of greatest actinic energy. (Page 329 discusses the effects of natural selection).

Albinism is a complete lack of pigment and differs genetically from white coat color. Melanism is an excess of pigment. Pigmentation in the human is a multiple factor in heredity, and inbreeding never gives a simple ratio.

4. Hair. Hair is one of the distinctive characteristics of the mammals, and from the evidence as to its phylogeny one may conclude that it was first a sensory rather than a protective structure. The long vibrissae, or lip hairs, help corroborate the belief. The hairs originate in the ectoderm of the embryo. A knot of cells pushes downward toward the dermis, the end forming a hollow vesicle. As development proceeds the cord of cells connecting the vesicle with the outer skin becomes tubular, thus making the hair follicle. The vesicle settles against the mesodermal papilla and invaginates around it, in this way coming into contact with a nervous and vascular supply. The hair grows by a proliferation of ectodermal cells from the bottom of the follicle, and as growth proceeds the hair is pushed up the follicle to the outside.

Most mammals are covered with hair except for a few areas -as the glans penis, lips, and soles of the feet. Cetacea are an exception to the rule, and the elephant, rhinoceros, and others have very sparse hair. The distribution of hair is typical for the species or race; in many species there are sex differences; 
and there are definite regional differences. Hair may be definitive in length or continue growing. The latter is usually more marked in the male than the female. There is no definite moulting of hair, though the covering tends to be thicker in winter than in summer in animals of cold climates. Hair is constantly being replaced in the individual, unless the growth cells at the base are killed, and as one falls it is soon replaced by a new one growing from the bottom of the follicle.

Each hair has a central medulla with concentric layers of cells around it. The young cells at the base of the hair are cuboidal, but as the hair is pushed upward the cells flatten and

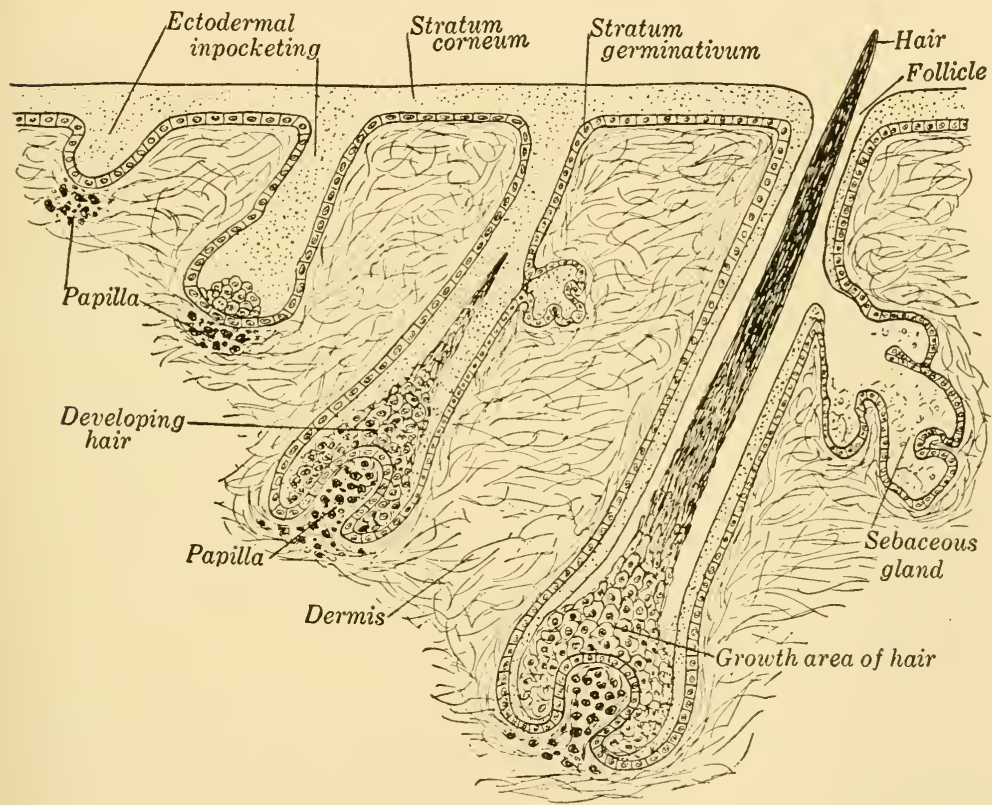

Fig. 53. Development of Hair. The four stages represent progressive embryonic growth, and are not found in the same specimen.

die. Those on the outside, the cuticular laver, overlap the more proximal ones and give the hair a scaly appearance. Many species, like the human, have smooth hair. Others have elongated cuticular scales and have a felting quality, like the wool of sheep. Some animals (rabbits and hares for example) have long 
hairs with an under layer of hairs which are used for making felt.

5. Glands. The glands of mammals are apparently new derelopments within the class. Anal or other scent glands may be present, but the principal integumentary glands are (1) sweat and (2) sebaceous, or oil. The sweat glands are simple tubes with the inner end highly coiled. The secretion assists in heat regulation by the evaporation of water on the skin, and they are to a certain extent excretory. Salts and a slight amount of nitrogen wastes are thrown out with the sweat. Dogs and some other animals lack the sweat glands, the heat regulatory function

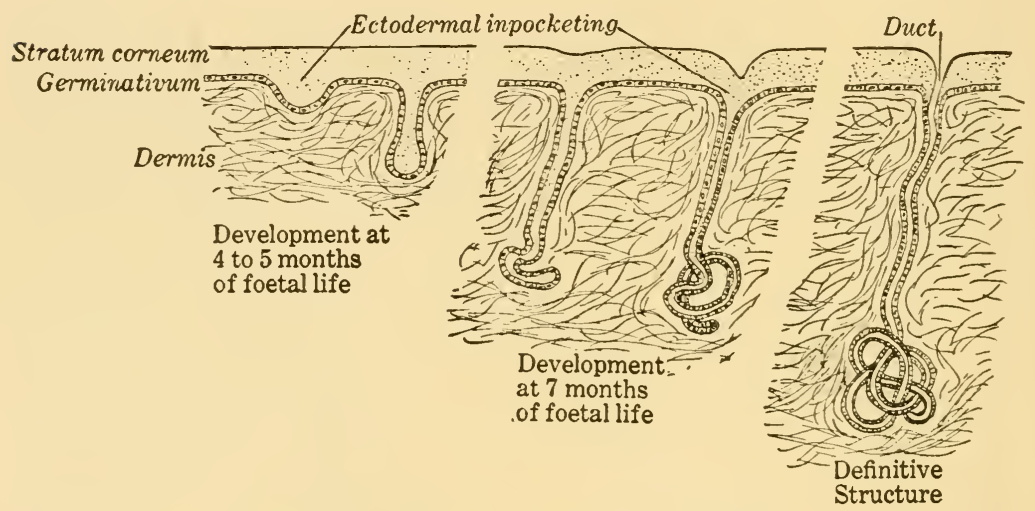

Fig. 54. Development of the Sweat Glands. (Adapted from Kollman).

being taken over by the vascular tongue and mouth, and the correlated loss of heat from the lungs.

The sebaceous glands empty into the follicles in which the hairs are located and keep the hair and skin oiled. These glands differ from most secretory epithelium cells in that the cell is destroyed when the secretion is thrown out. Consequently the gland repairs itself by cellular divisions in the layer next the basal membrane which bounds the epithelial covering.

Mammary, or milk, glands are diagnostic of the mammals. They are probably modified sweat glands. Although present in both male and female they are rarely functional in the male. In the female they are highly functional at the end of pregnancy. Both periodic and sex differences in function are controlled by 

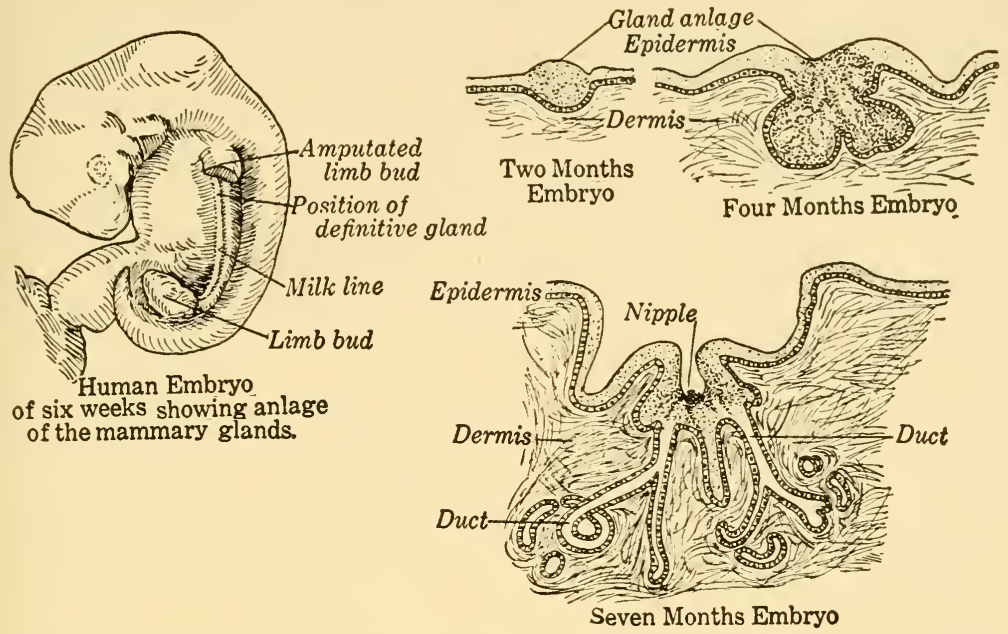

Fig. 55. Development of Mammary Glands. The embryo (left) shows the continuous milk line which degenerates in man, except in the pectoral region. Other mammals develop a series of mammary glands on either side. Super-numerary nipples appear in man as an anomaly. The cross sections through the milk line show three stages in the development of the glands and nipple. Compare with Fig. 54. (After Kollman, Tourneux and Basch.)

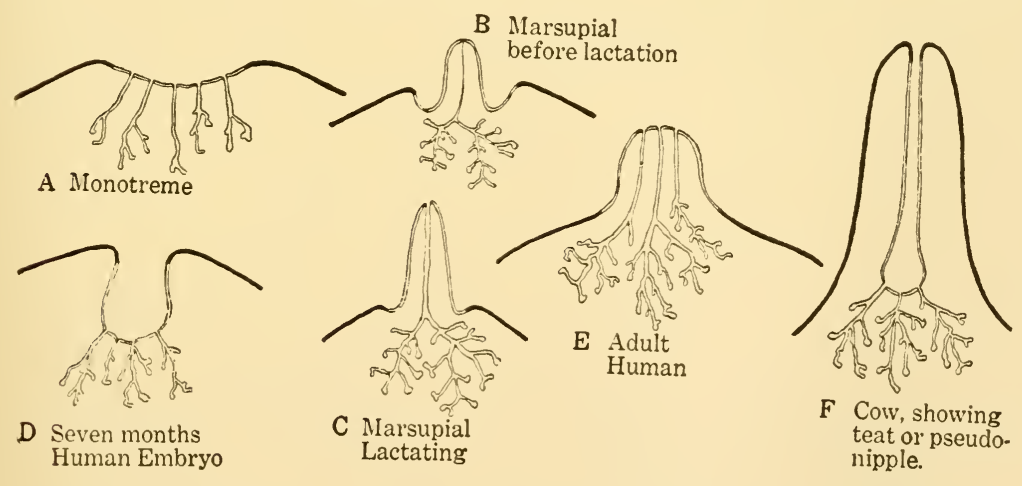

Fig. 56. Diagrammatic drawings of Mammary Glands and Nipples. A comparison of drawing D (Fig. 56) with the Seven Months Human Embryo in Fig. 55 will explain the relationships of structures. (After Weber). 
glands of internal secretion. Milk glands are normally found on the ventral surface of the body, varying widely as to number and position.

The monotremes (page 76) have the glands opening into slight depressions on the posterior abdominal wall. The young lap the milk, much as a kitten licks milk from a saucer. The marsupials (page 77) have developed teats to which the young become attached after birth. In the opossum there is a single gland duct leading into each teat.

The placentals usually have several ducts leading into a teat. In the more primitive type, as in the primates, the ducts empty directly to the outside at the end of the teat. In others, as the cow, there is an inpocketing of ectoderm at the nipple, forming a slight reservoir in the teat with the milk ducts opening proximally into it. The increased number of ducts in the placental gland furnishes a more abundant supply of milk to the more highly developed young.

The primitive placentals had a double row of mammae along the abdomen, but in the more specialized orders they are more limited in number and located either pectorally or inguinally. In the embryos of these animals there are usually several pair which do not develop; but accessory, functionless, nipples are frequently found in both sexes of the human and other placentals.

6. Horns. Horns and antlers, which are found in the artiodactyls (page 87), are distinct in development and structure. Horns have a permanent bony core with an epidermal covering, and the entire structure is permanent throughout life. The Long
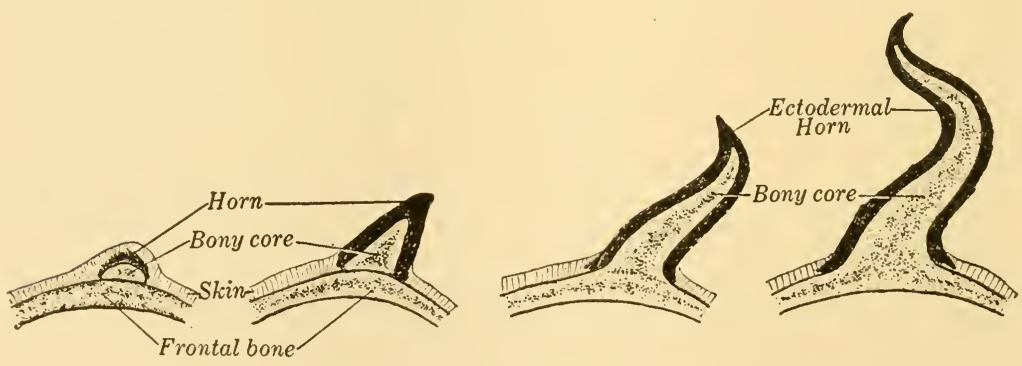

FIG. 57. Growth and Structure of Horns. Horns are permanent, with a bony core and an ectodermal cover. 
Horn steers of the western plains were almost an end product in horn development, some of them measuring eleven feet from tip to tip. After death the core shrinks and the epidermal cover is easily removed. The prong-horn antelope (Antilocapra) is an exception. This animal has a typical bony core in the horn, but the ectodermal cover is periodically shed.

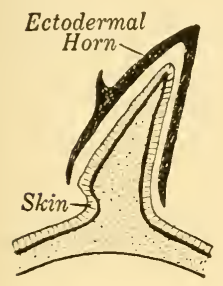

FIg. 58. Prong-horn Antelope (Antilocapra). An American artiodactyl with horns apparently intermediate between true horns and antlers. The bony core is permanent, but the ectodermal cover is shed annually. The diagram shows the horn at time of shedding, with the skin surrounding the core.

Antlers, on the other hand, are shed each year and replaced with larger ones until the deer, moose or elk approaches senility. The antlers are lost in the early spring and the new ones begin their derelopment soon after. Bone building cells congregate at the stub of the shed antler and growth begins. The food supply is gained from the vessels of the skin which is carried out over the growing antler. When full size is reached the skin constricts at the base of the antler, dies, and is rubbed off. The antler, consequently, is bone, and logically has its place with the skeletal system.

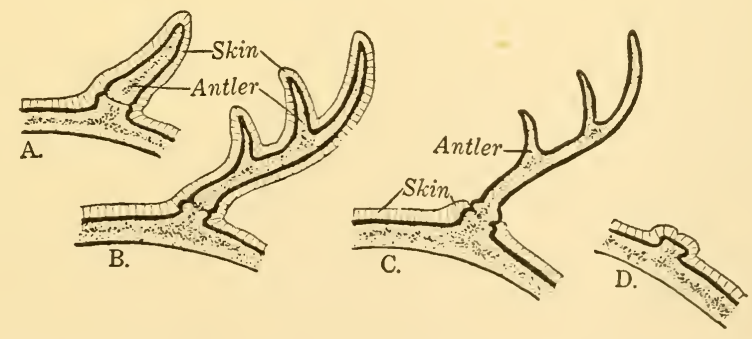

FIG. 59. Growth and Structure of Antlers. During the growth season the antler is covered with skin which is shed when full size has been reached. The antlers are shed annually, and the skin covers the growth base on the frontal bone (D). The growth process repeats itself during the spring and early summer. 
7. Other Structures. Under this heading are listed a few of the epidermal structures which are limited in distribution. (1) The horn of the rhinoceros has no core, and is composed of epidermal, hair-like fibers. (2) The whales which lack teeth have an ectodermal strainer in the mouth (page 91). The horny material is tough and flexible and was of commercial value in a past generation. (3) The ischial callosities on the rump of many Old IVorld primates are callous-like thickenings of the epidermis. These are genetic characters, and differ from the acquired callouses on the hands and feet of humans.

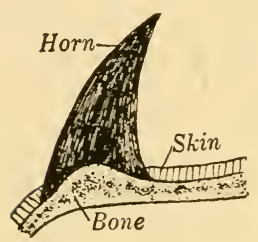

Rhinoceros Horn

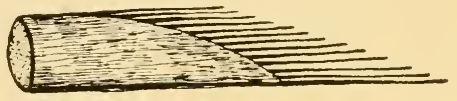

"Whale bone"

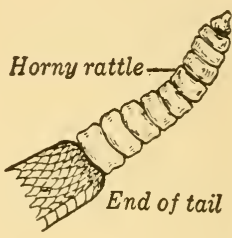

Rattle of Rattle-snake

FIG. 60. Other Ectodermal Structures of the Integument.

Located in the skin as ectodermal derivatives are a number of nerve receptors and organs of special sense. Perhaps the most prominent of these is the lateral line system of the fish and amphibia. The structure and relationships of the organs is discussed in Chapter XVIII, under Organs of Special Sense.

\section{Humax Skin}

The skin of man makes an excellent summary of the skin and its derivatives. The illustration gives the relationships of the two primary layers of the integument, and the position and structure of its outgrowths. The dermis is fairly thick and is of a loose fibrous structure. The stratum germinativum has a waved appearance, being several cell layers in thickness. Immediately above it is the pigment layer, the number of granules varying in different races and parts of the body. The thick outer layer is the stratum corneum. This last may be very thin, as on the lips, glans, etc. 
Friction ridges are found on the soles and palms. These are evidently remains of the scaly covering of the ancestral types. Following the work of Galton the study of finger prints has become an independent science. As no two hands entirely agree in configuration, but fall into definite groups, indexing is easy and identification is absolute. These ridges apparently change little throughout life, and if all hospitals adopted the methods of the more progressive ones in making finger or foot prints of the mother and newborn child before leaving the operating room, the too frequent litigations over mixed babies in hospitals would end immediately.

The sweat glands pass well into the dermis before coiling, and are distributed over the entire body without relationship to the hair follicles. The sebaceous glands, on the other hand, evidently developed in correlation with hair. In the human these are quite large. If the follicle of the hair is closed, the secretion continues and the gland may hypertrophy. The result is a spherical mass under the skin, the larger ones being called wens.

Technically speaking there are no "pores" in the skin. The only openings are the follicles and the sweat glands, and these end blindly in the dermis. There is no possibility of a respiratory function, although some excretion takes place. Therefore, the alleged cases of individuals dying from sealing the pores were caused by lead poisoning or other extraneous conditions. The fallacy is kept alive by soap manufacturers and the mothers of boys.

Hair distribution has been mentioned in relation to the sex differences and regional distribution. Related to the latter is pattern distribution on the body. It is common observation that the hair of the head grows away from the crown, and a secondary crown or "cow lick" is not uncommon. The body hair has similar patterns. In general terms, the direction of hair growth is the same as the scale direction of the reptile: (1) from anterior to posterior, and (2) from dorsal to ventral. On the human back the pattern lines are antero-posterior along the spinal column, but dorso-posterior on the sides. This is similar to the pattern of the other primates.

Embryologically hair arises in groups of three or four, again indicating a scale pattern as found in Manis. During later em- 
bryonic life the foetal body is covered with a deciduous lanugo, a soft thick coat of hair. As it cannot be interpreted in terms of function, the only explanation offered is that it is a hang-over from our hair ancestors. The pattern of the lanugo is the same as that of the anthropoids. 


\section{CHAPTER XI}

\section{SUPPORTING STRUCTURES}

THE vertebrate supporting structures include the endodermal notochord, and the mesodermal cartilage and bone. With these should be included the connective tissue sheaths of the latter and the ligaments which tie them together. These are necessary for proper function in protection and locomotion, and will be discussed under the leverage of muscles.

The original function of the skeleton was leverage, the structures serving for the attachment of muscles. With the development of the skull and vertebral column came a protective function as the bones surrounded the nerrous system; and in the land vertebrates the marrow of the long bones forms most of the corpuscles of the blood.

The vertebrate has an internal skeleton. The external skeleton of the insects and crustacea is perhaps more efficient so far as leverage is concerned, the muscular pull being more direct; but what the vertebrate lost in leverage was more than counterbalanced by flexibility of body parts.

The skeleton of the vertebrate is divided into two regions: (1) the axial skeleton including the skull and vertebral column, both of which develop around the notochord; and (2) the appendicular skeleton, including the skeletal girdles and the appendages. These two regions are developmentally independent, but where both exist they are functionally dependent upon each other.

\section{A. The Skull}

As was mentioned in the first part of the book, the vertebrate skull has a double origin, (1) from the primitive chondrocranium (cartilage skull) and (2) from the dermal plates or bones. The 
chondrocranium is phylogenetically and ontogenetically older, and will be discussed first.

The embryonic chondrocranium of the higher vertebrates has its counterpart in that of the cyclostome as an open trough in which the brain lies, with a cartilage bridge over the nerve cord at the posterior margin. The cupped hands with the tips of the thumbs touching will assist in visualizing the structure. In addition to the original trough there are three sensory capsules which develop synchronously. The most anterior capsule surrounds the olfactory nerves; the middle one is the eye, or optic, capsule; and the most posterior is the ear, or otic capsule which encloses the organs of equilibrium, and the true ear of the higher vertebrates. Olfactory and otic capsules form an integral part of the chondrocranium, but the eye capsule always remains free, leaving the eye movable.

The development of the chondrocranium is from two pairs of ventral cartilages, and three pairs of capsules, each arising as a separate cartilage center. The ventral cartilages lie on either side of the notochord, the posterior pair being the parachordal (beside the notochord), and the anterior are the prechordal. The three pairs of capsules arise slightly later. As the ventral cartilages grow, they fuse ventrally, surrounding the notochord, but leaving a ventral hole immediately anterior to the notochord. This opening remains in the higher forms as the pit for the pituitary gland.

The lateral margins of the basal cartilages then grow dorsally. In the meantime the olfactory capsule has surrounded the olfactory center, and the otic has surrounded the ectodermal ear structures. These soon fuse with the chordal cartilages to form a coherent unit. Cartilages then grow from either otic capsule toward the dorsal-median line and join to make the posterior "bridge" or synotic tectum, and the chondrocranium is complete. The posterior opening through which the nerve cord passes is the foramen magnum, and numerous smaller foramina are caused by the passage of nerves and blood ressels from the brain through the chondrocranium to the body tissues.

The dogfish chondrocranium is in many ways specialized. In addition to the simple trough a roof has developed, completely enclosing the brain except for the foramina. Also, a rostrum has 


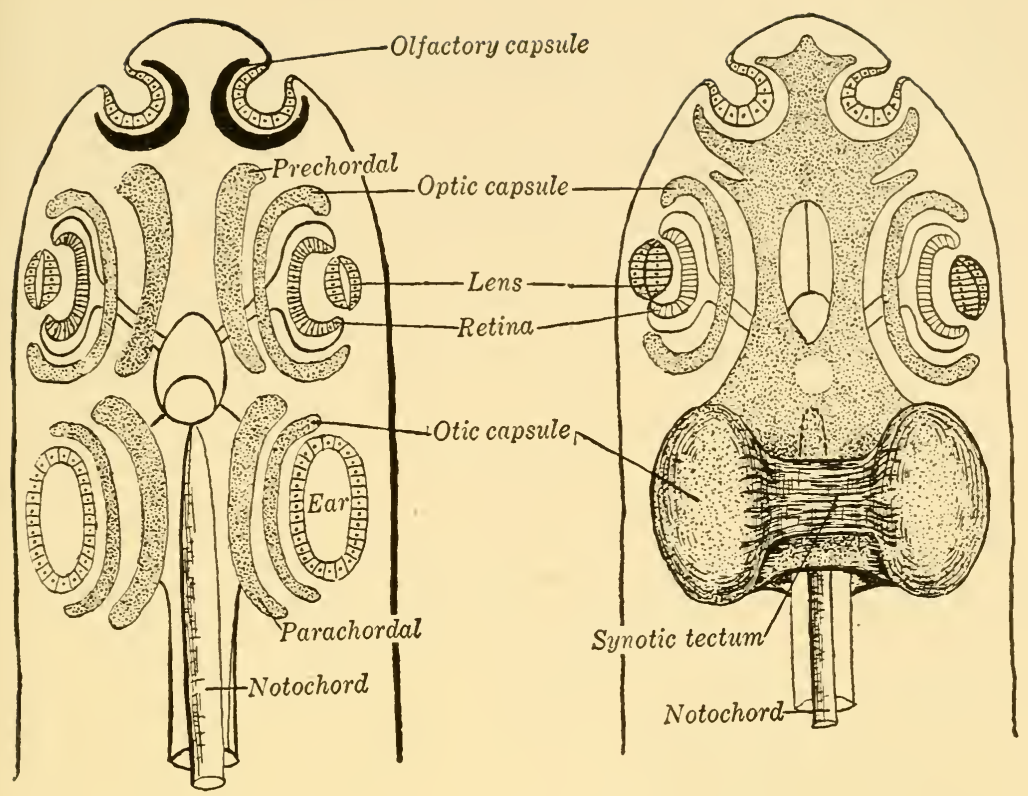
A. Chondrocranial
B. Early Chondrocranium Cartilages

Fig. 61. Development of the Chondrocranium. In (A) the cartilages are shown lying on either side of the notochord, and surrounding the organs of special sense; (B) shows the fusion of the cartilages to form the primitive chondrocranial trough.

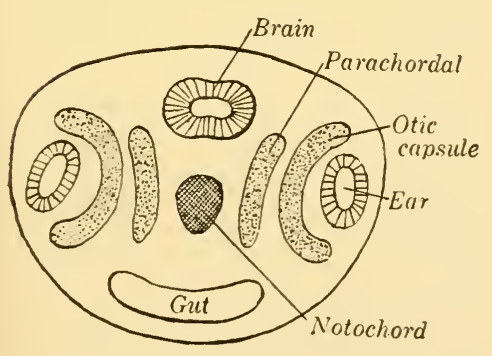

FIG. 62. Cross section of (A), Fig. 61 , through the otic region.

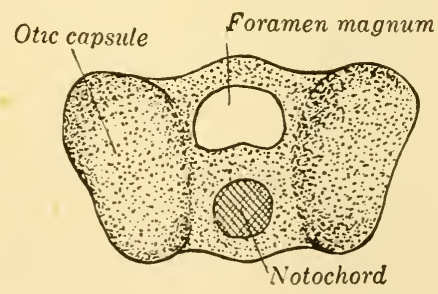

Fig. 63. Posterior View of (B), Fig. 61. The narrow synotic tectum is the only roof of the chondrocranium in the higher vertebrates. 
developed, supporting the anterior projection of the head; and there are a few minor specialized structures. The great advance which the early elasmobranchs made was in the gill, or visceral, arches. The first gill arch developed into jaws (upper and lower), and the second into the hyoid apparatus which functions in the dogfish as a suspensory cartilage for the jaws and a support for the throat and tongue. The suspensory cartilage is the hyomandibular. It is attached to the chondrocranium at the otic capsule, and passes laterally and slightly ventrally to the upper jaw. The spiracle of the dogfish is posterior to it. The upper jaw cartilage is the pterygo-quadrate, and the lower jaw is Meckel's cartilage.

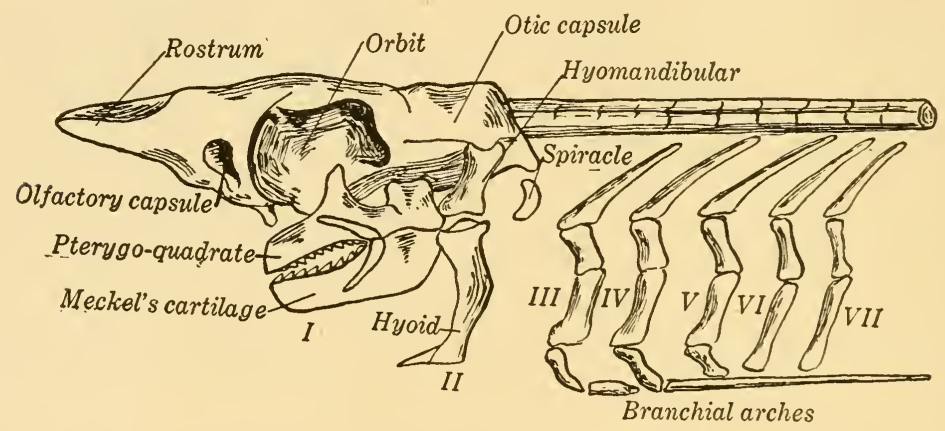

FIG. 64. Chondrocranium and Branchial Arches of the Dogfish. The jaws and hyoid apparatus are shown in position.

To summarize, the jaw apparatus of the elasmobranch is made up of three major cartilages: (1) the suspensory hyomandibular, (2) the pterygo-quadrate of the upper jaw, and (3) Meckel's cartilage of the lower.

The chondrostean skull (page 40) is useful in showing the probable evolution of the higher skull. The chondrocranium is like that of the dogfish, but the cartilages have become encased in bone. The upper jaw became immorably attached to the skull. The lower jaw was covered with the bony plates, except at the point of articulation with the upper jaw. The articulation remained between the pterygo-quadrate and Meckel's cartilages.

In the higher vertebrates the skull, as such, takes form. The chondrocranium becomes ossified, or replaced by bone tissue, and the dermal roofing bones fuse with the replacement bones of the 
chondrocranium. The student should again be reminded that the typical chondrocranium is like that described in the first paragraph, with the result that the roof of the skull is dermal in origin.

Ossification of the Chondrocranium. Bone replacement begins in a number of centers, arranged in transverse series. Due to this arrangement of the bones the skull was once considered as modified vertebrae, but embryology has dispelled this theory. Beginning posteriorly there are four series of bones.

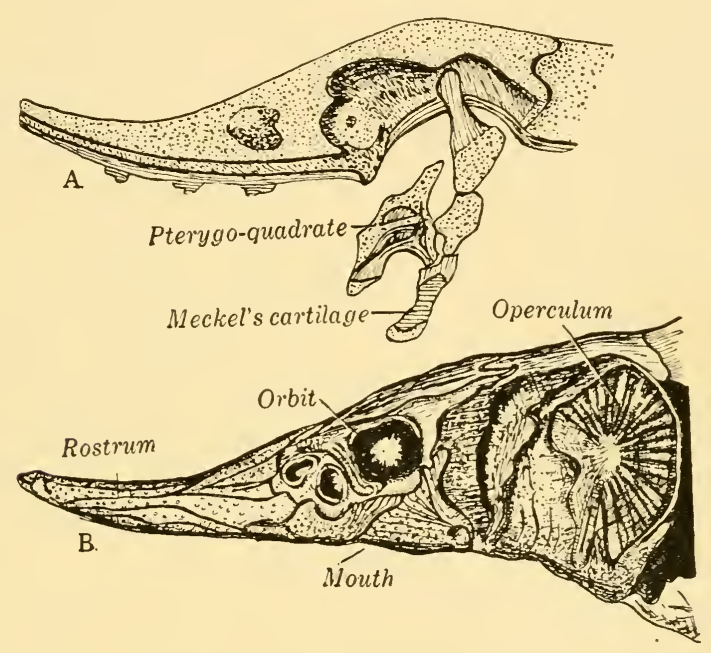

FIg. 65. The Chondrocranium and Dermal Skull of the Sturgeon (Order Chondrostei). (A) is the unossified chondrocranium. (B) shows the external appearance of the skull with the dermal bones overlying and completely covering the chondrocranium.

1. The cartilage surrounding the foramen magnum ossifies as four bones, the occipital complex. The ventral is the basioccipital, the lateral are the paired exoccipitals, and the dorsal is the supraoccipital.

2. In front of the occipital bones is the posterior sphenoid complex composed of three bones: the ventral basisphenoid, and the lateral paired alisphenoids. Recall the chondrocranium shape, and it will be clear that the alisphenoids form the sides of the skull. 
3. The anterior sphenoid complex is in contact with the former group, and like it has three bones: the unpaired presphenoid and the lateral orbitosphenoids. The latter are at the level of the optic capsule and form part of the orbit, or cavity, in which the eye rests.

4. The ethmoid group is most anterior, though covered by dermal bones. These bones are developed in part from the anterior half of the prechordal cartilages, and partly from the

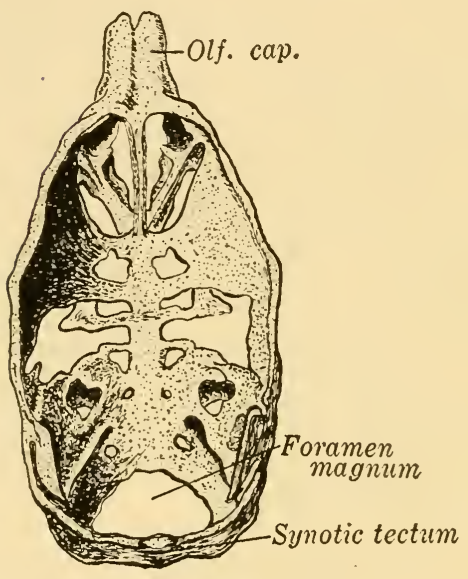

FIg. 66. Chondrocranium of the Pig. Centers of ossification appear in the chondrocranium soon after the first strands of bone appear in the dermal fibers. (After Mead).

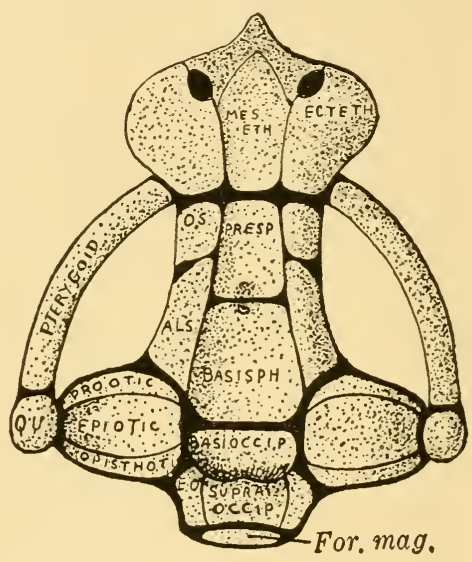

FIG. 67. Ossification of the Chondrocranium (Diagrammatic). The major regions are heavily outlined.

olfactory capsule. The ethmoid bones of the lower vertebrates are not as separate as in the mammals. In the latter group there is a ventral ethmoid; a median mesethmoid, forming a septum between the two nostrils; and the paired ectethmoids.

The otic capsule ossifies as five separate centers. In the lower vertebrates these are often separate. The lower mammals also have these otic bones defined, but there is a tendency toward fusion in the higher groups. In man the five embryonic bones form a single petrosal bone which fuses with a dermal bone to make the temporal. 
Jaws. In the bony fish, amphibia, reptiles and birds the pterygo-quadrate cartilage ossifies as the pterygoid and the quadrate bones. The latter is the ossified posterior end and therefore articulates with the lower jaw. The posterior end of Meckel's cartilage ossifies as the articular bone, being in contact with the quadrate. The anterior portion of this cartilage disappears with the growth and development of the dermal covering.

In the primitive reptiles there were seven bones on either side of the lower jaw, one replacement bone (articular) and six dermal bones. A comparative study shows that the dentary, the most anterior of the dermal bones, pushed farther back during the course of evolution, occupying more and more of the jaw. In the Theromorpha (page 59) the posterior elements, including the articular, are small; and in the Therapsida the dentary makes up practically all of the jaw, with the posterior bones a nipplelike structure at the end. The point of articulation is, however, between the quadrate and the articular. In one species of the therapsids there is a secondary point of articulation between the dentary and the squamosal, a dermal bone of the skull. The mammals have completely lost the old articulation. The lower jaw is composed of one bone, the dentary, and this articulates directly with the squamosal.

Dermal Bones of the Shull. As one would expect from their origin from dermal denticles and dermal plates, the covering bones of the skull have proceeded from the complex to the simple. The reverse has been true in the more fundamental systems. Therefore, clarity may be gained by describing the dermal bones of a simple mammal first, and comparing afterward. The opossum, a marsupial (page 78) is sufficiently generalized to serve the purpose.

The top of the skull is covered by four pairs of bones, the longitudinal suture, or point of contact between two bones, being the mid-dorsal line. From posterior to anterior these are: the dermal supraoccipital, completely fused with the replacement occipital and indistinguishable from it except in the embryo; (2) the parietals, covering the posterior part of the brain case and extending over the sides of the skull; (3) the frontals, the longest of the dorsal bones, bordering and extending into the 
orbits, or eye sockets; and (4) the nasals, which roof the nostrils or anterior nares.

On the side of the face are: (1) the squamosal, in contact with the parietal and occipital bones; and forming the temporal region and the bulla of the ear, and a part of the zygomatic arch, to which jaw muscles are attached; (2) the jugal which completes the arch below the orbit; (3) the maxilla, a large bone of the face, carrying most of the teeth and extending across the roof of the mouth to form the hard palate; (4) the premaxilla, which completes the skull anteriorly, bounds the ventral margin

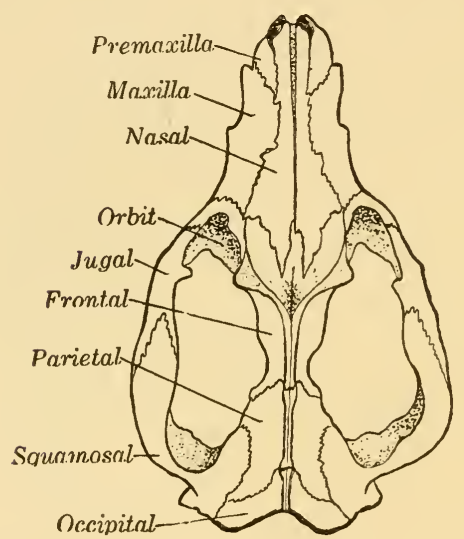

Fig. 68. Opossum Skull, Dorsal View.

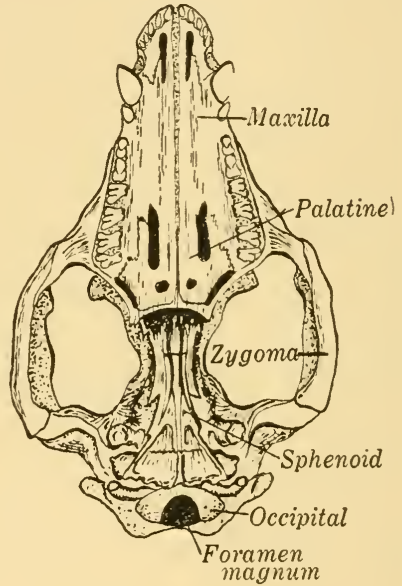

Fig. 69. Opossum Skull, Ventral View.

of the nostrils, and bears, the front teeth; and (5) the lacrimal, a small bone at the anterior margin of the orbit which is punctured by the duct from the lacrimal gland.

The ventral sicle of the skull shows few dermal bones except in the anterior region. The roof of the mammalian mouth is bony, and covered by the maxillae and the more posterior palatine bones. The formation of a hard palate has carried the internal opening of the nares back to the naso-pharynx. This is found only in the mammals, the mammal-like reptiles, and a few other reptiles. In the amphibia and most reptiles the nares open directly into the mouth cavity. 
The loss of dermal bones is more easily studied with pictures than description. The fish has been largely omitted on account of the complexity of the skull, the teleost particularly having little relation to other forms. The primitive fish had a more typical skull, which was complicated, however, by the bony operculum covering the gills. This gill cover is found in all the fish above the elasmobranchs, and one or two of the opercular bones were carried over to the earliest amphibia.

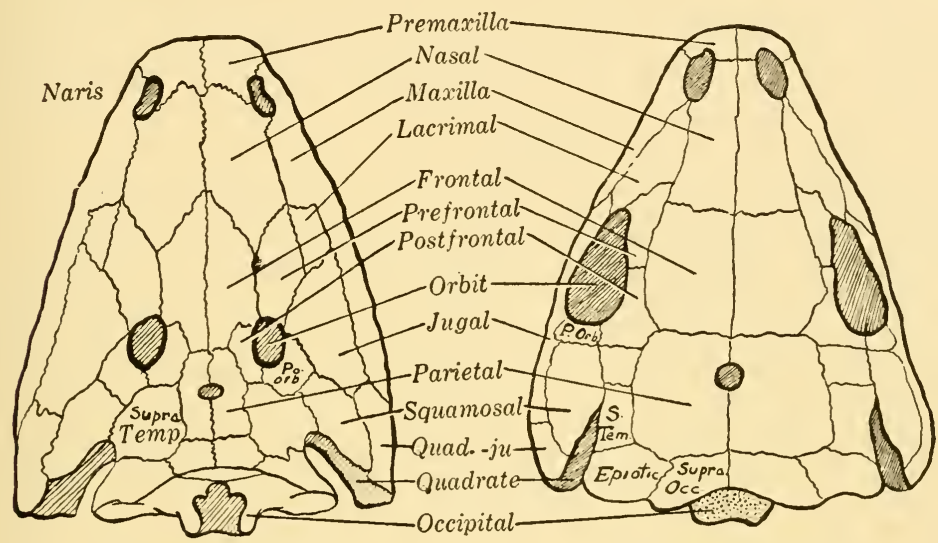

A. Capitosaurus (Stegocephalia)
B. Seymouria (Extinct Reptile)

Fig. 70. Primitive Skulls, Dorsal Views. (A) Capitosaurus (Order Stegocephalia) a primitive extinct amphibian. (B) Seymouria, a primitive, extinct, anapsid reptile.

The recent amphibia have a very specialized skull structure. The typical primitive form is illustrated by the Stegocephalia (page 50). The earliest amphibian (Archegosaurus) was extremely fish-like, resembling the fossil Crossopterygian in structure. The later members of this primitive order diverged in two directions, (1) toward the unarmored amphibia and (2) toward the reptilian form. Until recent research definitely placed the latter as amphibia they were classified as Pro-reptilia.

The reptiles continued the loss of bones, the elements of the skull becoming reduced from 76 to 26 in the mammal. The reduction in number was gradual, only two pairs of bones being found 
in the mammal-like reptiles which are unknown in the mammals: (1) the post-frontals and (2) the post-orbitals. The posterior jaw elements have been transferred into the skull as ear ossicles.

Evolution of the Skull. The evolution of the mammal skull is intimately correlated with the shifts of the dermal bones outlined above. In addition to the chance mutation of the bones, there are ontogenetic influences which depend upon the modification of other structures. The derelopment of the brain dictates the size of the calvarium, or brain case. Pathological conditions in the human demonstrate that the skull takes the size of a

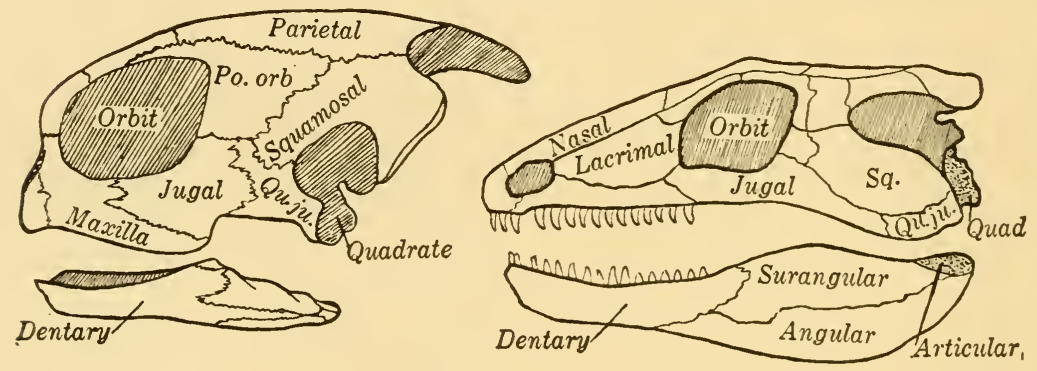

Turtle (Living Anapsid Reptile)

Seymouria (Extinct Anapsid)

Fig. 71. Skulls of Anapsid Reptiles. Side View. (A) Turtle, (B) Seymouria. Observe the lack of teeth and the greater depth of the turtle skull.

degenerate brain, and an increase in the amount of cerebral fluids causes a hypertrophy of the calvarium. In other words, although bone is the hardest tissue of the body, developmentally it is the most easily modified. A second influence is the shift in the muscles of the head and face. There is evidence that changed size and position of muscles directly influence the shape and position of bony elements. It is equally true that the reverse is true, and that bone changes cause the increase or decrease in the length of muscles.

The definitive vertebrate skull was laid down in the Stegocephalia. The brain case was very small, with a huge roof of dermal bones. The muscles of the jaw, neck and face were attached to the under side of the roof, and the skull was shallow 
dorso-ventrally and very wide. The majority of the skull space was taken by the lateral projections, and the huge mouth.

In the primitive reptile (Seymouria) the skull had deepened and shortened. At the posterior end there was an otic notch. The dermal roof was unbroken except for the anterior nares and the orbits. This is the type skull of the sub-class Anapsida (without an apse, or arch). The turtle is the living representative of this early group (page 60).

The next development in the skull was the breaking through of an opening (fenestra) in the roof, through which the temporal muscles of the jaw passed to the upper surface of the dermal bones. The brain case was enlarged, though still occupying a

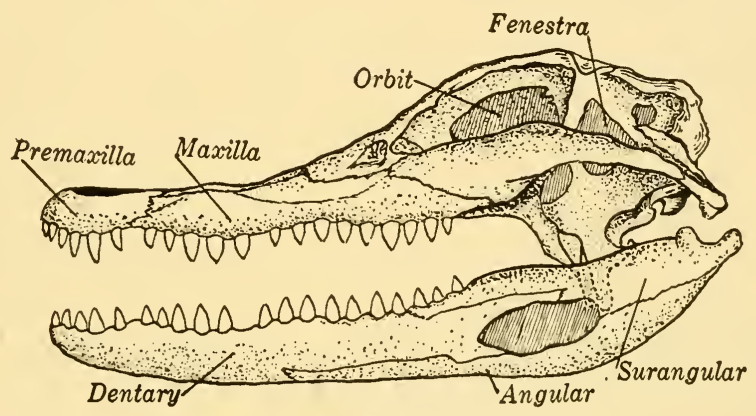

FIg. 72. Skull of Alligator, a Diapsid Reptile. The face is greatly elongated, and two pairs of fenestrae have developed in the temporal region.

small part of the total mass of the skull. This sub-class was called the Synapsida, through the mistaken belief that the opening was a fusion of two fenestrae. As mentioned (page 60) the mammal-like reptiles carried this development to its logical conclusion. The dinosaurs and Crocodilia were an offshoot from the early Anapsid stem, and with a few less well known orders form a sub-class, the Diapsida, which have two fenestrae on either side.

The Synapsida were the ancestors of the mammals. A study of the drawings will show that the fenestra became larger, leaving a lateral border of dermal bones, with the jaw muscles gaining a more prominent footing on the upper surface of the calvarium. The brain had enlarged, occupying more room in the skull, and the face had shortened materially. In the Therapsida 
the skull had practically assumed the mammal form. The ventral border of the skull below the fenestra had become a distinct zygomatic arch to which the outer jaw muscles (masseter muscles) were attached, while the temporal muscles had their origin on the side of the calvarium. The latter pass through the zygomatic opening to the jaw.
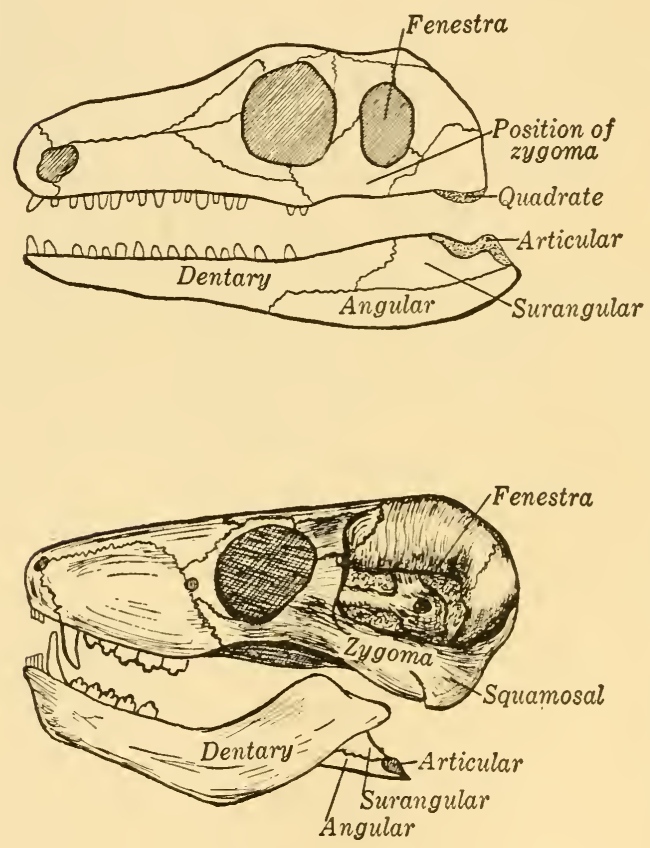

FIG. 73. Evolution of the Mammal Skull. (A) Primitive mammal-like reptile (Order Theromorpha); (B) Specialized mammal-like reptile (Order Therapsida); and (C) a mammal (Opossum). Observe the enlargement of the lateral fenestra, the growth of the brain case, and the loss of the posterior lower jaw bones.

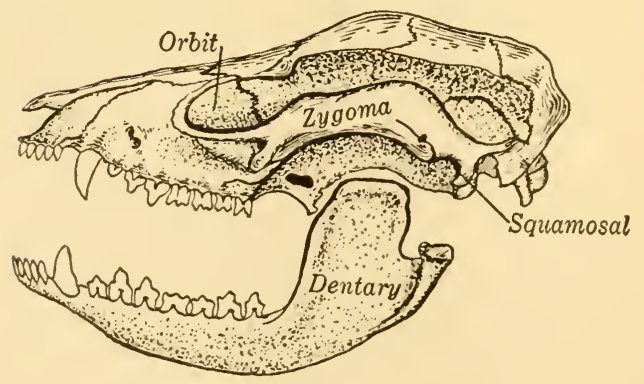


The general characteristics of the mammal skull have been discussed. The evolution of the human skull shape depended upon the shortening of the face and the great growth of the brain. These two movements automatically carried the calvari' $m$ upward and forward, until in the human a line drawn from the foramen magnum to the chin is at right angles to the vertebral

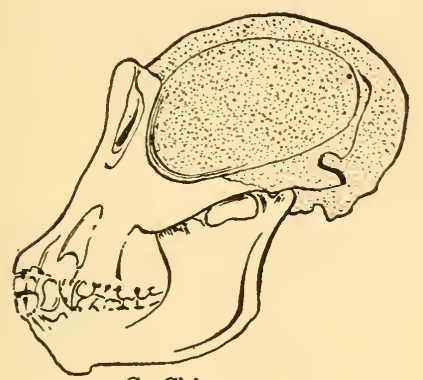

C. Chimpanzee
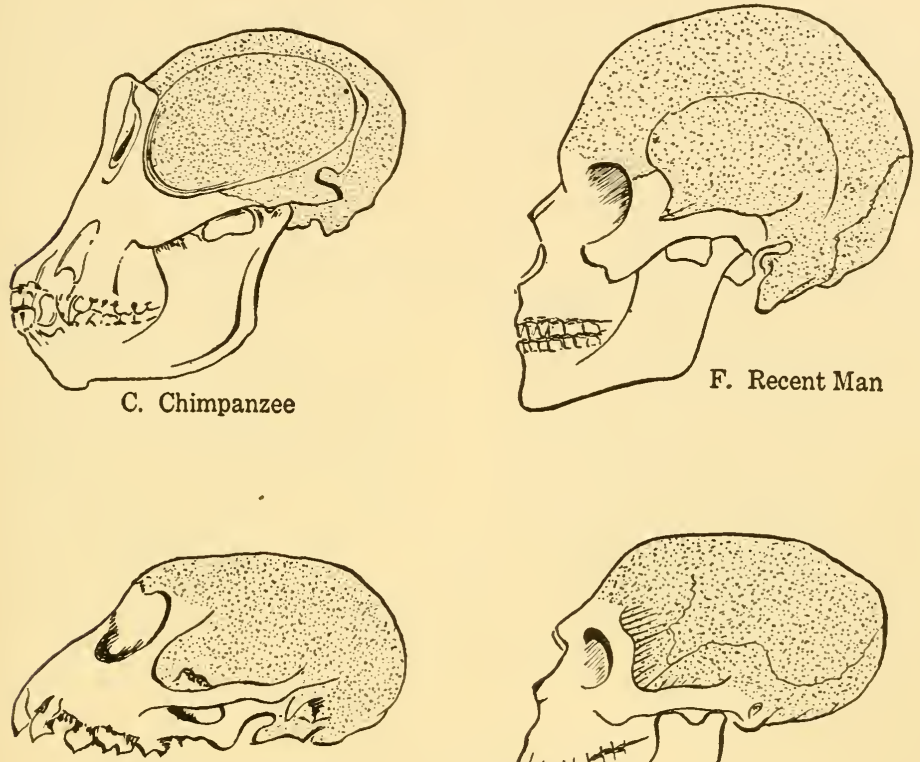

B. Old World Monkey
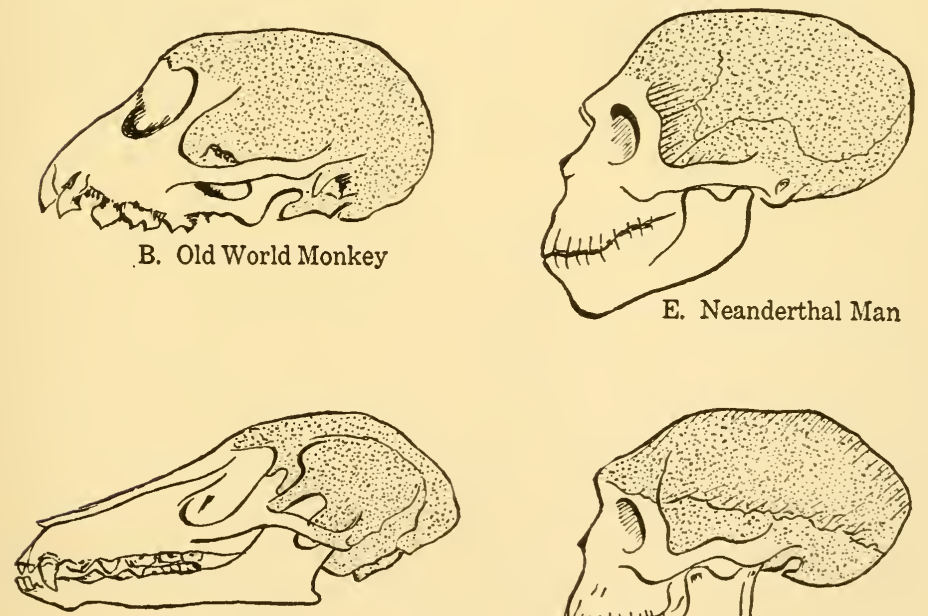

A. Lemur

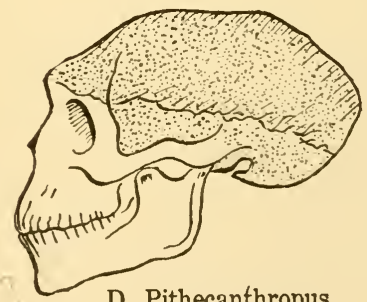

D. Pithecanthropus

FIG. 74. Skulls of Primates. Note the increase in size of the brain case (calvarium) and the shortening of the face. The calvarium is stippled. 
column. In other words, the mouth and face have been submerged under the calvarium. A comparison of the opossum, lemur, monkey, anthropoid ape, a primitive man, and the present man will demonstrate this line of evolution.

The Human Skull. The early embryology of the human follows the stages outlined under the development of the chondrocranium. As bone cells begin the replacement of cartilage, fine strands of bone can be seen in the deeper layers of the skin. These bone strands begin at definite centers, one for each bone of the skull. As the bony area spreads, the dermal bones come in

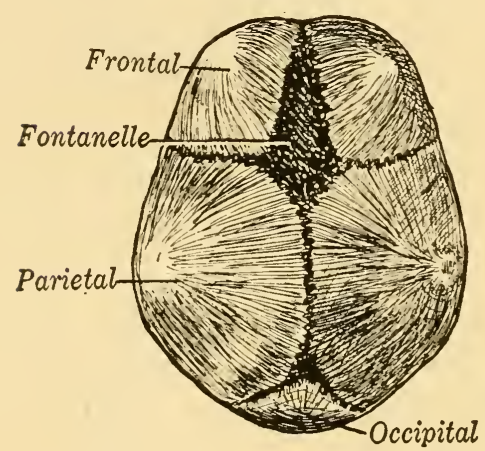

Top View

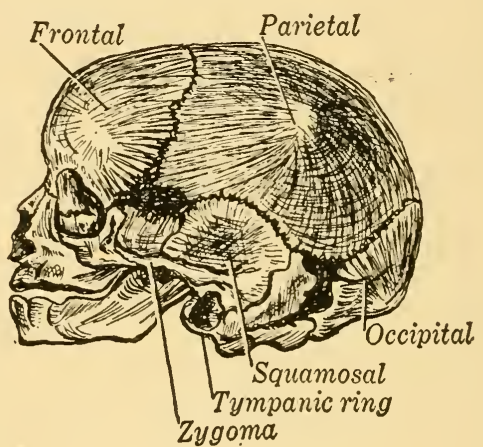

Side View

Fig. 75. Skull of a new born Human Infant.

contact at their rounded margin, and naturally leave spaces at the junction of four rounded corners. These spaces are the fontanelles. The "soft spot" on top of the young human is the fontanelle formed by the juncture of the parietals and frontals.

In structure the human skull is only a shortened, dorsally enlarged mammal skull. As in other mammals, spirals of bone have grown into the nasal cavities, arising from the ethmoids and the dermal bones. These serve the function of moistening and warming the air, and on them are located the olfactory sense endings. The olfactory nerves pass back toward the brain as a number of small nerves, each piercing the ethmoid bone which forms the anterior of the calvarium. This sieve-like bone is the cribriform plate. The nerve foramina are packed into a smaller area than in 
the lower forms, due to the antero-posterior shortening. One other structure is of phylogenetic interest. The pituitary opening: at the end of the notochord was described. From the dogfish to man this pit becomes closed ventrally to form a pit in the floor of the skull. In the mammal this pit is bounded anteriorly and posteriorly by sharp ridges of bone and is the sella turcica (Turk's saddle) in which the pituitary gland rests.

TeEth of Vertebrates. The vertebrate teeth are homologous with the dermal denticles of the elasmobranchs. In this group the

A
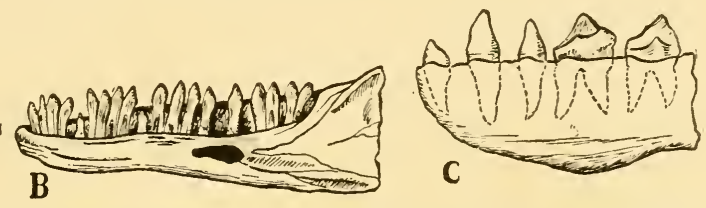

FIG. 76. Types of Tooth Attachment. (A) Acrodont, attached on biting margin of jaw; (B) Pleurodont, attached on inner side of jaw; and (C) Thecodont, the teeth sunk into cavities or thecae.

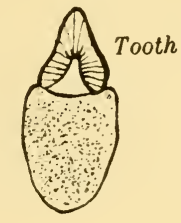

A. Acrodont

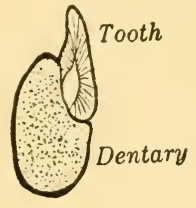

B. Pleurodont

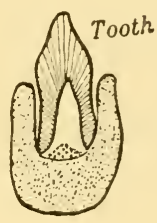

C. Thecodont

FIG. 77. Cross Sections showing Tooth Attachment.

teeth are continuous with the denticles of the jaws, and are embedded in the skin without any contact with the jaw cartilages. The teeth are all alike and can be replaced indefinitely.

With the development of bony jaws the teeth came in contact with the bones, and fused to the tooth-bearing bones either (1) on biting rims of the jaws, or (2) along, the inner sides of the bones. In the amphibia and primitive reptiles the teeth are all similar, although some may be slightly longer than the others.

The Crocodilia retain the tooth similarity, the homodont condition, but show advance in having the teeth sink into the jaw bones, fitting into sockets. The mammal-like reptiles also have teeth in sockets, and other advances. In these reptiles the teeth 
become heterodont, the front being differentiated from the back. In the higher (Therapsid) types there are front incisors, long canines, and primitive molars posteriorly. The evidence indicates that the number of replacing teeth was also reduced.

The mammals show the evolution of the teeth from the simple differentiated condition to the highly specialized condition found in many orders. The primitive mammals had molars with three points or cusps. In time accessory cusps developed, and so stable and definite are these cusps that the evolution of the different orders can be traced by their tooth structure.

The mammals have.two sets of teeth, the baby or milk teeth, and the permanent teeth which replace the first. The posterior molars are an exception to this rule. Those which are replaced are: (1) the incisors which are borne on the pre-maxillary bones of the upper jaw, and the homologous teeth of the lower; (2) the canines, the first tooth on the maxilla of the upper jaw, and the lower tooth which fits in front of it; and (3) the premolars, posterior to the canines, and having more than one cusp in the normal condition. (The Cetacea and some other animals are exceptions to this rule.) Posterior to the premolars are the permanent molars, which are not replaced after the original eruption of the tooth. The difference between premolars and molars often cannot be determined without this knowledge of development.

Dental Formula refers to the written formula expressing the number of different teeth in any particular species. In these formulae I represents incisor; $\mathrm{C}$, the canines; $\mathrm{P}$ equals the premolars, and $\mathrm{M}$ the molars. One side of the jaw is given, so that the formula is multiplied by two. The upper teeth are placed above a line, the lower below it, thus: $\mathrm{I}-\frac{2}{2} ; \mathrm{C}-\frac{1}{1} ; \mathrm{P}-\frac{2}{2}$; $\mathrm{M}-\frac{3}{3}$. This is the human formula, and when the numbers are added and doubled, it gives the normal thirty-two teeth of the species.

The primitive dental formula of the placental is: $\frac{3}{3} ; \frac{1}{1} ; \frac{4}{4} ; \frac{3}{3}$ -44 teeth in all. More specialized animals have many varia- 
tions of this formula, a general reduction in number being typical. The carnivores tend to lose the molars, and the rodents usually have no canines and anterior premolars. The loss of these teeth leaves a wide diastema, or space, between the anterior and posterior teeth. The Cetacea, which have teeth, always have an increased number.

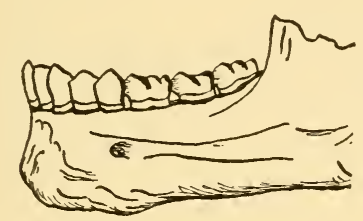

A. Man

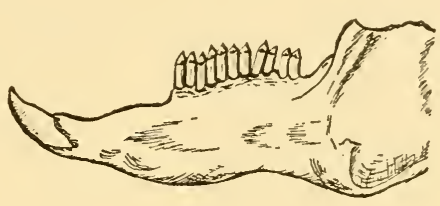

B. Rabbit

FIG. 78. Mammal Jaws, showing position of Teeth. Observe the wide space (diastema) between the incisors and premolars of the rabbit.

Development of Teeth. As one would suspect, the vertebrate teeth and the dermal denticles have may points of derelopment in common. (See page 109.) The ectoderm covering the body swings orer to line the lips, gums and part of the mouth cavity, and it is from the gum ectoderm that the dental vesicles develop. From the embryonic ectoderm a cord of cells pushes downward, the inner end enlarging to form a hollow vesicle. As this ectodermal vesicle meets the knot of mesodermal cells which forms a papilla, the resicle invaginates to form a double cup. The cells of both mesoderm and ectoderm then begin secreting an inorganic deposit of hard material. The mesodermal body of the tooth is the dentine, the ectodermal cover is the enamel. The latter is the hardest and most completely inorganic substance of the body. As the tooth takes form it is pushed upward until it breaks through the gum tissues. The papilla, which contains nerves and blood vessels, forms the pulp cavity inside the tooth. The tooth proper has neither nerves nor blood vessels, the pain from tooth decay or a dentist's drill being transmitted to the pulp cavity.

The majority of mammals have teeth with closed pulp cavities, and these teeth do not grow after eruption is completed unless the top of the tooth is worn off and the dentine forming 
cells are released. In such cases the tooth developing cells begin forming bone on top of the pulp cavity. And conversely, if dentine begins to fill the cavity, bone destroying cells normally resorb it as rapidly as it is developed.

Other mammals have certain teeth with an open pulp cavitythat is, the base remains wide open. Under these conditions the
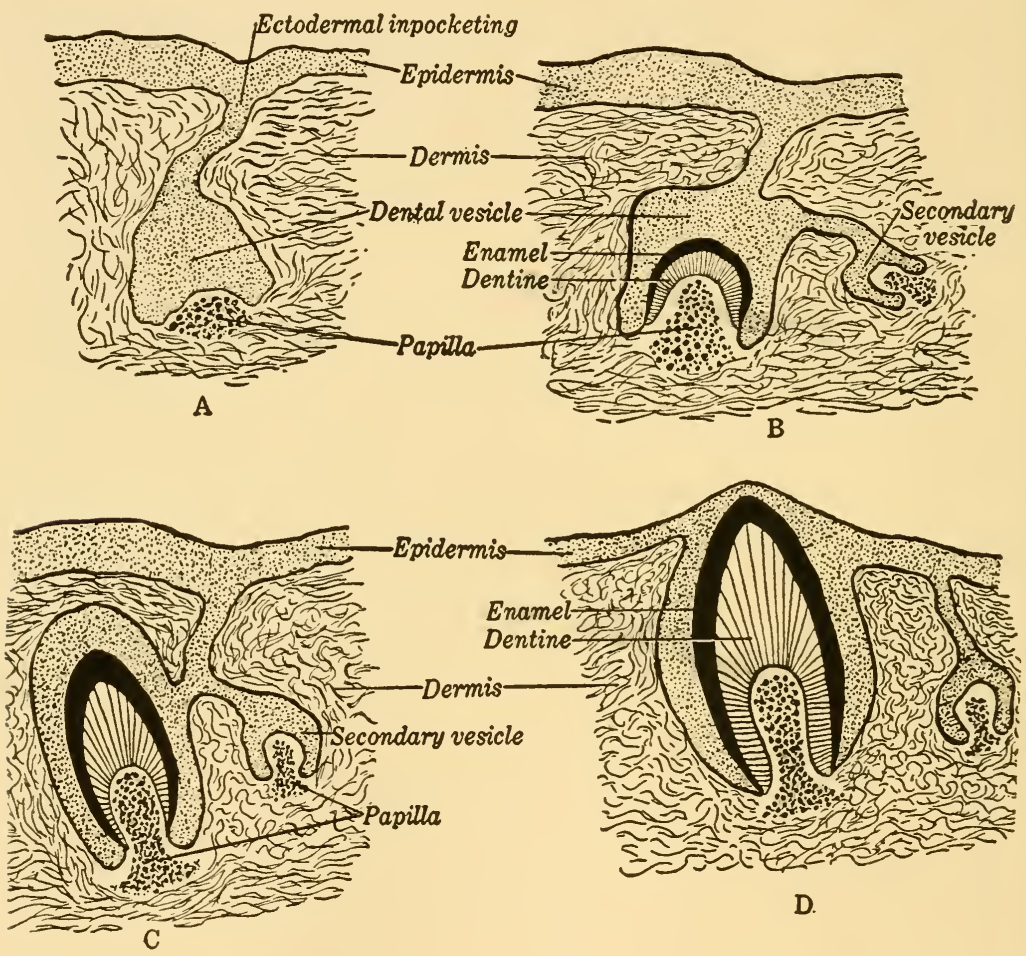

FIG. 79. Derelopment of Teeth (Man). Note the inpocketing of ectoderm (A) to form a dental resicle which meets the mesodermal papilla; the development of a secondary resicle and the beginning of tooth derelopment (B); and the growth up to the time of eruption (C) and (D).

dentine forming cells may continue to secrete without filling the inner cavity, and in that way killing the tooth. As dentine is laid down on the inner side of an open cavity, the tooth is pushed upward and continues growing throughout life. The incisors of rodents and several other animals continue their growth, 
but are normally worn down by wear against hard substances. When removed from their barren grazing lands the incisors of llamas have been known to grow so long that the animals died as a result. The canine tusks of the pig and some carnivores are

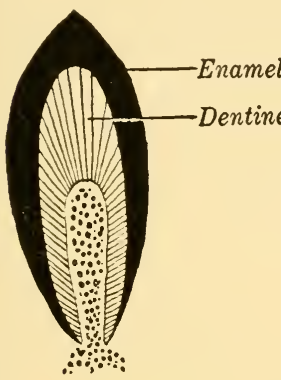

A. Closed Pulp Cavity

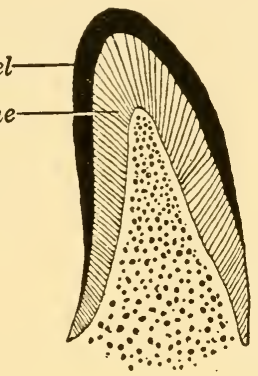

B. Open Pulp Cavity
Fig. 80. Structure of Incisor Teeth. Note the structure of the open pulp eavity which permits the continued upward growth of the tooth.

similar in structure. The tusks of the elephant are the second incisors of the upper jaw, lack enamel, and are the largest teeth known. Certain extinct elephants had tusks eleven feet long, weighing more than 250 pounds each.

\section{B. Visceral Skeleton}

The visceral, or branchial, skeleton is technically an entity, although it forms an important part of the skull and can be considered with the axial skeleton of the vertebrate. There is no proved homologue of the branchial arches below the elasmobranch fishes. The numerous gill bars of Amphioxus afford no clue as to the origin of vertebrate gill supports, while those of the jawless cyclostomes are too highly modified to be of much assistance. The latter group today has an intricate branchial basket which has little relationship. with the gill arches of the other vertebrates.

In the most primitive sharks there are nine visceral arches in the embryo, with rudiments of several more; but when the number of gills was reduced to the typical five, the number of arches was also reduced to seven. The anterior two of these became modified into jaws and supporting structures of the throat 
and tongue, the posterior five remaining as the branchial, or gill, arches.

The evidence for the homology between the jaws and the visceral skeleton is based upon several factors: (1) in the very early embryo of the dogfish the first visceral cartilage is clearly divided into two major portions, but these form a wide angle; (2) the second (hyoid) arch lies anterior to the spiracle which carries rudimentary gills; (3) the muscles of the jaw and hyoid arise with the branchial muscles from the hypomere as the visceral muscles; and (4) the innervation of these muscles completes the proof of homology. The muscles and nerves are

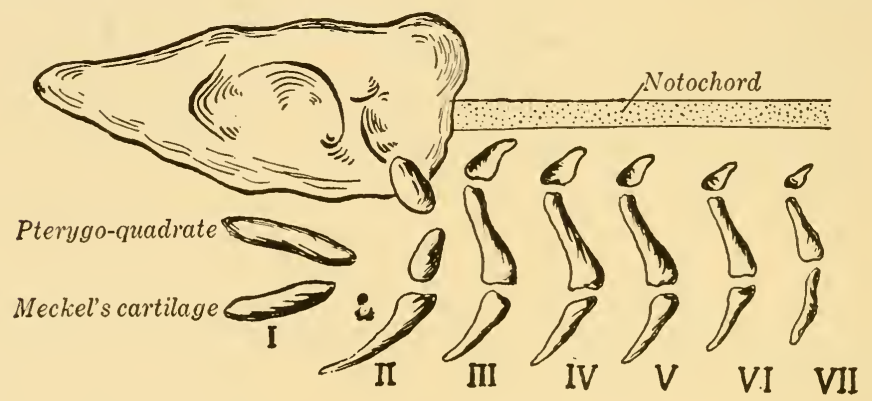

Fig. 81. Chondrocranium and Visceral Arches (Diagrammatic). Arches I and II form the jaws and hyoid apparatus; Arches III to VII form the branchial cartilages.

undoubtedly the strongest evidence. The illustration of the chondrocranium and visceral arches of the dogfish shows the relationships of these cartilages to the chondrocranium.

The enclosure of the upper and lower jaws by dermal bone has been discussed. With this development of the dermal skull the hyomandibular of the fish became a practically functionless structure, lying in close contact with the spiracle. This is the condition in the Crossopterygian fishes, the hyomandibular bone being very small.

The amphibia evolved an entirely new relationship between these structures. The outer (distal) end of the spiracle enlarges and engulfs the hyomandibular into its cavity, and a membrane of skin closes the outer opening of the cavity. In this way the midlle car of the amplibian is developed. The skin membrane 
is the tympanum or ear drum, and the opening into the pharynx is the Eustachian tube. Recall the position of the hyomandibular, its proximal end in contact with the otic capsule. Its position in the amphibia is unchanged, except for its inclusion within the middle ear. Therefore the hyomandibular of the fish becomes the columella of the amphibia, and is the ear ossicle which assists in transmitting sound waves from the tympanum to the inner ear which contains the organs of equilibrium and the rudimentary hearing apparatus.

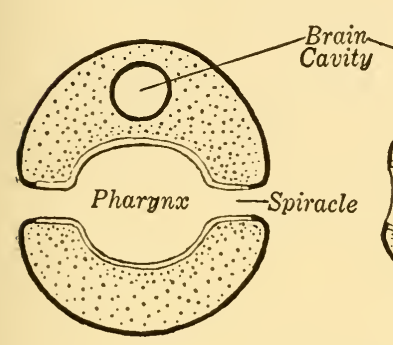

A. Dogfish

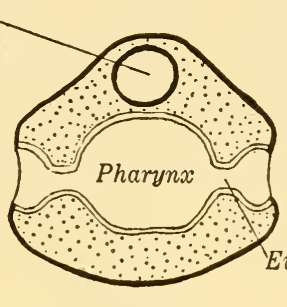

B. Frog

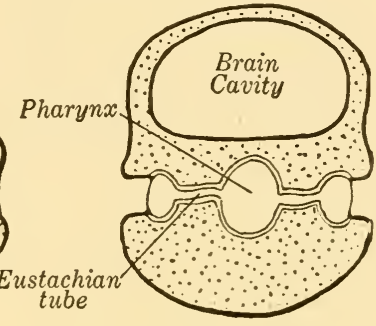

C. Mammal

FIG. 82. Cross sections of the Pharynx (Diagrammatic). The spiracle (A) develops into the middle ear and Eustachian tube (B), closed by the ear drum. The Mammal (C) shows the deep position of the ear drum and the elongated Eustachian tube.

In the reptiles and birds the same conditions exist in regard to the three replacement bones under discussion: (1) the articular, (2) the quadrate, and (3) the columella. In the mammallike reptiles the replacement bones of the jaws become smaller, and an accessory point of articulation develops between the dermal dentary and squamosal.

The embryo of the mammal demonstrates the final evolution of the three jaw cartilages. The hyomandibular cartilage remains as an ossicle of the middle ear, and is called the stapes in the mammal. The minute end of the pterygo-quadrate cartilage ossifies in close contact with the hyomandibular and is also included within the middle ear cavity as a second ossicle, the incus. The third mammalian ossicle, the malleus is a derivative of the ossified Meckel's cartilage, and is homologous with the articular bone of the amphibia and reptiles. 
Those relationships can be ascertained by staining the cartilages of the early embryo and clearing the tissues. It can be then demonstrated that the Meckel's cartilage passes from the lower jaw into the skull, and its posterior end is in contact with the quadrate cartilage. As growth continues the jaw part of the cartilage degenerates, and the posterior end ossifies as an ear ossicle. The relationships of the three cartilages is unchanged,

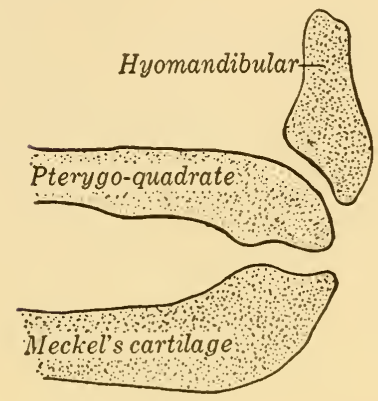

A. Elasmobranch

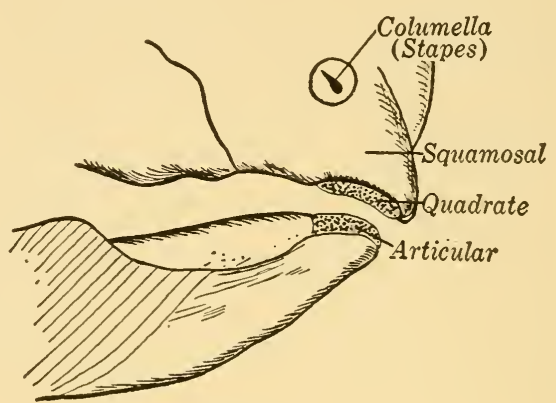

B. Reptile

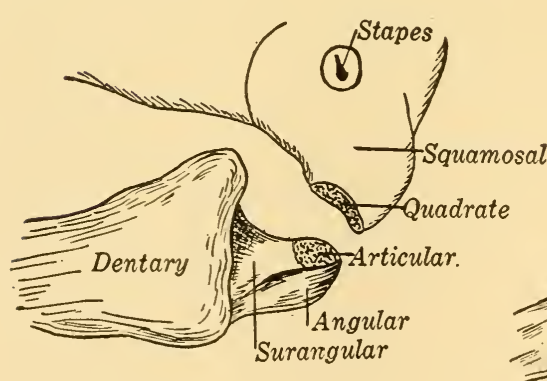

C. Mammal-like Reptile

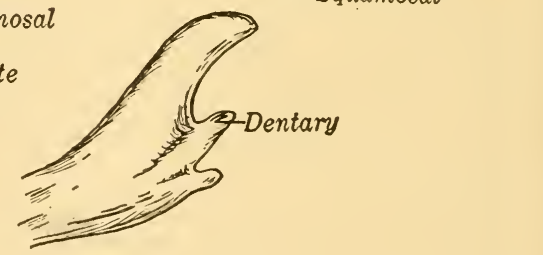

D. Mammal

Fig. 83. Jaw Articulation and the Derelopment of the Ear Ossicles. The Dogfish (A) has the three jaw cartilages. The typical reptile (B) has the skull encased in dermal bone, cartilage bones forming the jaw articulation, the hyomandibular being an ear ossicle; the mammal-like reptile (C) shows the back growth of the dentary; and the mammal (D) has the articulation between dermal bones, the quadrate and articular having passed into the middle ear as the incus and malleus. 
but their position has shifted. The homology is confirmed not only by the embryology of the cartilages, but by the muscles attached to the stapes and their innervation. The latter proofs are too complex for this discussion.

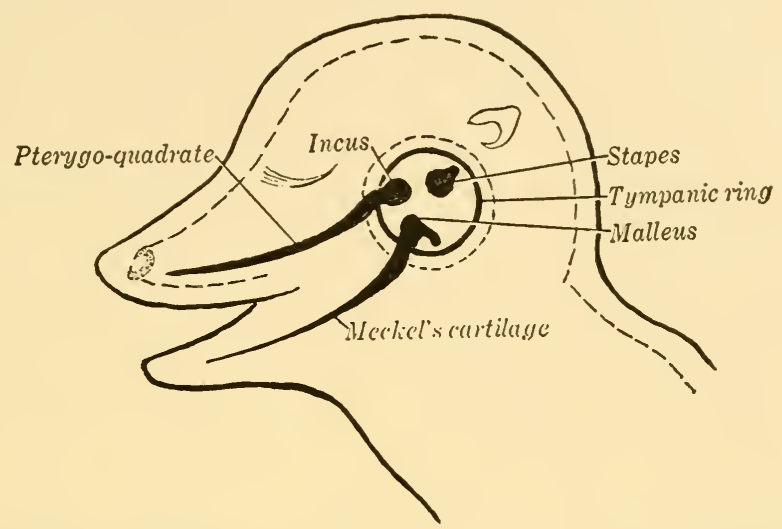

Fig. 84. Mammal Embryo with Unossified Jaw Cartilages. The proximal ends of the pterygo-quadrate and Meckel's cartilages lie within the tympanic ring and ossify as ear bones.

The posterior five arches, the branchial arches of the fish, eventually become transformed into the cartilages of the larynx. The primitive amphibia which retain gills naturally retain the branchial arches in reduced number, the others forming disconnected throat cartilages. The larynx is completed in the mammals and will be discussed under the respiratory system. The following chart shows the homologies in tabular form.

\begin{tabular}{|c|c|c|c|}
\hline & ELASMIOBRANCHS & $\begin{array}{l}\text { AMPHibia } \\
\text { Reptiles }\end{array}$ & Mamimals \\
\hline $\begin{array}{l}\text { Hyoman- } \\
\text { dibular }\end{array}$ & Jaw Suspension & $\begin{array}{l}\text { Columella, or Stapes } \\
\text { (Ear Ossicle) }\end{array}$ & $\begin{array}{c}\text { Stapes } \\
\text { (Ear Ossicle) }\end{array}$ \\
\hline $\begin{array}{l}\text { Pterygo- } \\
\text { quadrate }\end{array}$ & Upper Jaw & $\begin{array}{c}\text { Quadrate } \\
\text { (Jaw articulation) }\end{array}$ & $\frac{\text { Incus }}{\text { (Ear Ossicle) }}$ \\
\hline $\begin{array}{l}\text { Meckel's } \\
\text { Cartilage }\end{array}$ & Lower Jaw & $\begin{array}{c}\text { Articular } \\
\text { (Jaw articulation) }\end{array}$ & $\begin{array}{c}\text { Malleus } \\
\text { (Ear Ossicle) }\end{array}$ \\
\hline $\begin{array}{l}\text { Branchial } \\
\text { Cartilages }\end{array}$ & Gill supports & $\begin{array}{l}\text { Partly or entirely } \\
\text { lost } \\
\text { Throat supports }\end{array}$ & $\begin{array}{l}\text { Laryngeal } \\
\text { Cartilages }\end{array}$ \\
\hline
\end{tabular}




\section{Vertebrae}

The vertebral column is part of the axial skeleton. The individual vertebrae develop from mesodermal tissues which are laid down around the endodermal notochord. The latter becomes constricted in the elasmobranch fishes, and completely obliterated in the adults of the higher vertebrates.

Cyclostomes have the most primitive vertebrae of any class. Each vertebra is composed of six minute cartilages, three on either side, which have no connection with each other. The vertebrae are separated from each other by the myoseptum. It is difficult to ascribe any function to these discrete elements.

The Elasmobranchs have well developed, cartilaginous vertebrae which are biconcave and constrict the notochord in the

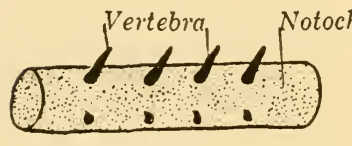

A. Cyclostome

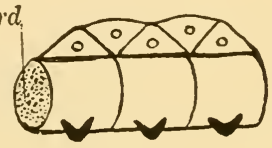

B. Dogfish

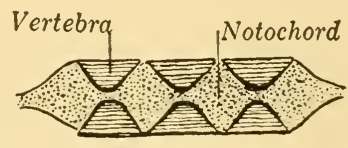

C. Dogfish (sagittal section)

FIG. 85. Cartilaginous Vertebrae. The Cyclostome vertebrae are isolated metameric cartilages; the Dogfish (B) has centra surrounding the notochord and a neural arch enclosing the nerve cord; (C) shows the centra constricting the notochord, leaving large discs of notochordal tissue between.

middle of each vertebra. The notochord is, however, continuous, being shaped in the adult like a string of beads-a narrow neck through the center of each vertebra, and a lens-shaped area fitting into the cavities of the two contiguous vertebrae. The circular part of the vertebra which surrounds the notochord is the centrum, and is evidently the most ancient part of the vertebra. Evidence of this is found in a small fossil (Paleospondylus).

The Elasmobranchs have also developed processes for protection of the nerve cord and for the attachment of muscles. On the dorsal side of each vertebra is an arch made of a pair of processes, through which the nerve cord passes. This is the neural arch of the vertebrae, and is found in all classes above the cyclostomes. In the Elasmobranch there are inter-neural cartilages which, with the neural arch, completely enclose the neural canal except where it is pierced by spinal nerves. On the lateral- 
ventral portion of the centrum is another pair of transverse processes serving as points of muscular attachment. In the caudal region of the dogfish these processes grow toward the ventral median line, fuse, and form a ventral haemal arch through which passes the aorta.

The vertebrae of the living Elasmobranchs are more specialized than those of many fossil fish and amphibia, and the

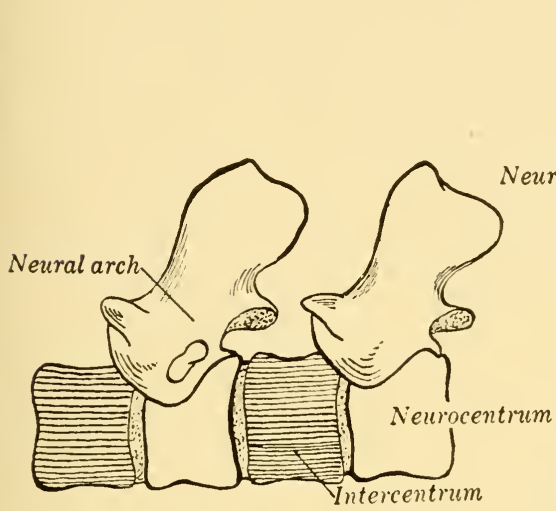

A. Primitive Stegocephalian (Cricotus)

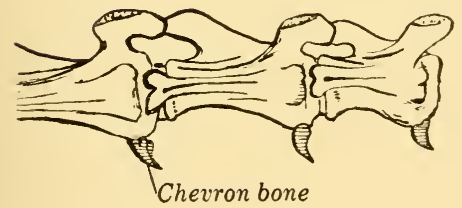

C. Tail Vertebrae of Cat

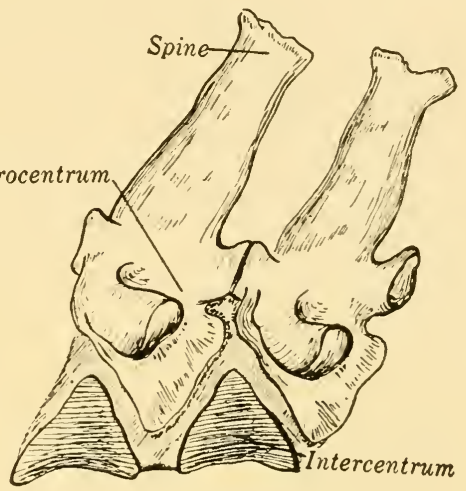

B. Stegocephalian (Eryops)

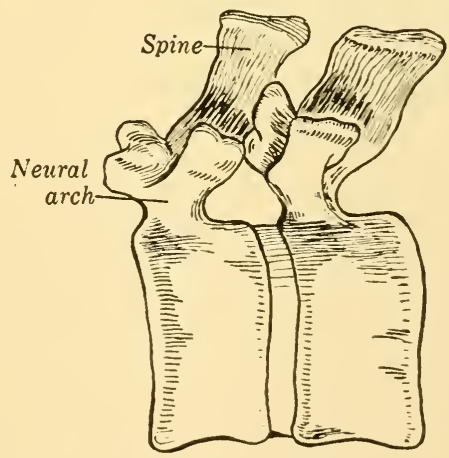

D. Lumbar Vertebrae of Mammal

FIG. 86. Comparative Anatomy of Bony Vertebrae. Primitive vertebrae ronsist of two centra in each borly segment (A); the intercentra are pushed ventrally (B) in many amphibia and reptiles; in the tail region of some mammals (C) the intercentra are left as cherron bones, and are lost completely in mammals without a tail. 
tail vertebrae of some recent fish. Considering these facts, and the embryology of the vertebrae, it becomes evident that the primitive animals had two vertebrae to each body segment. Wherever the condition is found the anterior vertebra has a neural arch and is called the neurocentrum; and the posterior is the intercentrum. This structure of the vertebrae was carried over from the early Crossopterygii to the Stegocephalian amphibian.

The more primitive fossil amphibia have the two alternating vertebrae, but the later fossils show a forward growth of the neurocentrum, with the intercentrum being pushed ventrally to form a triangular element. The mammal-like reptiles carried this destruction of the intercentrum a step farther, the intercentrum being very small. In the mammals the intercentrum is entirely crowded out in the body region, but remains in the caudal region of many species as the small chevron bones.

With the anterior growth of the neurocentrum, and its enlargement, the position of the vertebrae has changed. Originally the vertebrae lay between the myosepta, but the forward growth carried each into the more anterior body segment so that the myoseptum crosses its middle portion. In this way the vertebral number has been reduced to half, although metamerism has not been lost. Observe, however, that each body segment contains two halves of vertebrae, the posterior half of one, and the anterior half of another.

Above the fish the vertebrae displace the notochordal tissue completely, and articulate against each other. This is usually a ball and socket joint, a rounded projection of one vertebra fitting into a concavity of the adjacent one. The concavity may be either on the anterior or posterior face. The mammals have another modification, both faces of the centrum being flat. Movement is assisted by a disc of cartilage lying between the two faces.

Accessory processes are found in most groups of the vertebrates. In the reptiles these are rather simple. The mammal vertebrae are more complex. From the neural arch develop anterior and posterior zygapophyses, the anterior articulating with the posterior ones of the vertebra in front of it. This permits flexibility, but gives added strength. 
Vertebral Regions. The vertebrae of the land vertebrates can be divided into five more or less distinct regions: (1) the cervical, or neck; (2) the thoracic, bearing the pleural ribs; (3) the lumbar, lying in the lower abdominal region; (4) the sacral, to which the pelvic girdle attaches; and (5) the caudal, or tail, vertebrae.

Cervical vertebrae. In the lower forms these are only slightly modified from the more posterior ones. The higher reptiles show definite differentiation, connecting the primitive type and the
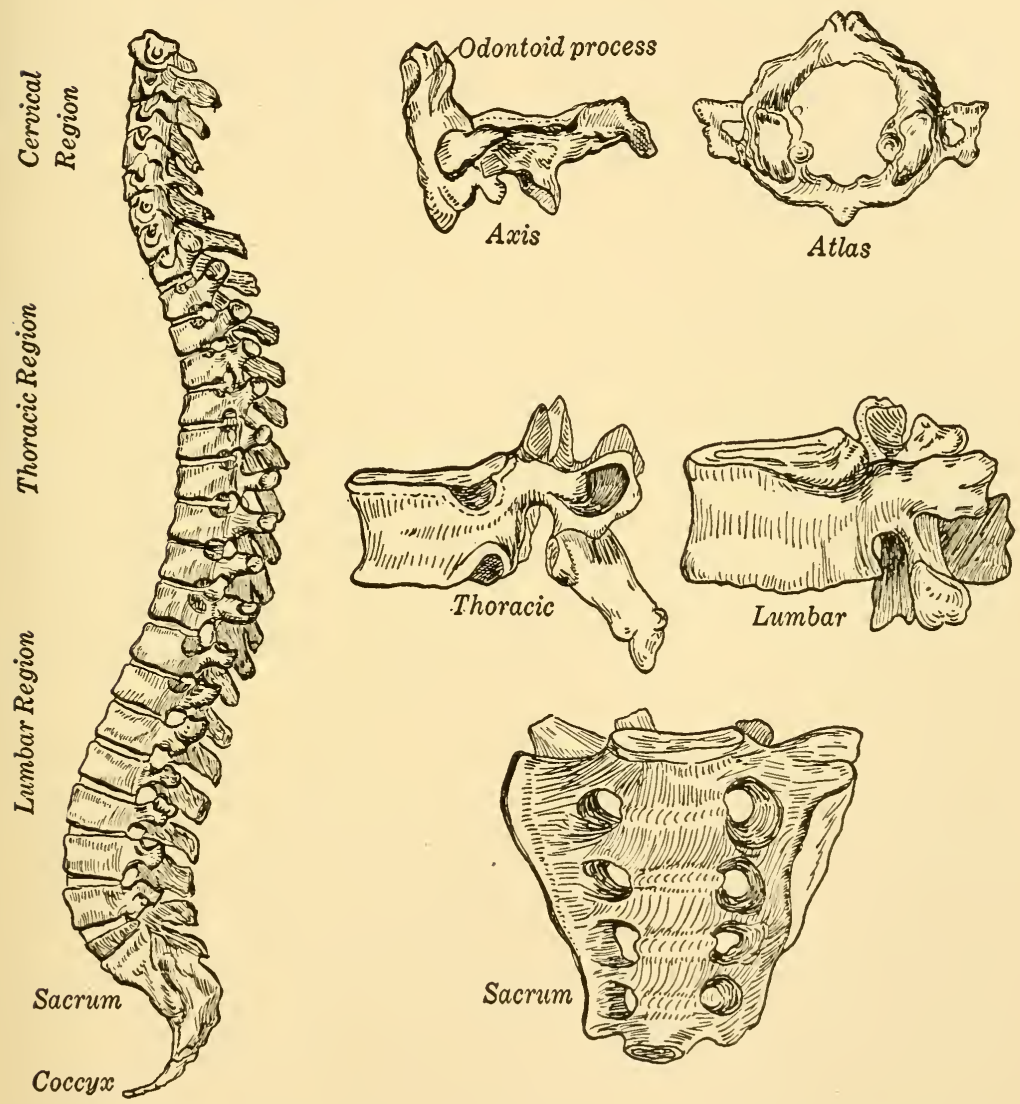

Fig. 87. Vertebral Regions. The spinal column of man with vertebrae from the different body regions. 
mammal. Typically there are seven vertebrae in the mammal, although this number is reduced in some whales, and increased in the edentates and one or two isolated species. However, the girafie, man, and most others have the same number, no matter how long the neck. All but the first two of these vertebrae bear cervical ribs which are fused by two points to the centrum, and through the canal thus formed runs the vertebral artery. In abnormal cases the cervical ribs (particularly the last two) may be long and movable.

The first cervical is highly modified to form the atlas which bears the head. The centrum of this vertebra fuses with the second vertebra in the mammal, and the atlas is left as a bony ring. The second cervical is the axis, the head rotating at the joint between the atlas and axis. The centrum of the first forms the odontoid process of the axis. The posterior five cervicals are very similar in structure.

Thoracic vertebrae are distinguished by the articular facets for the ribs. The spines of the neural processes are rather long, like those of the cervical ribs, and the muscles and tendons of the occipital region are attached to them. The ribs attached to these vertebrae meet the breast bone, or sternum.

Lumbar vertebrae of the mammal are heavy and have a stout neural spine. The transverse processes are directed toward the head, and there are no facets for rib attachment. In the lower vertebrates these lumbar vertebrae are hardly distinguishable from those of the thoracic region.

Sacral vertebrae have the pelvic girdle attached to them. The amphibia have only one vertebra which bears a modified rib for the girdle attachment, while the reptiles have two with sacral ribs. The mammal retains the reptilian number of true sacral vertebrae; but the ribs and the transverse processes of two, three or four other vertebrae become indistinguishably fused to form a heavy sacrum to which the pelvis becomes attached. The sacrum of the bird is very large, due to the fusion of seven or more vertebrae.

Caudal vertebrae are the simplest in structure. In most mammals only the first two or three bear any sign of transverse processes, and the more posterior ones consist of only a small centrum, the size of this element being reduced from anterior to 
posterior. The caudal vertebrae of the human are fused to form a terminal process, the $\operatorname{coc} y x$.

Embryology of the Vertebrae. The developmental history of the vertebrae varies in the different classes of vertebrates.

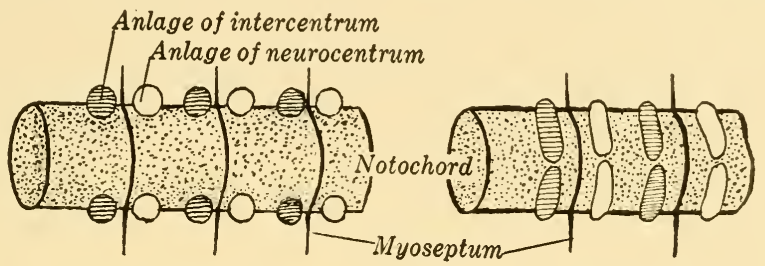

A. Early Stage

B. Later Stage

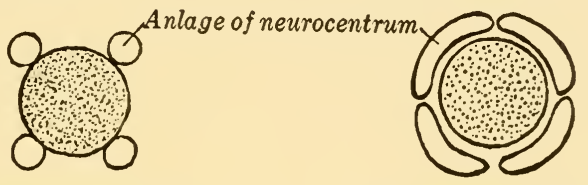

A'. Cross section of "A"

$B$ '. Cross section of " $B$ "

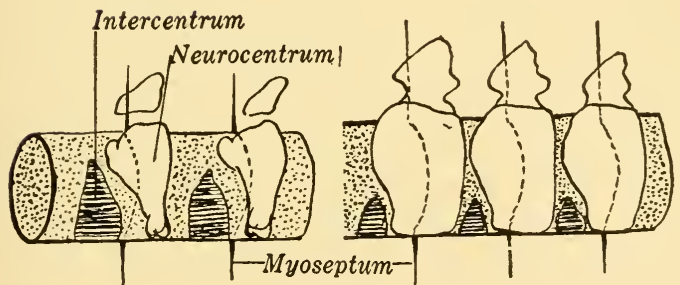

C.

D.

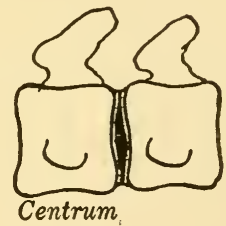

E.

FiG. 88. Embryology of the Vertebrae. (A) and (B) show the isolated cartilages forming rings around the notochord, equivalent to the intercentrum and neurocentrum. Drawings (C) and (D) show the intercentra being crowded ventrally during development, and lost in the definitive structure (E).

The following is a generalized description. The vertebrae first appear as eight minute cartilages in each body segment, forming two incomplete rings around the notochord. As these cartilages grow they fuse into two rings, thus parallelling the fossil history. The more anterior ring of each segment enlarges, developing a neural arch, and pushes anteriorly across the myoseptum. In 
this way the embryonic tissues of the ring in front of the neurocentrum (the posterior ring of the next segment) are crowded downward. This posterior ring of the segment is the homologue of the intercentrum, and in many mammals these ossify as the small chevron bones of the caudal region. The intercentra in the more anterior regions of the vertebral column are completely crowded out.

All vertebrates above the cyclostomes begin with similar cartilaginous vertebral blocks, but there is a differential development in different classes and even the regions of the body. The most primitive vertebrae ossified as two independent rings in each segment, and this condition is retained in the tail region of many fish. Others carried the growth of the neurocentrum further before complete ossification, as was shown above, while the human and some others have carried the process so far that the intercentral tissues are completely obliterated before ossification is completed.

\section{Ribs and Sternum}

The most primitive ribs are very small cartilages found in the elasmobranch fishes. The ribs develop in the myoseptum, and are in contact with the transverse processes of the vertebrae. The Crossopterygians retain these myoseptal ribs, but have an accessory pair with a different origin. The second pair is typical of the bony fishes and is the so-called "fish rib". Apparently it was not carried over to the amphibia, as the ribs of all the land vertebrates originate in the connective tissues between the muscle segments.

In the primitive amphibia the ribs articulated with two vertebrae, being in contact with the vertebral column at the point of articulation between the neurocentrum and the intercentrum. But, as the neurocentrum pushed anteriorly, the rib came to lie in contact with the middle of a single vertebra.

The amphibia and reptiles have movable ribs on all vertebrae from the third cervical to the last sacral. In the mammals the movable ribs are reduced to twelve, in the thoracic region. However, both the cervical and sacral vertebrae have small ribs fused to the centra. 


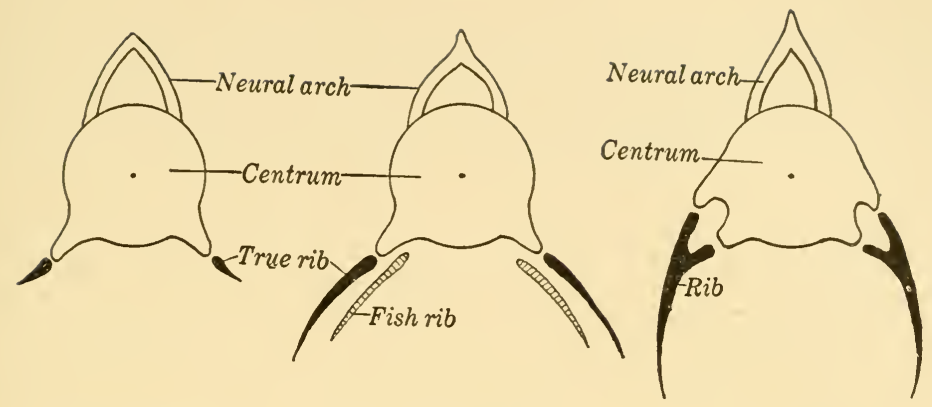
A. Dogfish
B. Crossopterygian
C. Amphibian

Fig. 89. Structure of Ribs. Evolution of the true (myoseptal) ribs; and the position of the "fish" ribs of the Crossopterygii and Teleostei.

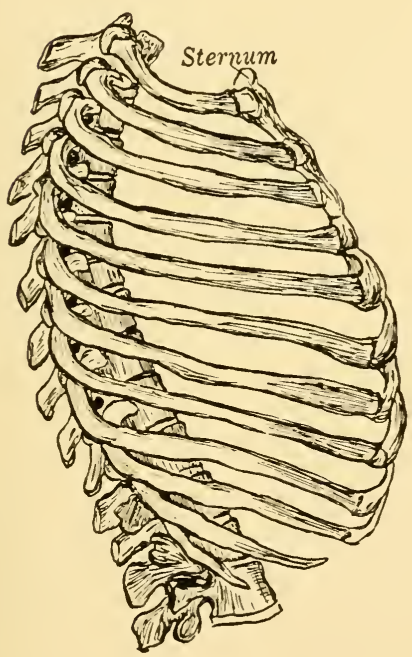

Fig. 90. Thorax of Man. The relationship between the ribs, the vertebrae and the sternum is shown.

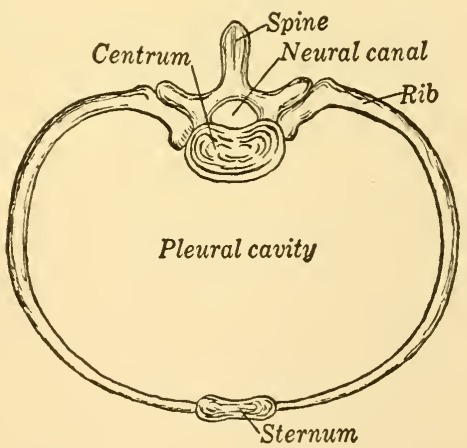

FIG. 91. Cross Section of the Thorax of Man. Compare with Figures 89 and 90. 


\section{E. Origin of the Appendages}

The pectoral and pelvic girdles together form the appendicular skeleton. With the girdle proper, which is the internal bracing structure, is included the appendage-either fin, leg, arm, or wing.

There are several theories to account for the origin of the paired fins from the finless, jawless ancestors of the fish, but the most acceptable to most anatomists is the fin fold theory. This theory requires an ancestral type with a continuous fin along either side of the body, beginning posterior to the gills and extending posteriorly and ventrally to a point posterior to the anus. At this point they fuse and continue to the end of the animal as the ventral and caudal fins. This animal is supposed to have developed metameric cartilage supports to give strength and rigidity to the long fin fold. Then, during evolution, the middle and most posterior regions of the paired fins disappeared, leaving wide pectoral and pelvic fins. In similar manner the continuous dorsal fin became reduced to two dorsal fins, and the upper portion of the caudal. The evidence from both palaeontology and embryology supports this theory.

The most ancient and primitive sharks are known only as fossils. Of these Cladoselache is the type form. Its notochord was continuous and its vertebrae only slightly better developed than those of the cyclostomes. Its most interesting feature was the appendages, the pelvic fin being more primitive than the pectoral. Each fin had a wide base and was half-moon shaped. The pelvic was supported by metameric, disconnected cartilages; while the pectoral had similar cartilaginous rays and a longitudinal series of small cartilages at their base. Eventually this basal series shortened to form the three basal cartilages found in the living sharks.

The embryological evidence is equally clear. In the elasmobranch embryo the first sign of the appendages is a double row of limb buds in the pectoral and pelvic regions, (page 158). Each limb arises from six or more segments. As these muscle buds grow each carries a spinal nerve with it, the entire structure extending distally as a wide, rounded embryonic limb. As the limb takes shape the base constricts and soon the definitive fin 


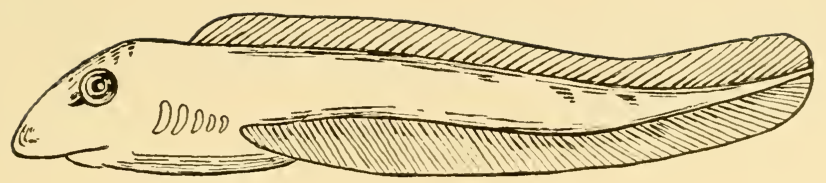

A.

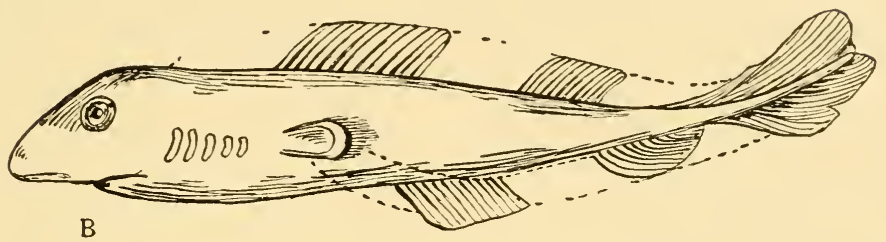

Fig. 92. Hypothetical Stages in the Development of Fins. The shortening of the single dorsal and paired lateral fins to form the discontinuous dorsal and lateral (pectoral and pelvic) fins according to the fin-fold theory.

(After Dean).

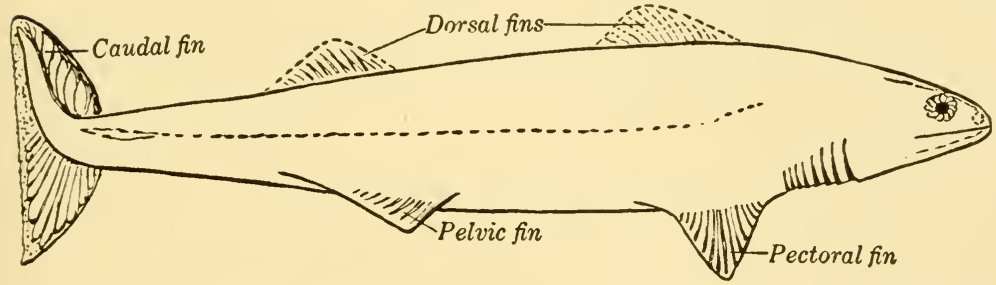

A. Side View

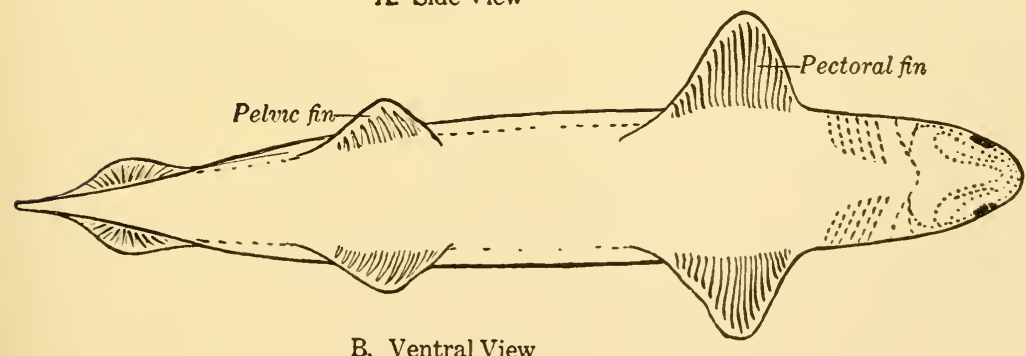

Fig. 93. Cladoselache, Side and Ventral Views. A primitive, extinct Elasmobranch with fins having wide bases and numerous radial cartilages. (After Dean). 
is formed. The limb development of the higher vertebrates is modified and less diagrammatic, but the origin from several segments is the cause of the large plexus of nerves in the limb of all vertebrates.

\section{F. Pectoral Girdle}

The recent sharks have a simple girdle and limb supports. The girdle is a pair of cartilages held tightly together in the mid-

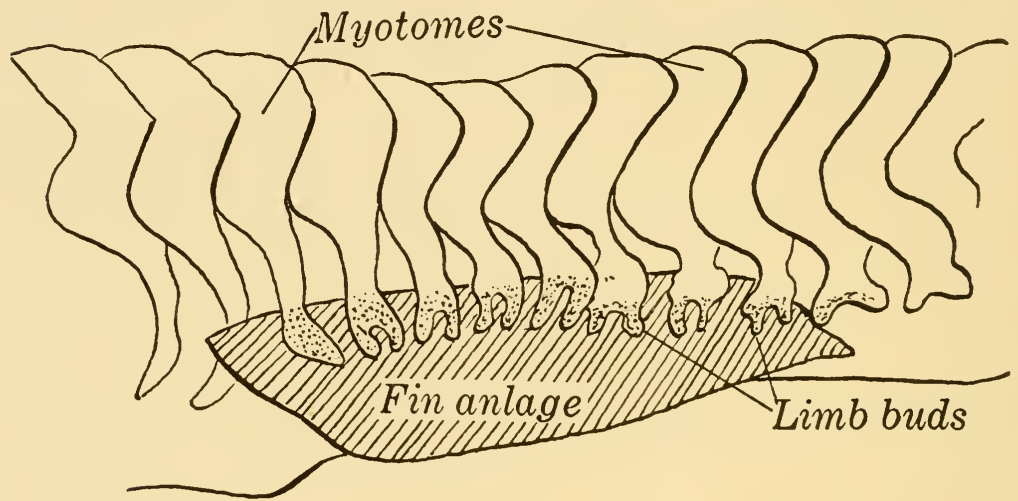

FIG. 94. Embryology of Fins. Note the numerous myotomes which collectively form a single appendage.

ventral line, and loosely anchored to the vertebrae with connective tissue. About the middle, on the posterior side, is a notch for the articulation of the fin cartilages. The region ventral to this notch is the coracoid, and in the higher forms is a separate bone. The dorsal portion is the scapula.

The fin is supported by three cartilages, the "pro-", "meso-" and metapterygium. Distal to these is a row of small cartilages. The dorsal and ventral fin muscles are attached to the cartilage supports. Distally the fin ends in a fleshless flap supported by dermal fin rays.

The most primitive crossopterygians known already had a pectoral fin showing evidence of its kinship with that of the land animals. The middle pterygial cartilage was largest, with the two others smaller and separated from the girdle. The more 
specialized members of this order had a bony skeleton which is clearly a pre-amphibian arrangement. The middle bone was large, forming the main support of the fleshy lobe (page 44), and distally were two other bones. These three bones are homologized with the proximal humerus and the radius and ulna of the forearm. Distal to these were small bones which evidently gave rise to the carpals of the wrist joint, and the metacarpals and

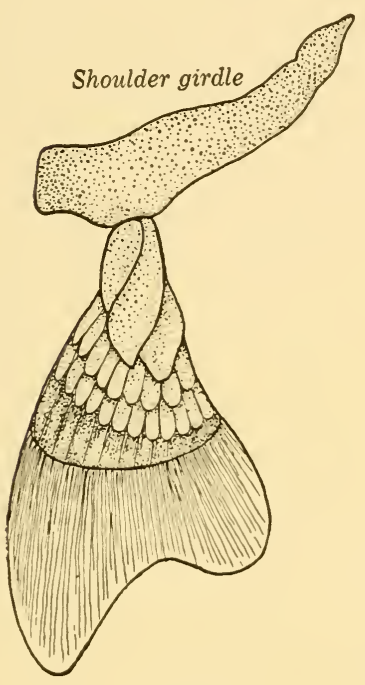

A. Dogfish

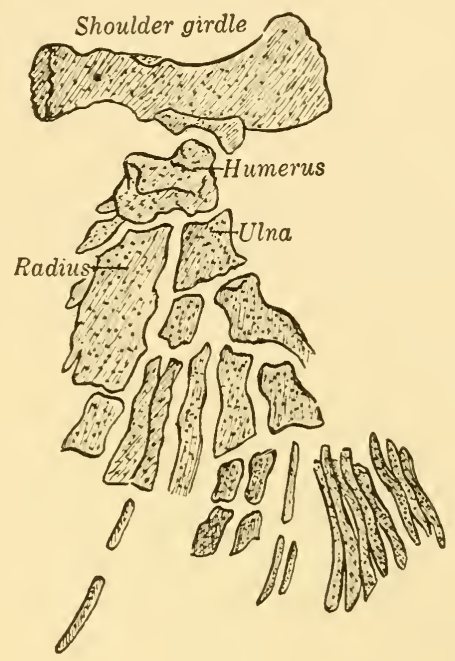

B. Primitive Extinct

Crossopterygian

Fig. 95. Stages in the Evolution of the Pectoral Fin. Compare the Dogfish fin with that of Sauripterus (Order Crossopterygii). The latter has all the elements of a form ancestral to the amphibia. (Sauripterus after Hussakoff).

bones of the digits. Two genera of these fish hare been carefully studied, Sauripterus and Eusthenopteron, the latter being illustrated.

The girdle of these fish had become ossified as two bones, the ventral coracoid and the dorsal scapula, which had kept their primitive positions. In addition to these replacement or cartilage bones, three small dermal bones had become attached to the scapula at its dorsal margin. These formed an attachment between the girdle and the head. Another pair of bones was added 
to the girdle of this animal, the clavicle, parallelling the coracoid on its anterior face, and also meeting its mate at the midventral line. It is unknown if this bone is of cartilage or dermal origin in the fish. In the frog it is of mixed origin, and in the higher forms the dermal part is apparently lost.

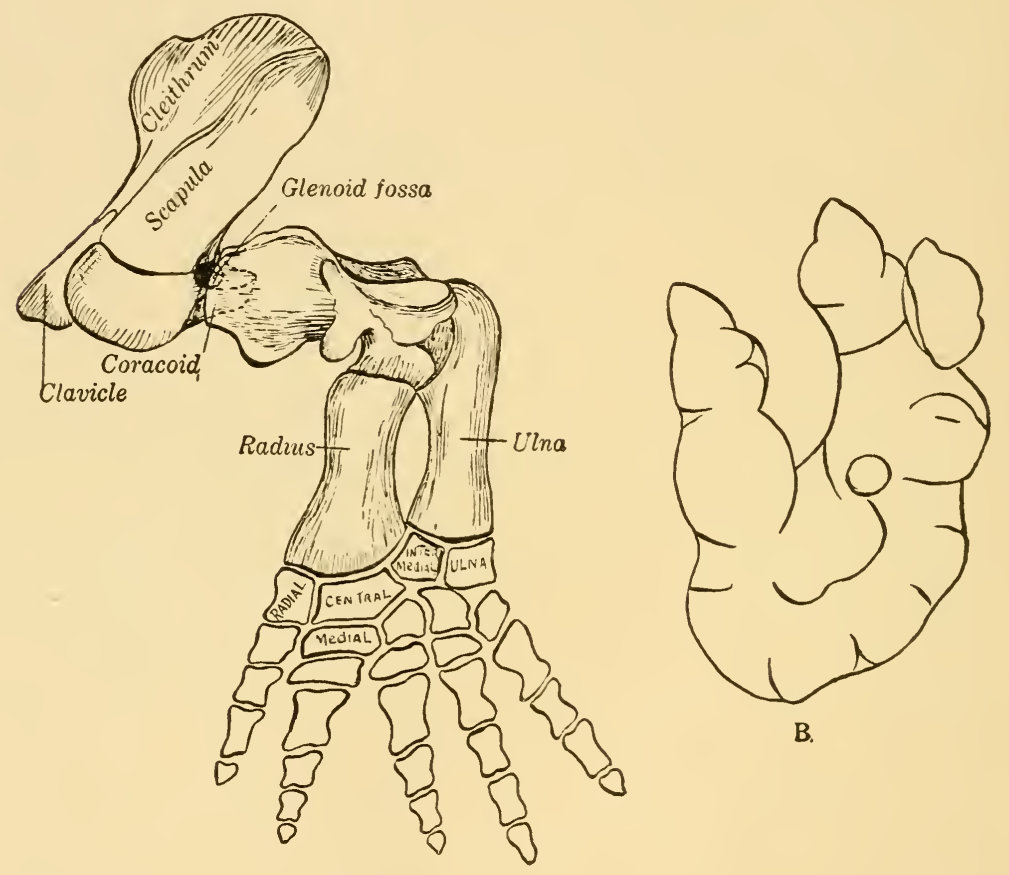

A

Fig. 96. (A) Shoulder Girdle and Appendage of Eryops (Order Stegocephalia), an extinct amphibian with a primitive girdle and a heary, short humerus. (B) The foot-print of Thinopus, the earliest record of land animals.

The crossopterygian girdle was carried over to the earliest amphibia almost in its entirety. The ventral clavicies and coracoids met in the midline; and the scapulae were connected with the head by small dermal bones. The latter were soon lost, for the connection was a liability under conditions where flexibility between head and shoulder were required. 
In the first known amphibia the bones of the arm and hand were specialized for land life and had assumed their typical tetrapod (four footed) form. The humerus was short, the radius was on the preaxial (thumb) border of the arm, and the ulna was on the postaxial border. The carpals were numerous. Little is known regarding the original number of digits. The oldest evidence is a foot-print made by an early amphibian (Thinopus), which might indicate a foot with three toes. It is more probable that the print was made by some five-toed animal walking on the margin of its foot. The crossopterygian larva rests on the outer (postaxial) border of its fins (page 44), and the young frog also holds the foot in this position. Therefore, as the earliest skeletons had the typical five toes, it is believed that Thinopus was simply an amphibian which had not become flat footed.

The first reptiles had lost the dermal bones, and had the "complete" girdle: (1) dorsal scapulae not connected with the head, and (2) coracoids and clavicles meeting (3) the median ventral sternum. The heavy coracoids held the humeri at right angles to the body, with the forearms bent forward. The recent reptiles are similar in structure. When quiet the body of the animal rests upon the ground, but when running the legs are pulled under the body as it is lifted from the ground.

This complete girdle was carried over to the monotremes with almost no changes, and is a large part of the evidence that these animals arose as a separate line of evolution. The mammallike reptiles had a marked reduction of the coracoid bones, with the result that the legs were drawn under the body into the running position, and in this respect were more like the higher mammals than are the monotremes. The evolution of the separate bones is traced below.

1. Conacoid. The mammal-like reptiles had greatly reduced coracoids which still met the sternum. The embryo of the living marsupials shows a similar condition, with cartilaginous coracoids meeting in the mid-line. But as development continues these cartilages recede from the sternal position and become attached to the scapula as coracoid processes. In the placentals the coracoid cartilages never meet the mid-line, and develop as processes of the scapula. 


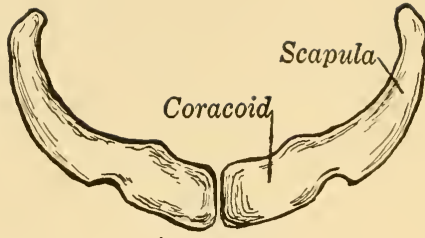

A. Dogfish
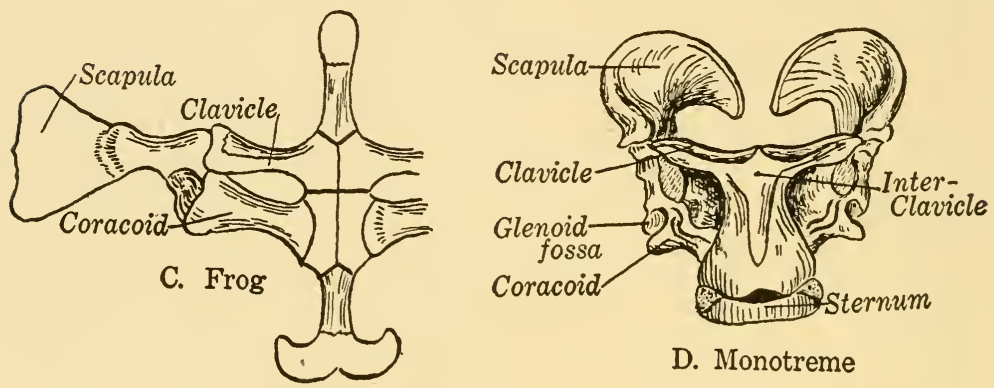

D. Monotreme

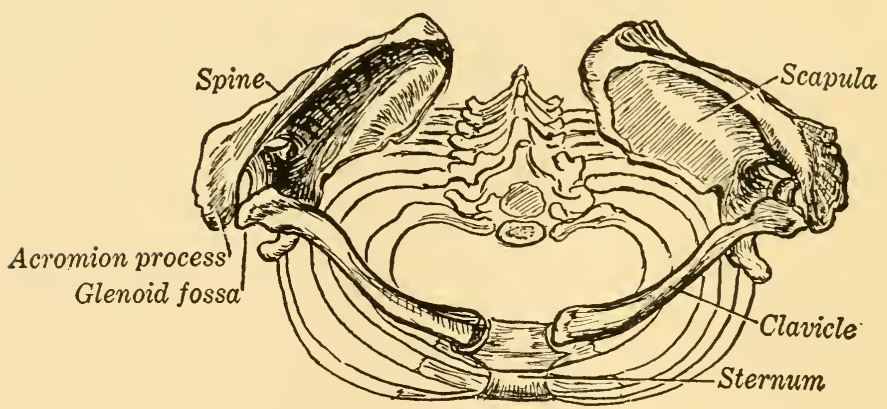

E. Man

Fig. 97. Comparative Anatomy of the Shoulder Girdle. The Dogfish has a single pair of cartilages meeting ventrally. The primitive reptile (B) and the Monotreme (D) have a complete girdle. The Placental Mammal (E) shows the reduction of the coracoid bone to a process of the scapula, and the development of the scapular spine. Diagrams (A), (B), (C) and (D) are ventral views; (E) is an anterior view. 
2. Scapula. In the reptiles the scapula is a flat, rather narrow bone. The monotreme scapula is no wider, but the anterior border is turned outward. The embryonic scapula of the marsupials is similar, and then develops an anterior extension of the blade of the bone, which leaves the upturned margin as the spine of the scapula. The spine divides the lateral face of the bone into two fossae in which shoulder muscles are attached. The placental scapula is similar, although the development is less diagrammatic.

3. Clavicle. The clavicle is the most recent and most variable bone of the shoulder girdle. It may be small or entirely absent. In the placentals there is a marked correlation between its size and the function of the pectoral limbs. In general terms, the bone is small or absent in running animals; while the climbing types retain a well developed clavicle. When the legs move in a single, antero-posterior, plane the clavicle is a liability; but when the limbs are moved in many directions the clavicle has selective value for muscular attachments, and for bracing the shoulder.

\section{G. Pelvic Girdle}

The evolution of the pelvic girdle is more obscure than that of the pectoral, and in the fish and amphibia is much simpler in structure. In the fish and earlier amphibia the anterior limbs carried a greater functional burden. In the fish the pectoral fin is the major balancer of the body, and in the primitive amphibia the head is raised from the ground on the fore limbs as the animal wriggles through the mud. These facts probably explain the greater selection exerted upon the anterior appendages.

Cladoselache gives the earliest clue to the development of the pelvic region, the fin being supported by isolated cartilages with no body supports. In the sharks the anterior rays have become fused into a relatively large cartilage on either side, the two meeting ventrally. Further evolution is indicated by the chondrostean fish, the ventral portion of each cartilage constricting off to form a paired ventral segment. In the living crossopterygian (Polypterus) these two segments have fused to 
form a small plate. The longer dorsal portions of the cartilages strongly resemble the femori of the land vertebrates.

A wide gap exists, however, between any known fish and the amphibia; and the anatomist is tempted to say that the true pelvis developed from the simple elasmobranch type after the evolution of the tetrapods. The primitive living urodeles have a flat ventral cartilage with a pair of posterior centers of ossification, and in addition, a pair of dorsal bones which are attached to the sacral vertebrae. The latter are the ilia. In certain frogs there is an additional pair of ventral ossifications. These

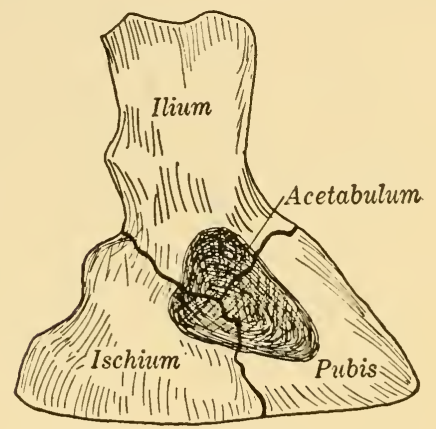

Primitive Extinct Amphibian (Stegocephalia)

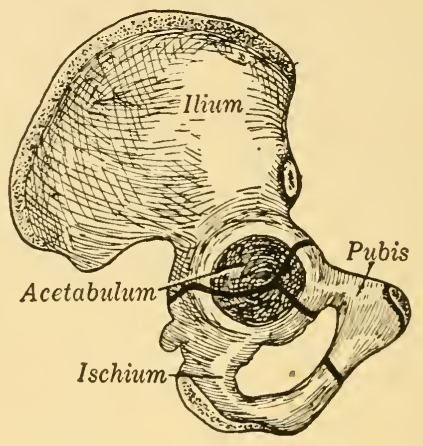

Human Embryo

FIG. 98. Evolution of the Pelvis. The primitive pelvis (A) consisted of three separate bones forming a solid plate. The higher animals develop an obturator foramen in the pubis and ischium. The mammal embryo has the sutures between the three bones, although the bones ankylose during development.

four ventral bones are the homologues of the anterior pubes and the posterior ischia of the higher animals. But the fact that the pelvic girdle of the Stegocephalia is clearly divided by sutures, renders any evidence from comparative anatomy doubtful.

The reptiles have a pelvis typical of all higher vertebrates. The acetabulum is a lateral depression in which the femur articulates, and is the center from which the three pelvic bones radiate. The dorsal ilia attach to two sacral vertebrae, and each pubis and ischium meets its mate in the mid-ventral line. Thus, including the sacrum, a complete circular girdle is formed which is decidedly stronger than the pectoral girdle, and conversely is less flexible. 
Further evolution is merely modification of this primitive plan. The pubic bones have developed along two main lines: (1) the primitive reptilian type with the pubic union, or symphysis, in an anterior position; and (2) the bird-like modification with the pubes growing posteriorly, parallelling the ischia. The mammal-like reptiles and mammals retained the primitive condition.

Homology of Parts. The girdles evidently arose independently of each other, and homology of parts is consequently difficult. The appendages, on the other hand, arise embryologically in the same manner and all the evidence points to a complete homology of parts.

\begin{tabular}{c|c|c}
\hline Region & Pectoral & Pelitc \\
\hline Upper limb & Humerus & Femur \\
\hline Lower limb & $\begin{array}{c}\text { Radius (preaxial) } \\
\text { Ulna (postaxial) }\end{array}$ & $\begin{array}{c}\text { Tibia (preaxial) } \\
\text { Fibula (postaxial) }\end{array}$ \\
\hline Wrist-Ankle & Carpals & Tarsals \\
\hline Palm & Metacarpals & Metatarsals \\
\hline Digits & Phalanges & Phalanges \\
\hline
\end{tabular}

The primitive tetrapod limbs are thought of as being held in a straight line at right angles to the rertebral column. Each limb had a joint between the upper and lower limb, the elbow of the pectoral limb, and the knee of the pelvic. The front leg was then rotated forward and under the body, and the elbow was forced posteriorly as the arm was flexed. Exactly the opposite occurred in the hind leg. The foot was carried posteriorly under the tail, and the knee joint was forced anteriorly. The limb position indicates the function. The front legs primitively act as a support of the head, and the pelvic limbs push the body along. The development of the pelvis and its appendages is in correlation with the greater function of the limbs as a loco- 
motor apparatus when the vertebrates left the water and muddy flats and became true land living animals.

\section{H. Specialization of the Limbs}

Modifications of the appendages begin with the fish, the two main lines of evolution having been traced, that is, the typical balancing fin, and the crossopterygian type. The amphibia arose as five-toed animals with short legs, and there are few specializations in the class except the loss of digits and entire limbs. Thus, in the recent amphibia, we have species without appendages, others lacking the hind legs, one with minute front and hind legs, and some lacking some of the digits. The typical amphibian has five digits on the pelvic limb, and four on the pectoral.

Among the reptiles the specializations become more pronounced. The snakes and some lizards have no limbs, the pythons having minute stubs on the degenerate pelvic girdle; and others lack certain digits. Among the fossil reptiles there are many adaptive radiations, many of which are parallelled in the mammals and may be considered under separate headings as functional adaptations. The student should keep in mind that these similar evolutionary changes are separate lines of specialization, and that there is no genetic relationship between the modifications in different classes. The typical five toes of the fossil amphibia and reptiles were inherited by the mammals, and any specializations for particular function have arisen independently.

1. Aquatic. Numerous orders of reptiles and mammals show varying adaptations for water life. Among the reptiles, the turtles (page 58) show many marked changes of the appendages. In addition to the shift in shape of the carapace (shell) the appendages become webbed, and in the sea-turtle the feet become flippers. The aquatic life was carried to its furthest extent in the extinct ichthyosaurs (fish-like lizards). These reptiles had flippers with the digits completely enclosed by the skin covering, the body was fusiform in shape, the tail was short with a terminal flattening, and the animals were ovo-viviparous (page 37). Apparently they lived their entire lives in the water.

The mammals, recent and fossil, show similar adaptations. 
The carnivores show almost every degree of aquatic specialization. Beginning with animals like the otter which spends a large part of its time in the water but has typical five-toed feet, a further degree of change is found in the Alaskan sea otter which spends most of its time floating on its back. The Pinnipedia (water living carnivores) form a separate sub-order and carry the modifications still further. The sea-lion and walrus
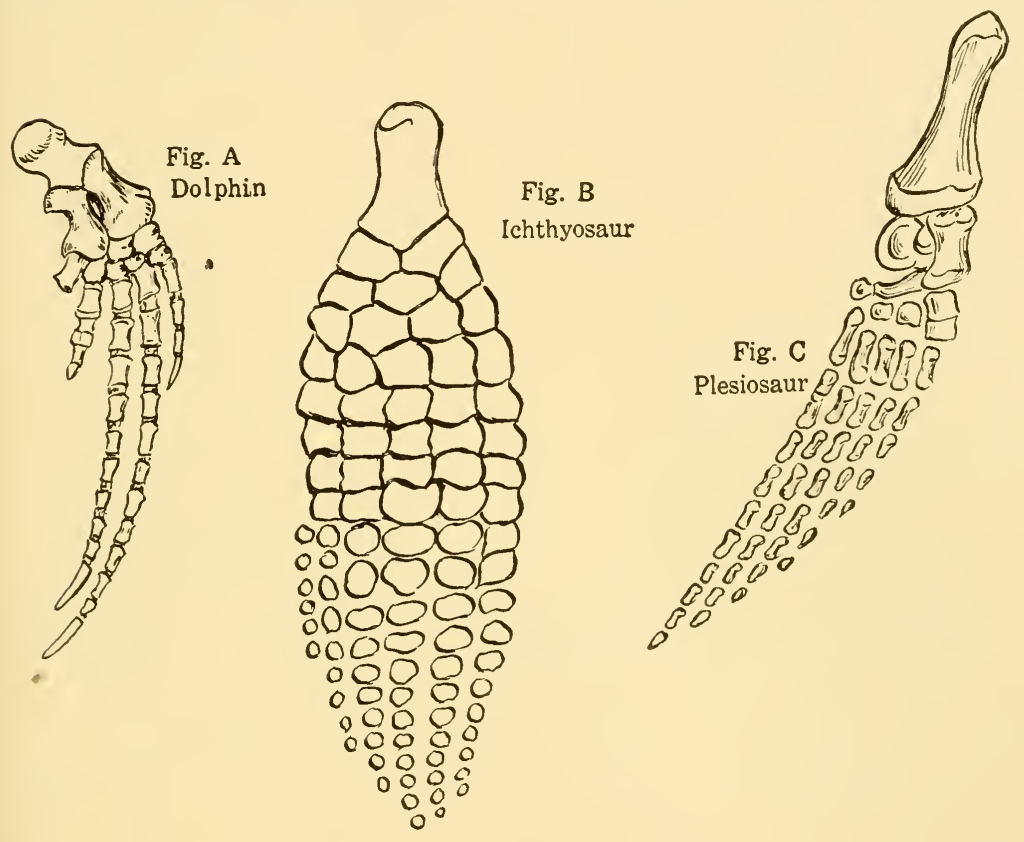

Fig. 99. Aquatic Adaptations of the Pectoral Appendages. The number of phalanges is increased, and in the more specialized forms the arm and hand form a flipper.

have flippers, both pairs of which can be used for locomotion on land. In the Atlantic seal the pelvic flippers are enclosed by skin for most of their length, and are functionless on land. The Cetacea and Sirenia (page 90) have carried modifications for water life to the ultimate extent. The front flippers have an increased number of digits, and the pelvic appendages have disappeared except in rare instances where a small femur is found. Although these animals are externally modified, they are 
fundamentally like other members of the class to which they belong. All come to the surface for air, and the mammals feed their young on milk.

2. Bipedal. Bipedal animals are found in all the three amniote classes. The pectoral appendages are small or highly modified, and the pelvic limbs are large. The weight of the body is thrown backward for balance. All living birds are completely bipedal. The same modification was found in many extinct reptiles, although the bird was not descended from any known

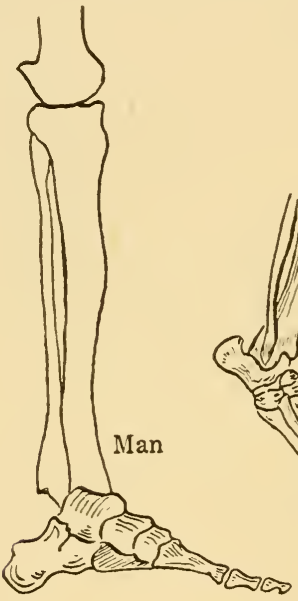

A. Plantigrade
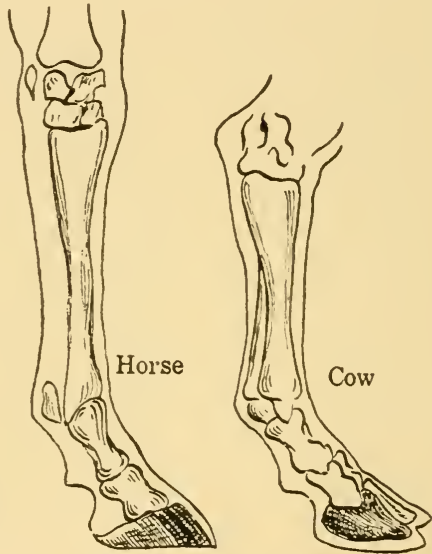

C. Unguligrade

Fig. 100. Walking and Running Modifications of the Appendages. The most marked specializations for running occur in the mammals. In this Class three major types are recognized, typical of mammals which walk (plantigrade), jump (digitigrade) or run (unguligrade).

group of this type. Both herbivorous and carnivorous reptiles were of this type, the latter being more frequent. Among the mammals the specialization has occurred in several unrelated groups. The marsupial kangaroos are highly modified for bipedal life; and man has similar locomotion, although there is less modification of the pectoral limb. The kangaroo has become adapted for a hopping motion. Man descended from an arboreal (tree living) group and has retained completely functional hands.

3. Cursorial. The running modifications have developed most prominently in animals living on dry plains where a pre- 
mium was placed upon speed. The limbs move in a single anteroposterior plane, and with this digital modifications have appeared. An animal on its toes runs more rapidly than one with its feet held flat. All grades are found in the placental mammals. Man, the bears, and many others walk in the plantigrade or flat-footed position, with the heel touching the ground. The carnivores and others have feet adapted for springing, with the weight resting on the ball of the foot, and the heel off the ground. The Artiodactyla and Perissodactyla (page 89) have carried the specialization to its furthest extent. These animals walk on the tips of the digits, only the terminal phalanx touching. This is the ultimate in ease of speedy motion. The evolution of the horse will be found in any biology text.

4. Arboreal. Tree-living modifications are of many types. The more primitive adaptation has bowed legs for holding the limb or tree trunk, with sharp claws. Among the primates, which have rounded nails, the specializations have been toward swinging from limb to limb, rather than a slow climbing. The primate condition has been termed brachiation. Long, well developed arms and fingers are typical of this group. The extreme specialization is found in two genera, one African and one South American, where the thumb is vestigial or lacking, and the other four fingers are very long. Mutations of the thumb are not infrequent, and the selective value of a small thumb is apparent. In jumping from limb to limb the thumb would often interfere with a proper landing; and a sudden fall from a tree-top, or an infected thumb from injury, are not conducive to longevity.

5. Flying. Under flying adaptations may be considered true flight and the volplaning modifications of sereral groups. Among the latter are the flying squirrels which have a fold of the body skin extending from the front to hind legs. The animal is enabled to descend at a flat angle and the jumping distance is greatly increased. True flight is found in all the amniote groups. The fossil pterodactyls ("wing fingers") had greatly elongated digits with a leathery flap connecting them. A similar modification has appeared among the placentals in the bats (page 86). This order has the digits elongated, with a thin membrane connecting them with the hind legs. The bats have speed, endurance and remarkable coordinations. Flight modifications in the birds 
are of an entirely different nature. The digits show a great degeneration, with a fusion of the carpals. Wing expanse is gained with the feathers.

There are other distinct modifications, for example digging or burrowing, and there are variations of the above. Specializations of the appendages are more frequent and more easily observed than in any other part of the body. Environment places a high

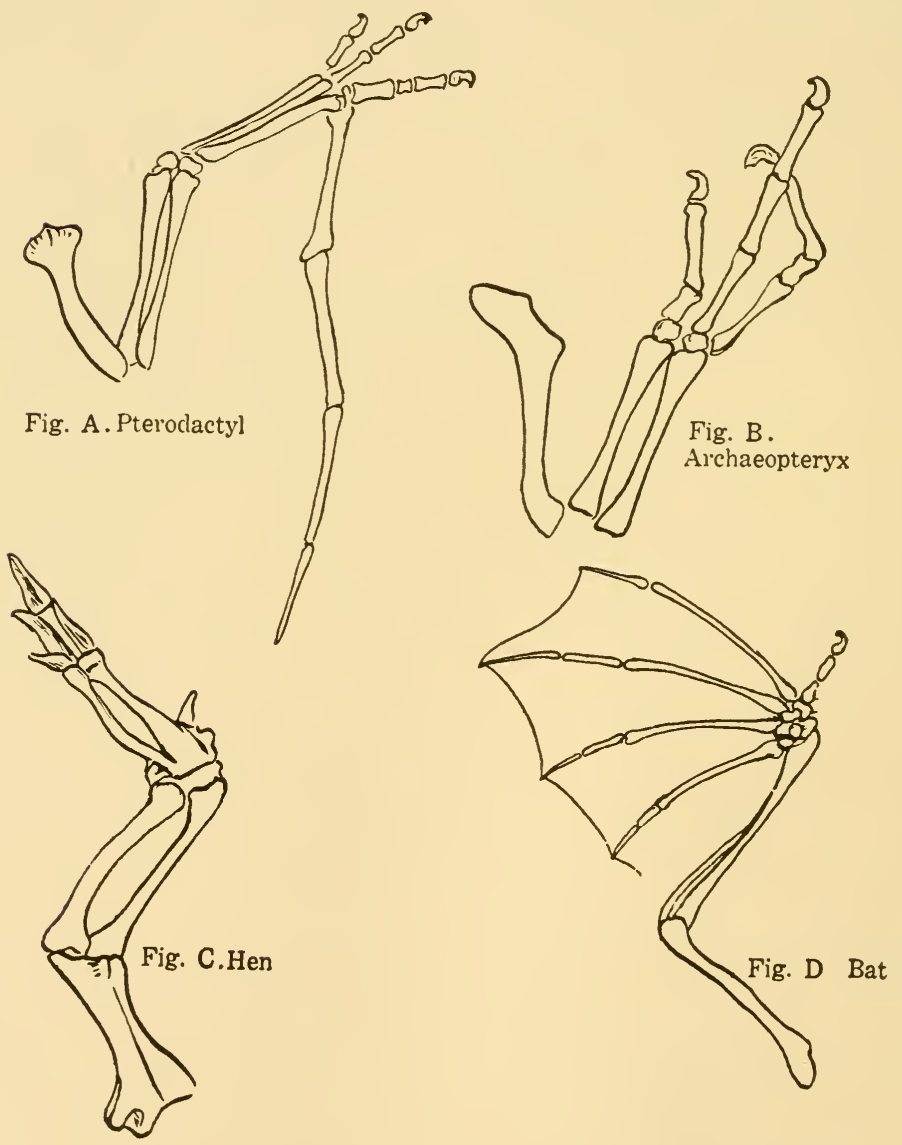

Fig. 101. Flying Adaptations. Flying Reptiles and Mammals (A and D) have elongated digits with membranous flaps stretched between them to give surface resistance. Increased area is given birds by the feathers. 
selective value upon locomotion, and at the same time degenerative changes are not necessarily fatal.

Due to the interdependence of the skeletal and muscular systems the bones may be modified by mutations in other tissues. An inherent degeneration of the muscles would undoubtedly affect the bones. The degeneration of the embryonic coracoid of the marsupial can be interpreted in this way, for there are no muscles attached to them. Conversely, the degeneration of a bone will cause developmental abnormalities in the muscles. The thumbless monkeys have all the thumb muscles, although they are vestigial and almost microscopic in some specimens. The interdependence of tissues is discussed in Chapter XIX.

\section{Development of the Skeleton}

The two developmental types of bone (dermal and replacement) have been defined. Either may give rise to the two anatomical types, long and flat bones. In the higher vertebrates few of the long bones are derived from dermal structures, these being limited to the dermal ribs and a part of the clavicle in a number of amphibia and reptiles. Flat bones include the body plates of the lower vertebrates, and the bones composing the skull of all bony vertebrates. Thus, the supra-occipital is flat, and composed in part of both replacement and dermal bone.

In addition to the above, bone is classified by histological structure into compact, or hard bone, and cancellous, or spongy. If a long bone is hemisected it will be seen that the head and the inner part of the shaft are made of coarse fibers which divide and anastomose, forming a spongy substance with many air spaces between the fibers. The heavy part of the shaft is a solid, compact structure. The flat bones are formed of two layers of compact bone, with an inner layer of spongy bone. The following classification will make this clear.

Classification of Bone

EMBRYOLOGICAL

Anatomical

Histological

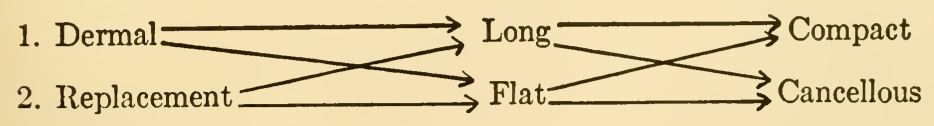


Dermal bone is limited in its distribution in the mammal, and its origin in the dermal fibers of the skin has been described. The student should recall that the osteoblasts deposit the bony substance around fibers which radiate from definite centers of ossification, growth in size being due to growth of the distal ends of the fibers and peripheral deposition of bone.

The locomotor elements of the skeleton, with a few minor exceptions, are replacement bone; and with the exception of the dermal bones which have been mentioned, the entire embryonic skeleton is laid down in cartilage which is gradually replaced by bone. However, it is a matter of common observation that growth continues long after embryonic life is ended, and that changes in bony configuration continue throughout life.

Bone cells are of two types: (1) osteoblasts, or bone building cells which secrete the organic fibers and the inorganic salts which give hardness to the bone; and (2) osteoclasts, or bone destroyers. Both types are at work, even after the limit of growth has been reached. The healing of a broken bone and the smoothing of the jawbone after a tooth extraction are illustrations which the student has observed.

The processes of growth can be demonstrated in a long bone of the arm. The embryonic cartilage is surrounded by a connective tissue sheath, the periosteum (called the perichondrium when surrounding cartilage). At about the middle of the shaft of the bone the periosteum breaks and blood vessels migrate into the cartilage, the osteoclasts destroying the cartilage tissue. From this central cavity the destruction proceeds toward either end of the bone, the cartilage being destroyed unevenly, so that finger-like processes are left projecting into the original cavity. Osteoblasts then begin secreting bone which fills the original cavity, and also the spaces at either side. As destruction proceeds, the cavities are filled with bone.

Secondary centers of ossification begin at either end of the cartilage producing the epiphyses which form the ends of each long bone. As the shaft continues to grow it soon approaches the terminal epiphyses. The skeleton of a young animal shows this condition, the epiphyses being separated from the shaft by a thin line of cartilage. Growth takes place in this cartilage area. Length of the long bone is determined by the time of 
fusion between the two, for when complete fusion occurs the growth stops. In the human this takes place, usually, between fifteen and eighteen years, taller individuals continuing to grow for a longer time than the short ones. Other animals may continue growth throughout life.

There is also a growth in diameter. This takes place in the periosteum, which lays down concentric layers of bony tissue, the outer layer being the younger. And as the bone grows outward, osteoclasts destroy the bone at the center, with the result that the inner marrow cavity becomes larger.

The growth and structure of bone, however, are not so simple as the above description. A cross section of a long bone will show that the outer layers are concentric, extending entirely around the shaft, but the major portion is composed of microscopic groups of concentric layers, each built around a canal in which lie an artery and vein. These small canals are the Haversian canals, and the canal and the concentric rings surrounding it form a Haversian system. As the osteoclasts eat out the peripheral layers, blood vessels with osteoblasts migrate in and lay down a long tube of bone. Other, smaller, layers are formed within the original one, until a new Haversian system is formed. In this case the inner ring is the youngest. Old systems are constantly being destroyed and new ones built. This can be demonstrated experimentally by feeding young animals a stain which colors the bone which is being laid down at the time of feeding. By alternating the stained rings with unstained, the order of destruction and rebuilding can be determined.

After the fusion of the epiphyses there is little further growth in size, the changes being internal. However, in pathological conditions bone may grow or be destroyed. A broken bone releases bone cells and growth proceeds rapidly. Diseases affecting the pituitary gland, which lies in the sella turcica of the skull, will cause bone growth later in life. This usually affects the jaw and the cartilaginous joints of the fingers.

Destruction is shown in old age by the thinning of the skull bones, and the degeneration of the angles of the jaw bones. The jaw of an old man is usually small and thin.

The size of the bones is influenced by glandular secretions, and these organs are also a factor in determining height. Proper 
nutrition and other environmental factors also influence height, particularly in species which have a definite size limit. Those which continue growth throughout life are less influenced by periods of semi-starvation. Malformations of bone may be due to injuries of the periosteum, or to improper feeding. Rickets, which causes bowed legs and other deformities, is caused by a lack of bone developing vitamines. There are, however, racial and individual differences in susceptibility to the condition. 


\section{CHAPTER XII}

\section{MLSCLLAR SYSTEM}

Muscle tissues are of mesodermal origin and are specialized for contractility. In all groups of animals above the coelenterates it is these tissues which are concerned with locomotion. In the vertebrates a study of the muscular system is usually limited to the striated skeletal muscles, for by common consent the smooth muscles are considered with the digestive tract, and the cardiac muscles with the vascular system.

\section{A. Developanent and Classification of Muscles}

The mesoderm arises as metameric blocks on either side of the notochord. Each of these segments soon becomes differentiated into epimere, an intermediate cell mass or nephrotome, and the hypomere (illustrations on page 68). From the hypomere arise the cardiac and smooth muscles, and the striated muscles of the gills; and also the mesenteries and peritoneum. The cavity which forms within the hypomere becomes the coelomic cavity of the body.

The epimere gives rise to the dermis of the skin, the connective tissues and bone, and the skeletal muscles. The last develop from the central portion of the epimere (the myotome) which grows ventrally between the peritoneum and the ectoderm of the embryo. When each myotome meets its mate from the opposite side the metameric structure of the body is completed. The myotomes are heavier in the dorsal region than in the ventral. Slightly later in development the myotomes are divided into dorsal and ventral regions by an axial line from anterior to posterior which parallels the ventral border of the notochord. The muscles which develop from the myotomes above this line are the epaxial group, the ventral muscles being the hypaxial 
group. Reference to Amphioxus will make the relationships clear. The embryos of all vertebrates are similar in this general plan, although the adults vary widely in the degree of specialization from this primitive condition.

The striated muscles are classified both by their origin and their position in the body. In the more primitive vertebrates the topographic arrangement of the muscles will usually indicate their origin and homology, but in the higher groups the position of the muscles has become more highly specialized. Therefore the anatomist must depend upon embryology and comparative anatomy to determine the correct relationships of the individual groups. Four major groups of skeletal muscles are usually recognized.

1. Axial muscles are the original metameric muscles which arise from the epimere and lie along the axis of the body. They are primitively metameric, although the metamerism is largely lost or obscured in the higher classes.

2. Branchiomeric muscles arise from the hypomere in connection with the branchial, or gill, cartilages. After the disappearance of the gills and the modification of the cartilages, these muscles are retained in the jaw and throat.

3. Appendicular muscles arise from the primitive axial group and grow distally on to the appendages. They are so highly modified that they are classified as a separate major group of muscles. Embryologically they can be traced to the epimere and are classified on the basis of their topographic arrangement.

4. Integumentary muscles arise from all three of the above groups. They become attached to the skin of the animal and form a distinct functional group.

\section{B. Nomenclature and Function}

There is no system of the body in which a knowledge of homologies between individual structures is as incomplete as in the muscles. The major topographic divisions can easily be followed from class to class, but homologies between individual muscles are not always certain. Homologies between reptiles and mammals are particularly difficult. This is caused by the fact that each muscle is not always clearly defined, for in the course 
of evolution a single muscle may divide to form several; and conversely, several separate muscles may fuse to form a single one. The three bases of homology are (1) comparative anatomy, (2) embryology, and (3) innervation. The last is, perhaps, the soundest basis of judgment.

The names of muscles are also confusing to students. The earliest study of anatomy was human, and the muscles were named according to their shape, position or function in man. These names rarely apply to the muscles of other animals. The more recent method of naming muscles by their points of attachment is an advance in the direction of simplicity. However, as the skeletal structure of the vertebrates has changed, the muscular attachments have changed with them; and a muscle which can be accurately homologized will have different names in the various classes of vertebrates. The result is that scientific accuracy is incompatible with simplicity of nomenclature, and the subject of homologies has been left largely in the hands of specialists in the field.

Structurally a muscle consists of muscle fibers bound together and attached at either end with connective tissues. Each fiber is surrounded with a microscopicaliy thin sheet of tissue, and groups of fibers are bound into bundles. Several of these bundles make a muscle, the entire structure being surrounded by a strong covering of connective tissues. These fibers completely interlace, and in this way bind the muscle into a strong functional unit.

A muscle is usually attached by a broad base known as the origin. It then widens to form. the belly of the muscle, and from this point narrows almost to a point at its insertion on a bone. The insertion is defined as the point of attachment on the part which is to be moved. Frequently the function of a muscle changes with the position of the animal, because of the change of leverage. The rectus abdominis, which extends from the pubic symphysis to the lower border of the ribs, illustrates the point. In man the contraction of this muscle tends to pull the ribs, and therefore the upper part of the body, downward; but in a rabbit, or a man when he is suspended by his arms, the muscle pulls the pelvis upward. Either end might be called the insertion. Structurally, however, the muscle arises on the pelvis as a thick, rounded mass, and spreads out on the ribs as a thin 
aponeurosis, or sheet of connective tissue. Similar changes in function are found in numerous other muscles. The important thing to understand is the usual or major function of a muscle or group of muscles.

The tendons and ligaments which attach the muscles to other structures (a bone or other muscle) are important in their function. All muscle fibers are attached by connective tissue strands to the periosteum of the bone or to other connective tissues. As a long muscle approaches its point of insertion the amount of connective tissue increases as the number of fibers decreases; until near its insertion only a band or cord of tendinous tissue is present. The fibers of the tendon become interwoven with those of the periosteum, in that way firmly attaching the muscle.

During the course of evolution the tendency has been for muscle groups to divide into smaller units, thus increasing the total number of separate muscles. Consequently, several muscles may be inseparable at the point of origin. In tracing the history of a muscle the progressive stages of the splitting can be found, varying from a single muscle which inserts by two or more tendons to those which are separate throughout their entire length. What appears to be the opposite condition is also found, a single muscle arising from several heads, or points of origin.

Although the muscles furnish the power in any movement, the skeleton gives form and rigidity to the body, flexibility being gained by the joints between the individual bones. The contraction of muscle results in various movements of the bones, acting upon them as any power acts upon a lever. A number of motions are possible, their range being limited by the structure of the joints and the articulations between bones. The more important skeletal movements follow.

1. Flexion is the formation of an angle between two bones. When the arm is bent at the elbow it is flexion between the upper and lower arm. Similarly the hand may be flexed in relation to the forearm, or the fingers flexed toward the palm.

2. Extension refers to straightening an angle already formed. After a part is flexed the extensors pull it back into the original position. 
3. Abduction is drawing an entire unit of structure from its median axis. When the arm is hanging at the side, and is then drawn upward and outward, it is abducted from the axis of the body. The thumb is abducted when it is drawn away from the axis of the arm; that is, away from the first finger.

4. Adduction is the opposite of abduction, the function of drawing a structure back into a position parallel with the axis.

5. Circumduction consists in swinging a unit of structure so that its distal end describes a circle. Holding the arm out from the body and moving it so that the fingers describe a circle fulfils the conditions.

6. Rotation, on the other hand, is turning a structure while its axis remains unchanged. The head may be turned without shifting the body axis; or, if the arm is held out as above described, it may be rotated slightly at the joint between the humerus and scapula.

7. Pronation is limited to the lower arm and leg. Hold the arm out with the palm up. Pronation turns the hand through 180 degrees so that the thumb is toward the median ventral line of the body.

8. Supination is the opposite of pronation, turning the thumb outward from the ventral line. Supination and pronation differ from rotation in that, in the former, the bones of the arm are twisted upon each other, and not rotated at a joint.

9. Elevation is raising a structure. The jaw is elevated when the mouth is closed, and the ribs are elevated when the chest is expanded.

10. Depression is lowering an elevated structure. Muscular contraction which depresses the lower jaw opens the mouth.

11. Dilation is enlarging a circular opening. The reflexive opening of the pupil of the eye is dilation.

12. Constriction is closing an opening. The muscles which cause constriction of openings are usually sphincter muscles, and are found in the so-called valves of the digestive tract.

Any movement of a skeletal part involves leverage. The levers of the vertebrate body fall into the three classes which are recognized in physics. The following symbols are used in the diagrams: 
1. $\mathrm{L}=$ Lever.

2. $\mathrm{W}=$ Weight, the object to be moved through the arc of a circle by the force exerted on the lever.

3. $\mathrm{F}=$ Fulcrum, the point upon which the lever moves.

4. $\mathrm{P}=$ Power, the force which moves the lever upon its fulcrum, and thus shifts the position of the weight. It is clear that the position of the fulcrum in relation to the weight and power will materially influence the speed and force with which the weight is moved.

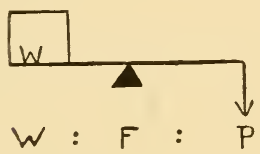

A. $1^{\text {st }}$ Order
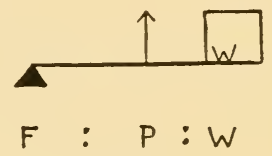

B. $2^{\text {nd }}$ Order
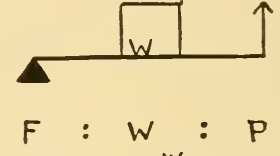

C. $3^{\text {rd }}$ Order $w$

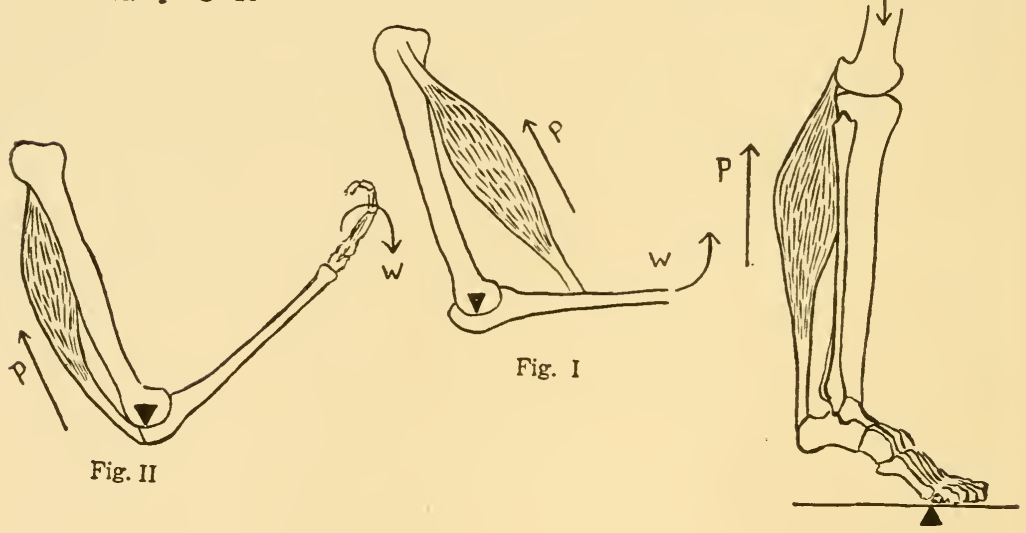

Fig. III

In Figure I it will be seen that when a weight is held in the hand, and the forearm is flexed, the leverage is like that of the second order (B). When (Diagram II) the back of the hand is pressed against an object as the weight to be moved, the extension of the arm observes the conditions of the first order lever (A). In Diagram III the ball of the foot is the fulcrum, the tibia and fibula support the weight of the body, and the calf muscles act as the power. This is like the third lever $(\mathrm{C}$ $\mathrm{F}: \mathrm{W}: \mathrm{P})$, and is powerful although the range of movement is limited and the motion is slow. 
Diagram III will show how muscle levers may be changed by shifting the function and therefore the fulcrum. If the heel is raised and the floor is tapped with the toe, the calf muscles function as in the first lever, $\mathbb{I}: \mathrm{F}: \mathrm{P}$. In this case the lever has remained the same, while the fulcrum has been shifted. Other cases involve a shift in the lever itself. Thus, a monkey may stand on the ground and pull the limb of a tree downward; or, he may hold a limb and pull his body upward. Essentially the same muscles are brought into play although the weights and levers have been changed.

Muscle groups are named for their more prominent function, although at times their action may be different. For example, the muscles on the palmar (ventral) side of the forearm are called flexors. Those on the back (dorsal) side are the extensors because their major function is to straighten an angle caused by the contraction of the flexor group. But when the hand is flexed at the wrist, the extensors may draw it into the position of a straight angle and then continue until the hand is in a position of dorsal flexion.

\section{Axial Muscles}

Amphioxus has the simplest possible arrangement of the axial muscles. Each segment is approximately equal to every other, and is a $\mathrm{V}$-shaped band extending from the dorsal to the rentral

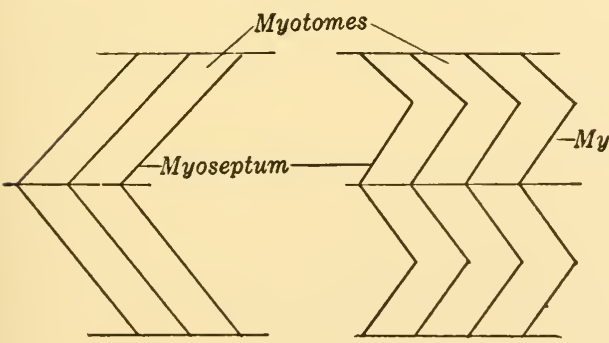

A. Amphioxus

B. Cyclostome

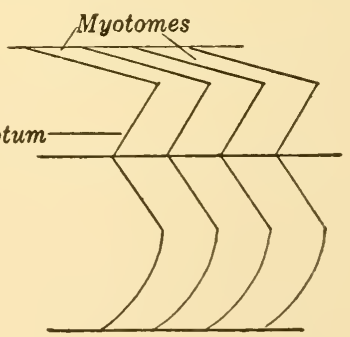

C. Dogfish

FIg. 102. Axial Muscles of Primitive Chordates (Diagrammatic). Note the bending and elongation of the myotomes in the Cyclostome and Dogfish. 
side. The dorsal portion is thick, and thins out ventrally. Separating each myotome from its neighbor is a myoseptum, a band of connective tissue, uniting adjacent muscle bands with each other and with the notochordal sheaths. The muscle fibers extend longitudinally, being attached with connective tissues to the myosepta. Therefore, when one side contracts the animal forms the arc of a circle. As the anterior end of the animal is more rigid than the caudal end, the alternate contraction of the two sides sets up a propeller-like motion and sends the animal forward.

The cyclostomes have more complex myotomes. The middle portion of the " $\mathrm{V}$ " is retained in place, the apex being at the axial line, but the dorsal and ventral portions are bent forward, giving a sigma shape to each myotome. Contractions thus tend to give a dorso-ventral as well as a lateral motion.

The myotomes of the dogfish are more complicated by the further bending and elongation of the dorsal part of the epaxial group. Each myotome is attached to several vertebrae, losing in part the simple metameric structure. The contraction of the myotome affects more than one segment of the body, giving greater flexibility to vertebral movements.

Future evolution, particularly in the land vertebrates, is in correlation with (1) the lack of a supporting water environment which diffuses shocks, and (2) the flexibility required by land life where locomotion is supplied by the legs rather than axial movements of the body. There is a progressive elongation and splitting of the myotomes, attaching them to a greater number of vertebrae, and giving a nicety of individual function impossible in the simpler forms.

The embryology of the mammal demonstrates many of these changes. The first myotomes are simple metameric structures, broken at the axial line. The epaxial group elongate, split, and become largely longitudinal in position. A great number of muscles result, some remaining as short muscles of small diameter, others fusing to form long muscles extending more than half the length of the vertebral column. These back muscles are classified into several distinct groups. In the anterior region are short occipital muscles which are attached to the head and cervical vertebrae. These are structurally continuous with the 
short vertebral muscles which attach to the spines of the vertebrae. Lateral to these in the anterior half of the body are the long muscles of the back, the longissimus dorsi, composed of a number of slips. In the posterior half of the back, in addition to the short spinal muscles, are two major groups: (1) the multifidus and (2) the sacro-spinalis, which pass from the sacrum and pelvic girdle to the transverse processes of the vertebrae, and give leverage to the back. This is, usually, the strongest muscle group of the body.

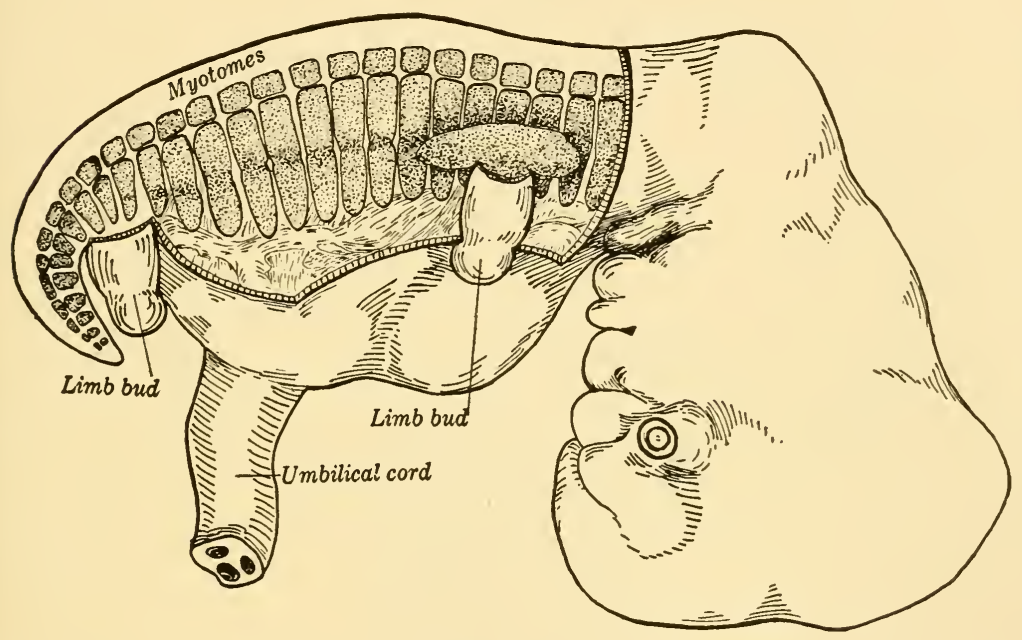

Fig. 103. Axial Muscles of the Human Embryo. The primitive myotomes bend, elongate and fuse to form the long axial muscles of the mammal.

The caudal muscles are continuations of the axial muscles. In the reptiles the body decreases in size toward the tip of the tail without any definite break in continuity at the pelvis. In the mammals the tail is less prominent, and does not serve as a locomotor or balancing organ as in the reptiles; and as the tail degenerates these muscles become less and less prominent. A functional exception exists in the South American monkeys, and other mammals with prehensile tails, for in these the caudal muscles are well developed to the tip; but in these animals the tail is approximately the same size throughout its length, and is 
clearly delimited from the body. In the higher anthropoids and man, in which the tail is vestigial, the caudal muscles are left as a small group of anal muscles, giving added support to the perineum in the upright walking position.

Certain of the epaxial muscles migrate to the ventral side of the vertebral transverse processes and pass posteriorly to the

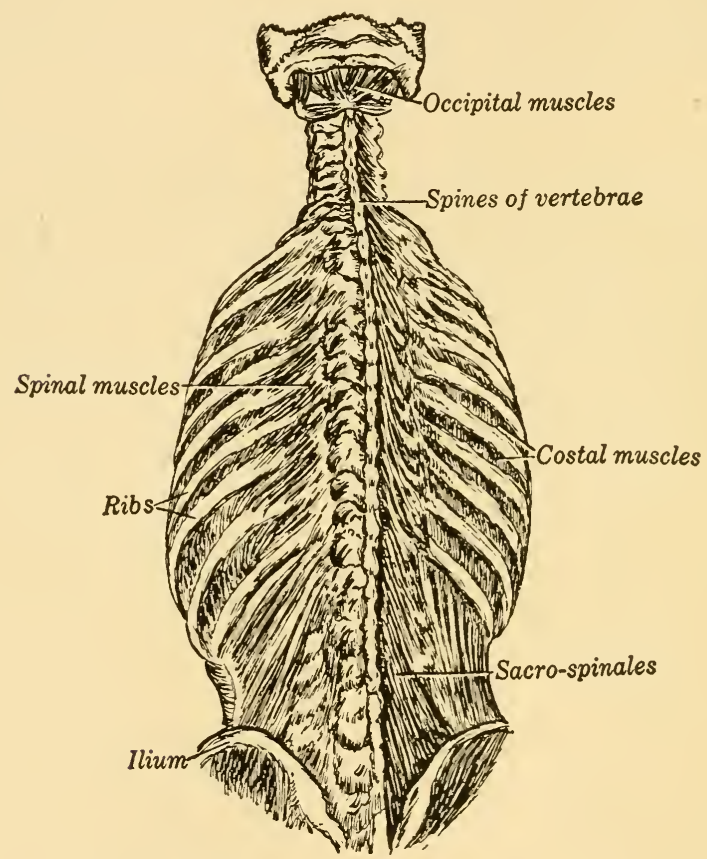

Fig. 104. Back Muscles of Man. The superficial muscles are shown dissected away. The left side of the figure shows muscles which are deeper in position than those on the right side.

pelvis and femur. This is the powerful ilio-psoas group, its homologue beginning in the urodele amphibia.

During the course of evolution the hypaxial region remains more primitive. The ventral elements of the myotome form the costal, or rib, muscles which are distinctly metameric. In most vertebrates they are limited to the thoracic region of the body. In the abdominal region the hypaxial muscles form the layers of the body wall and the rectus abdominis. The abdominal 
muscles are in three layers, (1) an inner transverse group with the fibers running dorso-ventrally; (2) the middle oblique fibers which are directed in an antero-ventral direction; and (3) the external oblique fibers which pass from the epaxial line in a postero-ventral direction. The fibers from either side meet a connective tissue septum, the linea alba in the midline. The rectus abdominis muscle is another part of the abdominal group. The fibers are longitudinal, arising on the pubic bones and extending

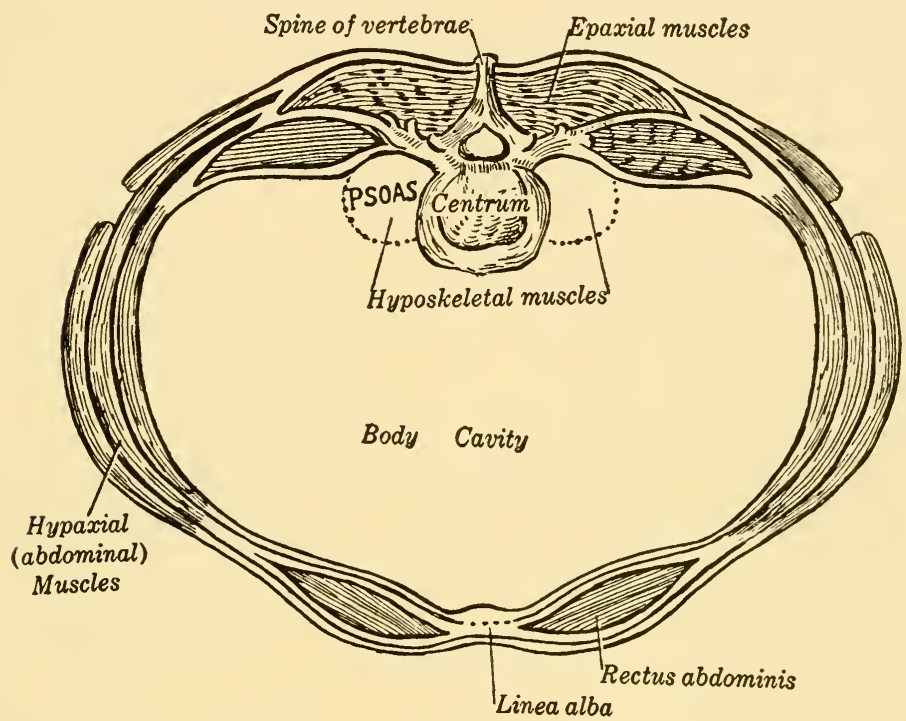

Fig. 105. Cross Section of a Mammal through the Lumbar region. The skin and viscera are removed, with the muscle groups in position.

anteriorly to the ribs, where they insert in a wide band. The hypaxial caudal muscles, like those of the epaxial region, undergo degeneration during their evolution, and are left in man as a few small muscles in the anal region.

\section{Branchioneric Muscles}

These striated muscles which derelop from the hypomere were originally attached to the gill bars. As the anterior two pairs of visceral arches dereloped into the jaws and hyoid ap- 
paratus, the muscles attached to them shifted their position and function. Those of the posterior five arches remained as elevators and depressors of the gill arches.

In the urodele amphibians there is a further degeneration of the arches, with correlated changes of function in the musculature. The anterior muscles become attached to the throat cartilages and the cranium. In the mammals the final evolutionary stages are found. The visceral muscles control the function of the jaw, the hyoid, and the larynx. Most of these muscles are very small, although those of the jaw are heavy, and those which pass posteriorly from the larynx to the sternum are fairly long and slim. If it were not for their embryological origin, and innervation from cranial nerves, they could be considered with the ventral axial group.

\section{E. Appendicular Muscles}

The origin of the appendicular muscles is intimately connected with the origin of the limbs. The entire appendage arises embryologically as two rows of metameric buds, one epaxial and one hypaxial, which grow distally from the mesodermal somites. (See page 158.) This diagrammatic metamerism in origin is found only in the fish. In more specialized vertebrates there is some fusion of the separate cell masses, until in the mammals each appendage arises as a mass of cells which comes from several segments and grows distally to form the limb. The complete homology between the limbs of different vertebrates is shown by the number of segments included and the spinal nerves which grow out into the appendage.

The appendicular muscles begin in all vertebrates as dorsal and ventral groups, and no matter how much torsion or specialization of the limb takes place, this relationship is maintained. After the full development of the limb the muscles fall into two divisions: (1) the extrinsic muscles which have their origin on the girdles, or other bones, and pass out to the limb where they insert; and (2) the intrinsic muscles which lie entirely on the appendage. The latter, even including the muscles of the digits, retain the primitive dorsal and ventral relationships. 
1. Pectoral limb. The extrinsic muscles of the pectoral limb are most primitive in the fish. They are fan-shaped groups of fibers, the apex inserting on the basal cartilages. In the urodeles the dorsal group becomes divided into several distinct muscles which can be homologized with those of the mammal: (a) an anterior sheet, the trapezius, arising on the spines of the vertebrae; (b) a larger, more posterior, muscle, the latissimus dorsi, which draws the forelimb posteriorly and slightly dorsally; (c) the deltoid, which lies between the two former muscles, and is an abductor of the arm; and (d) one or two other dorsal muscles

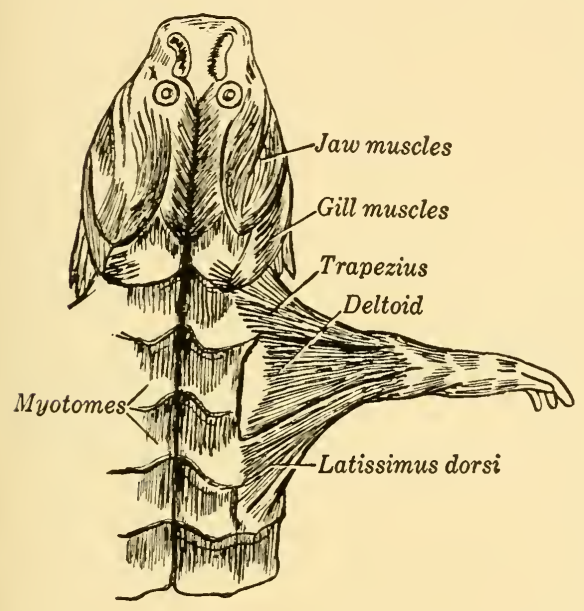

Fig. 106. Head and Shoulder of Necturus (Order Urodela). Note the metameric structure of the axial muscles, the simple arrangement of the extrinsic muscles of the arm, and the modification of the branchial musculature.

which are not clearly homologized. The ventral, pectoralis, muscles are almost undivided in the urodeles. During the course of evolution other muscles are added to the anterior group, and the pectoralis muscle is divided into two or three parts. All the muscles change their shape and relative size, but their position and function are very constant throughout the different classes.

The intrinsic muscles of the anterior limb undergo much greater specialization. In the dogfish these muscles arise on the basal cartilages and pass out to the distal cartilages as a group of separate slips or bands. With the development of tetrapod limbs in the urodeles the muscles take a more definite shape with a division of function. The ventral biceps, which flexes the forearm, is well developed and remains in the higher classes as 
a single muscle. The dorsal muscle group is a large undivided muscle in the lower forms. It becomes variously divided, the larger median part becoming the triceps of the mammal. The forearm of the urodele has the extensor, flexor, and pronator muscles developed. The pronator muscle remains relatively unchanged. The first two groups, however, become greatly divided, accounting for the highly developed use of the hands and fingers. The opposability of the thumb, which was supposed to be limited to man, has been shown to be a gradual evolution within

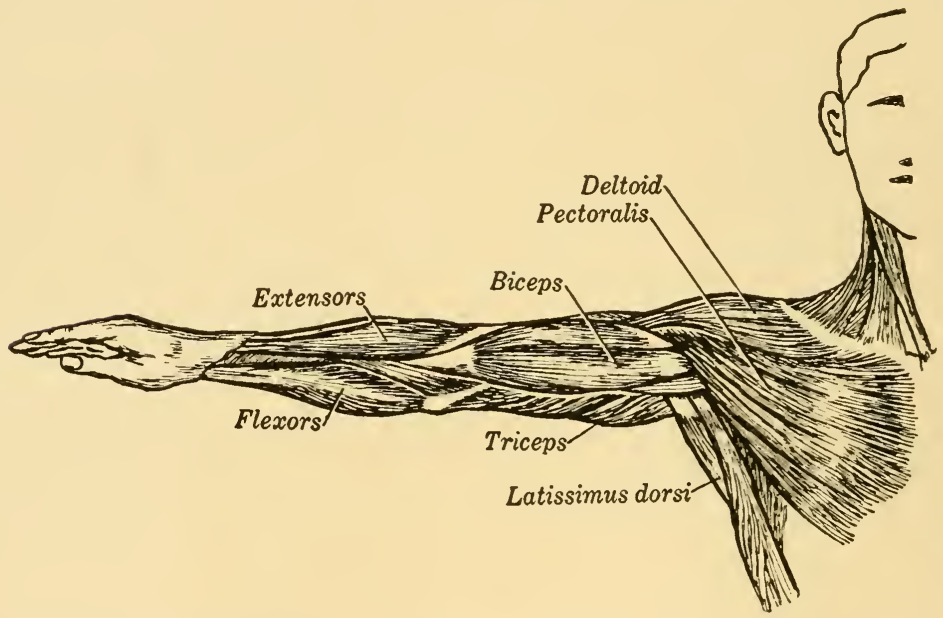

Fig. 107. Arm and Shoulder of Man, Ventral View. The ventral adductor and flexor muscles of the shoulder and upper arm are in normal position. The forearm is pronated to show the dorsal extensors and the ventral flexors of the hand.

the primates, and correlated with structural changes. In the lower primates the long adductors and abductors are tendinous slips from the extensor and flexor series. In the higher primates there is a progressive splitting off of the thumb muscles until in man there is complete independence of the thumb from the other digits. The short digital muscles are too complicated for an elementary discussion.

2. Pelvic limb. The extrinsic muscles of the posterior appendage, like the skeleton, are usually more modified in the land animals than are those of the anterior limb. Beginning with 


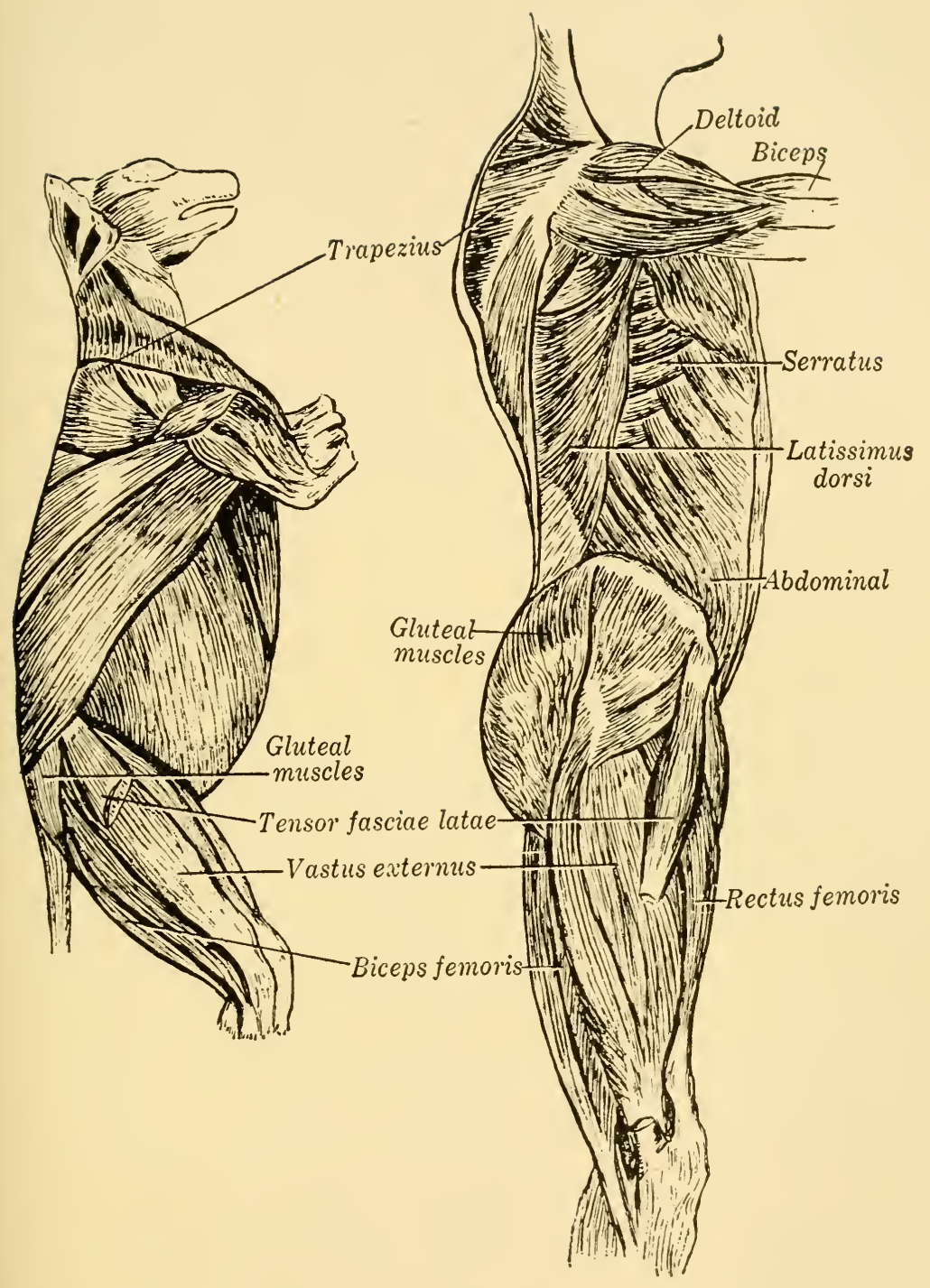

Fig. 108. Musculature of the Cat and Man. Side View. A comparison demonstrates the fundamental similarity, although the size and shape of the muscles have changed. 
the first urodeles the pelvic limbs were used for propulsion. When the knee was drawn toward the anterior part of the body, the torsion placed the dorsal muscles of the thigh in an anterior position. With this change the origins of the pelvic extrinsic muscles became compressed into narrower limits. This is particularly true of man and the higher anthropoids, where a more or less upright walking position has been assumed. In the mammals the rectus femoris on the anterior of the thigh; the adductor magnus which lies laterally; and the large gluteus group which form the buttocks, can be compared in position with the three dorsal muscles of the pectoral limb. In the lumbar region a large muscle mass migrates into a sub-skeletal position, with origins on the ventral side of the transverse processes of the vertebrae. This is a very constant group in all land vertebrates, and is the ilio-psoas group of the mammals. They insert on the femur near its head.

The intrinsic pelvic muscles are also more modified than the pectoral, particularly the more distal ones. In most vertebrates the foot is held in a permanent position of dorso-flexion. Therefore the dorsal (extensor) group increase the flexion of the foot against the shank; and conversely, the ventral (morphologically the flexor) gastrocnemius and other calf muscles pull the foot into a straight line with the limb. Reference should be made to the discussion of the evolution of the leg position. The calf muscles are usually much more powerful than the dorsal group. In the normal walking position of the vertebrate the push of the pelvic limb is gained by straightening the three angles-that between body and thigh, thigh and shank, and the shank and foot. The last gives the final push forward, particularly when the animal is in rapid motion.

\section{F. Integumentary Muscles}

The muscle slips which become attached to the skin arise from all three of the skeletal muscle groups. The integumentary muscles are of small importance when they occur in the reptiles, but they become quite prominent in most of the orders of mammals. A dog or horse can shake his skin without movement of the skeletal muscles. In the armadillo, which is covered with 
dermal plates, these muscles are very large. During the evolution of the primates the integumentary muscles of the body region become of increasingly less importance, until in man they have all but disappeared.

The panniculus carnosus is the major integumentary muscle of the body, and in most mammals is distributed over the entire back and sides of the animal. The panniculus of the lemurs is large, enveloping the body. In the lower monkeys it becomes restricted to a band on either side of the body, centering in the axillary and inguinal regions; and in the anthropoids it is further reduced to small slips which spread out from the axilla to the skin. The appearance of the panniculus in man is only occasional, and it is then limited to thin bands in the axilla or to elongated slips along the sternum.

The integumentary muscles of the neck and face, however, do not undergo the same degeneration. The alligator and some other reptiles have a well developed, transverse band of muscles in the neck, the sphincter colli. In the mammals this muscle divides to form two distinct groups: a neck muscle which retains the original name; and a larger portion, the platysma, which spreads over the face and moves the skin, scalp and ears. The mimetic muscles of the higher primates are derived from the platysma. These become progressively differentiated in the anthropoids and man. In the latter the muscles attached to the ears and scalp have degenerated, the ability to move either of these structures appearing only as an anomaly. The derivatives of the platysma which control the eyes, lips and facial expression, on the other hand, are more developed in man than in other mammals. As with other muscle functions, it is incorrect to say that frowning or smiling is limited to any one genus. The chimpanzee and gorilla have every facial expression known to man, although in many cases the coordinations are less well developed.

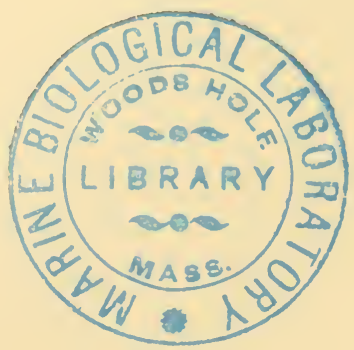




\section{CHAPTER XIII}

\section{DIGESTIVE SYSTEM}

Due to the fact that the respiratory organs, with a few minor exceptions, develop from the digestive tract, the two systems are frequently classified together as the digestive and respiratory system. Functionally, however, the two are widely different, and are technically separate systems. The intimate connection betrreen them is most apparent in the more primitive classes. In Amphioxus, the cyclostomes and the fish the oxygen used in metabolism is dissolved in the water, and both food and oxygen enter through the mouth. The excess water passes out from the pharynx through the gill slits and the food is swallowed. Actually, a limited connection is maintained in all vertebrates, the passages of the digestive and respiratory tracts meeting in the pharynx.

The digestive system includes the functions of food getting, digestion, absorption and defecation. The other nutritive processes include distribution of digested materials by the bloodvascular system, metabolism within the cells, and the excretion of waste products. Distribution and excretion are discussed in later chapters.

The teeth, which function in securing and crushing food materials, were included with the skull, for they belong anatomically with the skeletal system. The specializations of the teeth should be reviewed, for there is a close correlation between tooth structure, the anatomy of the digestive tract, and the type of food taken by the animal.

\section{A. Developaient}

The origin of the digestive tract begins with gastrulation, when the archenteron (primitive gut) is formed. Around this en- 
dodermal tube the mesodermal smooth muscles and mesenteries are added. The digestive tract in its completed form consists of the endodermal lining (the epithelium) and the contracting and strengthening tissues.

The blastopore, formed at the time of gastrulation, is posterior and forms the anus. The mouth later breaks through at the anterior end. The early embryonic tract is a straight tube opening to the exterior at either end. There is an infolding of ectoderm at both mouth and anus, with the result that the anal opening and
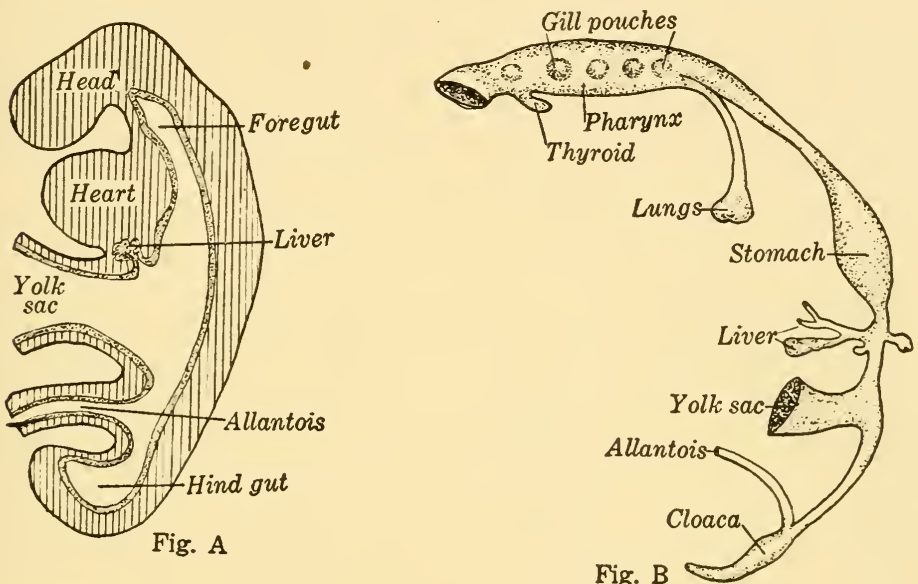

FIG. 109. Development of the Digestive Tract in Man. (A) The early embryo in sagittal section showing the elongation of the tract and its continuity with the yolk sac. (B) A later stage, the tract dissected out.

a part of the mouth cavity are lined with ectoderm. The latter is of particular interest in the development of teeth, which are covered with a layer of enamel secreted by ectodermal cells.

The primitive tube becomes lengthened and modified into more or less sharply defined regions. In the lower vertebrates the mouth cavity, the pharynx and the esophagus are practically continuous. There is more separation between the regions in the reptiles, particularly the alligators and allied members of the order; and in the mammals the three regions are clearly delimited. The stomach is developmentally a pouch-like enlarge- 
ment of the digestive tube. In most vertebrates a constrictor muscle surrounds the posterior end of the esophagus; and, except in the cyclostomes, the outlet of the stomach is guarded by a strong pyloric sphincter. The intestine is the longest portion of the tube. In this region the digestion of foods is continued and the absorption of nutrient materials takes place. The intestine is itself divided into regions. The stomach contents empty into the small intestine. Its anterior portion is the short duodenum into which the liver and pancreatic ducts empty, and is often classified as a separate part of the intestinal tract. In the higher vertebrates the small intestine joins a heavy-walled large intestine or colon which enters the rectum, an enlarged portion of the intestinal tract. In the fish, amphibia, reptiles, birds and monotremes the anus empties into the cloaca, an enlarged sac-like structure receiving the products of the digestive and urinogenital tracts. In the higher mammals the cloaca is divided so that the anus and urinogenital ducts empty separately.

The digestive glands develop as outpocketings from the gut. As in other glands, these vary from single secretory cells to large compound glands supported by masses of connective tissue. Of the latter, the pancreas and liver are the most important.

1. The pancreas develops as one or several dorsal outpocketings from the duodenal region of the intestine. In more primitive animals it is fairly compact, but in the higher vertebrates the pancreas is a large, diffuse glandular structure. The secretory acini drain into numerous ducts which fuse to form one, two, or three large ducts which carry the pancreatic juices to the in-

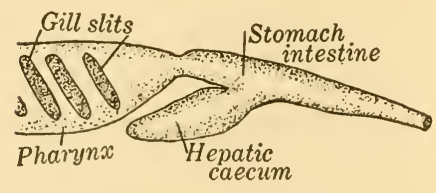

Fig. A Amphioxus

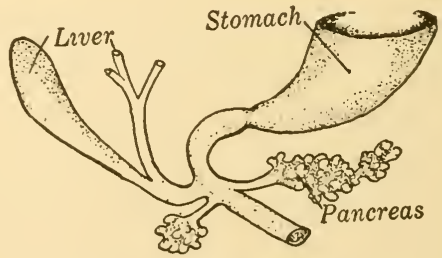

Fig. B Human Embryo

FIG. 110. Development of the Digestive Glands. (A) The stomach-intestine of Amphioxus with the hepatic eaecum. (B) The human tract, showing the endodermal outpocketings which give rise to the liver and pancreas. 
testine. The pancreas also functions as a gland of internal secretion (Chapter XIX).

2. The liver is a rentral outgrowth, almost opposite the pancreatic ducts. The homologous hepatic caecum of Amphioxus is similar in derelopment, but does not become highly glandular. The embryonic hepatic caecum of the vertebrates grows anteriorly, the distal end dividing to form a mass of tubular glands. The liver becomes attached to the diaphragm anterior to the stomach, but the large duct, which is the original outpocketing, empties into the duodenum. A comparison with Amphioxus will explain the relationships in the vertebrate.

The spleen is frequently considered with the digestive system although it is mesodermal in origin, and functionally a part of the vascular system. It has no duct leading from it. Its function is (1) a blood forming organ in the lower vertebrates, and (2) a blood corpuscle destroying organ in the higher.

\section{B. Development of Mesodermal Visceral Structures}

The student should refer to the diagrams of the embryology of Amphioxus and the reptile to understand the relationships of the digestive organs in the body, and the origin of their muscular walls and mesenteries. Recall that as the hypomere of the mesodermal somite grows distally from the notochord it divides into two layers, (1) the somatopleure (the outer or body layer) and (2) the inner visceral layer, or splanchnopleure. The cavity between is the coelomic cavity. As the splanchnopleure pushes in toward the median line the archenteron is separated from the notochord and forced ventrally. The right and left sides of the splanchnopleure enclose the gut and thicken to form the heary wall of smooth muscle with its peritoneal covering. Dorsally the layers join to form the thin, strong mesentery which suspends the gut from the dorsal wall of the body cavity. The thin layer of mesodermal epithelium lining the coelomic carity and covering the viscera is the peritoneum.

It is evident that any outgrowth from the digestive tract will push the mesodermal tissues out with it and become surrounded by connective tissues and peritoneum. Therefore, although structures appear to lie in the coelomic cavity, they are technically 
outside the peritoneum. Glands are usually suspended by long mesenteries; the kidneys are rarely completely surrounded by the peritoneum on the dorsal side; and the urinary bladder may have but slight contact with the peritoneal lining.

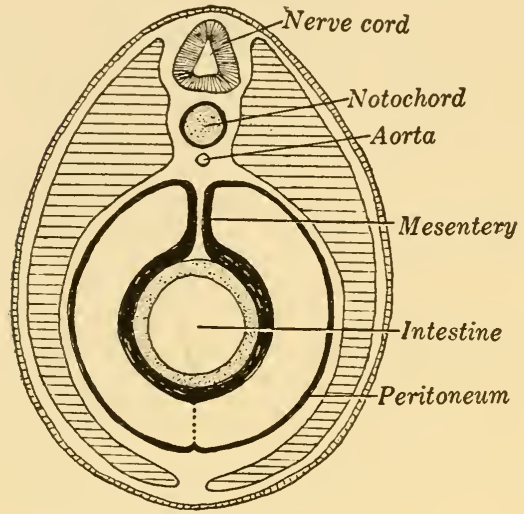

Fig. A

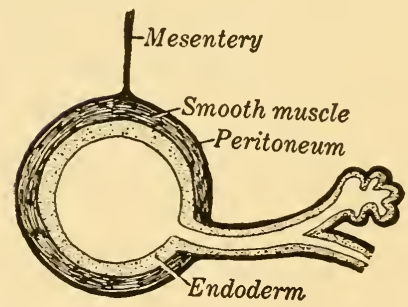

Fig. C

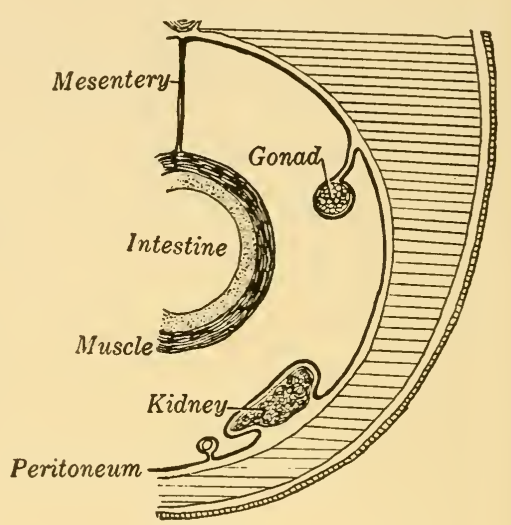

Fig. $B$

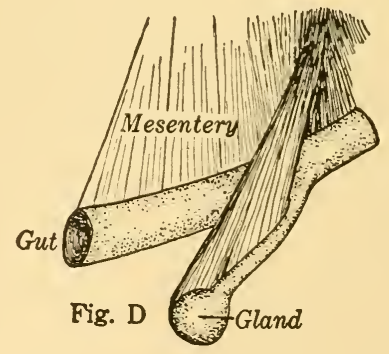

FIG. 111. Development of the Mesenteries and Peritoneum. (A) Shows the embryo in cross section showing the relationship between the peritoneum, mesentery and smooth muscles of the gut. (B) The same structures at a later stage, with glands developing in a retro-peritoneal position. The extensions of the mesentery are shown in cross section (C) and in side view (D).

When the mesentery develops in the embryo it is connected ventrally as well as dorsally; but except for a few isolated regions the ventral portion is soon lost. The dorsal mesentery (or simply "mesentery") becomes very complex. The lengthening of the intestine stretches it along the ventral margin so that it be- 
comes thrown into wide folds or ruffles, while the attachment along the dorsal median line remains only the length of the animal's body. The growth of glands further complicates its structure. Each, as it grows out, pulls the mesentery with it, so that the adult has numerous small supporting mesenteric folds. In the mammals a ventral pocket of the mesentery grows posteriorly from the stomach, forming an apron which covers the viscera. This is the fat laden omentum.

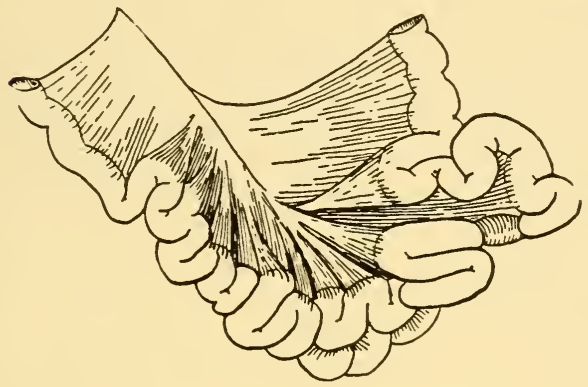

FIg. 112. Mesentery of a Cat. As the intestine grows and coils the rentral margin of the mesentery is thrown into folds.

\section{Modifications of the Digestive Tract}

The digestive tract as a whole has undergone so many modifications during the course of evolution that recent animals give slight evidence of the evolutionary history. The functions of the different regions of the tract remain very constant, but the structural variations are almost limitless. Within a family or order the specializations are usually not great; but there is little progressive evolution from class to class.

There is an excellent, although not perfect, correlation between the food habits of the animal and the length and shape of the intestine. The herbivores have a complex stomach, a long intestine, and usually a large caecum. In the carnivores the stomach and intestine are simpler in structure, the tract being decidedly shorter. This correlation is also found in a single order (for example the bats), in which the fruit eating varieties have longer intestines than the carnivorous ones. The blood eating bats have the shortest intestine known among the mammals. Even in a species there may be variations in correlation 
with diet, the Eskimos having a shorter intestine than the white races.

1. Mouth axd Pharryx. In the fish and primitive amphibia the mouth cavity and the pharynx are continuous, the latter being the larger of the two regions. With the disappearance of
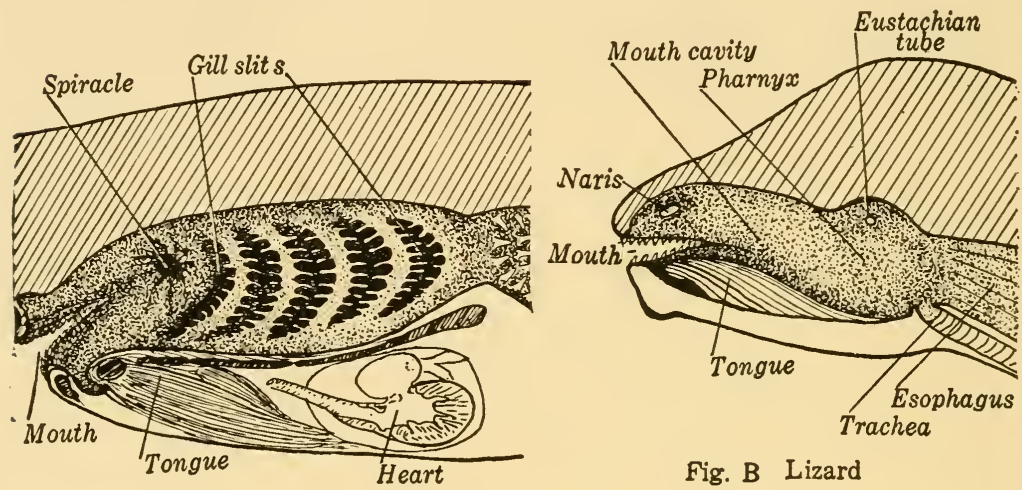

Fig. A Dogfish

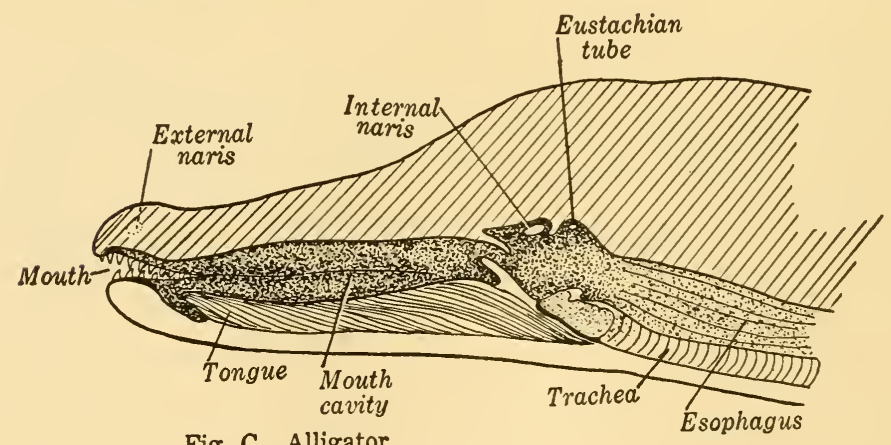

Fig. C Alligator

Fig. B Lizard

FIG. 113. Comparative Anatomy of the Mouth Cavity and Pharynx. In the Dogfish the cavity of the mouth and pharynx is continuous. A slight separation occurs in the lizard, and in the alligator folds of tissue separate the two.

the gill slits in the adult, which occurs in the more specialized amphibia and all the amniotes, the pharynx tends to be relatively smaller, and in the higher amniotes is separated from the mouth by a fold of tissue. In the mammals this is the soft palate.

The mouth is distinguished by the jaws with teeth. In the fish 
the skin covering the jaws forms the anterior margin of the mouth, but in the amphibia lips develop as folds of tissıe anterior to the gums of the jaws. The lips and cheeks are well developed in most reptiles, the Chelonia being an exception, and are highly specialized in the mammals. The birds lost the lips with the loss of teeth and the development of the beak.

The tongue, a ventral muscular organ, is found in most fish as a flat thickening above the hyoid apparatus. In the higher classes of vertebrates it becomes more specialized as to size and distensibility. The frog's tongue which is attached at the anterior margin and can be turned outward as a trap for insects, and the forked tongue of many reptiles are well known specializations. Particularly in the mammal, the tongue assists in swallowing and contains most of the taste buds.

The glands of the mouth are limited to simple mucous cells in the fish. More complex glands appear in the land vertebrates and have the double function of moistening the lips, mouth and food, thus assisting in getting and swallowing food; and secreting various enzymes. The glands are classified by their position as (1) labial, (2) buccal, (3) lingual (tongue) or sublingual, (4) mandibular or (5) submaxillary. The secretion varies widely in composition. The salivary secretion of the mammals is either serous or mucous. The poison of reptiles is a toxic secretion from specialized mouth glands, as is the gelatinous material used in the building of many birds' nests.

The gill slits are located in the pharynx, which is large in the fish. In the land vertebrates the posterior slits disappear, the most anterior remaining as the Eustachian tube and middle ear. This opens into the pharynx.

In mammals and some reptiles the pharynx is the common cavity for swallowing and breathing. The nasal cavities have been cut off from the mouth and enter the pharynx. The opening to the lungs is at the posterior end of the pharynx, and the mechanism which controls breathing and swallowing is located in this region.

2. Esophagus and Stomach. The esophagus is a straight tube leading from the pharynx to the stomach. Its length varies with neck length rather than any functional adaptation. The diameter tends to be larger in animals which swallow their food whole, 
as the reptiles and carnivorous mammals. The sphincter which separates the esophagus from the stomach is poorly developed in amphibia and reptiles, making regurgitation of food a simple matter; but in mammals the opening is small and the constrictor muscles are more completely under the control of the nervous system.

The stomach breaks the larger masses of food, and begins the digestion of proteins. The acids and digestive enzymes de-

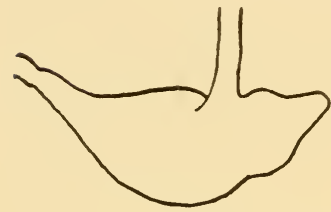

A. Rat

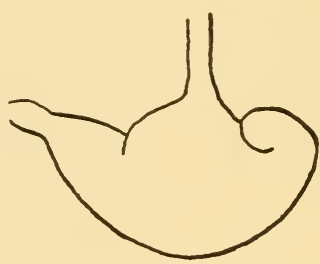

B. Pig

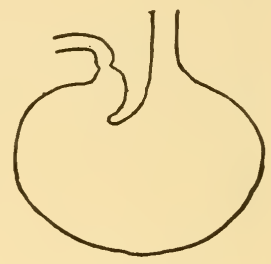

C. Dog

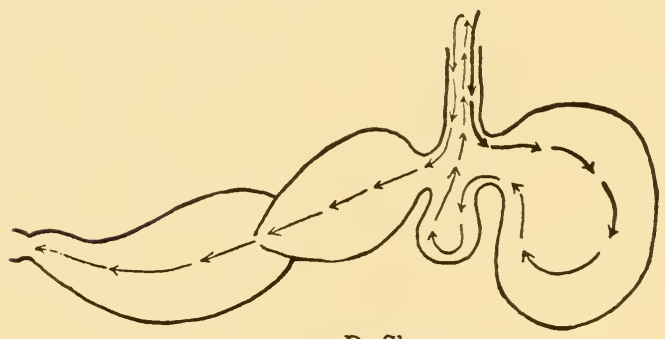

D. Sheep

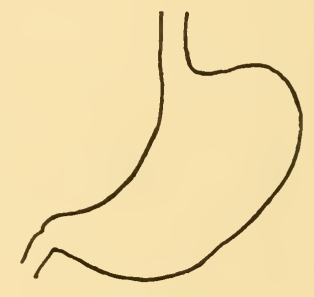

E. Human

FIG. 114. Structure of the Mammalian Stomach. The stomach is structurally simple in most mammals. Herbivorous mammals show more extreme modification. In (D), the sheep, the arrows show the course of food in a cud-chewing animal.

stroy the connective tissues which bind together the cells of muscles, and hasten the stomach and intestinal digestion. In correlation with this function the walls are generally thick and muscular, churning the mass until it is mixed with the digestive fluicks.

Anatomically, the simplest stomach is a slight enlargement, and in the cyclostomes is continuous with the intestine. This stomach-intestine is sufficient to digest the finely ground particles of flesh which are swallowed. In the vertebrates generally the 
carnivorous ones have a simpler stomach than those with herbivorous habits. Man is omnivorous in habit, but has a simple, pouch-like stomach, the anterior border being much shorter than the posterior. The cud chewing herbivores have a stomach divided into four distinct regions, the anterior acting as a reservoir for the unchewed herbs which are later regurgitated and chewed while the animal is at rest. In most vertebrates the stomach is terminated by the strong pyloric sphincter.

3. Intestine. The cyclostomes have a straight intestinal tube, not divided into anatomical regions. The dogfish intestine con-
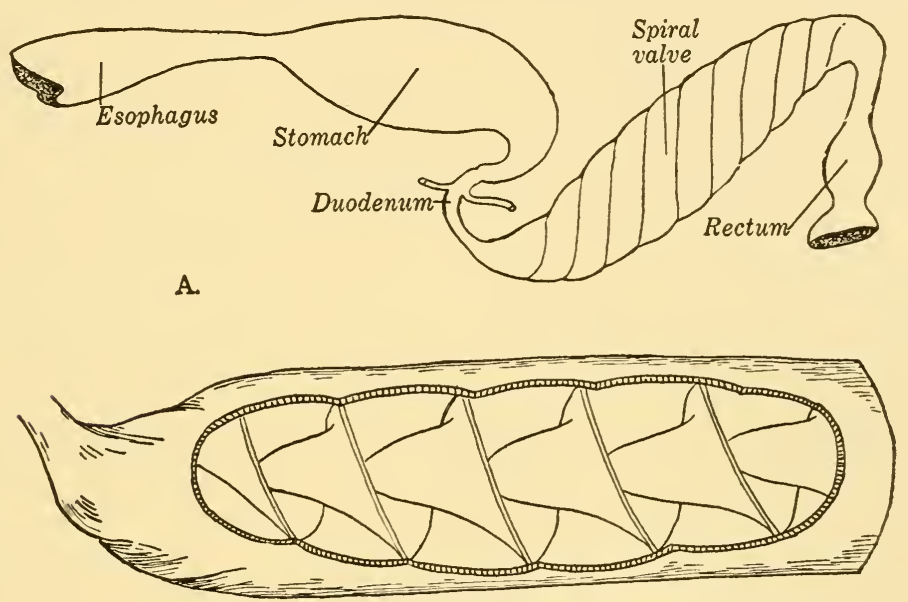

B.

FIG. 115. Digestive Tract of a Dogfish. (A) Shows the entire tract. (B) Shows the structure of the spiral ralve which greatly increases the absorptive surface of the small intestine.

sists of a short duodenum, a greatly enlarged spiral v'alve, and a short rectal region. The valve arises as a spiral infolding of the gut wall, strongly supported by connective tissues and corered with the digestive and absorbing epithelium. The structure gives a greatly increased functional surface. The spiral valve was carried over in modified form to the more primitive bony fish, including the Crossopterygii and a few of the Teleostei. In the higher vertebrates a coiling of the intestine gives the same functional result as the spiral value.

The mammalian intestine is distinctly divided into a thin- 
walled small intestine and a thick-walled large intestine or colon. The small intestine is not continuous with the large, but enters it at approximately a right angle some distance from its anterior, blind end. This blind pouch, the caecum, is large in the herbivores and a functional part of the intestine, for its bacterial con-

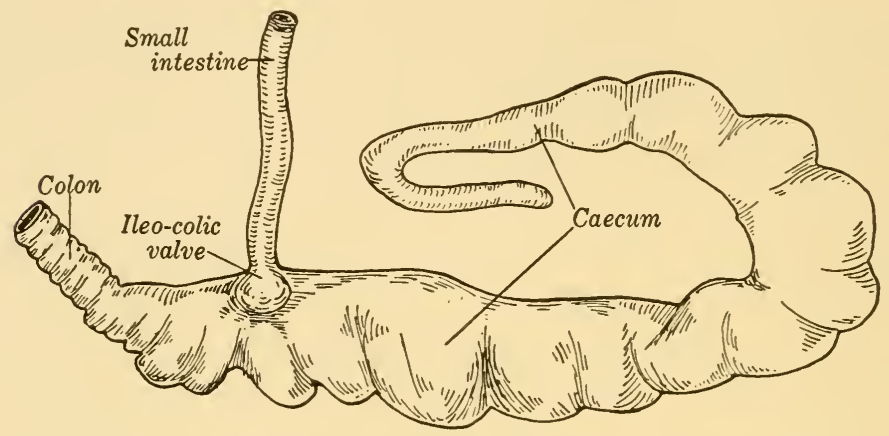

FIG. 116. Caecum of a Rabbit. Digestive caeca are found in many groups of vertebrates. In the mammals it usually is found at the ileo-colic juncture. In some herbirores the caecum contains cellulose-breaking bacteria.

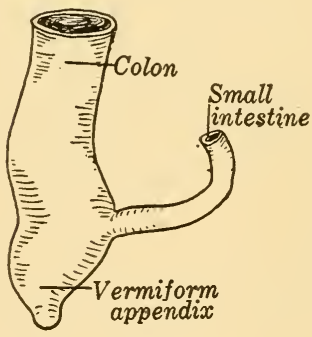

A. 2 months Foetus

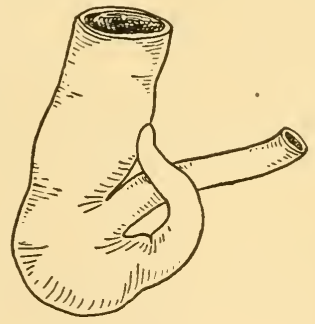

B. New born

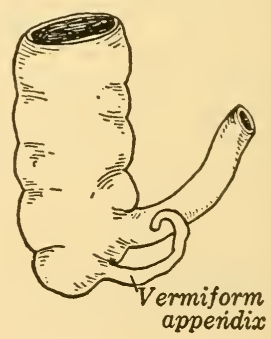

C. 4 Years

FIG. 117. Development of the Vermiform Appendix in Man. The digestive caecum degenerates in man, and is loft as a small appendix in the adult. (After Kiollman).

tent assists in breaking down the cellulose of plants. The caecum of the human is short and terminated by a small vermiform appendage. The evidence from embryology and the comparative anatomy of the primates indicates that the appendix is the vestigial remains of a once large caecum.

The rectum is the terminal enlarged portion of the intestine, 
and is a reservoir for the feces. The rectum terminates at the anus which is closed by the sphincter ani.

4. ClOACA. The cloaca develops from the enlarged part of the hind gut from which the allantois outpockets, and is partly lined by inpocketed ectoderm. With a few exceptions (as in some cyclostomes and fish) a cloaca is present in all vertebrate groups except the marsupials and placentals. In the last two sub-classes the cloaca is present in the embryo until a horizontal septum cuts the dorsal from the ventral part and separates the anus from the urinogenital openings.

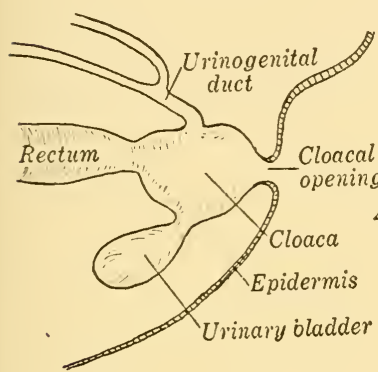

Fig. A

Adult Reptile

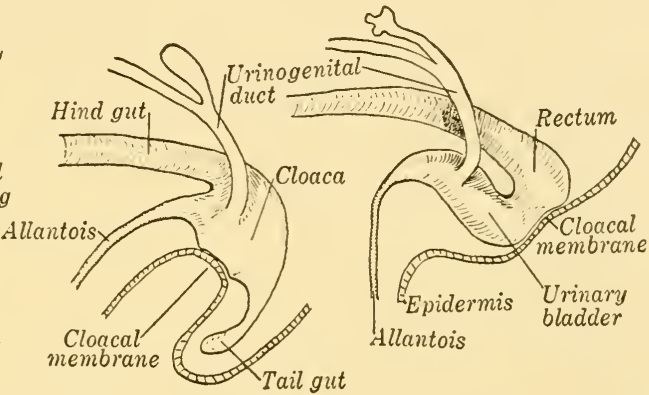

Fig. C

Fig. B

Mammal (embryo)

FIG. 118. Structure of the Cloaca. The cloaca remains undivided in the majority of vertebrates, (A). In the mammals ( $B$ and $C$ ) a horizontal constriction separates the dorsal digestive tract from the ventral urinary bladder.

\section{The Human Digestive Tract}

The human mouth cavity and esophagus are typically mammalian. The stomach is a simple sac slightly divided into two regions: the cardiac or larger portion; and the pyloric region which lies toward the right side and empties into the duodenum.

The small intestine averages about twenty-two feet in length, although variations of from sixteen to thirty-one feet have been noted. It is divided into the duodenum, about a foot long; the jejunum, the anterior two-fifths of the remainder and the ileum, 
the posterior three-fifths. The small intestine enters the colon at the ileo-colic valve.

The colon is nearly five feet in length, the ileo-colic valve which constricts the entrance of the ileum being about three inches from its blind end. The vermiform appendix averages a few inches in length. Its lumen, which opens into the end of the caecum, varies widely. At times the point of division between appendix and caecum is hardly determinable. The juncture of the ileum and colon, and the position of the appendix, is normally in the lower right side. From this point the colon passes anteriorly as the ascending colon; bends and lies across the body as the transverse colon; and on the left side near the spleen it bends posteriorly at the splenic flexure, and passes posteriorly as the descending colon. In the posterior part of the body cavity the colon makes a curved turn, the sigmoid flexure, and then enters the rectum. The latter is slightly less than six inches in length. 


\section{CHAPTER XIV}

\section{RESPIRATORY SISTEM}

Respiration is the process of taking in oxygen for metabolic processes, and giving off the excess carbon dioxide from the blood. In all vertebrates respiration is divided into (1) external respiration, the mechanism for getting oxygen from the surrounding medium; and (2) internal respiration, the passage of oxygen from the blood across the membrane of each cell of the body. Anatomically, respiration is usually limited to the organs which take the oxygen from the animal's environment.

Any moist, vascular membrane may serve as an organ of respiration. In the vertebrates there are a number of such respiratory membranes in isolated groups, but the most widely distributed organs are gills in the water-living groups, and lungs in the land vertebrates. Some amphibia, however, lack both lungs and gills, and other groups have various organs modified for respiration. Gills are the most primitive respiratory organs of vertebrates.

\section{A. Gills}

1. Origin of the Gill Slits. An outline of the development of gill slits was given on page 4 . The embryonic pharynx is relatively much larger than that of the adult, the difference being much more marked in the higher groups of vertebrates. In Amphioxus there is a great number of the endodermal pharyngeal pockets, each of which becomes secondarily divided into two. In the cyclostomes the number is reduced to fourteen for the primitive group, and seven for the lampreys. The latter number of gill slits was carried over to the primitive sharks, and in several living genera seven or six are found. In the dogfish the number is reduced to the definitive vertebrate number, five, with the 
first anterior pouch forming the spiracle. This structure in the dogfish carries a rudimentary gill on its posterior surface. In most of the higher fish the spiracle is completely lost, although it is retained by the crossopterygian fish, and from them inherited by the amphibia as the Eustachian tube and middle ear.

The embryos of all vertebrates develop the gill pouches and, with doubtful exceptions, these break through to the outside of
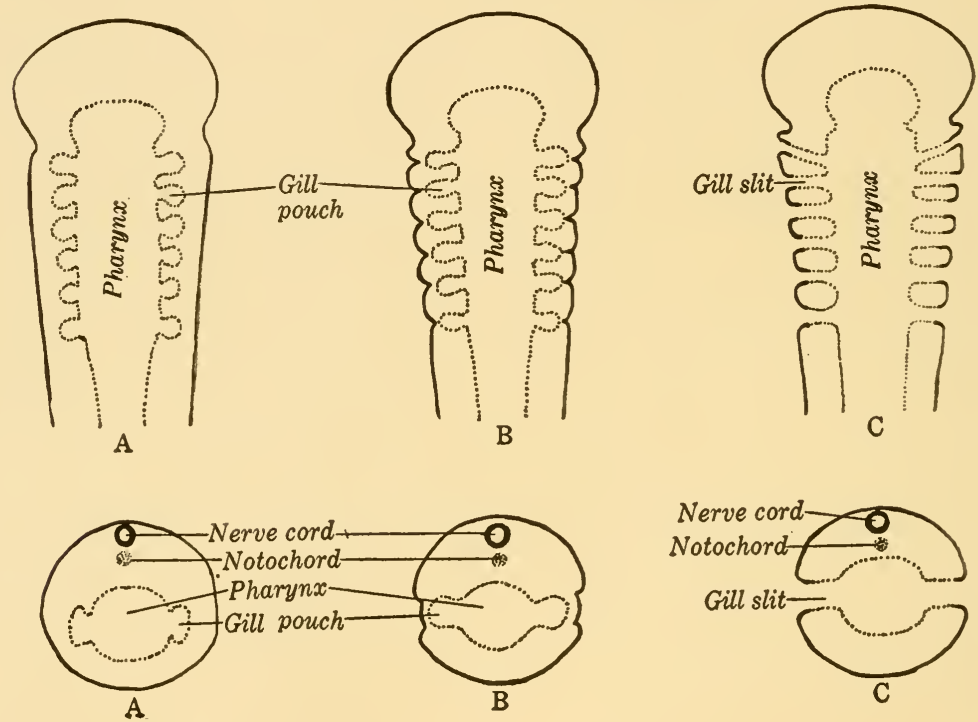

FIG. 119. Embryology of the Gill Slits (Diagrammatic). Drawings (A), (B), and (C) are in horizontal section. Cross sections of similar stages are placed immediately below. The gill pouches begin as outpocketings of the pharynx and meet invaginations of the ectoderm.

the animal as gill slits. Gills develop only in the cyclostomes, fish and amphibia, and are never found in amniote embryos. The septum between each gill pouch is supported by a gill bar, or branchial cartilage. These branchial cartilages become variously modified in the vertebrates. (See Visceral Skeleton, page 143.)

A horizontal section through the human embryonic pharynx illustrates the typical vertebrate condition. The five paired branchial evaginations extend laterally from the pharynx. With the exception of the first, these pouches are never functional; but from each pouch develops a proliferation of cells which be- 
comes differentiated into a glandular structure. These glands, which will be discussed in Chapter XIX, are ductless and function as glands of internal secretion. The derivatives of the gill pouches are:

I, the paired Eustachian tubes;

II, the palatine tonsils, which retain their position in the pharynx throughout life;

III, the thymus gland, the two halves of which unite and migrate posteriorly, the adult gland being located near the bifurcation of the trachea;

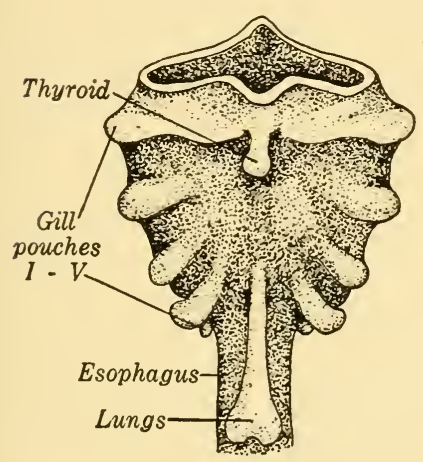

A Ventral View

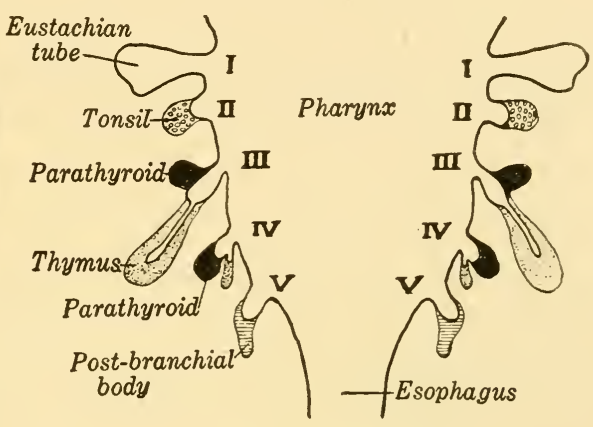

B. Longitudinal Section

FIg. 120. Derivatives of the Human Pharynx. The ventral view (A) shows the branchial pouches, the thyroid and lungs. The diagrammatic horizontal section shows the glandular derivatives of pouches I to $\mathrm{V}$.

III and IV, develop two pairs of small glands, the parathyroids, which lie against the thyroid cartilage of the larynx embedded in thyroid tissue; and

$\mathrm{V}$, the postbranchial bodies.

The development of the middle ear and Eustachian tube from the first pouch is a direct change of function of a structure. The pharyngeal glands are derivatives of the pouches, not modifications of the structures themselves.

2. Internal Gilis. Internal gills develop within the gill slits of the early chordates, the fish, and the larvae of most amphibia. In several amphibian groups they are carried over to the adult. 


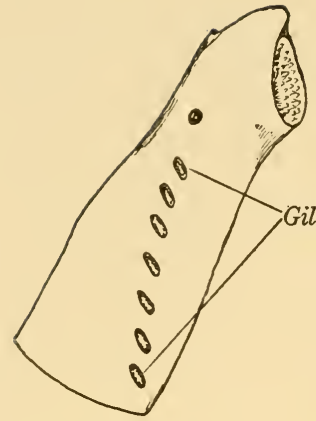

A. Cyclostome

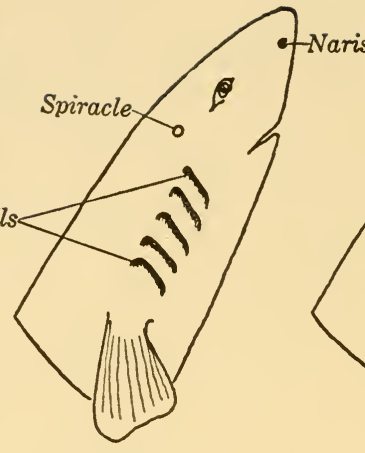

B. Shark

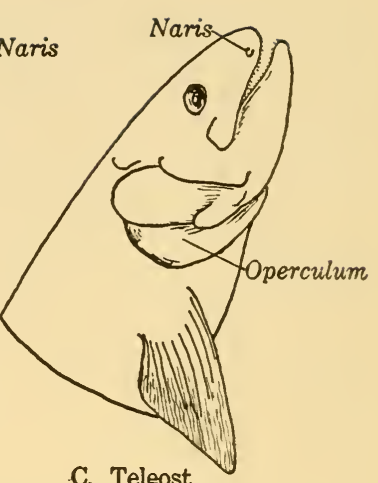

C. Teleost

Fia. 121. External Appearance of the Gill Region of Cyclostomes and Fish. In fish above the Elasmobranchii the gills are covered with a bony operculum. Compare Fig. 122.

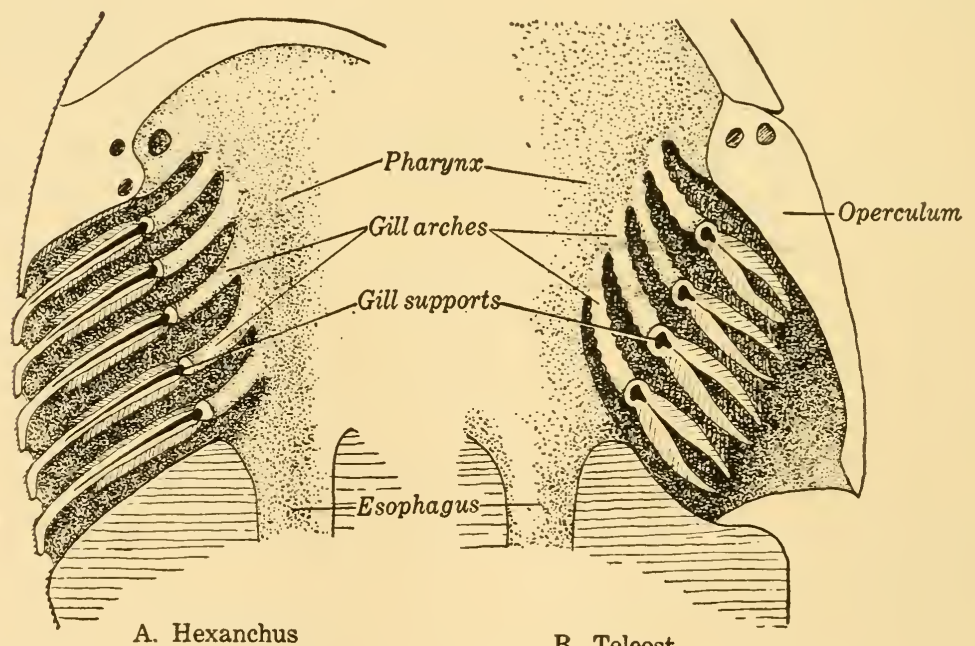

(primitive shark)

B. Teleost

FIG. 122. Horizontal Sections of the Pharynx. (A) Primitive Elasmobranch, showing the six gill slits, and (B) a typical Teleost with five gill slits covered by an operculum. In the Elasmobranch the septa extend to the outside. 
These internal gills may be covered entirely by endoderm, or by both endoderm and ectoderm.

In Amphioxus, it will be recalled, the afferent branchials break into capillaries in the gills, and as the blood stream slows down the exchange of gases takes place. The capillaries then re-collect into efferent branchials which pass into the dorsal aortae. This is essentially the same condition which prevails throughout the gill-breathing fish and amphibia. In the amniotes the relationships of the branchial arteries are the same, although they do not break into capillaries.

In the dogfish the gill is a paired structure, developing along the inner surface of the gill bar. The two halves, or hemi-branchs, of each gill lie in adjacent gill slits. Therefore the gills which lie in a gill opening belong to different branchial cartilages: one half belongs to the anterior bar, the other half to the next posterior gill support.

This relationship of the hemi-branchs is carried over to the higher groups. The greatest change which occurs in the more specialized fish is the modification of the septa separating the gills from each other. The septa of the dogfish extend from the pharynx to the outside of the animal, and the septum is covered externally by the body skin. Each gill slit, in other words,

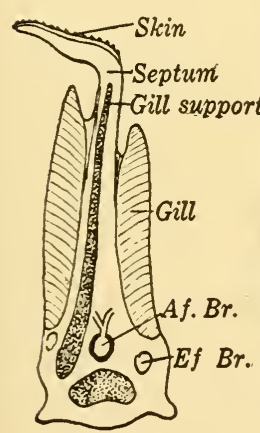
A. Dogfish
B. Sturgeon
(Chondrostei)

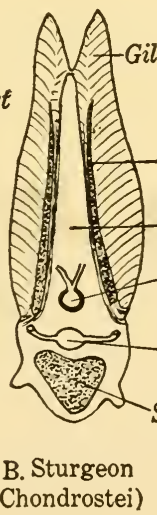

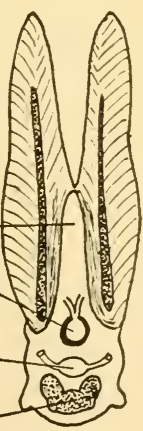

C. Gar pike (Holostei)

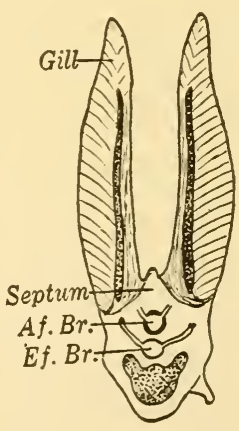

D Typical Teleost

FIG. 123. Cross Sections of Gills, showing reduction of the septum. 
is a simple opening through the body wall, connecting the pharynx with the exterior. In the Chondrostei (page 40) a bony covering, the operculum, shields the gill slits. In this group there is a degeneration of the distal part of the septum, so that the body covering of skin is no longer present in the region. The reduction of the septa proceeds further in the Holostei, and in the Teleostei the septa are reduced to a small fleshy membrane along the gill bar. This modification obscures the primitive and embryological origin of the gill slits. It gives, however, greater function to the gills, for they are protected by the operculum and the gill filaments are in freer contact with the water.

3. External Gills. External gills make their first appearance in the Crossopterygian and Dipnoan fish. In the larvae of these animals the gills develop on the outer side of the branchial septa. Their position is shown in the drawing on page 46. As the internal gills develop both types of gill are present for a time, and then the external disappear. Almost the same condition is found in the amphibia, the external gills being wholly ectodermal and appearing before the gill pouches break through. They are very prominent structures in the early larvae. In Crodeles with persistent gills both sets remain; but in the salamanders and anurans the external gills disappear when the internal gills and operculum develop. The operculum is a fleshy membrane not homologous with that of the fish. At metamorphosis the lungs replace the gills. Exceptions to this rule, due to accessory respiratory structures, will be discussed later.

The vascular supply of the external gills comes from the branchial arches by branches which pass out into the gills. With the disappearance of the gills the blood follows the more primitive course, circling through the gill support from the ventral to the dorsal aorta. The branchial arteries vary considerably in the different groups of amphibia, in correlation with the reduction in number of gills and the branchial arches. The second and fifth branchial pouches are rarely functional in amphibia.

\section{B. LUNGS}

Lungs are the functional respiratory organs of land animals. Their internal position keeps the epithelium moist. Like the gill 
slits they are a derivative of the digestive tract. In the fish, however, the connection between the two systems is closer. Water, the environmental medium, is drawn into the mouth with the food, and the dissolved oxygen of the water is taken up as it passes over the gills in its course to the outside. With the development of lungs a certain degree of separation begins between the digestive and respiratory tracts, although the environmental oxygen is drawn into the pharynx and passed into the respiratory tissues.

1. Evolution of the Luxgs. The first homologue of the lungs appears in the chondrostean fishes. In the sturgeon (page 41) a dorsal outpocketing of the gut develops in the region betrieen the pharynx and the esophagus. The distal portion of the diverticulum enlarges to form a swim-bladder, its duct maintaining a free connection with the gut throughout life. The organ is slightly vascular, the supply coming from the sixth branchial artery. In Amia (a holostean fish) the swim-bladder is highly vascular, and the connection with the gut remains in the adult.

From the simple, slightly vascular condition, the swim-bladder evolved in two directions: (1) toward the condition found in the teleost fishes, where the organ is non-vascular, loses its connection with the gut, and remains as a dorsal sac solely hydrostatic in function; and (2) toward a ventral position, the duct open, and the function respiratory. The latter condition is found in the Crossopterygii and Dipnoi which have a lung-like swim-bladder. Polypterus (a recent crossopterygian fish) has a swim-bladder which develops as a ventral outpocketing of the gut. The structure becomes bi-lobed and highly vascular, the blood supply coming from the sixth branchial as in the other fish. During the dry periods, or when the river waters become muddy, the fish gulps air and carries on respiration with the bladder. The Dipnoi (page 45) parallel the amphibia in development and have a swim-bladder which is functionally a lung. In the South American genus the structure is so specialized that the animal is unable to survive if kept submerged in water.

When the amphibia evolved the lung relationships of the crossopterygian ancestors were retained. The lung develops as a ventral outpocketing of the gut at the posterior end of the pharynx, and soon divides into two distinct lobes. The opening 


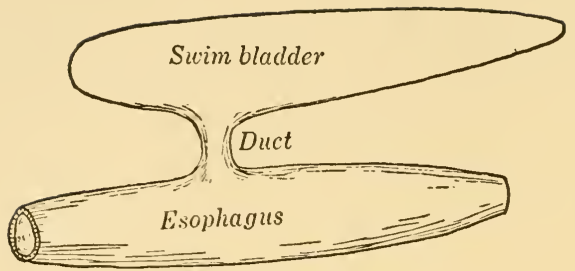

A. Sturgeon (Chondrostei)

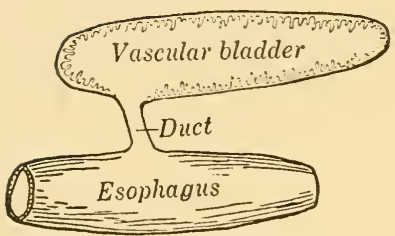

B. Gar pike (Holostei)

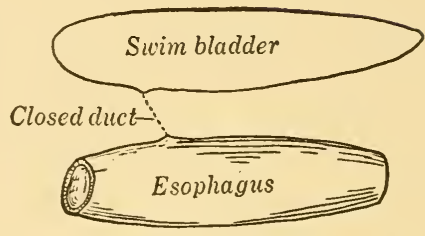

C. Typical Teleost

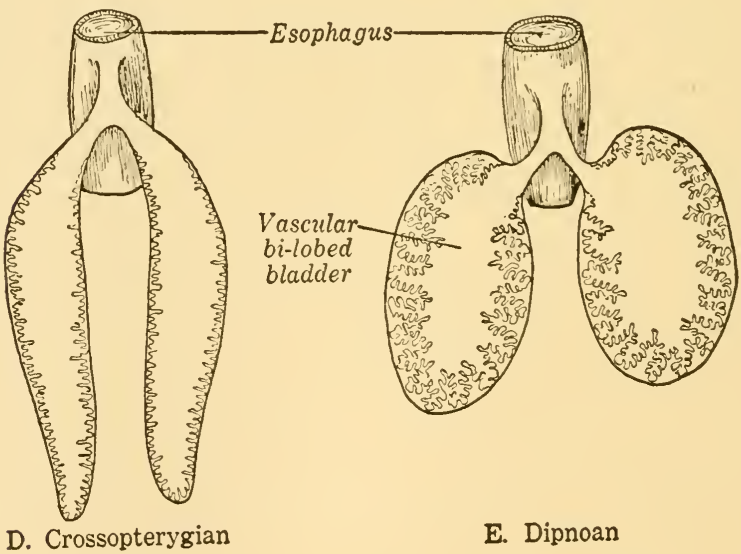

FIG. 124. Comparative Anatomy of the Swim Bladder. Observe the evolution toward a closed hydrostatic bladder in the Teleostei, and toward a ventral lung in the Dipnoi. (Adapted from Dean). 
from the pharynx is the larynx; the undivided upper portion of the tube is the trachea; and the two tubes into which the trachea divides are the bronchi. Urodele lungs are simple expansions of each bronchus, and are less lobulated than those of the dipnoan fish. During the larval period of development all amphibia have the gill and lung combination for respiration, and this double system is retained by many urodeles.

The homology of the swim-bladder is shown by (1) the comparative anatomy and function of the two structures; $(2)$ by the mode of embryological development, which is practically identical in the crossopterygian and dipnoan fish and the land vertebrates; and (3) the vascular supply. In all cases the supply is from the sixth aortic artery. It is only in the mammals that there is a complete separation of the pulmonary arteries, which arise as branches of the sixth branchial, and the ventral aorta.

2. Comparative Anatomy of the Lungs. Beginning with the unlobulated, elongated lungs of the primitive urodeles, the lungs evolved toward more complex divisions. Salamanders have the anterior part of the lungs divided into shallow pockets or alveoli, giving the lungs a honeycomb appearance. Each alveolus opens wide into the central cavity, but the low septa add to the absorptive area of the epithelium.

Frogs show a definite advance over the salamanders. The lungs are more rounded in correlation with their body shape, and the alveoli are deeper and cover the entire inner surface of the lungs. The toads, which live in drier areas, have lungs of the frog pattern; but the dividing septa close partly over the alveoli, giving them an almost spherical shape with a circular opening into the central lung cavity.

Sphenodon (a New Zealand primitive reptile) and some turtles have lungs which are no better developed than those of a frog. A definite advance in lung structure appears in the lizards. During development the alveoli move further from the central cavity, and are connected with it by small ducts, homologous with the bronchioles of the birds and mammals.

Alligators and crocodiles have the best developed lungs among the reptiles. As the lungs develop each bronchus divides into several branches, thus cutting the lung into several lobes, each with its own cavity. From the air cavity of each lobe numerous 
bronchioles are given off, each ending in a spherical alveolus.

A progressive degeneration of one lung is found in the lizards and snakes. In the primitive condition the lungs are bilaterally symmetrical; but in the elongated lizards one lung is definitely smaller than the other, and in the snakes one is either vestigial or lacking.

Although the lungs of flying birds are not so greatly lobulated

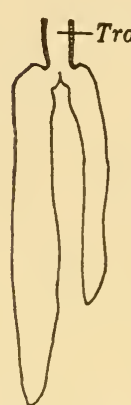

A. Necturus

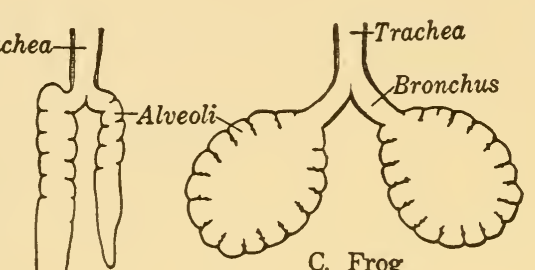

C. Frog

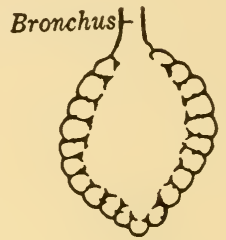

D. Toad

B.

Salamander
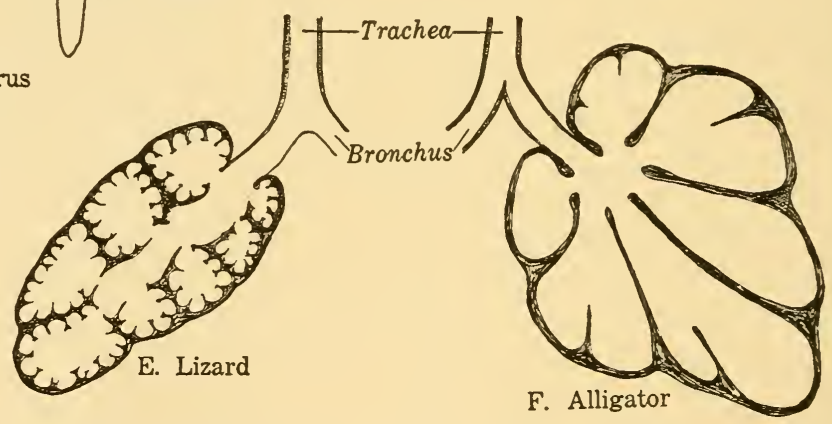

FIg. 125. Comparative Anatomy of the Lungs. There is a steady increase in number of bronchi and alv eoli. Each division of the Alligator lung is equivalent to a complete lizard lung.

as those of the mammals, their lungs are specialized for more efficiency. The organ is not of unusual size, but greater efficiency in gas exchange is effected by three pairs of air sacs. These sacs are continuations of bronchial ducts, which expand after passing through the lungs to form membranous sacs in the pleural, abdominal, and wing regions. As the air is drawn into the lungs it passes across the alveoli and into the sacs, greatly decreasing the specific gravity of the animal. The ramifications of the sacs 
into the humeri can be demonstrated by cutting the humerus of a pigeon and blowing smoke into the trachea. In addition to lightening the animal, the mechanism tends to lessen the total amount of air left in the lungs. Therefore the air, which is normally only slightly depleted of its oxygen content, passes across the vascular ducts at inspiration and expiration as it is forced into and from the air sacs.

Mammals have complexly lobulated lungs, with innumerable bronchioles and terminal alveoli. The structures have evolved so far that there is no sign of a central air cavity in any of the lobes. The idea of structure can best be gained by a description of the development of mammalian lungs. The human lungs begin in embryos of about 3.5 millimeters as a ventral groove along
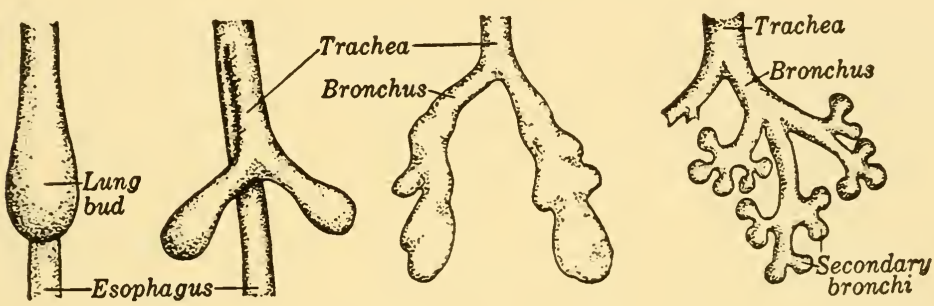

Fig. 126. Embryology of the Human Lung. The secondary bronchi divide minutely, each ending in an alveolus. (Adapted from His).

the esophagus. The posterior portion of the groove soon becomes separated from the floor of the gut and forms a blind tube opening into the pharynx. This opening is retained as the glottis. The posterior end divides into two lobes, the distal ends becoming vesicular. At this stage there is a trachea and two bronchi. The latter continue to divide, the early divisions forming the larger lobes of the lung. The bronchi continue to divide into smaller and smaller tubes until microscopic bronchioles are formed. Each bronchiole terminates in a spheroidal alveolus. The endodermal organ is enveloped and bound together by mesodermal tissues. Capillaries from the pulmonary arteries ramify around the smaller tubes.

3. Mechanisur of Breathixg. Most amphibian tadpoles take the air into the mouth and force it into the trachea by com- 
pressing the mouth and raising the tongue. This method is only slightly modified in the primitive lung breathing urodeles; but in the frogs the air is drawn into the mouth cavity through the nares which open immediately behind the teeth, and the air is forced through the glottis with the floor of the mouth. Reptiles, with the exception of the Chelonia, and Crocodilia, depend almost entirely upon the ribs as the respiratory mechanism. The contraction of the costal muscles forces the air from the lungs; and as the muscles relax the glottis opens and atmospheric pressure fills the lungs again. The crocodiles and alligators develop a muscular diaphragm which separates the pleural cavities from the abdominal cavity and is an aid in respiration.

The diaphragm is highly developed in the mammals. It is a dome-shaped band of muscle, the convex side being toward the pleural cavities. As the costal muscles contract, pulling the ribs downward and inward, the diaphragm relaxes and the air is forced from the lungs. The reverse process greatly increases the size of the chest, and air rushes in when the glottis is openerl. In the human, at least, diaphragmatic breathing has largely replaced costal breathing.

4. Development of the Laryx. The larynx is the supporting mechanism of the glottis and upper end of the trachea. During evolution the development of the larynx has been correlated with the development of cartilages supporting the trachea and bronchi. The cartilages of the trachea are incomplete dorsally. Those surrounding the lower portion where the trachea bifurcates, and also those surrounding the bronchi, are complete annular cartilages. The tracheal and laryngeal cartilages differ in origin, the latter being modified from the visceral (branchial) cartilages of the embryo. It will be recalled that the first and second visceral arches form the jaws and hyoid apparatus in all vertebrates above the cyclostomes, the posterior (branchial) cartilages supporting the gills. In the dipnoan and crossopterygian fish, where the swim bladder is modified into a respiratory organ, the opening of the trachea is unsupported; but with the reduction of the number of gill openings and gill cartilages in the amphibia, the larynx begins its development. The seventh visceral (fifth branchial) cartilages are the first to become modified. These form the arytenoid cartilages on either side of the glottis. 
The next adrance is in the reptiles. The arytenoids are of increased size, and the sixth visceral arches form an epiglottis. The paired cartilages migrate toward the ventral side of the glottis and unite to form the epiglottis which curves over the opening of the glottis. In addition, the third visceral (first branchial) cartilages fuse with the hyoid bone, forming the greater horns of the hyoid, and bringing the structure into close contact with the larynx. Another modification is the fusion of the anterior rings of the trachea to form a supporting cricoid cartilage of the larynx.

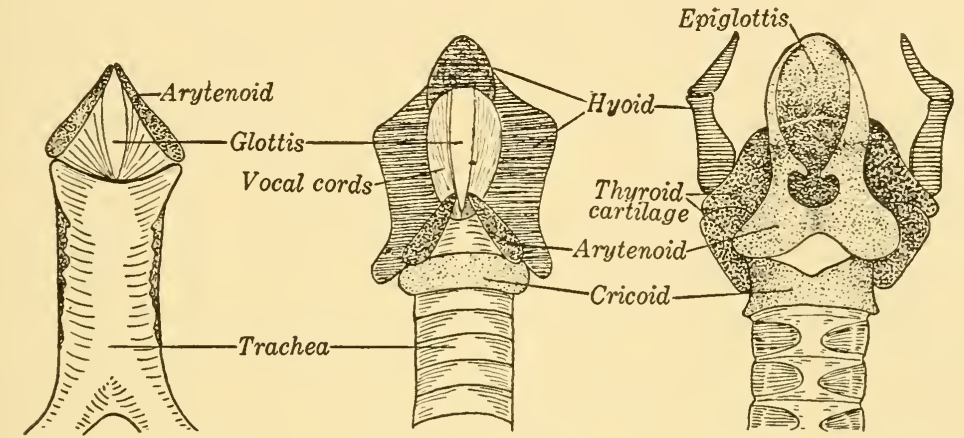

A. Necturus (Amphibia)

B. Tortoise (Reptilia)

C. Opossum (Mammalia)

FIG. 127. Development of the Larynx.

In the mammals the remaining cartilages of the branchial skeleton are brought into the larynx. The second and third (fourth and fifth visceral) cartilages unite across the ventral side of the larynx to form the thyroid cartilage. In the monotremes this cartilage is clearly separated into two parts. A similar division is found in the embryos of the higher mammals, but in these groups a complete fusion takes place during development.

The homologies of the first tro visceral arches were given in the section on the Visceral Skeleton, but are included here for clarity. The jaws and cartilaginous supports for the gills assumed their definitive condition in the elasmobranch fishes. Therefore, the posterior five visceral cartilages are called the branchial cartilages, and Visceral III is the same as Branchial I. 


\begin{tabular}{|c|c|c|c|c|}
\hline $\begin{array}{l}\text { Tisceral } \\
\text { Cartilage }\end{array}$ & \begin{tabular}{|c|} 
URodeles With \\
PERSISTENT Gills
\end{tabular} & $\begin{array}{c}\text { Other } \\
\text { AMPHibia }\end{array}$ & Reptiles & MamMaLs \\
\hline I & $\begin{array}{l}\text { Articular } \\
\text { Quadrate }\end{array}$ & $\begin{array}{l}\text { Articular } \\
\text { Quadrate }\end{array}$ & $\begin{array}{l}\text { Articular } \\
\text { Quadrate }\end{array}$ & $\begin{array}{l}\text { Malleus } \\
\text { Incus }\end{array}$ \\
\hline II & $\begin{array}{l}\text { Columella } \\
\text { Hyoid }\end{array}$ & $\begin{array}{l}\text { Columella } \\
\text { Hyoid }\end{array}$ & $\begin{array}{l}\text { Columella } \\
\text { Hyoid }\end{array}$ & $\begin{array}{l}\text { Stapes } \\
\text { Hyoid }\end{array}$ \\
\hline III & Functionless & Functionless & $\begin{array}{c}\text { Unites with } \\
\text { hyoid }\end{array}$ & $\begin{array}{l}\text { Unites with } \\
\text { hyoid }\end{array}$ \\
\hline IV & Branchial & Functionless & Functionless & $\begin{array}{l}\text { Thyroid } \\
\text { Cartilage }\end{array}$ \\
\hline V & Branchial & Functionless & Functionless & $\begin{array}{l}\text { Thyroid } \\
\text { Cartilage }\end{array}$ \\
\hline TI & Branchial & Functionless & Epiglottis & Epiglottis \\
\hline VII & $\begin{array}{l}\text { Arytenoid } \\
\text { Cartilages }\end{array}$ & Arytenoid & Arytenoid & Arytenoid \\
\hline
\end{tabular}

Tocal cords are found first in the anuran amphibia. These are membranous bands of tissue attached to the arytenoid cartilages, and partially closing the glottis on either side. The contraction or relaxation of these membranes by movements of the larynx give a limited change in pitch to the voice. This primitive
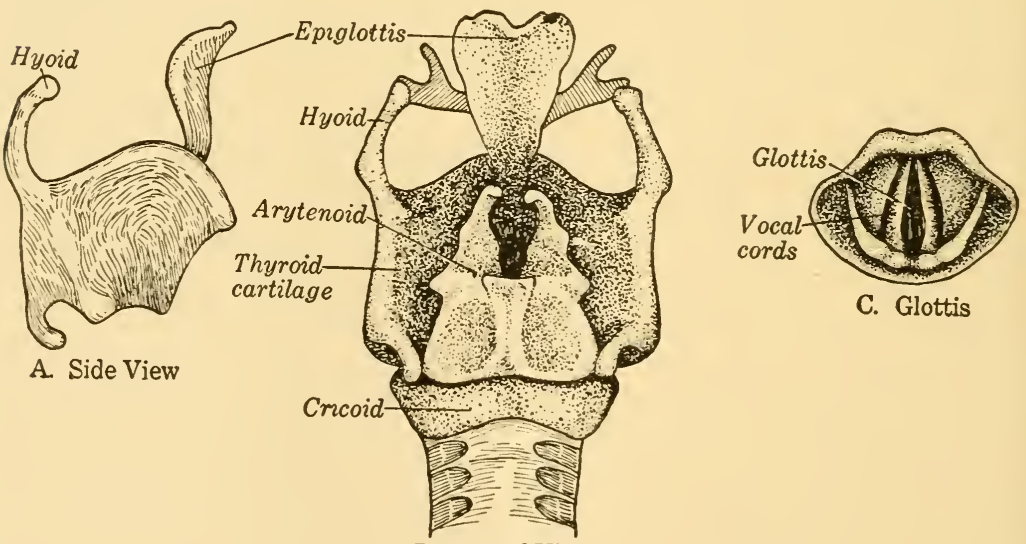

C. Glottis

B. Dorsal View

Fig. 128. Structure of the Human Larynx. (A) Shows the thyroid cartilage attached to the hyoid; (B) is a dorsal view with the esophagus removed; and $(\mathrm{C})$ shows the appearance of the glottis as seen through the mouth. 
voice mechanism is only slightly changed in the reptiles. In the mammals, however, with the development of the thyroid cartilages, the vocal cords stretch from the arytenoids to the thyroid cartilages. This increased length, correlated with the greatly developed muscular and nervous control of the larynx, gives the mammals a fuller control of pitch and tone. The principle of voice is based on the fact that a taut membrane vibrates more rapidly and with a higher pitch than one less tightly stretched. Differences in normal voice pitch in the mammals are due to the length of the membranes. Under equal tension a long cord will vibrate more slowly than a short one. The deeper voice of the male is caused by the growth of the larynx in a dorso-ventral direction at puberty. Length of larynx and depth of voice in a species are definitely correlated with the prominence of the Adam's apple.

The voice box of the birds is an entirely different structure, being located at the bifurcation of the trachea. This syrinx is a remarkably efficient organ as is demonstrated by parrots, mocking birds, and other birds which have ability for mimicry and a wide variation in pitch.

\section{Other Respiratory Structures}

In addition to gills and lungs, a number of animals have modifications of other structures which have a respiratory function. The only one of wide distribution is the skin which serves as a respiratory organ in most urodeles and many anura. In water living urodeles the cutaneous artery along the side of the body is larger than the pulmonary artery. Even the frog, when at rest and in a moist location, can secure sufficient oxygen through the skin. The most highly developed case of skin breathing is in some salamanders which lack both gills and lungs in the adult condition, and carry on their entire respiration through the skin. Cutaneous breathing is necessarily limited to those animals which have a very thin, highly vascular skin, and live in wet regions. For these reasons it is not found in any group above the amphibia.

An isolated, but interesting, modification is found in the South American dipnoans. As was mentioned on page 46, the pelvic 
appendages of the male become vascular and swollen during the breeding season and the animals are able to stay submerged for long periods while guarding the egg masses.

The sea turtle, although a lung breathing amniote, has two specializations which aid in respiration. Both the pharynx and cloaca are vascular and have thin epithelial coverings, and by keeping a constant stream of water passing into and out of these openings the animals can remain immersed for long periods without damage. Only a small amount of oxygen is needed, for the turtle at rest has a low metabolic rate and a minimum of respiratory function suffices.

There are no accessory respiratory structures in the birds and mammals, both of which have a heavy, dead skin. This is also true of the completely aquatic forms. Porpoises and whales come to the surface frequently for air. Some of the latter have a pharyngeal air chamber which gives a reserve supply to the animal while under water, but respiration takes place in the lungs. 


\section{CHAPTER XV}

\section{VASCULAR SYSTEM}

THE blood-vascular system is the distributing mechanism of the body. Food materials are absorbed by the epithelium of the digestive tract, and oxygen is diffused through the lining of the lungs, and these pass into the blood. In the more complex animals the means of distribution are very important, for substances necessary for metabolism cannot be absorbed directly by the individual cells of the organism. Therefore an efficient system of distribution has been vital in the evolution of the higher groups.

The chordates, and particularly the vertebrates, have a closed system. The epithelial lining of the vessels is continuous. The arteries divide to form capillaries, and the arterial capillaries become venous capillaries without any break. The blood, therefore, courses through unbroken channels. A certain amount of the vascular fluids seep through the capillary walls and surround the cells. These fluids are collected by the lymph vessels (page 243) which empty into, and have a lining continuous with, the veins.

The typical chordate system is found in Amphioxus. The ventral, pulsating artery (ventral aorta) functions as a heart and forces the blood forward along the ventral vessel lying under the pharynx. Paired branchial arteries pass from the aorta to the gills on either side, where each breaks into capillaries. The capillaries from each gill collect to form an efferent branchial artery, and these in turn form two dorsal aortae, one on either side of the notochord. At the posterior end of the pharynx these fuse to form a single median aorta lying ventral to the notochord. Arteries are given off to the muscles and viscera, supplying them with food and oxygen, taking up the waste products of metabo- 


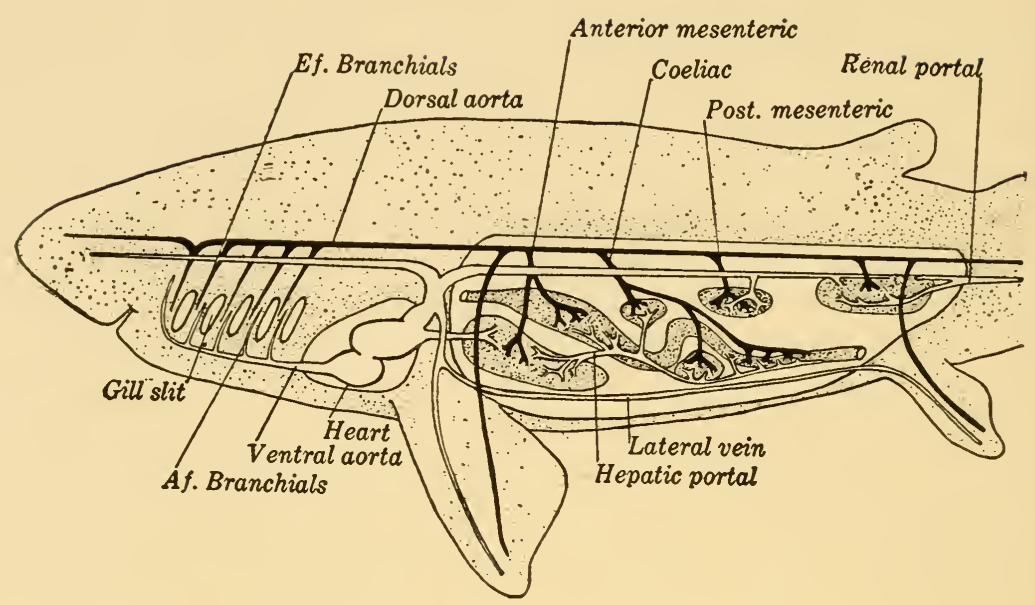

FIG. 129. Generalized Vascular System of the Elasmobranch. Only the larger ressels are shown, and the connections between arteries and reins are omitted.

lism, and collecting food materials from the intestine. Veins are formed from the capillaries and in Amphioxus there are two venous systems: (1) the subintestinal vein, which is homologous with the lateral body veins and the veins entering the liver (hepatic portal) of the higher vertebrates; and (2) the cardinal veins, a small group of vessels which drain the gonads and nephridia. The cardinal veins enter the subintestinal vessel anterior to its passage around and through the hepatic caecum. The combined vessel is continuous with the pulsating portion of the ventral aorta, completing the vascular system of the animal. (See the diagram of the blood system on page 19.)

\section{A. Vascular Sisteil of the Dogfish}

The heart of the dogfish is a muscular organ consisting of four chambers in a series, lying along the antero-posterior axis of the body. It is enclosed in a pericardial sac, a division of the primitive coelomic cavity. As the most anterior chamber and the most posterior are found only in the embryos of the higher classes of vertebrates, the heart is usually considered as consist- 
ing of only two chambers, one auricle and one ventricle. The ventricle is thick-walled and muscular, and is the pumping region of the heart. Anterior to the ventricle is the muscular conus arteriosus which continues anteriorly as the ventral aorta. The most posterior chamber is the sinus venosus which receives the blood from the veins and empties it into the thin-walled auricle, from which it passes to the ventricle.

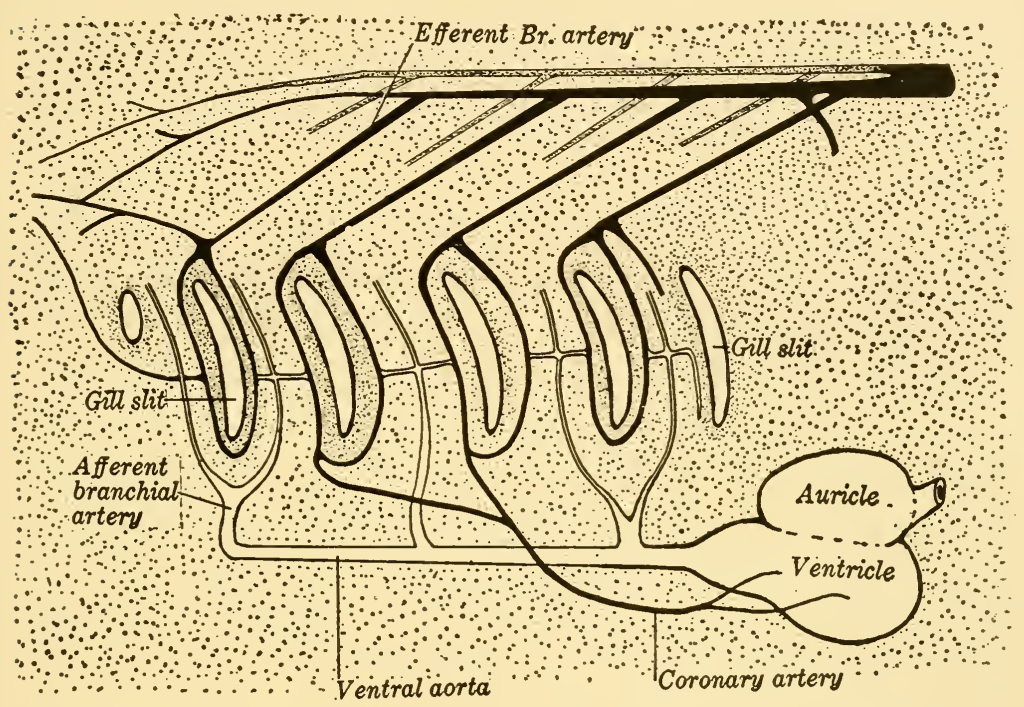

FIg. 130. Branchial Vessels of the Dogfish. Only the gross relationships of the left side are shown. The ressels break into capillaries in the gills.

The six pairs of afferent branchial arteries of the embryo (see page 235) are reduced to three in the adult. Beginning posteriorly, the fifth and sixth rise close together and in Squalus are fused for a short distance from their point of origin. The fourth embryonic arch passes into the gill arch as the second afferent artery of the adult. The second and third unite to form the first afferent artery, and then separate to supply the two anterior gill arches. The first afferent arch is not complete in the elasmobranchs, and forms the internal and external carotids. In Squalus these vessels appear to rise as branches from the first efferent artery. 


\begin{tabular}{c|c}
\hline $\begin{array}{c}\text { Afferent } \\
\text { Embryonic } \\
\text { Artery }\end{array}$ & Adulit Structure in Squalus \\
\hline I & Carotids \\
\cline { 2 - 2 } II & First afferent artery \\
\hline IV & Second afferent artery \\
\hline V & Third afferent artery \\
\hline
\end{tabular}

After the afferent branchials break into capillaries in the gills they re-collect as efferent branchial arteries. The four pairs of efferent branchials of Squalus form complete loops around each gill pouch, and then enter the paired dorsal aortae which forms a single vessel posterior to the pharynx.

From the first efferent branchials a pair of common carotid arteries passes forward, and these divide to form the internal and external carotids. These vessels supply the head and brain. Posterior to the carotids branches pass from the aortae to the mouth and esophagus; and posterior to these the large subclavians go to the pectoral fins. The hypobranchial arteries supply the muscles of the gills, and from them the coronary arteries pass to the heart and pericardium.

The body muscles are supplied by the small, metameric parietal arteries. The larger branches of the dorsal aorta, in order from anterior to posterior, are: (1) coeliac which divides into several branches, and goes to the liver, stomach, duodenum, pancreas, and parts of the ileum; (2) the anterior mesenteric artery to the left side of the intestine; (3) the gastro-splenic to the spleen and parts of the pancreas and stomach; (4) the posterior mesenteric supplies the rectal gland, rectum and cloaca; (5) the renal arteries to the kidneys; and (6) the iliac arteries which supply the pelvic fins. The dorsal aorta is continued into the tail as the caudal artery. In the female the oviducal arteries arise directly from the dorsal aorta.

Blood is returned to the heart through the veins, which will 
be traced from points of origin to the heart. Blood in the muscles of the body is collected by the paired lateral veins and a single cutaneous vein. These empty directly into the heart. The lateral veins are joined by the subclavian and iliac veins from the appendages. The head region is drained by the anterior cardinals and the jugular veins. These may unite before entering the heart, or enter separately.

The caudal vein drains the tail region, and at the level of the cloaca splits into two vessels which enter the kidneys and there break into capillaries. These two vessels are the renal portal veins. Observe that the blood passing to the excretory organs is both arterial and venous in origin. The nitrogenous wastes are taken from the arterial blood. The venous blood from the renal portal resorbs sugars from the kidney tubules, and collects the blood carried in by the metameric and renal arteries. It is here also that the tonus of the blood is maintained. The capillaries from the kidneys collect into a number of small veins along the median sides of the kidneys, and these form the large paired posterior cardinal vessels which pass anteriorly, close to the dorsal median line, to empty into the heart.

The digestive tract and mesenteries are drained by the hepatic portal system. Food materials are taken from the absorptive cells of the intestine and taken to the liver. There the hepatic portal vein breaks into capillaries and the sugars are to a large extent deposited as glycogen. The capillaries in the liver re-form as several vessels which unite to form the hepatic veins which enter the heart.

The large vessels carrying blood to the heart are classified as two groups: (1) the systemic veins which enter the heart directly, and (2) the portal veins which break into capillaries before entering the heart. In the lower groups of vertebrates the veins do not enter the auricle, but into a sinus venosus, the thinwalled posterior chamber. The sinus is extended laterally in the Elasmobranchs as the ducts of Cuvier. The sinus venosus is present in the embryos of all vertebrate classes, but grows progressively smaller as a definitive structure until it disappears entirely in the birds and mammals. 


\section{B. Embryonic Circulation of the Aminotes}

The embryonic development of the vascular system closely parallels the phylogenetic history. Therefore the embryonic circulation of the higher groups gives a generalized idea of the adult circulation of the lower forms. The following discussion is based upon the embryology of the chick which is essentially like that of the other amniotes, although the placentals have numerous minor modifications in correlation with placental nutrition. The student will recall that the reptile, bird and monotreme embryos develop on large yolked eggs with meroblastic cleavage; but that the marsupials and placentals have small ova which cleave holoblastically. The latter develop a yolk sac upon which the embryo grows in a typically amniote manner.

In large-yolked eggs the outline of the embryo is laid out on the blastodisc before the blood vessels begin development. The first sign of the vascular system is a group of rounded blood islands in the extra-embryonic mesoderm, each of which soon begins to branch. As lumina (internal cavities) appear, the branching arms fuse and the larger vessels begin to grow in toward the embryo, until a series of vessels is formed on either side of the embryo. Within the embryo a synchronous growth of vessels has formed a pair of ventral veins which grow outward. These unite with the extra-embryonic series of vessels and form the vitelline veins, one on either side, which carry food materials from the yolk to the embryo.

A continued growth of the ritelline veins carries them anteriorly to the region of the pharynx, where they turn dorsally and join the developing dorsal aortae. The dorsal aortae are at first paired throughout their entire length, and continue posteriorly into the caudal end of the embryo. Immediately posterior to the vitelline veins a group of capilliform vessels is given from each aorta. These pass outward to the yolk and make contact with the capillaries of the veins, thus completing the earliest circulatory system. The small arteries to the yolk later fuse to form a pair of large yolk sac arteries. At this time the fundamental outline of the circulatory system has developed: (1) a pair of incoming veins from the yolk to embryo; (2) an enlarged region in the anterior portion of these vessels, destined 

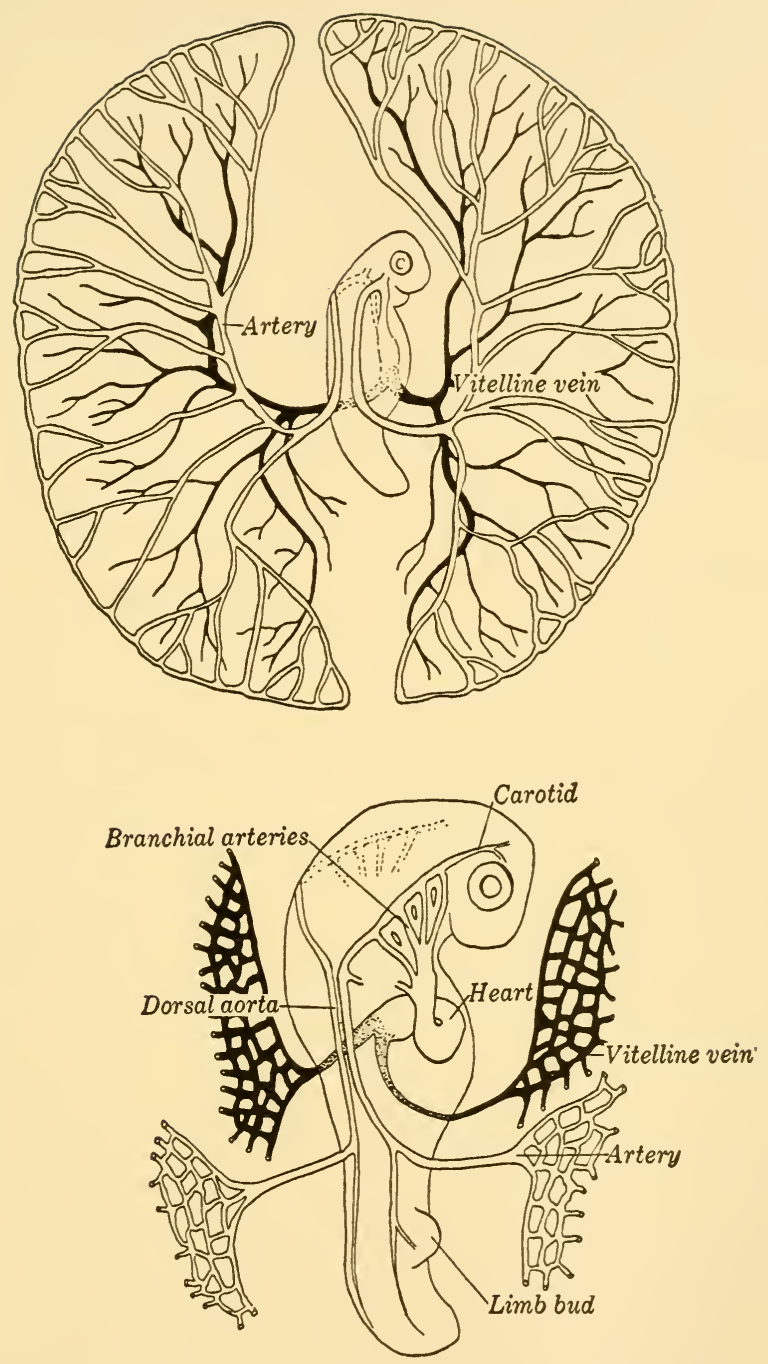

FIG. 131. Embryonic Circulation of the Chick. At the top is an early stage, showing the entire blastodisc. The lower drawing shows the torsion of the heart and the development of the branchial arteries. 
to become the heart; (3) the first aortic loop connecting the ventral vessels with the dorsal; (4) the paired dorsal aortae; and (5) branches from the aortae completing the circuit as they pass outward from embryo to yolk.

Modifications rapidly appear in the embryo. The vitelline reins begin a fusion to form the heart and a single ventral aorta; and the aortae fuse from the posterior end of the pharynx to the tail, forming a single dorsal aorta. Synchronously with this the carotid arteries grow anteriorly from the first aortic loop into the head region, and small arteries grow into the tissues from the dorsal aorta. The blood carried out to the body is returned to the developing heart by the cardinal veins. There are two anterior cardinals draining the head and brain, and two postcardinals which carry blood from the posterior tissues and the developing kidneys.

In the meantime the aortic loops have increased in number. The primitive gill outpocketings have appeared along the pharyngeal wall; and the aortic, or branchial, loops pass between them. At this stage the chick embryo is essentially like the dogfish adult. In the latter the branchial arches break into capillaries in the gills and form afferent and efferent branchial arches. In the amniotes the arches remain as continuous ressels which undergo various modifications.

Amniote embryos of this stage develop an allantois (page 69) from the posterior end of the gut. Veins and arteries are carried with it, and these eventually lie in contact with the chorion. Thus a vascular, moist membrane is next to the porous shell, and acts as a respiratory structure for the growing embryo. In placentals the allantois induces the development of a placenta (page 93) and the allantoic ressels have the same function as they do in the chick. In time the allantoic veins fuse with the vitelline veins as the omphalomesenteric veins which pass through the liver to the heart. The right omphalomesenteric disappears and the left remains as the hepatic portal vein which enters the liver and breaks into capillaries. The anterior ends of the original pair remain as the two hepatic veins.

At this stage the system of the amniote embryo does not differ essentially from that of the adult dogfish, with the exception of the allantoic vessels. Future changes in the embryo can best be 
treated in connection with the comparative study of the different regions of the system, and the developmental changes will be discussed in connection with the comparative anatomy of the vessels.

\section{Development of the Vascular System}

The student at this time should have a concept of the general structure of the vascular system. It is clear that the entire group of structures included in the system is a unified whole, both anatomically and functionally. For convenience the system can be divided into its integral parts and each treated separately. It should be borne in mind that development is synchronous, the entire system growing and developing as a unit in correlation with the growth and differentiation of the other organs.

1. Comparative Anatomy of the Heart. Amphioxus has a straight, muscular, pulsating ventral aorta. In the cyclostomes the heart is S-shaped, with a single dorsal auricle and a heavywalled ventricle. The blood from the systemic veins empties into a sinus venosus, from which it passes to the auricle, then to the ventricle and is forced into the ventral aorta. In the larva of the lamprey (page 31 ) the coelomic cavity surrounding the heart is partly cut off from the body coelom, and in the adult the separation is completed. This coelomic pocket is the pericardial cavity and is found in all the vertebrates.

The dogfish heart is essentially similar, with the addition of a conus arteriosus containing numerous cup-shaped semi-lunar valves to prevent the back flow of the blood from the ventral aorta. This muscular conus is gradually lost in the higher fish and the semi-lunar valves reduced to a typical three.

The amphibia make a definite advance in heart structure with the separation of the auricle into two distinct chambers, the dividing septum passing between the pulmonary veins which enter the auricle directly, and the sinus venosus which receives the body veins. A single auriculo-ventricular opening is present. The left auricle consequently contains aerated blood, and the right is filled with unaerated; but there is necessarily an admixture of blood as it enters the single ventricle. In this respect the amphibian heart is less efficient than that of the fish. The 
conus arteriosus is muscular and is usually guarded by several rows of valves.

The reptiles as a group differ from the amphibia in several important points: (1) the sinus venosus is small and usually not visible on the outside of the heart; $(2)$ the septum of the auricle passes through the auriculo-ventricular opening, cutting the valves into two groups; and (3) the ventricle is always more or

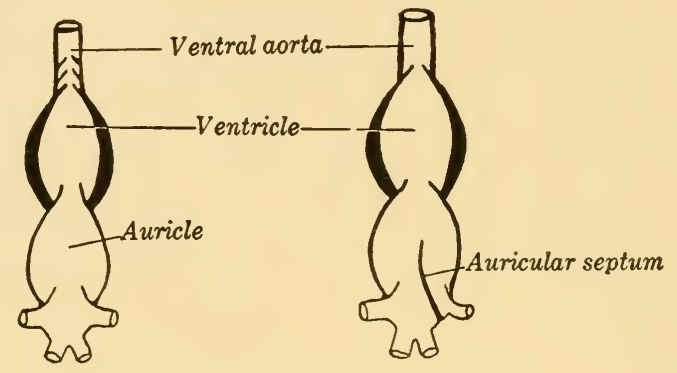

A. Dogfish

B. Crossopterygian

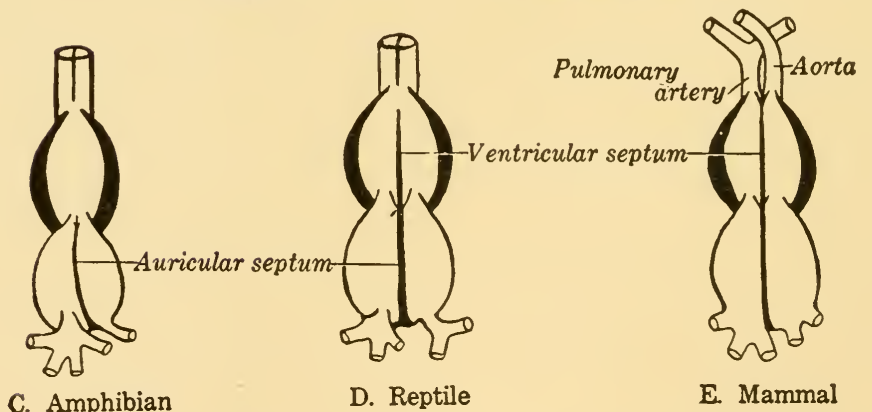

C. Amphibian

D. Reptile

E. Mammal

Frg. 132. Comparative Anatomy of the Heart (Diagrammatic). The twisting of the heart has been eliminated, the diagrams showing the hearts in their primitive position with the ventricle and the auricle posterior. Note the growth of the septum, with the eventual separation of the ventral aorta.

less divided by a septum into two chambers. The degree of separation in the ventricle varies widely in the different orders. In the turtles and lizards the ventricular septum is a semicircular band of tissue incompletely dividing the chamber. Blood from the right auricle is directed by flaps of the valves into the right (pulmonary) cavity, the aerated blood entering the left chamber of the ventricle. As the heart contracts the opening in the septum 
narrows and forces the unaerated blood of the pulmonary chamber into the pulmonary artery, and the aerated blood of the left chamber into the two loops (radices) of the ventral aorta. In the alligator the septum is complete, making a four-chambered heart, but there is an opening between the right and left ventral aortae which permits a slight admixture of bloods.

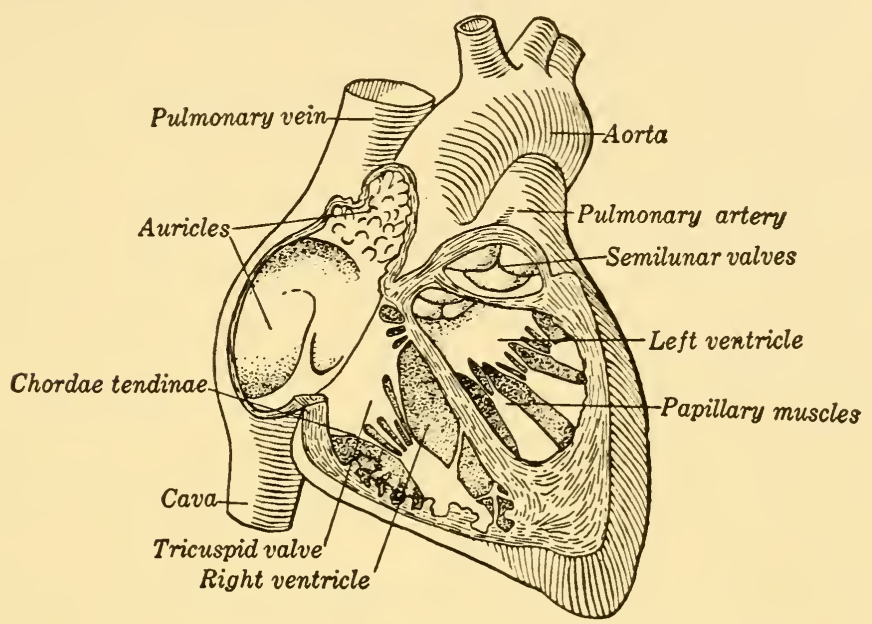

Fig. 133. Structure of the Mammal Heart. A portion is removed to show the internal structure. The ventricular septum curves toward the right, separating the semi-lunar valves of the aorta and pulmonary artery.

The mammal heart is structurally and functionally completely four-chambered. The sinus venosus is left as a vestigial band of tissue within the right auricle. The septum is complete, dividing the heart into left auricle and ventricle, and right auricle and ventricle. The auriculo-ventricular opening is guarded by two sets of valves, each valve being a flap of tissue attached to the inner periphery of the opening, and the loose edge held by strands of connective tissue (the chordae tendinae) which are attached to the wall of the ventricles. The left (mitral) valve has two cusps while the right (tricuspid) has three. The ventral aorta consists of a single loop (radix) and is completely separated from the pulmonary. Therefore the blood is never mixed, except in pathological conditions. The heart of the birds is essentially like that of the mammals. 
2. Development of the Mammal Heart. The heart begins as a fusion of the two vitelline veins, and is a single straight tube. As the heart region enlarges and becomes more muscular it assumes an S-shape, the posterior (auricular) end moving dorsally. The ventricular portion becomes heavily muscled and forces the blood into the ventral aorta as it is received from the auricles. Further growth of the ventricle forces it posteriorly, so that the heart soon has the appearance of the auricles being anterior and the ventricle posterior in position. If the student understands the twisting and growth of the heart, the primitive morphological relationships will be clear.

As the septa in the heart develop the ventricular septum completely cuts this chamber into two; but the auricular septum is left incomplete until after birth, this opening (the foramen ovale) between the two auricles being of functional benefit in embryonic circulation, for the blood is aerated in the placenta rather than in the lungs. If the foramen does not close soon after birth the blood is mixed as it enters the aorta and a cyanotic condition results in the individual. The so-called "blue babies" are persons affected by this anomaly.

3. Aorta And Aortic Arches. The ventral aorta is the ressel from the heart passing out of the pericardial cavity along the rentral side of the pharynx. In the early embryonic condition of all vertebrates the aortic, or branchial, arches pass dorsally to the paired dorsal aortae. The typical number of arches is six, although the cyclostomes and most primitive elasmobranch fishes have a greater number. Beginning with this primitive condition there are many modifications in the groups of vertebrates, but the ontogenetic and phylogenetic histories of the classes are closely parallel.

The elasmobranchs retain the most primitive condition. The major changes are: (1) the first arch forms the carotid arteries, and (2) the more posterior arches break into capillaries, thus forming the afferent and efferent arteries from the original vessel. Most sharks (see Squalus, page 223) are further modified by the fusion of vessels, so that only three leave the ventral aorta in the adult condition.

The urodele amphibians show a reduction in the number of arches, strongly resembling the modifications found in the dip- 


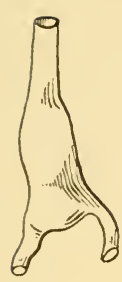

Fig. A

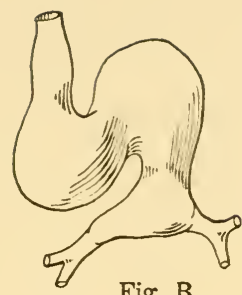

Fig. B

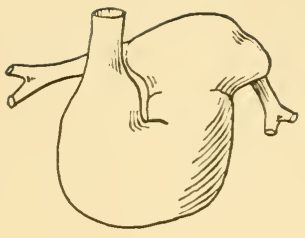

Fig. C

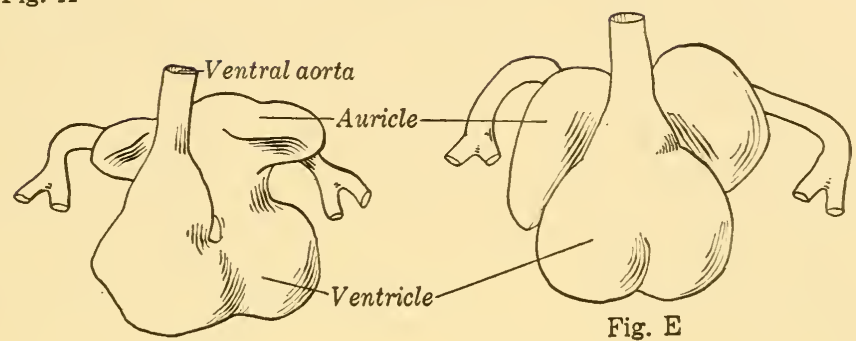

Fig. D

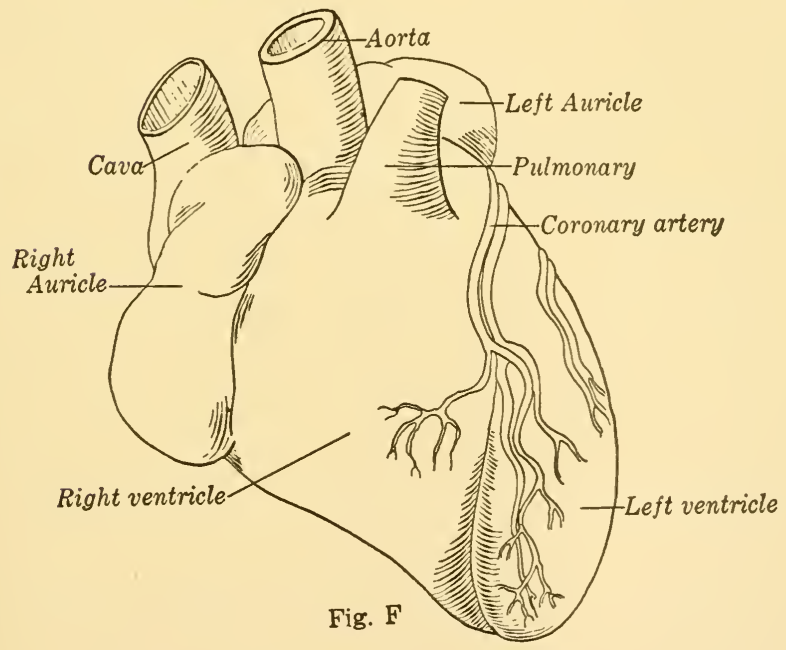

Fig. 134. Embryology of the Human Heart. Drawings (A) to (D) are shown in lateral-rentral view to show the bending and constriction of the organ. Drawing (E) is in ventral view. (F) Is the definitive structure, 
noan fish. The first and second arches are lost, the base of the third serving as the common carotid. The portion of each dorsal aorta between the third and fourth arches is greatly reduced, as is also the fifth arch. Large branches from the sixth arches have grown posteriorly to enter the lungs as the pulmonary arteries, and the dorsal segment of each sixth is greatly reduced. Therefore, most of the blood entering the sixth branchials is carried to the lungs, a small amount passing to the dorsal aorta in an unaerated condition. The pliaryngeal portion of the dorsal aorta is paired, the two radices being formed by the fourth branchial arches.

The anuran amphibia are more effectively specialized for land life. On either side the connection between the third and fourth arches has completely disappeared; the fifth arch is lost; and there is no connection between the pulmonary artery and the dorsal aorta. However, the pulmonary arteries remain as branches of the single ventral aorta, causing a further mixture of blood as it leaves the heart. The separation of aerated and unaerated blood is due to the anterior position of the former in the ventricle, and as the contraction of the heart forces it into the ventral aorta the first blood takes the line of least resistance into the large ventral vessel and the two radices of the aorta. When the unaerated blood leaves the heart, pressure has developed in the aortae and the pulmonary vessels are filled.

The typical reptile has a separation of the pulmonary arteries from the aorta, the separation accompanying the development of the ventricular septum. The pulmonary artery is cut from the ventral aorta by a longitudinal constriction, so that the two vessels leave the heart independently. Due to the structure of the ventricular septum the pulmonary vessel carries mostly unaerated blood.

In most reptiles the right radix of the aorta is larger and filled with almost unmixed blood. It is from this vessel that the carotids arise. The left radix carries partially mixed blood which is emptied into the dorsal aorta and carried to the tissues. The alligator has the most complete separation of blood of any reptile, the small opening between the pulmonary and aortic vessels permitting of only slight mixture.

In the birds the separation of aerated and unaerated blood is 


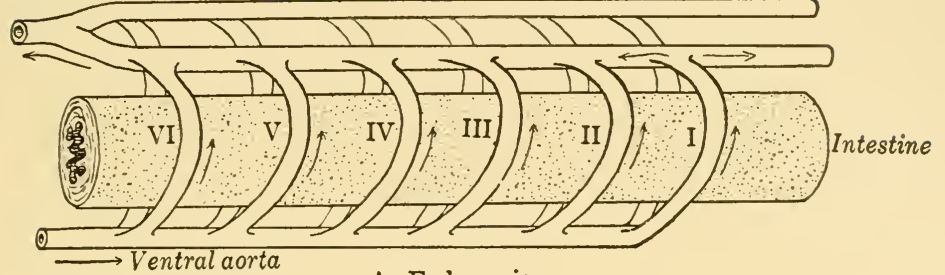

A. Embryonic

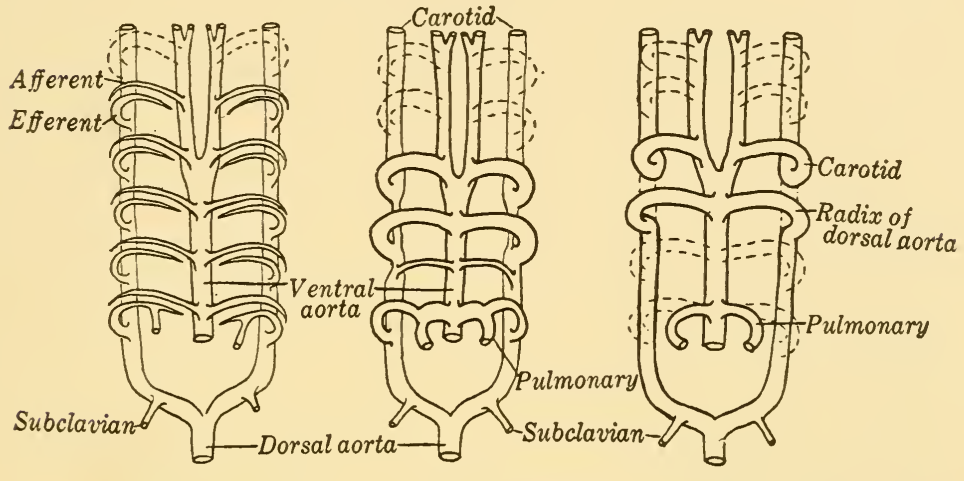
B. Elasmobranch
C. Urodele
D. Anura

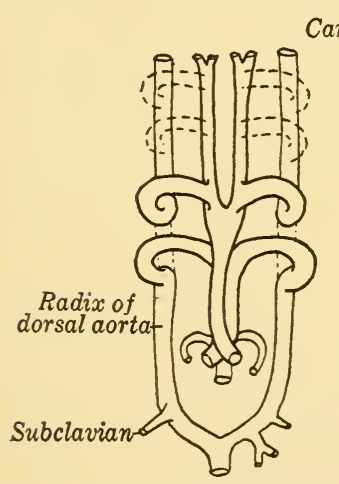

E. Reptile (primitive)

\section{Carotids}

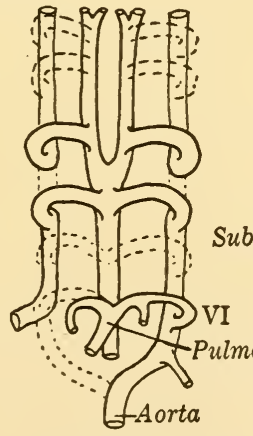

F. Mammal (embryo)

\section{Carotids}

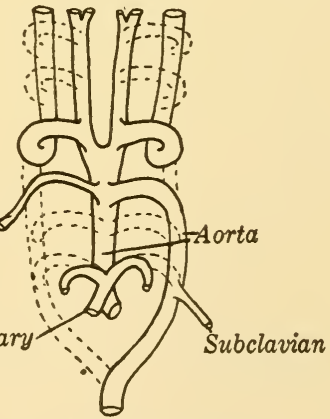

G. Mammal (adult)

Fig. 135. Comparative Anatomy of the Aortic Arches. Drawing (A) is the generalized embryonic condition; (B) shows the division of each arch to form an afferent and efferent branchial; (C) to (G) show the reduction of the arches in the different groups of vertebrates. 
complete. The left radix, which is less functional than the right in reptiles, has disappeared; the right remaining as the aorta of the birds. The base of the left fourth branchial (the left radix of the reptiles) remains as the left subclavian artery, supplying the wing and shoulder.

Mammals are essentially like the birds in the modification of the aortic arches, with the exception that the left radix remains as the aorta. The left subclavian, like that of the more primitive vertebrates, is a branch from the aorta, and the right subclavian is connected with the aorta by the base of the fourth branchial arch (the right radix of the reptiles). Following is a summary of the degeneration of the aortic arches of the mammal. Recall that in the embryo the rentral aorta is bifurcated from the level of the third arch to its anterior end. Each half of the anterior ventral aorta extends to the head as the external carotid, and the anterior portion of the dorsal aortae form the internal carotids. The common carotids are formed by the divided region of the ventral aorta between the third and fourth arches.

Arch I, disappears.

Arch II, disappears.

Arch III, connects the common carotid with the internal carotid.

Arch IV, forms: (1) the left gives rise to the "aorta"; and (2) the base of the right connects the aorta with the subclavian. Arch $V$, disappears.

Arch VI, forms the pulmonary artery. The dorsal half disappears, destroying the connection between the pulmonary and the aorta.

4. The Cardiac Cycle of the Mamalal. The foetal circulation of the mammal differs widely from that of the adult. The lungs are non-functional, respiration taking place in the placenta. Consequently, aerated blood comes into the heart through the veins, mixed with unaerated. Blood is carried back to the placenta through branches of the dorsal aorta, with the result that blood within the embryo is always mixed.

Blood is prevented from going to the unexpanded lungs by three mechanisms: (1) as the blood pours into the foetal auricles, most of that which falls into the right auricle passes through the opening in the auricular septum (foramen orale) and goes into 
the left ventricle; (2) the mammal embryo retains the primitive connection between the pulmonary artery and the dorsal aorta (the distal portion of the sixth arch) and this tends to shunt the blood away from the lungs; and (3) the pressure within the collapsed lungs prevents the blood from entering. The connecting vessel between the pulmonary artery and the aorta is called the ductus arteriosus (or ductus Botalli). It begins to degenerate soon after birth, but may remain open for some months in the longer lived animals. Breathing is initiated in the newborn mammal by a hypertension of carbon dioxide in the blood, and the ductus arteriosus degenerates in correlation with the function of the lungs.

The cardiac cycle depends upon the alternate contraction and relaxation of the heart and the flow of blood in one direction through the arteries and veins. The latter is made possible by the system of valves which direct the flow through the heart. These are: (1) the auriculo-ventricular valves separating the auricles from the ventricles; and (2) the semi-lunar valves at the base of the aorta and the pulmonary artery.

Assume that the heart muscles are completely relaxed. The blood then pours into the auricles from the systemic veins and passes directly into the ventricles. As the ventricles fill, a wave of contraction begins in the auricles, in the node of tissue formed by the primitive sinus venosus. This wave spreads downward, continuing into the ventricles. The swirl of blood in the ventricles has thrown the valves outward, and the ventricular contraction forces them upward and together, preventing the flow of blood backwards into the auricles. The continued contraction of the ventricles forces the blood into the aorta and pulmonary artery. Relaxation occurs in the same order as does the contractile wave, and as the pressure in the ventricle decreases the semi-lunar valves are forced open by the column of blood in the arteries. The fluid is unable to flow backward into the ventricle, and is forced into the smaller arteries by a peristaltic wave of contraction in the larger vessels which carries it into the capillaries. The heart completes its relaxation, and the cycle begins anew.

The closure of the valves is due to a differential pressure within the regions of the heart and arteries. The sounds of the heart are made by the snap of the valves as they close, the 
larger auriculo-ventricular valves making a slightly deeper note. Any weakness or hardening of the valves may cause them to close imperfectly and permit a backflow, the regurgitating blood causing a murmur as it rushes past the valves.

5. The Aorta and Its Branches. In their fundamental plan the branches of the aorta remain remarkably constant in the classes of vertebrates. The coronary arteries supply the heart. In the dogfish they arise from the efferent loops around the third gill pouch. In the amniotes they arise directly from the proximal portion of the ventral aorta. The origin of the carotid arteries, which are next in order from anterior to posterior, was discussed with the development of the branchial arteries.

The subclavian arteries supply the pectoral appendages. The vessel receives other names as it passes distally, the changes being based upon human terminology. It is the axillary artery as it passes the arm-pit; the brachial in the upper arm; and when it divides at the elbow the two main branches are the radial and ulnar. In the classes which have both radices of the aorta present, the subclavians are given off as branches from each side. The condition in the mammal and bird has been discussed.

Posterior to the subclavians the blood vessels can be divided into two groups: (1) the parietal and (2) the visceral. The parietal arteries are primitively metameric, although complete metamerism may be lost in the higher animals. In the thoracic region the parietal arteries pass to the muscles of the back and ribs, the latter being the costal arteries. Posterior to these are the lumbar and sacral arteries. The large iliac arteries, which supply the pelvis and pelvic limbs, are branches from the aorta. In most vertebrates the dorsal aorta is continued into the tail as the caudal artery.

The visceral vessels are: (1) the coeliac axis which divides into several large vessels supplying the liver, pancreas, stomach and upper parts of the intestine; (2) the anterior mesenteric which is more widely distributed in the mammal than in the dogfish, passing to most of the small intestine in the former class; (3) the posterior mesenteric which supplies the posterior part of the digestive tract, including the colon and rectum.

The amniotes have several visceral arteries arising directly 
from the dorsal aorta which are absent in the lower groups, or arise as branches from other vessels. The genital arteries are paired and supply the reproductive glands. The adrenal arteries go to the adrenal glands which are located near the kidneys; and the renal arteries go to the kidneys. The latter are present in the dogfish, but arise as very small, metameric vessels. In the amniotes these are collected into a few vessels, or a single pair as in the mammals.

6. The Srstemic Veins. The systemic veins are those which enter directly into the heart without breaking into capillaries. Of these systemic vessels, the anterior cardinals remain relatively unchanged. In the higher vertebrates they are known as the internal and external jugular veins. The internal pair drains the brain and upper portion of the head, the external pair drains the superficial musculature. In the mammal there are frequent anastomoses between the two veins, and their independent origin can be less clearly seen.

The lateral veins of the dogfish, which drain the sides of the body and the paired fins, disappear in the urodeles. However, the abdominal vein arises as a pair of veins which fuse, and this median vessel is considered homologous with the laterals. The alligators have two lateral veins. The other reptiles have an abdominal vein similar to that of the urodeles. In the mammals the lateral (or abdominal) veins practically disappear, and the subclavian veins join the jugulars before entering the heart.

The postcardinal veins are very prominent in the dogfish, passing anteriorly from the kidneys, and joining the anterior cardinals before entering the heart. The further evolution of these veins is intimately correlated with the development of the postcava, or posterior vena cava, and these two veins must be considered together. The postcava makes its first appearance in the dipnoan fish. In the urodele amphibia it is a large vessel passing from the anterior level of the kidneys to the right auricle. At the kidneys the ressel divides and passes to each kidney, taking over much of the function of the cardinals. The latter are small vessels which appear to be branches of the postcava, and pass anteriorly to enter the ducts of Cuvier, draining the intercostal muscles and thorax. Embryological development shows that the postcava grows posteriorly from the 
auricle and joins the cardinals. Therefore the posterior part of the postcava is morphologically the older cardinal vessels.

In the reptiles the cardinals lose their posterior connection with the postcava, and are small paired vessels draining the thoracic region. The postcava drains the posterior part of the body (the abdominal veins having disappeared) and the cardinal vessels have further degenerated. The left vein loses its connection with the jugulars, and develops one or more transverse connections with the right cardinal. The left postcardinal is called the hemiazygos; and the right half, which empties into the jugular, is the azygos. As in the reptiles, it drains the costal muscles.

The embryonic development of the veins of the mammal will review these changes. The embryo has two postcardinals carrying the blood from the primitive kidneys (mesonephroi) to the ducts of Cuvier. Subcardinal vessels develop on the median sides of the kidneys, taking over the smaller vessels of the postcardinals. The postcava grows posteriorly and joins the subcardinals, taking its definitive form. In the meantime the postcardinals have begun to degenerate in the kidney region, and the cross connections have developed. The left cardinal then degenerates along its anterior course, the blood from both cardinals emptying through the right azygos into the jugular.

7. The Renal Portal Srstem. A portal vein is one which breaks into capillaries before reaching the heart. In the dogfish the caudal vein divides and passes to either kidney, the lateral veins making a connection with the two renal portal vessels. Blood from the tail, therefore, may pass to the heart either through the kidneys, or around the kidneys into the lateral veins.

The vessels entering the kidneys break into capillaries around the tubules, with the result that both venous and arterial blood is received. The renal portal system is present, almost fully developed, in the amphibia; but with the degeneration of the cardinals in the reptiles the portal veins become less important. In the mammals the kidneys are supplied by large arteries, and the renal portal reins disappear.

8. The Hepatic Portal Srstex. It has been mentioned that the paired omphalo-mesenteric ressels pass anteriorly around the 
liver to the heart. As the liver tubules develop, the right omphalomesenteric is interrupted and breaks into capillaries. This condition persists for a short time, and then the left vein breaks into capillaries and the system is completed.

The earliest homologue of the hepatic portal is found in Amphioxus, where the subintestinal vein breaks into small vessels which re-unite before entering the ventral aorta. In the elasmobranch fishes the system is fully developed, and with minor changes remains in all the classes of vertebrates. Blood from the liver is carried to the heart by the two hepatic veins, which belong with the systemic vessels.

9. The Human Blood Srster. Blood leaves the heart through the aorta, which turns dorsally from right to left. A few inches from its base is given off the innominate artery which gives rise to the right common carotid and the right subclavian. Next is given off the left common carotid, and then the left subclavian. The development of the aortic arches will make the relationship clear. The external branch of the carotids passes to the muscles of the neck and face; the internal branch goes to the brain and its coverings. At the base of the brain the two internal carotids anastomose forming a circle of vessels.

The aorta passes posteriorly along the ventral side of the vertebrae, giving off the metameric parietal arteries to the ribs and body muscles and several esophageal branches. Next are the phrenic arteries to the diaphragm which separates the pleural cavities from the abdominal cavity. The large coeliac axis is next, going to the viscera, and then in order: (1) the superior mesenteric; (2) the paired suprarenal (adrenal) vessels; (3) the paired renals; (4) the paired spermatic (genital) arteries to the gonads; (5) the inferior mesenteric to the colon and rectum; and (6) the sacral artery. Immediately posterior to the last the aorta divides to form the two large iliac arteries which supply the pelvic limbs. The numerous branches of the latter need not be discussed.

The external jugular vein, after making connections with the internal jugular, enters the subclavian. The internal jugular fuses with the subclavian to form the innominate. The conditions are approximately the same on both sides. The two innominate 
veins then coalesce and form the superior vena cava which enters the right auricle.

The metameric costal reins are a part of the azygos, which empties into the superior vena cava. The posterior part of the body is drained by the inferior vena cava (postcava). The external and internal iliac veins fuse to form the paired common iliacs, which unite at the level of the sacrum to form the vena cava. This large vessel receives blood from the renal veins, which are joined by the spermatics. Next in order are the

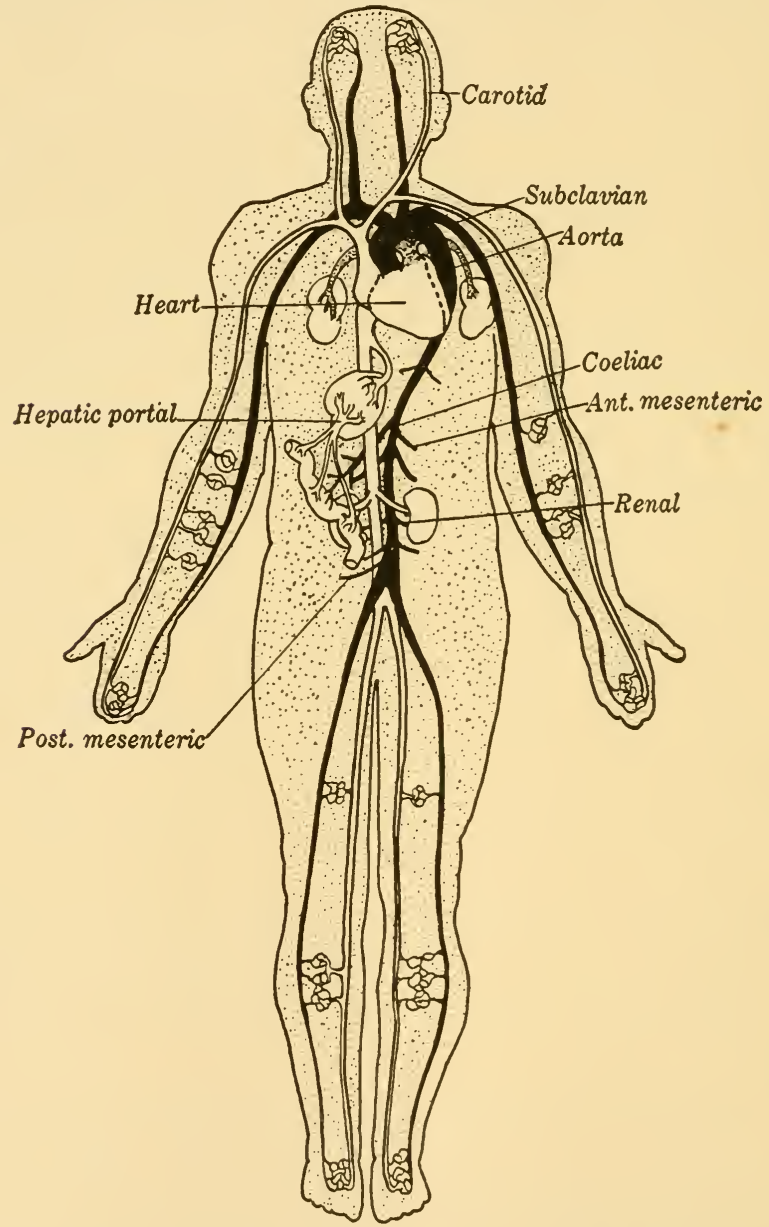

FIG. 136. Vascular System of Man (Diagrammatic). 
suprarenals and the phrenic reins. The rena cara then passes through the liver and empties into the auricle.

The hepatic portal system is composed of the following major branches: (1) the superior mesenteric; (2) splenic; (3) inferior mesenteric; (4) the gastric; and (5) the pyloric vein. The completed hepatic portal vessel is short, and as it reaches the liver it divides into right and left branches. The right is the larger vessel. In the liver the vessels break into capillaries and sinuses. The blood from the liver is collected into several small veins, and the two large hepatic veins which enter the vena cava before it passes through the diaphragm.

The heart consists of the four chambers typical of the mammals, the auricles being anterior and the ventricles posterior. It is roughly triangular in shape, the apex pointing downward, and located usually in the fifth intercostal space; that is, between the fifth and sixth rib cartilages. About one-third of the heart lies to the right of the median line, the axis of the organ passing from right to left. The aortic arch usually lies behind the sternum. There is considerable range in the size and shape of the heart, both being correlated with the general shape of the body, the tall thin individual tending to have a slender heart, while the broad type tends to have a shorter rounder one.

\section{Lrmphatic Srstem}

Although the veins and arteries of the vertebrate form a closed system, a certain amount of the blood fluid passes through the capillary walls, or is forced through their inter-cellular spaces. This fluid is the lymph and lacks most of the corpuscles, or cells, of the blood, and some of the coagulating elements. The lymph bathes each cell of the body and carries food materials and free oxygen. This fluid is collected from the tissues by a definite system of vessels, called the lymphatics, and is returned by them to the veins. Failure of the lymphatic system to function properly causes an accumulation of fluid in the tissues or body cavities, and the affected region becomes swollen or edematous. There are many other causes of edema (infections, heart trouble, and others) but during embryonic life lymphatic edema is perhaps most frequent. 
The lymphatic system also functions in collecting the fats absorbed by the digestive tract. Each villus which projects into the lumen of the intestine is supplied with a minute lymphatic duct in addition to the vein and artery. The fats pass through the lining epithelium into the lacteals, or lymphatic ducts of the villi, and through them into the main lymphatic stream. The whitish, fat-ladened fluid is linown as chyle and the lymphatics from the intestine are frequently called chyle ducts. As a result of these two functions of the lymphatic system, the lymph entering the veins has a high content of fats and waste products, and a low content of oxygen, carbohydrates and proteins.

Both in phylogeny and ontogeny there is a reduction in the extent of the lymphatic system. In the primitive vertebrate and in the embryo there are large irregular lymph sinuses, and these become reduced to a definite system of vessels, the system occupying relatively less space in the more specialized animal.

In the cyclostomes and fish the lymphatic capillaries collect into definite vessels which empty into large sinuses connecting with each other. The largest is below the vertebral column, lying around the dorsal aorta. Others are in the ventral body wall and underneath the skin. A single lymphatic vessel drains each of the sinuses, and these efferent ducts fuse to form four main ducts which empty into the veins. The anterior two join the veins at the junction of the jugulars with the subclavians, and the posterior two enter either the caudal or the iliac veins. At the point where the lymphatic vessel leaves the sinus there is a pulsating lymph heart forcing the lymph toward the veins. Each is protected by valves which keep the fluid flowing in the same direction.

The lymph hearts are retained by the amphibia, particularly the urodeles. In the anura (frogs and toads) the hearts are definitely smaller and the anterior hearts disappear. Almost the same condition is found in the lower reptiles. The crocodiles and alligators retain the two posterior hearts, but the large dorsal sinuses are reduced to thoracic lymph ducts. The birds lose the lymph hearts entirely in the adults, although there are both anterior and posterior connections with the venous system. In both reptiles and birds there is an increase in the number of the smaller lymphatic vessels, and the system takes on the appear- 
ance of a definite group of vessels leading from the inter-cellular spaces and digestive tract to the veins.

The mammalian lymphatic system shows the following changes: (1) the terminal capillaries ramify more fully through the tissues; (2) there are no definite sinuses with the exception of minute ones in several glands; (3) the posterior openings into the veins are lost, the entire body lymph entering the veins through the two anterior connections with the subclavians; (4) the area drained by the right lymphatic duct is limited to the head and thoracic region, while the left duct drains the left anterior half and the entire posterior part of the body; and (5) lymph nodes develop.

The lymph nodes of the mammal are ovoid masses of glandular connective tissue, each divided into a number of independent lobes or follicles. The lobe has a center of blood capillaries surrounded by small lymphatics. The connective tissue is characterized by rather close-packed small cells with relatively large nuclei, without any definite arrangement. The largest lymph nodes are in the neck, the axilla (arm pit), and groin. The lymph nodes have a double function, (1) the formation of certain white blood cells and (2) filtration of the blood. In many types of infection the lymph nodes swell due to the concentration of bacteria at these points. Similar masses of lymphatic tissue appear in other parts of the body: in the mesenteries; the intestine where they are called Peyer's patches; the thymus; and the adenoidal tissues of the naso-pharynx.

Developanent. The mammalian lymphatics begin development as several outpocketings from the veins at the juncture of the jugulars and subclavians. As these grow anteriorly and posteriorly lymph hearts appear, and from the latter many branches grow out into the tissues. The branches continue dividing until the entire system of vessels is formed, each lymphatic capillary ending blindly in the tissues. There is little evidence to support the theory that the lymphatic vessels have a double origin, with the capillaries arising as enlarged inter-cellular spaces independent of the outpocketings from the veins. The terminations of the lymphatic capillaries are closed, the lining: cells of all the vessels are similar and continuous, and the em- 
bryological evidence supports the jdea that the vessels arise from definite centers of growth.

As the lymph hearts of the embryo degenerate the lymph nodes develop, and all signs of sinuses disappear. The vessels and nodes are relatively more prominent in the young than in the adult. The chyle ducts enter the left lymphatic duct.

\section{E. Structure of the Blood}

The blood is a fluid tissue (page 103) acting as the distributing medium of the body. It is composed of discrete cells or corpuscles floating in a fluid medium. The latter is the plasma, and is composed of a clear straw-colored serum and fibrinogen. The fibrinogen when properly activated is the clotting element, precipitating and leaving the clear serum, thus coagulating the blood. Lymph is the serous portion of the fluid with less fibrinogen than is found in the normal plasma. The corpuscles of the blood are of two types, (1) red cells or erythrocytes, and (2) white cells or leucocytes.

LEUCocytes. The white cells of the blood are very similar throughout the vertebrates, although the relative proportion of the various cell types varies widely. The leucocytes are divided into two major groups: (1) agranulocytes which have a clear cytoplasm lacking granules; and (2) granulocytes which have the cytoplasm loaded with granules. The non-granular cells are the lymphocytes and the large mononuclear leucocytes. The granular cells include the mast cells of the blood and the polymorphonuclear cells.

1. Lymphocytes are small cells which arise in the lymph tissues, particularly the lymph nodes of the mammals. They have a single nucleus almost as large as the cell. In the human these form about twenty-five per cent of the Ieucocytes.

2. Large mononuclear cells have a single, often crescentic nucleus, and are the largest of the white corpuscles.

3. Mast cells have granules taking a basic stain, and are considered by many investigators as degenerate cells. They form less than one percent of the leucocytes in normal blood.

4. Polymorphonuclear leucocytes have nuclei which assume many shapes, often giving the appearance of being divided 
into several distinct nuclei. They are ameboid in appearance and are actively phagocytic (that is, they ingest foreign particles). These cells form about seventy-four per cent of all leucocytes in the human. The great majority show a neutral staining reaction, but a few take an acid stain. The granulocytes are classified according to their staining reactions, and the size of the granules.

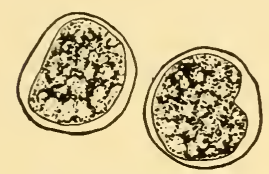

A. Lymphocyte

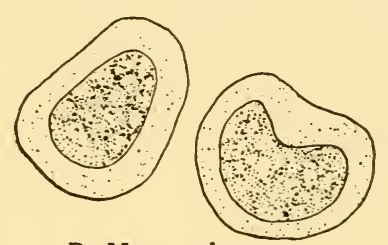

B. Mononuclear

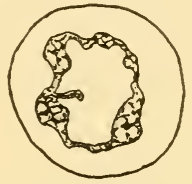

C. Neutrophile

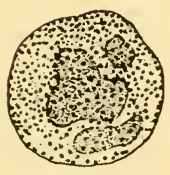

D. Mast

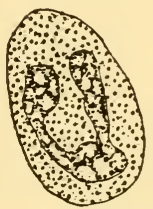

E. Acidophile
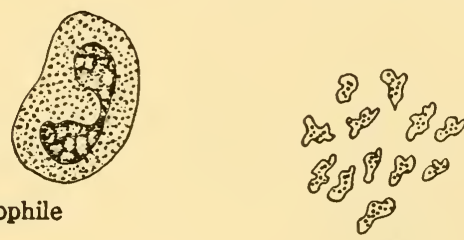

Fig. 137. Mammalian Leucocytes and Blood Platelets.

The proportion of leucocyte types is of clinical value, since the blood count indicates different physiological conditions. Most of the white cells are phagocytic, and therefore have a definite function in preventing infections. Pus is made of dead white cells and serum. In the human there are approximately 8,000 leucocytes in each cubic millimeter of blood.

ERYTHROCYTES. The red cells contain haemoglobin which has a strong affinity for oxygen, the combination of the two being an unstable chemical compound. Due to this instability the oxygen is liberated in the capillaries and passed to the tissues. Internal respiration depends upon the amount of oxrgen carried to the cells, and insufficient oxygenation may be caused either 
by a lack of haemoglobin in each cell, or by a lessened number of cells in the blood. In the normal human there is an average of four and a half to five million erythrocytes in a cubic millimeter of blood. There is a slight sex difference, the female averaging less than the male.

Erythrocytes vary markedly in structure in the different vertebrate classes. With the exception of mammals all vertebrates have nucleated red cells, and most of these are elliptical in shape. The largest known are found in the urodele amphibia. Although essentially the same shape in the reptiles and birds they grow progressively smaller and more numerous.

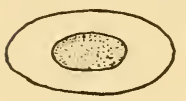

A. Fish

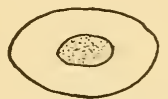

B. Amphibian

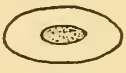

C. Lizard

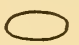

D. Camel

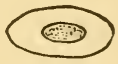

E. Bird

FIG. 138. Erythrocyte Types. Diagram (D) shows the enucleated, oval erythrocyte of the camel.
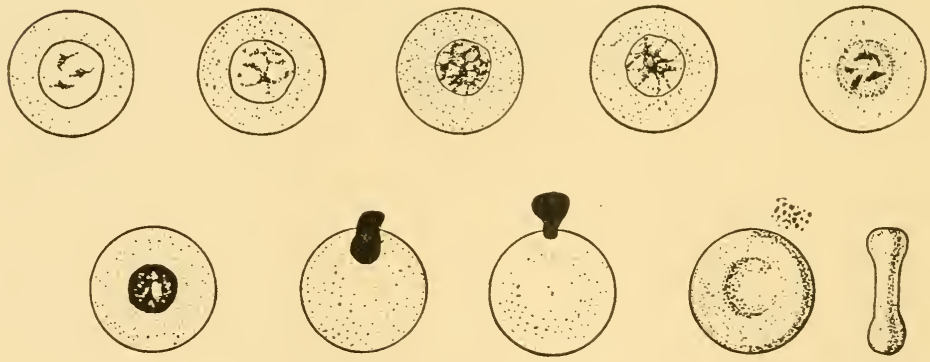

Fig. 139. Derelopment of Mammalian Erythrocytes. Diagrams illustrating the changes which occur in the marrow of the long bones; a surface view of a red cell with fragmented nucleus; and a side view of an erythrocyte.

All normal adult mammals have red cells without nuclei. These cells, as in all bony vertebrates, arise in the marrow of the long bones. In their early state these cells have nuclei, and are carried into the blood stream of the cmbryo as nucleated cells. But before birth (or shortly after in some species) the erythrocytes begin losing their nuclei before entering the blood. The nucleus of the maturing red cell is thrown out and disintegrates, the 
enucleated cell passing into the capillary. The functional life of the cell is evidently very short.

The erythrocytes are disc-shaped and bi-concave, and their size varies in different groups of mammals. Microscopic examination of the blood can often determine the genus from which it was taken, even in a dried specimen. The usual circular shape of the mammalian erythrocytes has an exception in the camel and its relatives. In these animals the red cells are elliptical in shape, although they agree with all other mammals in lacking nuclei. In pathological conditions any mammal may throw nucleated cells into the blood stream but these are rarely, if ever, numerous. 


\section{CHAPTER XVI}

\section{THE URINOGENITAL SYSTEM}

THE urinary and genital systems are considered as a unit in vertebrate anatomy because of their close association. Physiologically they are entirely different; but certain structures are common to both systems, and during the course of evolution some excretory (urinary) ducts became a part of the reproductive system. In the lower chordates there is no connection between the two systems, but in the cyclostomes an association begins and becomes progressively closer in the more specialized classes.

The essential structures of excretion and reproduction are (1) nephridia which collect wastes from the body and pass them to the outside, and (2) gonads in which the reproductive cells (ova and spermatozoa) develop. With the exception of the lower chordates there are always ducts for the conduction of wastes and reproductive cells to the outside. In the higher groups structures may develop (1) for the storage of wastes; (2) to permit the fertilization of the ora while they are still in the maternal body; and (3) for the retention of the fertilized ova during their development.

The nephridium is the functional unit of excretion. There is no evidence of homology between the nephridia of invertebrates and those of the vertebrates, although there is a functional similarity. In the primitive condition each is an independent structure composed of a head within the coelomic cavity, a short tubule for the conduction of wastes, and an excurrent pore opening on the outside of the animal. The head has a funnelshaped opening, the nephrostome, surrounded by cilia which beat in toward the lumen of the tubule. The nephridia are paired, metameric structures. This completely primitive condition is not found in any of the living chordates, but is present in some in- 
vertebrates. Amphioxus has retained the primitive metamerism and position of the nephridia, but the coelomic head differs from that found in the higher invertebrates and lower vertebrates. Instead of a nephrostome the head is terminated by numerous minute closed knobs. The tubules are short and each opens clirectly to the outside.

The gonads of the vertebrates are paired mesodermal glands, primitively located in the anterior part of the body, dorsal to the peritoneum. The female gonad is the ovary, that of the male is the testis. The sexes are separate in the majority of vertebrates, an individual developing only ova or spermatozoa. The cyclostomes and some of the teleost fishes, however, develop eggs and sperms in the same animal. The condition is called hermaphroditism. Although isolated cases have been described in the higher groups, most of the specimens are aberrant males or females which have some of the characters of the opposite sex.

\section{A. Organs of Excretion}

The earliest vertebrate nephridia do not open independently to the outside but enter a pair of pronephric ducts. The tubules of the nephridia begin in the mesodermal somite at the junction of the epimere and hypomere, in the region called the nephrotome (see illustration on page 68). The tubules on either side grow posteriorly and unite to form a pronephric duct. At the end of the pronephric region the duct continues its growth toward the posterior outlet. As the coelomic cavity develops the first vertebrate kidney, or pronephros, makes its appearance in the anterior part of the animal. The pronephridia are metameric and few in number. Nephrostomes grow toward the coelomic cavity and break through the peritoneum to enter the cavity. The two pronephric ducts parallel each other to a point near the anus, where they fuse to form a slight enlargement and leave the body through a single opening.

The excretion of wastes from the blood into the coelomic cavity is secured by small metameric arteries. These paired renal (kidney) arteries pass laterally and ventrally to the peritoneum where they coil and form a glomerulus, a knot of thin-walled capillaries. The glomerulus pushes into the coelomic cavity but 

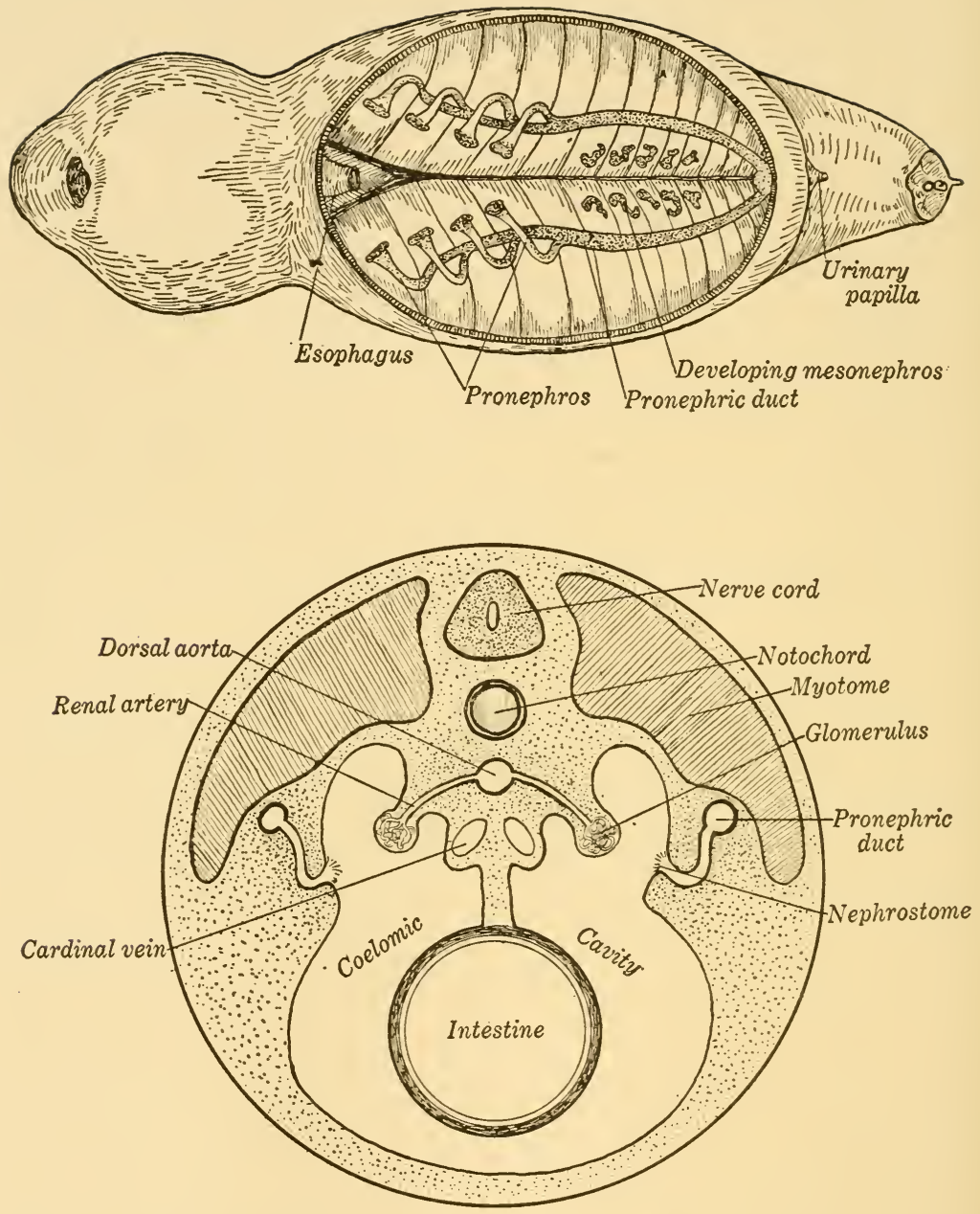

Fig. 140. Pronephros. Top, tadpole with body wall and riscera remored to show the position of the pronephros and pronephric ducts. The metameric mesonephros is shown beginning its development. Bottom, a diagrammatic cross section of a dogfish embryo, showing the structure and position of the pronephros. 
remains covered by the peritoneum, the waste materials being forced into the cavity by osmosis and blood pressure.

It will be seen that there is no direct connection in the pronephros between the artery and the nephridium. A physiological unity is attained by a slight longitudinal constriction of the cavity, forming a dorsal pocket which remains open to the main body of the coelom. Wastes passing into this pocket are collected by the open nephrostomes, and then enter the pronephric ducts to be conveyed out of the body. The pronephros is present in the embryos of all vertebrates. It functions in the young of the cyclostomes and is partially retained by the adult. It is also functional in those fish which pass through a larval stage (for example the crossopterygians and dipnoans) and in the tadpoles of the amphibia. It probably does not function in the embryos of amniotes. The pronephros is soon replaced by the mesonephros.

Mesonephros. The mesonephros develops posterior to the pronephros and is serially homologous with it. The mesonephridia are rapidly surrounded by connective tissues, and due to a process of budding of the primary tubules to form others the metameric character is lost. The mesonephric tubules do not develop a new pair of ducts but enter the pronephric ducts, and when the pronephridia degenerate it is called the mesonephric duct.

The most important advance is the development of a connection between the glomerulus and the excretory tubule. The renal arteries do not grow in toward the peritoneum but parallel with it, and when the glomerulus forms it is not in contact with the coelomic cavity. The glomerulus is met by a branch of the mesonephric tubule. This accessory tubule begins as an outpocketing from the original tubule at a point about halfway between the nephrostome and the entrance to the main duct. The branch grows dorsally and its distal end enlarges into a vesicle which presses against the glomerulus. The vesicle then invaginates, grows around the knot of blood vessels and practically encloses it, forming a Bowman's capsule. In this way the waste products are passed directly into the kidney tubules and not into the coelomic cavity; but any products of excretion which may be in the cavity are eliminated by the nephrostomes. The glomerulus 

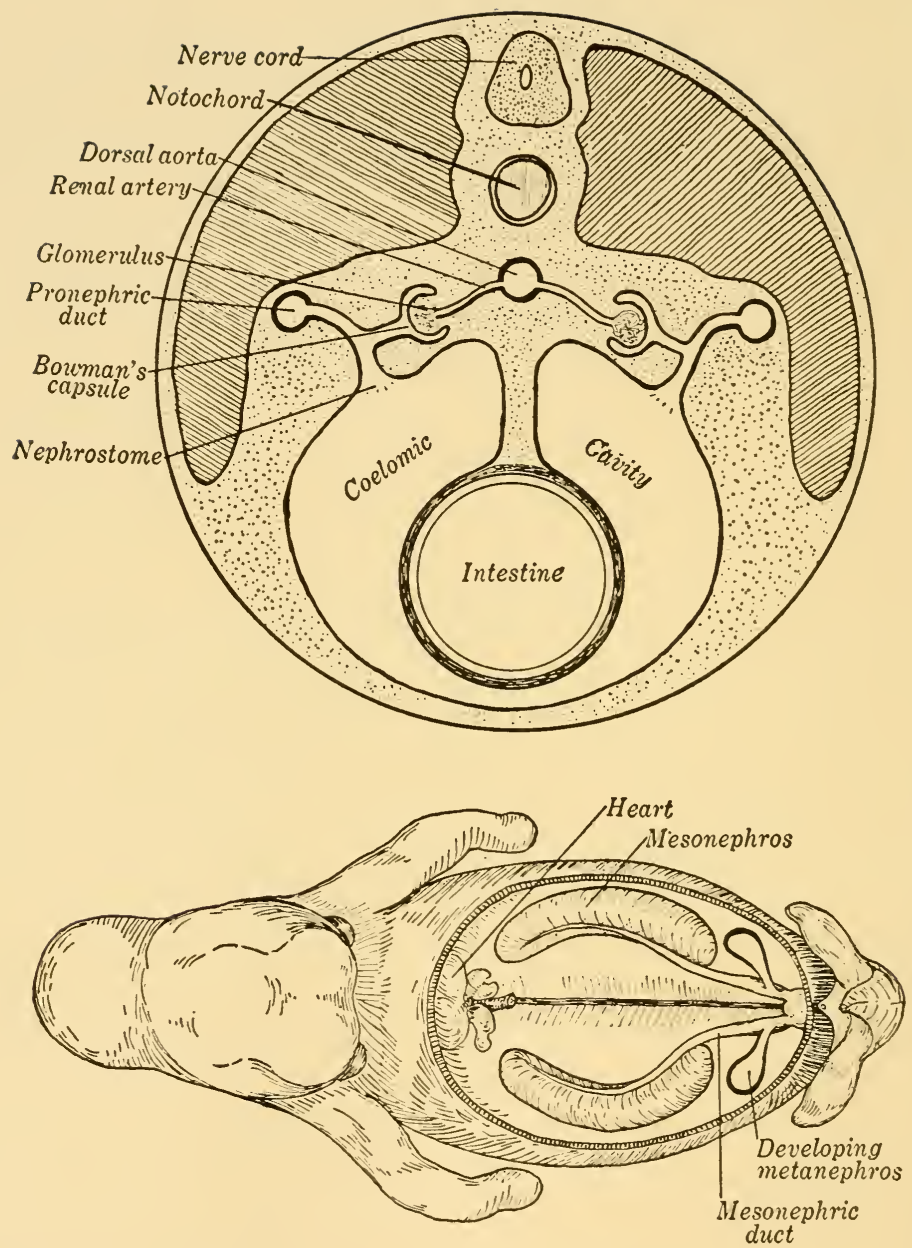

Fig. 141. Mesonephros. At top. diagrammatic cross section of amphibian embryo to show structure of mesonephros. At bottom, embryo of man with body wall and riscera remored to show mesonephros, with the metanephric outpocketings from the mesonephric ducts. 
and the Bowman's capsule together are known as a renal or Malpighian corpuscle. In amniote embryos the nephrostomes rarely, if ever, break through into the coelomic cavity.

The mesonephros is the functional kidney of the cyclostomes, fish and amphibia. It appears as a large body in amniote embryos and was first described by the anatomist Wolff. As a result of his discovery the mammalian mesonephros is known as the Wolffian body, and the mesonephric duct as the Wolffian duct. In the amniotes the mesonephros is merely a transitory embryonic

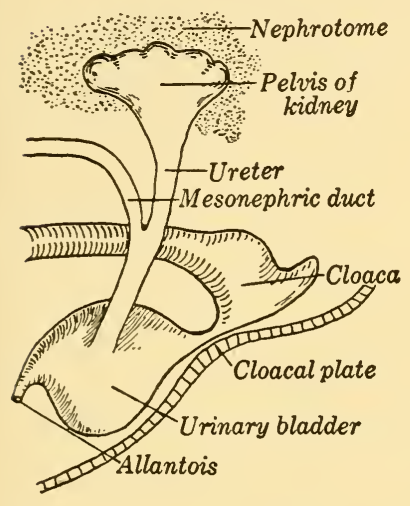

Fia. 142. Derelopment of the Metanephros. The metanephric outpocketing is shown in contact with the nephrotome. Compare

Fig. 118 for the earlier stages.

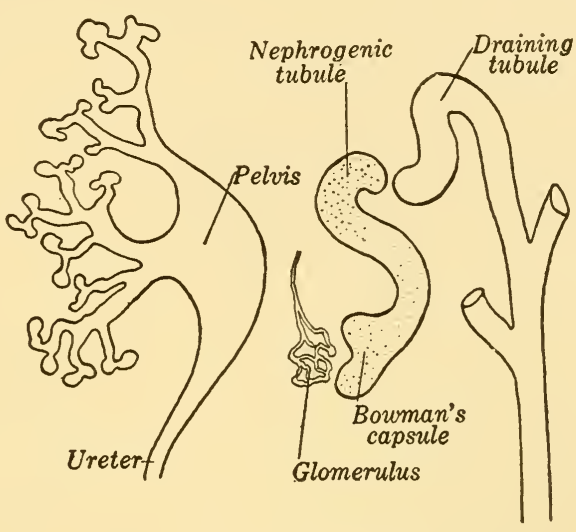

Fig. 142-a. The Derelopment of Collecting Tubules. The pelvis divides, the tubules joining the nephrogenic tubules (right). (From preparations by R. T. Kempton).

structure. A part of it, however, remains in the male in connection with the reproductive system. The metanephros is the functional kidney of the amniotes.

Metanephros. The metanephros and its draining ducts have a double origin. The renal corpuscles and the outer ends of the tubules arise from the primitive nephric anlagen, the nephrotomes, and are serially homologous with the mesonephric and pronephric kidneys which arise more anteriorly. The ureters and collecting tubules which drain the kidneys arise as paired outpocketings from the posterior ends of the mesonephric ducts. Immediately anterior to the fusion of the mesonephric ducts an 
evagination arises on either side and grows anteriorly toward the developing metanephros. The anterior end of each outpocketing enlarges and branches. Due to continued division the tubes become smaller and greatly multiplied until the terminal branches become the collecting tubules. These grow into and are surrounded by the nepliric tissues. The tubules of the renal corpuscles have been developing synchronously, each renal

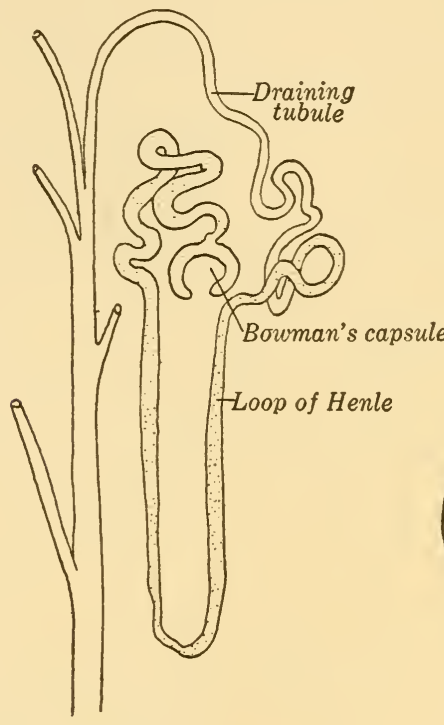

A. Single Tubule
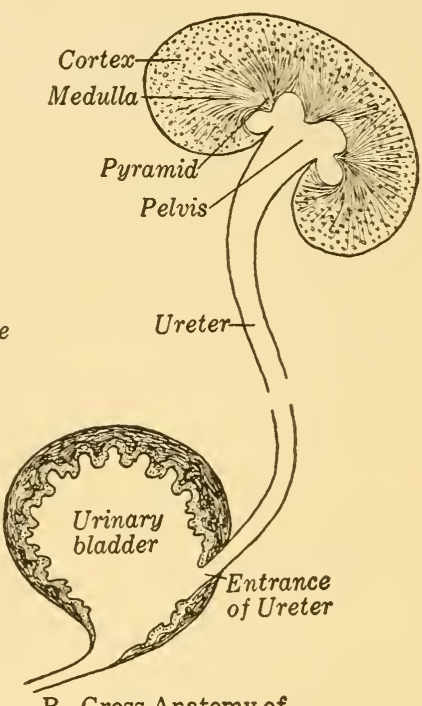

B. Gross Anatomy of Urinary Organs

Fig. 143. Structure of the Kidney. (A) A single tubule with the branching collecting tubule. (B) The gross anatomy of the kidney and bladder in long section.

tubule developing from a small vesicle which invaginates at one end to form a Bowman's capsule, and elongates at its other end to form a kidney tubule. The tubules from the Bowman's capsules unite with the collecting tubules, and the connection is completed.

The amniote kidney is therefore composed of an outer cortex from the nephrotome, and an inner medulla which arises from the branching ureter. The collecting tubules join to form larger tubes, and these empty into the pelvis of the kidney. The latter 
is an enlargement formed at the original point of branching. The pelvis narrows into the ureter which passes backward to its outlet from the animal's body.

The major differences between the metanephros and the mesonephros are: (1) the loss of the nephrostome; (2) the complete loss of metamerism in its origin; (3) the loss of a metameric blood supply and the development of paired renal arteries which leave the dorsal aorta and reach the pelvis before dividing; and (4) the development of new draining ducts and collecting tubules.

URINARY BladDER. The urinary bladder is homologous with the allantois. The latter develops as a ventral outpocketing from the posterior gut, and its proximal portion is incorporated into the cloaca. An allantoic bladder develops first in the amphibia; but in the embryos of these animals the body wall is continuous (enclosing the yolk sac and allantois) and the allantois is limited in size. In the adult amphibian this bladder is a posterior pocket from the cloaca and the urinary products fall into it as they pass from the mesonephric ducts.

In the reptiles and birds the allantois grows out as the embryonic breathing and excretory organ. When the body stalk closes after hatching, the proximal end of the allantois remains as a distinct urinary bladder which has the same relative position as in the amphibia. In the birds (with a few exceptions) the bladder is merely a division of the cloaca, known as the urodeum. The structure of the cloaca and bladder of the monotreme mammals is similar in all essentials.

The marsupials and placentals develop an allantois which is similar in development to that of the reptiles and birds; and, as in the other amniotes, it is the proximal end of this outpocketing which develops into the urinary bladder. In both groups during embryonic life the cloaca is present with its allantoic pocket, the anus and urinogenital ducts opening into it; but as development proceeds a constriction cuts the ventral bladder from the more dorsal rectum. See diagram on page 255 . The ureters enter the bladder. The urethra which carries wastes from the bladder to the outside is joined by the genital ducts of the male. In the female the urinary and genital outlets are separate in all vertebrates above the cyclostomes. 


\section{B. Development of the Urinogenital Systent}

The simplest urinogenital system among the rertebrates is found in the cyclostomes. In this class the mesonephros, and the few retained pronephric tubules, are drained by the mesonephric ducts which pass posteriorly and unite to form a urinogenital sinus which has a genital pore on either side. Consequently the coelomic cavity is connected with the outside of the body by the pores which open from the coelom to the sinus, and then to the outside through the urinogenital duct. The reproductive cells as they mature in the gonad break through the closely adherent peritoneum and fall into the coelomic cavity. With the movement of the animal they pass posteriorly, through the genital pores into the sinus, and out into the water with the urinary wastes. Fertilization is external. The system is structurally identical in the two sexes, the difference lying in the type of reproductive cells which are produced; and, although the same animal may produce both sperms and eggs, in most cases these mature at different times and self fertilization does not occur.

Dogfish. The embryo of the dogfish shows the fundamental vertebrate plan of the urinogenital system. There are two important changes from the system as found in the cyclostomes: (1) the genital pores lose their connection with the sinus, and remain as apparently functionless abdominal pores connecting the coelomic cavity with the cloaca; and (2) the pronephric ducts split longitudinally to form two ducts, (A) the mesonephric ducts and (B) a new pair of ducts, the Müllerian or oviducts. Each oviduct has an anterior ostium opening into the coelomic cavity, and a posterior outlet into the cloaca. The mesonephric tubules, as in the more primitive condition, drain into the mesonephric duct. This undifferentiated stage is found in the embryos of both sexes.

As development proceeds the female shows no material change. The Wolffian (mesonephric) ducts continue to drain the kidneys; they fuse posteriorly, and open to the outside through a urinary papilla which projects into the cloaca. The oviducts develop wide ostia in the anterior part of the coelom, and in Squalus these fuse in the adult to form a single opening. As the 


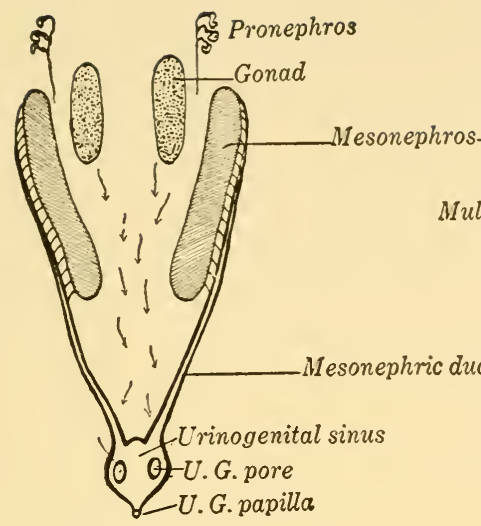

A. Cyclostome*

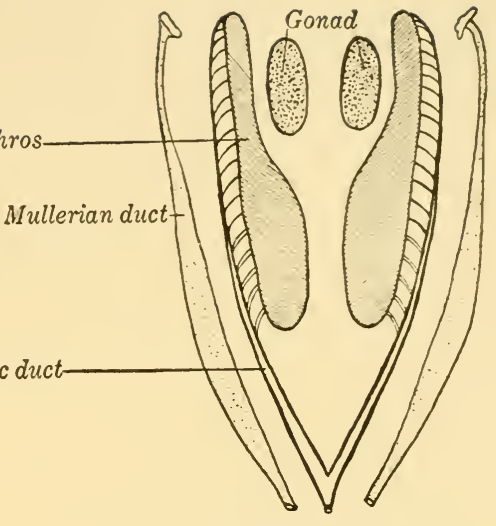

B. Undifferentiated Dogfish Embryo

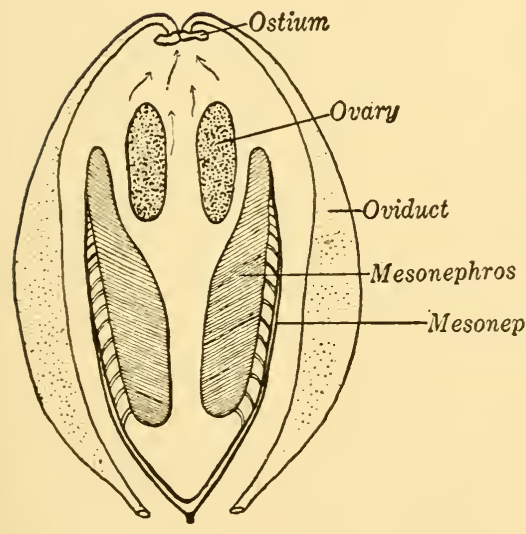

C. Female Dogfish

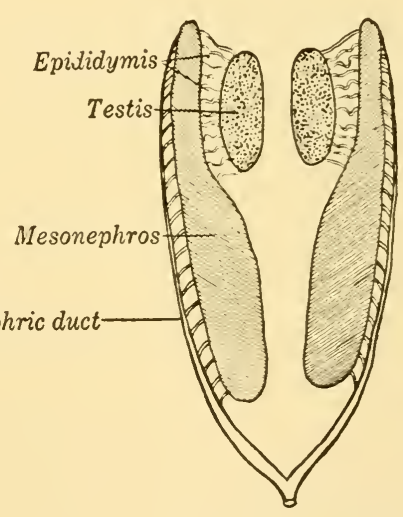

D. Male Dogfish

Fig. 144. Urinogenital System of Anamniotes. The Cyclostome condition is shown in (A), the arrows indicating the course of the reproductive cells from the gonads to the urinogenital pores. Drawing (B) is the undifferentiated stage in the Fish and Amphibia, with the definitive structure of the female (C) and the male (D). 
eggs break from the ovaries they enter the ostia and pass into the oviducts. The dogfish has internal fertilization and the eggs remain in the oviducts until the embryos are capable of independent life. This is true of most sharks. In the skates, rays, and most teleosts the eggs are thrown into the water and fertilized externally.

The male dogfish undergoes more radical changes during development. The testes remain in the primitive anterior position, and the mesonephric tubules of the anterior (sexual) portion of the kidney grow through the mesonephros and enter the gonad. The Wolffian ducts, therefore, function as both urinary and genital outlets. The papilla through which the united ducts leave the body is called the urinogenital papilla. The Müllerian ducts degenerate, and disappear entirely except for a small, vestigial mass of tissue.

The system as described for the dogfish is typical of that of the fish and amphibia. In the vertebrates above the Elasmobranchs the oviducts arise parallel to the Wolffian ducts, but from the same anlage. The essential changes during development are the same. In those fish which retain their eggs during development (the ovo-viviparous condition) the male develops specialized intromittent organs for placing the sperms in the oviducts, so that internal fertilization may take place. A few amphibia have internal fertilization but deposit their eggs in an early stage of cleavage; but in this group the sperms are usually deposited in capsules, and the female has a specialized mechanism for taking up the sperm capsules.

Anniotes. Amniote embryos develop oviducts and mesonephric ducts similar to those of the fish and amphibia. A slightly generalized drawing of the undifferentiated embryonic stage would apply equally to any vertebrate above the cyclostomes. Amniote embryos add, however, the metanephric kidney and the mesonephros gradually degenerates. In both sexes the Wolffian ducts lose their excretory function.

The female amniote loses the mesonephros and the mesonephric ducts, a small fragment of tissue remaining in the mesentery as a vestigial body. The Müllerian ducts remain as the oviducts and undergo various modifications in correlation 
with the reproductive habits of the animal, which may be (1) oviparous or egg laying; (2) ovo-viviparous, the retention of large-yolked eggs until the embryo develops; or (3) viviparous. In the last case the oviducts are specialized as uteri. The urinogenital system then consists of two practically independent systems: (1) the ovaries and oviducts; and (2) the kidney, ureter, allantoic bladder, and urethra.

The male amniote loses the Müllerian ducts during development, a small posterior fragment remaining as the prostate
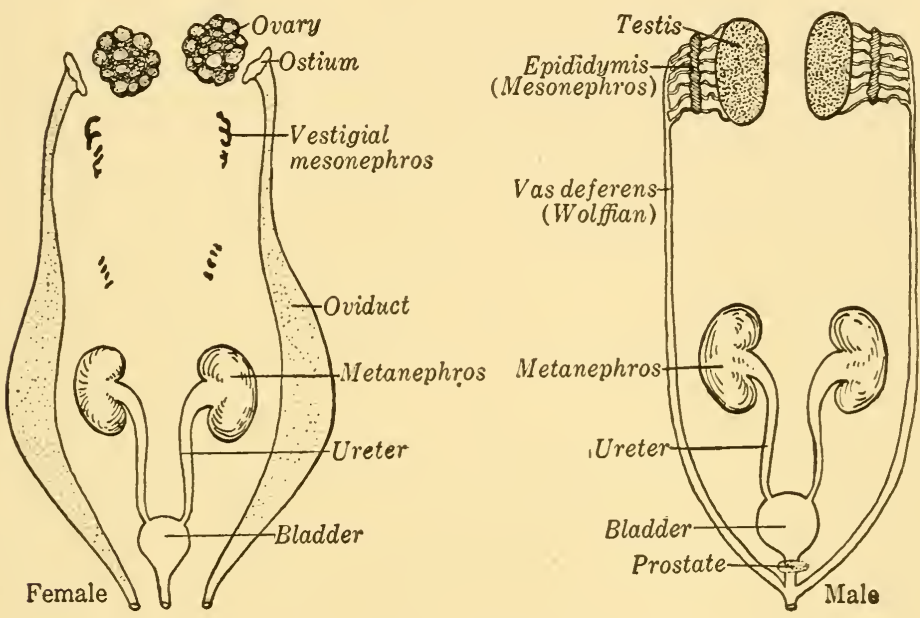

FIG. 145. Structure of the Amniote Urinogenital System. Diagram (B), Fig. 144, shows the undifferentiated stage with both Müllerian and Wolffian ducts.

gland which secretes a part of the seminal fluid. The metanephros carries on the excretory function and the posterior portion of the mesonephros disappears. The anterior portion of the mesonephros, which functions in the fish and amphibia as a sexual kidney, and the Wolffian duct remain in the amniotes as the efferent tubules and ducts of the testis. As in the anamniotes, the tubules burrow through the anterior part of the mesonephros into the testis, and these structures remain in the male after the mesonephros degenerates. The mass of tubules in 
connection with the testis is the epididymis, and the Wolffian ducts are the vasa deferentia (vas deferens is the singular).

All amniotes have internal fertilization, and a number of intromittent structures have developed. In the turtles, crocodilia, a few birds, and all mammals the copulatory organ is the penis which is homologous throughout these groups. The testes remain in the primitive position in the reptiles, birds, monotremes and a few placentals; the descent of the testes is described in the following section.

\section{Reproductive Structures of the Mammals}

The above description is a generalized discussion of the amniote system and applies particularly to the reptiles, birds and monotremes. The last, although mammals, are typically reptilian in their reproductive organs, both sexes retaining the cloaca, and the female having oviducts with separate openings on either side of the urinary papilla. In the marsupials and placentals the cloaca disappears and other genital structures make their appearance.

Female. With the division of the cloaca and the separation of the digestive and urinogenital tracts, the female mammals develop labia, or lips, guarding the external orifice of the urinogenital openings. These, particularly in the higher mammals, are double, consisting of outer and inner labia. Dorsally, within the labia, is the opening of the ragina which develops largely as a fusion of the posterior ends of the Müllerian ducts. Ventral to this is the single urethral opening; and at the ventral union of the inner labia is a small clitoris which is homologous with the penis of the male.

The greatest modification occurs in the evolution of the uterus, which retains the minute ova during development of the embryos. The union of the oviducts is very incomplete in the marsupials, with the result that the vagina is paired or separated by a tissue septum, which causes each half to persist as an almost independent unit. At the anterior end of each hemivagina there is a marked constriction with a cervical opening into the uterus. Sperms deposited in the vaginae during coitus 


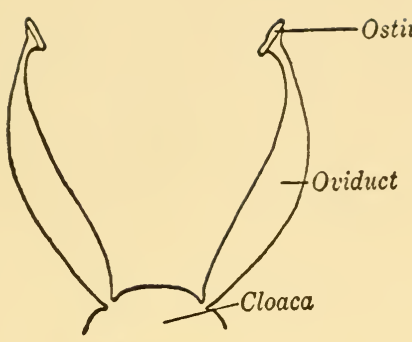

A. Monotreme

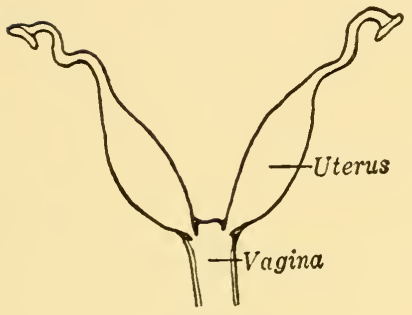

C. Rodent

DUPLEX UTERUS

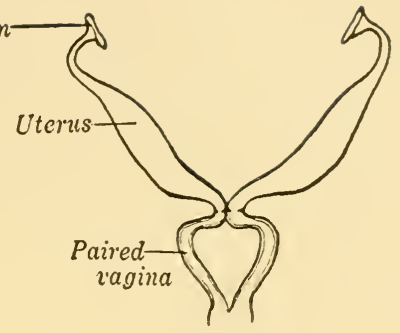

B. Marsupial (Opossum)

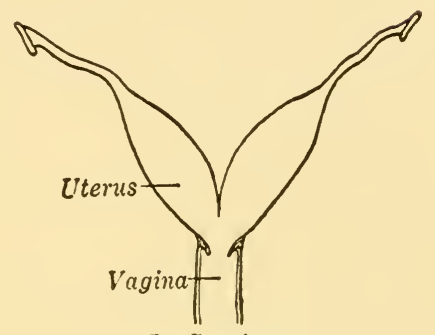

D. Carnivore BIPARTITE UTERUS

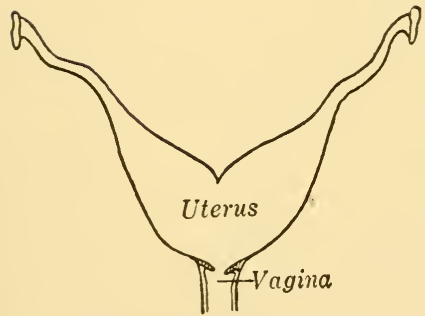

E. Artiodactyl BICORNUATE UTERUS

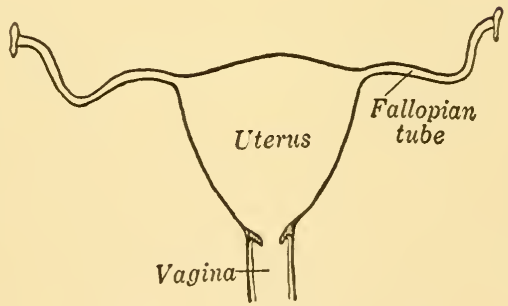

F. Human

SIMPLEX UTERUS

FIg. 146. Comparative Anatomy of the Oriducts and Uterus. Observe the loss of the cloaca and the fusion of the oriducts to form a ragina and uterus. 
pass through the cervical opening into the uteri and fertilize the ova in their anterior ends.

The vaginal septum disappears in the placentals. However, in the more primitive orders (insectivores and rodents) the two uteri are distinct and each has a cervical opening into the vagina. This is a duplex uterus. Further fusion of the two ducts forms a bipartite uterus (found in carnivores and certain families of other orders) with a single cervical opening, but the two uteri united only at their posterior ends. The bicornuate uterus occurs in horses, sheep, cors, and most other herbivorous animals, and is functionally a single uterus. The majority of animals of this type have only one embryo developing at a time and it occupies a median position in the uterus. Displacement occurs when twins develop. Mammals with duplex or bipartite uteri more frequently have a number of offspring at a birth.

The simplex uterus is a further step in the evolution of the organ, and is characteristic of the anthropoids and man. The uterus has a single median carity, with the small oviducts (Fallopian tubes of human anatomy) passing from the lateral ovaries to the uterus. The uterus is roughly triangular in shape, the apex being at the cervix.

Male. In the monotremes the seminal fluid containing the spermatozoa from the testes is thrown into a common urinogenital sinus and conducted through the grooved penis. The latter lies within the cloaca, but is protruded during coitus.

Two paired erectile bodies (the carernous bodies) are added to the penis in the marsupials, and the lateral walls of the groove grow medially to form a tube. The tube is in the unpaired median body of the penis, and is functionally a continuation of the urethra. A cross section of the organ appears as a single rentral body containing the urethra, and paired erectile bodies. The penis with its muscles is attached to the ischial bones, and distally is enclosed within a sheath of cutaneous tissue. The urethra opens through the glans, an enlargement of the median body.

The position of the penis shifts in the upper groups of mammals. In the monotremes it lies within the cloaca and closes the anal opening as it protrudes. With the disappearance of the 


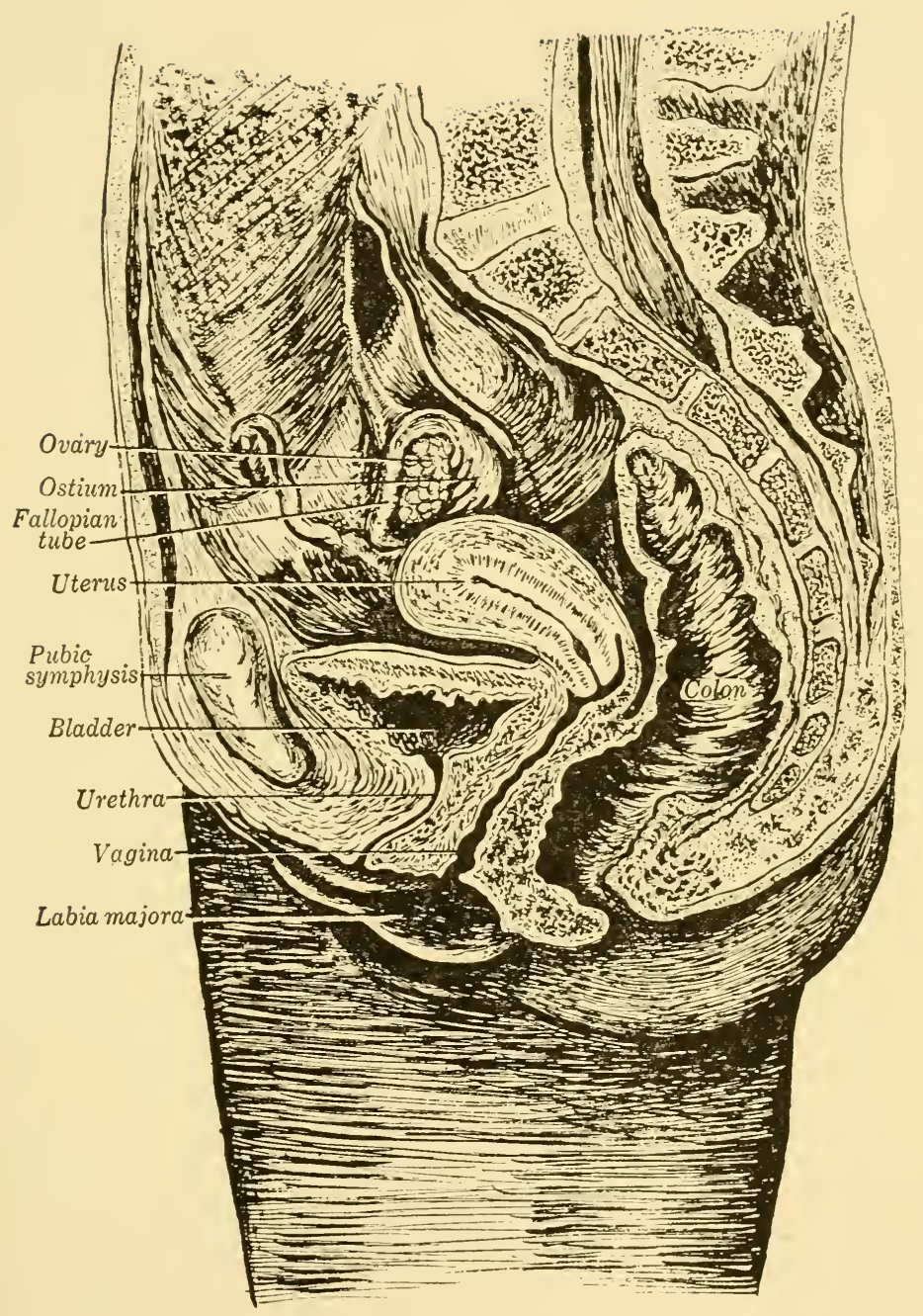

FIG. 147. Sagittal Section of Human Female. 
cloaca in the marsupials, the penis becomes enclosed within its own sheath, and projects posteriorly from its attachments on the pubic and ischial bones. The organ of the placentals is structurally the same, although it projects anteriorly from its

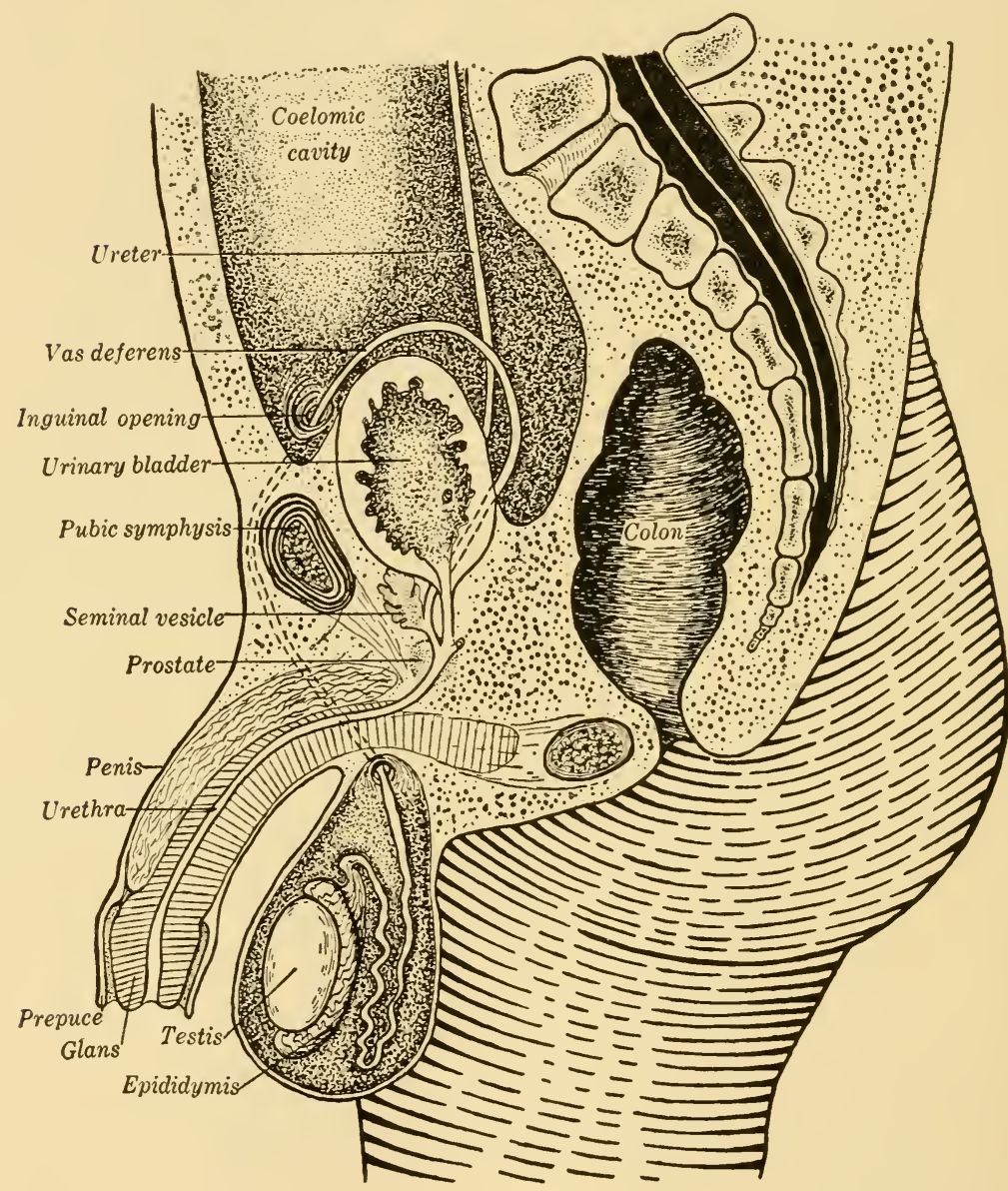

FIG. 148. Sagittal Section of Human Male.

origin on the pelvis. In the majority of the placentals the organ is enclosed within a preputial sheath and is protruded when the erectile bodies are engorged with blood. The penis is pendant (not enclosed within a sheath) in the bats and primates. 
Descent of the testes. The primitive position of the testes is within the body; but in the marsupials and the majority of placentals the testes descend into a scrotal sac which is homologous with the external labia of the female. The testes do not descend in the elephants, whales and sirenia, but this is evidently a specialization. In other mammals the sperm cells will not mature when kept in the body cavity. Descent of the testes is seasonal in many rodents, the glands remaining within the body except during the mating season; in some the testes may be withdrawn at will from the scrotum; while in others the connection between the scrotum and the body cavity is lost and the testes remain in position after descent.

The gonads arise in a retro-peritoneal position and are covered by the somatic layer of the peritoneum. At the posterior end

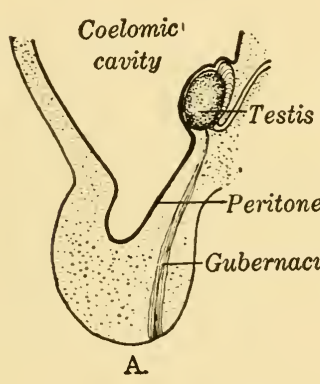

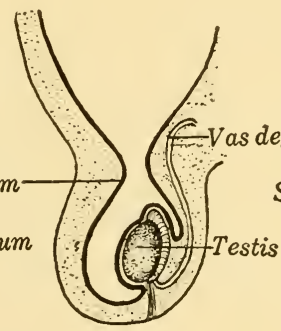

B.

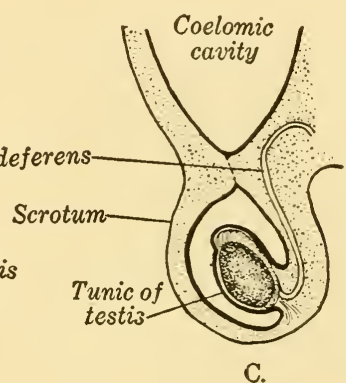

C.

Fig. 149. Descent of the Testes in the Mammal. As the testes descend from their body position, a pocket of the coelomic cavity is drawn into the scrotum. In some mammals the connection with the body cavity remains open as in (B); the connection is lost in man. (C).

of the embryonic gland there is a gubernacular ligament which passes from the testis to the scrotum. As growth proceeds the testes are drawn posteriorly, and the peritoneal covering is carried with them as the tunic of the testes. Shortly before (or after) birth in the human the testes pass through the openings of the body wall into the scrotum. The inguinal canals through which the glands pass are located in the groin, and the testes leave the body anterior and ventral to the pubic bones, passing between the pelvis and the skin. A pocket of the coelomic cavity is carried into the scrotum with them, and the somatic mesoderm 
comes in contact with the scrotal wall and develops the muscular lining of the sac. Each vas deferens passes from the epididymis over the pubic bone, through the inguinal canal, and around the urinary bladder to its entrance into the urethra.

The semen develops as a fluid medium for the sperms. These cells are motile and live best in an alkaline medium. Part of the fluid is secreted by the tubules of the testis and epididymis, but the major portion is secreted by several sets of glands located around the urethra. The prostatic fluid is strongly alkaline and neutralizes the urethral passage. Near the prostate are the paired seminal vesicles, and more distally, the small Cंowper's glands. Small lubricating glands, which have no connection with the seminal fluid, are found near the glans.

\section{Differentiation of Sex in the Human}

Except for the fundamental difference in sex as determined by the chromosomes at fertilization, much of sex differentiation is controlled by the secretion of the ductless glands. Sex characters are divided into the primary characters, those structures which are directly related to the reproductive function; and the secondary characters, which develop later and are simply manifestations of sex difference. The growth or degeneration of the different internal reproductive structures has been discussed. The following is limited to the external organs.

In the early, undifferentiated, human embryo the cloaca is present and the external orifice is bounded by nearly circular folds. Near the ventral margin of the fold appears a genital tubercle resembling a small papilla. Synchronously with the growth of this tubercle the anal opening is separated from the urinogenital region. The tubercle grows larger and the lateral folds become more prominent. At this stage the sexes are still externally undifferentiated.

From this time on the sexes rapidly take on their typical characteristics. In the male the tubercle enlarges and is joined by two small inner folds. The latter develop into the erectile or cavernous bodies of the penis. The tubercle forms the urethral body and the glans. The larger, outer, genital folds push ventrally, unite, and form the scrotum into which the testes descend. 


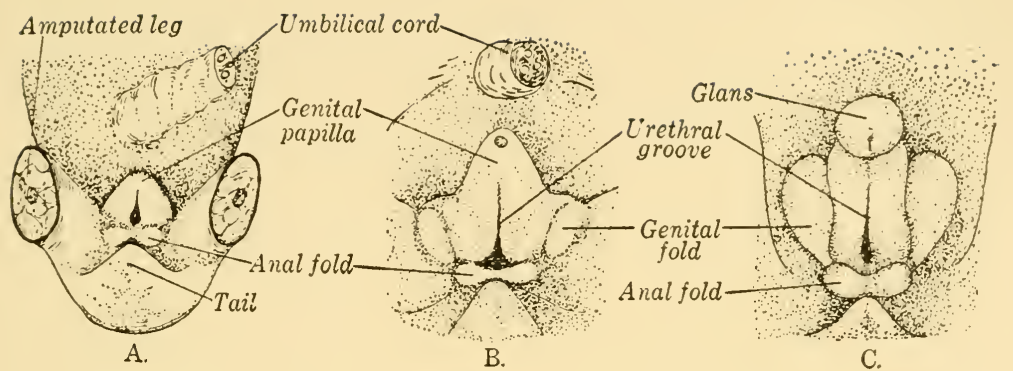

Fig. 150. Undifferentiated Stages of the External Genitalia in Man. (Adapted from Otis).
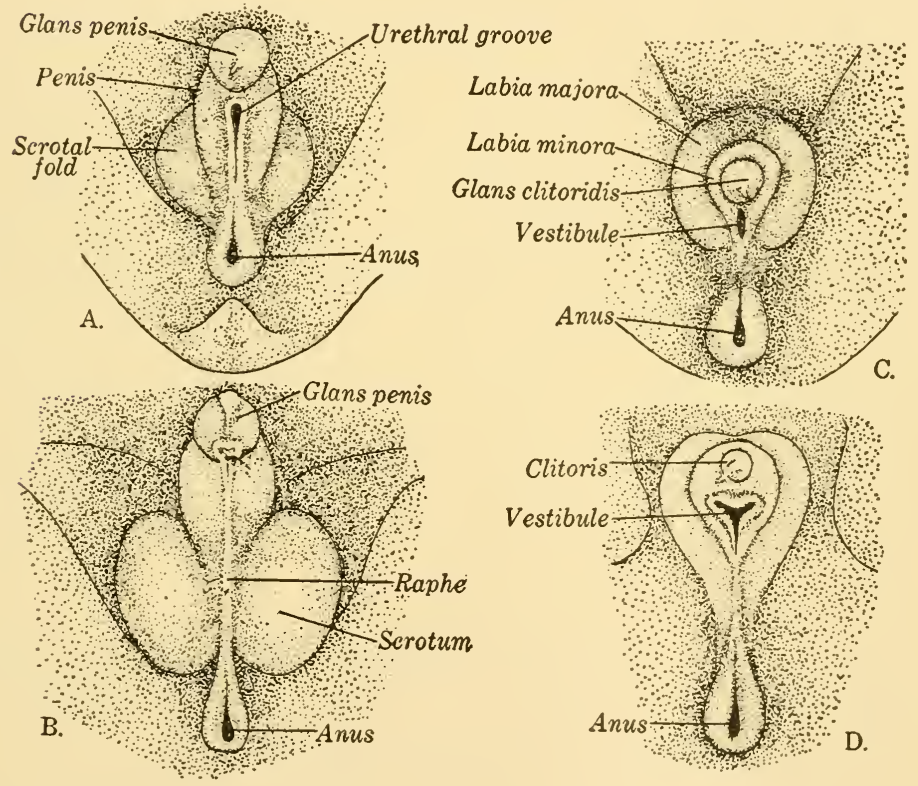

Fig. 151. Differentiation of Sex in Man. Diagrams (A) and (B) show the development in the male; diagrams (C) and (D) are the female. Compare undifferentiated stages in Fig. 150. (Adapted from Otis). 
The female is less differentiated from the embryonic condition. The tubercle remains as the small ventral clitoris; the primary genital fold forms the outer labia (labia majora); and the inner folds develoy into the inner labia (labia minora). The vaginal and urethral openings are enclosed by the paired labia.

\begin{tabular}{c|c|c}
\hline Embryonic Structure & \multicolumn{2}{|c}{ Definitive Structure } \\
\hline Undifferentiated & Male & Female \\
\hline Primary genital fold & Scrotum & Labia majora \\
\hline Inner genital fold & Erectile bodies & Labia minora \\
\hline Genital tubercle & Urethral body & Clitoris \\
\hline
\end{tabular}

These changes occur at an early stage in embryonic life, and further differentiation is a matter of slow growth until the time of puberty. At birth the sexes are practically identical in body form, but at the end of one or two years the child begins to assume the pectoral and pelvic shape characteristic of the sex. 


\section{CHAPTER XVII}

\section{NERVOUS SYSTEM}

THE nervous system is composed of cells specialized for the transmission of impulses, and acts as the coordinating mechanism of the body. Stimuli from one region are transmitted to other regions, and througl the function of the nerves the body is made to respond as a unified whole. So closely are the muscles correlated with the nerves that the two are physiologically grouped as the neuro-muscular system. Fven the involuntary bodily reactions are regulated by the nervous system.

\section{A. Development}

Nerve tissues are derived from the ectoderm along the dorsal portion of the embryo. The nervous system begins as a neural groove anterior to the blastopore (page 23). The lateral ridges of the groove grow medially until a tube is formed. The development progresses more rapidly at the anterior end, with the result that the brain becomes specialized into regions before the posterior end of the groove has closed to form a tube. As the neural tube

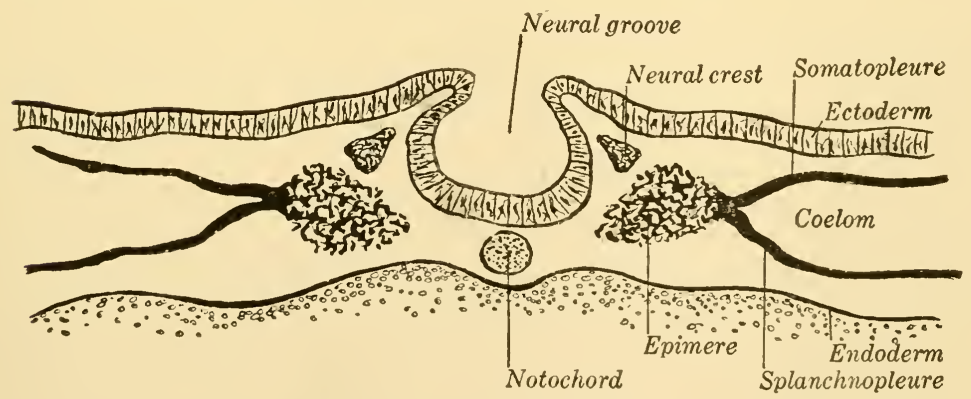

Fig. 152. Cross Section of Chick Embryo. 
separates from the covering ectoderm, small metameric masses of ectodermal tissue develop on either side of the tube along its dorsal periphery. These are the neural crests which give rise to the sensory cells of the metameric spinal nerves. This is the developmental beginning of the division of the nervous system into (1) the central nervous system composed of the brain and spinal cord; and (2) the peripheral nervous system. The latter includes the nerves and ganglia which develop from the central system and connect it with the organs of the body.

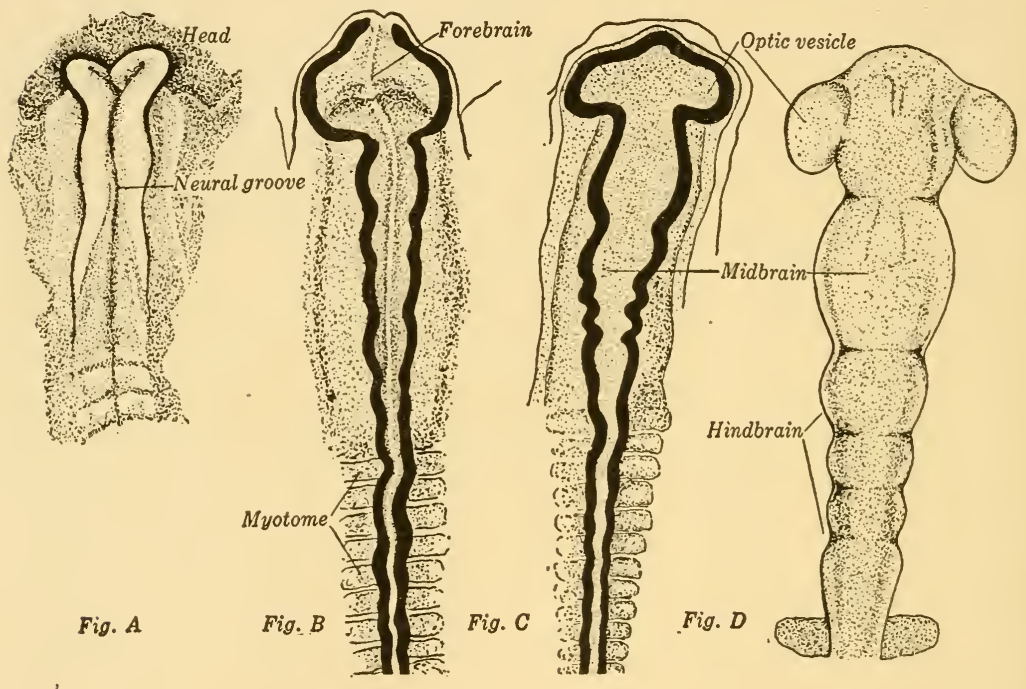

Fig. 153. Development of the Brain (Chick).

The brain and spinal cord are continuous, structurally and functionally, and the separation of the two is entirely arbitrary (the foramen magnum being accepted as the dividing line). The brain grows rapidly, two constrictions dividing it into three major regions: (1) the forebrain (prosencephalon); (2) the midbrain (mesencephalon); and (3) the hindbrain (rhombencephalon). From the forebrain arise the olfactory lobes, the cerebral hemispheres, and the diencephalon. The midbrain develops into the optic lobes; and the hindbrain gives rise to the cerebellum and medulla. The individual regions are discussed in later paragraphs. 


Prosencephalon $\quad\left\{\begin{array}{l}\text { Rhinencephalon (olfactory lobes) } \\ \text { Telencephalon (cerebrum and ventral } \\ \text { regions) } \\ \text { Diencephalon }\end{array}\right.$

Mesencephalon

Optic lobes

Rhombencephalon $\quad\left\{\begin{array}{l}\text { Metencephalon (cerebellum) } \\ \text { Myelencephalon (medulla) }\end{array}\right.$

As the brain develops in the early embryo flexures, or bends, appear. They are more prominent in the higher vertebrates and are retained throughout life, while in the lower vertebrates they are weak in the embryo and almost entirely disappear in the

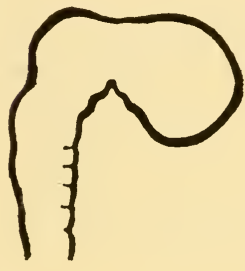

A. Primary (Apical)

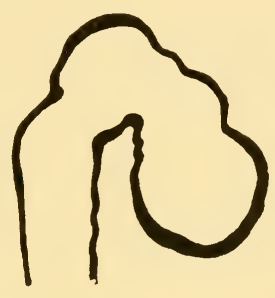

B. Secondary (Pontal)

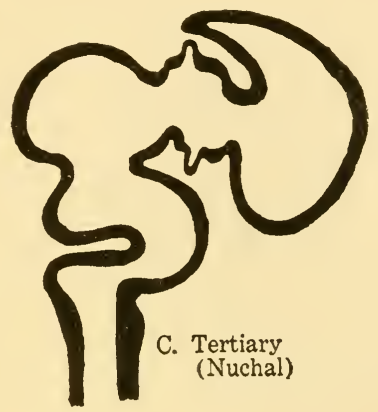

Fig. 154. Flexures of the Brain (Chick).

adult. The first, primary, flexure occurs in the midbrain, and the forebrain is bent ventrally until it lies at right angles to the cord. The second flexure is at the end of the medulla (nuchal flexure) and this bends the brain until the forebrain almost touches the cord, its anterior end pointing posteriorly. The third (pontal) flexure through the cerebellar region bends the brain backward toward its original position. In the fish and amphibia the third flexure carries the brain back into line with the antero-posterior axis of the body. In the amniotes the first and second flexures are progressively stronger; and in the birds and mammals the forebrain remains at right angles to the axis of the body.

The neurocoel is the cavity formed in the central nervous system when the groove closes. It is a typical characteristic of 
the chordates. The cavity remains small in the spinal cord, but as the brain grows the neurocoel expands and becomes enlarged into ventricles. Each region carries a ventricular cavity with it, but the ventricles of the brain are connected with each other and with the neurocoel of the cord. The ventricles are fluid filled and help supply the inner surfaces of the brain with oxygen and food.

The brain and cord are enclosed by connective tissue coverings, the meninges of the central nervous system. In the embryo this is a single meninx, closely adherent to the nerve cord, which carries blood vessels to the brain. The space between the meninx and the periosteum of the skull and neural arches
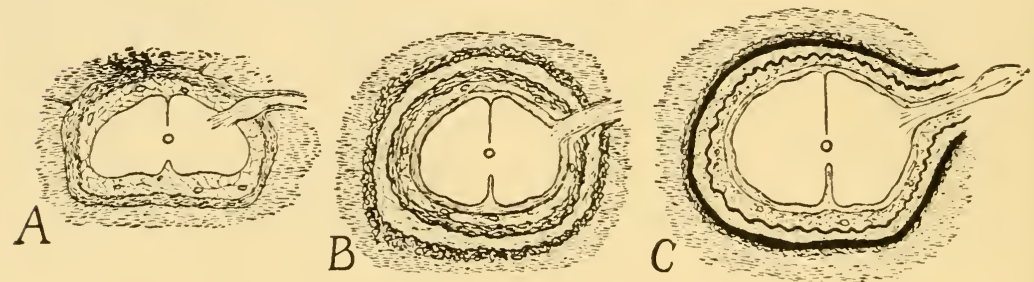

Fig. 155. Comparative Anatomy of the Meninges. The single dura spinalis of the dogfish (A) becomes double in the reptiles. A third membrane, the arachnoid, appears in the mammal (C).

is filled with fluid, and the two membranes are connected by fibers which pass freely between the two. This simple covering is retained by the fishes throughout life. Beginning with the amphibia there is an increasing complexity of the meninges. The meninx separates to form (1) a thin covering of the brain, the pia mater; and (2) a heavier outer layer, the dura spinalis. In the mammals the pia divides, a microscopically thin arachnoid layer being added as a meninx. The dura spinalis becomes the dura mater. This membrane lies against the periosteum of the bone and the two eventually (about the eighth year in the human) become inseparably united. The spaces between the membranes is always filled with fluid which is protective and nutrient. The meninges pass out over the spinal and cranial nerves, enclosing them as a sheath. 


\section{B. Units and Structure and Function}

The brain and spinal cord are composed of nerve cells and their fibers, and an ectodermal supporting tissue called neuroglia. The latter makes up the vast majority of the bulk of the central system. The nerve cell is the neurone, the anatomical unit of the nervous system.

A neurone is composed of (1) a cell body with its nucleus and cytoplasmic inclusions; (2) filamentous dendrites which receive the stimuli; and (3) a long axone which carries impulses

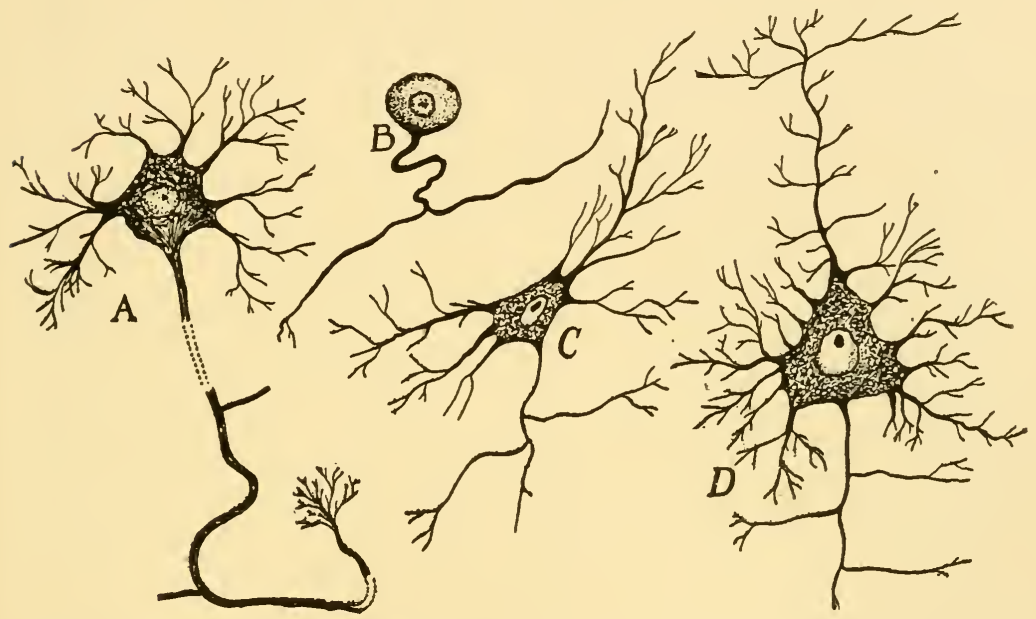

Fig. 156. Types of Nerve Cells. Motor Cell (A) from the ventral horn of the cord; Sensory Cell (B); and Brain Cells, (C) and (D).

from the cell body and (4) ends in a highly branched terminal arborization. Neurofibrils pass from the dendrites through the cell body and out to the end of the axone. The cell body is the center of metabolism. The dendrites, axone, and terminal arborization carry on the specialized function of the nerve tissues.

Neurones are divided by their structure into two large groups: multipolar cells which comprise the great majority, and unipolar sensory cells. The former vary enormously in structure, from the rather simple type found in the nerve cord to the highly complex cells of the cerebral region. In this type the 
dendrites are short and enter the cell body at several points, or poles, and pass out through the single axone. In the unipolar cells the dendrites are very long and the axone short. The relative length of the processes in the two types becomes clear if position and function are considered. The cell bodies lie in or near the spinal cord, and are connected with all the regions of the body through their neurofibrils. The sensory cells have long dendrites in contact with the periphery of the body, and short axones to the cord. The motor cells, lying in the cord, have short dendrites and long axones. The cells of the brain may vary in the relative length of dendrites and axone.

The axones give off collateral branches during their course. Axones and their collaterals which pass from and to the cord are usually covered with a fatty myelin sheath. The lipoid substance is enclosed in a delicate connective tissue sheath which is broken at irregular nodes. The sheath between the nodes is continuous and formed of two concentric tubes with the space between filled with the myelin substance. The cell bodies are bare. The unmyelinated fibers are those of the association neurones which do not leave the brain or cord, and the fibers of the sympathetic system.

Unmyelinated tissues have a pale, translucent appearance when cut and are known as the gray substance of the nervous system. The myelinated fibers have a waxy white appearance and form the white substance. A trans-section of the brain or cord will demonstrate the two types of neural substance.

Cell bodies are usually collected into definite masses. Any group of cells which is separated from other groups is a ganglion; and the groups of nerve cells which develop from the neural crests are therefore the spinal ganglia. If, however, cell groups are continuous or barely separated from one another, as in the brain and cord, they are termed nerve nuclei.

The functional unit of the nervous system is made by three or more neurones forming a reflex arc. External stimuli are usually received by specialized sensory cells, the receptors of sensations. The impulse is carried from the receptor to the dendrite of a sensory neurone, and is transmitted through the spinal ganglion into the cord. Small association neurones take the stimulus from the dorsal region of the cord to the motor cells 
located in the ventral portion of the cord. Dendrites receive the stimulus and a motor impulse is carried out over the axone to a muscle, gland, or other effector. This is the simplest type of nerve reaction. It is doubtful if a two-neurone arc is possible; and in the normal animal an arc as simple as the one described rarely occurs. Every sensory stimulus reaching the cord is carried to the brain, and numerous pathways are usually stimulated.

The anatomical connection between two neurones is the synapse. There is no evidence that the neurofibrils are in actual contact, the experimental evidence indicating that there is a definite space between. The gap apparently causes the impulse to travel in one direction, for experiments have shown that nerve fibers carry impulses in either direction with equal facility.

\section{The Cord and Spinal Nerves}

The spinal cord is more primitive than the brain and will be described first on account of its simplicity. In dissection the spinal cord of the primitive vertebrate appears as a tapering structure with two enlargements; one in the neck, the cervical enlargement, and a posterior one near the pelvic limbs, the sacral enlargement. Except in the most primitive types, the cord is divided both dorsally and ventrally by deep fissures. In transverse section the cord of the higher vertebrates is approximately circular, and almost divided into two halves. The fissures are caused by the unequal growth of the embryonic tube, the cord enlarging laterally and not dorso-ventrally. In the center is the neurocoel, surrounded by the tissue and fibers connecting the right and left sides. The peripheral region of the cord is white substance; and centrally is an $\mathrm{H}$-shaped region of gray substance, the neurocoel being in the center of the cross bar.

In the primitive vertebrate the spinal nerves leave the cord as paired, metameric structures. Each has a dorsal sensory root with its dorsal ganglion near the cord, and a ventral motor root. In Amphioxus and some cyclostomes (Petromyzon and others) these roots do not unite, but pass independently to the tissues of the body. In all other vertebrates the roots coalesce to form a single spinal nerve. 
The cord tends to shorten in the reptiles, and in the most primitive mammals barely reaches the sacrum. The tendency of the nerve tissues to become concentrated in the anterior region is described as cephalization. This centralization of function is most prominent in the higher mammals. The fish, amphibia and reptiles show a progressive development away from the

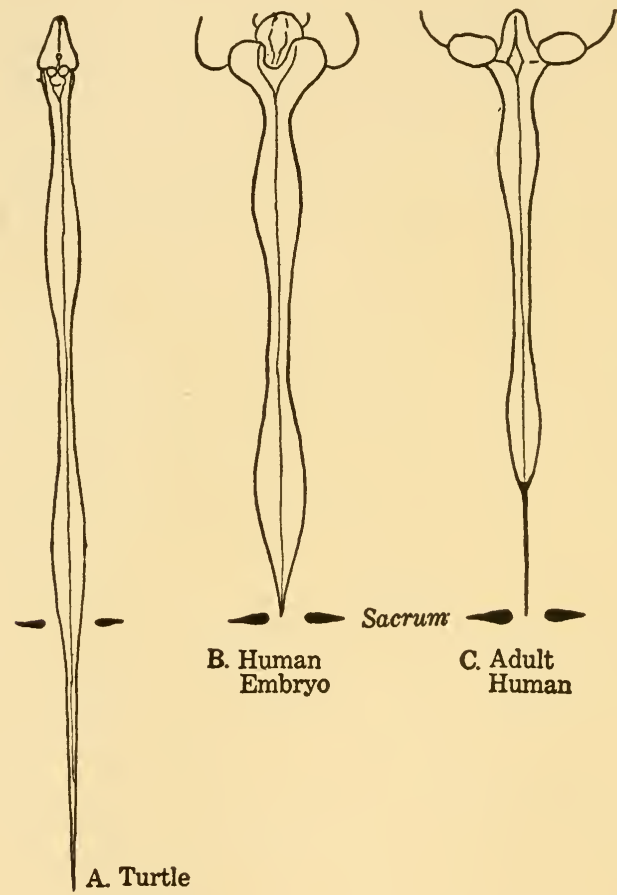

FIG. 157. Spinal Cords. Illustrating the shortening of the cord during evolution and during individual development.

spinal type of animal. The extinct dinosaurs, however, had a highly decentralized nervous system. The spinal centers were more important than the brain, and the selective value of this is apparent when one considers the great size of the animals. It has been estimated that in the largest dinosaurs it would require approximately five seconds for an impulse to travel from the tip of the tail to the brain, and back to the tail. 
With the shortening of the cord the spinal nerves become telescoped, and plexuses of nerves leave the two enlargements. This is most prominent in man and the anthropoids. In these animals the cord has shortened until it reaches only to the middle of the lumbar region, and is anchored to the pelvis with a connective tissue filum terminale. From the cervical enlargement arise the nerves supplying the pectoral girdle; and from the sacral (lumbar) enlargement comes a great plexus of nerves, the cauda equina of human anatomy. The nerves of the thoracic

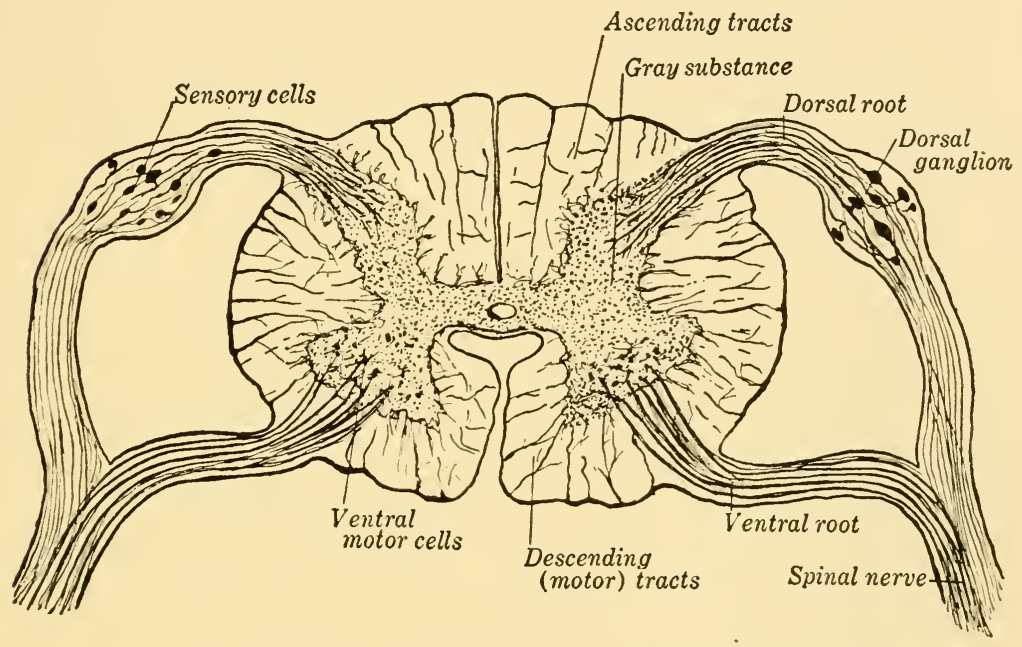

Fig. 158. Typical Section of the Spinal Cord of the Mammal.

region retain, to a large degree, the primitive metameric arrangement.

The white substance of the cord is composed of the long ascending fibers which pass from the spinal nerves up the cord to the brain; and the descending motor fibers from the brain to the motor cells of the cord. Recall that each impulse reaching the cord through a sensory fiber is transferred to the brain through - a myelinated collateral or another sensory fiber; and it becomes evident that the number of long ascending fibers increases at each spinal nerve. Similarly, the motor fibers from the brain decrease in numbers at each lower level due to the fibers which 
end at the spinal motor nerve cells. An examination of the cord sectioned through the two enlargements will show that there is more white than gray substance in the cervical region, and that the arrangement is exactly reversed in the sacral enlargement.

In a general way, the fibers of the cord which carry similar

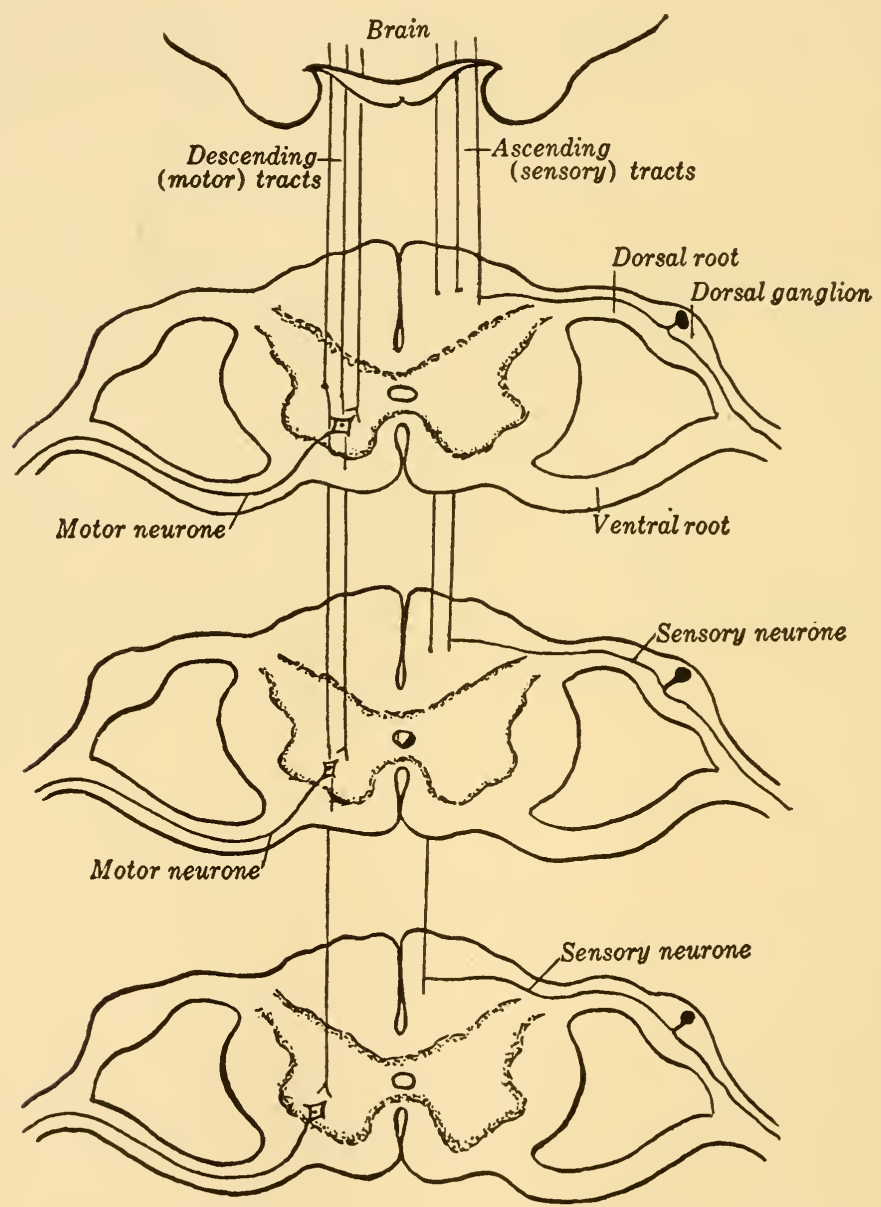

Fig. 159. Sections of the Cord at Different Levels. Sensory fibers are added at each spinal nerve, and motor fibers decrease in number at each more posterior level. Observe that the sensory 'tracts are concentrated in the dorsal region, and the motor tracts are mainly in the ventral half. 
types of impulse are grouped into tracts. The sensory fibers tend to be concentrated in the dorsal part of the cord, and the motor fibers in the ventral half. The division of the tracts is carried even further, particularly in regard to the sensory fibers. Fibers carrying specialized impulses (as tactile, pain, or kinesthetic sensations) are congregated into smaller tracts on

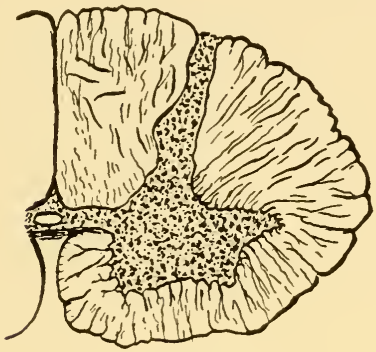

A. Cervical

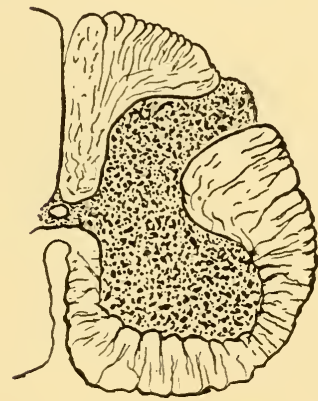

C. Lumbo-sacral

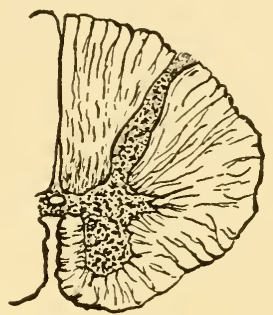

B. Thoracic

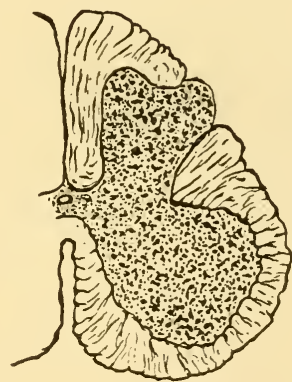

D. Sacral

Fig. 160. Sections of the Cord at Four Levels, showing the increase of fibers in the cervical region. The sacral enlargement controls the pelvis and pelvic limbs.

either side of the cord. The triangular region bounded dorsally by the periphery of the cord, and laterally by the entrance of the dorsal roots of the spinal nerves, is filled with the sensory fibers from the muscles carrying kinesthetic sensations. These fibers convey sensations of muscle position to the brain, and their loss causes a lack of coordination in the affected part of the body. The tactile and pain sensory fibers are located 
laterally, between the dorsal and ventral roots. In this same region, but more central in position, are a number of motor tracts. The ventral segment of the cord is almost entirely motor.

A destruction of the motor tracts causes a loss of voluntary action, for the connections between the brain and the motor nerves of the cord would then be lost. The reflex pathways would not be affected; but reflex movements would be exaggerated for the coordinating influence of the brain would be lost. If, however, the motor cells of the cord are destroyed, both voluntary and involuntary reactions would cease in the regions supplied by affected nerves. Motor impulses from the brain would continue to pass down the cord but could not be relayed to the effectors. As a result of any such destruction of cells the muscles, including those of the arteries, lose their power to react; and a degenerative condition follows. Infantile paralysis is caused by the destruction of ventral motor nerve cells.

Thus far the fibers of the cord and spinal nerves have been classified simply as motor or sensory; but each of these types can be divided into somatic fibers which pass to or from the muscles and skin, and visceral fibers to or from the internal organs. Each spinal nerve therefore contains four types of fibers: visceral and somatic motor, and visceral and somatic sensory. The visceral fibers form the connection between the central nervous system and the sympathetic ganglia.

\section{Srmpathetic System}

In addition to the central nervous system and the cranial and spinal nerves which arise from it, there are a number of visceral ganglia which are not affected by voluntary motor impulses. The nomenclature of this vegetative nervous system has never been standardized. The simpler terminology uses autonom$i c$ to describe this entire system of ganglia and nerves which are involuntary in action. The ganglia of the thoracic region form the sympathetic part of the system. Those ganglia which lie in the cervical and lumbar regions are the parasympathetic. The classification is based upon position and the physiological reactions of the ganglia.

Lying immediately ventral to the centra of the vertebrae, and 
to either side of the midline, is a metameric group of autonomic vertebral ganglia. These are connected in series with each other, and through the rami communicantes with the spinal nerves. Each ramus communicans is composed of the visceral motor and

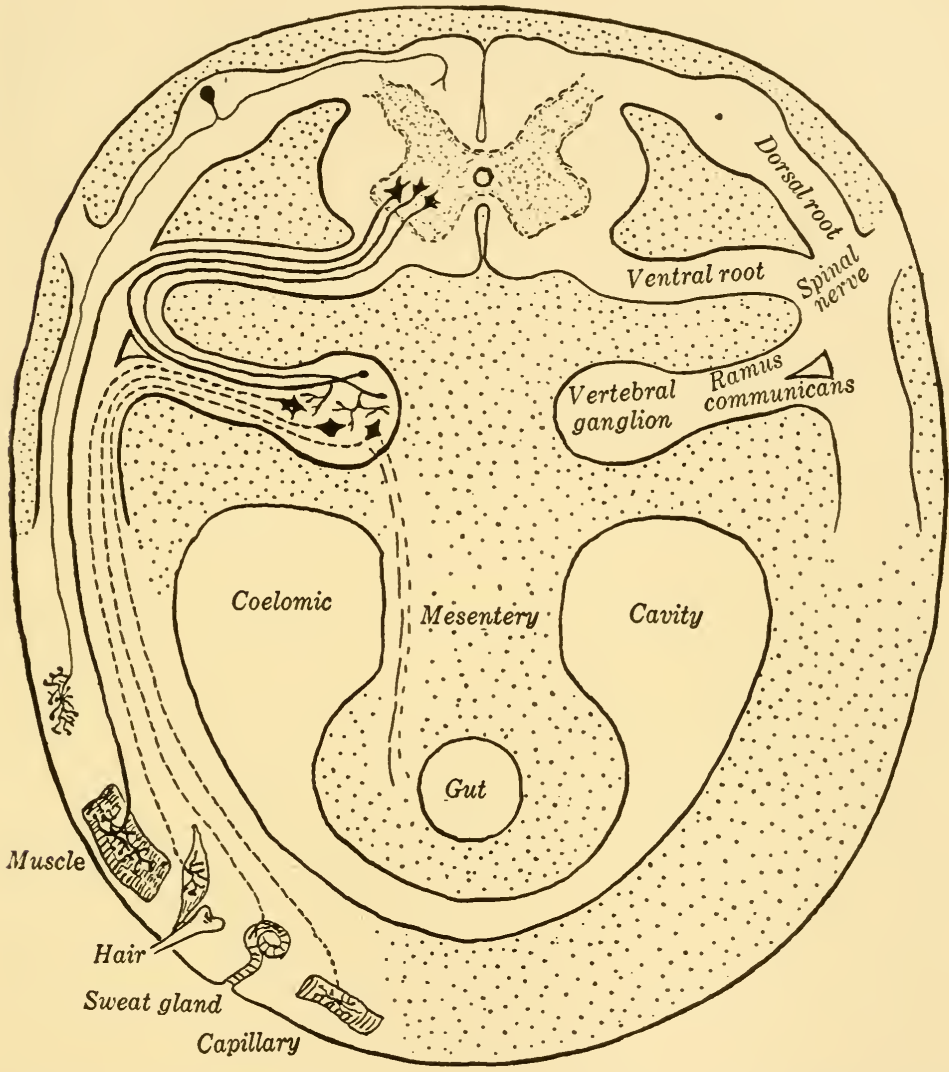

Fig. 161. Diagrammatic Cross Section of the Body. The pathways of the somatic and visceral nerves are shown. The dotted lines indicate the autonomic motor fibers from the vertebral ganglia to the integumentary region of the body.

the visceral sensory fibers of the spinal nerve. In addition, unmyelinated motor fibers pass from the ganglia to the somatic regions of the body, forming the gray ramus. These fibers pass to the skin with the somatic fibers of the cord and innervate the 
sweat glands, the muscles of the cutaneous capillaries, and the minute muscles of the hairs. Other fibers from the vertebral ganglia extend ventrally into the mesentery to the small prevertebral ganglia, and take impulses to and from the glands and smooth muscles of the viscera.

Although the autonomic system is not under "voluntary" control, it is intimately connected with the central nervous system and influenced by it. Any pain sensation in the deeper viscera is transmitted to the brain; and conversely, somatic sensory sensations may be transferred over to the visceral motor tracts. Motor stimuli from the brain may be sent to the autonomic ganglia in response to pain, visual or auditory sensations; and in turn cause cutaneous or visceral reactions. The lungs, heart and upper viscera are innervated by autonomic and also by cranial nerve fibers. Functionally the latter may be termed parasympathetic fibers, although they are antagunistic in their action to those of the autonomic ganglia. Cranial motor fibers in the viscera are usually depressors of action while the autonomic impulses are accelerators, and any imbalance between the two types of stimuli tends to cause a physiological upset in the organs affected.

Let us summarize the reactions. A sensation received in the skin through a receptor will be transferred over to a somatic sensory fiber to the cord. At this point it will be sent in two directions: (1) directly to a spinal motor nerve, and (2) to the brain. The reflex stimuli will go immediately from the cord to the nearby effectors; and following them, voluntary impulses will travel from the brain to the motor nerves. The time difference between reflex and voluntary movement is measurable; and in an animal such as the giraffe, with short legs and a long neck, a sensation received in the hind foot would return as a motor impulse from the cord before the sensory stimulus had reached the brain.

A sensory impulse may be, and usually is, transferred to the visceral motor fibers of the cord and sent to the vertebral ganglia. From this point motor impulses would be sent out to the deeper viscera and the somatic tissues innervated by these autonomic fibers. Pain sensations from the viscera travel to the vertebral ganglia, and from them over two pathways: 
over myelinated sensory fibers to the cord and thence to the brain where the sensation is registered; and (2) over unmyelinated motor fibers from the ganglion to the cutaneous regions. The latter cause blanching of the skin and profuse sweating. The autonomic reactions are influenced by the sensitivity of the nervous system, and to a certain degree by the conditioning (experience) of the individual. The voluntary response following the registration upon the brain is almost entirely controlled by training.

The study of nerve pathways differs from most anatomical systems in that knowledge of structure depends very largely upon physiological reactions. The function of a muscle is indicated by its attachments; but a study of the cord and its nerve tracts tells almost nothing as to regional function, and the pathways have been traced through the degeneration of fibers which follows injuries to the cord and brain.

\section{E. The Brain}

The brain of the lamprey (Cyclostomata) most nearly resembles the embryonic brain with its three simple divisions. But in this most primitive group there are clear indications of the division of the brain into more complex regions. From the forebrain come two pairs of outpocketing, anterior evaginations to form olfactory lobes, and dorsal cerebral hemispheres; and the hindbrain has a slight constriction which separates the anterior cerebellum from the medulla.

The embryos of the higher vertebrates develop a more complex structure: (1) both the fore- and hindbrains are distinctly divided into two regions; (2) complex evaginations appear in both these regions, with folds and creases which increase the surface area without greatly increasing the bulk of the entire structure; and (3) certain areas increase in thickness due to the growth of nerve nuclei and the invasion of nerve fibers.

The forebrain is divided into an anterior telencephalon and a narrow, thin-walled diencephalon. The former undergoes the greatest differentiation. Paired anterior outpocketings derelop into the olfactory lobes and nerves, with the lamina terminalis of the forebrain between them. Laterally grow out two optic 


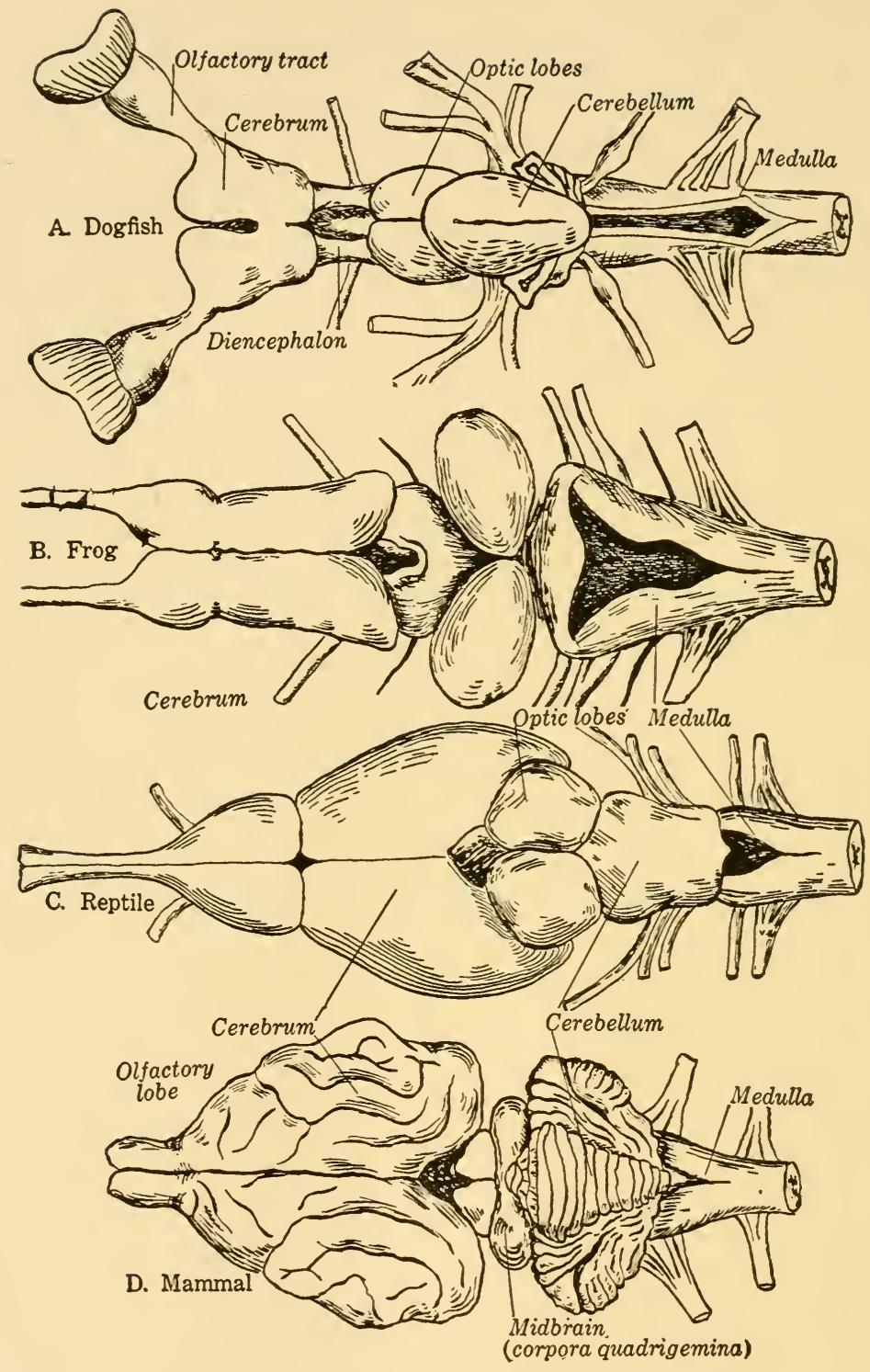

FIg. 162. Vertebrate Brains in Dorsal View. 

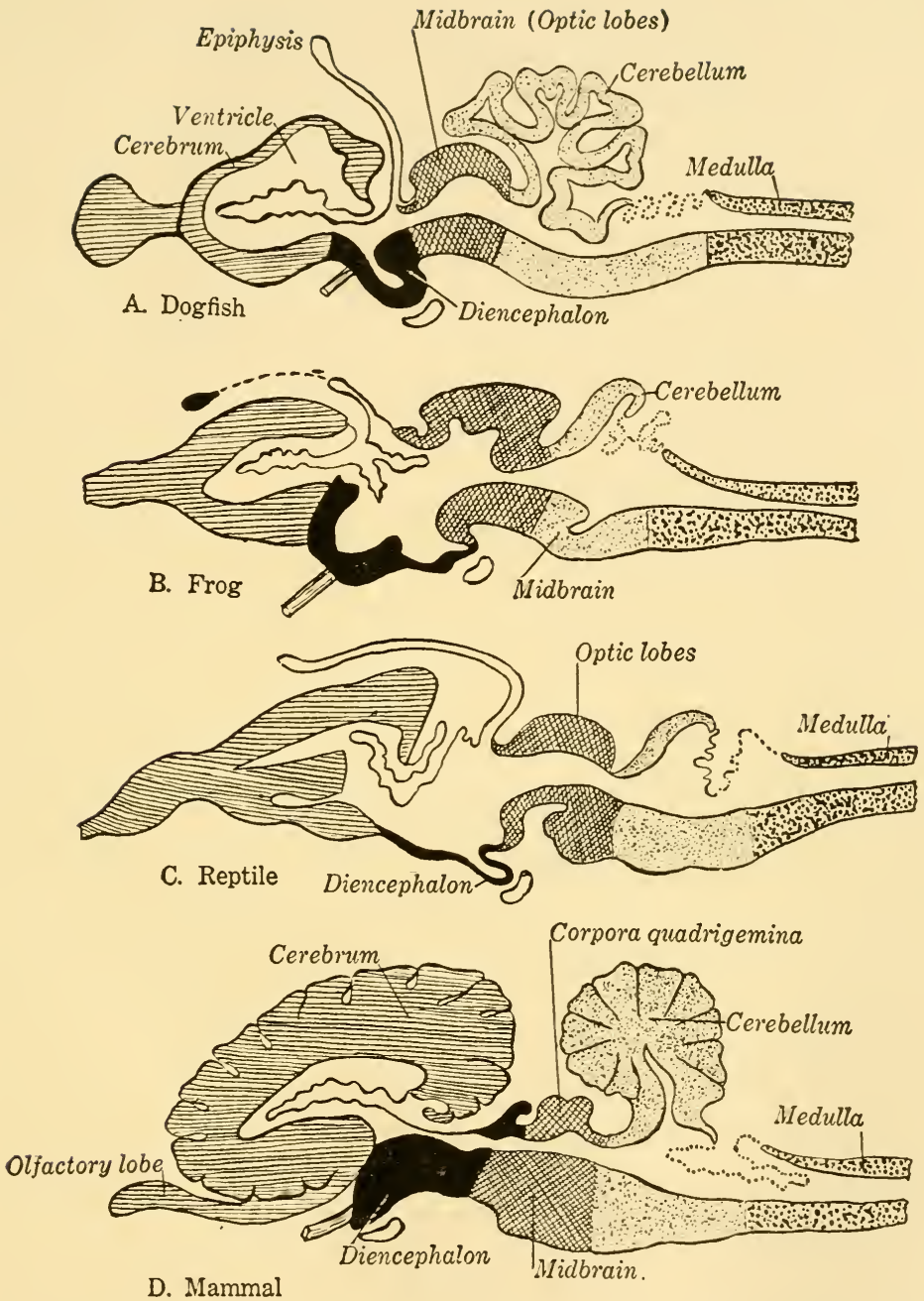

Fig. 163. Hemisected Vertebrate Brains. The olfactory and cerebral regions are lined; the diencephalon is black; the midbrain is cross lined, and the hindbrain is stippled. 
vesicles which form the optic stalks and the retina of each eye; and dorsally there are two pockets, the cerebral hemispheres, the ventricle of each connected with the original median ventricle of the forebrain by a wide foramen of Munro.

The forebrain was probably given over entirely to the olfactory function in the primitive ancestors of the vertebrates, for in the cyclostomes this function occupies practically all of this region; but in the elasmobranch fishes two lateral nuclei develop, the corpora striata. The dorsal covering, or pallium, is non-nervous, and functions only as a roof for the ventricle. During evolution the olfactory function becomes progressively less important and the development of the cerebral hemispheres becomes more and more prominent.

Cerebral Hemispheres. The telencephalon of the dogfish has the nuclei enclosed by the peripheral nerve fibers and non-nervous tissues. The amphibia keep the same general relationship with two advances, (1) a material increase in the size of the hemispheres, and (2) the invasion of the ventricular side of the hemispheres by nerve cells from the lateral nuclei. Both tendencies increase in the turtles and other lower reptiles. The nerve cells cover the inner surface of the pallium and collect along the median line as two distinct nuclei. The dorsal and median growth of the hemispheres has pushed them close together, so that the telencephalon is divided by a decp fissure resembling the fissure of the spinal cord. The cerebral pallia so far described are grouped together as archipallia, the primitive pallium with non-nervous tissue on the outside.

The neopallium is found first in the crocodilia. In these animals the nerve cells of the ventricular side migrate through the pallium to the outer surface and form a primitive cerebral cortex. A more extensive cortex is found in the birds and monotremes. In both groups the cerebral hemispheres are large, covered with a thick cortex, but perfectly smooth. Further progress is found in the marsupials. The cortical layer of cells multiplies more rapidly than the pallium grows, and the cortex becomes slightly creased and folded into convolutions. The evolution of the mammalian brain is an advance along the two lines indicated, growth in absolute size of the hemispheres, and multiplication of cortical cells. 

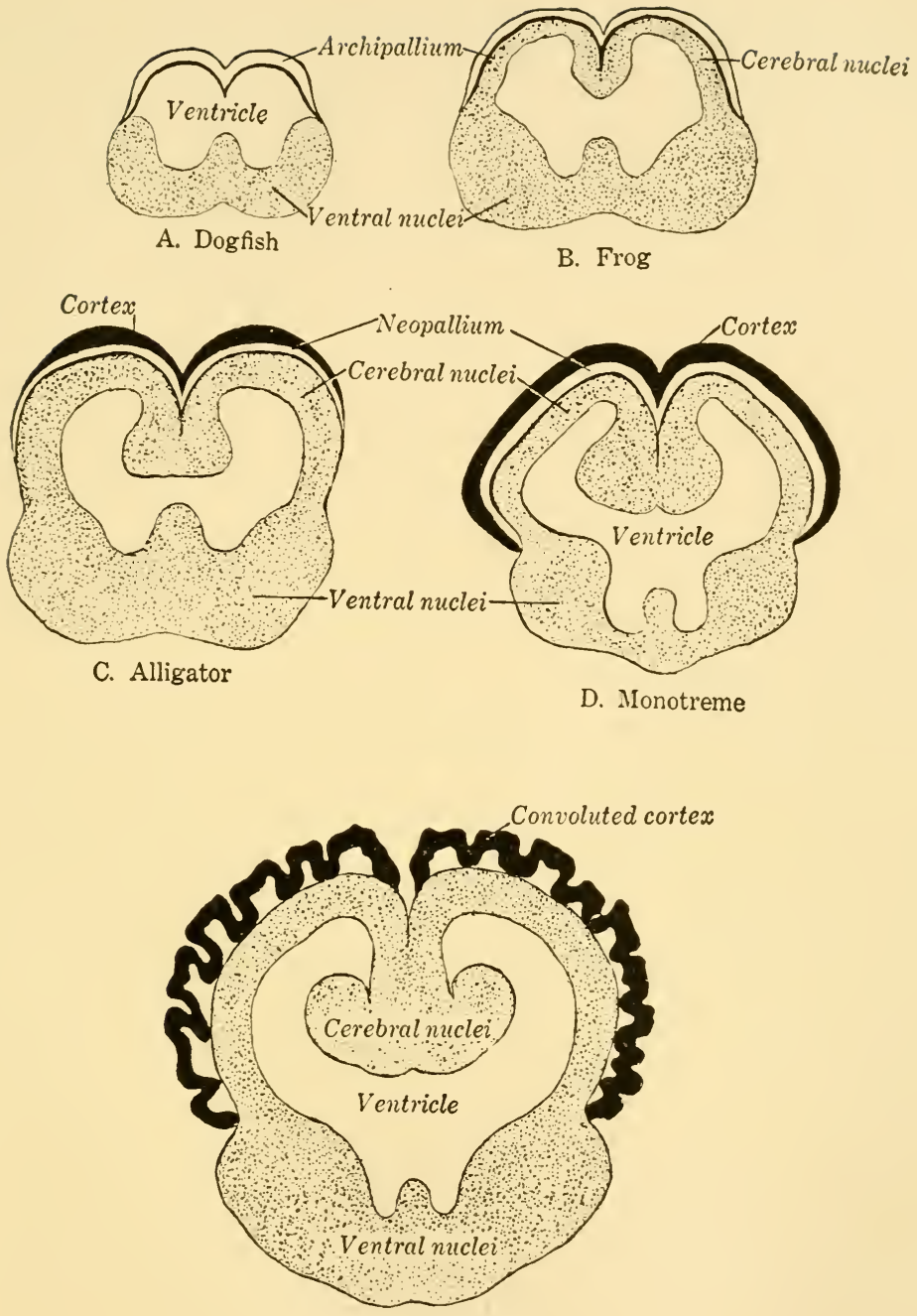

E. Primitive Placental

FIG. 164. Cross Sections through the Cerebral Hemispheres. Observe the development of cerebral nuclei in the Frog (B), and a cerebral cortex in the Alligator (C). The cortex increases in size in the Monotreme, and becomes convoluted in higher mammals. 
The development of the neopallium causes several important changes. One of the first forebrain centers to become separated from the olfactory function is the hippocampus, located as an important nucleus on the side of the telencephalon in the lower groups including the reptiles. In the mammals this region becomes infolded and covered by the cerebral hemispheres. The corpus striatum enlarges and is also pushed deeper into the brain tissue, along with smaller nuclei. With the continued growth of the cortex in the higher mammals the shallow creases multiply in number, and certain ones become deep fissures separating the cerebrum into fairly well-marked lobes. The four major lobes are the frontal forming the dorsal and anterior portion; the parietal posterior to the former; and the occipital as the most posterior portion of the cortex. The outer ventral region is the temporal lobe. The names are most applicable to the human, in which the lobes correspond generally to the skull bone of the same region.

Diencephalon. The diencephalon is a relatively small region between the telencephalon and the midbrain. The ventral region is invaded by the thalamic nuclei, important points of transfer for impulses from the brain to the cord. The diencephalon contains the large third ventricle, the older anatomists having numbered the right and left ventricles of the cerebral hemispheres one and two. The dorsal wall of the diencephalon is a thin membrane on which lies the choroid plexus of blood vessels. In most vertebrates the plexus sinks into the ventricular space, osmosis giving a constant supply of nutrient fluid to the rentricular spaces. This is the most important blood plexus of the brain.

From the diencephalon arise two evaginations, one dorsal and one ventral, which play an important part in growth and development. The dorsal epiphysis is a glandular tube in the cyclostomes and fish. From this structure, or from nerve cells which grow out with it, a third (or parietal) eye developed in the earliest amphibia. A dorsal eye was of selective value to mudliving animals with wide flat heads, and the structure was carried over to the primitive reptiles. Living amphibia have little evidence of the structure, but a number of reptile embryos derelop a dorsal optic vesicle which soon degenerates. The more primitive reptilian groups derelop not only an optic vesicle, but 
a primitive retina and lens. The eye extends to the surface through a median parietal foramen. Its highest development is seen in the most primitive living reptile (Sphenodon of New Zealand), but there is no proof that it is capable of visual function. The mammals show no evidence of a dorsal eye, the organ being glandular in structure and deeply hidden between the cerebral hemispheres. This pineal gland is discussed further in Chapter XIX.

The ventral outpocketing is the infundibulum. As it grows ventrally (in the vertebrates above the cyclostomes) it is met by an invagination of ectoderm from the stomodeal region. The stalk connecting the latter with the mouth is eventually cut off by the developing bones of the skull, and the combined organ becomes the pituitary gland which rests in the sella turcica of the skull. In the cyclostomes there is an infundibular evagination from the brain, but the anterior pouch (known in the embryology of the higher vertebrates as Rathke's pouch) remains separate, and is the naso-pituitary sac. In the lamprey the sac ends blindly, its tip lying immediately below the infundibulum.

Midbrais. The midbrain persists in a less modified condition than any primary part of the brain. In all classes except the mammals this region becomes the thick-walled, bi-lobed optic lobes. These lobes are the center of visual sensations. The optic vesicle arises from the forebrain, but the nerves which develop from the retina grow backward into the midbrain and carry light stimuli to the nuclei of the optic lobes.

The lobes are secondarily divided in the mammals by a transverse fissure, and the four bodies are called the corpora quadrigemina. The nuclei relay optic and auditory sensations from the organs of special sense to the cortex, the major centers of sight and hearing in the mammals being located in the cerebrum.

The ventricle of the midbrain is fairly large in the dogfish and widely connected with the third ventricle of the forebrain; but in the higher groups the growth of nuclei and the heary bands of fibers which pass rentrally as the cerebral nuclei increase, gradually crowd out the ventricular space. In the mammals this 
portion of the ventricle is a small tube, the iter or aqueduct of Sylvius.

Metencephalon. The metencephalon is the anterior portion of the hindbrain. From its dorsal region develops the cerebellum, the major seat of the nerves influencing equilibrium and coordination in the vertebrate.

The cerebellum is the first brain region to develop a cortical zone. In the primitive animals it is relatively small, with few fibers on the ventral side. In recent amphibia the cerebellum has undergone an interesting degenerative specialization, the

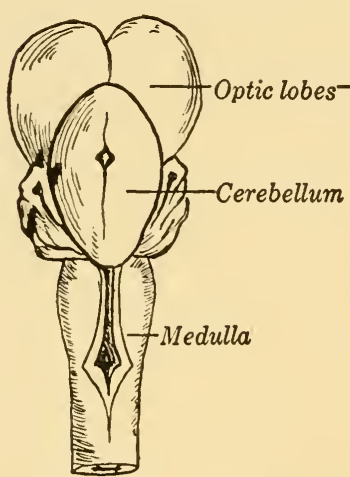

A. Dogfish

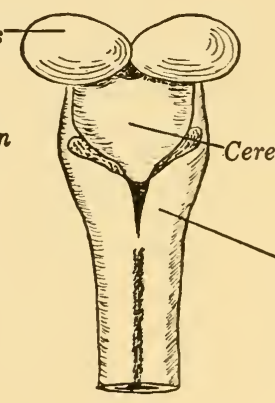

B. Lizard

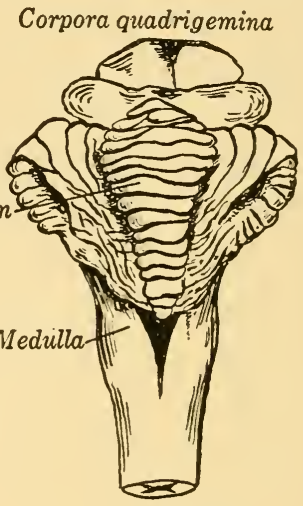

C. Mammal

FIG. 165. Midbrains and Hindbrains of Dogfish, Reptile and Mammal. The midbrain of the mammal is divided transversely to form the corpora quadrigemina.

region being very small, with a consequent loss of well coordinated movements. The higher reptiles and the birds have the central region of the cerebellum well developed, with lateral outgrowths (the flocculi) which increase the functional size of the structure.

The mammals add two lobes to the cerebellum. The original lobe becomes transversely creased and is known as the vermis, with the lateral lobes on either side. The flocculi remain, but are relatively less important than in the birds. The ventral part of the cerebellum is a great mass of transverse myelinated fibers, the pons Varolii, or more usually simply pons. 
Mrelexcephalox. The posterior part of the hindbrain is the medulla oblongata which is continuous with, and most resembles, the spinal cord. The ventricle of the medulla is continuous with that of the cerebellum, and is the large fourth ventricle of mammalian anatomy. The roof is thin and covered with a plexus of nutrient vessels which usually dip into the ventricular space.

The medulla is roughly triangular in shape, the ventral region being filled with the large tracts of nerve fibers which pass from the cord to the upper centers of the brain. In external view the most prominent are the pyramidal tracts, which are large bundles on the ventral surface. Other tracts and nuclei occupy the lateral expansions, and these nuclei are relay centers for impulses to the cerebellum and cerebrum. The posterior limit of the medulla is an artificial division from the cord, determined by the foramen magnum.

Commissures. A commissure is any large group of fibers crossing from one side of the brain to the other. Every impulse entering the body crosses to the opposite side before reaching the upper centers of the brain. Thus, the left half of the brain influences the right side, and the right influences the left. This is due to the decussation, or crossing, of the fibers. The fibers may cross at any level of the brain or cord, but the decussations in the cord are isolated and few in number. More generally the fibers from receptors pass up the cord on the original side of entrance, and cross in the brain stem.

The pons of the cerebellum is a large commissure, and others have been mentioned. In the cerebral region there are several commissures connecting one side with the other. The most ancient in a phylogenetic sense is the anterior commissure which lies in front of the third rentricle in the lamina terminalis. The amphibia develop a commissure dorsal to this one, the pallial commissure, connecting the hippocampal regions. Another develops in this region in the higher mammals, the corpus callosum. This large body of fibers lies dorsal to the ventricle and in longitudinal sections gives the appearance of a strong band of connective tissue. The corpus callosum has not been described in the monotremes; it is very weak in the marsupials; and increases in size in mammals with a well developed cerebral cortex. 
The commissure is an accurate indication of the number of cerebral cells, for each sends a fiber posteriorly and most of these cross in the cerebral commissure. The posterior commissure, crossing at the junction of the diencephalon and the midbrain, is also very primitive. The pons, connecting the two sides of the cerebellum, has been mentioned.

Exceptions must be made to the impression that all impulses eventually cross from one side of the central nervous system to the other. The incomplete crossing of optic fibers in the higher animals is discussed on page 308. Also, it will be understood that simple motor reflexes do not decussate, for a sensory stimulus reaching the cord is transferred to a motor cell on the same side; but the sensory fiber which carries the impulse to the brain when it enters the cord, crosses to the opposite side before reaching the higher centers. In the same way motor impulses from, the brain cross before reaching the ventral motor cells of the cord, and then pass out to the body. Therefore, destruction of fibers in the cord would be more liable to affect the same side of the body, whereas a destruction of brain tissue would affect the opposite side.

\section{F. Cranial Nerves}

The cranial nerves are those which pass out to the somatic regions of the body through foramina of the skull. There is evidence of two cranial nerves in Amphioxus, and it is thought that these are homologous with the first two (the olfactory and optic) nerves of the higher vertebrates. Synchronously with the development of a chondrocranium in the cyclostomes the number of cranial nerves increases to eight; and, as cephalization proceeds and the cranial cavity increases in size, the number of cranial nerves is increased to ten in the fishes and amphibia, and to twelve in the amniotes.

The above evidence, and the structure of the nerves themselves, have given rise to the theory that the cranial nerves posterior to the first two are modified spinal nerves, and that the brain is metameric in nature. According to this theory there are at least eight neuromeres, the first three corresponding to the three primary vesicles described above. The third brain 
pouch would then form the cerebellum, and the posterior five neuromeres would form the medulla.

Two other factors give evidence to the validity of the theory. (1) The appearance of muscle segments in the eye and ear regions indicates the former extension of metamerism in the higher vertebrates. (2) The structure of the cranial nerves is further evidence. Although most of the nerves are highly specialized, some being entirely sensory and others entirely motor, most of them are mixed like the spinal nerves; and some arise from the brain as several roots.

The cranial nerves are known by names which describe their function or location, and are also designated by numbers. These numbers are usually written Nerve I, Nerve II, etc. As the cranial nerves are very important, and vary widely in function, each will be treated separately.

Nerve I, Olfactory. The olfactory nerve is sensory and connects the olfactory lobe of the brain with the epithelium of the nasal sacs. It is usually considered that the epithelium of the sac gives rise to the receptors and their axones which extend posteriorly toward the brain. In many vertebrates, including the fish, reptiles and mammals, these axones are very short and make connection with the long dendrites extending outward from the olfactory lobe. In this case the nerve is really an olfactory tract, as is found in the elasmobranchs. In other vertebrates the sensory axones are long and pass toward the brain, forming an olfactory nerve. These axones meet the short dendrites of the brain nerves within the olfactory lobe.

Terminal Nerve. The terminal nerves lie mediad to the olfactory, and should be designated as Nerve I, but they were discovered after the more prominent nerves were numbered. Their function is unknown. They are found in most elasmobranchs and dipnoans, and in the embryos of many higher vertebrates, including man and other mammals. Apparently they are sensory nerves of the rostrum, whose function has been replaced in the higher groups by Nerves $\mathrm{V}$ and VII.

Nerve II, Optic. The optic nerve is formed by the axones of association neurones which connect the receptors of the retina (sensory layer of the eye) with the brain. The retina arises as an evagination from the primitive forebrain, and the nerves 
form a tract connecting this ganglionic mass with the higher centers. In the lower forms the optic axones pass through the diencephalon to the midbrain, but in the higher groups some of the fibers make connection in the thalamic nuclei of the diencephalon.

There is a complete decussation of the optic fibers in lower vertebrates, forming a complete optic chiasma, but in the mammals half the fibers cross to the opposite side while half do not cross. These relationships will be considered more fully in the chapter on organs of special sense.

Nerves III, IV, VI; Oculoyotor, Trochlear, Abducens. These are the motor nerves controlling the muscles of the eye. It is now known that each carries a small bundle of sensory fibers, transmitting proprioceptive muscle sensations to the brain.

The eye muscles arise from three small metameric muscle bundles, the most anterior (innervated by Nerve III) splitting to form four of the muscles. The second and third myotomes form one muscle each, with the result that each has a cranial nerve, while the oculomotor supplies the remaining four. The six eye muscles are arranged in two groups: (1) the rectus muscles, four in number, having their insertions equally spaced around the equator of the eye, and arising together on the inner surface of the eye socket; and (2) the two oblique muscles, one dorsal and one ventral, which pass from the eye to the anterior (median in the human) surface of the socket. The rectus muscles are the dorsal and ventral, and the anterior and posterior. In the human these are called the superior and inferior, and internal and external recti.

Nerve III, oculomotor, innervates the muscles arising from the first myotome: the dorsal and ventral recti; the anterior (internal) rectus; and the ventral oblique.

Nerve IV, trochlear, goes to the dorsal (superior) oblique. As these muscles, when they contract synchronously, pull the eyes medially and upward, the alternative name patheticus is often given them. The trochlear leaves the skull through the most dorsal of the nerve foramina.

Nerve VI, abducens, innervates the posterior (external) rectus. The fourth and sixth nerves are the smallest of the cranial nerves and are frequently lost in dissection. 
Nerve V. Trigeminal. The trigeminal is one of the largest of the cranial nerves, arising on the anterior part of the medulla. In the elasmobranchs there are four branches, each bearing a ganglion near its exit from the brain. The ganglia are distinct in the embryos of amphibia and amniotes, but soon fuse to form a single large Gasserian ganglion. Nerve V, with Nerve VII (Facial), supplies most of the face, the two parallelling each other throughout most of their courses.

Near the ganglion the nerve splits to form three branches: (1) the ophthalmic, a purely sensory branch, which derives its name from the fact that it passes through the orbit; $(2)$ the maxillary, also sensory, to the face and maxillary region; and (3) the mandibular, a mixed nerve, to the lower jaw and adjacent regions.

The ophthalmic branch is most prominent in the fish and urodele amphibia, in which groups it is associated with the lateral line system of sense organs (page 301). It is smaller in the anura and amniotes. This branch receives autonomic fibers, the exact relationships of which are not understood.

The maxillary nerve is larger in the groups lacking the lateral line, innervating the face and upper teeth.

The mandibular branch goes to the teeth and muscles of the lower jaw. It is a mixed nerve, receiving autonomic fibers; and, as it goes to the branchiomeric musculature (page 185), it may be considered a visceral nerve. In some reptiles and the mammals a small branch goes to the tongue.

Nerve VII, Facial. The facial is a mixed nerve, parallelling the fifth in much of its course. It arises from the medulla near the origin of the fifth. A sensory branch goes with the fifth to the tip of the tongue. The motor portion innervates the muscles of the neck and face.

Nerve VIII, Auditory. The auditory nerve is purely sensory, the fibers being distributed to the ear. In the fish it is a single nerve, the ear being only a balancing organ; but in the higher vertebrates the nerve has two branches, one to the organ of equilibrium and one to the hearing portion of the ear. The eighth nerve rises near the seventh, the ganglia of the two being fused in the higher classes. 
Nerve IX, Glossopharyngeal. The name is descriptive of the mammalian condition, the nerve being distributed to the muscles of the tongue and pharynx. In gilled vertebrates the nerve supplies the first gill slit, dividing into anterior and posterior branches. The anterior branch goes to the oral cavity and the hyoid muscles; the posterior branch goes to the muscles of the first gill arch, with a small branch to the taste buds of the primitive tongue. With the modification of the gill arches in the higher vertebrates, the nerve supplies the homologous muscles in the hyoid (which supports the tongue), some laryngeal muscles, the pharyngeal region, and some of the taste buds.

Nerve $\mathrm{X}$, Vagus. The vagus is the most widely distributed of the cranial nerves, supplying hypomeric (visceral) muscles. Vertebrates with gills have two major branches, (1) the lateral and (2) the branchio-intestinal. The former is a sensory branch in connection with the lateral line system. It persists throughout life in fish and urodele amphibia, but disappears at metamorphosis in the anura. The lateral branch is missing in the amniotes.

The branchio-intestinal nerve of gill bearing animals sends a branch to each of the gill slits posterior to the first. The main trunk passes posteriorly and innervates the heart, stomach, part of the intestine, and the swim bladder of the three groups which have one. There is a degeneration of the branchial nerves in the amniotes, the laryngeal and pharyngeal branches of the vagus apparently being homologous structures. The intestinal branch goes to the heart, stomach, parts of the intestine, and the lungs. The last is contributory evidence in the undoubted homology of swim bladder and lungs.

The vagus has numerous connections with the autonomic system, and the relationships are very close due to the innervation of organs by both types of fibers-cranial and autonomic. The relationships between the two parts of the nervous system were discussed under the sympathetic nervous system.

Nerve XI, Spinal Accessory. The accessory nerve is evidently a modified spinal nerve. Its origin is on both the posterior medulla and the spinal cord, the spinal roots fusing to form a nerve which passes into the cranium, joins the roots from the medulla, and then passes out through the foramen to the ster- 
nomastoid and cleidomastoid muscles, which unite to form one in the human. The nerve also innervates the trapezius.

Nerve XII, Hypoglossal. Like the accessory, the hypoglossal is motor. It has several roots on the cord which pass into the cranium and out to the retractors and muscles on the ventral side of the tongue and jaw.

The relationships of the cranial nerves and their evident homology with spinal nerves, have an interesting bearing on the metamerism of the face and head. The long discarded theory of Owen (1846) that the skull is metameric has no foundations in either evolution or embryology; but the metameric origin of the jaws and muscles of the face is now well established. First developed the hypocranial structures of the cyclostomes, and the metameric muscles of the eye. Following this the anterior gill arches became modified into jaws and hyoid apparatus, and the posterior gill clefts were pushed closer under the chondrocranium, with the inclusion of their nerves as the ninth and tenth cranial nerves. When the gill clefts disappeared in the amniotes, and the cartilages and muscles became incorporated into the laryngeal and neck region, two other spinal nerves came into the skull, making the twelve of the higher vertebrates. These nerves are all highly specialized, but their relationships correspond perfectly with the other evidence regarding the evolution of the skull. 


\section{CHAPTER XVIII}

\section{ORGANS OF SPECIAL SENSE}

ORGANS of special sense are the receptors of the body, receiving stimuli and passing them on to the sensory neurones. Through the latter, connection is made with the ganglia and nuclei of the sympathetic and central nervous systems. As the essential element of the receptor is the nerve ending, the sense organs are ectodermal in origin; but the majority become surrounded by complex accessory structures derived from the mesodermal connective tissue.

Receptors are located in the deeper tissues of the body (striated and smooth muscles, mesenteries and carities of the joints) and in the outer covering. The latter are more numerous and include the more complex sense organs. These may be either in the epidermis or embedded in the mesodermal supporting layers of the skin. There is, during the course of evolution, a general tendency toward assuming a deeper position.

A direct correlation exists between the diverse stimuli received and a specialization of the receptors to become specific for certain types of stimuli. Thus the vertebrates hare proprioceptive organs which respond to pressure; tactile cells or corpuscles; pain corpuscles; and the more specialized organs which receive chemical, or light and sound wave stimuli. The latter tend to be collected into close packed groups, while the skin and visceral endings are usually isolated corpuscles and are known as the nerve end apparatus.

Nerve endings. The simplest, and perhaps most primitive, type is the free nerve termination. The sensory fiber loses its myelin sheath and breaks into fine fibrils which ramify into the muscle or skin. This type is typical of striated muscle endings. Free terminations in the skin are more typical of the water living vertebrates than of the land living animals. 
End organs are more complex. The simplest type is the tactile corpuscle, the nerve ending at a tactile cell surrounded by a connective tissue cup. In other types the nerve may end in a minute plexus resembling a glomerulus and surrounded by a sheath. The largest corpuscles are those described by Pacini (Pacinian corpuscles) in which the nerve core is surrounded by concentric layers of connective tissue. These are easily demonstrated in sections of the mesentery, or the pancreas of the cat. They are large enough to be located without a lens.

\section{A. Lateral Line Organs}

In addition to the isolated cutaneous sense organs, the cyclostomes, fish, and gill-bearing amphibia have epithelial sense organs arranged in definite lines upon the head and body. The distribution of these lines varies greatly, although there is a basic arrangement of the primitive lines which is added to or modified in many ways. These lines radiate from the region of the ear. Two are facial, one above and one below the eye, converging toward the snout; a third is mandibular; and the fourth, which gives the name to the group of organs, lies along the axis of the body. This last follows generally the horizontal line separating the epaxial from the hypaxial regions of the muscle segments. The lateral line is parallelled by the lateral branch of the vagus nerve.

During development the organs appear as conical patches of modified epithelium, the base of the cone resting upon the dermal tissues. The central cells are sensory, each having a flagellum-like bristle projecting from the epidermis, and are surrounded by the supporting cells of the cone. The fibrils from the lateral nerve pass through the supporting cells and ramify around the sensory group.

In the cyclostomes and amphibia each cone sinks into a pit which is not directly connected with other depressions. In the fish these pits are modified to form connecting grooves or canals. In one group of the cartilaginous fishes the canals remain open in the adult; in the others the canals sink deeper and the lateral ridges close over to form canals which open to the outside through pores located above the sensory patches. The position of the 
canals and pores in relation to the scales differs widely. In a few fish the cranial canals run through the skull bones.

Many investigators have attempted to homologize the lateral line organs with other organs of special sense. Patches of lateral line receptors are thought to have migrated into the olfactory pits and mouth, and to have inpocketed to form the ear. However, as the system appears almost completely developed in anuran larvae, and then as completely disappears, including the lateral nerve; and, as the organs appear synchronously in the vertebrates, it seems more logical to the author to assume that these structures developed independently.

\section{B. Taste Buds}

The taste buds, in their individual structure, more nearly resemble the lateral line organs than any other sensory organs. The taste bud consists of a cone of supporting cells enclosing an inner cone of taste receptors. The latter lie in a depression in the supporting cells, connected with the outside through a taste pore. The taste buds are found in the oral cavity and pharynx. In the mammals they are most prominent on the tongue, where groups of taste buds are collected on relatively large papillae.

Taste is one of the chemical senses, the substance with a detectable taste being necessarily in solution. Most "taste" as recognized by the human is olfactory, taste being limited to elementary sensations of bitter, sweet, sour, salt, etc. As sweet and bitter can be more easily detected in different parts of the mouth, it is reasonable to conclude that there is a specialization of function in the different buds.

\section{Olfactory Sense}

The olfactory sense, like taste, is chemical. The odors detected by water living animals are in solution, and in land living vertebrates the gases become dissolved in the layer of moisture covering the epithelium. The sensory epithelium is located in olfactory pits, the axones of the receptors passing posteriorly to the dendrites of the olfactory lobe. 
The olfactory epithelium arises on the dorsal side of the embryonic head, and the region soon pits in to form olfactory sacs. The deeper layer of cells develops short dendrites which push between the supporting cells toward the lining of the sac; and axones which grow backward toward the olfactory lobe, thus forming the olfactory nerve.

The cyclostomes develop a single naso-pituitary pouch. The paired olfactory sacs and nerves lie near the dorsal region of the tube. The pituitary portion of the tube extends posteriorly to the level of the hypophysis of the brain, ending blindly in the lampreys, but opening into the mouth in the myxinoid cyclostomes.

The olfactory pits are paired in the fish and all higher vertebrates, with the pituitary pouch arising separately from the stomodeal region. As the chondrocranium develops the sacs are pushed anteriorly, and ventrally in fish with a rostrum. The sacs end blindly in the fish, and serve only an olfactory function.

In the amphibia the sacs have an origin similar to that of the fish, but the invaginations push ventrally toward the mouth cavity, and break through to form the internal nares, or choanae. The olfactory epithelium lies near the outer end of the tubes, the external nares, although accessory patches of sensory epithelium are near the internal opening. (This internal organ degenerates in higher vertebrates, and is degenerate and nonfunctional in the human).

The conditions in the early reptiles are hardly changed, with the exception of the development of lateral projections into the nasal passage. These conchae are homologous with the more highly developed turbinals of the mammals. The Crocodilia have more highly developed conchae, and in this order of reptiles the maxillary and palatine bones grow medially to form a hard palate, thus elongating the nasal passage so that the internal nares are at the back of the mouth near the pharynx.

There is a great increase in the sensory area in the mammals, although the olfactory lobes are relatively much smaller than in more primitive vertebrates, particularly the fish. The sensory epithelium is accommodated by the growth of turbinals, bony scrolls projecting into the nasal passage. The more anterior naso- and maxillo-turbinals, arising from the nasal and maxillary 
bones, are ordinarily not covered with olfactory epithelium, but with serous cells which tend to warm and moisten the air. The posterior ethmo-turbinals push in between those from the dermal bones, and carry the olfactory receptors.

The external nares are separated by a cartilage projecting anteriorly from the mesethmoid. Laterally the nares are enclosed by fleshy bands of tissue, which may be prolonged into a snout or, in the tapir and elephant, a greatly elongated proboscis.

Water living mammals tend to have a poorly developed sense of smell. The toothed whales lack even the olfactory nerves and the foramina of the cribriform plate. The primates also have a small sensory area, and in comparison with the rodents, carnivores, and most other orders of mammals an inefficient sense of smell.

Development in the Human. In the embryo of five weeks' development the mouth is wide, the nose is broad and flat, and the nostrils are widely separated and connected with the oral cavity by grooves. The median nasal region rapidly becomes elevated as the lateral masses of tissue grow medially, forcing the nares closer together until they are separated by a narrow septum and the passages look downward. The upper lip is formed by this median growth, completely cutting the nares from the mouth. The line of fusion is left in the adult as the median line of the upper lip. A failure of these tissues to coalesce causes hare lip, a common defect of the human. Less frequently the maxillary bones fail to meet in the mid-line and cleft palate results.

\section{The Eres}

The vertebrate eye differs from that of the invertebrate in the inversion of layers of the retinal cells. That is, the eve receptors of the vertebrate lie nearest the supporting cover, with the association neurones between the sensory layer and the source of light. The invertebrates have the direct method of receiving light rays directly against the sensory cells, the association neurones being deeper and next the supporting layers. But, after the vertebrate eve developed, there has been little 
change in the organ itself. The evolutionary changes have been in position, the optic nerves, and the protective structures around the eye.

ExibRYology. The eyes begin their development as lateral pouches from the forebrain before the posterior cord is closed. As each evagination continues to grow laterally the distal end enlarges into an optic vesicle which soon begins to inpocket and

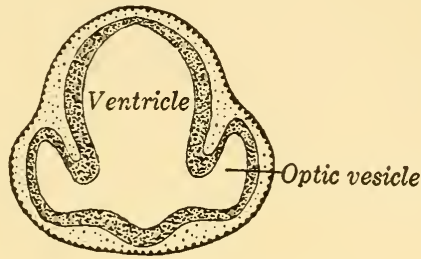

A.

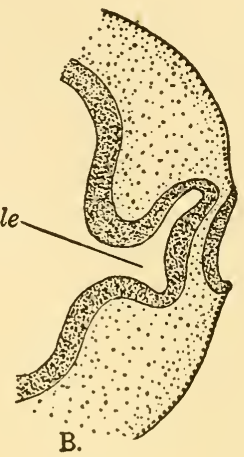

B.

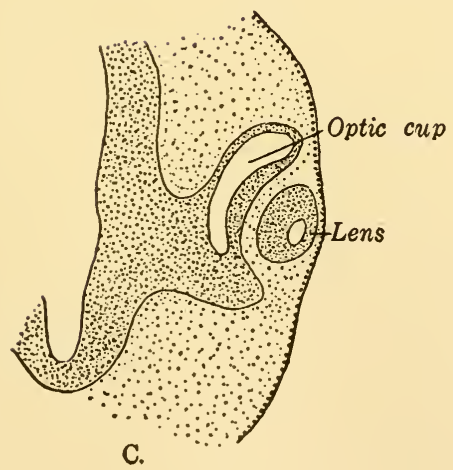

C.

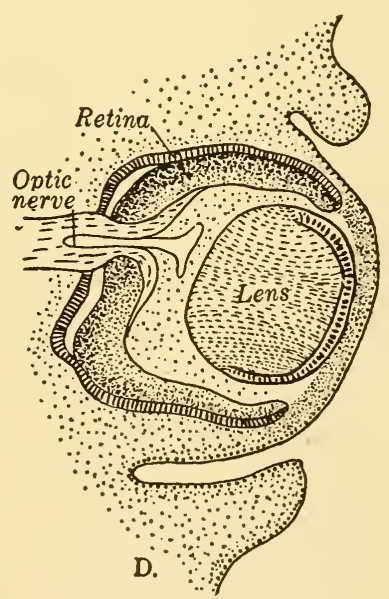

Fig. 166. Development of the Eye. (A) Section through the dereloping optic vesicles; (B) the invagination of the optic cup, and the epidermis to form the lens; (C) a later stage showing the lens; and (D) the embryonic eye. 
form a double layered optic cup. The cell layer lining the cavity of the optic cup (which will be spoken of as the inner layer) develops into the retina which contains the light sensitive cells. The outer, surrounding, layer of cells is thinner than the retinal layer, and develops into a pigmented coat protecting the retina. Synchronously with the derelopment of the optic cup, the covering ectoderm of the embryo thickens over this region, pushes inward, and becomes the lens of the eye, lying within the circular lip of the cup. As the lens pushes inward, the skin ectoderm closes over to form a continuous layer over the eye. In the completed structure this thin layer forms the epithelium of (1) the conjunctiva which surrounds the eye as folds of tissue, and (2) the cornea, the transparent part of the mesoderm which completely surrounds the eye ball. The continuity of this epithelium and the epidermis is demonstrated whenever a reptile sheds its skin, for the eye covering is moulted as an integral part of the shed epidermis.

The inner layer of the cup (that which receives light rays as they pass through the cornea and lens) becomes rapidly modified into sensory retinal cells and association nerve cells. The axones of the latter grow centrally toward the optic stalk which connects the eye with the brain, and through the stalk to the visual centers of the central nervous system. In this way the stalk is filled and transformed into the optic nerves, which become a nerve tract connecting the retinal ganglion with the central nuclei.

The original optic cup is surrounded by the cartilaginous optic capsule of the chondrocranium. This never fuses to the skull as do the other capsules, and thus permits free movement of the eye. The larger proximal portion of the capsule becomes thickened into the sclerotic protective membranes of the eye. The circular region over the lens forms the transparent cornea, covered externally by the ectodermal epithelium.

Structure of the Ere. The eye consists of (1) ectodermal retina, pigmented layer, lens, and outer epithelium; and (2) mesodermal iris which is pigmented, sclerotic protective membranes, and cornea. As the lens is attached along its equator to the eye membranes, the spherical hollow of the eye is divided into two cavities which are filled with a heary fluid. 
1. Retina. The retina consists of three rather distinct layers of cells: (1) an outer receptor layer (in contact with the pigmented layer); (2) a middle group of association neurones with short processes; and (3) an inner layer (next the cavity) of association cells with long axones which pass to the brain. Due to their shape the sensory cells are known as rod and cone cells. These are partially embedded in the pigmented layer. Stimuli are received by the rods and cones, transferred to the short association cells, and then passed to the inner association layer. Thus, a ray of light entering the eye passes through two layers of retinal cells before reaching the receptors. The axones of the inner layer of association neurones converge toward a central point where they enter the optic stalk. Near the exit of the optic nerve the axones are close packed and the area occupied by the nerve is not sensory. This is the blind spot of the eye. The area surrounding the blind spot is packed with sensory cells, and this region (the macula lutea) is extremely sensitive. Between the nerve cells of the retina are ectodermal supporting cells, resembling the similar neuroglia of the brain.

The pigmented layer, which lies in contact with the mesodermal covering, is very thin and becomes an integral part of the retina. The cells expand or shrink, assisting other eye structures in protecting the sensory cells from too intense light.

2. Choroid layer. The choroid layer is the inner portion of the optic capsule, lying in contact with the pigmented layer of the retina. It corresponds functionally with the pia mater of the brain. It is highly vascular and is the major nutrient source of the eye. The choroid is in close contact with the sclerotic layer throughout most of its area, but on the distal surface the two separate. The outer sheath is continued over the eye as the cornea; the choroid forms (1) the circular iris, and (2) the ciliary process. The latter arises at the point where the iris separates from the cornea, and is the muscular base to which the lens is attached. The ciliary muscles move the eye forward and backward and slightly adjusts its shape. The iris continues over the lens as a curtain with a central opening, the pupil of the eye. The change in pupillary size is caused by the constriction or dilation of the iris by its sphincter muscles. The iris is normally pigmented, giving color to the eye. The dark 
cavity of the eye gives the black appearance to the pupil. The pink eyes of albinos are caused by the lack of pigment in the iris, which permits the minute blood vessels to show and give their color to the eye.

3. Sclerotic layer. The sclerotic, or hard, layer is continuous over the entire eye, and forms both the proximal tough membranous covering and the cornea. The latter is very similar in all vertebrates. The sclerotic coat varies more widely in structure. In a number of reptiles and some birds the sclera becomes ossified into bony plates; but in all cases the eye remains freely movable.

4. Cavities. The attachment of the lens to the ciliary processes of the choroid layer separates the cavity of the eye into two distinct regions. The distal chamber between the cornea and the lens is filled with a refracting fluid, the aqueous humor. The larger space between the lens and retina is filled with a semi-solid vitreous body or humor. The origin of the vitreous body is in dispute; its function is evidently to maintain the almost spherical shape of the eye.

5. Function. Light rays passing through the cornea strike the lens, and its biconvex shape concentrates them on the retina. Each point of light and shade that is reflected to the eye is focussed separately so that the retina receives a mosaic picture. These stimuli are transmitted over the optic nerve and coordinated in the brain. If the rays come to a focus in front of or behind the retina the point of light becomes circular and the image is blurred. This is near-sightedness or far-sightedness.

Accommodation to distance is cared for by the movement of the lens and the slight ability to vary the shape of the focussing structure so that its convexity becomes greater or less. Light accommodation is adjusted by several structures. The function of the pigment layer has been mentioned. The major part of adjustment is cared for by the constriction of the iris. In the normal eye this reflex action admits only sufficient light to make a clear picture and prevent the blurring from very intense radiation.

Bifocal vision appears first in the mammals. In the lower groups the eyes are placed on either side of the head, and there is a complete optic chiasma. In the mammals, however, decus- 
sation is incomplete. The axones from the tro sides of the retina divide, so that the outer (posterior) half from the left eye and the inner (anterior) half from the right eye pass to the left side of the brain. The condition is similar in the right optic nerve. The complete nerve from the eye meets its mate from the other side just anterior to the pituitary body, and forms an incomplete chiasma.

Accessory Structures. The eyelids form protective covers for the eyes. The fish either lack or have very poorly developed dorsal and ventral lids; but a few sharks and teleosts have a third lid, the nictitating membrane, which arises on the anterior margin of the eye and mores posteriorly. The anura and most reptiles have all three lids developed; but in the mammals the membranous third lid becomes small, and in the human is left only as a small fold on the inner angle of the eye. The mammalian lids are fringed with stiff hairs, the lashes of the eye. The epidermal covering of the lids is continued over the inner surface as the conjunctiva. The lids and the surrounding skin are well supplied with muscles. The eyes are bathed by fluids from the lacrimal glands, the excess fluid passing to the nasal passages through the lacrimal ducts.

\section{E. Alditori Organs}

The ears undergo marked progressive changes in the different classes of vertebrates. Primitively the organ is concerned only with a sense of balance or equilibrium. This is true of the cyclostomes and fish, in which the ear is limited to the inner ear, consisting of the semicircular canals.

In all vertebrates the auditory apparatus arises as a thickening of the ectodermal covering of the embryo which sinks in as a hollow vesicle, a pore connecting it with the outside. The connection with the exterior remains open in the adults of some elasmobranchs, but closes in all the other groups. The cavity of the inner ear is filled with an endolymphatic fuid, and the cavity in which it rests is similarly filled with perilymph.

The most primitive ear is found in the lower cyclostomes, these animals having a single canal, flattened on the bottom and rounded dorsally. The tube is lined by epithelial cells with 
sensory processes, and the movement of fluid within stimulates the cells and indicates body position. The canals are paired in the lamprey and its relatives.

The definitive inner ear of the vertebrates is established in the elasmobranchs. To the two dorso-ventral canals a third horizontal one has been added, all of which empty into an en-
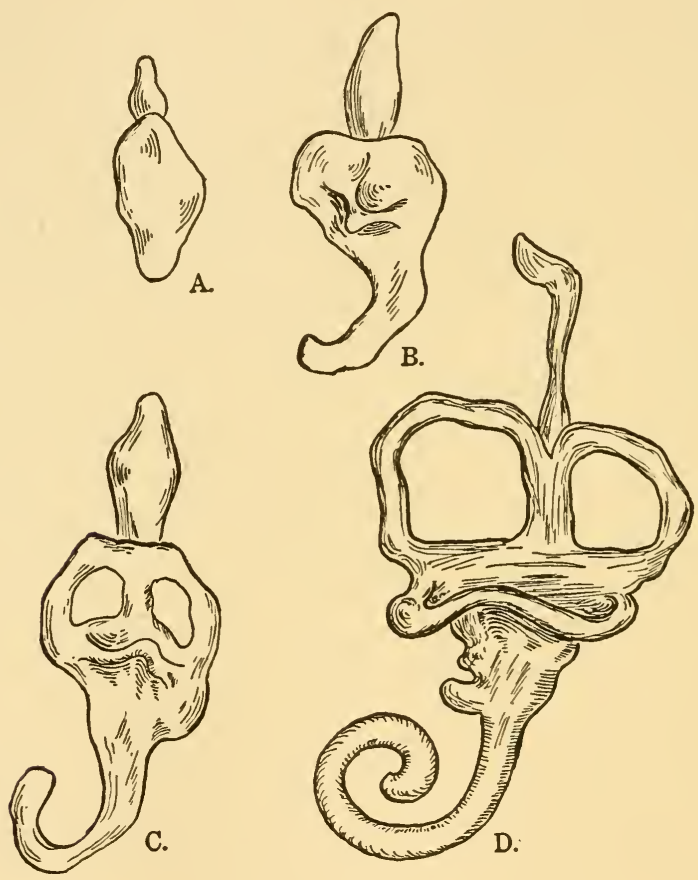

Fig. 167. Development of the Semicircular Canals (Mammal). (A) Shows the utriculus and endolymphatic duct; in (B) the first (transverse) canal has developed and the cochlea is elongating; (C) and (D) show further progress in development.

larged central cavity, the utriculus. From the ventral side of the utriculus develops a rounded sacculus which is of great morphological importance in the evolution of the higher vertebrates. Each canal has an enlarged ampulla at one entrance to the utriculus. The ampullae are at the ventral ends of the two vertical canals, and at the anterior entrance of the horizontal 
canal. The ampullae are characterized by large sensory areas with unusually long sensory processes on the cells.

The ear of the dogfish functions in the same way as do those of the cyclostomes. The endolymphatic fluid contains minute crystals of calcium salts (which in some fish are collected into a large otolith) and as the animal changes position the crystals are washed against the sensory hairs. This gives the impression of falling in that direction. The three canals of the typical vertebrate give a complete record of movement in any direction; and the dizziness which accompanies a whirling motion is due to centrifugal force throwing the fluid away from the center, and the impression made upon the brain of falling in all directions at once.

The amphibia develop a cochlea, a coiled outgrowth from the sacculus, which receives sound waves. The cochlea has its beginning in the fish where a small lagena, or pouch, projects from the ventral posterior portion of the sacculus. With the progressive growth of the cochlea the powers of hearing become greatly increased. In the mammal the cochlea is so highly coiled that it becomes spiralled, and contains the specialized organ of Corti. The function of this organ is speculative, for the birds, which lack the structure, perceive tones and pitch.

The inner ear is closely connected with the eighth, auditory, nerve which spreads over it and enlarges into ganglionated areas. The nerve is the essential organ of hearing, conveying the impressions to the brain, and the associated structures are the specialized receptors.

Middle Ear. The middle ear first becomes a definite structure in the anuran amphibia. It will be recalled that the first gill pouch develops into a spiracle in elasmobranchs and crossopterygian fish. In the anura and amniotes the pouch does not break through to the outside, but enlarges distally to form a middle ear cavity, the proximal portion remaining as the Eustachian tube. The external surface is covered by the tympanic membrane, a fusion of the branchial pouch and the body covering.

In the anura, reptiles, and birds, only the columella, or stapes, is present, the embryonic hyomandibular having been taken into the cavity. When the jaw articulation between the articular 
and quadrate is lost in the mammals, these bones are incorporated into the middle ear as the malleus and incus. The stapes is in contact with the inner ear, passing through a foramen in the bone and resting against a thin membrane of the utriculus. The malleus is in contact with the tympanic membrane with the incus lying between. The three assist in transmitting vibra-tions of the tympanum to the inner ear.

External EAR. The external ear is often limited to the conch, or shell-like funnel, which projects outward in most mammals. It may also include the external auditory meatus, the canal from the outside to the tympanic membrane.

The anura and lower reptiles have a tympanum flush with the surface of the body. In other reptiles the membrane sinlis below the surface; and in the crocodiles and birds the meatus is partially covered by flaps of tissue. The conch, a mechanism for concentrating the sound waves, appears first in mammals. It is supported by cartilages, and in the primitive condition rises to a dorsal point. Darwin's point, on the upper rim of the human ear, is a remnant of this primitive structure. The conch varies widely in size and shape. It is particularly large in bats. It tends to be small in animals adapted for water life, and has been secondarily lost in the Atlantic seals, sirenia and whales. 


\section{CHAPTER XIX}

\section{MECHANICS OF DEVELOPMENT}

Biologically considered, derelopment includes the growth of the individual from the fertilization of the egg to the time of death. Birth is a poor developmental landmark, for there is a wide variation in the degree of development at the time of birth or hatching. The amphibian hatches as an immature larva, while the reptile is usually highly organized and is anatomically a miniature adult. Similarly, the degree of differentiation in different groups of birds or mammals is extreme. Compared with a newborn calf, a marsupial at birth is an early embryo with a few specializations. And comparing man with his nearest relatives, the anthropoid apes, similar although less pronounced differences exist.

By carrying developmental processes over into postnatal growth it is found that the races of man grow and develop at different rates of speed. The changes in the individual are, howerer, continuous. Birth causes a complete shift in environment, with the attendant changes in the anatomy of the nutritive mechanisms, for the individual passes from a parasitic life to one of partial independence. But, although birth is one of the developmental landmarks, there are probably as great physiological changes at puberty as at birth. Therefore, a consideration of anatomy and the functions of structures necessarily depends upon the developmental changes which occur in the individual or the race.

The regulators of development fall naturally into three groups: (1) the differentiation which exists in the protoplasm of the egg; (2) the organizators which are developed by tissues or organs, and which influence the growth processes of other structures; and (3) the definite organs of internal secretion which develop during embryonic life and perhaps play the 
greatest rôle in postnatal growth and differentiation. There naturally is a great overlap in the influence of these regulators. Each tissue probably influences others throughout life, and the internally secreting (endocrine) glands exert an influence at a very early stage of development.

The differentiation which exists in the egg varies widely in the classes of vertebrates. In all there appears to be an anteroposterior specialization; and in some there is evidence that there is a transverse and dorso-ventral differentiation. The frog is an illustration of this highly regulative type of egg. In others the protoplasm is more generalized in nature, with less localization of developmental potentialities.

In the latter group the first cleavage apparently adds nothing to the specialization of parts already present. This is indicated by the so-called identical twinning, two individuals developing from a single fertilized ovum. It is well established that in Amphioxus each of the first four, or eight, cells has the potentiality of forming a complete animal. This has been proved true of some mammals, identical twinning being known to occur in a number of groups. The armadillos offer the best illustration of this process in the mammals. One species regularly develops four embryos from a single egg, and another species has eight young at a time when only one egg is given off from the ovary. In both cases the embryos are attached to a single placenta.

In all vertebrates, however, this generalized condition of the protoplasm disappears after a few cleavages; and with the formation of the primary germ layers each region becomes specialized and limited in its ability to develop various tissues. A biological determinism has taken place within the cells. But, although there is an apparent specialization controlling the fate of each group of cells, many internal and external factors may influence future growth. The external influences, such as mutilations, temperature and food supply, may be disregarded in this discussion. The internal influences, the organizators of the body, are more relevant to a discussion of anatomy.

Only the surface has been touched in the study of these organizing substances. Working upon the frog it has been found (Brachet and others) that the anterior region exerts a more 
powerful influence than the posterior portion. The removal of a fragment from the posterior part of an early embryo may affect the immediate surroundings; but the removal of a similar bit of cells from the anterior region will carry its influence throughout wide areas. As organs begin their development the organizing influence becomes more specific, both in regard to the extent of the area affected, and the reaction initiated in the neighboring structures. One or two cases will illustrate the principle involved.

During the development of the eye, the lens and the optic cup develop synchronously; but if the epidermal covering over the optic vesicle is removed, and epidermis from any other region of the animal's body is grafted in its place, a lens will develop in the normal position. The organizing substance which causes lens development is apparently not limited to the cup itself, but is present in the optic region of the brain. The converse of this experiment is the transfer of tissues carrying the more dominant organizator. The pelvic anlagen of early amphibian larvae can be removed and grafted against the vertebral column in a more anterior position. These anterior vertebrae will then develop sacral structures.

From the above it will be seen that there are two influences in the development of the organism as a unified whole: (1) the inherent potentiality of cells and tissues to develop along specific lines; and (2) the modifying influences of one tissue upon another. The nerves always arise from ectoderm, and the central nervous system from a limited region of the ectodermal covering; but the position and structure of the nerves are influenced by the mesodermal somites. Thus, in the growth of organs there is a definite interdependence between tissues, causing an orderly growth of the organism and its structures.

The third regulator of development, the ductless glands, do not function until the embryo is well formed. On the other hand, there is an imperfect line of demarcation between the organizing substances given off by tissues and the endocrine organs. The latter are usually limited to those glands which secrete specific hormones, and throw their secretions directly into the blood stream. These internally secreting glands develop synchronously with the other organs, but it is unknown when, during the course 
of development, they begin functioning. These glands develop from ectoderm, endoderm, or mesoderm; and in one case from two germ layers. In all cases the glandular, secreting, portion of the organ is supported by mesodermal connective tissues. The position, structure and function of the glands is equally diverse. Most of the endocrine organs have been mentioned previously in connection with the structural system with which they develop or are associated; but they are reviewed at this point as they may be considered together as a functional system of structures, although there may be no anatomical connection between them.

1. Pineal. The pineal gland arises as the epiphysis of the diencephalon, and is connected with the parietal eye in development. In its original condition in the fish, and in the mammals, it is solely glandular as far as its histological picture is concerned. Its function is not known.

2. Pituitary. As the stomodeal inpocketing meets the hypophysis of the diencephalon, the former spreads over the evagination from the brain and gives rise to the pituitary gland. The gland is divided into three portions, the anterior and posterior lobes being most important. The anterior part secretes a hormone (or hormones) which, among other effects, influences the growth of bone. An over secretion causes large heavy bones to be formed. If this occurs before the epiphyses are ossified, gigantism usually results, the degree depending upon the amount of secretion. The results differ if the over function occurs later in life. Those animals with a low secretion of the pituitary tend to be small boned; and, due to some correlation with the mechanism of carbohydrate metabolism, usually have an accumulation of fat.

Recent research has shown that the pituitary secretion, either independently or through some other gland, has a direct influence upon general differentiation and the development of sex. The metabolic influences are too complex to be considered here.

3. Thyroid. The thyroid gland is considered as homologous with the endostyle of Amphioxus. The development of the thyroid of the cyclostome from the endostyle of the larva has been described. In other vertebrates the gland develops as a median ventral outpocketing of the pharynx about the level of 
the second gill pouch, and the gland soon loses its connection with the pharyngeal cavity.

The thyroid was one of the first endocrine glands brought to the attention of research workers; for goiter, which is very prevalent in certain regions, is a pathological condition of the organ. The gland lies across the thyroid cartilage of the larynx and is composed of secreting vesicles surrounded by connective tissue. The thyroid secretion influences differentiation. In the mammal the lack of sufficient secretion results in a form of dwarfism (cretinism) which keeps the individual more or less infantile throughout life. Although the condition is corrected by feeding thyroid extract, there is evidence that the effect is caused by the influence of the thyroid upon the pituitary, and not a direct influence upon the tissues. The first experiments relating to differentiation were upon frog tadpoles (Gudernatsch). Feeding tadpoles bits of thyroid caused rapid metamorphosis. Larvae of bull frogs which normally would have remained as tadpoles for two years can be metamorphosed into small frogs in ten or twelve days. The thyroid gland also acts as a metabolic accelerator. Effects upon metabolism are greater when mammalian thyroid tissue is used, for the amphibia appear to lack, to a large extent, the essential radical in the thyroxin molecule.

4. Parathyroids. The parathyroid glands received their name from their position in juxtaposition with the thyroid gland. They arise as proliferations of cells from the gill pouches, and consist of four discrete structures. They secondarily become partially embedded in the thyroid, but there is no relationship between the two types of glandular tissue. The recent extraction of the effective hormone and the experiments which have followed, show that these glands influence calcium metabolism in the body. So far as the mammals are concerned, the proper secretion of the glands is not only necessary for the development of bone and teeth, but for the function of the muscles as well. In some animals accessory parathyroid tissues are present and carry on the function of the glands in extirpation experiments.

5. Thymus. The thymus arises as a paired gland from the branchial pouches. The two halves usually unite, and in the mammal the gland migrates posteriorly to a position near the 
bifurcation of the trachea. It is closely associated with the other lymphoid tissues of the body, and in the mammal a hypertrophy (overgrowth) of the thymus is correlated with an increased amount of other lymph tissues. In the human its absolute weight is greatest at about the time of puberty, although relatively it is largest at birth. Removal of the gland has so far had little effect upon the experimental animal, probably because of functional as well as structural similarity between the thymus and the lymph nodes. Clinical observations show that when the thymus is hypertrophied general development proceeds slowly. Feeding experiments on tadpoles (Gudernatsch) indicate the correctness of the observations on the human. The effects are the exact opposite of thyroid feeding, for thymus inhibits metamorphosis past the normal time of differentiation.

6. Pancreas. The pancreas is a duct gland emptying into the duodenum, with small "islands" of endocrine tissue scattered throughout its mass. The extract of the endocrine portion (the islands of Langerhans) is known as insulin. This hormone influences the absorption of carbohydrates by the cells, and thus the general carbohydrate metabolism. Diabetes is the pathological effect of improper function.

7. Adrenal glands. The adrenal glands of the mammal lie near the kidneys, and have a mesodermal cortex and an ectodermal medulla. The functions of the two are entirely different, and they may be considered as composites of two different glands. In the fish the mesodermal and ectodermal glands are separate. The ectodermal portion lies as two strips of chromaffin tissue in close contact with the sympathetic system of ganglia. The mesodermal glands are bands of inter-renal tissue parallelling the mesonephros. In the reptiles the two portions of the gland have come into close contact. In the mammals the ectodermal gland is completely surrounded by the mesodermal portion, forming a single structure.

The function of the cortex has not been solved, although extracts from it have been isolated which correct the effects of Addison's disease. In the mammal the removal of the cortical portion is followed by death. Clinical evidence indicates that the glands are related to anatomical development. A peculiar condition characterized by almost complete hairlessness and 
premature senility is always accompanied by degenerated cortical tissue.

The functions of the medullary portion of the adrenals is better understood. Adrenalin, the active substance of its secretion, has been isolated and widely used. The effects are upon the activity of the animal rather than upon growth and bodily form, the hormone activating the muscles and autonomic nervous systems.

8. Sex glands. The testis and ovary, like the pancreas, are both duct and ductless in their structure and function. The primitive function of the gonads is the production of reproductive cells; but in the land vertebrates (and probably in the more primitive rertebrates) certain cells take on an endocrine function. This function in the vertebrates is usually assigned to the interstitial tissue which lies between the tubules of the testis and the follicles of the ovary. In the latter organ, glandular bodies are developed after ovulation (corpora lutea) which secrete a hormone of great physiological importance. It influences the fixation of the fertilized ovum on the uterine wall, and the retention of the embryo in the uterus. In the rodents, at least, the degeneration of the corpora lutea is largely responsible for the termination of pregnancy; and their proper function helps initiate function in the mammary glands.

Knowledge of the anatomical effects of the gonads has been gained through castration and grafting experiments. The effects of gonad removal are more prominent in the male, the individual remaining in an undifferentiated stage with some female characteristics. If castration occurs in youth it affects the shape of the male pelvis, thorax and larynx.

Removal of the sex glands of either sex during early youth causes a delayed fusion of the epiphyses of the long bones with the shaft. As a result a cartilage growth area remains after the usual time of ankylosis. This permits continued growth of the long bones, and such individuals have unusually long appendages. Congenital, or very early, castration may result in apparent gigantism, but the height is in the limbs, not in the body.

Inter-relationships of the glands. It is always difficult to prove a specific, independent action for a gland secretion. This is particularly true for the hormones concerned with bodily growth 


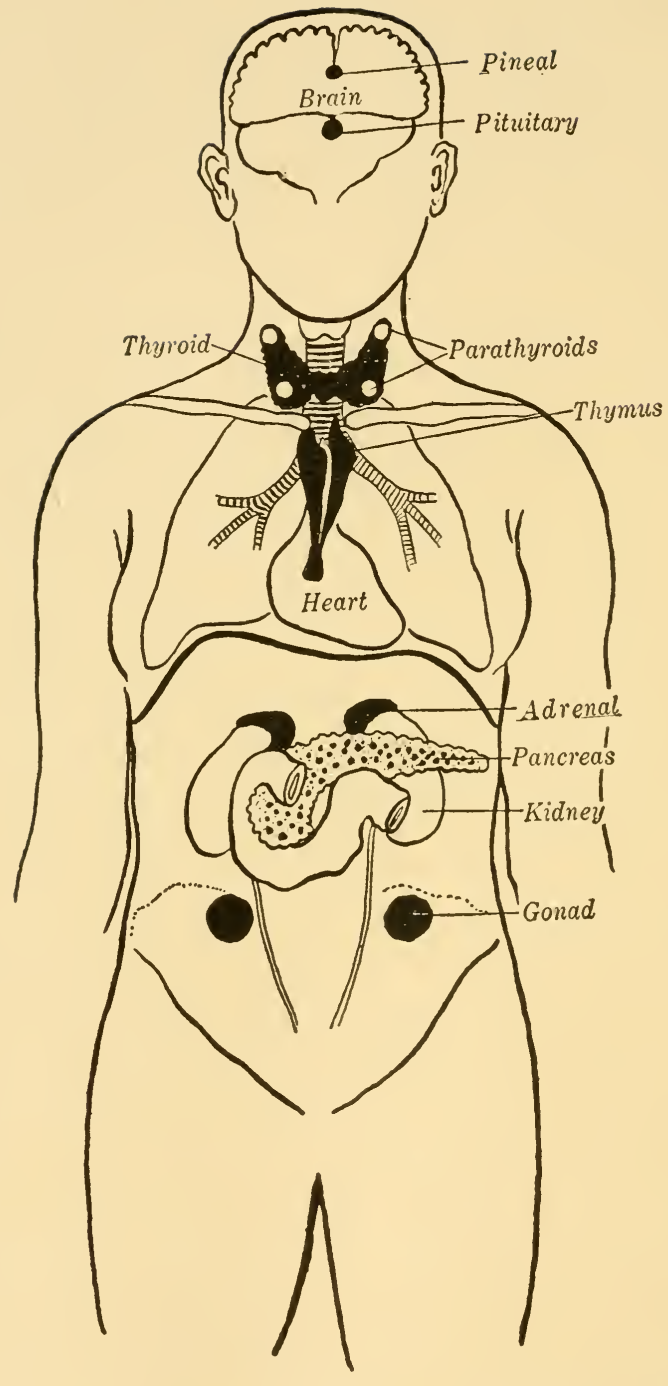

FIG. 168. Position of the Endocrine Glands in Man. 
and differentiation, for the same result is often obtained by the operative removal of different glands. Infantilism, for example, results from the removal of either the thyroid or the pituitary; and thyroidless animals may be brought to sexual maturity by the injection of the extract of the pituitary. In like manner the removal of the sex glands affects the structure and function of the pituitary, and the excision of the pituitary prevents the full development of the gonads.

So frequently and so intimately is the pituitary linked with the glands causing morphological changes that it is often called the "master gland". It affects sex development and bodily size, the effects appearing together in one individual, or independently of each other; and, conversely, other glands are necessary for the proper function of the pituitary.

From our present knowledge it seems justified to speak of the balance of glandular function; and any imbalance existing in the group will cause widespread morphological and physiological upsets. The subject is still in its infancy, and far-reaching conclusions are not warranted by the evidence.

Conclusion. The three mentioned regulators of development are not independent or mutually exclusive. The original pattern within the egg may be modified in its expression by the organizators, and both may be shifted by the function of the ductless glands; but under normal circumstances all work interdependently and synchronously. The endocrines are, perhaps, most readily affected by external, environmental causes; but in the final analysis all are but the expression of the inherited tendencies, the genetic pattern. The frequent statements regarding the "effects of the endocrines upon evolution" are but another way of saying, "the effects of heredity upon the glands, and thus upon evolution".

Gland complexes are as definitely inherited as are eye color or hair conditions. A recent discovery (MacDowell) proves that a single point mutation may affect the pituitary so that infantilism results; and although the individual may be brought to normality by gland injections, the genetic mutation remains unaffected and is passed on in a Mendelian manner. Therefore the endocrines, like all other known characters, are the expression of heredity modified by environment. 



\section{PART III}

\section{EVOLUTION OF THE VERTEBRATES}

The Greek philosophers and scientists, under the influence of Empedocles and Aristotle, realized that species of plants and animals were not immutable, and that special creation could not account for the innumerable changes which have occurred among living organisms. But after the destruction of Greek civilization in Greece and Alexandria science became static, or retrogressed, under the influence of tradition and authority. With the revival of Greek culture evolution again crept into the philosophical literature; but it was not until Descartes carried the mechanistic theory to its logical conclusion that any coherent effort was made to controvert the prevailing idea that each species was created as an unchanging unit.

So rock-bound had the dogma of special creation become that Buffon, one of the first revivers of the evolutionary theory, found it necessary in the middle of the eighteenth century to qualify his scientific speculations with the statement that all divine creations were immutable. Toward the end of the century the grandfather of Charles Darwin, Erasmus Darwin, conjectured that "one and the same kind of living filament is and has been the cause of all organic life".

A few years after Darwin, Lamarck in 1809 published his Philosophy of Zoology, a work which has gained permanence as the first clear statement of the theory. Unfortunately the influence of the dead Linnaeus and the contemporary Cuvier were sufficient to obscure the results of the great naturalist's observations; and it was not until fifty years later that the theory of evolution was placed upon a firm foundation.

Although during the years between 1809 and 1859 a number of papers had been published which gave hints of a workable theory as to the cause of evolution, the essential facts of erolution remained unnoticed until 1858 when two papers were read before the Royal Society in London. One was by Wallace who had been working in the East Indies, and the other by Charles Darwin who for twenty years had carefully collected data in England and during a long scientific royage. 
Briefly, Darwin's ideas can be stated as the theory of natural selection of the variations which always occur in all species. Because experimental research in physiology and genetics was still undreamed of, he tentatively accepted the Lamarckian theory of the inheritance of acquired characters which come about due to environment and the use and disuse of structures. He accounted for this by saying that each cell of the body sends fragments of itself, or "genes", to form the germ cells. When such variations appear, as it is known that they do, whatever their cause, certain ones will be saved; because, (1) far more individuals are born than can survive and reproduce; (2) there is a constant struggle for existence due to shortage of food and space; and (3) there will be a selection by nature, natural selection, of those animals which are most fit for existence.

\section{Bases of Evidence}

Although evidence for organic evolution has been accumulating since the first observations on natural history were preserved, it was not until Darwin began correlating the facts of different sciences that the evidence was placed upon a sound and coordinated basis. Early research in biology was limited to a study of nature, to the naming and cataloguing of specimens. This led to living organisms being grouped into phyla and classes, and the outcome was speculation as to the relationships of genera. From these random speculations and hypotheses grew the theory of evolution, which is now supported by all the biological and geological sciences.

Arranged in approximate chronological order, the sciences which have contributed to the theory are: (1) Comparative Anatomy; (2) Geographical Distribution, the study of organisms in relation to their distribution on the earth's surface; (3) Palaeontology, the study of fossils; (4) Embryology; (5) Genetics, the study of heredity; and (6) Physiology, the function of cells and tissues.

The student has studied the theoretical background of evolution in General Biology. The following chapters briefly discuss geographical distribution in relation to the vertebrates; the geological record, and the adaptive radiations of the vertebrates. 


\section{CHAPTER XX}

\section{GEOGRAPHICAL DISTRIBUTION}

IT HAS long been known that the life on two islands separated by a narrow strip of water might differ more than the plants and animals of northern Europe and Canada. Geographical distribution attempts to explain these similarities and differences, and depends upon geography and geology for the solution. The general term distribution is divided into time distribution, or palaeontology, which studies the relative periods in history when certain groups have lived; and geographical distribution which is limited to living organisms and their relative position on the earth's surface. The latter is frequently divided into vertical distribution, life from the depths of the ocean to the tops of the mountains; and horizontal distribution, the spatial relationships on the surface of the earth. The present discussion will confine itself largely to the horizontal distribution of vertebrates and its causes.

Geographical distribution depends largely upon two factors: (1) the migration of animal groups, and (2) the paths which they have followed. Migration would tend to bring animals into new environmental conditions, with the elimination of those least adapted to the particular conditions; and in time, with the slow shifting of the earth's surface, new species would evolve. Naturally the greatest similarity in animals would be found under conditions where free migration is possible.

There are several causes for the migration of animals. Passive migrations (when animals are carried by winds, water currents, or floating masses of débris) are of slight importance except among the fish and birds. A too swift current which sweeps fish from their original location would start a new center for their multiplication and spread; and a storm may carry birds miles away from their natural habitat. Some sea birds have in this 
way been carried miles inland to fresh water lakes. Many cases are known where mammals have been transported across straits and large bodies of water on floating ice, logs, or tiny floating islands of matted roots and earth which have broken from their anchorage on the shore. Under these conditions a single pregnant female would start a new colony, provided the climate and other conditions were suitable.

An inherent tendency toward roaming activity would cause a marginal extension of range. The localized area occupied by a specific group is gradually enlarged at the periphery. An extension of the principle is a longer migration of a group. Although there is a tendency among higher vertebrates to return to the same location, which might militate against permanent residence in the new environment, it is not unusual for animals or groups of animals to wander off and become lost.

According to Darwin the greatest cause of migration is the struggle for existence. Survival of one group means death for another, because the food supply of a region is soon exhausted. Only a perfect balance between plants and animals, and a complete limitation of the birth rate, could prevent this. As neither has ever been found in nature, it follows that there is a constant warfare between species, and individuals of the same species, for a proper supply of food and a place to live. Certain individuals would undoubtedly wander and start new colonies in new regions.

Migration pathways are equally important, and always a group would find obstacles in the way. Such natural obstacles are barriers. The effectiveness of barriers depends upon the habits and specializations of the animals involved. Mountain ranges would not stop birds in their flight, but dry rocky slopes would effectively prevent the migration of reptiles and amphibia. On the other hand, mountains might aid migration if the range lay north and south. To illustrate, urodele amphibia are unadapted for extreme heat; but in southerly migrations these animals would ascend the slopes where the mean temperature is lower, and would go to higher altitudes as they moved to lower latitudes. These conditions are known in such ranges as the Rockies, where the urodeles are found in the higher valleys of Mexico, many miles south of their normal range. The same mountain range 
effectively stops east and west migrations, for the urodele cannot go over the mountains. Most groups of animals find high or rocky mountains insuperable barriers.

Bodies of water are other effective barriers. Few mammals will swim far in salt water, although fresh water is not so effective in stopping them. Recently several deer were found four miles off the coast of New Jersey in an exhausted condition, and when the ticle or current is with them these animals have been known to swim twenty miles in the ocean. Howerer, studies of the mammals on islands indicate that only a few miles of salt water acts as a complete barrier.

Deserts, or a lack of water, are as difficult to cross as a superabundance of water. Only animals highly adapted to drought can stand the desert. Reptiles succeed better than either amphibia or mammals, although certain toads and mammals have become specialized for life in very dry regions.

Other barriers of physiological nature are less well understood. Throughout a large area of central Europe there is a complete lack of urodeles, correlated with a high lime content in the soil. Some mammals seem to avoid areas which lack a supply of salt, and alkalis in the water supply prevent spread into other regions. It is unknown how far the chemical nature of the soil and water has affected migration; but as the soil dictates the plant life, herbivorous animals would naturally seek plant food to which they were formerly adapted, and in this indirect way the soil would affect animal life.

Pathways from one region to another must be present or all islands would be limited to birds and flying mammals. This we know is not true. America is completely cut off from Asia, yet the animal life of North America is very similar to that of northern Europe and Asia. Evidently a land bridge at one time existed between Alaska and Siberia. The long stretch of the Aleutian Islands which leaves a relatively short space of open water between Alaska and Kamchatka would permit the migration of human beings in canoes; and in view of the fact that most of the islands are volcanic, and the region is in a condition of geological flux, the existence of a land bridge in recent times is more than mere speculation. A rise or fall of a few hundred feet in the land surface would make a land bridge; and the er- 
idence is that it opened and closed regularly during the Tertiary period. The existence of other connections between continents is more hypothetical, except for the island groups in the Pacific. Australasia is undoubtedly a drowned area, the high lands and peaks being left as the large and small islands.

With the shifting of the earth's surface barriers and bridges may rise or disappear. The repeated shifting of the coast in Italy and other regions during historical times illustrates the (geologically speaking) rapid changes which occur; and the fossilized sea shells on the rim of the Grand Canyon bear testimony to the great upheavals which have formerly taken place.

Any change of the earth's surface may be a gradual shifting of the land's weight, or a sudden revolutionary process. A small area or an entire region may sink, leaving a fault line to mark the clean-cut division between the two. Such fault lines are more prominent in volcanic regions where violent earthquakes are more or less frequent; but the most stable lands of the earth show sign of faults where one part of the land sank far below the other.

Australasia was cut from the continent of Asia by a series of faults. The general course of the major fault was determined by Wallace and is known as Wallace's Line; but more recent research has shown that the division is not so regular as was thought half a century ago. However, Bali and Lombok, two islands less than twenty miles apart, have faunas more different than those of Europe and Canada, and even than those of Eastern Asia and the Mississippi Valley.

The sinking of the lands of the southern Pacific occurred after the marsupials had become widely distributed and before the placentals had arisen. The result is that in Australasia no placentals exist except those carried into the region by man, and a few bats and rodents (and the latter may have gone with the early Malay settlers). The other mammals are monotremes and marsupials. The latter, safe from competition with the more active placentals, evolved in many directions. Almost all the groups of placentals are parallelled in external form. Some have the gnawing incisors of the rodents; others are wolf-like; others resemble insectivores; and others resemble nothing known among 
the higher group. It is one of the finest examples of adaptive radiations known among the mammals.

The opposite conditions are found among the marsupials which exist in America. Most of them failed to meet the competition with animals with better brains, and placentae which better equipped the young for the struggle of life. Those which remained were the highly generalized, nocturnal opossums which survived by hiding from their enemies. They eked out a precarious existence while the placentals conquered the earth. The American opossum is skeletally almost identical with the Cretaceous marsupials; more generalized than any mammal known to Australia.

Human migrations have been less controlled by natural barriers, but human enemies have been a telling influence. The Eskimos have been a thorn in the flesh of anatomists who contend that pigmentation and climate are definitely correlated. These arctic dwellers are a yellow-skinned race with apparently no physical adaptation for their environment. They are a Mongolic race, but not of the warlike Tartar type. When they migrated across the Alaskan chain of islands and settled in the north, they evidently attempted to find a southern home, for relics of their civilization are found along the Pacific coast. Warlike Indians drove them back and they remained in the frozen north because they could not fight their way out, not because they particularly liked the neighborhood.

The same evidence could be adduced as to the location of the pigmies in the valleys of the Amazon and the Congo. Small, low thyroid, unwarlike, they were never a match for the taller tribes of the plains. And when forced into an unfit environment, climate and disease soon selected a race which was adapted for survival. In this way barriers, both natural and human, have largely dictated the course of racial and cultural history. 


\section{CHAPTER XXI}

\section{THE GEOLOGICAL RECORD}

Assuming that the earth began its development as a central core which grew by the accretion of matter from space-and millions of meteors still reach our atmosphere every day-the force of gravity would be sufficient to hold an atmosphere when the sphere reached a diameter of slightly more than three thousand miles. As soon as gases and water had collected around the earth, winds and rains would be present, and the erosion of the surface would begin.

With an increase in diameter there would be an increase of pressure at the center, and heat would be generated, probably sufficient to transform the core into the state of a "solid gas". If then there were any reduction of pressure, these minerals would become liquid. The crust of the earth is reasonably firm and rigid, but fissures and weak lines develop which permit the potentially molten interior to push upward toward the surface. It follows that volcanic or igneous rocks would then be formed as part of the crust, due to the cooling and crystallization of the laval outcrops. Following the laws of crystallization, the more rapid the cooling process the smaller the crystals, and igneous rocks can be found which form a progressive series from those which resemble glass, to others composed of huge crystals. All volcanic flows do not reach the surface and erupt at a volcanic peak. Some are columnar masses, others are vertical planes which open and fill a crack; but the majority of such flows intrude between layers of rock already formed, and are left as great horizontal sheets of igneous rocks. It follows that all types of volcanic intrusion will vary in size from minute lines to great sheets which cover miles of territory.

The sedimentary rocks are the ones which interest the evolutionist. The meteoric rocks were the original mass, and they 
were cut and metamorphosed by igneous intrusions before the accumulation of an atomosphere; but as soon as winds and rains appeared these original rocks were decomposed and washed or blown into the valleys. Synchronously the accumulated moisture would fill the depressions and form lakes and seas, which would be gradually filled by the sediment of sand and mud. It is in these deposits that the remains of plants and animals are caught and buried. Rocks are formed from the sedimentary layers as a result of pressure and cementation. Layers of sand become sandstone, mud becomes shale, and deposited calcium carbonates are turned into limestone. The last are particularly interesting, for it is generally conceded that limestone deposits show the presence of living organisms. The carbon dioxide of metabolism unites with the calcium oxide nearly always found in water, and calcium carbonate is precipitated. Therefore, the fact that highly altered limestones are among the earliest known rocks, indicates the presence of some form of life much older than any recognizable fossils.

Sedimentary rocks are usually laid down in strata. And change in composition of the sediment would cause a distinct line of demarcation. Even so slight a change as the fallen leaves of autumn would distinguish those strata from the clearer ones of spring. When the strata are undisturbed the older ones are at the bottom, and this condition is found in many places of the earth. But fortunately for the palaeontologist, the movements of the earth's surface have tilted the strata, or turned them on end, so that miles of strata can be studied which would never be found if each stratum had remained in place. The earth movements are so vast that the relative position is rarely disturbed, and the sequence of history can be traced. A more disturbing problem for the palaeontologist is presented when a region shows that the ocean bottom has risen above the surface, been eroded, and then sunk again with more recent strata above. In such cases the line of erosion (unconformity) is usually distinct, and the gap can be partially filled from a knowledge of other undisturbed strata.

By a study of the rocks a surprisingly clear record of the earth's story has been deciphered. After the fundamental position of the strata had been determined and the fossil life care- 
fully studied, any new region discovered could be quickly and accurately identified by the remains of living organisms found embedded.

In the very nature of things the record is, and probably always will be, incomplete. Each year new data are added as new fossils are found and studied; but at best the record can be compared to an ancient parchment, its writing partially erased and re-written upon until the process had been repeated several times.

\section{A. The Preservation of Fossils}

Fossils are the remains, impressions, or petrifactions of living organisms embedded in rock by natural means. This definition has to be slightly qualified or amplified; for the twigs, beads and bits of pottery which are placed in the heavily charged waters of Yellowstone Park or Auvergne, France, would not be considered fossils, whereas the mammoths embedded in the ice of Siberia would be.

It is not necessary that the remains be exceedingly old nor "petrified". A smaller percentage than is usually thought are actually turned to stone. An unusual case of such preservation is the Siberian mammoth, some of which are so well preserved in the strata of ice that one authenticated case of its flesh being eaten is on record, and travellers would have one believe that it is of frequent occurrence. The statement is not unbelievable. These extinct elephants existed in such enormous numbers that the ivory of their tusks is an article of commerce.

In most of the skeletal remains of mammals there has been no replacement of bone by other substances, although the spaces and cavities have been filled with soluble carbonates or silicates. Microscopic sections show the minutest canaliculi. Similarly the shells of mollusks, and other hard structures, are often simply enclosed by the rock.

Impressions are also fossils. Fossil leaves and plants are usually formed by the darkening of the rock by the contained carbon. The imprint of the outline and veins was formed when a thin film of mud was laid on the leaf, and eventually pressed into stone. Jelly-fish, although about ninety-eight per cent 
water, are preserved as impressions in the sand, every structure being sometimes shown with perfect accuracy. And in this class of fossils would be placed the footprints of long extinct reptiles.

Petrifaction is the slow infiltration of water bearing dissolved mineral matter which gradually replaces the structure of the plant or animal. It is not difficult to understand how the cell spaces of a tree might be filled with silica as the protoplasm oozed away; and later, as the walls decayed, these in turn be replaced with quartz, so that thinly ground sections show the perfect woody structure. It is more difficult to understand how animal tissues were so perfectly preserved. Some crinoids (Phylum Echinodermata) have been silicified, and sections show the delicate ampullae in position, as well outlined as in the living animal.

Conditions for Fossilization. The calcareous shells of sea living animals are most abundant, for these simply remained buried in the sand or mud of the sea. If that particular region of the coast were sinking, new layers of sand, mud, or limestone would be laid above and the shell surrounded by potential rock. The shell would be preserved; or, more frequently, the lime dissolved away and the space filled with mineral matter. This latter makes a natural cast of the shell which is as perfect as the preserved animal.

Fish also would be preserved by falling to the bottom and being quickly covered before the body had disintegrated or been eaten. The layer of mud and water would prevent much of the bacterial action, and the imprint would be saved indefinitely. The essential is quick burial to prevent separation of parts, and the packing down of the superimposed stratum of earth.

Land animals are much less frequently fossilized, for conditions are not so propitious. Most animals at death are eaten by others, or the winds and rain scatter the bones so that they decay before being preserved. The destruction by carnivorous animals is very important. Mammalian fossils show the grooves made by the gnawing teeth of rodents; and a herbivorous dinosaur (American Museum of Natural History) has vertebrae with grooves which exactly fit the tooth spacing of a carnivorous dinosaur found close by. Birds, as would be expected, are the rarest of fossils, most of those found being water living varieties. 
The great number of animals found demonstrates the great length of time involved, and the abundance of material which might have been preserved. Water living amphibia and reptiles would sink into the mud. Some animals were covered by sudden landslides. Animals which roamed dry regions with constantly blowing sand would be buried, as illustrated by a group of primitive pug-like animals, the mother and her litter being almost in place. If the region were very arid the animal might be mummified before being covered. A dinosaur (Trachodon) has been found with the leathery skin preserved almost intact. The quickest death would be falling into quicksand or deep bogs of peat. A similar hazard to animal life were pits of asphalt, a particularly fine example being found in California where a great mass of bones indicates that many animals became mired in the sticky material and were buried. The evidence shows that carnivorous animals lost their lives as they attempted to eat others which had been caught in the thick natural oil. When pits of asphalt or sinks of quicksand can be located the bones of extinct animals are found enormously concentrated. Another rich source is the bottom of a cave in which animals lived, the packed down dung often disclosing layer after layer of animal remains, giving a chronological history of life in the caves.

Fossils are frequently well preserved. It is a fallacy, encouraged by those who do not understand the methods of palaeontology, that "animals are reconstructed from a single fragment". When the complete skeleton of an animal is known it is true that a larger bone from a similar animal indicates a larger individual; but reconstructions from incomplete animals are made by comparison with known species, and while minor discrepancies may later come to light the method is accurate and scientific. And the readiness with which the palaeontologist admits any error preserves the science from bigotry and tradition.

Microscopic Anatomy. The preservation of bone and wood and invertebrates has been mentioned. Other structures are equally well preserved as to microscopic structure. The histology of fish scales is studied as accurately in fossils as in living animals; the joint and vertebral tendons of some dinosaurs are 
shown in all their relationships; and even striated muscle has been found. The last was described in a fossil shark (Cladoselache) by Dean. This could hardly be petrifaction for the animal was buried in mud, and the discovery evidences the marked difference in the chemical composition between the light and dark bands of the tissue. Such illustrations could be multiplied many times, despite the fact that palaeontological histology has hardly been touched.

In conclusion it may be said that the anatomy of many fossils is almost as well known as though the animals were living. The attachment of muscles leaves a roughness on the bone, and in this way the musculature can be "dissected" with great accuracy. Casts of the calraria show the gross anatomy of the brain, even to the convolutions; and with these facts in hand the activity and the habits of the animal are more than speculation, although one has to be guarded in conclusions regarding the analogy between living animals and the dead.

\section{B. Geological Time Table}

The known sedimentary rocks have been divided into great eras. Each of these divisions, which covered enormous lengths of time, is divided into periods, and each of these into minor subdivisions. Thus, by locating the stratum in which it occurs, the relative age of the fossil is known. There is a remarkable similarity in the grade of life found in the same period, no matter where on the earth's surface it is found. There are exceptions to this rule, as illustrated by the distribution of mammals in Australia, but in such cases other animals and the sequence of strata make identification accurate.

The scientist is not as much concerned over the number of years involved as he is in the position of the strata, but many attempts have been made to give approximate dates. The biologists made the effort to determine the number of years which would be required for certain erolutionary processes to take place, but recent genetic research has shown that rays which are present in light will greatly increase the rate of mutations in the germplasm. Stratigraphers, by studying seasonable depositions of minute strata, gave an idea of the time required 
for the great strata and periods. More recently physicists have used the degeneration of radio-active minerals as evidence regarding the time elapsed since particular periods were laid down. All the evidence agrees that the earliest fossil bearing rocks are billions of years old. Estimates vary widely, but compared with the entire time involved a few million years is no more than a day in the life of a geologist.

Major ERAS of Time

\begin{tabular}{c|c}
\hline \hline Era & Dominavt Life \\
\hline Cenozoic & Mammals, including man \\
\hline Mesozoic & Reptiles. Beginning of mammals \\
\hline Palaeozoic & $\begin{array}{c}\text { Earliest reptiles } \\
\text { Amphibia } \\
\text { Fish }\end{array}$ \\
\hline Proterozoic & All invertebrate phyla \\
\hline Archeozoic & $\begin{array}{c}\text { Marine Invertebrates } \\
\text { (Arthropods, Sponges, Protozoa) }\end{array}$ \\
\hline & $\begin{array}{c}\text { Indirect evidence of Life } \\
\text { garaphites, with "fossil algae".) }\end{array}$ \\
\hline
\end{tabular}

These eras are arranged according to their relative age, the most ancient at the bottom. The Cenozoic includes time up to the present. Man evolved from the anthropoid stem in the late Cenozoic, probably half a million years ago. Estimates as to the age of the Archeozoic vary from one to several billion years.

The Archeozoic and Proterozoic, which probably represent more time than the other three combined, are only interesting to the vertebrate anatomist in that they may in time give the clue to the origin of the chordates. The vertebrates were not autochthonous, but trace their history to the most ancient forms of life. Evidences of their existence, however, begin in the Palaeozoic era; no remains or impressions of the earlier soft bodied chordates having been found. It is more than possible that such remains were preserved, for a very small, cyclostome- 
like fossil (Palaeospondylus) is known from the middle of the Palaeozoic.

The following tables, beginning with the oldest vertebrate era, are divided into Periods or major divisions; while the last table, covering the Cenozoic, is further divided into Epochs. The Cenozoic is the age of the rise of mammals and is of most interest in the evolution of man. In these tables dominant life refers to the plants and animals which are (1) most abundant; or (2) had reached the highest degree of specialization, with particular reference to the vertebrate line. It is not inferred that the invertebrate phyla were not represented.

\section{Palaeozoic Time}

\begin{tabular}{|c|c|c|}
\hline Periods & Beginnisg of New Lives & Dominant Life \\
\hline Permian & Reptile radiation & Amphibia \\
\hline Pennsylvanian & Earliest Reptiles & Amphibia \\
\hline Mississippian & Higher Amphibia & Amphibia \\
\hline Devonian & $\begin{array}{c}\text { Amphibia (?) } \\
\text { (Thinopus) } \\
\text { Fish } \\
\text { (Ray-finned, } \\
\text { Dipnoi } \\
\text { Crossopterygii) }\end{array}$ & Fish \\
\hline Silurian & Sharks & $\begin{array}{l}\text { Higher invertebrates } \\
\text { (Most abundant) }\end{array}$ \\
\hline Ordovician & $\begin{array}{l}\text { Armored "Cyclostomes" } \\
\text { (Ostracoderms) }\end{array}$ & $\begin{array}{c}\text { Molluses } \\
\text { (Cephalopods) }\end{array}$ \\
\hline Cambrian & $\begin{array}{l}\text { (?) Chordates } \\
\text { Invertebrates }\end{array}$ & $\begin{array}{c}\text { Trilobites } \\
\text { Brachiopods }\end{array}$ \\
\hline
\end{tabular}


Mesozorc Time

\begin{tabular}{c|c|c}
\hline Periods & Begriving of New Lines & Dominant Life \\
\cline { 2 - 3 } Cretaceous & $\begin{array}{c}\text { Placentals } \\
\text { (Insectivores) } \\
\text { Marsupials } \\
\text { (Opossums) }\end{array}$ & Reptiles \\
\hline Jurassic & $\begin{array}{c}\text { Sub-Marsupials } \\
\text { Birds }\end{array}$ & Reptiles \\
\hline Triassic & $\begin{array}{c}\text { First Mammals } \\
\text { Dinosaurs } \\
\text { Pterosaurs }\end{array}$ & Reptiles \\
\hline
\end{tabular}

Cexozorc Trane

\begin{tabular}{|c|c|c|c|}
\hline Periods & Ероснs & Beginxing of New Lines & Dominant Life \\
\hline Quarternary & Pleistocene & Homo sapiens & Man \\
\hline \multirow{5}{*}{ Tertiary } & Pliocene & $\begin{array}{c}\text { Early Man } \\
\text { Most Genera of mammals }\end{array}$ & Proboscidia \\
\hline & Miocene & $\begin{array}{c}\text { Sub-families of existing } \\
\text { mammals }\end{array}$ & Artiodactyla \\
\hline & Oligocene & $\begin{array}{c}\text { Establishment of existing } \\
\text { families }\end{array}$ & Rhinoceroses \\
\hline & Eocene & $\begin{array}{c}\text { Rodents } \\
\text { Perissodactyls }\end{array}$ & Perissodactyls \\
\hline & Paleocene & $\begin{array}{l}\text { Carnivores } \\
\text { Primates }\end{array}$ & Archaic Mammals \\
\hline
\end{tabular}

A new line is considered as beginning when fossils of undoubted relationships have been found in a particular period. Evidently the group had more primitive ancestry, and as research continues in the fossil bearing beds the origin of lines is being steadily pushed farther back in history. The sharks, for example, are listed as having arisen in the Silurian Period although their ancestry must go back into the Ordovician; for their rise to dominance is synchronous with that of the armored fish (Antiarchs and Arthrodires). These are undoubted fish, although gill-arch jaws are not present. 


\section{CHAPTER XXII}

\section{ADAPTIVE RADIATIONS OF THE VERTEBRATES}

IT is significant that no biological science has offered evidence which in any way controverts the theory of evolution (stated many years before controlled conditions were used in experimental research) and that each has contributed positive evidence for the theory. From these rather diverse sciences an evolutionary tree has been worked out. Cndoubtedly the major portion of the evidence as to the course of evolution has come from palaeontology, the other sources of evidence confirming the fossil story.

Many phylogenetic trees, particularly in the older books, are shown as a large main stem with small branches on either side. This pine tree analogy does not fit the evidence. The semivining "Wandering Jew" is more illustrative. From an original stem are given off many shoots which take root and grow. Each in turn divides, and as the new branches grow the older stems tend to die or become unproductive and fail to develop new buds. The plant spreads in all directions, rapidly in some places, more slowly in others, depending upon the viability of the shoot and the environmental conditions. In time only the terminal shoots are left, with a few old stems to show the lines of growth.

Similar have been the adaptive radiations of the vertebrates, and as a result many of the ancestral stems are imperfectly known. Eventually, perhaps, palaeontologists will find the "missing links" which complete the story; but as long as hiatuses exist there will be a fertile field for speculation. It is not true that a knowledge of ancestral groups is lacking, but that there are few completely generalized individual specimens. This does not interfere with the weight of evidence, for the student is constantly amazed at the great number of specimens which have been found and prepared for study.

It is incorrect to speak of any living group as being an339 
cestral to another, except in the inexact sense that one may be much nearer the common stem. A distant cousin could not be an ancestor, and it is well to keep in mind that in actual number of years each evolutionary line is as old as any other. Some of the lines have remained much more like the early stock than others, and may be used as representatives of the common stem. Amphioxus is an animal of this type. The present members of the cephalochordate group have many specializations which have been added as adaptations to changing conditions; but in fundamental anatomy and embryological development they are remarkably generalized in nature, and form a perfect beginning for the family tree of the vertebrates.

Only fairly generalized animals will exist through violent environmental changes. Many specializations fit a group for only one type of life, and here lies one of the reasons for the great number of races which have evolved and then passed completely from the earth. But when the dominant animals of the time become extinct, or of no great importance, some apparently insignificant race survives and gives rise to some new dominant type. To this class belong the mammal-like reptiles. Small, rapidly moving, seemingly unfit for existence when in competition with the dinosaurs, they were the parent stem of the mammals which survived a revolution of the earth's surface and became the dominant life of the following era.

The diagrams (pp. 337-8) show the major groups of vertebrates, and the lines from which they came. Some of the lines are left unconnected, to indicate that the earliest ancestors are not known, and the period in which they arose is undetermined. This is more true of the earlier lines, both for the reason that they were less likely to be preserved, and the older the stratum the more alteration there would be in the fossil bearing rocks.

\section{A. Origin of the Vertebrates}

In the first two chapters is an outline of the Chordate theory of vertebrate evolution. This was included there in view of the fact that the pre-vertebrates are of undoubted chordate relationships, and because the author feels that this is by far the best established hypothesis of vertebrate evolution. 
The Hemichordata and Urochordata of the present day are highly specialized, but they show the three diagnostic characters of the vertebrates (dorsal hollow nerve cord, notochord, and pharyngeal gill slits) in a simple, yet progressive, condition. The theory does not, however, account for their evolution from the invertebrates; and it is admitted that no clear evidence exists as to the parental stem of the phylum.

The greatest difficulty to be overcome in deriving vertebrates from invertebrates is the position of the body and the internal organs. The invertebrate has a ventral, solid nerve cord, and the currents of the large blood vessels are exactly opposite those of the vertebrate. Also, the retinal cell layers of the vertebrate are inverted, while those of the invertebrate have the sensory cells toward the source of light-except in the Cephalopods (Phylum Mollusca). The chordate theory presupposes that the first chordates came from a race in which definite eyes were not well developed, or were lacking, and that the vertebrate eye is a new development. The adherents of this theory have not been entirely fair in stating that other theories derive a vertebrate "by turning an invertebrate inside out and upside down."

The oldest theory of vertebrate origins traced their ancestry to the Annelids (earthworm group), and found many points of comparison. (1) "Dorsal" and "ventral" in any given animal are arbitrary terms, the mouth being considered ventral, and an annelid might easily reverse his position. The nerve cord and blood ressels would then be in the proper position; and (2) a new mouth is believed to have developed, leaving the old mouth as a vestigial structure-the neuropore found in Amphioxus. (3) A new anus developed anterior to the old, which is supported by the fact that a post-anal gut is found in the caudal end of many vertebrate embryos. (4) The notochord evolved from strands of connective tissue which are found along the ventral aorta of the annelid, a point which is not corroborated by the endodermal origin of the chordate structure. (5) The gill slits are homologized with the external openings of the anterior nephridia, and the fact that no nephridia develop in the pharyngeal region of the vertebrates is used as evidence. However, with all the apparent evidence, most embryologists and comparative 
anatomists accept the chordate theory rather than the annelid theory of vertebrate development.

Another theory traces the vertebrates back to the Nemertines, a group of worms somewhat related to the flatworms. In this case it is not necessary to turn the animal over, for the nerve cord has two lateral branches and a small dorsal one. The vertebrate would be evolved by the great overgrowth of the dorsal branch with the modification of the lateral branches into the paired ragi. It has been shown that the lateral nerves of this worm have two branches, and these are supposed to be homologous with the intestinal and lateral branches of the tenth cranial nerve. Unfortunately the other organs do not show as much similarity as does the nervous system.

Patten and others have developed the theory that the vertebrates have come from an Arachnid line (spiders, scorpions, the extinct euripterids, etc.). The Horseshoe crab is the closest living relative of the extinct line which is used as the basis for the theory. The theory automatically discards the primitive chordates, leaving Amphioxus and the cyclostomes as degenerate animals of no phylogenetic importance; for the vertebrates are supposed to have arisen as armored cyclostomes with appendages directly from an arachnid line. The theory gains strength from recent research on the Ostracoderms, or armored cyclostomes. Casts of this fossil group show resemblances to the extinct arachnids which cannot be easily dismissed. Patten derives the vertebrate jaws from the anterior appendages of the arachnid. The objections to the theory are numerous. There are no known cases of a group so highly specialized giving rise to another highly specialized one. Further objections are: (1) the reversal of layers of the eyes; (2) the dorsal chitinous armor of the arachnid remains dorsal when the animal turns over; (3) the arachnid shell becomes bony without intermediate evolution; (4) the arachnid appendages arise from a single somite, the vertebrate appendages from several; and (5) the general embryological history of the two groups is entirely different.

Gaskell, in England, working on crustacean materials, attempted to overcome the difficulties of turning the animal over, and developed the theory that a new gut evolved. There are two major points in his theory. (1) The primitive gut was 
enclosed by the lateral growth of the flat nerve cord and left as the neurocoel of the vertebrate. (2) As it was necessary to get rid of the numerous appendages along the body, and as they were found to secrete small amounts of digestive enzymes, he conceived of them fusing along the bottom and thus forming the intestinal tract.

With all the evidence stated these theories do not appear as fanciful as a brief summary would indicate. However, when the three diagnostic characters of the chordates are found in such primitive condition in living chordates, it seems to the author unreasonable to search for the ancestral line among specialized phyla which have no embryological similarity with the vertebrates. As stated previously, these early ancestors would hardly be preserved as fossils; but, like other worms of the Cambrian Period, would be known to us only as burrows in the primeval sands.

\section{B. Adapted Radiations of the Early Chordates}

It is believed that the chordate stock had reached an Amphioxus-like form in the Cambrian Period. This would give three main evolutionary lines: (1) the most primitive which has survived as the two widely different groups of Hemichordates; (2) the ancient, but now highly specialized, Urochordates (Tunicates); and (3) the line with a complete notochord which has survived as Amphioxus and the vertebrates. The last apparently gave rise in the Cambrian to two distinct lines: (A) the cyclostomes and the armored ostracoderms, the latter shorring more relationships with the cyclostomes than with the other vertebrates, and both being more closely similar to Amphioxus than to the fish; and (B) the Elasmobranchs. There is also much eridence that the bony fish had arisen before the end of the Cambrian.

The earliest sharks known have a reduced number of gill arches, and the jarts are already formed from the first pair of branchial cartilages. Cladoselache, which has been described, supplies invaluable evidence as to the fin-fold theory. The most generalized sharks still living have seven gill slits, and others six. These groups show the beginnings of the heterocercal (upturned) tail. 
The shark-like structure of the sturgeon (Chondrostei) makes it and its relatives a good starting point for the development of the Holostei and the Teleostei. The two former have always been rather limited in their distribution. The teleosts (most of the recent fish) have evolved in every conceivable direction. Some forms are eel-like; others have become flattened dorsoventrally into wide, slow moving fish; others have flattened laterally until they are almost transparent; a few are so shortened that they consist of hardly more than a head and a very short body; the soles and flounders undergo a torsion during development; and many of the deep-sea fish are strangely disproportioned. The teleosts represent the extreme specializations toward "ichthyization", and represent fish-like characters as we know them today.

Early in the Silurian the lobe-finned fish (Crossopterygii) developed from the primitive group. These and the Dipnoi parallelled each other, and became "de-ichthyized" in character as the swim-bladder evolved into a functional lung. In their early history the crossopterygians were more generalized, and one small group developed the rudiments of a humerus, radius and ulna. The dipnoans, on the other hand, were specialized from earliest known time. The teeth had lost the generalized character necessary for further evolution, and the fins had a median line of bones with rays on either side. The latter could not have evolved into a vertebrate hand and arm, and specialization apparently could proceed only in the direction which it has taken-toward degeneration.

\section{Evolution of the Anphibia}

The evidence for the evolution of the amphibia from the crossopterygian fish has been summarized under separate chapter headings. This will be recalled as (1) the retention of the spiracle, which became the middle ear; $(2)$ the structure of the pectoral fin; (3) the swim-bladder with its ventral "trachea" and its bi-lobed, vascular lungs; (4) the blood supply of the lungs coming from the sixth aortic arch; (5) external gills in the larva, and the general similarity between the tadpoles of 
the Crossopterygii and amphibia; and (6) the marked similarity between the positions of the head bones in the tro groups.

In view of the extraordinary similarity between an amphibian tadpole and a fish larva, both structurally and functionally, the embryological transition from fish to amphibian is simpler than that from amphibian to reptile. The most difficult point to understand is the derelopment of feet and toes, and this has been partly bridged by the discovery of crossopterygian fossils (Sauripterus and Eusthenopteron). The most fish-like amphibian yet discovered (Eogyrinus) is elongate; covered with bony plates; the head bones have the crossopterygian pattern; and there is a large cleithrum, a typical bone of the fish, connecting the shoulder girdle with the skull. The vertebrae are of the primitive type with two distinct rings to each segment.

The fossil Stegocephalia (the first amphibia) evolved a number of specializations. The line leading toward the living amphibia is soon lost, the legless Apoda most resembling the Stegocephalia in skull type and in having minute scales beneath the skin. Others of the Stegocephalia became reptilian in body shape, and until larval forms were discovered these were known as Pro-reptilia.

The fossils supply evidence as to the evolution of the skeleton from amphibia to reptiles, but this gives us no clue as to the evolution of the amniote type of development. The gap is partly filled by the type of egg cleavage, gastrulation, and early development of the Apoda. The egg is relatively large and yolkladen and cleavage is partly meroblastic, the dorsal blastoderm being a layer of columnar cells. Gastrulation is almost reptilian, and is an essential link in understanding the process in reptiles and birds. A small area on the edge of the blastoderm folds under and the inturned tissue grows forward as the endodermal layer. It will be recalled that gastrulation in the chick begins as a bit within the blastodermic disc.

Although this does not account for the origin of the amnion, it is not difficult to understand how the yolk mass became greater and the blastoderm relatively smaller. This being admitted, the overgrowth of amniotic folds is not the enormous transition that might at first be supposed; for, not only is the embryo spread out as a sheet with its archenteron open to the yolk 
sac, but the allantoic bladder is well developed in the amphibia. Under the conditions of a large yolk the ventral body wall would not interfere with the continued growth of the allantois, and its enlargement into a breathing organ would be possible. It is probable that the first reptiles laid their eggs in moist places with the yolk surrounded by a gelatinous capsule. It is certain that the typical embryological membranes were dereloped before the recent reptilian sub-classes were separated from the original stock.

\section{Radiations of the Reptiles}

Seymouria, of the Lower Permian Period, an era when the amphibia were still the dominant type of vertebrate life, is the most primitive reptile known. The skull is wide and flat, the brain cavity very small in proportion to the total size, the posterior margin is indented by an otic notch across which the tympanum was stretched, and the dorsal bones retain the primitive arrangement. In the vertebrae both an intercentrum and neurocentrum are present, the former being relatively large, the latter having short dorsal arches. The ribs articulated with both inter- and neurocentrum, and become progressively smaller, extending on past the sacrum to the tail vertebrae. A single sacral rib is enlarged for the attachment of the pelvis.

The more primitive characters of the first reptiles were soon lost. The skull became deeper, the ribs were less numerous, the intercentrum was smaller, and the typical two sacral ribs became attached to the ilium. These reptiles were lacking fenestrae in the dermal covering of the skull, openings through which the muscles of the jaw became attached to the dorsal surface of the skull roof. This group forms the stem of reptile evolution. The turtles are the only living representatives of the group, the Subclass Anapsida.

Two other sub-classes sprang from the early anapsid reptiles, the Parapsida and the Synapsida. The former have a single pair of dorsal fenestrae, and a deep notch on the margin of the skull between the maxilla and the quadrate. The lizards and snakes are the living representatives of the parapsid reptiles.

Synapsid reptiles had a single lateral fenestra in the dermal 
roof of the skull. This group evolved in two major directions. The smaller group evolved toward the mammal-like reptiles, and eventually gave rise to the Class Mammalia. The other line developed a second pair of fenestrae and form the reptilian subclass Diapsida.

From the diapsid reptiles came the great reptilian groups of the Age of Reptiles: (1) the dinosaurs which dominated the earth for millions of years. Some were the largest land animals known, some were marvellously armored, and others were bipedal, swift, carnivorous and powerful. (2) The Pterosaurs were carnivorous flying reptiles which became extinct with the destruction of the dinosaurs. Also included in this sub-class are (3) Ichthyosaurs, ovo-viviparous animals highly specialized for water life; (4) the living Sphenodon of New Zealand, sole representative of a once large group; and (5) the Crocodilia, distributed throughout the tropical and semi-tropical regions of the earth, which with their semi-aquatic habits survived in large numbers.

The birds, an offshoot of the diapsid stock, have left few fossil remains. Archaeopteryx, the first known bird, lived in the Jurassic. Toothed, water living birds have been found in the Upper Jurassic and Cretaceous; but the immediate ancestors of the birds are almost unknown.

There are two problems which face the student of vertebrate distribution in the transition from the Mesozoic to the Cenozoic - the destruction of the great reptiles and the survival of the mammals. The latter is more easily understood. Small, generalized, active, slinking mammals could avoid the larger reptiles during the many changes of the earth's surface; and their generalized structure would have adapted them for new environments. There are several theories for the practical extinction of the larger reptiles. "Racial senescence" is used to express the tremendous specialization of most of them, with their size, or armor, or bony excrescences; but it is doubtful if a race does become senile and die without other cause. Certain lizards today, although smaller, have the same weird growths. Another theory (Schuchert) accounts for the disappearance of the dinosaurs as due to climatic changes. The late Cretaceous was a period of elevation in America, at least, with the Rocky Moun- 
tains in process of formation accompanied by the regression of the great inland sea which covered the Great Plains. The wide marshes which bordered the sea were the home of many herbivorous dinosaurs and their carnivorous enemies. The destruction of the herbivores would cut off the food supply of the carnivores. Botanical evidence indicates that the late Cretaceous was characterized by a much cooler climate than had formerly existed, and this may have been a contributing factor. In any event, with the disappearance of the larger and more dangerous reptiles, the conflict between the reptiles and the mammals became less unequal and the better coordinated mammals won.

\section{E. From Reptile to Mammal}

Fossil remains give the story of the evolution of the mammals from mammal-like reptiles. Unfortunately one is limited to the skeletal remains in drawing conclusions from this group, for the physiological changes were vastly more important, and here one can depend only upon analogy with living groups.

A higher basal metabolism is well correlated with the increase of brain structure, and the calvaria of the ancestral reptile group shows that their brains had kept pace with their other mammalian characters. Equally good analogies are found in the structure of the appendages and girdles. The typical reptile, with large coracoids and his humerus and femur almost at right angles to his body, moves rapidly for only a short time; and the reptile therefore gorges himself at one meal and lies down for days or weeks of rest. But with a higher metabolic rate more frequent eating is necessary, and this is correlated in all forms with a running, active type of body. The Therapsid (mammallike) reptiles had a skeleton indicative of swift, prolonged motion. The body was slender, the coracoids were small, and the elbows and knees were drawn in towards the vertebral axis of the body.

Further evidence is from analogy with the higher reptiles and more primitive mammals. It is evident from living mammals (armadillos and Manis) that hair and scales exist at the same time. Hairs probably arose as sensory structures, and surely have small function in an animal like Manis; but in the higher 
forms they assist in maintaining a constant temperature. Further aid in temperature control would be supplied by the diaphragm, a structure which is well developed in the Alligator and typical of the mammals.

The embryological processes are further evidence of the kinship between reptiles and mammals. The monotremes lay large yolked eggs with meroblastic development like that of the reptile. The young are hatched by the body heat of the mother. But in the marsupials and placentals the egg is microscopic and holoblastic. These eggs are structurally very dissimilar from those of a reptile; and yet, as the egg develops, a yolk sac is formed and the embryo assumes the spread-out condition of the reptile with the archenteron connected with the yolk sac. The heart begins its development from paired vitelline veins, and many other developmental similarities exist which can be accounted for in no other way except that it is a repetition of a reptilian scheme.

But so far removed from the other mammals does the ancestry of the monotremes appear, that the mammals are thought to have arisen as two separate lines from forms which are called "reptiles" rather than mammals. Aside from much other evidence, the bony structure makes this more than plausible. The shoulder girdle of the monotreme is more reptilian than that of the higher Therapsida. The monotreme skull and ear ossicles, however, are mammalian.

The evolution of the face has been outlined. The therapsid reptiles had an enlarged calvarium; a shortened, deepened skull; eyes which were focussed forward and not outward; and a heterodont dentition. The face and skull were mammalian with the exception of a few reptilian bones.

The embryology of the ear ossicles was discussed in the section on the visceral skeleton. The skull of the Therapsida supplies the anatomical evidence. The lower jaw of the primitive reptiles is formed of six bones, the dentary being the most anterior. With the rise of the mammal-like reptiles the dentary becomes the largest, and the other bones are pushed far to the proximal end, or are lost. In the higher therapsid types the articular, angular and surangular form a small group posteriorly. The articulation of the jaws remains between the quadrate 
and the articular, both cartilage bones. In at least one known form, a second point of articulation has developed between the dentary and the squamosal. Both are functional, thus disposing of the old argument that the transition animal "could neither eat nor hear".

Until comparatively recent research showed the embryological mechanism for the evolution of the vertebrae, this was another point which depended entirely upon palaeontology for its proof. It has been stated that in the primitive condition there are two vertebrae in each body segment (an intercentrum and a neurocentrum) with the rib developing in the myoseptum. This condition is found in the earliest amphibia. The intercentrum grows progressively smaller, until in the mammal it is present only as small cherron bones in the tail region. In tailless animals they are entirely lacking.

Embryologically the vertebrae begin as eight centers of cartilage in each segment, forming two incomplete rings and corresponding to the isolated condition of the vertebrae of the cyclostomes. Soon two rings are formed in the notochordal sheaths, the anterior ring of each segment developing a neural arch. These neurocentra push anteriorly, crowding the soft tissues of the intercentra downward, until each neurocentrum articulates with the next anterior one. In the Therapsida the intercentra are left as small triangular bones between the neurocentra. In the mammals they are pushed out completely except when left as chevron bones.

Detailed research has deciphered the history of each bone of the body in its evolution from fish to mammal; the changes and the homologies of the muscles are known; and eren the minutiae of embryology have been studied and found to fit perfectly into the picture. At best these facts can be but briefly stated in an elementary text, and the student is referred to more technical books and papers.

\section{F. Mammals}

Fossils have been found in the Triassic Period which appear to be mammals or an intermediate stage. They are probably the forerunners of the mammals which are known from the Juras- 
sic, contemporaries of the dinosaurs. The earliest mammals were about the size of mice. When the first Jurassic mammalian jaw (Amphitherium) was discorered more than a century ago Curier called it a small opossum, and little evidence has since appeared to change the classification. In the Cretaceous appear numerous definite marsupials, roughly contemporary with the early Insectivora.

In the earliest strata of the Paleocene there is a fullblown mammalian fauna. These were all small, generalized, and dominantly placental. From the insectivorous stock had developed two other of the living orders, the carnivores and the primates. The latter were apparently herbivorous or omnivorous. The primates of the Paleocene were lemuroid in structure, and from this group during the succeeding periods evolved the lemurs, monkeys, anthropoids and man.

During Eocene time most of the land mammals of the present time had their beginning. On the plains were the four-toed (and later three-toed) ancestors of the horse. The leathery armadillos had attained fair size; and the Artiodactyla, the eren-toed ungulates, had begun their radiations. The archaic carnirores had attained larger size, these creodonts disappearing in the Oligocene Period, their smaller and more generalized relatives surviving as the present Carnivora. In this period arose the largest of the early mammals, the herbivorous, horned, Titanotheres.

The following succession of strata show the rise of hundreds of genera, and a number of orders. Eleven of the known orders completely disappeared in the struggle for existence, although sixteen orders have survived to the present. These surviving orders have become adapted to nearly every type of environment. For their adaptive radiations the student is referred to Scott's A History of the Land Mammals in the Western Hemisphere.

\section{G. Erolution of Max}

The first primates appeared in the Paleocene Period, are lemuroid in structure, and appeared almost synchronously in Europe and America. All were adapted for arboreal life. The orbits were large, indicating large and (by analogy with the 
present primitive stock) nocturnal eyes. The brain case is not highly expanded, the cerebellar region being relatively greatest, and has not grown forward over the long primitive face. The teeth are almost like those of the insectivores.

The Old World monkeys appear first in the Oligocene strata of Egypt and evidently gave rise to the higher primates. They soon became adapted for sitting upright in a tree; the brain enlarged; the dental formula was typical for the recent group (two premolars and three molars); and the foreleg became distinctly arm-like. The adaptive radiations which followed led to the development of many specialized forms: (1) completely arboreal types lacking thumbs, the specialization appearing independently in both Africa and South America; (2) longtailed forms with short faces; (3) the baboons and mandrills with long, dog-like heads and brilliant coloration, the most aberrant of the primates; and (4) the genera which are more like the human in structure.

The anthropoid stem appears to have split from the ancestral type in the Oligocene period. Dryopithecus, found in Europe, is sufficiently generalized in its structure that it could easily have been the stem from which the anthropoids and man evolved. The skeleton shows that the line leading toward the anthropoids and man was becoming less arboreal during early Miocene times.

A skull found in Southern Africa in recent years adds further evidence to the relationships between the African anthropoids and man. This primate, Australopithecus, is evidently a young, highly developed anthropoid, but an animal which has more human characteristics than either the chimpanzee or the gorilla. It probably lies near the common ancestral stem, but with the group which eventually gave rise to man.

The most famous human fossil known is that discovered in Java by Dubois, Pithecanthropus erectus. Since its discovery in 1891 it has been extensively studied and shown to be definitely human, of a very primitive type. The skull cap shows a cranial capacity of only a few ounces less than that of the minimum for the normal human, and although the brain was extremely primitive it had evolved more than halfway between the upper limit for the gorilla and the lower limit for the recent human. 
The brain was nearly fifty per cent larger than that of the maximum for the gorilla, but smaller than that of even the Australian bushman. If, on the other hand, the brain of the white race is used as the standard for comparison, with an average brain weight of forty-eight ounces, Pithecanthropus lies about halfway between the gorilla and man.

The other physical characteristics of the "apeman of Java" are equally primitive. The femur shows that the race walked with knees bent, but that they stood in an erect position, not with the knuckles on the ground as do the higher anthropoids. The face and teeth protruded, the facial angle being more primitive than that of the bushman. With this projecting face there were heavy ridges over the eyes and a receding forehead, giving the race an ape-like appearance. Pithecanthropus is considered a specialized branch from the human stem, not the ancestral stock of the present humans.

Another recent find is the skull of Sinanthropus from China. It is the remains of a distinct human, although considered as too far removed from the present races of man to be included in the same genus. The Piltdown man, which is known from fragments of a skull and a jaw found in England in 1913, has a larger brain case than Sinanthropus, but shows highly specialized characters. The jaw is powerful, with large canines, and slopes directly back from the base of the teeth instead of having a protruding point as in the typical human. These characteristics are distinctly ape-like. It appears from the evidence that Sinanthropus lies nearer the human stem than does the Piltdown man (Eoanthropus), the latter belonging to a specialized race which has entirely disappeared.

In the middle of the last century the fragments of a primitive skeleton were discovered in Germany and placed in the same genus with the present race but in a different species. It was named from the valley in which it was found, Homo neanderthalensis. Since that time bones and even entire skeletons have been found in different parts of Europe and Asia Minor, showing that the race extended from Germany to Spain, and eastward to the Sea of Galilee. Associated with the fossils in caves and other excavations have been found crude chipped 
stone implements, evidently knives and scrapers, the earliest record of man's cultural beginning.

The Neanderthal man was short in stature, measuring about five feet four inches in height, and walked with knees slightly bent. The head was large but did not extend much above the level of the eyes, the forehead being receding. The orbital ridges were heary as in other primitive men, the bridge of the nose was flat, and the jaws protruded in front. The lorrer jaw had a slight chin which was not as prominent as that of the present race. He most resembled the bushmen of Australia, man's most primitive living relative. With the rise of the present species, Homo sapiens, the men of the earliest stone age were probably forced to take refuge in marshes, mountains and other inaccessible places. It is interesting that in several such spots in Europe, untroubled by the ravages of invasion and immigration, groups still live who have heavier orbital ridges and more protruding jaws than are found in their neighbors.

With the rise of the Cro-Magnon men, a tall race with large heads, stone work became an art. No longer were they limited to crude implements of flint, but awls, knives, spears and arrows were made of finely chipped flint and polished bone. From this time on culture grew. Close-grained rock was hammered and polished to make axes and other implements, and fragments of pottery jaws are found with their remains. From very early times the walls of the caves were decorated with finely drawn pictures of animals of the chase, many of which have long since become extinct. At first these drawings were in black and white, and then colors were added. In the later drawings the artists show a knowledge of perspective. Sea shells, bones and teeth were fashioned into ornaments for the body, and domestic and hunting implements were decorated with beautifully sculptured designs.

Later development is not the province of anatomy. The CroMagnon men were of the same species as the present race, and there is no evidence that man has evolved either physically or mentally since the beginning of these early cave dwellers. The time element involved, however, is so short that there is no reason to believe that the evolution of man has ceased; but as to the course which it may follow, it is impossible to prophesy. 


\section{APPENDIX I}

\section{BOOKS FOR REFERENCE}

THE following list makes no pretensions of being a bibliography of vertebrate anatomy. The student who wishes to go to the original sources should have the advice of his instructor. The references included will be found in most college libraries, and can be read without a great expenditure of time.

\section{PART I}

Parker and Haswell: Text-book of Zoology, Volume II.

A book of general reference for the classification of the Chordata. There is an excellent chapter on the structure and development of Amphioxus.

Cambridge Natural History.

One of the best references for natural history, with minor discussions of anatomical details. The several volumes on the vertebrates cover the subject thoroughly.

Newman: Vertebrate Zoology.

A brief, but inclusive, treatment of natural history, with sufficient anatomical detail to show the evolutionary implications of the subject.

Willey: Amphioxus and the Ancestry of the Vertebrates.

A specialized discussion of the early chordates and their relationship with the vertebrates.

McEwen: Vertebrate Embryology.

A textbook covering the details of comparative embryology, discussed systematically, and beginning with Amphioxus.

Jenkinson: Vertebrate Embryology.

A systemic treatment of comparative embryology, more technical than the former reference. It is particularly valuable for the section on mammalian development.

\section{PART II}

Parker and Haswell: Text-book of Zoology. Volume II.

The book includes a thorotigh description of the anatomy of vertebrate types, with sections on the comparative anatomy of each class. Although not treated in a systemic manner, it is one of the best references for the details of anatomy. 
AREY: Developmental Anatomy.

An embryological treatment of mammalian anatomy, with discussions of chick anatomy. One of the best references for the organogenesis of systems.

Darison: Anatomy of the Cat.

A brief treatment of the anatomy of the cat, with discussions of general mammalian anatomy.

SCHAFER: Textbook of Histology.

A condensed text covering microscopic anatomy. It is also valuable for discussion of technique at the end of each chapter.

Broom: The Origin of the Human Skeleton.

A technical book dealing with the vertebrate skeleton, excellent for

a study of fossil amphibians and reptiles.

JAYNe: Mammalian Anatomy.

A reference book on the skeleton.

BaIley: A Text-book Histology.

A general reference for histology, the chapter on the nerrous system being particularly valuable.

Vincent: Internal Secretions and the Ductless Glands.

One of the few books giving the comparative anatomy and evolution of the endocrine organs.

SCHAFER: The Endocrine Organs.

A more recent work in two volumes on the function of the glands of internal secretion.

\section{PART III}

Nordenskiold: The History of Biology.

An accurate and recent book, more technical in treatment than the following.

Locy: Biology and Its Makers.

A well written volume, giving biographical data and the contributions of those who are included.

Schuchert: Historical Geology (Vol. II of Pirsson and Schuchert). One of the best works on palaeontology for the student.

Scотт: A History of the Land Mammals in the II'estern Hemisphere.

A book which is rather limited in scope, and in many places tech- nical in treatment. It does not include recent research, but is well illustrated.

Morgan: Evolution and Genetics.

A brief discussion of the bearing of genetics upon evolution.

Plunkett: Outlines of Modern Biology.

One of the best discussions of the physiological aspects of evolution. 


\section{APPENDIX II}

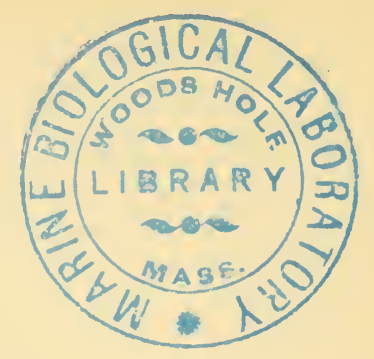

\section{CLASSIFICATION OF THE CHORDATA}

THE following classification includes the living classes and orders of the Chordates, and the fossil orders which are considered most important. All fossil groups are indicated with an asterisk (*). In an effort to indicate equivalence of phylogenetic rank it is often necessary to group animals into Superclasses, Super-orders, or Super-families. Division or Grade are also used to show such relative positions. Although this is undoubtedly the more scientific procedure, there is frequently a lack of agreement among taxonomists; therefore, for simplicity, these divisions are used as little as possible in this scheme of classification.

With the exception of the Order Primates, which is classified to family, only a few of the more important families of the vertebrates are included. The basis of selection is the need of the student in his laboratory work, rather than scientific importance.

\section{PHYLUM CHORDATA}

Sub-phylum I. HEMICHORDATA

Class 1. Enteropneusta (Balanoglossus, and other "Acorn Worms").

Class 2. Pterobranchia (Two little known genera).

Sub-phylum II. UROCHORDATA

Class 1. Tunicata (Tunicates).

Sub-phylum III. CEPHALOCHORDATA

(Contains only a few genera, including Amphioxus lanceolatus). 


\section{Sub-phylum IV. VERTEBRATA or CRANIATA}

Class 1. Cyclostomata

Sub-class 1. CYCLOSTOMI

Order 1. Myxinoidea (Myxine, Bdellostoma)

Order 2. Petromyzontea (Petromyzon marinus, the lamprey; Lampetra, and others.)

Sub-class 2. OSTRACODERMI*

Order 1. ANASPIDA *

Order 2. Osteostraci*

Order 3. Heterostraci *

Class 2. Pisces

Sub-class 1. ELASMOBRANCHII

Order 1. Cladoselachit * (Cladoselache $*$ )

Order 2. Pleuropterygit *

Order 3. ACANTHOdi *

Order 4. Selachir (Squalus, Mustelus, and many others. Includes several hundreds of genera of sharks, skates, and rays; both living and fossil).

Order 5. Holocephali (an aberrant group, often given the rank of a sub-class.)

Sub-class 2. TELEOSTOMI

Order 1. Chondrostei (Acipenser, Scaphyrhynchus, Polyodon and numerous fossils, including most primitive Actinopterygii.)

Order 2. Holostei (Lepidosteus, Amia, and many fossils).

Order 3. Teleostei (a heterogeneous assemblage, technically divided into several orders.)

Order 4. Crossopterrgir (Eusthenopteron*, Sauripterus*, Polypterus, Calamoichthys.)

Sub-class 3. DIPNOI

Order 1. Sirenoidei (Neoceratodus, Protopterus, Lepidosiren, and some closely allied fossils.)

Sub-class 4. PLACODERMI*

Order 1. ANTIARCHI *

Order 2. ARthrodira*

Class Amphibia

Order 1. Stegocephalia * (Archaegosaurus*, and others.)

Order 2. Urodela (Includes those with persistent gills, Necturus, Siren; Cryptobranchus; and the salamanders and newts, Amblystoma, Triturus.)

Order 3. Anura (The frogs and toads; Rana, Bufo.)

Order 4. Gymnophiona (The caecelians, or legless amphibia.)

\section{Class Reptilia}

Sub-class 1. ANAPSIDA

Order 1. Сотylosatria * (Seymouria*)

Order 2. Cheionia (Turtles, Chelone, Chrysemys; and the land tortoises.)

Sub-class 2. SYNAPSIDA *

Order 1. THEROMORPHA *

Order 2. Therapsida * (Cynognathus*, Ictidopsis*) 
Sub-class 3. DIAPSIDA

Order 1. RhYNchocephalia (Sphenodon)

Order 2. CRocodilia (Alligator, Crocodilus)

Order 3. Divosauria *

Order 4. Pterosalria*

Sub-class 4. PARAPSIDA

Order 1. LACERTILIA (Lizards)

Order 2. Ophidia (Snakes)

Order 3. Ichthrosacria * (Aquatic reptiles).

Order Plesiosalria (Probably a separate sub-class, between the Synapsida and Parapsida).

\section{Class Aves}

Sub-class 1. ARCHAEORNITHES * (Archaeopteryx).*

Sub-class 2. NEORNITHES

(The birds with shortened tail and bird-like wings are divided into the fossil tooth-bearing birds, and the recent birds which lack teeth. The latter include the (1) Ratitae, with more or less degenerate wings and a smooth sternum; and (2) the Carinatae, or flying birds, with a keel (carina) on the sternum. There are

Class Mammalia twenty-eight orders of the Neornithes.)

Sub-class 1. PROTOTHERIA

Order 1. Monotremata (Ornithorhynchus, Echidna.)

Sub-class 2. METATHERIA

Order 1. Marsupialia (The American opossum, Didelphys; and the Australian marsupials, kangaroos, wombat, etc).

Sub-class 3. EUTHERIA

Order 1. INsectivora (The moles, shrews, etc.).

Order 2. Primates

Sub-order 1. Lemuroidea (Lemur, Tarsius, Chiromys).

Sub-order 2. Anthropoidea

Family 1. Hapalidae (Hapale, the marmoset)

Family 2. Cebidae (South American monkeys)

Family 3. Cercopithecidae (Old World monkey's; Macacus, Cercopithecus and the baboons, Cynocephalus).

Family 4. Simiidae (The anthropoid apes; Gibbons, Hylobates; orangs, Simia; chimpanzee, Pan; gorilla, Gorilla; Australopithecus*)

Family 5. Hominidae (Pithecanthropus*; Eoanthropus*; Sinanthropus*; and Homo).

Order 3. Chiroptera (The bats)

Order 4. Dermoptera (One genus, Galeopithecus).

Order 5. Tubulidentata (Edentates; the Aard rarks).

Order 6. Pholidota (An edentate, Manis).

Order 7. Хexirthra (Ant-eaters, Armadillos; sloths). 
Order 8. Rodentia (Rat, capybara, beaver; for the rabbit see Lagomorpha).

Order 9. LAGoMoRpHa (Rodent-like animals, with two pairs of incisors; Lepus, the rabbit).

Order 10. CaRnivora

Sub-order 1. Fissipedia (Canis, Felis, and other carnivores adapted for land life.)

Sub-order 2. Pinnipedia (Walrus, Sea-lion, Seals).

Order 11. Hyracoidea (Hyrax).

Order 12. Cetacea (The whales and porpoises).

Order 13. Sirenia (The dugong and manatee).

Order 14. Proboscidea (The elephants, Mastodon*, Mam$m o t h *)$.

Order 15. Perissodactyla (Horses, Eohippus,* Merychippus,* Mesohippus,* Equus; Zebras; Asses; Rhinoceros).

Order 16. Artiodactrla (Cattle, Bos; Bison; Deer; Hippopotamus). 


\section{APPENDIX III}

\section{GLOSSARY}

AN ALPHABETICAI list of technical terms and systematic names, with their derivation and meaning. The following abbreviations are used: G., Greek; L., Latin; F., French.

A, or AN (G. without), a prefix combined with any terms; Anura; acentrous.

$A B$, (L.), away from; abduct; aboral.

ABDOMEN (L.), the body region containing the viscera; in mammals limited to the region between the diaphragm and the pelvis. abducens (L. $a b$, plus duco, to lead), Cranial Nerve VI.

ABDUCT (L. abduco, lead away from), to carry away from a given point. ACAnthias (G. acantha, a horn), a genus of sharks; also the specific name of Squalus acanthias, the American dogfish.

ACENTRous (G. a, plus kentron, a center), usually in reference to a vertebra lacking a centrum; e. g., the atlas.

ACETABULUM (L. a cup), the socket in which the head of the femur articulates.

ACIPENSER (L. acipenser, a sturgeon), a genus of chondrostean fish, the most primitive living bony fish.

ACoustic (G. akoustikos, related to hearing), pertaining to the auditory structures or function; acoustic nerve.

ACROMION (G. acros, top, plus omos, shoulder), the prolongation of the spine of the scapula.

ACUTE (L. acuo, to sharpen); in anatomy, sharp when applied to a process or angle; in medicine, having a short serere course.

AD (L. toward, upon), a prefix; adduct, adrenal.

ADDUCT (L. ad, plus duco, to lead), to carry toward; adductor.

ADIPOSE (L. adeps, fat) an adjective referring to fat, as adipose tissue.

adrenal (L. ad, upon, near; plus renes, kidney) ductless glands near the kidneys.

ADRENALIN, the active hormone secreted by the adrenal medulla.

AFFERENT (L. ad plus fero, to bear) a nerve, blood vessel, or other structure leading toward a given position.

albiNo (L. albus, white) an individual genetically lacking pigment in the skin and its derivatives.

ALI (L. ala, a wing) a prefix, as in alisphenoid.

Allantors (G. allas, allanto, sausage, plus eidos, form) an outpocketing of the archenteron, posterior to the yolk sac. The embryonic respiratory organ of the reptiles and birds. 
ALveolus (L. a small cavity) a small cavity or pocket, as the socket of a tooth, or the air pocket of the lung. Plural, alveoli; adjective, alveolar.

Amblystoma (G. amblys, blunt plus stoma, mouth) a genus of urodele amphibians; an important form in experimental anatomy.

Amia (G. amia, a variety of tuna fish) a genus of the Holostei; a fish more resembling the teleosts than other genera of the order. The name is a misapplication of terms.

Ammocetes (G. ammo, sand, plus coetes, sunk in) the larral stage of the lamprey (a cyclostome) formerly thought to belong to a different family.

AMNION (G. amnion, a foetal membrane) a membrane enclosing the embryo in the reptiles, birds and mammals.

Ammiota, or AmNiotes (see amnion) a group of the vertebrates which includes those which develop an amnion and allantois.

Axxiotic fluid, the fluid secreted by the embryo which fills the amniotic cavity.

Aмpнiвia (G. amphi, double, plus bios, life) a class of vertebrates which hatch as gill-breathing larvae, and usually metamorphose into lung breathing adults.

AMPHiCOELOUs (G. amphi, plus koilos, hollow) a structure concave at both ends, applied to vertebrae.

Amphioxus (G. amphi, plus oxys, sharp) a vertebrate-like lower chordate, of the Sub-phylum Cephalochordata.

AMPHIPLATYAN (G. amphi, plus platys, flat) flat on both ends, applied to vertebrae; the mammalian type.

Amphiuma (G. amphi, plus pneuma, breath) a genus of urodele amphibia with rudimentary legs, and retaining gill slits.

AMPUlla (L. a flask) a bladder-shaped enlargement, as the ampullae of the semicircular canals.

ANAlogous (G. ana, according to, plus logos, proportion) similar in function, but not necessarily alike in genetic relationship; opposed to homologous. Two structures may be analogous, homologous, or both. The wings of a bird and those of a bat are both analogous and homologous.

Axamia (G. an, plus amnion) vertebrates which do not develop an amnion during embryonic development; the cyclostomes, fish, and amphibia.

ANAstomosis (G. several openings) a union, or running together, as of two or more veins or other structures.

Axkrlosis (G. ankylos, bent) a union or knitting together of two or more bones or parts of bones.

ANTE (L. before) in front of, anterior.

ANтhro (G. anthropos, man), a prefix referring to human; anthropology; Pithecanthropus.

Anthropoidea (G. anthropos, plus cidos, form), a sub-order of primates including the higher apes and man.

Anura (G. an, plus oura, tail), an order of tailless amphibia, the frogs and toads.

AxUs ( $L$. a ring), the posterior opening of the intestinal canal.

AORTA (G. aeiro, to lift), the large artery leaving the heart.

Apoda (G. a, plus pous, foot), see Gymnophiona. 
APONEURosis (G. apo, from, plus neuron, a sinew), a broad flat fascia or sheet of tissue, attaching a muscle.

APSIS (G. an arch), a combining form; synapse, Anapsida, Diapsida.

AQUA (L. water), a combining form; aqueous, aqueduct.

ARACH (G. a spider's web).

ARBOREAL (L. arbor, a tree), pertaining to trees, as tree-living.

ARACHNOID (G. arach, plus eidos, form), the rery thin, middle covering of the brain, between the pia and dura mater.

ARChaEOPTERYX (G. archos, ancient, plus pteron, wing), a fossil bird of the Jurassic Period, with teeth and a reptilian tail.

Archegosaure's (G. archos, plus sauros, a lizard), a primitive genus of extinct stegocephalian amphibia.

ARCHENTERON (G. archos, plus enteron, gut), the embryonic digestive tract, formed by gastrulation.

ArcheozoIC (G. archos, ancient, plus zoon, animal), the most ancient sedimentary rocks, followed in succession by the Proterozoic.

ARCUALIA (G. arcus, a bow), the cartilaginous anlagen of the vertebrae, eight arcualia appearing in each segment.

ARTHRos (G. a joint), a combining form; arthritis, Arthrodira.

Artiodactyla ( $G$. artios, even, plus daktylos, finger, toe), an order of herbirorous mammals with the axis of the leg between the second and third digits; cows, deer, sheep, pigs, and hippopotami.

ARTtENoID (G. arytaina, a funnel), a pair of laryngeal cartilages lying posterior to the thyroid cartilage.

atlas (G. tlao, to bear), the first cervical vertebra.

ATRIUM (L. entrance chamber), an outer cavity, as the atrium (auricle) of the heart; or the specialized outer cavity of Amphioxus or the tadpole.

ATROPHY (G. $a$, without, plus trepho, nourish), a wasting or withering of the body or any of its parts.

ALRICLE (L. diminutive of auris, ear), the pinna of the ear, or the receiving chamber of the heart.

Australopithecus (L. auster, south, plus G. pithekos, ape), a fossil anthropoid found in Africa, with human characteristics.

Atто (G. self), a prefix; autonomic, autointoxication, autostylic.

Aves (L. birds), a class of vertebrates with feathers.

AXILLA (L. diminutive of axis), the armpit.

AXIS (L.), a line around which parts are symmetrically arranged; as the antero-posterior axis of the body.

AXONE (G. axon, axis), the efferent fiber of a nerve cell.

AZYGos (G. a, without, plus zygon, a yolic), a mammalian vein homologous with the cardinals. Azygous (adjective), occurring singly.

Balanoglossus (G. balanos, acorn, plus glossa, tongue), a genus of marine, worm-like, Hemichordates; the "acorn worms".

BASI (L. base), a prefix, basidorsal, basioccipital.

Bdellostoma (G. bdellion, a variety of plant, plus stoma, mouth), a genus of myxinoid cyclostomes from the Pacific.

BI (L. two), a prefix, biceps, bi-lobed.

BIos (G. life); a combining form; biology, amphibia, biogenetic.

BLAST (G. blasteo, to sprout), a combining form denoting formation or development. 
BLASTOCOEL (G. blastos, a sprout, plus koilos, cavity), the cavity of the blastula, the segmentation cavity.

BLASTODERM (G. blastos, plus derma, skin), the germinal membrane giving rise to the embryo; found in vertebrate eggs with meroblastic cleavage; blastoderm is in distinction to extra-embryonic tissue.

BLASTULA (G. dim. of blastos), a hollow sphere of cells formed by early cleavages; in the amniotes generally, a flat plate of cells.

BODY STALK, an embryonic stalk connecting the embryo with the yolk sac; its external cell layers are continuous with those of embryo and amnion.

BRACHIAL (L. brachium, arm), an adjective referring to the arm or its structure.

BRACHY (G. short), a prefix; brachycephalic, brachydactylous.

BRANCHIA (G. branchia, gills), a combining form in hemibranch, branchial, Branchiostoma; branchia may be used as a noun.

BRONCHIOLE (G. dim. of bronchos, windpipe), a minute bronchial tube. BRONCHUS (G. bronchos, windpipe), one of the larger divisions of the trachea.

BUCCA (L. cheek or mouth); as buccal cavity.

BULLA (L. a bubble), a hollow bony growth, as the tympanic bulla.

BURSA (L. purse or wallet), a pouch or sac, as the bursae of a joint.

CAECUM (L. caecus, blind), a pouch open at only one end; particularly the pouch at the juncture of the small with the large intestine.

CALVARIUM (L. skull), the brain case.

Cambrian (L. Cambria, for Northern Wales), the oldest geological period of the Paleozoic Era.

CANINE (L. canis, dog), the first mammalian tooth posterior to the incisors; they are unusually long in the carnivores.

CAPILLARY (L. capillus, a hair), any hair-like structure; particularly the smallest blood ressels lying between the arterioles and renules.

Capitosaurus (L. caput, head, plus G. sauros, a lizard), a genus of extinct stegocephalian amphibia.

Carboniferous (from carbon, or coal measures), a general name for the upper periods of the Paleozoic Era, in which most of the coal beds are found.

CARDIA (G. kardia, heart), pertaining to the heart; cardiac, pericardium.

CArina (L. keel), a projecting, keel-like structure, as the carina of a dogfish chondrocranium, or the keel of a bird sternum.

Carinatae (L. carina, a keel), a sub-class of birds having a keel on the sternum, the flying birds. See Ratitae.

Carnivora (L. caro, (carn), flesh, plus voro, to derour), an order of flesh-eating mammals with large canine teeth; dogs (Canidae), cats (Felidae), bears (Ursidae), etc.

CAUDA (L. tail), usually in adjectival form; caudal, caudad; or, cauda equina.

Cenozorc (G. kainos, new, plus zoon, animal), the most recent of great geological eras, following the Mesozoic.

CEntrum (L. center), a heary central region of a vertebra, from which spring the spinous and transverse processes.

Cephale (G. kephale, head), a combining form; cephalic, cephalad. 
CEPHalization (G. kephale), the tendency of nerve tissues to collect in the anterior part of the body.

CERCos (G. kerkos, tail), as suffix in heterocercal, homocercal.

CERATo (G. keras, horn), as prefix in ceratohyal, etc.

Ceratodus (G. keras, plus odous, tooth), an extinct genus of lung fishes (Dipnoi); sometimes Neoceratodus.

CEREBELlum (L. dim. of cerebrum, brain), the anterior development from the hindbrain.

CEREBRUM (L. cerebrum, brain), the dorsal growth of the telencephalon; the higher centers of the brain.

CERrix (L. neck), a neck-like opening, the cervix of the uterus; as adjective, cervical, applying to neck.

Cetacea (G. ketos, whale), an order of mammals, including the porpoises and whales.

Chelonia (G. chelone, tortoise), an order of reptiles; turtles, etc.

CHIASMA (G. a cross mark), usually in reference to the optic chiasma or crossing of the nerve fibers from the retina.

Chimeroids (G. chimaira, monster), an order (Holocephali) of elasmobranch fishes. They are very aberrant relatives of the sharks.

Chiroptera (G. cheir, hand, plus pteron, wing), an order of flying mammals, the bats.

Chlamydoselache (G. chlamys, a mantle, plus selachos, shark), a genus of primitive, six-gilled sharks from the coast of Japan.

CHoANA (G. a funnel), the internal and external openings of the nares.

CHONDROCRANIUM (G. chondros, cartilage, plus kranion, skull), the cartilaginous skull of cyclostomes and elasmobranchs; and the embryonic skull of higher embryos, around which the dermal bones are laid down. The chondrocranium ossifies in groups above the Chondrostei.

chondros (G. cartilage), a combining form; perichondrium; Chondrostei.

Chondrostei (G. chondros, plus osteon, bone), an order of bony fishes, including the sturgeon and spoon-bill (Polyodon), in which the chondrocranium remains unossified. The most primitive living bony fish.

CHORDA (L. a cord or string), a combining form; notochord, Chordata.

Chordata ( $\mathrm{L}$. chorda), a phylum of animals, including the vertebrates and three other sub-phyla.

CHORION (G. membrane), the outer embryonic membrane, developed synchronously with the amnion; and until fusion is completed, continuous with it.

chyмe (G. juice, fluid), a suffix, as in mesenchyme, referring to embryonic cells before tissue formation has taken place.

CILIARY (L. cilium, eyelid), near, or associated with, the eye or eyelashes.

Cladoselache (G. clado, branch or twig, plus selachos, shark), a genus of extinct sharks with fins attached to the body by broad bases; an important link in the fin-fold theory.

CLAST (G. klao, to break), a suffix as in osteoclast, the bone destroying cells.

CLIToris (G. kleio, to close), a structure of the female genitalia, homologous with the urethral body of the penis.

CloACA (L. cloaca, a sewer), the common opening of the digestive and urinogenital openings; found in many fish and amphibia, reptiles, birds, and monotreme mammals. 
COELOM (G. koilos, a carity), the carity formed between the somatopleure and splanchnopleure of the hypomere; the body carity.

сомма (G. to cut off), a suffix referring to a dividing sheath; myocomma. condylus (L. knuckle), a condyle or projection.

conjunctiva (L. conjunctus, to join together), the ectodermal epithelium connecting the outer covering of the eyelids with the covering of the cornea.

CORACoID (G. korakoeides, like a crow's beak), particularly the ventral pair of bones in the pectoral girdle, lost in the higher mammals except for the coracoid process of the scapula.

Corium ( $L$. leather), the dermal portion of the skin.

CORNEA (L. corncus, horny), the outer coat of the eyeball, derived from ectoderm and mesoderm.

CORNUA (L. horn); as, cornu (horn) of hyoid or uterus; as adjective, cornuate.

CORPUS (L. body), corpora, plural; corpora quadrigemina, corpus luteum. CORPUS LUTEUM (L. corpus, plus luteo, yellow), cellular bodies formed on the ovaries after the rupture of a follicle.

cortex (L. bark); anatomically, the outer portion; cerebral, renal, or adrenal cortex.

Costa (L. rib); costal cartilages, intercostal spaces.

CRANIAL (G. kranion, skull), the skull, specifically the brain case or calvarium; cranial nerves. As a noun, cranium.

Cretaceous (L. creta, chalk), the most recent period of the Mesozoic Era, following the Jurassic; described first in the chalk measures of England.

CRETIN (F. cretin, an idiot), a type of infantilism, partially corrected by thyroid feeding.

Cribriform (L. cribrum, a sieve), the sieve-like ethmoid plate through which the olfactory nerves pass.

Crocodilia (G. krokodeilos, crocodile), an order of diapsid reptiles including the crocodile, alligator, cayman and gavial.

Cro-Magnos (town in France), a race of recent men (Homo sapiens), the care dwellers of France and Spain.

CRossor ( $G$. fringe), a combining form.

Crossopteryail (G. crossoi, plus pteron, a wing or fin), an order of bony fishes from which the amphibia evolved.

crypto (G. hidden), cryptorchid, Cryptobranchus.

Cryptobranchus (G. kryptos, hidden, plus branchia, gills), a genus of American amphibia; the largest living amphibian (of Japan) is sometimes included in the same genus.

Cutis (L. skin); cutaneous, of or pertaining to the skin.

crclos (G. a circle), a combining form; cycloid, cyclostome.

Crclostomata (G. kyklos, round, plus stoma, mouth), a class of rertebrates lacking jaws; the most primitive rertebrates.

Crnogiathus (G. kynikos, doglike, plus gnathos, jaw), a genus of extinct therapsid, or mammal-like, reptiles.

cyst (G. kystis, a bladder), a membranous sac or vesicle; the cystic lobe of the liver.

сүто (G. kytos, a hollow space), a combining form; eytology, leucocyte.

DACTYL (G. daktylos, a finger), a combining form; pterodactyl, brachydactyl. 
DEFERo (L. to carry away), as vas deferens.

DELPHIN (L. dolphin), in Delphinus, a cetacean.

Delphos (G. uterus), a combining form; Didelphys, the opossum; Monodelphia, a variant name for Placentalia.

DELToID (G. fourth letter of alphabet), shaped like the letter delta, triangular.

DENDRON ( $G$. tree), a stem in dendrite, dendritic.

DENS (L. tooth); dental, dentary, dentine.

DERMA (G. skin); dermal, dermis; relating to skin.

DeRMATOMe (G. derma, plus toma, a cut), the portion of the epimere giving rise to the skin.

Devorian (from Devon, England, where the strata were described), a

Paleozoic period lying between the Silurian and Mississippian.

DI (G. two); digastric, dimorphic, didelphic, diapsid.

DIA (G. dia, between, through); diaphragm, diapedesis.

DIAPHRAGM (G. dia, through, plus phragnymi, enclose), a diriding membrane; the diaphragm of the ear; or, a muscular partition between the thoracic and abdominal carities.

DIASTEMA (G. interval), a wide space between teeth, usually caused by developmental suppression of the intervening teeth.

DIGIT (L. digitus, a finger), a finger or toe; digital.

DIMORPHISM (G. di, plus morphe, form), existing under two distinct forms.

Dinosauria (G. deinos, terrible, plus sauros, lizard), an order of extinct diapsid reptiles.

Diplos (G. double), diploblastic, diploid.

Dipnor (G. di, plus pneo, to breathe), a sub-class of fishes; the lung fishes with gills and lungs.

DORSAL (L. dorsum, back), pertaining to the back of an animal.

DUco (L. to lead), a combining form; adduct, abduct, aqueduct.

DUODENum (L. duodeni, from duodecim, twelve), the part of the intestine immediately following the stomach, in reference to its length in the human.

DURA (L. hard, tough), the heavy external membrane surrounding the central nervous system; dura spinalis, dura mater.

Echidna (G. echinos, a hedgehog, or G. echidna, an adder), a genus of monotremes of Australasia, the spiny anteater.

Ест, Есто (G. outer, outside), a prefix; ectoplasm, ectoderm.

ECTODERM (G. ektos, plus derma, skin), the outer cell layer of an embryo.

Edentata (L. e, without, plus dens, a tooth), an order of placentals with degenerate teeth. The order is an artificial group, and is technically divided into three distinct orders.

EFFECTOR (L. efficio, to carry out, to perform), a structure or organ transforming motor impulses into motor action.

EFFERo (L. to carry away from), a combining form; an efferent nerve or blood vessel leading away from a given point; efferent branchials; vasa efferentia.

ELASwo (G. elasmos, a plate), a combining form; flat, plate-like.

Elasmobranchi (G. elasmos, plus branchia, gills), a sub-class of fishes including the sharks, skates and rays. Their skeleton is entirely of cartilage. Several extinct orders are known.

ENAMEL (L. smaltum, enamel), the ectodermal covering of the teeth, the hardest substance of the body. 
END, ExDo (G. within, inside), a prefix.

ENDOCRINE (G. endo, plus krino, to separate), pertaining to the ductless or internally secreting glands.

ENDODERM (G. endo, plus derma, slin), the inner primary germ layer of cells; the lining of the archenteron, formed at gastrulation.

Exdostrle (G. endo, plus stylos, a column), the rentral pharyngeal groove of Amphioxus and the lamprey larva, homologous with the thyroid gland.

Exteron ( $G$. intestine), the primitive gut; a combining form, archenteron, enteritis.

Eocexe (G. eos, dawn, plus kainos, new), an early geological period of the Cenozoic Era. The Paleocene now includes the oldest strata of the Cenozoic.

EP, EPI (G. upon, over), a prefix; epiphysis, epidermis.

EPAXIal (G. ep, plus axis), above the axis of the body; particularly the dorsal half of the myotome.

EPIDERMIS (G. epi, plus derma, skin) the outer ectodermal layer of the skin. EPIDIDYMis (G. epi, plus didymos, testicle) the mass of efferent tubules of the testes, homologous with the mesonephric tubules; the rasa efferentia.

EPIMERE (G. epi, plus meros, part), the upper part of the primitive mesodermal somite, separated from the hypomere by the nephrotome; it gives rise to the myotome, dermatome, and sclerotome.

EPIPHYSIS (G. epi, plus phyo, to grow), a dorsal growth or outpocketing; the epiphysis of the diencephalon (pineal); or the center of ossification at the end of a long bone.

ERYTHRo (G. red); combining form in erythrocyte, Erythrogaster, erythema.

Esophagus (G. oiso, will bear, plus phagein, to eat) the food tube, leading from the pharynx to the stomach.

EтHMOID (G. ethmos, sieve, plus eidos, like), the anterior group of replacement bones in the chondrocranium, including the cribriform plate.

Eustachian (Eustachio, an Italian anatomist, 1534-1574); the Eustachian tube, connecting the pharynx with the middle ear; a modified gill slit.

EVAGiNation (L. $e$, out from, plus vagina, a sheath), an outpocketing from a hollow structure.

Ex (L. out, outside), external, excretory, exophthalmic.

Excreta (L. ex, plus cerno, to separate) waste materials excreted by the body; distinguished from secretions, having a bodily function; and feces, waste products which have not been digested.

ExCRETORY (L. see excreta), any gland or duct concerned with excretion.

FALCIFORM (L. falx, a sickle), curved like a sickle; as, falciform cartilage or ligament.

Fallopiax (Fallopio, Italian anatomist, 152?-1562), Fallopian tube, in human anatomy the duct connecting the ovary with the uterus; developmentally the upper end of the Müllerian or oviduct.

FASCIA (I. a band), a sheet of connective tissue investing an organ or attaching a muscle. 
FENESTRA (L. window), any window-like opening; the diapsid reptiles have two fenestrae in the roof of the skull.

FERO (L. to carry or bear), suffix in afferent, efferent.

FILUM (L. a thread), combining form; filiform, filoplume, filum terminale. FLocculus (L. floccus, a piece of wool), the lateral outgrowths on either side of the cerebellum.

FOLLICLE (L. folliculus, diminutive of follis, a bag), any minute cavity, sac or tube; as, the follicle from which a hair grows.

FoNTANEL (L. diminutive of fons, a fountain), an unossified area in the embryonic or infant skull; the space where four dermal bones have not met.

FORAMEN (L. an opening, from foro, to bore through), an opening through a membrane or bone; the foramen ovale in the embryonic auricular septum; or, the foramina of the skull.

FosSA (L. fossus, dug out), a shallow pit or depression; the fossae of the scapula; the temporal fossa; or the endolymphatic fossa.

FRONTAL (L. frons, the brow), pertaining to the forehead.

FUNDUS (L. bottom); the bottom or basal part; the rounded bottom of a hollow organ; the fundus of the stomach.

GANGLION (G. a tumor), a group of nerve cells, set off to themselves, serving as a center of nervous influence.

GANoID (G. ganos, bright, plus eidos, like), a shiny substance found in the scales of certain fish; the ganoid fishes include widely different forms, the Chondrostei, Holostei, and Crossopterygii.

GASTER (G. stomach), as combining form (gastro, gastero) referring to the stomach; gastric, mesogaster, etc.

GASTRULA (L. diminutive of gaster), an embryo at the stage when two germ layers are present, endoderm and ectoderm, formed by gastrulation.

GENITAL (L. genitalis, from gigno, to beget) pertaining to the reproductive organs; genital papilla.

GENUS (L. origin), a division in classification, a more coherent group than a family, but more diverse than a species. Generic names are capitalized: Homo sapiens.

GERM (L. germen, a sprig; germino, to sprout), a germ cell; in combining forms, a primary source.

GERMINATIVUM (L. germino), the basal ectodermal layer of the skin in which growth proceeds.

GLANS (L. acorn), the rounded end of the clitoris or penis, derived from the genital papilla.

GLOMERULUS (L. a ball of yarn), an agglomeration of vessels forming a rounded mass; specifically, the coiled ressel of a renal corpuscle.

GLOSSA (G. tongue), a combining form; glossopharyngeal, Balanoglossus, hypoglossal.

GLotTIS (G. glotta, tongue), the opening from the pharynx into the trachea.

GNaThos (G. jaw), a combining form; agnathous, Gnathostome.

Givathostomata (G. gnathos, plus stoma, mouth), vertebrates with jaws; distinguished from the Agnathostomata or cyclostomes.

GoNAD (G. gonos, seed), a reproductive gland, either testis or ovary. 
Graafias (de Graaf, a Dutch anatomist, 17th Century), specifically the graafian follicle which surrounds the orum in the ovary.

GRADUS (L. gradus, step, from gradior, to walk); as plantigrade, digitigrade, unguligrade.

GUBERNACULU ( $\mathrm{L}$. a rudder), the short ligament in the mammalian embryo, attached to both testis and scrotum.

Gymnophiona (G. gymnos, naked, plus ienai, to go), an order of legless amphibia, primitive in skull structure, and haring minute dermal scales in the skin. Synonymous with Apoda.

HAEмo (G. haima, blood), a combining form; variant of hemo.

HaENIOGLOBIN (G. haima, plus L. globus, a globe), a blood protein containing iron, with an affinity for oxygen.

Hatteria; see Sphenodon.

Heloderya (G. helo, a nail head, plus derma, skin), a genus of lizards of the Southwest U. S. and Mexico; the only poisonous lizards known.

HEMI (G.-L. half), a prefix; hemibranch, hemisection.

HeMichordata (G. hemi, plus chorda, a string), a sub-phylum of chordates with a small anterior homologue of the notochord; includes Balanoglossus and other forms.

hepar (G. liver), a combining form; hepatic, heparin.

HERBIVRous (L. herba, grass, plus voro, to devour), pertaining to animals which eat only regetable matter.

HERMAPHRODITE (G. Hermaphroditus, the son of Hermes and Aphrodite) an individual combining both sexes; hermaphroditic animals are rarely self fertilizing.

HETERo ( $G$. different), a prefix; heterodont, heterocercal.

Heterocercal ( $\mathrm{G}$. hetero, plus kerkos, tail), fish with the tail upturned at the posterior extremity, typical of the sharlss.

HetERodont ( $\mathrm{G}$. hetero, plus odous, tooth), having teeth of different shapes; i.e., incisors, canines, premolars, molars.

Holo (G. holos, whole, entire), a prefix, as holoblastic.

HOLOBLASTIC (G. holos, plus blastos, a germ), a type of cleavage in which the entire cell divides; opposed to meroblastic.

HoLocEphali (see Chimera).

Holostei (G. holos, plus osteon, bone), an order of bony fishes including Amia and the gar-pikes, structurally intermediate between Chondrostei and Teleostei. The name is in reference to the ossification of the chondrocranium, and its fusion with the dermal bones.

номо (G. homos, the same), a prefix opposed to hetero. See heterodont and heterocercal.

Howro (L. man), a genus of the higher primates including all recent races of man. The only existing species is H. sapiens. See Neanderthal and Cro-Magnon.

HoMOLOGE (G. homologeo, to agree), a structure which agrees with another in genetic origin. Adjective, homologous. See analogy.

Hormone (G. hormao, to excite), "a chemical messenger" secreted by an organ and influencing another.

HYAL (G. hyalos, glassy), clear or translucent; as hyaline cartilage.

IrYID (G. $y$, plus eidos, form, like the letter upsilon), derived from or pertaining to the hyoid bone-derelopmentally the second visceral arch. 
нур, нуро (G. hypo, under, below), a prefix in hypoglossal, hypophysis, hypo-secretion.

hrpaxial (G. hypo, plus axis), see epaxial.

HTPER (G. over, above), a prefix, hyperpharyngeal, hyper-secretion.

h YPERTRophy (G. hyper, plus trepho, nourish), an orergrowth of an organ or structure. See atrophy.

HYPoMere ( $G$. hypo, plus meros, a part), the rentral part of the primitire mesodermal somite, giring rise to the somatopleure and splanchnopleure. See epimere.

HYPOPHYSIS (G. hypo, plus phyo, to grow), a rentral outpocketing; specifically, the ventral evagination of the diencephalon which meets Rathke's pouch from the stomodeum, the two developing into the pituitary gland.

HrRacoines (G. hyrax, a shrew-mouse), an order of mammals, the "conies", including the type genus Hyrax.

ICHтнY (G. ichthys, a fish), a combining form, as ichthyology, Pterichthys.

IchtHYopsida (G. ichthys, plus opsis, appearance), rertebrates which resemble fish, the fishes and amphibia.

Ichthrosaurs (G. ichthys, plus saurus, lizard), an order of extinct, aquatic reptiles.

IGNEOUS (L: ignis, fire), formed through the action of heat; igneous rocks are consolidated from a molten state. Those which solidify at the surface are volcanic.

ILEUM (G. eilo, to twist), the posterior portion of the small intestine, meeting the large intestine at the ileo-colic juncture.

ILICM (L. flank), a bone of the pelris.

ixcido (L. to cut into), incisure, incisor.

INFCNDiblLu (L. a funnel), the stalk by which the pituitary is attached to the brain.

INGUINAL (L. inguin, groin), pertaining to the groin; the inguinal region, or inguinal canal. The mammalian testicles descend through the inguinal canals to the scrotum.

INTEGLAENT (L. intego, to corer), the skin of a vertebrate and its derivatives.

INTER (L. between), prefix; intercostal, intervertebral.

INTRA (L. within), prefix, intracellular.

INvagivation (L. in, in to, plus vagina, a sheath), an inpocketing or folding in of a structure; as, the invagination of the regetal pole of a blastula to form the gastrula.

Iso (G. isos, equal), a prefix implying equal degree; as isotonic, isogamy. ITER (L. a passage); specifically, the rentricle of the midbrain, which in the mammals is a narrow tube between larger ventricles.

JEJCNEM (L. jejunus, hungry), that portion of the small intestine of the mammal, extending from the duodenum to the ileum.

JoIvт (L. junctus, from jungo, to join), an articulation between two bones, the structure of the joint limiting the motion between the parts.

JUG.sL (L. jugum, a yoke), a bone of the skull assisting in the formation of the zygomatic arch. 
JUGULAR (L. jugulum, the collar bone), the major vein of the neck, draining the head region, and homologous with the anterior cardinal.

Jurassic (from Jura Mts. between France and Switzerland), the middle period of the Mesozoic Era, between the Triassic and Cretaceous.

LABIA (L. labium, a lip), a lip or lip-like structure; the labia of the cerebral hemispheres, orer the corpus callosum; the structures surrounding the mammalian female genitalia-the labia majora and minora, homologous with the scrotum and erectile bodies of the male.

LACERTILIA (L. lacerta, lizard), an order (or sub-order in some classifications) of reptiles including lizards.

LACRIMA (L. a tear), a combining form, denoting tears or structures associated with them; lacrimal glands, lacrimal bone, lacrimal duct.

LACUNA (L. lacus, a basin or lake); specifically, the spaces in cartilage or bone containing the indiridual cells.

LAMPREY (L. lambo, lick, plus petra, rock), See Petromyzontia.

LARYNGEAL (L. larynx, a gullet), adjectival form of larynx; laryngeal cartilages.

LARYNX (L. gullet), the cartilaginous and bony structures surrounding the glottis, containing the rocal cords.

LATissimus (L. broadest), as latissimus dorsi, a muscle of the back.

LATUS (L. side or broad), a stem and combining form; lateral, lateralis, along the side.

LEPIS (G. a scale), combining form; Cheirolepis, Lepidosteus.

Lepidosiren (G. lepis, plus siren, a mermaid), a South American genus of dipnoan fish, the most modified of the lung fishes.

Lepidosteus (G. lepis, plus osteon, bone), a genus of Holostean fish with bony plates arranged in a tile-like manner; the gar-pike and its relatives.

LEvATOR (L. levo, to raise), a muscle which raises a structure; the Levator scapuli; the levators of the jaw; or costal levators.

LINEA (L. a line); in anatomy, usually a connective tissue line separating two muscle groups; linea alba.

Lixgual (L. lingua, tongue), pertaining to the tongue; lingual muscles; lingual glands.

LiThos (G. stone), a combining form; otolith; lithographic limestone.

LUMB.AR (L. lumbus, loin), the body region between the thoracic and sacral regions.

LUNA (L. moon), a combining form; lunatum; semilunar valves.

LUTEA (L. clay-yellow), a descriptive word; macula luteum; corpora lutea.

LYMPH (L. lympha, clear water), blood fluids which have passed through the capillary walls and into the lymphatic vessels; it lacks the erythrocytes, and clots more slowly than blood.

LYMPHOCYTE (L. lympha), leucocytes which develop in the lymph nodes, the smallest of the white cells.

macro (G. makros, long, large), a prefix, opposed to micro; as, macroscopic anatomy.

MALlEUs (L. a hammer), descriptive of the shape in the mammalian ear ossicle; develops from the posterior end of Meckel's cartilage; homologous with the articular bone. 
Malpighian (from Malpighi, an Italian anatomist, 1628-1694); Malpighian (renal) corpuscle.

MAMMA (L. breast), the breast of a mammal; a combining form, mammary glands, mammary artery.

Mammalia (L. mamma), a class of vertebrates with mammary glands and hair.

MANATEE (Spanish manati, a sea cow), a genus of aquatic mammals, Order Sirenia.

MANDIBLE (L. mandibula, jaw), the lower jaw of a vertebrate.

Manis (L. manes, good), a genus of Old World edentates; the scaly ant-eater; a mammal covered with large horny scales.

Marsupialia (L. marsupium), a sub-class and order of mammals which lack a placenta, and have an abdominal pouch in which the immature young remain for some time after birth.

MARSUPIUM (L. a pouch), a combining form, marsipobranch, marsupial.

masseter (G. maseter, a chew), one of the larger muscles of the jaw.

MASTOID (G. mastos, breast, plus eidos, form), a rounded protuberance, as the mastoid process of the skull.

MAXimus (L. greatest).

MEATUS (L. meatus, a passage, from meo, to go), any conspicuous passage, as the external meatus of the ear.

Meckel's CARTilage (from Meckel, a German anatomist, 1781-1833), the cartilage of the lower jaw of the vertebrates; above the elasmobranchs the cartilage is surrounded by dermal bones; its posterior end ossifies as the articular bone. See malleus.

MEDIUS (L. middle); as, median, toward the middle.

MENinges (G. meninx, a membrane); plural of meninx.

MENiNX (G. a membrane), a connective tissue covering of the central nervous system; pia mater, arachnoid, and dura mater in the mammals.

MERo (G. meros, part), a combining form; epimere, hypomere.

MERoBlastic (G. meros, plus blastos, germ), a type of cleavage in which only the protoplasm divides, leaving the yolk mass uncleaved; typical of large yolked eggs.

mes, MEso (G. mesos, middle), a prefix; mesorchium, mesoderm.

MESENTERY (G. mesos, plus enteron, intestine), the band of tissue which suspends the viscera, developing from the hypomere, and continuous with the peritoneum.

mesodery (G. mesos, plus derma, skin), the middle layer of the three primary germ layers, lying between the ectoderm and endoderm.

mesomere (G. mesos, plus meros, a part), the small portion of the mesodermal somite connecting the epimere with the hyomere; equiralent of nephrotome.

mesonephros (G. mesos, plus nephros, kidney), the middle kidney of the vertebrate; it develops posterior to the pronephros, and at a later date; and anterior to the metanephros; the functional kidney of the anamniotes.

MET, meta (G. over, after, on the farther side of), a combining word. See following definitions.

metanephros (G. meta, plus nephros, kidney), the functional kidney of amniotes, partly serially homologous with the more anterior kidneys. See mesonephros. 
IETAPLEURAL (G. meta, plus pleura, side), specifically the folds of tissue along the sides of Amphioxus; metapleural folds.

minimus (G. least), used as a limiting adjective.

MINOR (L. less), descriptive adjective; as pectoralis minor or p. major.

Miocene (G. meion, less, plus kainos, recent), a geological period of Tertiary (Cenozoic) times, between the Oligocene and Pliocene.

Mississippian (from Mississippi Valley where it was first described), a period of late Paleozoic time; the lower strata of the rocks formerly included as the Carboniferous Period.

MITRAL (G. mitra, a turban; a divided bishop's hat), relating to the bicuspid valves in the left auriculo-ventricular opening of the mammalian heart.

MOLAR (L. molaris, belonging to a mill), the posterior, permanent, teeth of a mammal.

Moxo (G. single), a combining form.

Monotremata (G. mono, single, plus tremato, an opening), a sub-class of mammals from Australasia; egg laying mammals with a cloaca, or single opening for the anus and urinogenital outlets.

MORPH (G. morphe, form), a combining word.

MORPHOLOGY (G. morphe), the study of structure or form; more frequently used in the sense of derelopmental anatomy.

Mullerian (from Müller, a German anatomist, 1801-1858), specifically the Müllerian ducts of the mammal embryo which give rise to the uteri; same as oviduct.

Mustelus (L. mustela, weasel), a genus of sharks; the smooth dogfish. MYELON (G. marrow), a combining form referring to marrow or a marrowlike substance; the myelin sheath of neurones; myelocytes.

wyo (G. mys, muscle), a combining form; myology, the study of muscles; myocomma; myotome.

myocomm (G. mys, plus komma, a segment), a connective tissue band separating two myotomes.

ryотоме (G. mys, plus tomes, a cut), a muscle segment or somite.

Mrxine (G. myxa, slime), a genus of myxinoid cyclostomes.

MyxinoideA (G. myxa, plus eidos, like), a sub-class of cyclostomes, in many respects more primitive than the lampreys; the "slimy eels".

IrzoN (G. sucker), a combining form; Petromyzon, the lamprey $\mathrm{Ol}^{\circ}$ "stone sucker".

NARIS (L. nostril), singular of nares; the opening of the air passages, both internal and external.

Neaxderthal (a valley in Prussia), an extinct race of man; $H$. neanderthalensis.

Necturus (G. nelitos, to swim, plus oura, tail), a genus of urodele amphibia with external gills throughout life; a typical form in laboratory dissections.

NEO (G. neos, new), a combining form referring to recent development.

Neopallium (G. neos, plus palli, a mantle or cover), the cover of the cerebral hemispheres which has a cortical region; opposite of archipallium.

Nephros (G. kidney), a combining form; mesonephros; nephric tubule. Nephrostome (G. nephros, plus stoma, mouth), the ciliated opening of a pronephric or mesonephric tubule into the coelomic cavity. 
NEPHRoTome (G. nephros, plus toma, a cut), the anlage of the kidney tubules; the mesomere.

NEURocoel (G. ncuron, a nerve, plus koilos, hollow), the cavity of the nerve cord; homologous, and continuous, with the ventricles of the brain.

NEURox (G. a nerve), a combining form; neurone; neurology.

IIctitating (L. nicititatus, from nicto, to wink), the third eyclid; moving from the anterior (median) margin toward the posterior; usually small in the mammal.

Noто (G. notos, the back), a combining form; notochord.

Notochord (G. notos, plus chorde, a string), the endodermal rod of tissue ventral to the nerve cord of the Chordata; it becomes surrounded (or obliterated) by the mesodermal vertebrae.

NuchA (I. nucha, nape of neck), a combining form in reference to the neck region; nuchal ligament; nuchal flexure of the brain.

oblongata (L. ob, before, plus longus, long), specifically the medulla oblongata.

obTurator (L. obturo, to close), a structure that closes off a passage; specifically, the structures which close the obturator foramen of the pelvis.

occiput (L. back of head); as adjective, the occipital region; or occipital bones, which surround the foramen magnum.

occlo (L. oculus, eye), a combining form; oculomotor; oculist.

oDos (G. odous, tooth), a prefix referring to teeth; as a suffix, don, or dont; odontoblast; homodont: thecodont.

OLFACTORY (L. olfactoitum, a smelling bottle), pertaining to the sense of smell; olfactory nerves; olfactory capsule.

Oligocene (G. oligos, few or small, plus kainos, new), a geological period of Cenozoic time, following the Eocene.

ouestum (L. oment, a fold), an extension of the mesenteries which folds over the viscera.

oxro (G. shoulder), a combining form; omohyoid.

oxтo ( $G$. being), a combining form; ontogeny; ontogenetic.

oxtogesy (G. onto, being, plus genesis, origin), the development of the individual; opposed to phylogeny, the development of the race.

operCULAR (L. opercul, a cover), a protective cover; specifically, the bony covering of the gills in fish above the elasmobranchs.

Opнidia (G. ophis, a serpent), a sub-order (or order) of reptiles, including the snakes.

opнтнALMIC (G. ophthalmos, eye), of or pertaining to the eye.

ORAL (L. os, mouth), pertaining to the mouth.

ORBICUlo (L. orbis, a circle), a circular structure; specifically muscular; orbicularis oris of the mouth.

oRBIT (L. orbis, circle), specifically the eye socket; orbital ridges.

Ordovician (from Ordovices, a Celtic tribe of Wales); a geological period of the early Paleozoic, between the Silurian and Deronian.

OrNithorhyNCHUS (G. ornis, a bird, plus rhis, nose), a genus of monotreme mammals: the "Duckbill Platypus".

oRTHogenesis (G. orthos, straight, plus genesis, beginning), the theory which considers evolution to have progressed in a straight line; rather than as a fluctuating, branching development. 
оRTHOS (G. straight), a combining form; orthogenesis, Orthoptera, orthopedic.

osteo (G. osteon, bone), a combining form; osteoblast; osteoclast; osteology:

osteoblast (G. osteon, plus blastos, a germ), a bone building cell; opposed to osteoclast (G. klao, to break), a bone destroying cell.

ostium (L. ostium, a door), specifically the opening of the oviduct into the body cavity.

Ostracodermi (G. ostrac, shell, plus derma, skin), an extinct order of armored vertebrates; they were formerly classified with the fish, but are now generally considered as being more related to the cyclostomes.

oTIC (G. otikos, ear), pertaining to the ear; otic capsule.

отоцітн (G. otikos, ear, plus lithos, stone), a concretion in the utriculus of the inner ear of some vertebrates, which stimulates impulses by hitting the sensory hairs.

ovARY (L. ovum, egg), the female gonad; the organ in which the reproductive cells develop.

oviduct (L. ovum, egg, plus ductus, leading), the duct receiving the ova and conducting them to the outside or to the uterus.

ovum (L. egg); plural ova; the female reproductive cell.

oxys (G. sharp), a combining form; Amphioxus.

PALATAL (L. palatum, palate), relating to the palate; either hard palate, or soft palate.

Paleocese (G. palaios, ancient, plus kainos, new), the most ancient of the Cenozoic strata; the first geological period following the Mesozoic.

PALliu M (L. palli, a mantle), a covering orer a carity; specifically, the roof of the cerebral hemispheres. See neopallium.

pancreas (G. pas, all, plus kreas, flesh), a digestive gland emptying into the duodenum, with "islands" of endocrine tissue which secrete insulin.

PAPILLA (L. a nipple), any small, nipple-like structure; the papillae of the tongue; or, the mesodermal dental papilla which forms the dentine of the tooth.

PARA (G. para, besides), a combining form.

PARIETAL (L. paries, wall); the parietal bones of the skull; the parietal foramen.

PECTORAL (L. pectoralis, breast), relating to the upper thoracic region; pectoral muscles.

PELVIC (L. pelvis, a basin), pertaining to a pelvis; the bony girdle of the posterior limb; the enlarged part of the ureter attached to the kidney. PENIS (L.) the male copulatory organ, homologous with the clitoris of the female.

Pencspluanian (from Pennsylvania, where first described), one of the later geological periods of Paleozoic times; the upper strata of the Carboniferous. See Mississippian.

P'ERI (G. around), a prefix; peripharyngeal grooves; pericardium.

PERICARDIAL (G. peri, plus kardia, heart), a membranous sac containing fluid surrounding the heart; morphologically a part of the body cavity. 
PERINEUM (G. perineon (?)), the pelvic region including the digestive and urinogenital outlets.

PERIPHeral (G. peri, around, plus phero, to bear), that which lies near the margin, away from the center; the peripheral nerres, in distinction from the central nerrous system.

Perissodactrla (G. perissos, odd, plus daktylos, digit), an order of herbivorous mammals, including the horse, rhinoceros, zebra, etc.

PERITONeAL (G. peri, plus teino, to stretch), the membrane corering the intestines and mesenteries, and lining the body carities.

Permian (from Perm, a prorince of Russia), a geological period including the most recent strata of the Paleozoic Era; it is followed in succession by the Triassic Period of the Mesozoic.

petro (G. petros, rock), a combining form; petrosal, Petromyzon.

Petronyzon (G. petros, plus myzon, sucker), a genus of lampreys. See Petromyzontia.

Petronyzontia (from Petromyzon, the type genus), a sub-class of cyclostomes, including the lampreys.

PHAG (G. to eat), a combining form; that which eats, as phagocyte.

PHARYNGEAL (G. pharynx, throat), the region of the digestive tract between the mouth cavity and the esophagus; the region from which the gill pouches derelop.

PHYLOGENY (G. phylon, tribe, plus genesis, beginning), the history of a race; opposed to ontogeny; phylogenetic is the adjective.

phrLu (G. phylon, tribe), a large group of animals or plants; a major division of a kingdom; the Phylum Chordata.

PHYSOS (G. a bubble, from phyo, to grow), a combining form; epiphysis; hypophysis.

PIA (L. tender), the more delicate membrane corering the brain, carrying blood ressels; pia mater.

PINEAL (L. pinea, a pine cone), an endocrine gland on the dorsal side of the diencephalon. See epiphysis.

PINNA (L. feather), a wing or fin; specifically, the external ear.

Pisces (L. plural of piscis, fish), a class of vertebrates, the fish.

Pithecanthropus (G. pithekos, ape, plus anthropos, man), a genus of extinct, ape-like men; the fossilized remains having been found in Java. P. erectus.

Pituitary (L. pituitarius, from pituita, phlegm), an endocrine gland of the brain, derived partly from the diencephalon and partly from a stomodeal invagination.

PLACENTA (L. a cake, in reference to its discoidal shape), an embryonic organ of the placental mammals, forming the embryonic attachment to the uterine wall.

PLANTigRade (L. planta, sole of foot, plus gradior, to walk), referring to animals which walk with the sole of the foot on the ground; humans or bears.

Pleistocese (G. pleistos, much, plus kainos, new), the most recent geological period, the uppermost strata of the Cenozoic.

Plesiosauria (G. pleistos, much, plus sauros, lizard), an order of extinct, aquatic reptiles.

PLEURA (G. side), a combining form; metapleural; pleural cavities.

PLEXUS (L. interweaving), a group of interlacing or anastomosing nerves or blood vessels. 
Pliocene (G. pleion, more, plus kainos, new), a Cenozoic geological period above the Miocene; the Quarternary (Pleistocene) follows it.

PNEUMA (G. air), a combining form, referring to breathing in anatomy;

Dipnoi, pneumogastric.

PODA (G. pous, foot), a combining form; Apoda, Tetrapoda.

POLY (G. polys, many), a prefix; polydactyl; polyembryony.

Polypterus (G. polys, plus pteron, fin or wing), a genus of recent crossopterygian fish found in Africa.

PoNs (L. a bridge), specifically the pons Varolii, a large commissure of the brain.

PORTAL (L. porta, a gate), veins which break into capillaries before entering the heart; renal portal; hepatic portal.

POST (L. after, behind), a prefix; posterior; post cardinal.

PRE (L. before), prefix; precava; premolar.

PREPUCE (L. praeputium, from pre, plus G. posthion, penis) the foreskin of the penis covering the glans; specifically that of the primates.

Primates (L. primus, first), an order of mammals including the lemurs, monkeys, anthropoid apes and man.

PRo (L. before, in front of), a prefix; procoelous, pronate.

Proboscidea (G. pro, before, plus bosko, to feed), an order of mammals with a proboscis or prolonged snout, including the African and Indian elephants.

PRoctodeum (G. proktos, anus, plus daio, to divide), the region of the anus lined by invaginated ectoderm.

PRoNephros (G. pro, plus nephros, kidney), the first embryonic kidney, and the most anterior one; it disappears in the adults of all vertebrates except the cyclostomes.

prostate (G. prostates, one in the front rank), a seminal gland of the higher vertebrates (specifically the mammal) located near the exit of the urethra from the bladder; the homologue of the Müllerian ducts.

Proterozorc (G. protos, first, plus zoon, animal), a Pre-cambrian Era, later than the Archeozoic, covering an enormous length of time.

Pterodactyla (G. pteron, wing, plus daktylos, finger), a group of extinct flying reptiles with membranes stretched between the fingers.

pteron (G. wing or fin), a combining form; pterygoid, Polypterus.

PTERYGo-QUADRATE (G. pteron, plus L. quadratus, square), the cartilage of the upper jaw in rertebrates; its posterior end ossifies as the quadrate bone; homologue of the incus.

PUBIC (L. pubes, grown up), the lower hypogastric region, covered with hair at maturity; pubic symphysis.

PULMONARY (L. pulmon, lung), relating to the lungs; pulmonary arteries or veins.

PYLORUS (G. pyloros, gate keeper), the rentral portion of the stomach; pyloric sphincter, the constrictor muscle between the stomach and duodenum.

QUADRATE (L. quadratus, square), the bone of the upper jaw articulating with the articular of the lower jaw. See pterygo-quadrate.

RADIAL (L. radius, a ray), pertaining to a ray, radial cartilage; radius, a bone of the forearm on the pre-axial side. 
RAMUS (L. branch), a branch or outgrowth; as, the ramus of the mandible; the aortic rami of the amphibia and birds; the ramus communicans of the peripheral nerves.

RAPHE (G. rhaphe, a seam), a seam-like appearance of an organ; the median line of the body; the dividing line of the scrotum.

R.atit.Ae (L. ratitus, like a raft), a sub-class of birds lacking a carina on the sternum; the non-flying birds including the ostrich, emu, kiwi, etc.

RECTUM (L. rectus, straight), the lower, enlarged portion of the intestine. RENAL (L. renes, kidneys), pertaining to the kidneys; renal corpuscles; renal portal.

Reptilis (L. reptilis, from repo, to creep), a class of vertebrates, covered with ectodermal scales, including turtles, lizards, snakes, dinosaurs, etc.

RESPIRATORY (L. re, back, plus spero. to breathe). relating to breathing; obtaining oxygen from the surrounding medium.

RETINA (L. rete, a net), the cell layer of the eye containing the receptors of light impulses.

RETRO (L. backward), that which is back or behind another structure; retro-peritoneal.

RHINAL (G. rhis, nose), relating to the nose or snout; rhinencephalon; rhinocoel; rhinitis.

Rhyschocephalia (G. rhynchos, snout, plus kephale, head), an order of reptiles from New Zealand; the most primitive living reptiles.

Rodextia (L. rodo, to gnaw), an order of mammals with two gnawing incisors in each jaw; includes rats, beavers, capybara, etc. Rabbits are usually included in a separate order (Lagomorpha) as they have four incisors and other divergent characters.

Rostrum (L. beak), a projecting snout, or any projecting process; specifically, the cartilage supporting the snout of the dogfish.

RUMINANTS (L. rumen, throat), an artificial grouping of mammals, including the herbivorous land mammals which chew a cud.

sacculus (L. diminutive of saccus, a sac), a small, pouch-like structure; the sacculus of the ear.

SACRUM (L. sacrum, from sacer, sacred; the part offered in sacrifice), the lower end of the rertebral column, attached to the pelvic girdle; sacral, pertaining to the sacrum.

SAgitTAL (L. sagitta, an arrow), a median plane along the antero-posterior axis; a longitudinal section may be to either side of the median line.

SAlamaxdriva (G. salamandra, salamander), a group of urodele amphibia, including the salamanders which lose their gills in the adult stage, and are generally land liring.

SALIVARY (L. saliva, spit), pertaining to the glands which secrete saliva. SAURopsida (G. sauros, lizard, plus opsis, appearance), a group term which includes the reptiles and birds.

SACRe's (G. sauros, a lizard), a combining form, referring to lizard, or lizard-like; Ichthyosaurs; Dinosaurs, etc.

SCLERA (G. skleros, hard), a combining form, implying that which is hard, or which gives rise to hard structures; sclerotic, sclerotome.

sclerotome (G. skleros, hard, plus toma, a cut), that part of the epimere which gives rise to the vertebral column and connective tissues. 
SCRotum (L. scrotum, pouch), the pouch in which the mammalian testes lie; the scrotum is homologous with the labia majora of the female. SEDIMENTARY (L. sedimentum, settling), in geology, referring to the rocks which have been deposited in water or land depressions. The original materials may be carbonates, mud, sand, or gravel which are cemented or pressed into rock formations. They are the fossil bearing rocks.

Selachir (G. selachos, a shark), a group of elasmobranch fish, including the sharks.

SELLA (L. a seat or saddle), sella turcica, the depression of the skull in which the pituitary rests.

SEMI (L. half), combining form; semilunar, semipermeable, semispinalis.

SEMILUNAR (L. semi, plus luna, moon), halfmoon-shaped; specifically the valves guarding the orifices of the arteries learing the heart.

SEMINAL (L. semen, seed), pertaining to the fluid medium of spermatozoa, secreted by the testicular tubules and the glands associated with the ducts; semen is the fluid.

SEPTUM (L. sepes, a fence), a dividing partition; the septum between the auricles; myosepta.

Silurian (from Silures, a tribe in England), a geological period of the Paleozoic, above the Ordovician, and below the Devonian.

Sinanthropus (G. Sinai, Chinese, plus anthropos, man), a genus of extinct men, a skull of which was discovered near Peiping.

sinus ( $L$. a bend or curve), in anatomy, a carity in a bone or an enlargement of a blood ressel; the sinuses of the head and face are cavities in the bones, as the frontal or maxillary sinuses; also, lymphatic sinuses; sinus venosus.

Sirenia (G. seiren, a mermaid), an order of herbirorous aquatic mammals, including the dugong and manatee.

soma (G. body), a combining form; somatic, somatopleure.

somatopleure (G. soma, plus pleura, side), the layer of the hypomere lying next the ectoderm; it gires rise to the somatic peritoneum and a part of the mesenteries.

somite (G. soma, body), a body segment; a metamere.

Sprevodon (G. sphen, a wedge, plus odous, tooth), a genus and only living representative of the order Rhynchocephalia, the most primitive recent reptile.

SPHENOID (G. sphen, a wedge, plus eidos, form), a wedğe-shaped bone or process; specifically, the sphenoid bones of the skull, developing from the chondrocranium.

SPIRACLE (L. spiraculum, an air hole), the modified first gill slit of the sharks; the structure was carried orer to the Crossopterygii, and to the tetrapods as the middle ear and Eustachian tube.

SPLANCH (G. splanchnon, viscera), a combining form; splanchnic, splanchnopleure.

SPLANCH NOPLEURE (G. splanchnon, plus pleura, side), the layer of the hypomere in contact with the endoderm; it encloses the archenteron and gives rise to the smooth muscle, mesentery, and visceral peritoneum. The anterior part develops the heart and branchial (visceral) muscle.

Squalus (L. fish), a genus of small sharks; Squalus acanthias is the spiny dogfish. 
SQUAMA (L. a scale), a combining form; squamous epithelium.

Squanata (L. squama), an order of reptiles including the lizards and snakes; it is sometimes divided into Lacertilia and Ophidia.

STAPES (L. a stirrup), one of the ear ossicles; the name is descriptive of the shape in the mammal. The stapes is homologous with the columella, and originally the hyomandibular.

stego (G. cover), a combining form; Stegosaur, covered with plates.

Stegocephalia (G. stego, plus kephale, head), an extinct order of amphibia; the group from which the more recent ones developed.

stervo ( $G$. breast), referring to the breast. The sternum is the unpaired bone in the median ventral line to which the ribs are attached.

stoma (G. mouth), a combining form; stomodeum, nephrostome.

stomodeum (G. stoma, plus daio, divide), the ectoderm-lined region of the mouth carity.

STRATUM (L. spread out, from sterno, to spread). In anatomy, a layer or sheet of tissue, as stratum Malpighii. In geology, a layer or sheet of sedimentary rock. Stratified refers to a series of layers, one above the other.

STRIA (L. a furrow), used alone or as a combining form; corpora striata; striated muscle.

sub (L. under), a prefix implying under the following stem; subintestinal, subclavian.

sulces (L. sulco, to plow), a groove or furrow of the brain.

SUPER (L. over), a prefix; superior.

SUPRA (L. above), a prefix; supraorbital; supraoccipital.

sYM, sYN (G. together), a prefix; synotic, sympathetic; syncytium.

symphysis (G. sym, plus phyo, to grow), a union between two parts; pubic symphysis.

syхотіс (G. syn, plus otikos, ear), the cartilaginous connection between the otic capsules in the chondrocranium, bounding the foramen magnum.

syrivx (G. pipe), the voice box of the birds, located at the bifurcation of the trachea.

TELEO (G. teleos, whole), a prefix; Teleostei.

TEMPORAL (L. tempus, temple), the region of the skull dorsal and posterior to the eye.

TENDON (L. tendo, to stretch), a connective tissue band attaching a muscle.

TENTORIUN (L. tentori, spread like a tent), a partition of the dura mater pushing between the cerebellum and the cerebral hemispheres.

TEstes (L. testis), the male reproductive glands; the testis is the gland; the testicle is the gland with its accessory structures.

THECODONT (G. theke, a case, plus odous, tooth); referring to teeth which are embedded in sockets.

Therapsida (G. theros, a wild beast, plus apsis, an arch), an order of extinct mammal-like reptiles with heterodont dentition.

Theromorpha (G. theros, beast, plus morphos, form), an order of synapsid reptiles, less mammal-like than the Therapsida.

THORAX (L.), the anterior part of the body enclosed by the ribs, a term of mammalian anatomy. 
THYMUs (G. thymon, thyme), a gland of lymphoid tissue, arising as proliferations of cells from the gill pouches, and migrating posteriorly (in the mammal).

тHYRoId (G. thyreos, a shield), an endocrine gland arising as a median evagination from the floor of the pharynx; homologous with the endostyle.

томн (G. a section), a suffix, meaning a cut or segment; sclerotome; myotome.

TRABECULA (L. a small beam), a supporting band of connective tissue; also, the finger-like cartilaginous processes during the development of bone.

TRACHEA (G. trachys, rough), the windpipe from the glottis to the bifureation.

TREMA (G. an opening), a combining form; monotreme, post trematic, pretrematic.

TRI (L. three), a prefix; trituberculate, tricuspid.

Triassic (G. trias, from treis, three), a geological period of the Mesozoic, the lowest strata, followed by the Jurassic and Cretaceous.

TUBER (L. a swelling), a rounded swelling or prominence; tuber cinerium.

TUNIC (L. tunica, a tunic), a covering or investing sheet, the tunic of the testis; also as a combining form, Tunicata.

Tunicata (L. tunica), a sub-phylum of chordates, the Urochordata. The tunicates have a notochord in the tail region during embryonic life, then become sessile and develop a tunic.

Turcica (L. Turcus, Turk), in sella turcica, the Turk's saddle; see sella.

TYMPANIC (G. tympanon, a drum), a drum-like membrane; specifically, the ear drum, or tympanum.

ULNA (L. ulna, elbow), the post-axial bone of the forearm, against which fits the humerus; its proximal end is the olecranon process.

UMBILICAL (L. umbilicus, navel), pertaining to the umbilicus; the umbilical cord connects the embryo with the placenta.

UxGUlatA (L. ungula, a hoof), an artificial grouping of mammals, including those which walk on the tips of their toes (the orders Artiodactyla and Perissodactyla).

UNGULigrade (L. ungula, a hoof, plus gradior, to walk), a foot position, with the sole of the foot entirely off the ground, and standing on the tips of the toes.

UNI (L. one), a prefix; unilateral, uniform.

URA (G. oura, tail), a combining form; Urodela, Anura.

uRETER (G. ouron, urine), the metanephric duct leading from the kidney to the urinary bladder.

URETHRA ( $\mathrm{G}$. ouron, urine), the duct leading from the bladder to the outside; in the male it is joined by the vasa deferentia.

URINogenital (L. unina, urine, plis genitalis, genital), the organs associated with the excretory and reproductive functions.

Urodel. (G. oura, plus delos, evident), an order of amphibia with a well developed tail, including salamanders, newts, and the gilled amphibia.

Uterus ( $\mathrm{L}$. the womb), a mammalian structure homologous with the oviducts, in which the embryos develop. 
UTRICUlus ( $\mathrm{L}$. a little bag), the cavity of the inner ear, from which the semicircular canals arise.

vagina (L. a sheath), in mammalian anatomy the outer part of the reproductive canal; it is homologous with the ends of the oviducts; it is connected with the uterus through the cervical opening. raGus ( $\mathrm{L}$. wandering), the tenth cranial nerre.

ras (L. a duct), specifically the ducts connected with the testes; the rasa efferentia form the epididymis; the paired vasa deferentia conduct the sperms to the urethra.

VENA (L. a rein), as rena cara; renous; sinus venosus.

VENTRICLE (L. diminutive of venter, belly); specifically the cavities of the brain; or the muscular chambers of the heart.

VERMIS (L. a worm), as the vermis of the cerebellum; vermiform appendix.

TERTEBRa (L. a joint), the single structural units of the vertebral column. vilLI (L. villus, shaggy hair), the hair-like processes lining the intestine.

VISCERA (L. viscus, an internal organ), the internal organs, including the digestive tract and the glands.

vitelliNe ( $\mathrm{L}$. vitellus, yolk), specifically the paired embryonic veins which form the heart.

vitreous (L. glassy), in vitreous humor of the eye.

Wolffian (from Wolff, a German anatomist, 1733-1794), the mesonephric ducts of the amniotes from which develop the vasa deferentia. Also, the mesonephros, or Wolffian body.

zoox (G. animal), a combining form; spermatozoa; zoology; Cenozoic. ZYG, ZYGo (G. zygon, yoke), combined in zygomatic arch, zygote, etc. ZYGOMATIC ARCH (G. zygon), the cheek bone of the mammals and mammal-like reptiles; homologous with the ventral margin of the skull, and formed by the enlargement of the lateral fenestra of the skull. 



\section{INDEX}

Abducens nerve, 296

Acetabulum, 164

Adaptive Radiations, Def., 7

Adrenal glands, 318

Age of Reptiles, 80

Albinism, 116

Allantois, 56, 67, 68, 92, 228

Alligator, 61

Dermal plates, 112

Skull, 135

Vascular system, 234

Amblystoma, 49, 52

Amnion, 56, 67, 345

Amphibia, 48

Cleavage, 345

Dermal plates, 110

Evolution, 344

Glands, 111

Muscles, 187

Nares, 132

Ribs, 155

Skull, 133

Vascular system, 235

Vertebrae, 149

Amphioxus, $14 \mathrm{ff}$.

Embryology, 21

Muscles, 181

Skin, 107

Spinal nerves, 277

Vascular system, 221

Analogy, Def., 7

Anapsida, Skull, 134

Annelid Theory, 341

Anthropoidea, 82

Anthropoids, Evolution, 352

Antilocapra, 121

Antlers, 121

Anura, 52

Aorta, Branches of, 38

Aortic Arches, 232

Apoda, 50

Dermal plates, 110
Aponeurosis, 178

Appendages

Aquatic, 166

Bipedal, 168

Cursorial, 168

Flying, 169

Homology, 165

Origin, 156

Appendicular Skeleton 125

Appendix, vermiform, 202, 204

Apteryx, 72

Aquatic adaptation, 166

Arachnid Theory, 342

Arboreal adaptation, 169

Archaeopteryx, 71

Evolution, 347

Wing, 170

Archegosaurus, 50

Skull, 133

Archenteron

Amphioxus, 22

Frog, 53

Reptile, 66

Archipallium, 288

Armadillo, 87

Artiodactyla, 87

Atrium, Amphioxus, 27

Auditory nerve, 297

Auditory organs, 309

Aves, 71

Axial skeleton, 125

Balanoglossus, 11

Barriers, geographical, 326

Bats, 86

Wing, 170

Bifocal vision, 308

Bilateral symmetry, Def., 5

Bipedal adaptations, 168

Birds, 71

Embryology, 75

Integument, 114 
Bladder, urinary, 257

Blastopore

Amphioxus, 23

Frog, 53

Reptile, 66

Blastula

Amniote, 64

Amphioxus, 22

Frog, 53

Reptile, 64

Blood, 103

Islands, 226

Structure, 246

Body stalk, 67

Bone

Cells, 172

Malformations, 173

Structure, 173

Tissue, 102

Types, 171

Bowman's capsule, 253

Brain

Comparative Anatomy, 285

Development, 272

Flexures, 273

Branchial arches, 143, 147

Derivatives, 232

Dogfish, 223

Branchial arteries, 221, $232 \mathrm{ff}$.

Evolution, 232 ff.

Branchial skeleton, 206, $216 \mathrm{ff}$.

Breathing, 215

Bron hioles, 214

Buffon, 323

Caecum, digestive, 202

Calvarium, 134

Carina, 72

Carinates, 72

Carnivora, 84, 85

Cartilage, 101

Cauda equina, 279

Cephalization. 5, 278

Cephalochordata, 15

Cerebellum, 292

Cerebral Hemispheres, 288

Cetacea, 90

Appendages, 167

Cheiroptera, 86

Chelonia, 57

Chevron bones, 150
Chondrocranium

Cyclostome, 30

Development, 125

Ossification, 128

Chondrostei, 40, 344

Dermal plates, 110

Skull, 128, 129

Chordata, Def., 3

Classification, 8

Choroid layer, 307

Cladoselache, 37,343

Appendages, 156

Pelvic fin, 163

Classification, 10

Clavicle, 163

Claws

Mammal, 115

Reptile, 113

Clitoris, 262

Cloaca, 76, 194, 203

Cochlea, 311

Coelomic cavity Amphioxus, 24

Vertebrate, 195

Colon, 202

Commissures, 293

Connective tissues, 100

Coracoid, 158, 161

Cornea, 306

Corneum, stratum, 108

Corpora quadrigemina, 291

Corpus callosum, 293

Corpus striatum, 288, 290

Cranial nerves, 294

Cretinism, 317

Cribriform plate, 138

Crocodilia, 60, 61, 135

Cro-Magnon Man, 354

Crossopterygii, 44, 344, 345

Dermal plates, 110

Fin, 159

Gills, 210

Larva, 161

Ribs, 154

Skull, 48

Cryptobranchus, 50, 51

Cursorial adaptations, 168

Cyclostomes, 29

Brain, 285

Chondrocranium, 126

Glands, 111, 112

Intestine, 201 
Cyclostomes-Continued

Muscles, 181

Spinal nerves, 277

Urinogenital system, 258, 259

Vertebrae, 148

Darwin, Charles, 323

Decussation, 293

Degenerate, Def., 7

Dental formula, 140

Dermal bone, 103, 110 of skull, 131

Dermal denticles. 35, 108

Dermal plates, $109 \mathrm{ff}$.

Ostracodermi, 33

Teleostomi, 39

Dermis, 107

Development, regulators, 313

Diaphragm, 216

Diapsida, 60

Diencephalon, 290

Digestive glands, 318

Development, 194

Digestive system

Amphioxus, 16

Development, 193

Human, 203

Digestive tract, modifications of, 196

Digitigrade, 168, 169

Dipnoi, 45, 344

Gills, 210

Dogfish

Brain, 286

Chondrocranium, 126

Dermal denticles, 108, 109

Digestive tract, 198, 201

Fin, 159

Gills, 209

Muscles, 183

Urinogenital system, 258

Vascular system, 222

Vertebrae, 148

Dura mater, 274

Ear, external, 312

Ear, inner, 309

Cyclostome, 32

Ear, middle, 144, 311

Ear ossicles, 145 ff., 311,349

Homologies, 147

Echidna, 77, 78

Edentates, S6
Elasmobranchii, 36

Elephant, 89

Tusks, 143

Embryology

Amphioxus, 21

Bird, 75

Cyclostome, 32

Frog, 52

Marsupial, 79

Placental, 92

Reptile, 64

Enamel, 108

End organs, 301

Endocrine glands, 316, 320

Endolymph, 309

Endostyle

Amphioxus, 16

Tunicate, 13

Enterocoels, 24

Eogyrinus, 345

Epaxial muscles, 175

Epidermis, 107

Epididymis, 262

Epimere

Amphioxus, 24

Development, 175

Epiphysis

Bone, 172

Brain, 290

Epithelial tissues, 99

Erythrocytes, 247

Esophagus

Development, 193

Structure, 199

Eustachian tube, 145, 206, 207, 311

Eutheria, 80

Excretory organs, 251

Extensors, 181

External gills

Crossopterygii, 44, 45

Dipnoi, 45,46

Tadpole, $5 j$

Eye, 304

Cavities, 308

Embryology, 305

Function, 308

Lids, 309

Muscles, 296

Structure, 306

Facial nerve, 297

Feathers, 114 
Fenestra, of skull, 135

Filum terminale, 279

Finfold theory, 156

Fins, origin, 156

Fishes, 35

Flexors, 181

Flexures of brain, 273

Flying adaptations, 169

Fontanelles, 138

Fossils

Microscopic anatomy, 334

Preservation, 332

Friction ridges, 123

Frog embryology, 52

Gastrula

Amniote, 64

Amphioxus, 22

Frog, 53

Placental, 92

Generalized, Def., 6

Genitalia

Development, 268

Female, 262

Male, 264

Geological Periods, 336

Geological time table, 335

Gila monster, 61

Gill pouches, derivatives of, 207

Gill slits, 4

Amphioxus, 17

Origin, 205

Primary, 28

Reptile, 56

Gills

External, 210

Internal, 207

Septum, 208

Glands

Digestive, 194

Endocrine, 316 ff.

Genital, 268

Mammalian, 118

Salivary, 199

Glands, types, 111, 112

Glans, 264

Glomerulus, 251

Glossopharyngeal nerve, 298

Gonads, 251

Function, 319

Gubernaculum, 267
Hair

Distribution, 123

Structure, 116

Hard palate, 132, 303

Haversian system, 102, 173

Heart

Cardiac cycle, 236

Comparative Anatomy, 229

Dogfish, 222

Embryology, 232

Valves, 229

Hemichordata, 11, 343

Heterocercal tail, 39, 40, 42

Heterodont dentition, 139

Holoblastic cleavage, 64

Holocephali, 37

Holostei, 40, 344

Dermal bone, 110

Hominidae, 84

Homocercal tail, 42

Homodont dentition, 139

Homology, Def., 7

Hoofs, 115

Horns, 120

Human skull, 137, 138

Hyomandibular, 128

Hypaxial muscle, 175

Hypoglossal nerve, 299

Hypomere, 24, 175, 195

Ichthyosaurs, 166,347

Igneous rocks, 330

Infundibulum, 291

Inguinal canal, 267

Insectivora, 81

Intestines, 201

Jaws

Articulation, 146

Elasmobranch, 128

Evolution, 131

Origin of, 144

Kangaroo, 79

Kidney, 256

Lacertilia, 61

Lacteals, 244

Lamarck, 323

Land bridge, 327

Lanugo, 124 
Larynx, 213, 216

Lateral Line Organs, 301

Lemuroidea, 82

Leucocytes, 246

Limb buds, 156

Liver, 194, 195

Lizards, 61

Lungs

Anatomy, 213

Development, 215

Erolution, 211

Lymph Hearts, 244

Lymph nodes, 245

Lymphatic system, 243

Development, 245

Malpighian corpuscle, 255

Malpighian layer, 107

Mammal-like reptiles, 59

Skull, 135

Teeth, 139

Mammals, 76

Embryology, 92

Erolution, 348, 350

Mammary glands, 118

Man, Erolution, 351

Manis, 87, 348

Integument, 115

Marsupials, 77

Embryology, 79

Radiations, 328

Meckel's cartilage, 128

Medulla, 293

Meninges, 274

Meroblastic cleavage, 64

Mesenteries, 195

Mesoderm, Frog development, 55

Mesonephros, 253

Metamerism, Def., 5

Metanephros, 255

Metatheria, 77

Metencephalon, 292

Midbrain, 291

Middle ear, 48, 144, 311

Monkeys, 81, 82, 83

Monotremes, 76, 78

Mammary glands, 120

Pectoral girdle, 162

Mouth

Development, 193

Structure, 198

Müllerian Ducts, 258
Muscles

Abdominal, $184 \mathrm{ff}$.

Adductor magnus, 190

Appendicular, 186

Attachment of, 177

Axial, 183

Biceps, 187

Branchiomeric, 185

Caudal, 183

Classification, 175

Costal, 184

Deltoid, 187

Development, 175

Epaxial, 175

Extensors, 181

Extrinsic, 186

Eye, 296

Flexors, 181

Gastrocnemius, 190

Gluteus, 190

Gross structure, 176

Hypaxial, 175

Ilio-psoas, 184, 190

Integumentary, 190

Intrinsic, 186

Latissimus dorsi, 187

Leverage, 180

Longissimus dorsi, 183

Metameric arrangement, 182

Mimetic, 191

Multifidus, 183

Nomenclature, 176

Panniculus carnosus, 191

Pectcralis, 187

Pelric, 188

Platysma, 191

Rectus abdominis, 185

Rectus femoris, 190

Sacro-spinalis, 183

Sphincter colli, 191

Trapezius, 187

Triceps, 188

Muscle tissues, 103

Myelencephalon, 293

Myotomes, 17, 175, 182

Myxinoidei, 32

Nails, 115

Nares, 303

Naso-pituitary sac, 30, 32, 303

Neanderthal man, 137, 353

Necturus, muscles, 187 
Neopallium, 288

Nephridia, 250

Nephrotome, 175, 251

Nerve cells, 275

Nerve tissues, 104

Nerve tracts, 281

Nerrous system

Brain, 285

Development, 271

Sympathetic, 282

Neural groove, 271

Amphioxus, 22

Frog, 53

Reptile, 66

Neurocoel, 274

Neurone, 275

Notochord, Def., 4

Oculomotor nerve, 296

Olfactory nerve, 295

Olfactory organs, 302, 304

Omentum, 197

Operculum, 133, 208

Ophidia, 63

Opossum skull, 131

Optic capsule, 126, 127, 306

Optic chiasma, 296, 308

Optic lobes, 291

Optic nerve, 295

Optic vesicle, 305

Organ formation, 105

Organizators, 314

Origin of vertebrates, 340

Ornithorhynchus, 77, 78

Osteoblasts, 172

Osteoclasts, 172

Ostracodermi, 30, 32

Ostrich, 72

Ovary, 260, 319

Oriparous, 37, 61

Oro-viviparous, $37,61,260$

Owen, 299

Paedogenesis, 52

Palaeospondylus, 30, 148, 337

Pallium, 288

Pancreas, 194, 318

Parapsida, 60

Parathyroid glands, 207, 317

Parietal eye, 290

Pectoral girdle, 158

Pelvic girdle, 163, 164
Penis, 262, 264

Perameles, 95

Pericardium, 229

Periosteum, 172

Perissodactyla, 89

Peritoneum, 195

Petrifaction, 333

Petromyzon, 31, 32

Pharynx, structure, 198

Pia mater, 274

Pigment, 115

Piltdown man, 353

Pineal gland, 291, 316

Pithecanthropus, 137, 352

Pituitary gland, 139, 291, 316

Placenta, 80, 93

Placentals, embryology, 80, 92

Plantigrade, 169

Polypterus, 44

Porpoise, 90

Primates, 81

Primitive, Def., 6

Proboscidea, 89

Pronephros, 251

Prototheria, 76

Pterodactyl, wing, 170

Pterygo-quadrate, 128

Pyloric sphincter, 201

Ramus communicans, 283

Ratites, 72

Rattlesnake, 62, 63, 122

Rays, 37, 38

Receptors, 300

Rectum, 202

Renal portal vein, 240

Replacement bone, 110

Reptiles

$$
\begin{aligned}
& \text { Age of, } 347 \\
& \text { Embryology, } 64 \\
& \text { Evolution, } 346
\end{aligned}
$$

Reptilia, 56

Respiration, Def., 205

Retina, 306, 307

Rhinoceros, 89, 122

Rhynchocephalia, 60

Ribs, 154

Rodentia, 85

Sacculus, 310

Salivary glands, 199

Sauripterus, 159, 345 
Scales

Dermal, 110

Mammal, 115

Reptile, 113

Scapula, 158, 163

Sclerotic layer, 308

Scrotum, 267

Sebaceous glands, 117, 118, 330

Sedimentary Rocks, 330

Segmentation cavity, 21

Sella turcica, 138

Semen, 267

Semicircular canals, 309

Sex differentiation, 268

Sex glands, 319

Seymouria, 58, 134, 316

Sinanthropus, 353

Sirenia, 90

Skates, 37, 38

Skeleton, development, 171

Skin, 107

Human, 122

Skull

Dermal bones, 131

Development, 125

Evolution, 134

Fenestrae, 135

Reptile, 346

Snakes, 62, 63

Somatopleure, 195

Specialized, Def., 6

Sphenodon, 61

Spinal accessory nerve, 298

Spinal cord, structure, 277

Spinal nerves, 277

Spiracle, 39, 144

Spiral valve, 39, 201

Splanchnopleure, 195

Spleen, 195

Stegocephalia, 50, 345

Dermal plates, 110

Pelvis, 161

Skull, 134

Vertebrae, 149

Sternum, 154

Stomach

Development, 193

Modifications, 199

Strata, formation, 331

Sweat glands, 118, 123

Swim bladder, 40, 45, 211

Sylvius, aqueduct of, 292
Sympathetic system, 287

Synapsida, 60

synotic tectum, 126

Systematic, Def., 97

Systemic, Def., 97

Taste buds, 302

Teeth, 139 ff.

Teleostei, 42, 344

Teleostomi, 39

Tendons, 17s

Terminal nerve, 295

Testes

Descent, 267

Function, 319

Therapsida, 59

Jaws, 131

Skull, 136

Theromorpha, 59, 60

Jaws, 131

Skull, 136

Thinopus, 160, 161

Thorax, 155

Thymus, 207, 317

Thyroid, 33, 207, 316

Time table, geological, 33 Ј

Tongue, 199

Trigeminal nerve, 297

Trochlear nerve, 296

Turbinal bones, 303

Tunicates, 13

Turtles, 57

Skull, 134

Twinning, 314

Unguligrade, 169

Ureter, 255

Urethra, 257

Urinogenital system, development, 258

Urochordata, 13, 343

Urodela, 50

Uterus, 262

Utriculus, 310

Vagina, 262

Vagus nerve, 298

Valves, of heart, 231

Vascular system

Amphioxus, 19

Developinent, 229

Dogfish, 222 
Vascular system-Continued

Embryonic, 226

Heart, 229

Human, 242

Lymphatics, 243

Ventral aorta, 221

Vas deferens, 262

Veins

Hepatic portal, 240

Renal portal, 240

Systemic, 239

Vitelline, 226

Ventricles, brain, 274

Vertebrae, 148 ff., 350
Vertebral ganglia, 283

Visceral skeleton, 143

Vitelline veins, 226

Viviparous, 37

Vocal cords, 218

Wallace, 323

Line, 328

Whales, 90

Appendages, 167

Teeth, 122

Wolffian body, 255

Zygomatic arch, 136 













